\title{
Microscopic Origin of the 0.7-Anomaly in Quantum Point Contacts
}

Florian Bauer

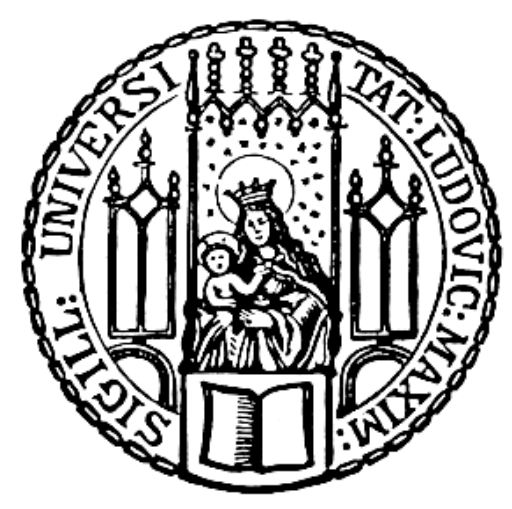

München 2014 



\section{Microscopic Origin of the 0.7-Anomaly in Quantum Point Contacts}

Florian Bauer

Doktorarbeit

an der Fakultät für Physik

der Ludwig-Maximilians-Universität

München

vorgelegt von

Florian Bauer

aus Ulm

München, den 8.7.2014 
Erstgutachter: Professor Jan von Delft

Zweitgutachter: Privatdozent Dr. Stefan Ludwig

Tag der mündlichen Prüfung: 29.07.2014 


\section{Contents}

Contents $\quad$ v

Zusammenfassung in deutscher Sprache $\quad$ ix

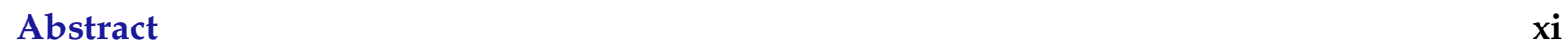

1 Introduction 1

2 Quantum Point Contacts and the 0.7 Anomaly 5

2.1 Heterostructures and the Split Gate Geometry . . . . . . . . . . . . . . . . 5

2.2 The noninteracting $\mathrm{QPC} \ldots \ldots \ldots \ldots \ldots \ldots$

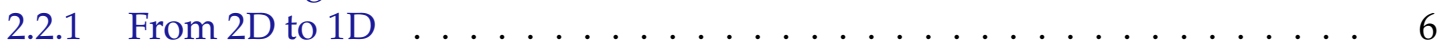

2.2 .2 Conductance Quantization . . . . . . . . . . . . . . 8

2.2.3 Concrete form of the 1D Potential . . . . . . . . . . . . . . 9

2.3 The Role of Interaction . . . . . . . . . . . . . . . . . . . . . . . . 11

2.3.1 Effective Interaction strength . . . . . . . . . . . . . . . . . . . 11

2.3.2 Effective one-dimensional Interaction . . . . . . . . . . . . . . . . 12

2.3 .3 Discrete model with Interactions . . . . . . . . . . . . . . . . . . 13

2.4 The origin of the 0.7 -anomaly $\ldots \ldots \ldots \ldots \ldots \ldots \ldots \ldots$

2.4 .1 Related Models . . . . . . . . . . . . . . . . . . . . . . 15

2.5 Experimental features of the 0.7 -anomaly $\ldots \ldots \ldots \ldots \ldots$

2.5 .1 Temperature Dependence . . . . . . . . . . . . . . . . . . . 17

2.5.2 Dependence on Magnetic Field . . . . . . . . . . . . . . . . . . . . . 18

2.5.3 Finite Source-Drain Voltage . . . . . . . . . . . . . . . . . . . . 18

2.5 .4 Low Energy Scale . . . . . . . . . . . . . . . . . . . . . . . . . 21

2.5 .5 Shot Noise . . . . . . . . . . . . . . . . . . . . 22

2.6 Other Theoretical Models . . . . . . . . . . . . . . . . . . . . . . 22

2.6 .1 Spin Polarization . . . . . . . . . . . . . . . . . . . 22

2.6.2 Kondo Model - Formation of a localized State . . . . . . . . . . . . . . . 25

3 Functional Renormalization Group $\quad 27$

3.1 Green's and Vertex Functions . . . . . . . . . . . . . . . . . . . . 28

3.1.1 Definitions of Green's and Vertex Functions . . . . . . . . . . . . . . . 28

3.1.2 Relation between Vertex and Green's Functions . . . . . . . . . . . . . . . 29 
3.2 The fRG Equation . . . . . . . . . . . . . . . . . 32

3.2.1 Flow Equation of the Generating Functionals . . . . . . . . . . . . . . . . 32

3.2.2 Flow equation for the Vertex Functions . . . . . . . . . . . . . . . . 34

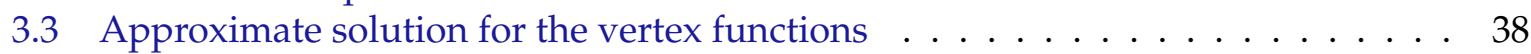

3.3 .1 Truncation . . . . . . . . . . . . . . . . . 38

3.3 .2 Specifying the Action . . . . . . . . . . . . . . . . . 38

3.3.3 $\Lambda$-dependence of the free Propagator . . . . . . . . . . . . . . 39

3.3 .4 Initial Condition . . . . . . . . . . . . . . . . . . . . . . . . 39

3.3.5 Morris' Lemma . . . . . . . . . . . . . . . . . . . . . . . . . . . . . 41

3.3.6 Further Approximations - Publication . . . . . . . . . . . . . . . . . . 42

4 Conductance for Interacting Fermi Systems 55

4.1 fRG in Keldysh formalism . . . . . . . . . . . . . . . . . . . . . . . . . 55

4.1 .1 Specifying the Action . . . . . . . . . . . . . . . . . 56

4.2 Conductance Formula - Publication . . . . . . . . . . . . . . . 57

4.3 Problems of the Matsubara Formalism . . . . . . . . . . . . . . . . . . . 82

4.3.1 Zero Temperature . . . . . . . . . . . . . . . . . . . . . 82

4.3 .2 Finite Temperature . . . . . . . . . . . . . . . . . . 82

5 Microscopic origin of the 0.7 anomaly - Publication 85

6 The 0.7 Anomaly in the Presence of Spin Orbit Interactions - Publication 123

7 Conclusion \& Outlook $\quad 137$

$\begin{array}{ll}\text { Bibliography } & 139\end{array}$

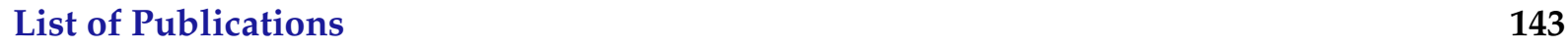

$\begin{array}{ll}\text { Curriculum Vitae } & 145\end{array}$

$\begin{array}{ll}\text { Danksagung } & 147\end{array}$ 


\section{List of Figures}

1.1 First measurement of the conductance quantization of QPCs: Conductance of a QPC as a function of applied gate voltage. The conductance increases stepwise in integer multiples of $G_{Q}=2 e^{2} / h$. Inset: Gate structure of the QPC. The metallic gates (shaded area) are placed on top of a 2DES. Reprinted figure from van Wees et al. (1988), copyright (1988) by the American Physical Society. . . . . . . . . . . .

1.2 First report of the 0.7-anomaly by Thomas et al. (1996). Left: conductance as a function of applied gate voltage for different temperatures. The first conductance step shows a shoulder-like step at around $0.7 G_{Q}$, which becomes more pronounced with higher temperatures. Right: conductance as a function of applied gate voltage for different magnetic fields. With increasing field, the 0.7 shoulder develops into the spin resolved conductance step. Reprinted figure from Thomas et al. (1996), copyright (1996) by the American Physical Society. . .

2.1 Schematic growth structure of the GaAs/AlGaAs heterostructure used in our NATURE publication (chapter 5, Bauer et al., 2013). The lowest layer of GaAs has a thickness of $800 \mathrm{~nm}$, followed by the structure shown in the schematic. The resulting potential energy $V(z)$ in $z$-direction (i.e. in growth direction) for electrons in the conduction band, w.r.t. the chemical potential (dashed black line) is shown as solid black line. Where the potential is below the chemical potential, the electron density $n$ (red line) is nonzero, forming a 2DES below the interface between the GaAs and the AlGaAs layer indicated as red area in the schematic. On top of the sample, metallic gates are attached indicated as gray area. The gates consist of 5nm Ti followed by 30nm Au. Data from Borowsky (2011) and from Stefan Birner. . . . . . . . . . . . . . . . . . . . . . . . .

2.2 a Illustration of a two-dimensional potential $V(x, y)$ resulting from a QPC geometry. Electrons are confined in $y$ direction, leading to quantized states with energies $E_{n}$ indicated by thick black lines in the center of the potential. Separation of variables leads to an effective one-dimensional potential, indicated as green line. b Illustration of the one-dimensional potential $V^{1 \mathrm{D}}(x)$ from a as a function of $x$. Electronic states on the left/right side are occupied up to the chemical potential $\mu_{L} / \mu_{R}$. Electrical current $I$ is carried within the voltage window $e V_{\mathrm{sd}}=\mu_{l}-\mu_{R}$. 
2.3 a Potentials $V^{(2)}(x)(2.13)$ and $V^{(4)}(x)(2.14)$ as a function of $x$. b Conductance $G / G_{Q}$ as a function of potential height $V_{g}$ for the potentials shown in a in corre-

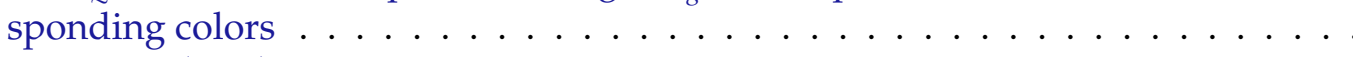

2.4 a LDOS $\rho(x, \omega)$ as a function of position $x$ and energy $\omega$, measured w.r.t. the chemical potential $\mu$ (black dashed line) for the parabolic potential $V^{(2)}(x)(2.13)$ with $V_{g}=0$ (black solid line). $\mathbf{b}$ LDOS line like in a but for the center of the QPC $\rho(x=0, \omega)$ (black solid line) and for a homogeneous one-dimensional system (black dashed line), and one partical transmission probability $\mathcal{T}(\omega)$ as a function of energy $\omega$ (red line). c Reprinted figure from Sloggett et al. (2008) (but in our notation): conductance $G$ as a function of gate Voltage $V_{g}$ for different temperatures $T$ between $T=0$ (uppermost curve) and $T=0.3 \mathrm{~K}$ (lowest curve), copyright (2008) by Springer-Verlag. . . . . . . . . . . . . . . . . . . . .

2.5 a Measured conductance $G$ as a function of applied gate voltage $V_{g}$ for different temperatures at zero magnetic field. $\mathbf{b}$ Same as in $\mathbf{a}$ but for different magnetic fields at the lowest temperature $T=20 \mathrm{mK}$. Data from Borowsky (2011).

2.6 a Non-linear conductance $G_{\mathrm{nl}}=\partial I / \partial V_{\mathrm{sd}}$ as a function of applied voltage $V_{\mathrm{sd}}$ for different gate voltages $V_{g}$ in the regime where the linear conductance $G / G_{Q}$ takes values between zero and one. Data from Borowsky (2011). b simplified circuit diagram of the measurement setup. The current $I$ resulting from an applied voltage $V_{\text {exp }}$, not only is determined by the resistance of the QPC $R_{\mathrm{QPC}}$, but also by the resistance of the wiring and measuring devices $R_{1}$ and $R_{2}$. Applying a voltage increases the chemical potential on both sides $\mu_{L}$ and $\mu_{R}$, but with a different magnitude. The voltage is defined by the resulting difference of the chemical potential $e V_{\text {sd }}=\mu_{R}-\mu_{L} \ldots \ldots \ldots \ldots$

2.7 a Conductance (black line), field scale $B_{*}$ (red line) and temperature scale $T_{*}$ (blue line) on a log-scale as a function of applied gate voltage $V_{g}$. Data from Borowsky (2011). b Shot-noise factor as a function function of conductance $G$. Reprinted figure from DiCarlo et al. (2006), copyright (2006) by the American Physical Society. . . . . . . . . . . . . . . . . . . . . .

2.8 Geometric construction of the solution of equation (2.44) (red line) and equation (2.45) (green line). The solutions of these equations is the intersection with the identity (black line). . . . . . . . . . . . . . . . . . . . .

2.9 a Quantum dot structure reprinted from Goldhaber-Gordon et al. (1998), copyright (1998) by Nature Publishing Group. b Non-linear conductance $G_{n l}=$ $d I / d V_{\mathrm{sd}}$ as a function of applied voltage $V_{\text {sd }}$ for different temperatures between $15 \mathrm{mK}$ (black line) and $900 \mathrm{mK}$ (red line). Reprinted figure from van der Wiel et al. (2000), copyright (2000) by AAAS. . . . . . . . . . . . . . . . . . . . 


\section{Zusammenfassung in deutscher Sprache}

Diese Dissertation behandelt ein Phänomen im Bereich der Halbleiter-Nanostrukturen, der 0.7 Anomalie in Quantenpunktkontakten (QPCen). Dieses ist seit 1996 bekannt, bisher jedoch nicht eindeutig erklärt. Seit den 80ern ist es möglich Strukturen auf Basis von Halbleitern zu bauen, deren Größe sich im Bereich von Nano- bis Mikrometer befindet. Eine der einfachsten Strukturen ist der QPC, eine kurze und schmale, und somit punktförmige Verbindung zwischen zwei ausgedehnten Elektronensystemen. Solche QPCe sind oft Teil komplizierterer Strukturen, und befinden sich damit in nahezu allen Halbleiter-Nanostrukturen. Daher ist es essentiell, jeden Aspekt dieser QPCe zu verstehen.

Die zentrale Eigenschaft des QPCs ist die Quantisierung seines Leitwerts: Abhängig von einer angelegten Gatterspannung, welche die Breite des QPCs bestimmt, ändert sich der Leitwert stufenweise in ganzzahligen Vielfachen des Leitwertquantums $G_{Q}=2 e^{2} / h$, wobei $e$ die Elektronenladung und $h$ die Planck-Konstante ist. Diese Quantisierung kann in dem Bild nichtwechselwirkender Elektronen erklärt werden: In eindimensionalen Systemen ist die elektronische Zustandsdichte indirekt proportional zu deren Geschwindigkeit - in Bereichen in denen sich Elektronen besonders langsam bewegen, ist die Anzahl von Zuständen pro Energieintervall entsprechend grösser.

Im Gegensatz dazu gibt es in QPCen Phänomene, die nicht in einem Einteilchenbild verstanden werden können, und deren Ursprung kontrovers diskutiert wird. Die prominentesten sind in einem Parameterbereich, in dem gemessene Leitwertkurven eine schulterartigen Zwischenstufe bei etwa $0.7 G_{Q}$ zeigen. Diese Phänomene sind unter den Namen 0.7-Anomaly bekannt. In diesem Parameterbereich zeigen viele Grössen, wie elektrischer und thermischer Leitwert, Rauschen und Thermoelektrizität anomales Verhalten als Funktion externer Parameter wie Magnetfeld, Temperatur oder angelegte Spannung. In dieser Arbeit wird ein Modell vorgestellt, dass die Eigenschaften der 0.7 Anomalie in theoretischen Rechnungen reproduziert und damit eine intuitive Erklärung für den mikroskopische Ursache der Phänomene ermöglicht. Dabei werden störungstheoretische Methoden, die funktional Renormierungsgruppe (fRG), sowie zweite Ordnung Störungstheorie, verwendet.

Für den Bereich der ersten Leitwertstufe wird der QPC durch ein eindimensionales wechselwirkendes System beschrieben, wobei der QPC selbst durch eine Potentialbarriere dargestellt wird. Ist die Barriere höher als das chemische Potential ist der Leitwert nahe bei Null, ist sie niedriger geht der Leitwert gegen $G_{Q}$. Wechselwirkende Effekte sind besonders stark 
wenn die lokale Zustandsdichte (LDOS) am chemischen Potential hoch ist, wenn also die Ladungsträger beim überqueren der Potentialbarriere besonders langsam sind. Dieser Fall tritt ein, wenn das Barrierenmaximum nur knapp unterhalb des chemischen Potentials ist. Der Leitwert ist dort bereits kleiner als $G_{Q}$, jedoch größer als $G_{Q} / 2$. Dieser Zusammenhang erklärt unmittelbar das Auftreten unerwarteter Effekte im Bereich der 0.7 -Schulter und liefert eine natürliche Erklärung des Auftretens der 0.7-Anomalie. Damit lassen sich die beiden wichtigsten Phänomene von QPCe, die Leitwertquantisierung und die 0.7-Anomalie, auf ein und die selbe Eigenschaft eindimensionaler Systemen zurückführen: die Zustandsdichte ist indirekt proportional zur Geschwindigkeit.

Unsere besten theoretischen Ergebnisse wurden mit einem eigens entwickelten Näherungsschema, der gekoppelten Leiternäherung (CLA), innerhalb der fRG erzielt. Üblichereise wird die unendliche Hierarchie der fRG Flussgleichungen nach der zweiten Ordnung abgebrochen. Damit erhält man eine gekoppelte Differentialgleichung für die Selbstenergie und den Zweiteilchenvertex. Im Falle inhomogener Systeme benötigt man eine gewisse räumliche Auflösung von $N \sim 100$ Punkten. Ohne weitere Näherung geht die Anzahl der unabhängigen Variablen, die den Zweiteilchenvertex representiereni, mit $\mathcal{O}\left(N^{4}\right)$. Die CLA nutzt aus, dass sich innerhalb der fRG der Zweiteilchenvertex als Summe seiner Kanäle darstellen lässt. Beschränkt man die Kopplung dieser Kanäle für Systeme mit einer kurzreichweitigen Wechselwirkung, reduziert sich die Anzahl der unabhängigen Variablen des Zweiteilchenvertex auf $\mathcal{O}\left(N^{2}\right)$.

Bedauerlicherweise ist es derzeit nicht möglich, Leitwerte bei endlicher Temperatur oder endliche angelegte Spannung mit fRG zu berechnen. Daher verwenden wir für diese Parameter Störungstheorie in zweiter Ordnung im Keldysh Formalismus. Um eine entsprechende Leitwertformel herzuleiten, benutzen wir die fRG Gleichungen in ihrer Eigenschaft als exakte Relationen für die Ableitung der Selbstenergie bezüglich eines Parameters.

Die Arbeit wird abgeschlossen mit einer Vorhersage für die 0.7-Anomalie in Gegenwart von Spin-Orbit Wechselwirkung (SOI). Ist die charakteristische Energie der SOI von der selben Grössenordnung wie die geometrische Energieskala des QPC, hängt die Form der Leitwertstufe bei endlichem Magnetfeld sehr stark von der relativen Richtung von SOI und Magnetfeld ab. Die theoretischen Vorhersagen werden ebenfalls mit der fRG in der CLA erzielt. 


\section{Abstract}

A Quantum point contact (QPC) is a one dimensional constriction, separating two extended electron systems allowing transport between them only though a short and narrow channel. The linear conductance of QPCs is quantized in units of the conductance quantum $G_{Q}=2 e^{2} / \mathrm{h}$, where $e$ is the electron charge and $h$ is Planck's constant. Thus the conductance shows a staircase when plotted as a function of gate-voltage which defines the width of the channel. In addition measured curves show a shoulder-like step around $0.7 G_{Q}$. In this regime QPCs show anomalous behaviour in quantities like electrical or thermal conductance, noise, and thermopower, as a function of external parameters such as temperature, magnetic field, or applied voltage. These phenomena, collectively known as the 0.7-anomaly in QPCs are subject of controversial discussion.

This thesis offers a detailed description of QPCs in the parameter regime of the 0.7-anomaly. A model is presented which reproduces the phenomenology of the 0.7-anomaly. We give an intuitive picture and a detailed description of the microscopic mechanism leading to the anomalous behavior. Further, we offer detailed predictions for the behavior of the 0.7 -anomaly in the presence of spin-orbit interactions.

Our best theoretical results were achieved using an approximation scheme within the functional renormalization group (fRG) which we developed to treat inhomogeneous interacting fermi systems. This scheme, called the coupled ladder approximation (CLA), allows the flow of the two-particle vertex to be incorporated even if the number of interacting sites $N$, is large, by reducing the number of independent variables which represent the two-particle vertex from $\mathcal{O}\left(N^{4}\right)$ to $\mathcal{O}\left(N^{2}\right)$. 


\section{Chapter 1}

\section{Introduction}

In the last decades fabrication of semiconductor nano-devices improved in many ways, such that nowadays it is standard to build devices which allow charge control on the level of single electrons. One way to fabricate such devices is to attach metallic gates on top of a heterostructure, e.g. GaAs/AlGaAs, which forms a two-dimensional electron system (2DES). At temperatures below $100 \mathrm{mK}$, where electron-phonon scattering is small, the mean free path of electrons in such structures is in the micron regime, allowing investigation of phase-coherent many-body quantum phenomena.

One of the building blocks of such nano-devices is the quantum point contact (QPC). Depending on the context, QPCs can serve different purposes. If the contact is almost completely closed, it provides a tunnel contact, which can be used to built quantum dots (QDs) (GoldhaberGordon et al., 1998). A QPC where the first mode is half open, is used to detect charge (Field et al., 1993) or as an electron beam splitter in quantum Hall bars (Ji et al., 2003).

In order to understand devices with a higher complexity it is essential to understand the

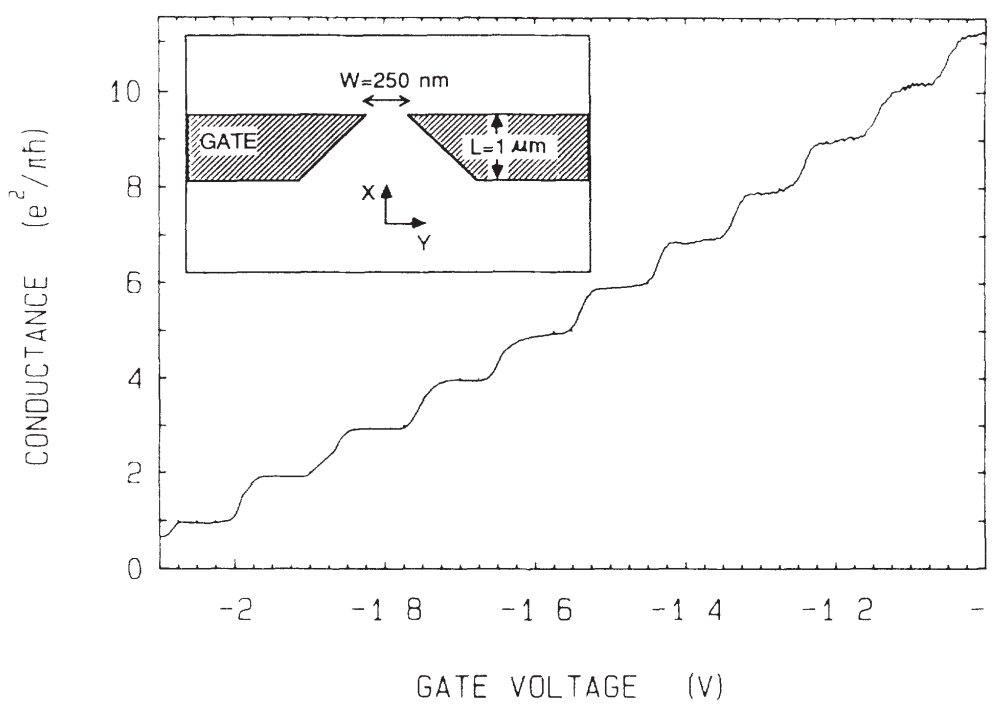

Figure 1.1: First measurement of the conductance quantization of QPCs: Conductance of a QPC as a function of applied gate voltage. The conductance increases stepwise in integer multiples of $G_{Q}=$ $2 e^{2} / h$. Inset: Gate structure of the QPC. The metallic gates (shaded area) are placed on top of a 2DES. Reprinted figure from van Wees et al. (1988), copyright (1988) by the American Physical Society. 

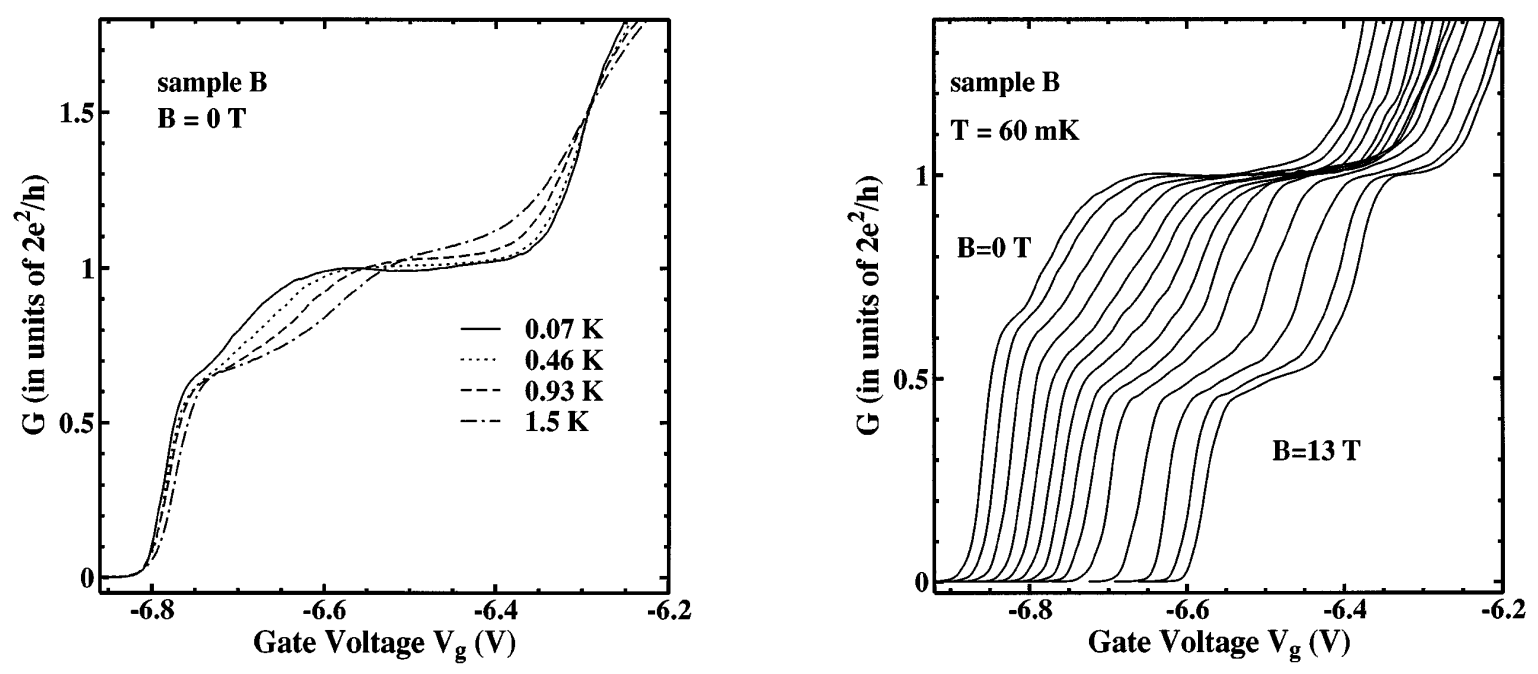

Figure 1.2: First report of the 0.7-anomaly by Thomas et al. (1996). Left: conductance as a function of applied gate voltage for different temperatures. The first conductance step shows a shoulder-like step at around $0.7 G_{Q}$, which becomes more pronounced with higher temperatures. Right: conductance as a function of applied gate voltage for different magnetic fields. With increasing field, the 0.7 shoulder develops into the spin resolved conductance step. Reprinted figure from Thomas et al. (1996), copyright (1996) by the American Physical Society.

properties of their building blocks. Many properties of QPCs are well understood. E.g. its quantized conductance has been predicted (Landauer, 1957) long before it was first measured by van Wees et al. (1988) and Wharam et al. (1988). Figure 1.1 shows the results of van Wees et al. (1988). The inset shows the split-gate structure lying above a 2DES, which is the most common way to build a QPC. Applying negative voltage on the two metallic gates (shaded area) depletes electrons underneath them, such that electrons can cross only through the narrow channel between the gates. As a consequence of the quantization in the direction transversal to the current the conductance of this QPC is quantized in units of $G_{Q}=2 e^{2} / h$, where $e$ is the electron charge and $h$ is Planck's constant. The applied gate voltage determines the number of modes contributing to the electrical current such that the conductance increases stepwise as a function of applied gate voltage.

In addition to these well-understood properties of QPCs, there are also properties whose microscopic origin is still under controversial discussion, such as the shoulder like step at $0.7 G_{Q}$ shown in figure 1.2. This feature was already present in the data by van Wees et al. (1988), but was not entirely shown (see figure 1.1). Thomas et al. (1996) were the first who reported that this is a generic feature of QPCs. Later this shoulder became known as the "0.7-anomaly", and hundreds of experimental papers presented all kinds of anomalous behavior for quantities such as the electrical and thermal conductance as a function of external parameters such as temperature, magnetic field or applied voltage, all related to the 0.7-anomaly (Micolich, 2011).

Despite the extensive amount of experimental results, no theoretical explanation could be presented which found approval by the entire community. The most prominent interpretations 
of the 0.7-anomaly are the idea of spin polarization, proposed by Thomas et al. (1996), and the Kondo model proposed by Cronenwett et al. (2002) and Meir et al. (2002). However these interpretations are not able to explain the whole phenomenology of the 0.7-anomaly. So if we are not able to understand a device as simple as a QPC, the question arises, weather we are able to understand more complicate setups, especially if they are built from QPCs. In this work we will argue, that a one-dimensional system with a potential barrier representing the QPC, explains all properties of the QPC, once interactions are taken into account, and thus we do understand the QPC.

Although this model is somehow the most obvious description of a QPC (see chapter 2), at the time this project started several years ago, there were hardly any theoretical calculations using this type of model. The reason for this might be, that getting reliable results for that kind of model is a real challenge if interactions are not small. Most methods that are able to treat models with large interactions are not able to treat systems with a potential that has spatial structure. So most of the work for this thesis and also most of the ongoing work in this project is dedicated to develop methods that are both reasonable fast, and reasonable accurate when used to calculate transport properties. The latter turns out to particularly difficult if the temperature is nonzero, which of course is desirable since the behavior with temperature is one of the central aspects of the 0.7-anomaly.

We approached this problem by using different perturbative methods. One of them, the functional renormalization group ( $f R G$ ), is more reliable at large interactions, but in the current status of its implementation only applicable at zero temperature (an extension of the fRG approach to finite temperature is topic of ongoing work). Further we used second order perturbation theory (SOPT) to get results at finite temperature on the cost of being less reliable at large interactions. A nice review of the fRG approach was written by Metzner et al. (2012). Though these approaches have a relatively simple analytic structure, numerically they are quite challenging, due to the extended spatial structure.

A broad introduction into the field of QPCs is given in chapter 2. We set the stage by introducing a typical experimental sample. Then we derive the conductance quantization by reformulating the two-dimensional model into a set of effective one-dimensional models. We discuss the potential form representing the QPC, and the role of interaction. With this motivation of the model we are ready to give a heuristic explanation of the microscopic origin of the 0.7 -anomaly. We do this by simply examining the properties of a one-dimensional model and without presenting any calculations including effects of interactions. The following summary of the most prominent experimental results is explained on the same heuristic level. The chapter is completed by introducing other theoretical models.

In chapter 3, we derive the equations of the functional renormalization group (fRG) approach used to calculate conductance and susceptibilities at zero temperature. Therefore we present a compact but complete and generic derivation of the fRG flow equations. At the end of the chapter we introduce the approximation scheme developed to treat interacting inhomogeneous one-dimensional models, in the form of a publication (see also Bauer et al., 2014).

A general formula to calculate conductance at finite temperature in Keldysh formalism is derived in chapter 4 , where we use the exact fRG equations in order to differentiate the self energy w.r.t. the applied voltage. The main part of this chapter is a draft of a paper which will 
published soon(see also Heyder, 2014).

The heart of this thesis is chapter 5, which contains a reprint of our NATURE publication (see also Bauer et al., 2013), and the extensive supplementary information. This publication clarifies the microscopic origin of the 0.7-anomaly, including detailed microscopic calculations. These calculations are compared with experimental measurements made by the group of Stefan Ludwig.

Subsequently in chapter 6 we predict how the magnetic field dependence of the 0.7-anomaly changes in the presence of strong spin orbit interactions. This work will be published soon (see Goulko et al., 2014). 


\section{Chapter 2}

\section{Quantum Point Contacts and the 0.7 Anomaly}

\subsection{Heterostructures and the Split Gate Geometry}

2DES made from heterostructures are the most frequently used ones. By arranging different layers of semiconductor materials, a potential is configured such that the electron density in the conduction band is finite only at a interface between two layers, in a way that only the lowest state is occupied. As a result the direction perpendicular to the layers, to be called $z$-direction, affects the dynamics of the system only by an overall phase factor, and the dynamics of the system is purely two-dimensional. In this work we consider mainly QPC made of $\mathrm{GaAs} / \mathrm{AlGaAs}$ hetero-structures. This material provides 2DES with a very high quality, and

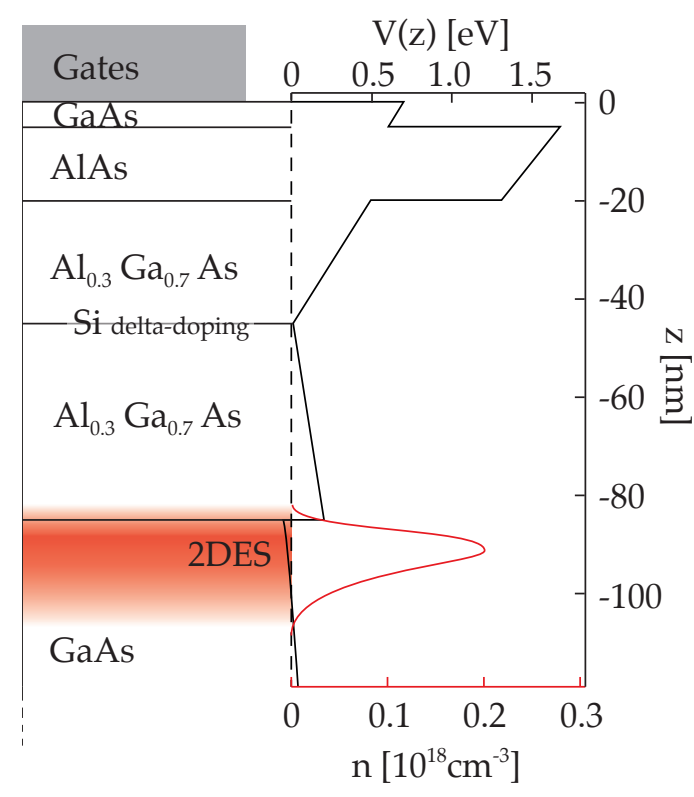

Figure 2.1: Schematic growth structure of the GaAs/AlGaAs heterostructure used in our NATURE publication (chapter 5, Bauer et al., 2013). The lowest layer of GaAs has a thickness of $800 \mathrm{~nm}$, followed by the structure shown in the schematic. The resulting potential energy $V(z)$ in $z$-direction (i.e. in growth direction) for electrons in the conduction band, w.r.t. the chemical potential (dashed black line) is shown as solid black line. Where the potential is below the chemical potential, the electron density $n$ (red line) is nonzero, forming a 2DES below the interface between the GaAs and the AlGaAs layer indicated as red area in the schematic. On top of the sample, metallic gates are attached indicated as gray area. The gates consist of $5 \mathrm{~nm}$ Ti followed by 30nm Au. Data from Borowsky (2011) and from Stefan Birner. 
thus it is well suited to investigate generic properties of QPCs. Figure 2.1 shows the schematic stucture, as well as the electrostatic potential and the density for electrons in the conduction band of the sample used in our NATURE publication (chapter 5, Bauer et al., 2013), made by Werner Wegscheider. The lowest state resulting from the triangular potential at the interface of the GaAs and the AlGaAs layers is $7 \mathrm{meV}$ below the chemical potential. The second state is $20 \mathrm{meV}$ above the chemical potential and we can neglect thermal excitations to this level at temperatures below $10 \mathrm{~K}$.

This 2DES can be further confined by attaching metal gates on top of the sample. Applying voltages to these gates changes the potential energy for electrons in the 2DES. The geometry of these gates can be shaped on the scale of several nanometers. In this way almost arbitrary structures can be built. Limiting factors are the number of different voltages that can be applied at the same time and the topology of the gates. The latter however, is improving continuously due to novel gating techniques, such that more and more complicated patterns can be built. However, in this work we are more interested in the simplest gate structure, the split-gate structure, which forms a QPC.

Split gates can have all kinds of forms, e.g. a trapezoidal form used by van Wees et al. (1988) (see inset of figure 1.1), but also rectangular or round forms are used. A higher tunability is achieved by multiple gates on each side allowing to control the length of the QPC. Such a device is used in our NATURE publication (chapter 5, Bauer et al., 2013, see figure 1 a there), where an additional top-gate enables control over the width of the QPC. The split gate divides the 2DES in two areas and allows electronic transport only through a narrow channel, the QPC.

\subsection{The noninteracting QPC}

All basic properties of a QPC, in particular its quantized conductance, are well understood within a noninteracting model. This picture is well established and can be found in textbooks e.g. Heinzel (2003, chapter 7.2). Hence it is reasonable to assume that if these basic properties can be found in an experimental realization of a QPC, interactions are not too strong, such that they do not dominate physical properties, but rather alter them w.r.t. the noninteracting behavior. So we will derive and justify the model for a QPC from the noninteracting point of view. In the last step we will add an interaction term. Such a model does not incorporate every aspect of the experimental realization of a QPC. If a certain experimental aspect cannot be explained by the chosen model one has to go back to this very point and reconsider every assumption made in the process.

\subsubsection{From $2 \mathrm{D}$ to $1 \mathrm{D}$}

We consider a QPC built by 2DES with a split gate geometry described in section 2.1. We assume that the electrons in the 2DES can be described by a two-dimensional Schrödinger equation with a quadratic dispersion and mass $m$. The top gates define a static electrostatic potential $V(x, y)$ :

$$
E \psi(x, y)=-\frac{\hbar^{2}}{2 m}\left(\partial_{x}^{2}+\partial_{y}^{2}\right) \psi(x, y)+V(x, y) \psi(x, y)
$$



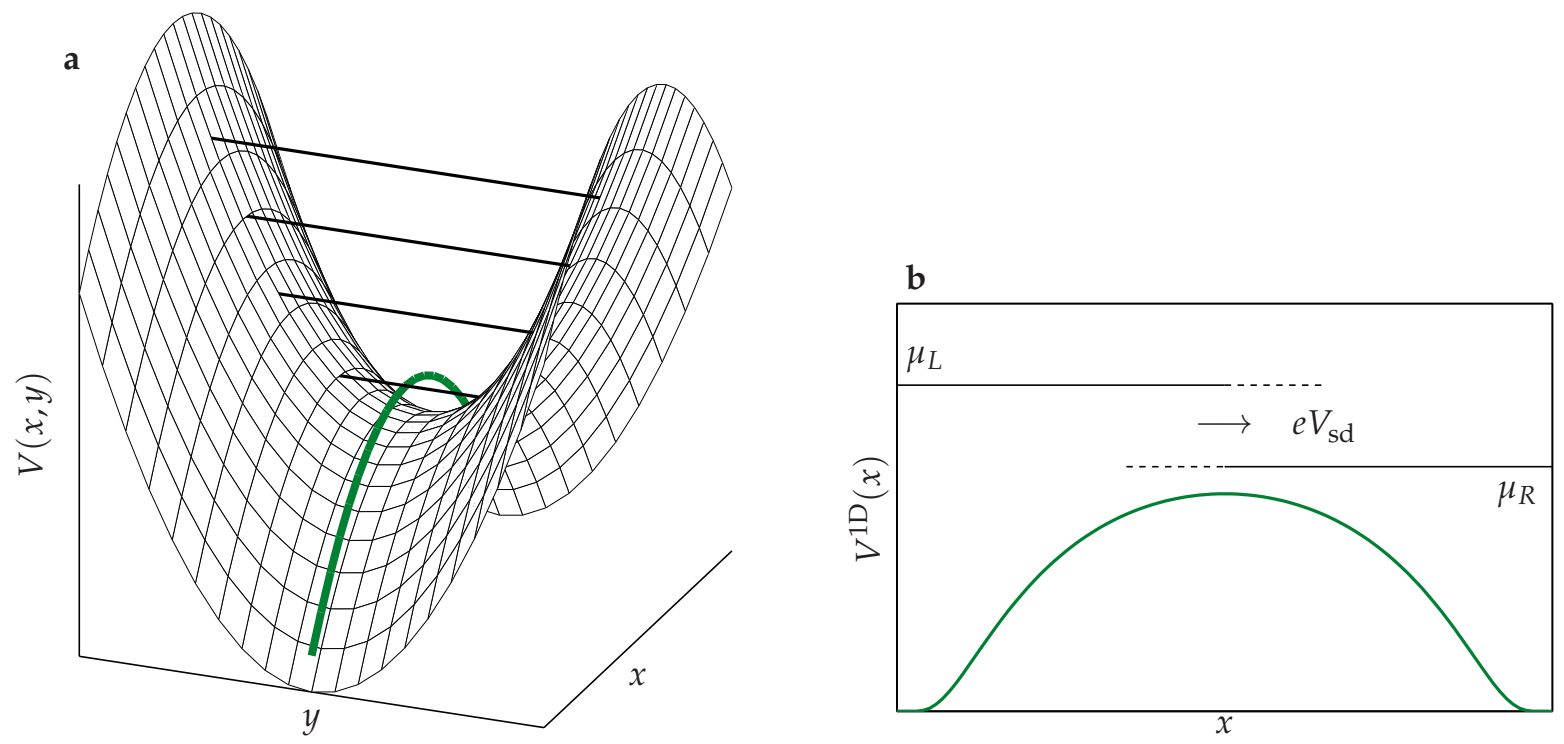

Figure 2.2: a Illustration of a two-dimensional potential $V(x, y)$ resulting from a QPC geometry. Electrons are confined in $y$ direction, leading to quantized states with energies $E_{n}$ indicated by thick black lines in the center of the potential. Separation of variables leads to an effective onedimensional potential, indicated as green line. $\mathbf{b}$ Illustration of the one-dimensional potential $V^{1 \mathrm{D}}(x)$ from a as a function of $x$. Electronic states on the left/right side are occupied up to the chemical potential $\mu_{L} / \mu_{R}$. Electrical current $I$ is carried within the voltage window $e V_{\text {sd }}=$ $\mu_{l}-\mu_{R}$

The potential $V(x, y)$ is illustrated in figure 2.2 a. We take $x$ to be the transport-direction of the QPC and assume that electrons are confined in $y$-direction, such that for a given $x$, the wave-function $\phi_{n}^{x}(y)$ solves the equation

$$
E_{n}(x) \phi_{n}^{x}(y)=-\frac{\hbar^{2}}{2 m} \partial_{y}^{2} \phi_{n}^{x}(y)+(V(x, y)-V(x, y=0)) \phi_{n}^{x}(y),
$$

with discrete eigen energies $E_{n}(x)$. These energies are indicated by horizontal lines within the $2 \mathrm{D}$ potential in figure 2.2 a. Their spacing growths with decreasing width of the QPC. If the potential changes smoothly with $x$, such that the cross section of the potential in $y$-direction changes slowly with $x$ so that we can neglect the derivatives of $\phi_{n}^{x}$ w.r.t. $x$, the ansatz

$$
\psi(x, y)=\phi_{n}^{x}(y) \psi(x),
$$

gives

$$
E \psi=-\frac{\hbar^{2}}{2 m} \partial_{x}^{2} \psi+\left(V(x, y=0)+E_{n}(x)\right) \psi
$$

Hence, for each mode $n$ this results an effective one-dimensional potential

$$
V_{n}^{1 \mathrm{D}}(x)=E_{n}(x)+V(x, y=0),
$$


This defines a purely one-dimensional system, where the $y$-dependence of the potential only enters via the offset $E_{n}(x)$. This fact is illustrated in figure 2.2 a: The green line shows the effective potential of the lowest mode $V_{1}^{1 \mathrm{D}}(x)$ which has an offset w.r.t. the 2D potential.

The approach discussed above is exact if the potential can be decomposed as $V(x, y)=$ $V_{x}(x)+V_{y}(y)$. A special case of this form is the saddle point potential $V(x, y)=\frac{m}{2 \hbar^{2}} \Omega_{y}^{2} y^{2}-$ $\frac{m}{2 \hbar^{2}} \Omega_{x}^{2} x^{2}$, which will always be a good approximation for the leading orders. This purely parabolic potential is one of the few cases which can be solved analytically and is discussed in Büttiker (1990).

\subsubsection{Conductance Quantization}

The next step is to derive the current and the conductance of such a one-dimensional system. I will do this following the original paper of Landauer (1957) for the special case of a onedimensional system. This approach may appear a little oldfashioned, in fact we present a modern approach in chapter 4 following Meir and Wingreen (1992), however, the derivation presented here provides a deeper understanding about why conductance is quantized, and we will see later on that the origin of the quantized conductance is also the origin of the 0.7anomaly.

Since electrons are fermions, all states up to the Fermi energy, i.e. the chemical potential $\mu$, are occupied, implying that their kinetic energy can be quite large. Thus in a semiclassical picture electrons move constantly through the sample. However, to get a finite net-current a voltage has to be applied to produce an imbalance between the chemical potential of two reservoirs. Due to that imbalance there are more electrons moving from, say left to right, than vice versa. This is illustrated in figure $2.2 \mathbf{b}$ : the current $I$ flowing over a potential barrier is determined by the voltage-window $\mathrm{eV}=\mu_{R}-\mu_{L}$ defined by chemical potential of the left $\mu_{L}$ and the right $\mu_{R}$ reservoir.

Each electron with energy $\omega$, moving with velocity $\pm v(\omega)$ carries the elementary charge $e$. The number of electrons per energy is given by the density of states, $\rho_{n}(\omega)$. The distribution of the right/left moving electrons, i.e. electrons with positive/negative velocity is given by the Fermi-distribution function of the left/right reservoir $f_{L} / f_{R}$. Whether or not an electron with energy $\omega$ is transmitted through the one-dimensional system is given by the one particle transmission probability $\mathcal{T}_{n}(\omega)$, determined by the one-dimensional potential $V_{n}^{1 \mathrm{D}}(2.5)$. Integrating overall energies $\omega$ and summing over all modes $n$ gives the total current:

$$
I=\sum_{n} e \int d \omega \rho_{n}(\omega) v_{n}(\omega) \mathcal{T}_{n}(\omega)\left[f_{L}(\omega)-f_{R}(\omega)\right]
$$

In one dimension, the density of states is given by

$$
\rho(\omega)=2 \cdot \frac{1}{2 \pi} \frac{d k}{d \omega},
$$

(the 2 is due to spin degeneracy) and the velocity is

$$
v(\omega)=\frac{1}{\hbar} \frac{d \omega}{d k} .
$$


Together this yields

$$
I=2 \sum_{n} \cdot \frac{e}{h} \int d \omega \mathcal{T}_{n}(\omega)\left[f_{L}(\omega)-f_{R}(\omega)\right]
$$

At zero temperature, the conductance is

$$
G=\frac{\partial}{\partial V_{\mathrm{sd}}} I=\frac{2 e}{h} \frac{\partial}{\partial V_{\mathrm{sd}}} \int_{\mu-e V_{\mathrm{sd}} / 2}^{\mu+e V_{\mathrm{sd}} / 2} d \omega \mathcal{T}_{n}(\omega) \stackrel{V_{\mathrm{sd}}=0}{=} \frac{2 e^{2}}{h} \sum_{n} \mathcal{T}_{n}(\mu),
$$

where $V_{\text {sd }}$ is the source-drain-voltage. If $\mathcal{T}_{n}(\mu)$ is either one or zero with $N=\sum_{n} \mathcal{T}_{n}(\mu)$ the conductance is

$$
G=N G_{Q}
$$

Consequently $G$ is a stepwise increasing function of the gate voltage $V_{g}$ (see figure 1.1). Note that the fact that $G$ is a quantized number originates from the relation between the density of states and the velocity of Fermionic particles in one dimension:

$$
\rho \propto \frac{1}{v}
$$

\subsubsection{Concrete form of the 1D Potential}

To complete the considerations on the noninteracting model, I will discuss the form of the one-dimensional potential $V^{1 \mathrm{D}}(2.5)$ for a single mode of the QPC. From now on we will only consider one-dimensional potentials, hence we omit the superscript 1D. Since the potential has a global maximum in the center of the QPC (otherwise the transmission would show sharp resonances) we can choose our coordinates, such that the maximum is at $x=0$, implying that the potential has no component linear in $x$. Further we assume, that the potential is symmetric around its maximum. Since the transmission will be dominated by the lowest-order terms we will examine two potential forms shown in figure 2.3: A purely parabolic potential given by

$$
V^{(2)}(x)=V_{g}+\mu-\frac{m}{2 \hbar^{2}} \Omega_{x}^{2} x^{2}
$$

(black line), and a purely quartic potential,

$$
V^{(4)}(x)=V_{g}+\mu-\frac{m^{2}}{4 \hbar^{4}} \Omega_{x}^{3} x^{4}
$$

(red line). The potential height $V_{g}$, mimics the role of gate voltage and is defined such that when $V_{g}=0$, the potential maximum is at the chemical potential, $m$ is the effective mass of the electron and $\Omega_{x}$ is the characteristic geometric energy scale characterizing the effective barrier width, $l_{x}=\sqrt{\frac{\hbar^{2}}{2 m \Omega_{x}}}$. The form for a generic even integer power $n$ of $x$ reads as

$$
V^{(n)}(x)=V_{g}+\mu-\Omega_{x}\left(\frac{x}{2 l_{x}}\right)^{n} .
$$



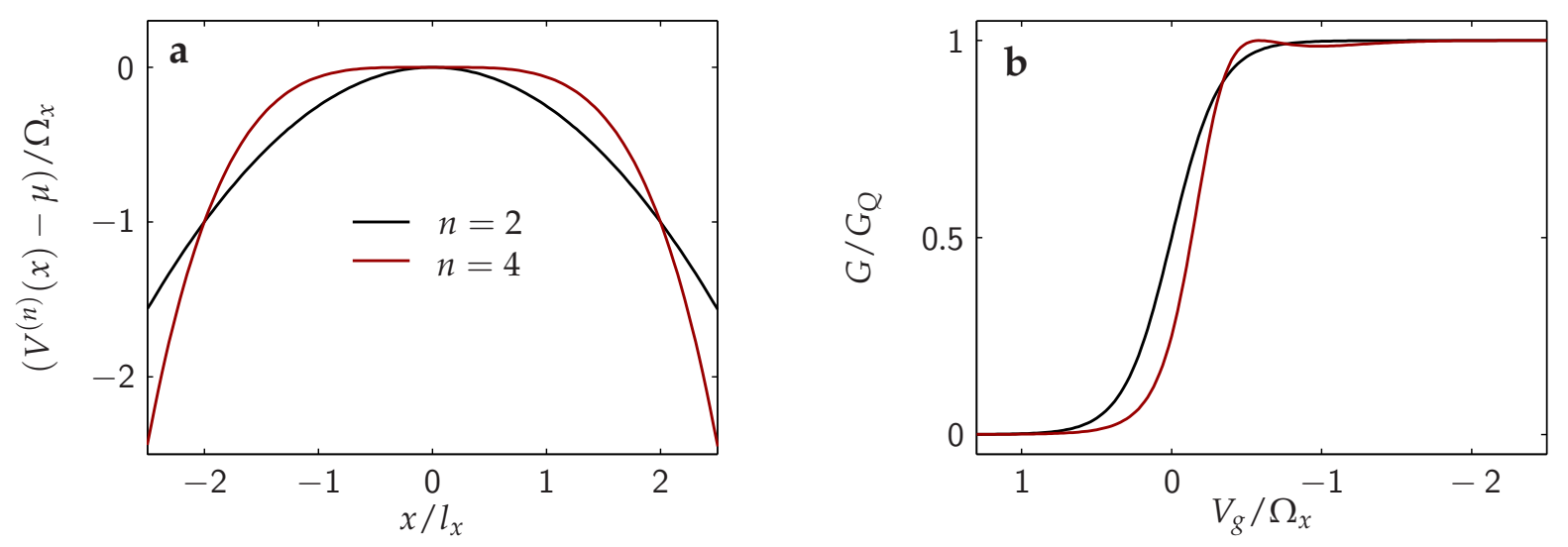

Figure 2.3: a Potentials $V^{(2)}(x)(2.13)$ and $V^{(4)}(x)(2.14)$ as a function of $x$. b Conductance $G / G_{Q}$ as a function of potential height $V_{g}$ for the potentials shown in a in corresponding colors

Figure $2.3 \mathbf{b}$ shows the conductance $G / G_{Q}$ at zero temperature as a function of gate voltage $V_{g}$ for the quadratic potential (black line) and the quartic potential (red line).

We first note the salient features of such potential barriers: The conductance $G / G_{Q}$ goes from one to zero if the potential height $V_{g}$ crosses the chemical potential from below. The width of this transition is proportional to the geometric energy scale $\Omega_{x}$, as can be derived by a dimensional analysis, up to a dimensionless pre-factor. Once the maximum of the potential is larger than the chemical potential, electrons can only tunnel through the barrier. This tunneling is exponentially suppressed with barrier height.

The conductance $G / G_{Q}$ of the quadratic potential (black line) has a smooth monotonic transition from one to zero. For the purely parabolic form given by equation (2.13), the conductance is known analytically (Büttiker, 1990): $G\left(V_{g}\right) / G_{Q}=\mathcal{T}(\mu)$ with

$$
\mathcal{T}(\omega)=\left[e^{-2 \pi\left(\omega-\mu-V_{g}\right) / \Omega_{x}}+1\right]^{-1} .
$$

In contrast, the conductance of the quartic potential (figure 2.3 a red line) shows oscillations for negative gate voltage. This fact seems surprising, since the potential is completely smooth and one would not expect any backscattering, which causes an oscillatory behavior in the transmission as a function of energy. We learn from this simple example, that oscillations in the conductance do not necessarily point to a non-adiabatic potential. Conversely, however,we can conclude that if the conductance shows nice plateaus, without any oscillations, it is a profound assumption that the potential is smooth and has a large leading quadratic term. For that reason, most of our research is focused either on the purely parabolic potential (2.13), as discussed in section 3.3.6 H, or the gaussian form used in chapter 5, which also has a large leading quadratic dependence:

$$
V(x)=\left(V_{g}+\mu\right) \exp \left(-\frac{\left(x / x_{0}\right)^{2}}{1-\left(x / x_{0}\right)^{2}}\right)
$$


Of course, many experimental conductance plots do show small oscillations in the plateau similar to the conductance of the quartic potential shown as red line in figure $2.3 \mathbf{b}$. However, including non-parabolic, and / or non-adiabatic potentials, would increase the parameter-space extensively. In this work we therefore focus on the generic features, even present for a "perfect" realization of a QPC. A first theoretical insight into the subject of non-parabolic QPC-potentials, is given in Heyder et al. (2014), where we study the crossover from a QPC to a QD.

\subsection{The Role of Interaction}

Before proceeding with the discussion of QPCs we comment on the role of interaction, when it becomes important, and what the correct effective one-dimensional form is.

\subsubsection{Effective Interaction strength}

Interaction is a dimensional quantity, thus a priori one cannot make any statement whether it is large or not. Such a statement can only be made about a dimensionless quantity. For the interaction the relevant dimensionless quantity is the interaction times density of states at the chemical potential. In order to make this statement plausible, we perform a simple cartoon calculation: Let us consider the spin susceptibility,

$$
\chi=\left.\partial_{B}\left(n_{\uparrow}-n_{\downarrow}\right)\right|_{B=0},
$$

where $n_{\sigma}$ is the number of electrons with spin $\sigma$ in the system. Above, the only consequence of spin was to give a factor of two in the DOS (2.7). A magnetic field lifts the spin degeneracy and the DOS explicitly depends on spin $\sigma$ : Due to the Zeeman energy, the DOS $\rho$ gets an linear shift in energy $\rho^{\sigma}=\rho(\omega+\sigma B)$, where $\sigma=\uparrow, \downarrow$ or,+- , and $B$ represents the Zeeman energy. Lets first discuss the case where the interaction $U$ is zero. Then

$$
\begin{aligned}
\chi & =\left.\partial_{B} \int d \omega f(\omega)\left(\rho_{\uparrow}(\omega)-\rho_{\downarrow}(\omega)\right)\right|_{B=0} \\
& \left.\stackrel{T=0}{=} \partial_{B} \int_{-\infty}^{\mu} d \omega(\rho(\omega+B / 2)-\rho(\omega-B / 2))\right|_{B=0} \\
& =\rho(\mu),
\end{aligned}
$$

which is the Pauli-susceptibility. Next we calculate the effects of interactions to first order. For simplicity we take the interaction $U$ as constant. Further since the spin-susceptibility is the response factor associated with the force coupled to the spin degree of freedom, we take $U$ as the component of interactions which couples to the spin, i.e. the exchange part of the interaction, which is a consequence of Pauli's principle (see e.g. Sakurai, 1993, chapter 6.3). Then the energy in first order $\omega_{\sigma}^{(1)}$ of an electron with spin sigma is given by its bare value $\omega_{\sigma}^{(0)}$ less the Coulomb energy to first order, i.e. the noninteracting occupation of the opposite spin $n_{\bar{\sigma}}^{(0)}$ times the interaction $U$ :

$$
\omega_{\sigma}^{(1)}=\omega_{\sigma}^{(0)}-n_{\bar{\sigma}}^{(0)} U
$$


Defining an effective magnetic field which absorbs the effects of interaction

$$
B^{\text {eff }}=\omega_{\uparrow}^{(1)}-\omega_{\downarrow}^{(1)}=\omega_{\uparrow}^{(0)}-\omega_{\downarrow}^{(0)}+\left(n_{\uparrow}^{(0)}-n_{\downarrow}^{(0)}\right) U=B+\left(n_{\uparrow}^{(0)}-n_{\downarrow}^{(0)}\right) U,
$$

the spin-susceptibility is given by

$$
\chi=\left.\partial_{B}\left(n_{\uparrow}-n_{\downarrow}\right)\right|_{B=0}=\left.\rho(\mu) \partial_{B} B^{\text {eff }}\right|_{B=0}=\rho(\mu)(1+\rho(\mu) U) .
$$

As a result the spin-susceptibility is enhanced by the factor $(1+\rho(\mu) U)$, thus interactions become important if $\rho(\mu) U$ is of the order one. A similar chain of argument can be made for each response quantity in any order of interaction. Note, that here the DOS $\rho$ is the total DOS integrated over space.

\subsubsection{Effective one-dimensional Interaction}

The world is three-dimensional, and so is the interaction between electrons, even if they live in a one-dimensional system. Their interaction is determined by the three-dimensional solution of the Poisson equation, the Coulomb potential:

$$
U\left(\mathbf{r}_{1}, \mathbf{r}_{2}\right)=\frac{e^{2}}{4 \pi} \frac{1}{\left|\mathbf{r}_{1}-\mathbf{r}_{2}\right|} .
$$

This two particle interaction cannot be transformed into a one-dimensional interaction by simply replacing the three-dimensional position vectors $\mathbf{r}_{1 / 2}$ with the one-dimensional positions $x_{1 / 2}$. This would yield an infinite changing energy for any two overlapping wave functions.

In order to get the effective one-dimensional interaction for a specific system, one has to integrate out the other space-dimensions, in our case the $y$ - and $z$-dimension. To do so, one has to know the confining wave functions in these directions, $\phi^{x}(y, z)$, provided one can make a separation of variables in the spirit of equation (2.3). Then the effective one-dimensional interaction is

$$
U\left(x_{1}, x_{2}\right)=\frac{e^{2}}{4 \pi} \int d z_{1} d z_{2} d y_{1} d y_{2} \frac{\left|\phi^{x_{1}}\left(y_{1}, z_{1}\right)\right|^{2}\left|\phi^{x_{2}}\left(y_{2}, z_{2}\right)\right|^{2}}{\sqrt{\left(x_{2}-x_{1}\right)^{2}+\left(y_{2}-y_{1}\right)^{2}+\left(z_{2}-z_{1}\right)^{2}}} .
$$

In Lunde et al. (2009), this integral has been carried out analytically, for a two-dimensional saddle-point potential and setting $z_{2}-z_{1}=0$. The latter is of course a simplification, which still can lead to an infinite charging energy. For most cases this integral has to be carried out numerically, however, this goes beyond the scope of this work. We deduce two main properties of the one-dimensional interaction from this integral form.

First, the interaction $U\left(x_{1}, x_{2}\right)$ depends explicitly on both positions $x_{1 / 2}$ and cannot be written as a function of their difference $x_{2}-x_{1}$. Second, the interaction is larger for those positions where the confinement in $y$ and $z$ direction is stronger, and thus the square of the absolute value $\left|\phi^{x_{1}}\left(y_{1}, z_{1}\right)\right|^{2}$ is larger. This again reflects the fact that the interaction times the DOS is the relevant strength of interaction. 
Note, that equation (2.23) and thus equation (2.24) applies only for the vacuum. In a typical setup, the QPC is surrounded by metallic gates, which have a large influence due to their high charge density. In order to get a realistic potential, one has to apply the method of mirror charges, which screens the potential.

Such a realistic treatment of interaction is beyond the scope of this work. We rather investigate the effects of a very short-ranged interaction.

\subsubsection{Discrete model with Interactions}

In order to calculate physical quantities, especially for the case where interactions are taken into account, it is convenient to discretize the one-dimensional model (2.4). The model Hamiltonian in continuous space without spin reads as

$$
H_{0}=-\frac{\hbar^{2}}{2 m} \partial_{x}^{2}+V(x)
$$

We set $x=j a$, where $a$ is the lattice-spacing and $j \in \mathbb{Z}$ is the position index. The discrete version of the potential then is $V_{j}=V(j a)$ and the first and second spacial derivatives read as

$$
\begin{aligned}
& \partial_{x} \psi(x)=\frac{\psi((j+1) a)-\psi(j a)}{a} \\
& \partial_{x}^{2} \psi(x)=\frac{\psi((j+1) a)-2 \psi(j a)+\psi((j-1) a)}{a^{2}} .
\end{aligned}
$$

Now we can write the Hamiltonian in the discrete form using second quantization, and add a Zeeman term

$$
H_{0}=\sum_{j, \sigma}\left[\left(V_{j}-\frac{\sigma}{2} B+2 \tau\right) d_{j \sigma}^{\dagger} d_{j}-\tau\left(d_{j+1 \sigma}^{\dagger} d_{j \sigma}+\text { h.c. }\right)\right],
$$

where $d_{j \sigma}^{+}\left(d_{j \sigma}\right)$ creates (annihilates) an electron with spin $\sigma$ on site $j$. We combined the prefactors into an energy called hopping amplitude,

$$
\tau=\frac{\hbar^{2}}{2 m a^{2}} \text {. }
$$

The total Hamiltonian is given by

$$
H=H_{0}+H_{\text {int }}
$$

with a short-ranged, i.e. onsite interaction given by

$$
H_{\text {int }}=\sum_{j} U_{j} n_{j \uparrow} n_{j \downarrow},
$$

where $n_{j \sigma}=d_{j \sigma}^{\dagger} d_{j \sigma}$ counts the number of electrons on site $j$ with spin $\sigma . U_{j}$ is larger where the confinement is smaller, i.e. it is largest in the center of the QPC and goes to zero where the system is actually two-dimensional. For this purely onsite interaction the exchange interaction is equal to the density-density interaction. If the interaction is long-ranged the exchange interaction is smaller than the density-density interaction. Hence in the present model the effects at finite fields are accentuated relative to spin symmetric effects, such as temperature effects or charge-susceptibility. 


\subsection{The origin of the 0.7 -anomaly}

After having discussed the noninteracting picture of QPCs we address properties which cannot be explained without considering many-body effects. The most prominent of these properties are all related to the regime where the conductance takes values around $0.7 G_{Q}$, and thus are collectively known as the 0.7 anomaly. Anomalous in this context means, that the features associated with the 0.7 anomaly cannot be explained in a noninteracting picture. In this section we will give an intuitive explanation for why interactions are important this regime. Later in this chapter, in section 2.5, we will address these features one by one, and explain on the intuitive level why they are affected by interactions. In chapter 5 we will corroborate these statements by detailed calculations which are also published in our NATURE article (Bauer et al., 2013).

First we define the regime of interest: When the gate voltage is tuned such that the conductance takes values between $0.5 G_{Q}$ and $0.9 G_{Q}$, we call the QPC "sub-open". The potential height $V_{g}$, measured w.r.t. the chemical potential, then has a small negative value (see figure 2.3):

$$
0>V_{g} \gtrsim-\Omega_{x} / 2 \text {. }
$$

In this sub-open regime electrons at the chemical potential move particular slowly in the center of the QPC, since their kinetic energy $\omega_{k}$ gets as small as the difference of the chemical potential and potential maximum, $\omega_{k}^{\min }=\mu-V(0)=-V_{g}$. This low kinetic energy has an important impact in the DOS, which is inversely proportional to the velocity as stated in equation (2.12). As a consequence the density of states at the chemical potential is particularly high in the center of the QPC. Thus the effective interaction $U \rho(\mu)$ in this regime is large, leading to the anomalous behavior.

This simple chain of arguments

« slow electrons - large DOS - large effective interaction - anomalous behavior »

is the essence of our explanation for the 0.7-anomaly. This argument explains why this regime is distinct and why one cannot apply the noninteracting picture here. As a direct consequence this picture explains why all expectations derived under the assumption of non-interacting particles fail in the sub-opne regime.

Of course these arguments explain why interactions are crucial and thus why the noninteraction picture breaks down. In order to provide a convincing argument, that this picture is indeed correct, one needs to reproduce as many features as possible with this model. Most of this work is dedicated to pursuing this goal. The first step is to get a detailed understanding of the noninteracting DOS. Figure 2.4 a shows the local DOS in grey scale as a function of energy $\omega$ and position $x$ for a parabolic potential barrier equation (2.13) (black line) with $V_{g}=0$. In regions where electrons are slow, the LDOS is enhanced (dark region). In a classical picture the velocity goes to zero at the classical turning-point and thus DOS and therefore the strength of interaction diverges. However, quantum-mechanically in an inhomogeneous system the velocity can never reach zero. Hence the divergence in the DOS is smeared out. The scale on which this happens is given by the geometric energy-scale $\Omega_{x}$. The maximum of the DOS lies $\mathcal{O}\left(\Omega_{x}\right)$ higher then the potential where its value is proportional to $\sqrt{m / \hbar^{3} \Omega_{x}}$ (see 2.4 b black solid 

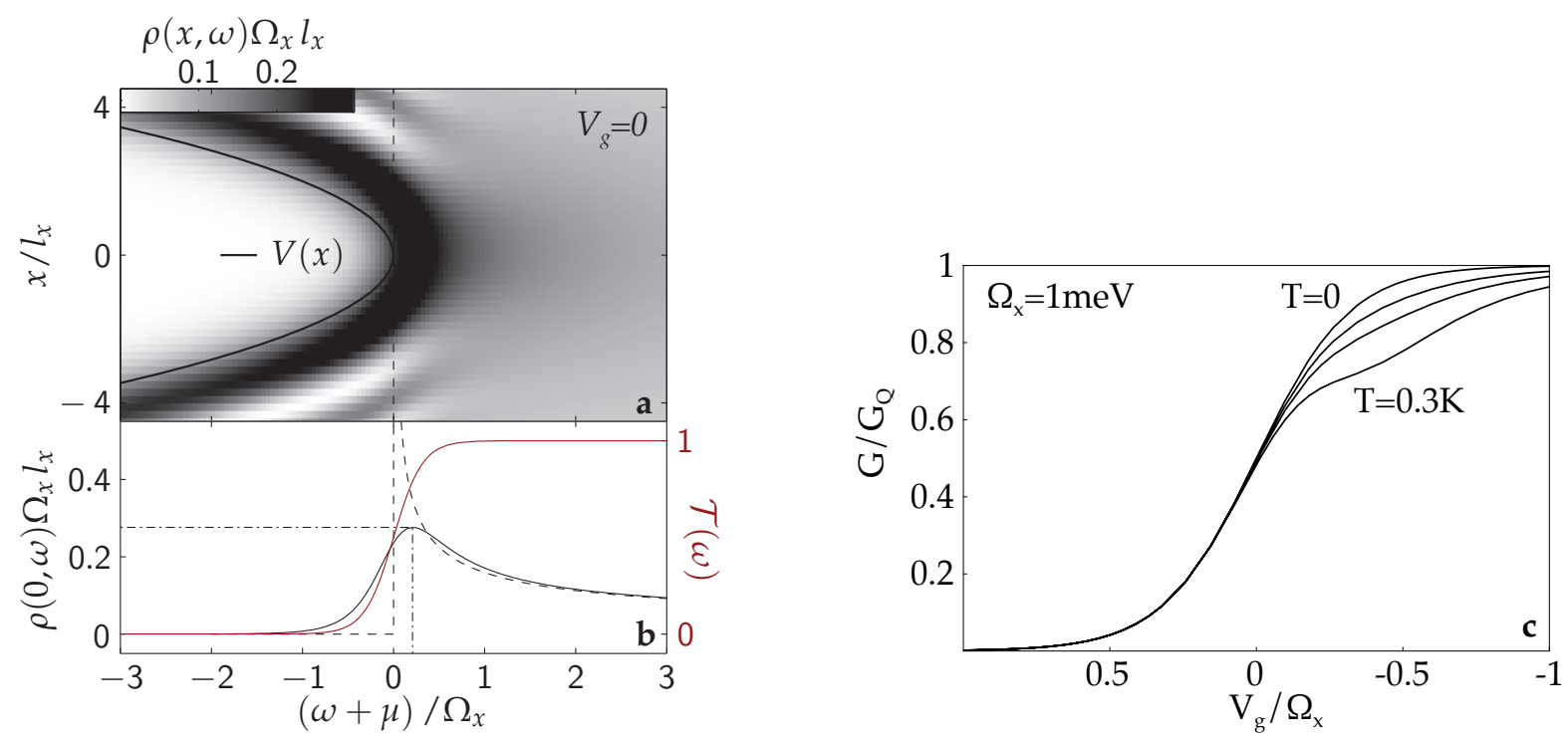

Figure 2.4: a $\operatorname{LDOS} \rho(x, \omega)$ as a function of position $x$ and energy $\omega$, measured w.r.t. the chemical potential $\mu$ (black dashed line) for the parabolic potential $V^{(2)}(x)(2.13)$ with $V_{g}=0$ (black solid line). $\mathbf{b}$ LDOS line like in a but for the center of the QPC $\rho(x=0, \omega)$ (black solid line) and for a homogeneous one-dimensional system (black dashed line), and one partical transmission probability $\mathcal{T}(\omega)$ as a function of energy $\omega$ (red line). c Reprinted figure from Sloggett et al. (2008) (but in our notation): conductance $G$ as a function of gate Voltage $V_{g}$ for different temperatures $T$ between $T=0$ (uppermost curve) and $T=0.3 \mathrm{~K}$ (lowest curve), copyright (2008) by Springer-Verlag.

line), meaning that the DOS is highest in the middle of the sub-open regime (compare red line in $2.4 \mathbf{b})$.

It is worth noting, that both, the conductance quantization, and the 0.7 -anomaly are results of one distinctive feature of electrons in one dimension: their density of states is inversely proportional to their velocity.

\subsubsection{Related Models}

The picture presented above provides a natural explanation for the origin of the anomalous behavior in the sub-open regime. This point of view has already been presented by others, e.g. Sloggett et al. (2008) or Lunde et al. (2009), however, despite its simplicity, it wasn't published prominently and was rarely cited. We will briefly discuss these two works.

\section{Kinetic equation}

Sloggett et al. (2008) have the same line of arguments, which they express via the "the conducting wave-functions" which become peaked "enhancing the electron-electron interaction". 
Further they argue that "away from the contact the interaction is strongly suppressed by screening". Hence the only input into their calculation is the LDOS in the center of the QPC of a parabolic potential barrier (black solid line in figure $2.4 \mathbf{b}$ ). This LDOS enters in the purely negative correction to the non-interaction conductance. The final result of this calculation in shown in figure $2.4 \mathbf{c}$. This is an impressive example of how the right intuition for the physical situation can directly lead to astonishing results, which shows an almost perfect agreement with experiments (compare e.g. figure 1.2 or figure 2.5). The downside of such a calculation is that it is constructed such that the correction to the conductance is purely negative and strongest in the sub-open regime. Thus it compulsorily leads to this results, not giving any additional corroboration for the physical picture. Further, the assumption that the zero temperature $(T=0)$ conductance is equal to the non-interaction one and that interaction are only present in the center of the QPC is a huge simplification. Nevertheless, the calculation can serve as a nice illustration of the very convincing argument.

\section{Momentum-nonconserving backscattering using WKB-Wavefunctions}

Another analytic calculation for a purely parabolic potential was presented by Lunde et al. (2009). This calculation uses semiclassical Wentzel, Kramers, Brillouin (WKB) wave-functions (see e.g. Sakurai, 1993, chapter 2.4), thereby taking into account the real-space structure of the system. The semiclassical approximation is only valid if the kinetic energy $\omega_{k}$ is larger than $\Omega_{x}$, since in this approximation the squared amplitude of the wave-function, i.e. the LDOS, diverges like $1 / \sqrt{\omega_{k}}$. In their approach Lunde et al. (2009) only take Fock-like diagrams into account, which have an influence only at finite temperature and finite source-drain-voltage, but not at finite magnetic field. An interaction enhanced backscattering as a function of magnetic field would have to be described by Hartree-type diagrams, which, they do not treat explicitly but they absorb into the bare potential. Further, the Hartree type diagrams would also alter the temperature and voltage dependence. They show, amongst others, that a single momentum-non-conserving scattering process, where two electrons approach the contact and one electron gets reflected while the other gets transmitted is the leading process which increases exponentially when the potential top approaches the chemical potential. This process leads to an enhanced backscattering in the sub-open regime, qualitatively explaining the anomalous behavior.

\subsection{Experimental features of the 0.7-anomaly}

Next, we will give a brief overview of the various experimental features, which are related to the 0.7 -anomaly. This overview does not claim to be complete or detailed in every aspect, but rather mentions only the most important features and those which are connected to our work. For a comprehensive summary of experimental results as well as a brief overview of theoretical publications see Micolich (2011). 

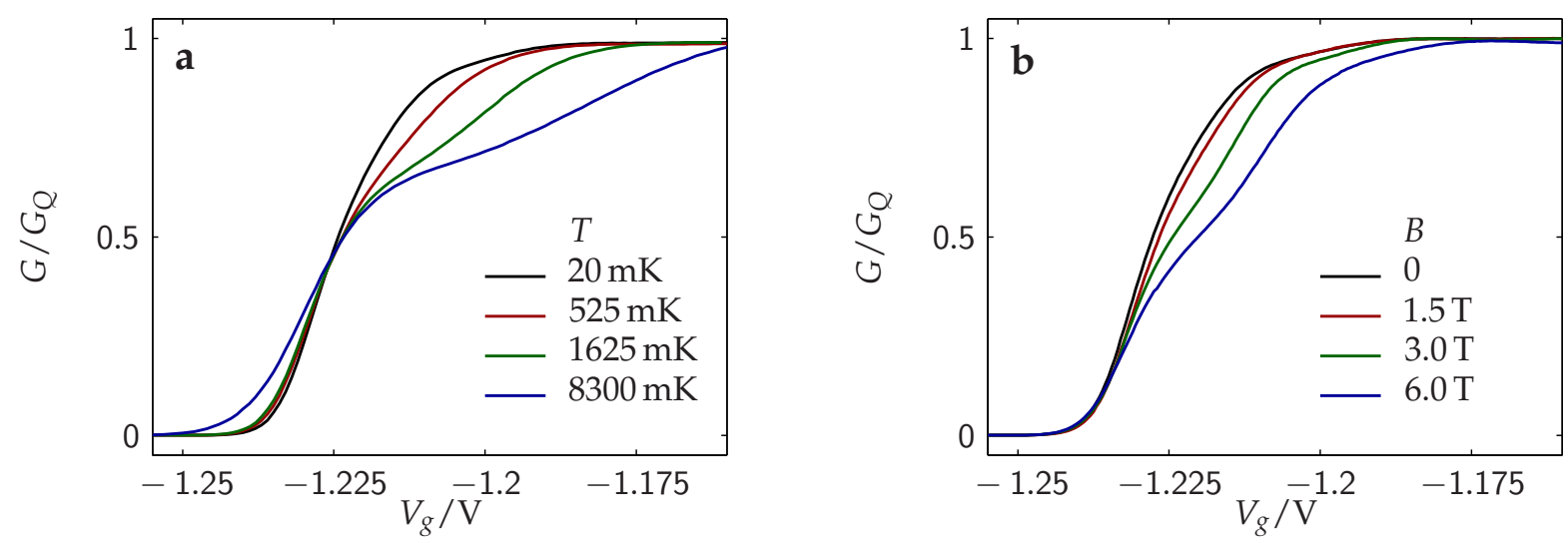

Figure 2.5: a Measured conductance $G$ as a function of applied gate voltage $V_{g}$ for different temperatures at zero magnetic field. $\mathbf{b}$ Same as in $\mathbf{a}$ but for different magnetic fields at the lowest temperature $T=20 \mathrm{mK}$. Data from Borowsky (2011).

\subsubsection{Temperature Dependence}

At temperatures around $1 \mathrm{~K}$, measured linear conductance curves of most clean QPCs show a pronounced shoulder at around $0.7 G_{Q}$, as shown in figure 2.5 a green line, and in figure 1.2 a (see also Thomas et al., 1998). This shoulder gradually weakens if temperature is lowered. Since in most measurements the temperature is large enough to see this shoulder, this effect is certainly the most prominent one and is the reason why the phenomenology in this parameter regime is known as the 0.7 -anomaly.

In the noninteracting picture, the effect of temperature is to smear out the conductance in a symmetric fashion. This smearing out gets relevant when the temperature is of the order of the geometric energy scale $\Omega_{x} /(2 \pi)$. Typically $\Omega_{x} \sim 1 \mathrm{meV}$ wich means $\Omega_{x} /(2 \pi) \sim 2 \mathrm{~K}$. So in order to explain the temperature-dependence in this regime one needs to invoke interacting many body effects. As explained in detail in chapter 5 (see also Bauer et al., 2013) our picture gives a natural explanation why the conductance is affected most in the sub-open. The large LDOS leads to a combination of elastic backscattering due to additional charge at finite temperature, and strong inelastic scattering due to the large phase-space in the temperature window around the fermi-surface.

A very important question is how the conductance curve is shaped in the limit where temperature goes to zero. In experiments it is of course impossible to reach zero temperature, but modern dilution refrigerators can reach temperatures down to several milli-Kelvin, which would be low enough to give an answer to this question. However, at these temperatures energy transfer from electrons to phonons is very inefficient, and the temperature of the electron bath is not necessarily equal to the phonon temperature. The former is heated by the measuring process and by external wiring. Hence, the electronic temperature is often higher than the dilution temperature, which is usually stated in experimental publications as temperature. In the measurements shown in figure 2.5 a black line, it was ensured, that the shape of the conductance curve has saturated as a function of temperature, and will hardly change if temperature 
is lowered even further. This shows, that the conductance curve is still asymmetric at zero temperature. This feature is reproduced by our calculations using fRG, and we give a detailed explanation in chapter 5 (see also Bauer et al., 2013).

\subsubsection{Dependence on Magnetic Field}

In this work we only consider the situation where the magnetic field is parallel to the plane of the 2DES. Hence, the magnetic field does not couple to motion of the electrons, but to the spin degree of freedom. A finite magnetic field lifts the degeneracy of spin up and spin down, leading to a conductance that is quantized in units of $e^{2} / h=G_{Q} / 2$. This is seen in experiments at large fields (blue line in figure $2.5 \mathrm{~b}$ ). In contrast to the noninteracting picture, where the spin up and spin down conductance step are shifted in $V_{g}$ by the same amount in opposite directions, in the measurements the position of the first conductance step is almost unchanged w.r.t. zero magnetic field, while the second step is strongly shifted. This shift corresponds to an energy which is about two to three times larger than the bulk Zeeman energy implying an enhanced electon $g$-factor (Koop et al., 2007). From the noninteracting picture one expects that the two spin-degenerate conductance steps should be identical in shape. The experimental data shows a shoulder at around $0.7 G_{Q}$ at low fields, similar to the 0.7 -shoulder at finite temperature. All these features point to a large exchange interaction in the QPC, as given by an interaction term (2.30).

These features are reproduced by our calculations using fRG and SOPT, and their origin is explained in detail section 3.3.6 (see also Bauer et al., 2014) and chapter 5 (see also Bauer et al., 2013), using a simple density-density interaction argument: at finite magnetic field, spin up electrons flow into the QPC, such that spin down electrons not only have to overcome the Zeeman energy but also the Coulomb repulsion of the additional spin up electrons. Since the inflow of spin up electrons depends on the DOS, this effect is strongest in the sub-open regime. This intuitive picture might suggest that it is sufficient to perform a purely Hartreetype calculation to get these results. This turns out to be true, but including Fock-type diagrams into the calculation improves the agreement with experimental data.

Furthermore, we discuss the interplay of magnetic field with spin orbit interaction (SOI) in chapter 6 . The latter has an influence on the dispersion relation, and thus alters the DOS, $\rho=\partial k / \partial \omega$, leading to additional features if the spin orbit energy $E_{\mathrm{SOI}}=\alpha^{2} m / 2 \hbar^{2}$ (where $\alpha$ characterizes the strength of spin orbit coupling (see e.g. Winkler, 2003)) is of the order of the geometric energy scale $\Omega_{x}$. We present detailed theoretical predictions; their future experimental tests will judge the validity of the model presented in this work.

\subsubsection{Finite Source-Drain Voltage}

So far, we only considered the linear response conductance, i.e. the limit where the applied source-drain voltage, $V_{\mathrm{sd}}$, goes to zero. At finite voltage, the non-linear conductance $G_{\mathrm{nl}}=$ $\partial I / \partial V_{\text {sd }}$ shows a large variety of features. Figure 2.6 a shows $G_{\mathrm{nl}}$ as a function of applied voltage for different gate voltages. For those values of gate voltage where the linear conductance $G / G_{Q}$ rises from zero to one, the nonlinear conductance has a well pronounced narrow maximum at zero voltage, the zero-bias peak (ZBP). This feature has been attributed to interaction 

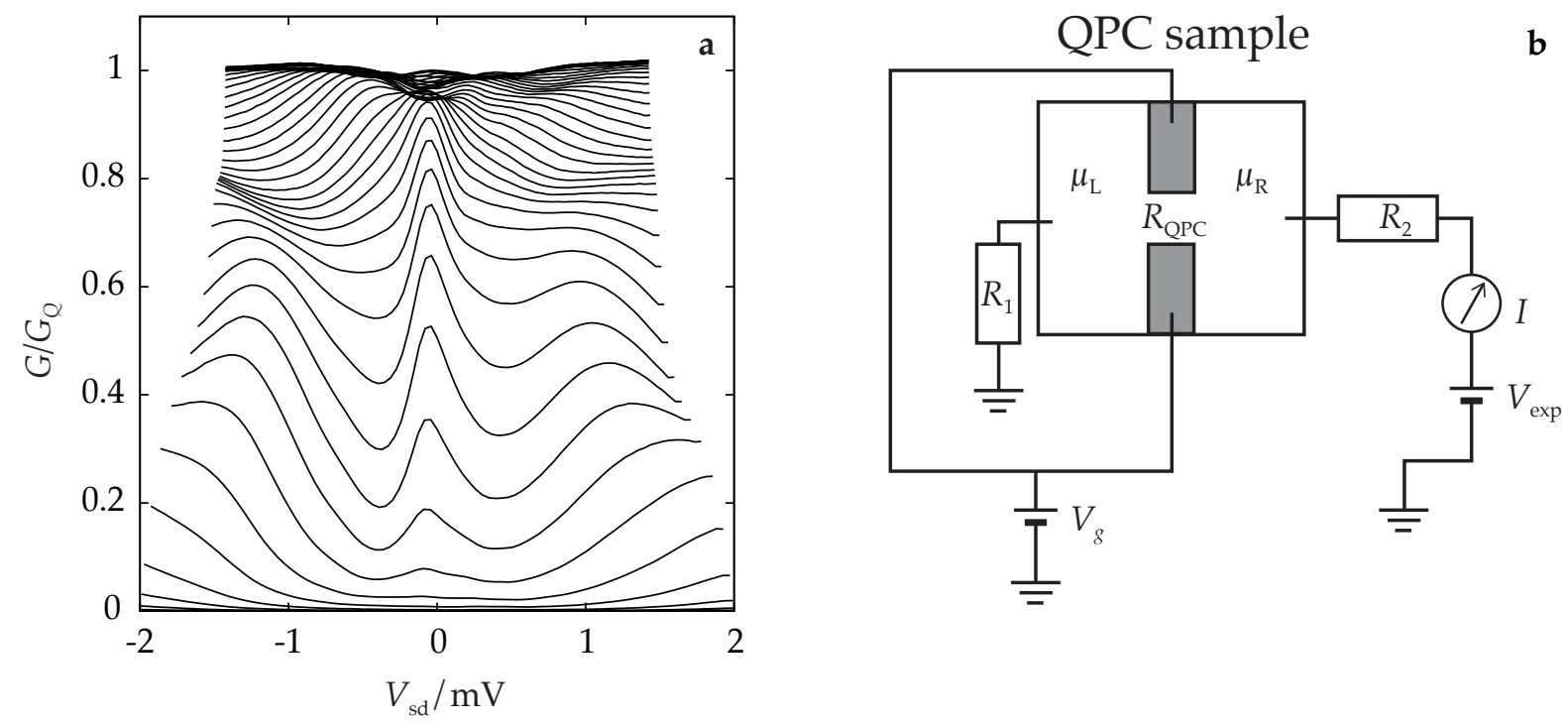

Figure 2.6: a Non-linear conductance $G_{\mathrm{nl}}=\partial I / \partial V_{\mathrm{sd}}$ as a function of applied voltage $V_{\mathrm{sd}}$ for different gate voltages $V_{g}$ in the regime where the linear conductance $G / G_{Q}$ takes values between zero and one. Data from Borowsky (2011). b simplified circuit diagram of the measurement setup. The current $I$ resulting from an applied voltage $V_{\text {exp }}$, not only is determined by the resistance of the QPC $R_{\mathrm{QPC}}$, but also by the resistance of the wiring and measuring devices $R_{1}$ and $R_{2}$. Applying a voltage increases the chemical potential on both sides $\mu_{L}$ and $\mu_{R}$, but with a different magnitude. The voltage is defined by the resulting difference of the chemical potential $e V_{\mathrm{sd}}=\mu_{R}-\mu_{L}$

effects, which is not entirely correct. Consider the non-interacting, non-linear zero-temperature conductance following equation (2.10)

$$
G_{\mathrm{nl}}=\frac{G_{Q}}{2}\left[\mathcal{T}\left(e V_{\mathrm{sd}} / 2\right)+\mathcal{T}\left(-e V_{\mathrm{sd}} / 2\right)\right]
$$

With the transmission (2.16) this conductance has a ZBP for $V_{g}<0$, i.e. when $G>0.5 G_{Q}$. To be more general, there is a ZBP in the non-interacting conductance whenever $\mathcal{T}(\omega)$ is a concave function of $\omega$ around $\omega=0$.

Interactions modify this in two ways: the width of the ZBP is much smaller than in the non-interacting picture, and a ZBP arises even for gate voltages where $\mathcal{T}(\omega)$ is convex. These two features are reproduced by our calculations using SOPT, and the microscopic mechanism, which is similar to the mechanism at finite temperature, is explained in detail in chapter 5, (see also Bauer et al., 2013).

Next, we will discuss the validity of the theoretical model at finite voltage. In the above equation (2.32), we assumed that the voltage is applied symmetrically, i.e. $\mu_{L}=-e V_{\mathrm{sd}} / 2$ and $\mu_{R}=e V_{\text {sd }} / 2$. However, this is typically not the case in the experimental setup. Figure $2.6 \mathbf{b}$ shows a simplified circuit diagram of a typical setup. Usually all voltages are applied w.r.t. a 
common ground. Due to the fact that the wiring on both sides of the QPC sample has a finite resistance, $R_{1}$ and $R_{2}$, an applied voltage $V_{\exp }$ increases the chemical potentials $\mu_{L}$ and $\mu_{R}$ on both sides. The resistance of the QPC, $R_{\mathrm{QPC}}$, dominates the total resistance, however, $R_{1}$ and $R_{2}$ are of order $2 \mathrm{k} \Omega$, which is non-negligible w.r.t. $R_{\mathrm{QPC}}$, which is of order $1 / G_{Q} \simeq 12.9 \mathrm{k} \Omega$. Expressing the chemical potentials in terms of the source-drain voltage $e V_{\mathrm{sd}}=\mu_{r}-\mu_{L}$ gives

$$
\mu_{L}=\frac{R_{1}}{R_{\mathrm{QPC}}} e V_{\mathrm{sd}}, \quad \mu_{R}=\left(\frac{R_{1}}{R_{\mathrm{QPC}}}+1\right) e V_{\mathrm{sd}},
$$

where both $\mu_{L}$ and $\mu_{R}$ have the same sign as $V_{\text {sd }}$. Nevertheless the conductance as a function of applied voltage is almost symmetric (see figure 2.6 a). Using these chemical potentials in the theoretical calculation would yield completely asymmetric curves, even if interactions would be taken into account. The major difference between the experiment and the theoretical model is that in the experimental setup, the leads are extended 2DESs. Due to their extension they also act like gates on the QPC potential. In the model we assumed that the latter is static, and results only from the gate structure controlled by $V_{g}$.

Let's estimate the effect of voltage onto the potential. The potential at finite voltage will be the sum of the bare potential and a potential resulting from the two leads:

$$
V(x)=V_{0}(x)+V_{l}(x) .
$$

We assume that $V_{l}$ changes linearly between the two leads over their spatial separation $d$ :

$$
V_{l}(x)=\mu_{L}+\frac{V_{\mathrm{sd}}}{d}(x+d / 2) \text {. }
$$

Then

$$
\begin{aligned}
V(x) & =V_{g}-m \frac{\Omega_{x}^{2}}{2 \hbar^{2}} x^{2}+\mu_{L}+\frac{V_{\mathrm{sd}}}{d}(x+d / 2) \\
& =V_{g}+\mu-m \frac{\Omega_{x}^{2}}{2 \hbar^{2}}\left(x-\frac{e V_{\mathrm{sd}} \hbar^{2}}{d m \Omega_{x}^{2}}\right)^{2}-\Omega_{x}\left(\frac{l_{x}}{d}\right)^{2}\left(\frac{e V_{\mathrm{sd}}}{\Omega_{x}}\right)^{2},
\end{aligned}
$$

with $\mu=\mu_{L}+\frac{V_{\mathrm{sd}}}{2}=\frac{\mu_{L}+\mu_{R}}{2}$. Hence, for small applied voltage we can indeed measure everything w.r.t. the average chemical potential $\mu$, with $\mu_{L / R}=\mu \mp \frac{V_{\text {sd }}}{2}$. For large voltages $e V_{\text {sd }} \gtrsim \Omega_{x}$ higher order terms will become important. In addition to the non-linear term in equation (2.36), there will be non-linear terms in $V_{l}(x)$. All these terms will depend on many details. An estimate for these terms would require solving a three-dimensional Poisson equation, which is beyond the scope of this work. In this work we only consider the case $e V_{\text {sd }}<\Omega_{x}$.

One of the features at large voltage is the side-peak structure arising for conductance values around $0.5 G_{Q}$ (see figure 2.6 a). This feature is reminiscent of the Hubbard side peak arising for quantum dots in the Kondo regime (see section 2.6.2). We are not able to reproduce this feature using SOPT. The reason is that, additionally to the question of the appropriate potential at large voltage, there are theoretical issues in this parameter regime: The SOPT is an approach that is perturbative in the interaction and is less reliable or large energies, and thus for large voltage. Whether or not the proposed model reproduces this feature, has to be answered with a different method, more suited for this regime, and is left for future studies. 

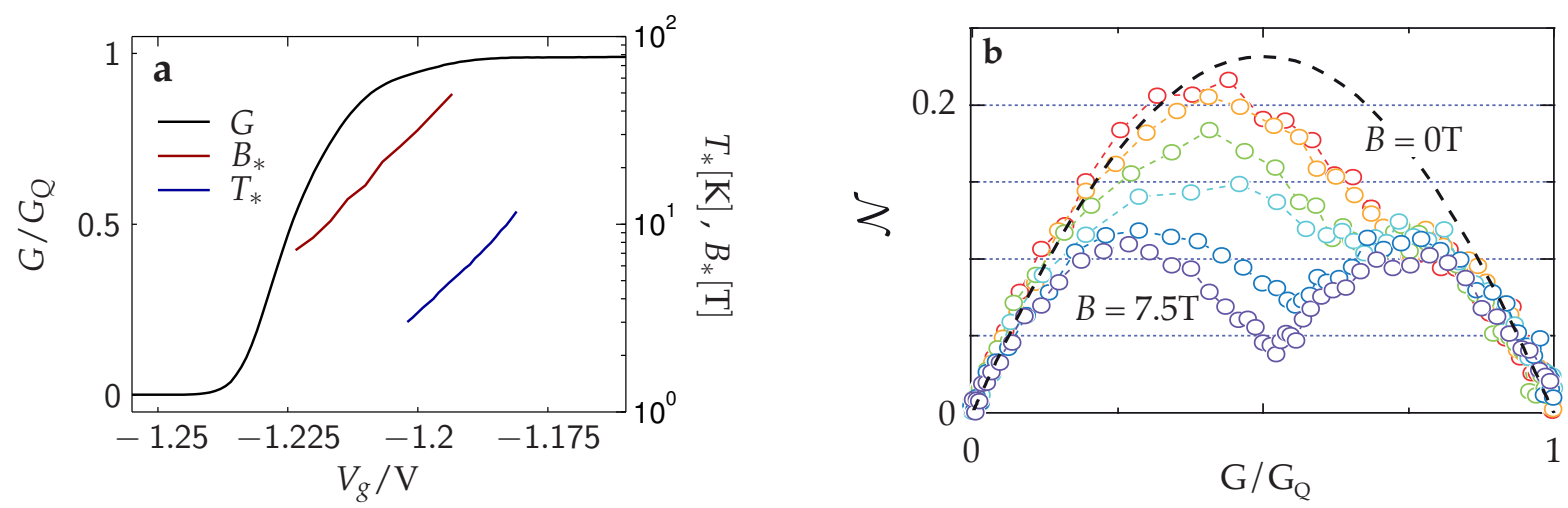

Figure 2.7: a Conductance (black line), field scale $B_{*}$ (red line) and temperature scale $T_{*}$ (blue line) on a log-scale as a function of applied gate voltage $V_{g}$. Data from Borowsky (2011). b Shot-noise factor as a function function of conductance $G$. Reprinted figure from DiCarlo et al. (2006), copyright (2006) by the American Physical Society.

\subsubsection{Low Energy Scale}

The dependence of the conductance on temperature $T$, magnetic field $B$ and voltage $V_{\text {sd }}$, at low values, can be analyzed in terms of the low-energy scales $T_{*}, B_{*}$ and $V_{\mathrm{sd} *}$ defined via the quadratic response of the conductance on these parameters:

$$
\frac{G_{\mathrm{rl}}\left(T, B, V_{\mathrm{sd}}\right)}{G(0,0,0)}=1-\left(\frac{T}{T_{*}}\right)^{2}-\left(\frac{B}{B_{*}}\right)^{2}-\left(\frac{V_{\mathrm{sd}}}{V_{\mathrm{sd} *}}\right)^{2}
$$

Such an analysis was first made by Cronenwett et al. (2002) for temperature and voltage. Motivated by the Kondo effect, they called these scales Kondo temperature $T_{K}$, and fitted the temperature dependence with the function

$$
G=\frac{e^{2}}{h}\left(f\left(\frac{T}{T_{K}}\right)+1\right), \quad f(x)=\left[1+\left(2^{1 / s}-1\right) x^{2}\right]^{s},
$$

with $s=0.22$, which is also motivated by the Kondo effect. Using this function they assumed, that the conductance $G / G_{Q}$ always reaches unity at $T=0$, and goes to $\frac{1}{2}$ for large temperature. However, these assumptions do not hold general. In the regime where the former does hold, this definition is equivalent to our definition of $T_{*}$.

These scales, $T_{*}, B_{*}$ and $V_{\mathrm{sd} *}$, turn out to depend exponentially on gate volage in the subopen regime

$$
T_{*} \propto B_{*} \propto V_{\mathrm{sd} *} \propto e^{c V_{g}},
$$

shown for $B_{*}$ and $T_{*}$ in figure 2.7 a ( $c$ is a geometry dependent constant). In first instance this may indeed sound reminescent of the Kondo-scale $T_{k}$ seen in QDs, for which $T_{K} \propto e^{c_{K} V_{g}^{2}}$. The conclusion of Cronenwett et al. (2002) was that this scale dependence is a result of interactions. However, the non-interacting transmission for a parabolic QPC (2.16), directly leads to the 
exponential dependence, due to the occurrence of the exponential $\omega$-dependence. A detailed calculation can be found in section S-5.A in the supplementary information of our NATURE publication (see chapter 5 or Bauer et al., 2013). The dependence of the low energy scale on interaction and geometry is discussed in section S-5.B and S-5.C respectively.

\subsubsection{Shot Noise}

DiCarlo et al. (2006) presented shot noise data measured for the first conductance step of a QPC. The results are shown in figure $2.7 \mathbf{b}$. In the regime where the conductance takes values around $0.7 G_{Q}$, the shot-noise is reduced with respect to its non-interacting value (Blanter and Büttiker, 2000)

$$
\mathcal{N}=\frac{1}{2} \sum_{\sigma} \mathcal{T}_{\sigma}\left(1-\mathcal{T}_{\sigma}\right)
$$

shown as dashed line in figure 2.7. If $\mathcal{T}_{\uparrow}=\mathcal{T}_{\downarrow}$ and $T=0$ equation (2.40) still holds at finite interaction. So if there is no spin polarization (see section 2.6.1), the reduction of shot noise is a finite temperature effect, where the shot noise is difficult to calculate, and goes beyond the scope of this work. At $T=0$ we can reproduce the evolution of the shot noise factor with magnetic field $B$ using fRG, as shown in our NATURE publication (see chapter 5, Bauer et al., 2013, see also section S-3.A of the supplementary information).

\subsection{Other Theoretical Models}

Over the years many physicists have approached the problem of the 0.7-anomaly, and thus a lot of ideas for what might be the origin of this phenomena came up. Some of these ideas are still being pursued. At the moment there is a controversial discussion, about what really happens inside a QPC in the regime of the 0.7-anomaly. We will briefly introduce two of the most prominent models.

\subsubsection{Spin Polarization}

In the first publication where the anomalous behavior, which later became known as the 0.7anomaly, was identified, Thomas et al. (1996) also introduced the first idea about what might be its origin: They suggested that the current is spin polarized, such that one spin-species fully contributes to the conductance while the other spin species is partially reflected, leading to a conductance between $0.5 G_{Q}$ and $G_{Q}$. This view arose, because the double-step structure, which can be explained at large magnetic fields (see section 2.5.2) is still present at zero field. Thomas et al. (1996) draw the conclusion, that there might be a spontaneous symmetry breaking leading to an intrinsic magnetic field, explaining the phenomena. This argument is corroborated by theoretical calculations, e.g. by Berggren and Yakimenko (2002). Most of the published calculations are performed in an two-dimensional system within a three-dimensional environment with Coulomb interaction, however, very similar results can be obtained for a one-dimensional system with point-like interaction. Interactions are incorporated in a self-consistent Hartree 


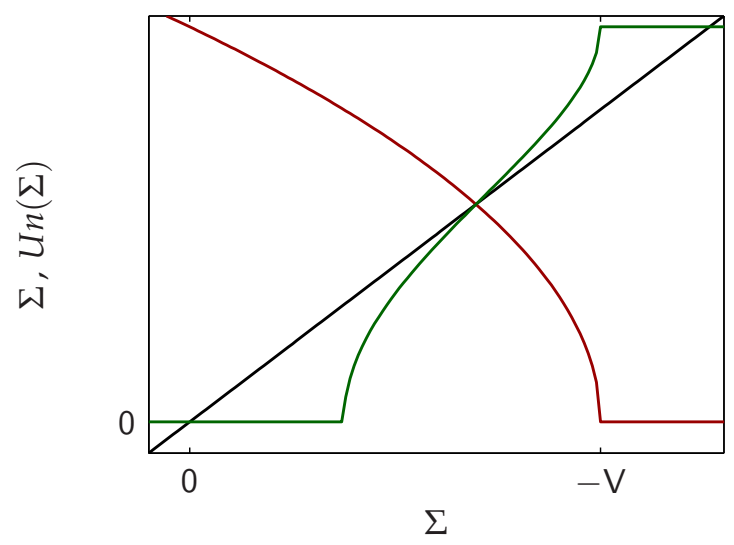

Figure 2.8: Geometric construction of the solution of equation (2.44) (red line) and equation (2.45) (green line). The solutions of these equations is the intersection with the identity (black line).

approximation resulting in an effective potential

$$
V^{\mathrm{eff}}(x)=V(x)+\Sigma(x),
$$

where $V$ is the bare potential, measured w.r.t. the chemical potential, and $\Sigma$ the self-energy describing the effects of interaction. The self-consistent Hartree-equation for $\Sigma$ reads as

$$
\Sigma=U \cdot n(\Sigma)=U \int d \omega f(\omega) \rho(\omega, \Sigma),
$$

where $U$ is the interaction, $n(\Sigma)$ the density which depends on the self-energy, $f$ the fermi function and $\rho$ the DOS. This calculation is relatively simple, and its result can be illustrated by a homogeneous one-dimensional system where

$$
n \propto \sqrt{-V-\Sigma}
$$

which is nonzero only for $V<0$. Absorbing all pre-factors into the interaction $U$ gives

$$
\Sigma=U \sqrt{-V-\Sigma} .
$$

Figure 2.8 illustrates the solution of this equation: both the left hand side (black line) and the right hand side (red line) of this equation are plotted as a function of $\Sigma$. The unique solution is the intersection of these lines. We now add the spin degree of freedom and set the interaction $U$ equal to the exchange interaction, then

$$
\Sigma^{\sigma}=U \cdot n^{\bar{\sigma}}(\Sigma)=U \sqrt{-V-\Sigma^{\bar{\sigma}}}=U \sqrt{-V-U \sqrt{-V-\Sigma^{\sigma}}},
$$

whose right hand side is plotted as green line in figure 2.8 , and intersects with the identity (black line) in three points, providing three solutions of equation (2.45). One of them is equal to the solution of the spin symmetric equation (2.44), while the other two provide symmetry broken solutions.

To incorporate the QPC potential one can use the model equation (2.29), i.e. the chain model with onsite interaction. Due to the inhomogeneity the DOS $\rho(\omega)$ is smeared out (see figure 2.4). 

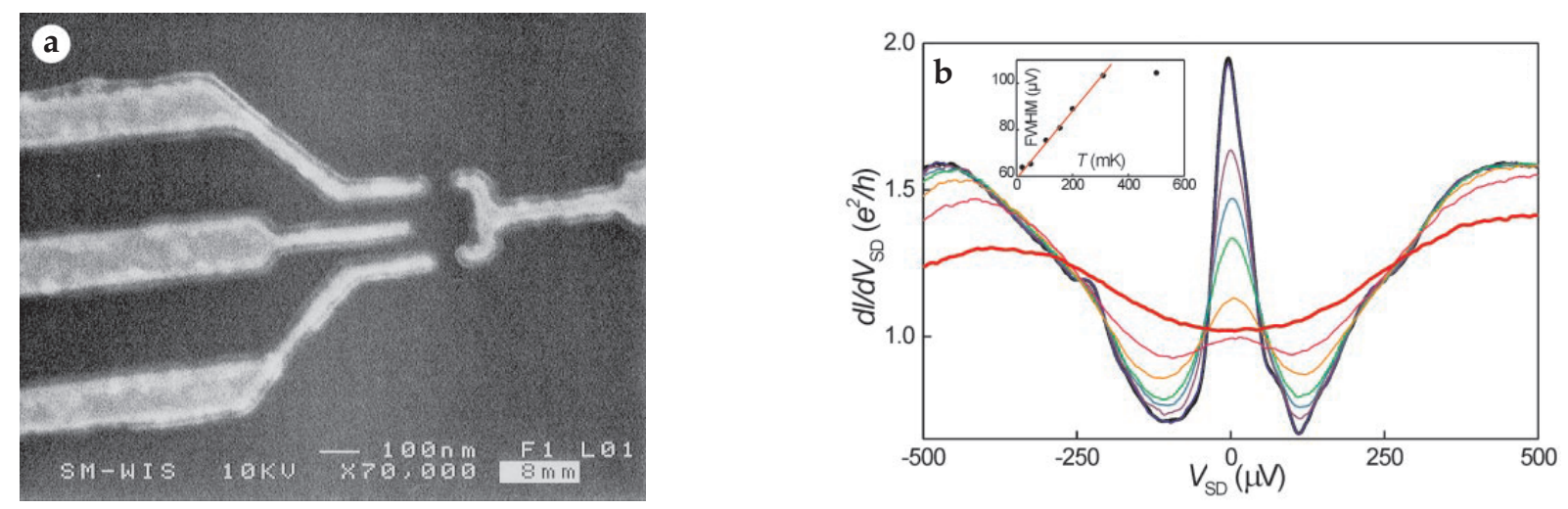

Figure 2.9: a Quantum dot structure reprinted from Goldhaber-Gordon et al. (1998), copyright (1998) by Nature Publishing Group. b Non-linear conductance $G_{n l}=d I / d V_{\text {sd }}$ as a function of applied voltage $V_{\mathrm{sd}}$ for different temperatures between $15 \mathrm{mK}$ (black line) and $900 \mathrm{mK}$ (red line). Reprinted figure from van der Wiel et al. (2000), copyright (2000) by AAAS.

This has two consequences: First, the density $n(\Sigma)$ is also smeared out, and thus the smallest solution for $\Sigma$ is nonzero. Second, the DOS at the chemical potential $\rho(\mu)$ is bounded and symmetry-broken solutions exist only if $U \rho(\mu)>1$.

Solving the self-consistent Hartree equations in two dimensions does not lead to any additional qualitative features. Nevertheless a two-dimensional calculation is useful to derive the QPC-potential from first principles. We believe however, that the symmetric solution is the physical solution, while the other two solutions are artifacts of the approach. This assumption is corroborated by experimental results, which point to a Fermi-liquid behavior. For a detailed discussion see section S-2.C in the supplementary information of our NATURE article (see chapter 5, Bauer et al., 2013).

To summarize, the idea of spin polarization is actually based on the same or similar model as equation (2.29). Further, as in our scenario, the relevant scale for the interaction is $U \rho(\mu)$. What differs is the interpretation of what happens for such a model. In contrast to the proponents of the spontaneous spin polarization scenario, we do not believe that there is any kind of symmetry breaking in a one-dimensional system.

The above calculation strikes a nerve of a numerical issue: Suppose you want to incorporate effects of interactions via the self-consistent Hartree equation (2.42), and want to get the symmetric solution. An iterative algorithm however, will always lead to the symmetry-broken solution (if the latter exists, i.e. if $U \rho(\mu)>1$ ). At zero magnetic field, this is not a big problem, since one can solve the equation with the boundary condition $\Sigma^{\uparrow}=\Sigma^{\downarrow}$, and as a consequence the solution is unique. However, at finite magnetic field, there are three solutions and in our experience numerical algorithms tend to flow into the solution corresponding to the symmetry broken solution at zero field. 


\subsubsection{Kondo Model - Formation of a localized State}

In 2002 two publications, an experimental paper by Cronenwett et al. (2002), and a theoretical paper by Meir et al. (2002), linked the 0.7-anomaly to the Kondo effect seen in quantum dots (QDs). A QD is a small island for electrons, which is weakly coupled to an electron bath with continuous dispersion. A QD can be realized in a 2DEG by a structure such as that shown in figure 2.9, which is essentially a series of two QPCs. The number of electrons in the center can be tuned by an additional independent gate.

The idea is that in the regime of the 0.7-anomaly, a localized state forms inside the QPC. The anomalous behavior then is explained in terms of the Kondo effect. The Kondo effect is a very complicated, but well-understood many-body state, which is present in quantum dots and other kinds of artificial and non-artificial atoms, which have two degenerate states, and large coulomb repulsion which prevents them from being fully occupied. A nice introduction into this subject is given by the review of Kouwenhoven and Glazman (2001).

The conclusion that the 0.7-anomaly is related to the Kondo effect was drawn mainly because of the similarities in the shape of the conductance as a function of source-drain voltage. The nonlinear conductance as a function of applied voltage for a QD is shown in figure 2.8. Its shape is similar to that of the non-linear conductance of a QPC, shown in figure 2.6. However, there are important differences beween these curves. In a $Q D$, the conductance $G / G_{Q}$ at zero voltage reaches unity. ${ }^{1}$ This is a central aspect in the Kondo effect: The local electron forms a many-body singlet state with the electrons in the bath, lying at the Fermi energy. As a result at zero temperature there is no backscattering. This is not the case in the QPC, and all other properties of QPCs differ in many aspects from the properties of QDs. In order to make concrete this idea, Meir et al. (2002) proposed a modified model for the localized state of a QPC, where in contrast to the common model for a $\mathrm{QD}$, the localized state is only coupled to the energies of the bath, which are larger then the energy of the localized state. However, until now no rigorous calculation has been presented, which shows, that this model indeed reproduces the phenomenology of the 0.7-anomaly. Further, no microscopic mechanism has been presented which leads to this asymmetric coupling.

In our opinion, up to now there is no conclusive evidence that supports the theory that the 0.7 anomaly can be explained in terms of a localized state. The similarities of these two effects originate in the fact, that both non-interacting systems have a peaked DOS: a Lorenz-curve in the case of the QD and the smeared van Hove singularity (see figure 2.4) in the case of a QPC. The differences between these two systems can be explained by the many differences of the non-interacting DOS (see discussion in section S-4.C of the supplementary information of our NATURE article, chapter 5).

The fact that the 0.7-anomaly is not related to a localized state, does not imply, that the formation of a localized state inside a QPC is not possible. In long QPC or quantum wires it is expected that electrons form a Wigner crystal at low density, which is shown e.g. by Güçlü et al. (2009). In this regime, the QPC is expected to show all features of a QD, which are different from the features of the 0.7-anomaly. Since there is a continuous transition between the two extrema, namely the 0.7 regime and the formation of a localized state, one can also get some

\footnotetext{
${ }^{1}$ note that in figure 2.8 , the conductance is plotted in units of $e^{2} / h=G_{Q} / 2$.
} 
kind of mixing of these two situations. A detailed discussion of this transition is beyond the scope of this work; however, we claim that all these effects can be understood in terms of a continuous modification of the potential, which will result in a continuous deformation of the DOS, from the smeared van Hove singularity to a peak with Lorenzian shape. 


\section{Chapter 3}

\section{Functional Renormalization Group}

In this chapter we derive the fRG equations, and describe the approximations used to treat one-dimensional chain models with on-site interactions. We use the fRG in its one-particle irreducible representation, i.e. a set of hierarchical ordinary differential equations in the vertex functions. The terminology "one-particle irreducible" originates from the fact that vertex functions have the diagrammatic property of being one-particle irreducible. In this work diagrammatic concepts are only used for illustrative purposes. A diagrammatic definition of these objects in the context of $f R G$, as well as a purely diagrammatic derivation of $f R G$, is given in the $\mathrm{PhD}$ thesis of Jakobs (2009). In this chapter we review the derivation of the fRG equation within the functional integral formulation. In the following we presume a detailed knowledge of functional integrals in the coherent state representation using Grassmann algebra, which is introduced in the book of Negele and Orland (1988) in the Matsubara formalism, and in the review of Kamenev and Levchenko (2009) in the Keldysh formalism. Both formalisms are introduced in the context of fRG in the thesis of Karrasch (2010).

We will define Green's and vertex functions via the action $S$ (section 3.1), and derive the fRG equations (section 3.2) without defining the action $S$ explicitly. The formalism isi fully specified, once the action has been defined, which we will do before introducing the approximations (section 3.3).

We are only interested in describing electrons, thus we restrict ourselves to fermions. An introduction to fRG in Matsubara formalism for both fermions and bosons can be found in the lecture notes of Meden (2002).

The derivation of the fRG equations presented in this chapter is a generalized version of the derivation presented in my diploma thesis (Bauer, 2008). The latter is based on the lecture notes of Meden (2002) and the diploma thesis of Karrasch (2006). In his PhD thesis, Karrasch (2010) generalized his presentation to simultaneously apply to the Matsubara and Keldysh formalism. I will do the same here, with some minor differences in definitions and notations. I recommend his thesis for those who are interested in more details and examples. 


\subsection{Green's and Vertex Functions}

\subsubsection{Definitions of Green's and Vertex Functions}

Let $S$ be an action, $\{\psi\}$ and $\{\eta\}$ sets of Grassmann variables (see Negele and Orland, 1988, chapter 1.5 for a precise definition) and $\bar{\psi}_{k}$ the conjugate of $\psi_{k} . k$ is a composite index, carrying all phase-space information, i.e. time or energy, quantum numbers etc.. $(\bar{\psi}, \eta)$ is a scalar product defined by $(\bar{\psi}, \eta):=\sum_{k} \bar{\psi}_{k} \eta_{k}$. The action defines the generating functional of Green's functions:

$$
\mathcal{W}(\{\bar{\eta}\},\{\eta\})=\frac{1}{\mathcal{Z}} \int \mathcal{D} \bar{\psi} \psi \exp (-\kappa S(\{\bar{\psi}\},\{\psi\})-(\bar{\psi}, \eta)-(\bar{\eta}, \psi)) .
$$

where $\int \mathcal{D} \bar{\psi} \psi$ is a coherent-state path integral (see Negele and Orland, 1988, chapter 2.2). $\mathcal{W}$ defines the $m$-particle Green's function

$$
\begin{aligned}
G_{m}\left(k_{1}^{\prime}, \ldots, k_{m}^{\prime}, k_{1}, \ldots, k_{m}\right): & =\left.\kappa^{m} \frac{\delta^{m}}{\delta \bar{\eta}_{k_{1}^{\prime}} \cdots \delta \bar{\eta}_{k_{m}^{\prime}}} \frac{\delta^{m}}{\delta \eta_{k_{1}} \cdots \delta \eta_{k_{m}}} \mathcal{W}(\{\bar{\eta}\},\{\eta\})\right|_{\bar{\eta}=0=\eta} \\
& =\kappa^{m} \frac{1}{\mathcal{Z}} \int \mathcal{D} \bar{\psi} \psi \psi \bar{\psi} \exp (-\kappa S) \\
& =(-\kappa)^{m}\left\langle\psi_{k_{1}^{\prime}} \ldots \psi_{k_{m}^{\prime}} \bar{\psi}_{k_{1}} \cdots \bar{\psi}_{k_{m}}\right\rangle .
\end{aligned}
$$

where $\kappa$ is a complex number. In this work we mainly use the Matsubara formalism, for which $\kappa=1$ (see Negele and Orland, 1988, chapter 2.2). Further we use the fRG equation of the self-energy, to derive a conductance formula in Keldysh formalism, where $\kappa=i$ (see Kamenev and Levchenko, 2009, chapter 5.1). My definition of the scalar product $(\cdot, \cdot)$ differs by a factor of $\kappa$ from the definition given in Karrasch (2010). This affects the definition of $\mathcal{W}$ but not the definition of the Green's functions.

The action shall have a term purely quadratic in $\psi, S_{0}$, and a term quartic in $\psi, S_{\text {int }}{ }^{1}$

$$
S=S_{0}+S_{\text {int }} .
$$

Let $G_{m}^{0}$ be the $m$-particle Green's function of $S_{0}$, with the shorthand $\mathcal{G}^{0}=G_{1}^{0}$ to be called bare Green's function, then $S_{0}$ can be written as

$$
S_{0}=-\left(\bar{\psi},\left[\mathcal{G}^{0}\right]^{-1} \psi\right)
$$

Further we define the m-particle connected Green's function,

$$
\begin{aligned}
G_{m}^{c}\left(k_{1}^{\prime}, \ldots, k_{m}^{\prime}, k_{1}, \ldots, k_{m}\right): & =\left.\kappa^{m} \frac{\delta^{m}}{\delta \bar{\eta}_{k_{1}^{\prime}} \cdots \delta \bar{\eta}_{k_{m}^{\prime}}} \frac{\delta^{m}}{\delta \eta_{k_{1}} \cdots \delta \eta_{k_{m}}} \mathcal{W}^{c}(\{\bar{\eta}\},\{\eta\})\right|_{\bar{\eta}=0=\eta} \\
& =(-\kappa)^{m}\left\langle\psi_{k_{1}^{\prime}} \cdots \psi_{k_{m}^{\prime}} \bar{\psi}_{k_{1}} \cdots \bar{\psi}_{k_{m}}\right\rangle_{c},
\end{aligned}
$$

\footnotetext{
${ }^{1} S_{\text {int }}$ can of course also contain terms quadratic in $\psi$.
} 
with

$$
\mathcal{W}^{c}=\ln \mathcal{W}
$$

and the vertex functions

$$
\gamma_{m}\left(k_{1}^{\prime}, \ldots, k_{m}^{\prime} ; k_{1}, \ldots, k_{m}\right)=\left.\kappa^{-m} v^{-m+1} \frac{\delta^{m}}{\delta \bar{\phi}_{k_{1}^{\prime}} \cdots \delta \bar{\phi}_{k_{m}^{\prime}}} \frac{\delta^{m}}{\delta \phi_{k_{1}} \cdots \delta \phi_{k_{m}}} \Gamma(\{\bar{\phi}\},\{\phi\})\right|_{\bar{\phi}=0=\phi},
$$

where $v$ is a constant and $\Gamma$ is the Legendre transform of $\mathcal{W}^{c}$,

$$
\Gamma(\{\bar{\phi}\},\{\phi\})=-\mathcal{W}^{c}(\{\bar{\eta}\},\{\eta\})-(\bar{\phi}, \eta)-(\bar{\eta}, \phi)+\kappa\left(\bar{\phi},\left[\mathcal{G}^{0}\right]^{-1} \phi\right)
$$

with

$$
\phi_{k}=-\frac{\delta}{\delta \bar{\eta}_{k}} \mathcal{W}^{c}(\{\bar{\eta}\},\{\eta\}), \quad \bar{\phi}_{k}=\frac{\delta}{\delta \eta_{k}} \mathcal{W}^{c}(\{\bar{\eta}\},\{\eta\})
$$

Note that in (3.8) we added an extra term to the common definition of a Legendre transformation. This modification influences $\Gamma$ only in the term quadratic in $\phi$, and thus the oneparticle vertex $\gamma_{1}$. It is needed to be consistent with the usual diagrammatic definition of vertex functions: The $n$-particle vertex functions are defined by the sum of all one-particle irreducible diagrams with $n$ amputated incoming lines and $n$ amputated outgoing lines. This definition will not be used later on, for a precise definition of lines and diagrams see Jakobs (2009). Further we included the prefactor $v^{-m+1}$ in the definition of the vertex functions, which is not present in the corresponding definitions in the works of Karrasch (2010) or Metzner et al. (2012). We included this factor in order to be more flexible, e.g. in section 3.3 we want to define the vertex functions to have all dimension of energy, which for the definition of the action (3.57) is only possible with such a term.

\subsubsection{Relation between Vertex and Green's Functions}

In this section we will derive some identities which show the relations between vertex and Green's functions. These identities will be used in the derivation of the fRG equations.

\section{General Relations}

The fields defined in (3.9) satisfy the identities

$$
\begin{aligned}
& \frac{\delta \phi_{k^{\prime}}}{\delta \phi_{k}}=\delta k_{k, k^{\prime}}=\frac{\delta \bar{\phi}_{k^{\prime}}}{\delta \bar{\phi}_{k}}, \\
& \frac{\delta \phi_{k^{\prime}}}{\delta \bar{\phi}_{k}}=0=\frac{\delta \bar{\phi}_{k^{\prime}}}{\delta \phi_{k}} .
\end{aligned}
$$


Differentiating the generating functional of the vertex function with respect to these fields yields

$$
\begin{aligned}
\frac{\delta}{\delta \phi_{k}} \Gamma(\{\bar{\phi}\},\{\phi\}) & \stackrel{3.8}{=} \sum_{q}\left[-\frac{\delta \mathcal{W}^{c}}{\delta \eta_{q}} \frac{\delta \eta_{q}}{\delta \phi_{k}}-\frac{\delta \mathcal{W}^{c}}{\delta \bar{\eta}_{q}} \frac{\delta \bar{\eta}_{q}}{\delta \phi_{k}}+\bar{\phi}_{q} \frac{\delta \eta_{q}}{\delta \phi_{k}}-\frac{\delta \bar{\eta}_{q}}{\delta \phi_{k}} \phi_{q}-\kappa \bar{\phi}_{q}\left[\mathcal{G}^{0}\right]_{q, k}^{-1}\right]+\bar{\eta}_{k} \\
& \stackrel{3.9}{=} \bar{\eta}_{k}-\kappa \sum_{q} \bar{\phi}_{q}\left[\mathcal{G}^{0}\right]_{q, k}^{-1}
\end{aligned}
$$

and

$$
\begin{aligned}
\frac{\delta}{\delta \bar{\phi}_{k}} \Gamma(\{\bar{\phi}\},\{\phi\}) & \stackrel{3.8}{=} \sum_{q}\left[-\frac{\delta \mathcal{W}^{c}}{\delta \eta_{q}} \frac{\delta \eta_{q}}{\delta \bar{\phi}_{k}}-\frac{\delta \mathcal{W}^{c}}{\delta \bar{\eta}_{q}} \frac{\delta \bar{\eta}_{q}}{\delta \bar{\phi}_{k}}+\bar{\phi}_{q} \frac{\delta \eta_{q}}{\delta \bar{\phi}_{k}}-\frac{\delta \bar{\eta}_{q}}{\delta \phi_{k}} \phi_{q}+\kappa \bar{\phi}_{q}\left[\mathcal{G}^{0}\right]_{q, k}^{-1}\right]-\eta_{k} \\
& \stackrel{3.9}{=}-\eta_{k}+\kappa \sum_{q}\left[\mathcal{G}^{0}\right]_{q, k}^{-1} \phi_{q} .
\end{aligned}
$$

Differentiating (3.11) with respect to $\bar{\phi}_{k^{\prime}}$ and (3.12) with respect to $\phi_{k^{\prime}}$ and solving both equations for the first term on the r.h.s. yields

$$
\begin{aligned}
\frac{\delta \bar{\eta}_{k}}{\delta \bar{\phi}_{k^{\prime}}} & =\frac{\delta^{2} \Gamma}{\delta \bar{\phi}_{k^{\prime}} \delta \phi_{k}}+\kappa\left[\mathcal{G}^{0}\right]_{k^{\prime}, k}^{-1}, \\
\frac{\delta \eta_{k}}{\delta \phi_{k^{\prime}}} & =-\frac{\delta^{2} \Gamma}{\delta \phi_{k^{\prime}} \delta \bar{\phi}_{k}}+\kappa\left[\mathcal{G}^{0}\right]_{k, k^{\prime}}^{-1} .
\end{aligned}
$$

Note the different order of $k$ and $k^{\prime}$ of the free propagator. Using these identities one gets

$$
\begin{aligned}
\delta_{k, k^{\prime}} & =\frac{\delta \phi_{k}}{\delta \phi_{k^{\prime}}} \stackrel{3.9}{=}-\frac{\delta}{\delta \phi_{k^{\prime}}} \frac{\delta \mathcal{W}^{c}}{\delta \bar{\eta}_{k}}=-\sum_{q}\left[\frac{\delta \eta_{q}}{\delta \phi_{k^{\prime}}} \frac{\delta^{2} \mathcal{W}^{c}}{\delta \eta_{q} \delta \bar{\eta}_{k}}+\frac{\delta \bar{\eta}_{q}}{\delta \phi_{k^{\prime}}} \frac{\delta^{2} \mathcal{W}^{c}}{\delta \bar{\eta}_{q} \delta \bar{\eta}_{k}}\right] \\
& =\sum_{q}\left[\left(\frac{\delta^{2} \Gamma}{\delta \phi_{k^{\prime}} \delta \bar{\phi}_{q}}-\kappa\left[\mathcal{G}^{0}\right]_{q, k^{\prime}}^{-1}\right) \frac{\delta^{2} \mathcal{W}^{c}}{\delta \eta_{q} \delta \bar{\eta}_{k}}-\frac{\delta^{2} \Gamma}{\delta \phi_{k^{\prime}} \delta \phi_{q}} \frac{\delta^{2} \mathcal{W}^{c}}{\delta \bar{\eta}_{q} \delta \bar{\eta}_{k}}\right]
\end{aligned}
$$

and in the same way

$$
\begin{aligned}
\delta_{k, k^{\prime}} & =\frac{\delta \bar{\phi}_{k}}{\delta \bar{\phi}_{k^{\prime}}}=\sum_{q}\left[\left(\frac{\delta^{2} \Gamma}{\delta \bar{\phi}_{k^{\prime}} \delta \phi_{q}}+\kappa\left[\mathcal{G}^{0}\right]_{k^{\prime}, q}^{-1}\right) \frac{\delta^{2} \mathcal{W}^{c}}{\delta \bar{\eta}_{q} \delta \eta_{k}}-\frac{\delta^{2} \Gamma}{\delta \bar{\phi}_{k^{\prime}} \delta \bar{\phi}_{q}} \frac{\delta^{2} \mathcal{W}^{c}}{\delta \eta_{q} \delta \eta_{k}}\right] \\
0 & =\frac{\delta \bar{\phi}_{k}}{\delta \phi_{k^{\prime}}}=\sum_{q}\left[-\left(\frac{\delta^{2} \Gamma}{\delta \phi_{k^{\prime}} \delta \bar{\phi}_{q}}-\kappa\left[\mathcal{G}^{0}\right]_{q, k^{\prime}}^{-1}\right) \frac{\delta^{2} \mathcal{W}^{c}}{\delta \eta_{q} \delta \eta_{k}}-\frac{\delta^{2} \Gamma}{\delta \phi_{k^{\prime}} \delta \phi_{q}} \frac{\delta^{2} \mathcal{W}^{c}}{\delta \bar{\eta}_{q} \delta \eta_{k}}\right] \\
0 & =\frac{\delta \phi_{k}}{\delta \bar{\phi}_{k^{\prime}}}=\sum_{q}\left[-\left(\frac{\delta^{2} \Gamma}{\delta \bar{\phi}_{k^{\prime}} \delta \phi_{q}}+\kappa\left[\mathcal{G}^{0}\right]_{k^{\prime}, q}^{-1}\right) \frac{\delta^{2} \mathcal{W}^{c}}{\delta \bar{\eta}_{q} \delta \bar{\eta}_{k}}-\frac{\delta^{2} \Gamma}{\delta \bar{\phi}_{k^{\prime}} \delta \bar{\phi}_{q}} \frac{\delta^{2} \mathcal{W}^{c}}{\delta \eta_{q} \delta \bar{\eta}_{k}}\right] .
\end{aligned}
$$

We can write the last four equations in one compact equation:

$$
\left(\begin{array}{cc}
\frac{\delta^{2} \Gamma}{\delta \bar{\phi} \delta \phi}+\kappa\left[\mathcal{G}^{0}\right]^{-1} & \frac{\delta^{2} \Gamma}{\delta \bar{\phi} \delta \bar{\phi}} \\
\frac{\delta^{2} \Gamma}{\delta \phi \delta \phi} & \frac{\delta^{2} \Gamma}{\delta \phi \delta \phi}-\left[\mathcal{K}\left[\mathcal{G}^{0}\right]^{-1}\right]^{T}
\end{array}\right) \cdot\left(\begin{array}{cc}
\frac{\delta^{2} \mathcal{W}^{c}}{\delta \bar{\eta} \delta \eta} & -\frac{\delta^{2} \mathcal{W}^{c}}{\delta \bar{\eta} \delta \bar{\eta}} \\
-\frac{\delta^{2} \mathcal{W}^{c}}{\delta \eta \delta \eta} & \frac{\delta^{2} \mathcal{W}^{c}}{\delta \eta \delta \bar{\eta}}
\end{array}\right)=1 .
$$


This identity can be written as

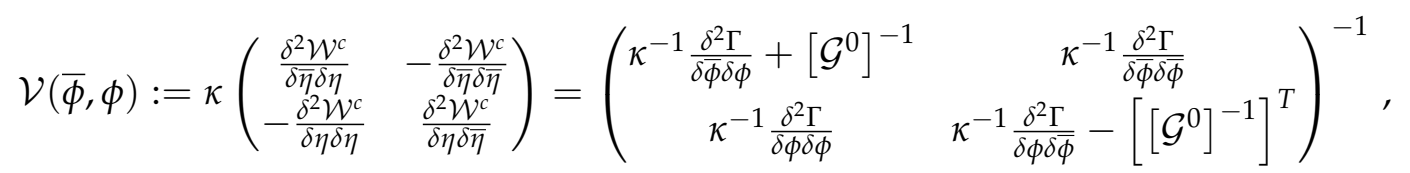

where we introduced an abbreviation for this matrix. This equation connects the two generating functionals $\mathcal{W}^{c}$ and $\Gamma$, thus we will need it later on.

\section{Relations for One and Two Particle Vertex Functions}

To show relations between vertex and Green's function we set the external sources to zero and assume we're not in a symmetry breaking phase, i.e.

$$
\left.\frac{\delta^{2} \mathcal{W}^{c}}{\delta \bar{\eta} \delta \bar{\eta}}\right|_{\eta=0=\bar{\eta}}=\left.\frac{\delta^{2} \mathcal{W}^{c}}{\delta \eta \delta \eta}\right|_{\eta=0=\bar{\eta}}=\left.\frac{\delta^{2} \Gamma}{\delta \bar{\phi} \delta \bar{\phi}}\right|_{\phi=0=\bar{\phi}}=\left.\frac{\delta^{2} \Gamma}{\delta \phi \delta \phi}\right|_{\phi=0=\bar{\phi}}=0 .
$$

The $(1,1)$ element of $\mathcal{V}$ then provides

$$
G_{1}^{c}: \stackrel{3.5}{=} \frac{\delta^{2} \mathcal{W}^{c}}{\delta \bar{\eta} \delta \eta}=\left[\kappa^{-1} \frac{\delta^{2} \Gamma}{\delta \bar{\phi} \delta \phi}+\left[\mathcal{G}^{0}\right]^{-1}\right]^{-1} .
$$

Comparing this with Dyson's equation,

$$
G_{1}=\mathcal{G}=\left[\left[\mathcal{G}^{0}\right]^{-1}-\Sigma\right]^{-1}
$$

where we introduced the short-hand $\mathcal{G}=G_{1}$ and $\Sigma$ is the self energy. Using the fact that for the one-particle propagator the linked cluster theorem provides $G_{1}^{c}=G_{1}$, one gets the identity

$$
\gamma_{1}=\kappa^{-1} \frac{\delta^{2} \Gamma}{\delta \bar{\phi} \delta \phi}=-\Sigma
$$

Note that without the last term in equation (3.8), the definition of $\gamma_{1}$ would be altered to $\gamma_{1}=$ $\mathcal{G}^{-1}$, which would contradict the usual diagrammatic definition.

Finally, we state the relation between two-particle vertex and Green's function. To this aim we differentiate equation (3.14) twice, first with respect to $\phi_{l}$ and second with respect to $\bar{\phi}_{l^{\prime}}$ and multiply the result with $\kappa^{-1}$. Setting the fields to zero yields

$$
\begin{aligned}
0 & =\left.\sum_{q} \kappa^{-1}\left[\frac{\delta^{4} \Gamma}{\delta \bar{\phi}_{l^{\prime}} \delta \phi_{l} \delta \phi_{k^{\prime}} \delta \bar{\phi}_{q}} \frac{\delta^{2} \mathcal{W}^{c}}{\delta \eta_{q} \delta \bar{\eta}_{k}}+\left(\frac{\delta^{2} \Gamma}{\delta \phi_{k^{\prime}} \delta \bar{\phi}_{q}}-\kappa\left[\mathcal{G}^{0}\right]_{q, k^{\prime}}^{-1}\right) \sum_{s, s^{\prime}}\left(\frac{\delta \eta_{s}}{\delta \phi_{l}} \frac{\delta \bar{\eta}_{s^{\prime}}}{\delta \bar{\phi}_{l^{\prime}}} \frac{\delta^{4} \mathcal{W}^{c}}{\delta \bar{\eta}_{s^{\prime}} \delta \eta_{s} \delta \eta_{q} \delta \bar{\eta}_{k}}\right)\right]\right|_{\phi=0=\bar{\phi}} \\
& =-v \sum_{q} \gamma_{2}\left(l^{\prime}, q, k^{\prime}, l\right) \mathcal{G}_{k, q}-\sum_{q, s, s^{\prime}}[\mathcal{G}]_{q, k^{\prime}}^{-1}[\mathcal{G}]_{s, l}^{-1}[\mathcal{G}]_{l^{\prime}, s^{\prime}}^{-1} G_{2}^{c}\left(s^{\prime}, k, q, s\right)
\end{aligned}
$$

where we again used $G_{1}^{c}=\mathcal{G}$ and equation (3.13). Solving for the two-particle vertex provides

$$
v \gamma_{2}\left(k_{1}^{\prime}, k_{2}^{\prime}, k_{1}, k_{2}\right)=-\sum_{q_{1}^{\prime}, q_{2}^{\prime}, q_{1}, q_{2}}[\mathcal{G}]_{k_{1}^{\prime}, q_{1}^{\prime}}^{-1}[\mathcal{G}]_{k_{2}^{\prime}, q_{2}^{\prime}}^{-1}[\mathcal{G}]_{q_{2}, k_{2}}^{-1}[\mathcal{G}]_{q_{1}, k_{1}}^{-1} G_{2}^{c}\left(q_{1}^{\prime}, q_{2}^{\prime}, q_{1}, q_{2}\right)
$$




\subsection{The fRG Equation}

In the following we will derive the fRG equation in the one-particle irreducible version, i.e. differential equations of the vertex functions w.r.t. a parameter called $\Lambda$. $\Lambda$ will be a parameter of the bare Green's function $\mathcal{G}^{0, \Lambda}$, and can either be a physical parameter like temperature or magnetic field, or an artificial additional parameter. This involves a large class of possible flow schemes. Another scheme, not contained here, would be the interaction flow proposed by Honerkamp et al. (2004). Since $\mathcal{G}^{0}$ plays a special role here, we write the action as indicated in the last section as

$$
S=-\left(\bar{\psi},\left[\mathcal{G}^{0, \Lambda}\right]^{-1} \psi\right)+S_{\mathrm{int}}
$$

To simplify notation we introduce the following shorthands:

$$
Q^{\Lambda}=\left[\mathcal{G}^{0, \Lambda}\right]^{-1}, \quad \dot{Q}^{\Lambda}=\partial_{\Lambda}\left[\mathcal{G}^{0, \Lambda}\right]^{-1}
$$

\subsubsection{Flow Equation of the Generating Functionals}

In order to arrive at a differential equation for the vertex functions we will set up a differential equation for their generating functional $\Gamma$, which we will expand on both sides in powers of its external fields $\phi \bar{\phi}$. Comparing orders of $\phi \bar{\phi}$ will lead to the desired equation.

Since the generating functional of the vertex function is defined via the generating functional of the connected Green's functions, we will need the following derivative:

$$
\begin{gathered}
\frac{d}{d \Lambda} \mathcal{W}^{c, \Lambda}=\frac{d}{d \Lambda} \ln \left(\frac{1}{\mathcal{Z}_{0}^{\Lambda}} \int \mathcal{D} \bar{\psi} \psi \exp \left[\kappa\left(\bar{\psi}, Q^{\Lambda} \psi\right)-\kappa S_{\text {int }}-\left(\bar{\psi}, \eta^{\Lambda}\right)-\left(\bar{\eta}^{\Lambda}, \psi\right)\right]\right), \\
\mathcal{Z}_{0}^{\Lambda}=\int \mathcal{D} \bar{\psi} \psi \exp \left[\kappa\left(\bar{\psi}, Q^{\Lambda} \psi\right)\right] .
\end{gathered}
$$

Note that the normalisation of the functional differs from the previous definition (3.2,3.6). The free propagator now depends on $\Lambda$, hence this is also the case for $S_{0}, \mathcal{Z}_{0}, \mathcal{W}$ and $\mathcal{W}^{c}$, as indicated by the superscript $\Lambda$. Furthermore we require $\eta$ and $\bar{\eta}$ to have a $\Lambda$-dependence, chosen such that the fields $\phi$ and $\bar{\phi}$, which are the natural variables of $\Gamma$, are $\Lambda$-independent. As a result $\eta$ and $\bar{\eta}$ depend on $\Lambda$ via (3.11) and (3.12).

To decompose the derivative we write it as

$$
\begin{aligned}
\frac{d}{d \Lambda} \mathcal{W}^{c, \Lambda} & =\sum_{k, k^{\prime}} \dot{Q}_{k, k^{\prime}}^{\Lambda} \frac{\delta \mathcal{Z}_{0}^{\Lambda}}{\delta Q_{k, k^{\prime}}^{\Lambda}} \frac{\partial \mathcal{W}^{c, \Lambda}}{\partial \mathcal{Z}_{0}^{\Lambda}}+\sum_{k, k^{\prime}} \dot{Q}_{k, k^{\prime}}^{\Lambda} \frac{\delta \mathcal{W}^{c, \Lambda}}{\delta Q_{k, k^{\prime}}^{\Lambda}} \\
& +\sum_{k}\left[\frac{d \eta_{k}^{\Lambda}}{d \Lambda} \frac{\delta \mathcal{W}^{c, \Lambda}}{\delta \eta_{k}^{\Lambda}}+\frac{d \bar{\eta}_{k}^{\Lambda}}{d \Lambda} \frac{\delta \mathcal{W}^{c, \Lambda}}{\delta \bar{\eta}_{k}^{\Lambda}}\right] .
\end{aligned}
$$

Here we already replaced $\frac{d Q^{\Lambda}}{d \Lambda}$ by $\dot{Q}^{\Lambda}$, since $Q^{\Lambda}$ explicitly depends on $\Lambda$. 
For the last sum we can use the definition (3.9) of the fields - mind the minus sign in the definition of $\phi$.

$$
\begin{aligned}
& \sum_{k} \frac{d \eta_{k}^{\Lambda}}{d \Lambda} \frac{\delta \mathcal{W}^{c, \Lambda}}{\delta \eta_{k}^{\Lambda}}=\sum_{k} \frac{d \eta_{k}^{\Lambda}}{d \Lambda} \bar{\phi}_{k}=-\left(\bar{\phi}_{k}, \frac{d \eta_{k}^{\Lambda}}{d \Lambda}\right) \\
& \sum_{k} \frac{d \bar{\eta}_{k}^{\Lambda}}{d \Lambda} \frac{\delta \mathcal{W}^{c, \Lambda}}{\delta \bar{\eta}_{k}^{\Lambda}}=-\sum_{k} \frac{d \bar{\eta}_{k}^{\Lambda}}{d \Lambda} \phi_{k}=-\left(\frac{d \bar{\eta}_{k}^{\Lambda}}{d \Lambda}, \phi_{k}\right) .
\end{aligned}
$$

Furthermore we see from (3.30a) that $\frac{\partial \mathcal{W}^{c, \Lambda}}{\partial \mathcal{Z}_{0}^{\Lambda}}=-\frac{1}{\mathcal{Z}_{0}^{\Lambda}}$ and with

$$
\frac{1}{\mathcal{Z}_{0}^{\Lambda}} \frac{\delta \mathcal{Z}_{0}^{\Lambda}}{\delta Q_{k, k^{\prime}}^{\Lambda}}=\kappa \int \frac{\mathcal{D} \bar{\psi} \psi}{\mathcal{Z}_{0}^{\Lambda}} \bar{\psi}_{k} \psi_{k^{\prime}} e^{-\kappa S_{0}^{\Lambda}}=-\kappa \int \frac{\mathcal{D} \bar{\psi} \psi}{\mathcal{Z}_{0}^{\Lambda}} \psi_{k^{\prime}} \bar{\psi}_{k} e^{-\kappa S_{0}^{\Lambda}} \stackrel{3.2}{=} \mathcal{G}_{k^{\prime}, k}^{0, \Lambda},
$$

we conclude that

$$
\sum_{k, k^{\prime}} \dot{Q}_{k, k^{\prime}}^{\Lambda} \frac{\delta \mathcal{Z}_{0}^{\Lambda}}{\delta Q_{k, k^{\prime}}^{\Lambda}} \frac{\partial \mathcal{W}^{c, \Lambda}}{\partial \mathcal{Z}_{0}^{\Lambda}}=-\operatorname{Tr}\left(\mathcal{G}^{0, \Lambda} \dot{Q}^{\Lambda}\right)
$$

The minus sign in (3.34) results from interchanging the two fields $\psi$ and $\bar{\psi}$. The same appears in the following:

$$
\begin{aligned}
& \frac{1}{\mathcal{W}^{\Lambda}} \sum_{k, k^{\prime}} \dot{Q}_{k, k^{\prime}}^{\Lambda} \frac{\delta}{\delta Q_{k, k^{\prime}}^{\Lambda}} \int \frac{\mathcal{D} \bar{\psi} \psi}{\mathcal{Z}_{0}^{\Lambda}} e^{\left[\kappa\left(\bar{\psi}, Q^{\Lambda} \psi\right)-\kappa S_{\text {int }}-\left(\bar{\psi}, \eta^{\Lambda}\right)-\left(\bar{\eta}^{\Lambda}, \psi\right)\right]} \\
& =-\frac{\kappa}{\mathcal{W}^{\Lambda}} \sum_{k, k^{\prime}} \dot{Q}_{k, k^{\prime}}^{\Lambda} \int \frac{\mathcal{D} \bar{\psi} \psi}{\mathcal{Z}_{0}^{\Lambda}} \psi_{k^{\prime}} \bar{\psi}_{k} e^{\left[\kappa\left(\bar{\psi}, Q^{\Lambda} \psi\right)-\kappa S_{\text {int }}-\left(\bar{\psi}, \eta^{\Lambda}\right)-\left(\bar{\eta}^{\Lambda}, \psi\right)\right]} \\
& =\frac{\kappa}{\mathcal{W}^{\Lambda}} \operatorname{Tr}\left(\dot{Q}^{\Lambda} \frac{\delta}{\delta \bar{\eta}} \frac{\delta}{\delta \eta} \mathcal{W}^{\Lambda}\right)=\frac{\kappa}{\mathcal{W}^{\Lambda}} \operatorname{Tr}\left(\dot{Q}^{\Lambda} \frac{\delta}{\delta \bar{\eta}} \mathcal{W}^{\Lambda} \frac{\delta}{\delta \eta} \ln \mathcal{W}^{\Lambda}\right) \\
& =\frac{\kappa}{\mathcal{W}^{\Lambda}} \mathcal{W}^{\Lambda}\left[\operatorname{Tr}\left(\dot{Q}^{\Lambda} \frac{\delta}{\delta \bar{\eta}} \mathcal{W}^{c, \Lambda} \frac{\delta}{\delta \eta} \mathcal{W}^{c, \Lambda}\right)+\kappa \operatorname{Tr}\left(\dot{Q}^{\Lambda} \frac{\delta^{2}}{\delta \bar{\eta} \delta \eta} \mathcal{W}^{c, \Lambda}\right)\right] \\
& =\kappa\left(\bar{\phi}, \dot{Q}^{\Lambda} \phi\right)+\kappa \operatorname{Tr}\left(\dot{Q}^{\Lambda} \frac{\delta^{2}}{\delta \bar{\eta} \delta \eta} \mathcal{W}^{c, \Lambda}\right) .
\end{aligned}
$$

Putting all together provides

$$
\begin{aligned}
\frac{d}{d \Lambda} \mathcal{W}^{c, \Lambda}= & -\operatorname{Tr}\left(\mathcal{G}^{0, \Lambda} \dot{Q}^{\Lambda}\right)+\kappa \operatorname{Tr}\left(\dot{Q}^{\Lambda} \frac{\delta^{2} \mathcal{W}^{c, \Lambda}}{\delta \bar{\eta} \delta \eta}\right) \\
& +\kappa\left(\bar{\phi}, \dot{Q}^{\Lambda} \phi\right)-\left(\bar{\phi}_{k}, \frac{d \eta_{k}^{\Lambda}}{d \Lambda}\right)-\left(\frac{d \bar{\eta}_{k}^{\Lambda}}{d \Lambda}, \phi_{k}\right) .
\end{aligned}
$$


This makes it easy to differentiate $\Gamma$ :

$$
\begin{aligned}
\frac{d}{d \Lambda} \Gamma & =-\frac{d}{d \Lambda} \mathcal{W}^{c, \Lambda}-\left(\bar{\phi}_{k}, \frac{d \eta_{k}^{\Lambda}}{d \Lambda}\right)-\left(\frac{d \bar{\eta}_{k}^{\Lambda}}{d \Lambda}, \phi_{k}\right)+\kappa\left(\bar{\phi}, \dot{Q}^{\Lambda} \phi\right) \\
& =\operatorname{Tr}\left(\mathcal{G}^{0, \Lambda} \dot{Q}^{\Lambda}\right)-\kappa \operatorname{Tr}\left(\dot{Q}^{\Lambda} \frac{\delta^{2} \mathcal{W}^{c, \Lambda}}{\delta \bar{\eta} \delta \eta}\right) \\
& =\operatorname{Tr}\left(\mathcal{G}^{0, \Lambda} \dot{Q}^{\Lambda}\right)-\operatorname{Tr}\left(\dot{Q}^{\Lambda} \mathcal{V}^{(1,1)}\right) .
\end{aligned}
$$

\subsubsection{Flow equation for the Vertex Functions}

For convenience we leave out the index $\Lambda$ from now on, but we consider all functions to have an implicit $\Lambda$-dependence. To get the $(1,1)$ matrix element of $\mathcal{V}$ we use equation (3.20) and expand the r.h.s. around the full propagator $\mathcal{G}$ :

$$
\mathcal{V}=\left(\begin{array}{cc}
\mathcal{K}^{-1} \frac{\delta^{2} \Gamma}{\delta \bar{\phi} \delta \phi}+\left[\mathcal{G}^{0}\right]^{-1} & \mathcal{K}^{-1} \frac{\delta^{2} \Gamma}{\delta \bar{\phi} \delta \bar{\phi}} \\
\mathcal{K}^{-1} \frac{\delta^{2} \Gamma}{\delta \phi \delta \phi} & \mathcal{K}^{-1} \frac{\delta^{2} \Gamma}{\delta \phi \delta \bar{\phi}}-\left[\left[\mathcal{G}^{0}\right]^{-1}\right]^{T}
\end{array}\right)^{-1}
$$

To get a dependence of the full propagator we insert a fat zero containing the self energy and thus we can use the Dyson equation to bring the full propagator into play:

$$
\kappa^{-1} \frac{\delta^{2} \Gamma}{\delta \bar{\phi} \delta \phi}+\left[\mathcal{G}^{0}\right]^{-1}=\kappa^{-1} \frac{\delta^{2} \Gamma}{\delta \bar{\phi} \delta \phi}-\gamma_{1}+\gamma_{1}+\left[\mathcal{G}^{0}\right]^{-1}=\kappa^{-1} \frac{\delta^{2} \Gamma}{\delta \bar{\phi} \delta \phi}-\gamma_{1}+[\mathcal{G}]^{-1}
$$

Since we will use it in the following, it is convenient to define an abbreviation for the first two terms:

$$
\mathcal{U}:=\kappa^{-1} \frac{\delta^{2} \Gamma}{\delta \bar{\phi} \delta \phi}-\gamma_{1}
$$


Now we can factor out $\mathcal{G}$ :

$$
\begin{aligned}
& \mathcal{V}=\left(\begin{array}{cc}
\mathcal{U}+[\mathcal{G}]^{-1} & \mathcal{K}^{-1} \frac{\delta^{2} \Gamma}{\delta \bar{\phi} \delta \bar{\phi}} \\
\mathcal{K}^{-1} \frac{\delta^{2} \Gamma}{\delta \phi \delta \phi} & -\mathcal{U}^{T}-\left[[\mathcal{G}]^{-1}\right]^{T}
\end{array}\right)^{-1} \\
& =\left[\left(\begin{array}{cc}
{[\mathcal{G}]^{-1}} & 0 \\
0 & -\left[[\mathcal{G}]^{-1}\right]^{T}
\end{array}\right)+\left(\begin{array}{cc}
\mathcal{U} & \mathcal{K}^{-1} \frac{\delta^{2} \Gamma}{\delta \bar{\phi} \delta \bar{\phi}} \\
\mathcal{K}^{-1} \frac{\delta^{2} \Gamma}{\delta \phi \delta \phi} & -\mathcal{U}^{T}
\end{array}\right)\right]^{-1} \\
& =-\left[1-\left(\begin{array}{cc}
-\mathcal{G} & 0 \\
0 & {[\mathcal{G}]^{T}}
\end{array}\right)\left(\begin{array}{cc}
\mathcal{U} & \mathcal{K}^{-1} \frac{\delta^{2} \Gamma}{\delta \bar{\phi} \delta \bar{\phi}} \\
\mathcal{K}^{-1} \frac{\delta^{2} \Gamma}{\delta \phi \delta \phi} & -\mathcal{U}^{T}
\end{array}\right)\right]^{-1}\left(\begin{array}{cc}
-\mathcal{G} & 0 \\
0 & {[\mathcal{G}]^{T}}
\end{array}\right)
\end{aligned}
$$

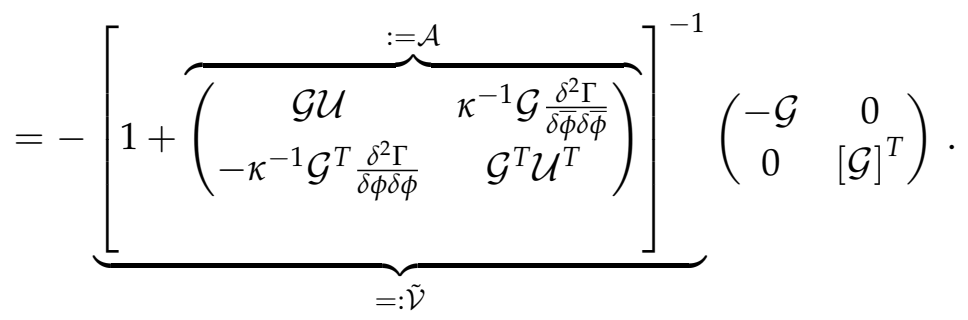

With this definition $\mathcal{V}^{(1,1)}=\tilde{\mathcal{V}}^{(1,1)} \mathcal{G}$ and we can write the differential equation (3.38) as

$$
\frac{d}{d \Lambda} \Gamma=\operatorname{Tr}\left(\mathcal{G}^{0, \Lambda} \dot{Q}^{\Lambda}\right)-\operatorname{Tr}\left(\mathcal{G} \dot{Q}^{\Lambda} \tilde{\mathcal{V}}^{(1,1)}\right)
$$

Now we expand $\tilde{\mathcal{V}}$ into a taylor series around $\mathcal{A}=0$ :

$$
[1+\mathcal{A}]^{-1}=1-\mathcal{A}+\mathcal{A} \mathcal{A}-\mathcal{A} \mathcal{A} \mathcal{A}+\ldots
$$

Inserting the definition of $\mathcal{A}$ and taking the $(1,1)$-matrix element provides

$$
\tilde{\mathcal{V}}^{(1,1)}=1-\mathcal{G} \mathcal{U}+\left(\mathcal{G U} \mathcal{G} \mathcal{U}-\kappa^{-2} \mathcal{G} \frac{\delta^{2} \Gamma}{\delta \bar{\phi} \delta \bar{\phi}} \mathcal{G}^{T} \frac{\delta^{2} \Gamma}{\delta \phi \delta \phi}\right)-(\mathcal{G} \mathcal{G} \mathcal{G} \mathcal{G}+\ldots)+\ldots
$$

where the brackets group the orders of expansion. Using the definition of the vertex functions (3.7) we can expand their generating functional

$$
\Gamma=\sum_{m=0}^{\infty} \frac{(-\kappa)^{m} v^{m-1}}{(m !)^{2}} \sum_{k_{1}^{\prime} \ldots k_{m}^{\prime}} \sum_{k_{1} \ldots k_{m}} \gamma_{m}\left(k_{1}^{\prime}, \ldots, k_{m}^{\prime} ; k_{1}, \ldots, k_{m}\right) \bar{\phi}_{k_{1}^{\prime}} \ldots \bar{\phi}_{k_{m}^{\prime}} \phi_{k_{m}} \ldots \phi_{k_{1}} .
$$

Note the minus signs when performing the functional derivative: the factor $(-1)^{m}$ cancels all minus signs due to permuting the $\bar{\phi}$-derivatives through the $\phi$ 's. Inserting (3.46) into the definition of $\mathcal{U}(3.41)$ gives

$$
\mathcal{U}_{q^{\prime}, q}=\sum_{m=1}^{\infty} \frac{(-\kappa)^{m} v^{m}}{(m !)^{2}} \sum_{k_{1}^{\prime} \ldots k_{m}^{\prime}} \sum_{k_{1} \ldots k_{m}} \gamma_{m+1}\left(k_{1}^{\prime}, \ldots, k_{m}^{\prime}, q^{\prime} ; k_{1}, \ldots, k_{m}, q\right) \bar{\phi}_{k_{1}^{\prime}} \ldots \bar{\phi}_{k_{m}^{\prime}} \phi_{k_{m}} \ldots \phi_{k_{1}} .
$$

$\mathcal{U}$ is a matrix, indicated by the indices $q^{\prime}, q$. These quantum numbers appear in each vertex. Note that due to the differentiation all indices are shifted by one and the first summand cancels (see (3.41)). So $\mathcal{U}$ is at least of second order in the fields and does not depend on $\gamma_{0}$ and $\gamma_{1}$. 


\section{The Zero-Particle Vertex Function}

For completeness we write down the differential equation for $\gamma_{0}$ also known as free energy. To this end we collect all terms on the r.h.s. of (3.43) that do not depend on $\phi$ or $\bar{\phi}$. For $\tilde{\mathcal{V}}^{(1,1)}$ this is only the constant 1 . Thus we get

$$
\frac{d}{d \Lambda} \gamma_{0}=v \operatorname{Tr}\left(\mathcal{G}^{0, \Lambda} \dot{Q}^{\Lambda}\right)-v \operatorname{Tr}\left(\mathcal{G} \dot{Q}^{\Lambda}\right)
$$

\section{The One-Particle Vertex Function}

Now we are in a position to set up the differential equation for the $m$-particle vertex starting from (3.38), and expressing its right hand side using equation (3.42) and (3.45). In (3.45) only the second term is linear in $\bar{\phi} \phi$. This can be seen from the expansion of $\mathcal{U}(3.47)$, by noting that $\mathcal{G} \frac{\delta^{2} \Gamma}{\delta \bar{\phi} \delta \bar{\phi}} \mathcal{G}^{T} \frac{\delta^{2} \Gamma}{\delta \phi \delta \phi}$ contains only quadratic terms in $\bar{\phi} \phi$. Thus we find

$$
\frac{d}{d \Lambda} \gamma_{1}\left(k^{\prime}, k\right)=v \sum_{q^{\prime}, q}\left[\mathcal{G} \dot{Q}^{\Lambda} \mathcal{G}\right]_{q, q^{\prime}} \gamma_{2}\left(k^{\prime}, q^{\prime} ; k, q\right)
$$

To make the notation clear we replaced the trace by a sum over all quantum numbers, but in the following we will use the shorthand notation:

$$
\left[\gamma_{m}\left(k_{1}^{\prime}, \ldots, k_{m-1}^{\prime}, \cdot ; k_{1}, \ldots, k_{m-1}, \cdot\right)\right]_{q^{\prime}, q}=\gamma_{m}\left(k_{1}^{\prime}, \ldots, k_{m-1}^{\prime}, q^{\prime} ; k_{1}, \ldots, k_{m-1}, q\right)
$$

Furthermore, we use the convenient definition

$$
\mathcal{S}:=\mathcal{G} \dot{Q}^{\Lambda} \mathcal{G}=\mathcal{G} \partial_{\Lambda}\left[\mathcal{G}^{0, \Lambda}\right]^{-1} \mathcal{G}
$$

to be called single scale propagator. This name originates from the fact that in many flow schemes $\mathcal{S}$ describes propagation with a narrow energy interval. With this definition equation (3.49) becomes

$$
\frac{d}{d \Lambda} \gamma_{1}\left(k^{\prime}, k\right)=v \operatorname{Tr}\left[\mathcal{S} \gamma_{2}\left(k^{\prime}, \cdot ; k, \cdot\right)\right]
$$

Note that the derivative of the vertex contains only one-particle irreducible diagrams, as is the case for the vertex itself. However the r.h.s. is not independent of $\gamma_{1}$, since $\mathcal{G}$ and thus $\mathcal{S}$ depends on it. 


\section{The Two-Particle Vertex Function}

To be able to write down the flow equation for $\gamma_{2}$, we consider on the following relations, which follows directly from (3.46):

$$
\begin{aligned}
& \frac{\delta^{2} \Gamma}{\delta \bar{\phi} \delta \bar{\phi}}=\sum_{m=2}^{\infty} \frac{m(m-1)(-\kappa)^{m} v^{m-1}}{(m !)^{2}} \sum_{k_{1}^{\prime} \ldots k_{m-2}^{\prime}} \sum_{k_{1} \ldots k_{m}} \gamma_{m}\left(\begin{array}{c}
\left.k_{\overline{1}^{\prime}}^{\prime}, \ldots, k_{m-2}^{\prime}, \cdot, \cdot ; k_{1}, \ldots, k_{m}\right) \\
\bar{\phi}_{k_{1}^{\prime}} \ldots \bar{\phi}_{k_{m-2}^{\prime}} \phi_{k_{m}} \ldots \phi_{k_{1}}
\end{array}\right.
\end{aligned}
$$

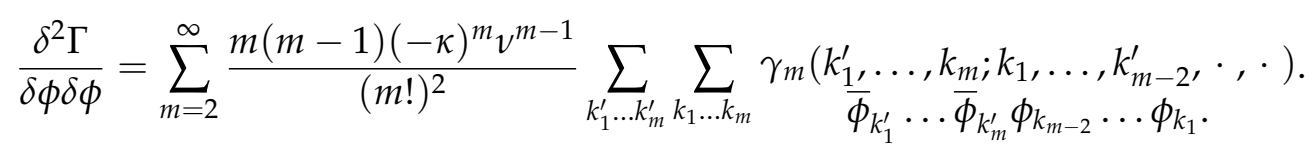

As long as we have not a broken symmetry, all cross-terms in $\mathcal{G} \frac{\delta^{2} \Gamma}{\delta \phi \delta \phi} \mathcal{G}^{T} \frac{\delta^{2} \Gamma}{\delta \phi \delta \phi}$ that contain a different number of derivatives w.r.t. $\phi$ and $\bar{\phi}$ vanish. Note that the flow equation (3.43) with the expansion of $\tilde{\mathcal{V}}$ (3.45) only contains products of (3.53), (3.54) and $\mathcal{U}$ (3.47). Consequently, the derivative of the $m$-particle vertex contains vertices up to order $m+1$.

To set up the differential equation for $\gamma_{2}$ we need all terms in (3.45) that are proportional to $\overline{\phi \phi} \phi \phi$. As one can easily convince oneself they only appear in

$$
\mathcal{G U}-\kappa^{-2} \mathcal{G} \frac{\delta^{2} \Gamma}{\delta \bar{\phi} \delta \bar{\phi}} \mathcal{G}^{T} \frac{\delta^{2} \Gamma}{\delta \phi \delta \phi}+\mathcal{G} \mathcal{U} \mathcal{G}
$$

The first term is proportional to $\gamma_{3}$. The second and third are quadratic in $\gamma_{2}$ :

$$
\begin{aligned}
\frac{d}{d \Lambda} \gamma_{2}\left(k_{1}^{\prime}, k_{2}^{\prime} ; k_{1}, k_{2}\right)= & \operatorname{Tr}\left(\mathcal{S} \gamma_{3}\left(k_{1}^{\prime}, k_{2}^{\prime}, \cdot ; k_{1}, k_{2}, \cdot\right)\right) \\
& +v \operatorname{Tr}\left(\mathcal{S} \gamma_{2}\left(\cdot, \cdot ; k_{1}, k_{2}\right) \mathcal{G}^{T} \gamma_{2}\left(k_{1}^{\prime}, k_{2}^{\prime} ; \cdot, \cdot\right)\right) \\
& -v \operatorname{Tr}\left(\mathcal{S} \gamma_{2}\left(k_{1}^{\prime}, \cdot ; k_{1}, \cdot\right) \mathcal{G} \gamma_{2}\left(k_{2}^{\prime}, \cdot ; k_{2}, \cdot\right)\right) \\
& -v \operatorname{Tr}\left(\mathcal{S} \gamma_{2}\left(k_{2}^{\prime}, \cdot ; k_{2}, \cdot\right) \mathcal{G} \gamma_{2}\left(k_{1}^{\prime}, \cdot ; k_{1}, \cdot\right)\right) \\
& +v \operatorname{Tr}\left(\mathcal{S} \gamma_{2}\left(k_{2}^{\prime}, \cdot ; k_{1}, \cdot\right) \mathcal{G} \gamma_{2}\left(k_{1}^{\prime}, \cdot ; k_{2}, \cdot\right)\right) \\
& +v \operatorname{Tr}\left(\mathcal{S} \gamma_{2}\left(k_{1}^{\prime}, \cdot ; k_{2}, \cdot\right) \mathcal{G} \gamma_{2}\left(k_{2}^{\prime}, \cdot ; k_{1}, \cdot\right)\right)
\end{aligned}
$$

This procedure can be continued to get differential equations for the higher order vertex functions. The hierarchy of these equations is infinite: Because of the second term $\mathcal{G U}$ in the expansion of $\mathcal{V}^{(1,1)}$, equation (3.45), the derivative of the $m$-particle vertex functions depends on all vertices up to order $m+1$. 


\subsection{Approximate solution for the vertex functions}

In the last section we derived exact differential equations for the vertex functions $\gamma_{1}$ and $\gamma_{2}$. These equations can be used to calculate an approximate solutions for these vertex functions, applying the following strategy. Suppose, that for a given value of $\Lambda=\Lambda_{\text {initial}}$, all vertex functions are known, and for another value of $\Lambda=\Lambda_{\text {final }}$, the $\Lambda$-dependent bare propagator is the physical propagator $\mathcal{G}^{0 \Lambda_{\text {final }}}=\mathcal{G}^{0}$, then integrating the vertex functions from $\Lambda_{\text {initial }}$ to $\Lambda_{\text {final }}$ gives the solution for the vertex-functions.

\subsubsection{Truncation}

In practice, the fRG flow equations cannot be solved exactly, since they form an infinite hierarchy of differential equations. Thus in order to apply this strategy, one has to make approximations. The most common approximation, in this context, which we also apply here, is the truncation of the flow equation in $n$-th order: The derivative of all $m$-particle vertices with $m>n$ is set to zero. This approximation, to be called fRGn, can be justified as follows: At $\Lambda_{\text {initial }}$ all vertices except the two-particle vertex are zero and all higher vertices are generated by the two-particle vertex at least to power $n+1$. Hence fRG $n$ is exact to $n$-th order in the interaction. So as long as the effective interaction can be regarded as small, all higher vertices can be neglected. If at any value of $\Lambda$, the flow equation generates an $n$-particle vertex that can not be regarded as small or even is divergent, this approximation breaks down. In most cases the only practical approximation schemes are fRG1 and fRG2, and for models involving a large number $N$ of interacting states, such as the chain model considered in this work, fRG2 runs into problems, since the two-particle vertex is represented by $\mathcal{O}\left(N^{4}\right)$ independent variables. This complication can be resolved by using further approximations for models with a local interaction, as explained in section 3.3.6 (see also Bauer et al., 2014).

\subsubsection{Specifying the Action}

For the rest of this chapter we will use the fRG equations in the Matsubara formalism and in the frequency representation and hence set $\kappa=1$. Further we consider fermions in real space. The latter is discretized (see section 2.3.3), such that the position is described by a site index. Thus the composite-index reads as $k=\left(\omega_{n}, j, \sigma\right)$, where $\omega_{n}=i \pi(2 n+1) T$, with $n \in \mathbb{Z}$, is a purely imaginary discrete Fermionic Matsubara frequency, $T$ is the temperature, $j$ is the site-index and $\sigma=\{\uparrow, \downarrow\}$ or $\{+,-\}$ the spin. We define the action as

$$
\begin{aligned}
S=\sum_{i j \sigma n} \bar{\psi}_{\sigma i}\left(\omega_{n}\right)\left[\mathcal{G}^{0}\right]_{\sigma, i j}^{-1}\left(\omega_{n}\right) \psi_{\sigma j}\left(\omega_{n}\right) & \\
& +T \sum_{\substack{i j \sigma \sigma^{\prime} \\
n m n^{\prime} m^{\prime}}} U_{i j} \delta_{n+m, n^{\prime}+m^{\prime}} \bar{\psi}_{\sigma i}\left(\omega_{n}\right) \bar{\psi}_{\sigma^{\prime} j}\left(\omega_{m}\right) \psi_{\sigma i}\left(\omega_{n}^{\prime}\right) \psi_{\sigma^{\prime} j}\left(\omega_{m}^{\prime}\right)
\end{aligned}
$$

with

$$
\left[\mathcal{G}^{0}\right]_{\sigma, i j}^{-1}\left(\omega_{n}\right)=\left(\omega_{n}-h_{i j}^{\sigma}+\mu\right) e^{\omega_{n} 0^{+}}
$$


where $h^{\sigma}$ and $U$ are real symmetric matrices and $\mu$ is the chemical potential. This action conserves spin and frequency. Here the action is defined without deriving it from first principles (for a derivation see e.g. Meden, 2002), to do so, one has to define the Fourier expansion of the Grassman fields:

$$
\psi_{\sigma i}\left(\omega_{n}\right)=\sqrt{T} \int_{0}^{1 / T} d \tau e^{\omega_{n} \tau} \psi_{\sigma i}(\tau)
$$

The prefactor $\sqrt{T}$ defines the dimension of the fields, and the prefactors of the matsubara sums in the action.

In order to make the notation short, we will use the following conventions: In some cases we denote the frequency-dependence of the Green's and vertex functions explicitly, e.g. $\mathcal{G}_{k, k^{\prime}}(\omega)$. In this case $k, k^{\prime}$ are considered not to carry the information of the frequency. The same hold for the spin, e.g. $\mathcal{G}_{k, k^{\prime}}^{\sigma}$. If the indices $k, k^{\prime}$ are not written at all, e.g. $\mathcal{G}(\omega)$, then the Green's and vertex function are considered to be matrices or tensors w.r.t. these indices.

In general, the frequency is a complex number, however most of the time we use one of two special cases: When we write the frequency with an index $\omega_{n}$, then it is a purely imaginary discrete fermionic Matsubara frequency $\omega_{n}=i \pi(2 n+1) T$. In contrast, a frequency without index $\omega$ is considered a real, continuous variable, if not specified explicitly otherwise.

\subsection{3 $\Lambda$-dependence of the free Propagator}

Next we will specify the $\Lambda$-dependence of the free propagator. Note that the only restriction we made is that there has to exist some $\Lambda_{\text {initial }}$ for which the value of all vertices has to be known. One possibility is $\mathcal{G}^{\Lambda_{\text {initial }}}=0$. Then all vertices except the two-particle vertex are zero.

At low temperatures the relevant excitations are located near the Fermi surface. Thus when turning on $\mathcal{G}^{\Lambda}$, it is intuitive to incorporate large energies first. We use the Matsubara frequency cutoff

$$
\mathcal{G}^{0, \Lambda}\left(\omega_{n}\right)=\Theta_{T}\left(\left|\omega_{n}\right|-\Lambda\right) \mathcal{G}^{0}\left(\omega_{n}\right),
$$

where $\Theta_{T}$ is a step-function broadened on the scale $T$ :

$$
\Theta_{T}(x)=\left\{\begin{array}{lc}
0 & x<-\pi T \\
\frac{1}{2}+\frac{1}{2 \pi T} x & |x| \leq \pi T \\
1 & x>\pi T
\end{array} .\right.
$$

\subsubsection{Initial Condition}

For $\mathcal{G}_{0}^{\Lambda_{\text {initial }}}=0$

$$
\Gamma^{\Lambda_{\text {initial }}}(\{\bar{\phi}\},\{\phi\})=\kappa S_{\text {int }}(\{\bar{\phi}\},\{\phi\}) .
$$

For a derivation see Meden (2002). We now fix $v=T$, to enshure that $\gamma_{2}$ has dimension of energy. Comparing the quartic terms of equation (3.46) and (3.57), we obtain

$$
\begin{aligned}
\gamma_{2}^{\Lambda_{\text {initial }}}\left(k_{1}^{\prime}, k_{2}^{\prime} ; k_{1}, k_{2}\right)= & \delta_{n_{1}^{\prime}+n_{2}^{\prime}, n_{1}+n_{2}} U_{j_{1} j_{2}} \\
& \times\left(\delta_{j_{1} j_{1} j_{2} j_{2}^{\prime}} \delta_{\sigma_{1}^{\prime} \sigma_{1}} \delta_{\sigma_{2}^{\prime} \sigma_{2}}-\delta_{j_{1} j_{2}^{\prime}} \delta_{j_{2} j_{1}} \delta_{\sigma_{1}^{\prime} \sigma_{2}} \delta_{\sigma_{2}^{\prime} \sigma_{1}}\right)\left(1-\delta_{j_{1} j_{2}} \delta_{\sigma_{1} \sigma_{2}}\right) .
\end{aligned}
$$


We introduce a shorthand for the socalled bare action

$$
v=\gamma_{2}^{\Lambda_{\text {initial }}}
$$

Since for the action (3.57) $S_{\text {int }}$ is purely quartic in the fields, all other vertex functions are zero:

$$
\gamma_{m}^{\Lambda_{\text {initial }}}=0 \quad(m \neq 2) .
$$

This is correct for $\Lambda_{\text {initial }}=\infty$, however numerically it is not possible to start the flow at infinity. Due to the slow convergence of the single scale propagator $\mathcal{S}$ at large frequencies, the integration form $\Lambda=\infty$ to $\Lambda=\Lambda_{0}$ yields a finite contribution for $\gamma_{1}$, which has to be calculated analytically. $\Lambda_{0}$ is then the starting point of the numerical integration, chosen much bigger than all relevant energies and such that $1 / \Lambda_{0}$ is smaller than the desired numerical accuracy.

Because of the slow convergence of $\mathcal{S}$, the factor $e^{\omega_{n} 0^{+}}$in the definition of the bare Green's function (3.58) is relevant for the limit $\omega \rightarrow \infty$. Integrating the differential equation of $\gamma_{1}$ (3.52) from infinity to $\Lambda=\Lambda_{0}$ gives

$$
\begin{aligned}
\Sigma_{k, k^{\prime}}^{\Lambda_{0}}\left(\omega_{n}\right) & =-T \lim _{\epsilon \rightarrow 0} \int_{\infty}^{\Lambda_{0}} d \Lambda \sum_{q, q^{\prime}, n^{\prime}} \mathcal{S}_{q, q^{\prime}}^{\Lambda}\left(\omega_{n^{\prime}}\right) v\left(k^{\prime}, q^{\prime} ; k, q\right) \\
& =-\frac{1}{2 \pi} \lim _{\epsilon \rightarrow 0} \int_{\infty}^{\Lambda_{0}} d \Lambda \sum_{\omega= \pm \Lambda} \sum_{q, q^{\prime}} e^{i \omega \epsilon} \frac{\delta_{q^{\prime}, q}}{i \omega} v_{k^{\prime}, q^{\prime} ; k, q}+\mathcal{O}\left(\Lambda_{0}^{-1}\right) \\
& =-\frac{1}{\pi} \sum_{q} v_{k^{\prime}, q ; k, q} \lim _{\epsilon \rightarrow 0} \int_{\infty}^{\Lambda_{0}} d \Lambda \frac{\sin \Lambda \epsilon}{\Lambda}+\mathcal{O}\left(\Lambda_{0}^{-1}\right) \\
& =-\frac{1}{\pi} \sum_{q} v_{k^{\prime}, q ; k, q}[\underbrace{\lim _{\epsilon \rightarrow 0} \int_{0}^{\Lambda_{0}} d \Lambda \frac{\sin \Lambda \epsilon}{\Lambda}}_{=0 \text { independent of } \Lambda_{0}}-\lim _{\epsilon \rightarrow 0} \underbrace{\int_{0}^{\infty} d \Lambda \frac{\sin \Lambda \epsilon}{\Lambda}}_{=\frac{\pi}{2} \text { independent of } \epsilon}]+\mathcal{O}\left(\Lambda_{0}^{-1}\right) \\
& =\frac{1}{2} \sum_{q} v_{k^{\prime}, q ; k, q}+\mathcal{O}\left(\Lambda_{0}^{-1}\right) .
\end{aligned}
$$

In the second line we used

$$
\begin{aligned}
\int_{\infty}^{\Lambda_{0}} d \Lambda \mathcal{S}_{q, q^{\prime}}^{\Lambda}\left(\omega_{n}\right) & =\int_{\infty}^{\Lambda_{0}} d \Lambda\left[\mathcal{G}^{\Lambda} \partial_{\Lambda}\left[\mathcal{G}_{0}^{\Lambda}\right]^{-1} \mathcal{G}^{\Lambda}\right]_{q, q^{\prime}} \\
& =\frac{1}{2 \pi T} \int_{\infty}^{\Lambda_{0}} d \Lambda \sum_{\omega= \pm \Lambda}\left(e^{i \omega 0^{+}} \frac{\delta_{q, q^{\prime}}}{i \omega}+\mathcal{O}\left(\omega^{-2}\right)\right) \\
& =\frac{1}{2 \pi T} \int_{\infty}^{\Lambda_{0}} d \Lambda \sum_{\omega= \pm \Lambda} e^{i \omega 0^{+}} \frac{\delta_{q, q^{\prime}}}{i \omega}+\mathcal{O}\left(\Lambda_{0}^{-1}\right)
\end{aligned}
$$

Note, that $\Lambda$ is a continuous variable, whereas $\omega_{n}$ is not. Replacing $\omega_{n}$ by the continuous variable $i \omega$, with $\left|\omega_{n}-i \omega\right|<\pi T$ gives an error of $\mathcal{O}\left(\omega^{-2}\right)$. The same error is obtained by neglecting $h$ and the feedback of $\Sigma$ in $\mathcal{S}$. 
Neglecting terms of $\mathcal{O}\left(\Lambda_{0}^{-1}\right)$ provides the final version of the initial condition:

$$
\begin{aligned}
\Sigma_{k^{\prime}, k}^{\Lambda_{0}}=-\gamma_{1}^{\Lambda_{0}}\left(k^{\prime}, k\right) & =\frac{1}{2} \sum_{q} v_{k^{\prime}, q ; k, q} \\
\gamma_{2}^{\Lambda_{0}}\left(k_{1}^{\prime}, k_{2}^{\prime} ; k_{1}, k_{2}\right) & =v_{k_{1}^{\prime}, k_{2}^{\prime} ; k_{1}, k_{2}} \\
\gamma_{m}^{\Lambda_{0}} & =0 \quad(m>2)
\end{aligned}
$$

\subsubsection{Morris' Lemma}

In most cases we will use the flow equations in the limit $T=0$. This limit has to be performed carefully, since a combination of $\delta$ - and $\Theta$-functions appear, which is an application of Morris (1994) lemma, which we state without proof: For a product of a $\delta$ and $\Theta$-functions, where both are limits of smoothed functions

$$
\delta=\lim _{\epsilon \rightarrow 0} \delta_{\epsilon}, \quad \Theta=\lim _{\epsilon \rightarrow 0} \Theta_{\epsilon}
$$

the following holds:

$$
\delta_{\epsilon} f\left(\Theta_{\epsilon}\right) \rightarrow \delta \int_{0}^{1} f(t) d t
$$

In equation (3.51) we defined the operator $\mathcal{S}$, which now can be simplified, since we have specified the cutoff. Whenever the operators depend on the cutoff we indicate this by the superscript $\Lambda$, thus if we write $\mathcal{G}^{0}$ we mean the cutoff-free bare propagator.

$$
\begin{aligned}
\mathcal{S}^{\Lambda} & =\mathcal{G}^{\Lambda} \partial_{\Lambda}\left[\mathcal{G}^{0, \Lambda}\right]^{-1} \mathcal{G}^{\Lambda} \\
& =\frac{1}{1+\Theta \mathcal{G}^{0} \gamma_{1}^{\Lambda}} \Theta \mathcal{G}^{0}\left[\mathcal{G}^{0}\right]^{-1} \frac{1}{\Theta^{2}} \delta \frac{1}{1+\Theta \mathcal{G}^{0} \gamma_{1}^{\Lambda}} \Theta \mathcal{G}^{0} \\
& =\frac{\delta}{\left(1+\Theta \mathcal{G}^{0} \gamma_{1}^{\Lambda}\right)^{2}} \mathcal{G}^{0} \\
& =\delta \partial_{\Theta} \frac{1}{1+\Theta \mathcal{G}^{0} \gamma_{1}^{\Lambda}} \Theta \mathcal{G}^{0} \\
& =\delta(|\omega|-\Lambda) \partial_{\Theta} \mathcal{G}^{\Lambda},
\end{aligned}
$$

where we used (3.60) and

$$
\mathcal{G}^{\Lambda}=\frac{1}{\left[\mathcal{G}^{0, \Lambda}\right]^{-1}+\gamma_{1}^{\Lambda}}=\frac{1}{1+\Theta \mathcal{G}^{0} \gamma_{1}^{\Lambda}} \Theta \mathcal{G}^{0} .
$$

Hence the single scale Green's functions reads

$$
S^{\Lambda}(i \omega)=\delta(|\omega|-\Lambda) \tilde{\mathcal{G}}^{\Lambda}
$$

with

$$
\tilde{\mathcal{G}}^{\Lambda}=\frac{1}{\left[\mathcal{G}^{0}\right]^{-1}+\gamma_{1}^{\Lambda}} .
$$


Note that $\mathcal{G}^{0}$ has no $\Lambda$-dependence. Further the product of the single scale and the common Green's functions gives

$$
S^{\Lambda}\left(i \omega_{1}\right) G^{\Lambda}\left(i \omega_{2}\right)=\delta\left(\left|\omega_{1}\right|-\Lambda\right) \Theta\left(\left|\omega_{2}\right|-\Lambda\right) \tilde{\mathcal{G}}^{\Lambda}\left(i \omega_{1}\right) \tilde{\mathcal{G}}^{\Lambda}\left(i \omega_{2}\right)
$$

\subsubsection{Further Approximations - Publication}

Due to the large number of interacting sites in the model (2.29) one needs to make further approximations. A calculation scheme suitable for dealing with inhomogeneous lattice systems, called the coupled ladder approximation (CLA) has been published in the journal Physical Review $B$. In this publication we also use the fRG equations to derive a formula for the spin susceptibility. Thereby we use the property of the fRG equations to yield exact relations for the derivative of the vertex functions w.r.t. a physical parameter. The publication is completed with an analysis of the reliability of the CLA for the model of a QPC. Below we include a reprint of this publication (Bauer et al., 2014). 


\title{
Functional renormalization group approach for inhomogeneous interacting Fermi systems
}

\author{
Florian Bauer, Jan Heyder, and Jan von Delft \\ Arnold Sommerfeld Center for Theoretical Physics and Center for NanoScience, Ludwig-Maximilians-Universität München, \\ Theresienstrasse 37, D-80333 München, Germany
}

(Received 15 November 2013; revised manuscript received 17 December 2013; published 21 January 2014)

\begin{abstract}
The functional renormalization group (fRG) approach has the property that, in general, the flow equation for the two-particle vertex generates $\mathcal{O}\left(N^{4}\right)$ independent variables, where $N$ is the number of interacting states (e.g., sites of a real-space discretization). In order to include the flow equation for the two-particle vertex, one needs to make further approximations if $N$ becomes too large. We present such an approximation scheme, called the coupled-ladder approximation, for the special case of an onsite interaction. Like the generic third-order-truncated fRG, the coupled-ladder approximation is exact to second order and is closely related to a simultaneous treatment of the random phase approximation in all channels, i.e., summing up parquet-type diagrams. The scheme is applied to a one-dimensional model describing a quantum point contact.
\end{abstract}

DOI: 10.1103/PhysRevB.89.045128

PACS number(s): 71.10.-w, 05.10.Cc, 05.60.Gg, 12.38.Bx

\section{INTRODUCTION}

The calculation of properties of an inhomogeneous interacting quantum system requires adequate care regarding a proper description of its spatial structure: for a lattice model, the resolution of a potential landscape, without generating additional finite-size effects, typically requires an extension of $\sim 10^{2}$ sites per spatial dimension. If, in addition, the strength of interactions can not be regarded as "weak," a reasonable approximation scheme must involve detailed information about higher-order correlations. This usually demands a huge effort for modern computers, both in memory and speed. Thus, for a system with nontrivial spatial structure, any approximation scheme necessarily involves a tradeoff between computational feasibility and accuracy.

In Ref. [1], we introduced such a scheme, both reasonably fast and accurate up to intermediate interaction strength, within the framework of the one-particle-irreducible version of the functional renormalization group (fRG) [2-10]. The goal of this paper is to supply a detailed description of this approximation scheme, called the coupled-ladder approximation (CLA), which is implemented within the context of generic, third-order-truncated fRG. In the latter, the flow of the three-particle vertex is set to zero, while the flow equation of the two-particle vertex (which we will call "vertex flow" in the following) is fully incorporated. This vertex flow has to be incorporated if interactions can not be considered small. In general, this constitutes a computational challenge since the vertex generated by this flow involves a large number $\mathcal{O}\left(N^{4}\right)$ of independent functions, each depending on three frequencies, where $N$ is the number of sites of the interacting region. As a result, the flow equations involve $\mathcal{O}\left(N^{4} N_{\mathrm{f}}^{3}\right)$ independent variables, where $N_{\mathrm{f}}$ is the number of discrete points per frequency used in the numerics. Previous schemes that included the vertex flow for models with large $N$ made use of an additional symmetry, e.g., Refs. [5,6] described systems with a weak spatial inhomogeneity (either changing adiabatically with position, or confined to a small region), which could be treated as a perturbation, so that its feedback to the vertex could be neglected. The resulting equations for the vertex were solved in the momentum basis, exploiting the fact that the single-particle eigenstates could approximately be represented by plane waves. However, this is not possible for models with strong inhomogeneities. Our CLA scheme was developed to include the vertex flow for such models. It extends the idea of Refs. [7,11], where the CLA was introduced to parametrize the frequency dependence of the vertex for the single-impurity Anderson model, i.e., $N=1$, which reduces the number of independent variables for that model to $\mathcal{O}\left(N_{\mathrm{f}}\right)$. We show that the CLA can be applied to parametrize the spatial dependence of the vertex for models with a purely local interaction. The number of independent variables that represent the spatial dependence of the vertex then reduces to $\mathcal{O}\left(N^{2}\right)$, and the total number of independent variables representing the vertex to $\mathcal{O}\left(N^{2} N_{\mathrm{f}}\right)$. The CLA scheme is exact to second order $[12,13]$ and effectively sums up diagrams of the random phase approximation (RPA) of all three interaction channels.

To illustrate the capabilities of our CLA scheme, we apply it, as in Ref. [1], to a one-dimensional chain modeling the lowest submode of a quantum point contact (QPC), a short constriction that allows transport only in one dimension. Its conductance is famously quantized [14-16] in units of $G_{Q}=$ $2 e^{2} / h$. In addition to this quantization, measured conductance curves show a shoulder at around $0.7 G_{Q}$. In this regime, quantities such as electrical and thermal conductance, noise, and thermopower have anomalous behavior [17-19]. These phenomena are collectively known as the " 0.7 anomaly" in QPCs.

In Ref. [1], we showed that the 0.7 anomaly is reproduced by a one-dimensional model with a parabolic potential barrier and a short-ranged Coulomb interaction. We presented a detailed microscopic picture that explained the physical mechanism which causes the anomalous behavior. Its origin is a smeared van Hove singularity in the density of states (DOS) just above the band bottom which enhances effects of interaction causing an enhanced backscattering. We presented detailed results for the conductance at zero temperature, obtained using fRG in the CLA. These numerical data were in good qualitative agreement with our experimental measurements and showed that the model reproduces the phenomenology of the 0.7 anomaly. In this paper, we set forth and examine the approximation scheme in detail. We present additional numerical data to verify the reliability of the method for the case where it is applied to the model of a QPC. For this, we 
present and compare data obtained by different approximation schemes within the fRG, showing that the phenomenology is very robust, and can even be obtained by neglecting the vertex flow. However, including the vertex flow using the CLA reduces artifacts and gives an insightful view on the spin susceptibility. For the latter, we finally present a detailed quantitative error analysis.

\section{MICROSCOPIC MODEL}

The approximation scheme presented in this paper can be applied to any model Hamiltonian that can be written in the following form:

$$
H=\sum_{i j, \sigma} h_{i j}^{\sigma} d_{i \sigma}^{\dagger} d_{j \sigma}+\sum_{j} U_{j} n_{j \uparrow} n_{j \downarrow},
$$

where $h^{\sigma}$ is a real, symmetric matrix, $d_{j \sigma}^{\dagger}\left(d_{j \sigma}\right)$ creates (annihilates) an electron at site $j$ with spin $\sigma(=\uparrow, \downarrow$ or,+- , with $\bar{\sigma}=-\sigma$ ), and $n_{j \sigma}=d_{j \sigma}^{\dagger} d_{j \sigma}$ counts them (in general $j$ can represent any quantum number, however, for simplicity we refer to it as a site index throughout the paper). In order to apply the CLA, the necessary property of this Hamiltonian is a short-ranged interaction. In principle, the approximation scheme can be set up for an interaction with finite range (over several sites), however, since the structure then becomes very complicated we will only discuss the case of a purely local, i.e., onsite interaction in this paper as given by Eq. (1). Whereas the system can extend to infinity, it is crucial that the number of sites $N$ where $U_{j}$ is nonzero is finite and not too large, as discussed in Sec. III H. If the system is extended to infinity, the effect of the noninteracting region can be calculated analytically using the projection method (see the Appendix and Refs. $[8,20]$ ). An extension to a Hamiltonian that is complex Hermitian and nondiagonal in spin space, needed, e.g., to include spin-orbit effects, is straightforward. In contrast, applying the scheme to spinless models, for which the interaction term has to be nonlocal to respect Pauli's exclusion principle, is more complicated.

\section{III. fRG FLOW EQUATIONS}

In this section, we describe the functional renormalization group (fRG) approach that we have employed to treat a translationally nonuniform Fermi system with onsite interactions, such as described by Eq. (1). We use the one-particleirreducible (1PI) version of the fRG [2,21]. Its key idea is to approximately sum up a perturbative expansion, in our case in the interaction, by setting up and numerically solving a set of coupled ordinary differential equations (ODEs), the flow equations, for the system's 1PI $n$-particle vertex functions $\gamma_{n}$. This is typically done in such a way that the effects of higher-energy modes, lying above a flowing infrared cutoff parameter $\Lambda$, are incorporated before those of lower-energy modes lying below $\Lambda$. This yields a systematic way of summing up parquet-type diagrams for the two-particle vertex and for calculating the self-energy. $\Lambda$ serves as flow parameter that controls the RG flow of the $\Lambda$-dependent vertex functions $\gamma_{n}^{\Lambda}$ from an initial cutoff $\Lambda_{i}$, at which all vertex functions are known and simple, to a final cutoff $\Lambda_{f}$, at which the full theory is recovered.
This idea is implemented by replacing, in the generating functional for the vertex functions $\gamma_{n}$, the bare propagator $\mathcal{G}_{0}$ by a modified propagator $\mathcal{G}_{0}^{\Lambda}$,

$$
\mathcal{G}_{0} \rightarrow \mathcal{G}_{0}^{\Lambda}, \quad \text { with } \quad \mathcal{G}_{0}^{\Lambda_{i}}=0, \quad \mathcal{G}_{0}^{\Lambda_{f}}=\mathcal{G}_{0},
$$

constructed such that $\mathcal{G}_{0}^{\Lambda}$ is strongly suppressed for frequencies below $\Lambda$. The $\Lambda$ dependence of the resulting vertex functions $\gamma_{n}^{\Lambda}$ is governed by an infinite hierarchy of coupled ODEs, the $R G$ flow equations, of the form

$$
\frac{d}{d \Lambda} \gamma_{n}^{\Lambda}=\mathcal{F}\left(\Lambda, \mathcal{G}_{0}^{\Lambda}, \gamma_{1}^{\Lambda}, \ldots, \gamma_{n+1}^{\Lambda}\right),
$$

where $\gamma_{1}=-\Sigma$ is the self-energy and $\gamma_{2}$ the two-particle vertex. At the beginning of the RG flow, the vertex functions are initialized to their bare values

$$
\gamma_{2}^{\Lambda_{i}}=v, \quad \gamma_{n}^{\Lambda_{i}}=0 \quad(n \neq 2)
$$

while their fully dressed values, corresponding to the full theory, are recovered upon integrating Eqs. (3) from $\Lambda_{i}$ to $\Lambda_{f}$.

The infinite hierarchy of ODEs (3) is exact, but in most cases not solvable. In the generic, third-order-truncated fRG, all $n$-particle vertex functions with $n \geqslant 3$ are neglected

$$
\frac{d}{d \Lambda} \gamma_{n}=0 \quad(n \geqslant 3)
$$

and the resulting flow equations for $\gamma_{1}^{\Lambda}$ and $\gamma_{2}^{\Lambda}$ are integrated numerically. Due to this truncation, fRG is in essence an "RG-enhanced" perturbation expansion in the interaction, which will break down if $U$ becomes too large. In fact, the flow equations can be derived by a purely diagrammatic procedure, without resorting to a generating functional, as explained in Ref. [22]. The diagrammatic structure is such that the flow of the self-energy and three different parquet channels (i.e., three coupled RPA-like series of diagrams) are treated simultaneously, feeding into each other during the flow (as discussed in more detail below). This moderates competing instabilities in an unbiased way. We also mention that this approach has been found to be particularly useful to treat models where infrared divergences play a role [3] (although the latter do not arise for the present model).

The following statements in this section hold for most, however, not for every flow parameter. For that reason, we explicitly define the $\Lambda$ dependence at this point. If a different fRG scheme is used, one should carefully check all relations. The general idea should be applicable for all fRG schemes. We use fRG in the Matsubara formalism. In the following frequencies with subscripts $n, n^{\prime}, n_{1}$, etc., are defined to be purely imaginary:

$$
\omega_{n}=i T \pi(2 n+1) .
$$

We introduce $\Lambda$ as an infrared cutoff in the bare Matsubara propagator

$$
\mathcal{G}_{0}^{\Lambda}\left(\omega_{n}\right)=\Theta_{T}\left(\left|\omega_{n}\right|-\Lambda\right) \mathcal{G}_{0}\left(\omega_{n}\right), \quad \Lambda_{i}=\infty, \quad \Lambda_{f}=0,
$$

where $\Theta_{T}$ is a step function that is broadened on the scale of the temperature $T$.

For a derivation of the fRG flow equations, see, e.g., Refs. [3,5]; very detailed discussions are given, e.g., in 
Refs. [8,23], for a diagrammatic derivation see Ref. [22]. The flow equation for the self-energy reads as

$$
\frac{d}{d \Lambda} \gamma_{1}^{\Lambda}\left(q_{1}^{\prime}, q_{1}\right)=T \sum_{q_{2}^{\prime}, q_{2}} \mathcal{S}_{q_{2}, q_{2}^{\prime}}^{\Lambda} \gamma_{2}^{\Lambda}\left(q_{2}^{\prime}, q_{1}^{\prime} ; q_{2}, q_{1}\right)
$$

where $q_{1}, q_{2}$, etc., label the quantum number and the fermionic Matsubara frequency. Here, $\mathcal{S}^{\Lambda}$ is defined in terms of the scaledependent full propagator $\mathcal{G}^{\Lambda}$ :

$$
\begin{aligned}
\mathcal{S}^{\Lambda} & =\mathcal{G}^{\Lambda} \partial_{\Lambda}\left[\mathcal{G}_{0}^{\Lambda}\right]^{-1} \mathcal{G}^{\Lambda} \\
\mathcal{G}^{\Lambda} & =\left[\left[\mathcal{G}_{0}^{\Lambda}\right]^{-1}-\Sigma^{\Lambda}\right]^{-1}
\end{aligned}
$$

For later convenience, we divide the two-particle vertex $\gamma_{2}$ in four parts:

$$
\gamma_{2}^{\Lambda}=v+\gamma_{p}^{\Lambda}+\gamma_{x}^{\Lambda}+\gamma_{d}^{\Lambda}
$$

where $v$ is the bare vertex and $\gamma_{p}^{\Lambda}, \gamma_{x}^{\Lambda}$, and $\gamma_{d}^{\Lambda}$ are called the particle-particle channel $(P)$, and the exchange $(X)$ and direct $(D)$ contributions to the particle-hole channel, respectively. They are defined via their flow-equations with $\gamma_{y}^{\Lambda_{i}}=0$ :

$$
\frac{d}{d \Lambda} \gamma_{2}^{\Lambda}=\frac{d}{d \Lambda}\left(\gamma_{p}^{\Lambda}+\gamma_{x}^{\Lambda}+\gamma_{d}^{\Lambda}\right)
$$

Explicitly, these flow equations have the following forms:

$$
\begin{aligned}
\frac{d}{\Lambda} \gamma_{p}^{\Lambda}\left(q_{1}^{\prime}, q_{2}^{\prime} ; q_{1}, q_{2}\right) & =T \sum_{q_{3}^{\prime}, q_{3}, q_{4}^{\prime}, q_{4}} \gamma_{2}^{\Lambda}\left(q_{1}^{\prime}, q_{2}^{\prime} ; q_{3}, q_{4}\right) \mathcal{S}_{q_{3}, q_{3}^{\prime}}^{\Lambda} \mathcal{G}_{q_{4}, q_{4}^{\prime}}^{\Lambda} \gamma_{2}^{\Lambda}\left(q_{3}^{\prime}, q_{4}^{\prime} ; q_{1}, q_{2}\right), \\
\frac{d}{d \Lambda} \gamma_{x}^{\Lambda}\left(q_{1}^{\prime}, q_{2}^{\prime} ; q_{1}, q_{2}\right) & =T \sum_{q_{3}^{\prime}, q_{3}, q_{4}^{\prime}, q_{4}} \gamma_{2}^{\Lambda}\left(q_{1}^{\prime}, q_{4}^{\prime} ; q_{3}, q_{2}\right)\left[\mathcal{S}_{q_{3}, q_{3}^{\prime}}^{\Lambda} \mathcal{G}_{q_{4}, q_{4}^{\prime}}^{\Lambda}+\mathcal{G}_{q_{3}, q_{3}^{\prime}}^{\Lambda} \mathcal{S}_{q_{4}, q_{4}^{\prime}}^{\Lambda}\right] \gamma_{2}^{\Lambda}\left(q_{3}^{\prime}, q_{2}^{\prime} ; q_{1}, q_{4}\right), \\
\frac{d}{d \Lambda} \gamma_{d}^{\Lambda}\left(q_{1}^{\prime}, q_{2}^{\prime} ; q_{1}, q_{2}\right) & =-T \sum_{q_{3}^{\prime}, q_{3}, q_{4}^{\prime}, q_{4}} \gamma_{2}^{\Lambda}\left(q_{1}^{\prime}, q_{3}^{\prime} ; q_{1}, q_{4}\right)\left[\mathcal{S}_{q_{4}, q_{4}^{\prime}}^{\Lambda} \mathcal{G}_{q_{3}, q_{3}^{\prime}}^{\Lambda}+\mathcal{G}_{q_{4}, q_{4}^{\prime}}^{\Lambda} \mathcal{S}_{q_{3}, q_{3}^{\prime}}^{\Lambda}\right] \gamma_{2}^{\Lambda}\left(q_{4}^{\prime}, q_{2}^{\prime} ; q_{3}, q_{2}\right) .
\end{aligned}
$$

Here, the higher-order vertices $\gamma_{n} \geqslant 3$ have already been set to zero.

\section{A. Frequency parametrization}

Due to energy conservation, the frequencies in Eqs. (8) and (12) are not independent:

$$
\begin{aligned}
\gamma_{1}\left(q_{1}^{\prime}, q_{1}\right) & \propto \delta\left(\omega_{n_{1}^{\prime}}-\omega_{n_{1}}\right), \\
\gamma_{2}\left(q_{1}^{\prime}, q_{2}^{\prime} ; q_{1}, q_{2}\right) & \propto \delta\left(\omega_{n_{1}^{\prime}}+\omega_{n_{2}^{\prime}}-\omega_{n_{1}}-\omega_{n_{2}}\right) .
\end{aligned}
$$

In the case of the two-particle vertex, this gives a certain freedom to parametrize its frequency dependence. The natural choice, as will become apparent later on, is to parametrize it in terms of three bosonic frequencies:

$$
\begin{aligned}
& \Pi=\omega_{n_{1}^{\prime}}+\omega_{n_{2}^{\prime}}=\omega_{n_{1}}+\omega_{n_{2}}, \\
& X=\omega_{n_{2}^{\prime}}-\omega_{n_{1}}=\omega_{n_{2}}-\omega_{n_{1}^{\prime}}, \\
& \Delta=\omega_{n_{1}^{\prime}}-\omega_{n_{1}}=\omega_{n_{2}}-\omega_{n_{2}^{\prime}} .
\end{aligned}
$$

Note that due to their definition in terms of purely imaginary frequencies, the bosonic frequencies are imaginary too. Conversely, the fermionic frequencies can be expressed in terms of the bosonic ones:

$$
\begin{array}{ll}
\omega_{n_{1}^{\prime}}=\frac{1}{2}(\Pi-X+\Delta), & \omega_{n_{2}^{\prime}}=\frac{1}{2}(\Pi+X-\Delta), \\
\omega_{n_{1}}=\frac{1}{2}(\Pi-X-\Delta), & \omega_{n_{2}}=\frac{1}{2}(\Pi+X+\Delta) .
\end{array}
$$

\section{B. Neglecting the vertex flow}

For the purpose of treating the inhomogeneous model of Eq. (1), we take the quantum number that labels Green's functions and vertices to denote a composite index of site, spin, and Matsubara frequency $q_{1}=\left(j_{1}, \sigma_{1}, \omega_{1}\right)$, etc. Since the bare propagators are nondiagonal in the site index, the number of independent variables $\gamma_{2}^{\Lambda}\left(q_{1}^{\prime}, q_{2}^{\prime} ; q_{1}, q_{2}\right)$ generated by Eq. (12) is very large $\mathcal{O}\left(N^{4} N_{\mathrm{f}}^{3}\right)$, where $N_{\mathrm{f}}$ is the number of Matsubara frequencies per frequency argument kept track of in the numerics.

The simplest way to avoid this complication is to neglect the flow of the two-particle vertex:

$$
\frac{d}{d \Lambda} \gamma_{2}=0
$$

This scheme, to be called fRG1, yields a frequencyindependent self-energy, which, for the case of local interaction, is site diagonal. It is exact to first order in the interaction.

\section{Coupled-ladder approximation}

For models where the interaction can not be considered small, we introduced a novel scheme in Ref. [1], to be called dynamic fRG in CLA, to incorporate the effects of vertex flow. In the following, whenever the vertex flow is included, we treat it using the CLA, thus calling this approximation dfRG2, to distinguish it from fRG1, and from a static fRG scheme including the vertex flow sfRG2 to be introduced later. The dfRG2 scheme exploits the fact that the bare vertex

$$
\begin{aligned}
& v\left(j_{1} \sigma_{1}, j_{2} \sigma_{2} ; j_{3} \sigma_{3}, j_{4} \sigma_{4}\right) \\
& \quad=U_{j_{1}} \delta_{j_{1} j_{2}} \delta_{j_{3} j_{4}} \delta_{j_{1} j_{4}} \delta_{\sigma_{1} \bar{\sigma}_{2}} \delta_{\bar{\sigma}_{3} \sigma_{4}}\left(\delta_{\sigma_{1} \sigma_{3}}-\delta_{\sigma_{1} \sigma_{4}}\right)
\end{aligned}
$$

is purely site diagonal, and parametrizes the vertex in terms of $\mathcal{O}\left(N^{2} N_{\mathrm{f}}\right)$ independent variables.

To this end, we consider a simplified version of the vertex flow equation (12), where the feedback of the vertex flow is neglected: on the right-hand side we replace $\gamma_{2}^{\Lambda} \rightarrow v$. If the feedback of the self-energy were also neglected, this would be equivalent to calculating the vertex in second-order perturbation theory. As a consequence, all generated vertex 
contributions depend on two site indices and a single bosonic frequency. They have one of the following structures:

$$
P_{j i}^{\sigma \bar{\sigma}}(\Pi):=\gamma_{p}^{\Lambda}\left(j \sigma \Pi-\omega_{n^{\prime}}, j \bar{\sigma} \omega_{n^{\prime}} ; i \sigma \Pi-\omega_{n}, i \bar{\sigma} \omega_{n}\right)
$$

$$
\bar{P}_{j i}^{\sigma \bar{\sigma}}(\Pi):=\gamma_{p}^{\Lambda}\left(j \sigma \Pi-\omega_{n^{\prime}}, j \bar{\sigma} \omega_{n^{\prime}} ; i \bar{\sigma} \Pi-\omega_{n}, i \sigma \omega_{n}\right)
$$

$$
\begin{aligned}
& X_{j i}^{\sigma \bar{\sigma}}(\mathrm{X}):=\gamma_{x}^{\Lambda}\left(j \sigma \mathrm{X}+\omega_{n^{\prime}}, i \bar{\sigma} \omega_{n} ; i \sigma \mathrm{X}+\omega_{n}, j \bar{\sigma} \omega_{n^{\prime}}\right) \\
& \underset{\mathcal{O}\left(v^{2}\right)}{\mathrm{X}+\omega_{n^{\prime}}} \underbrace{\mathrm{X}+\omega_{n^{\prime \prime}}}_{\substack{j \bar{\sigma} \\
\omega_{n^{\prime}}}} \underbrace{\mathrm{X}+\omega_{n}}_{\substack{i \bar{\sigma} \\
\omega_{n}}}
\end{aligned}
$$

$$
X_{j i}^{\sigma \sigma}(\mathrm{X}):=\gamma_{x}^{\Lambda}\left(j \sigma \mathrm{X}+\omega_{n^{\prime}}, i \sigma \omega_{n} ; i \sigma \mathrm{X}+\omega_{n}, j \sigma \omega_{n^{\prime}}\right)
$$

$$
D_{j i}^{\sigma \sigma}(\Delta):=\gamma_{d}^{\Lambda}\left(j \sigma \Delta+\omega_{n^{\prime}}, i \sigma \omega_{n} ; j \sigma \omega_{n^{\prime}}, i \sigma \Delta+\omega_{n}\right)
$$

$$
D_{j i}^{\sigma \bar{\sigma}}(\Delta):=\gamma_{d}^{\Lambda}\left(j \bar{\sigma} \Delta+\omega_{n^{\prime}}, i \sigma \omega_{n} ; j \sigma \omega_{n^{\prime}}, i \bar{\sigma} \Delta+\omega_{n}\right)
$$

These second-order terms do not depend on the frequencies $\omega_{n}$ and $\omega_{n^{\prime}}$. Now note that no additional terms are generated if we allow for a vertex feedback within the individual channels in Eqs. (12a), (12b), and (12c), i.e., if we take the flow equation of $\gamma_{a}(A)(a=p, x, d$ and correspondingly $A=\Pi, \mathrm{X}, \Delta)$ and replace the feedback of the vertex on the right-hand side by

$$
\gamma_{2}(\Pi, \mathrm{X}, \Delta) \rightarrow v+\gamma_{a}(A)
$$

This scheme is equivalent to solving RPA equations for the three individual channels $P, X$, and $D$ (see Sec. III I), with an additional feedback of the self-energy via Eq. (9).

Note that if $i=j$ in Eq. (18), the terms $a$ and $c, b$ and $f$ as well as $d$ and $e$ have the same structure w.r.t. their external site and spin indices. As a result, it is possible to account for an interchannel feedback in the vertex flow without generating additional terms if the feedback is restricted to purely site diagonal terms. As in Ref. [11], we avoid frequency mixing by limiting the interchannel feedback to the static part of the vertex, i.e., the vertex contributions are evaluated at zero frequency when fed into other channels. Putting everything together, the approximation scheme is defined by replacing the vertex on the right-hand side of the flow equation $\dot{\gamma}_{a}^{\Lambda}$ by (12):

$$
\gamma_{2} \rightarrow v+\gamma_{a}(A)+\left[\gamma_{b}(0)+\gamma_{c}(0)\right] \delta_{j_{1} j_{2}} \delta_{j_{1}^{\prime} j_{2}^{\prime}} \delta_{j_{1} j_{1}^{\prime}},
$$

where $a, b, c$ are cyclic permutations of $p, x, d$, and $A, B, C$ are the corresponding cyclic permutations of the frequencies $\Pi, X, \Delta$. Since this equation is the central definition of this paper, we explicitly write it for each of the three channels:

$$
\begin{aligned}
& \dot{\gamma}_{p}(\Pi): \gamma_{2}\left(j_{1}^{\prime}, j_{2}^{\prime} ; j_{1}, j_{2} ; \Pi, \mathrm{X}, \Delta\right) \\
& \rightarrow v+\gamma_{p}(\Pi)+\left[\gamma_{x}(0)+\gamma_{d}(0)\right] \delta_{j_{1} j_{2}} \delta_{j_{1}^{\prime} j_{2}^{\prime}} \delta_{j_{1} j_{1}^{\prime}}, \\
& \dot{\gamma}_{x}(\mathrm{X}): \gamma_{2}\left(j_{1}^{\prime}, j_{2}^{\prime} ; j_{1}, j_{2} ; \Pi, \mathrm{X}, \Delta\right) \\
& \rightarrow v+\gamma_{x}(\mathrm{X})+\left[\gamma_{p}(0)+\gamma_{d}(0)\right] \delta_{j_{1} j_{2}} \delta_{j_{1}^{\prime} j_{2}^{\prime}} \delta_{j_{1} j_{1}^{\prime}}, \\
& \dot{\gamma}_{d}(\Delta): \gamma_{2}\left(j_{1}^{\prime}, j_{2}^{\prime} ; j_{1}, j_{2} ; \Pi, \mathrm{X}, \Delta\right) \\
& \rightarrow v+\gamma_{d}(\Delta)+\left[\gamma_{p}(0)+\gamma_{x}(0)\right] \delta_{j_{1} j_{2}} \delta_{j_{1}^{\prime} j_{2}^{\prime}} \delta_{j_{1} j_{1}^{\prime}} .
\end{aligned}
$$

This scheme generates a self-energy and a vertex which are both exact to second order in the interaction. To see this we note that first, the fRG flow equations without any truncation are exact, and second, in the fRG truncation (5) and in the CLA (20) the neglected terms are all of third or higher order in the interaction.

\section{Symmetries}

As can readily be checked, these flow equations respect the following symmetry relations:

$$
\begin{aligned}
& \mathcal{G}_{i j}^{\sigma \Lambda}\left(\omega_{n}\right)=\mathcal{G}_{j i}^{\sigma \Lambda}\left(\omega_{n}\right)=\left[\mathcal{G}_{i j}^{\sigma \Lambda}\left(-\omega_{n}\right)\right]^{*}, \\
& \Sigma_{i j}^{\sigma \Lambda}\left(\omega_{n}\right)=\Sigma_{j i}^{\sigma \Lambda}\left(\omega_{n}\right)=\left[\Sigma_{i j}^{\sigma \Lambda}\left(-\omega_{n}\right)\right]^{*}, \\
& P_{j i}^{\sigma \bar{\sigma}}=P_{j i}^{\bar{\sigma} \sigma}=P_{i j}^{\sigma \bar{\sigma}}, \quad \bar{P}_{j i}^{\sigma \bar{\sigma}}=\bar{P}_{j i}^{\bar{\sigma} \sigma}=\bar{P}_{i j}^{\sigma \bar{\sigma}}, \\
& P_{j i}^{\sigma \bar{\sigma}}=-\bar{P}_{j i}^{\sigma \bar{\sigma}}, \\
& X_{j i}^{\sigma \sigma^{\prime}}=X_{i j}^{\sigma \sigma^{\prime}}=\left[X_{j i}^{\sigma^{\prime} \sigma}\right]^{*}, \quad D_{j i}^{\sigma \sigma^{\prime}}=D_{i j}^{\sigma \sigma^{\prime}}=\left[D_{j i}^{\sigma^{\prime} \sigma}\right]^{*}, \\
& X=-D, \\
& P_{j i}^{\sigma \bar{\sigma}}(\Pi)=\left[P_{j i}^{\sigma \bar{\sigma}}(-\Pi)\right]^{*}, \quad X_{j i}^{\sigma \sigma^{\prime}}(\mathrm{X})=\left[X_{j i}^{\sigma \sigma^{\prime}}(-\mathrm{X})\right]^{*} \\
& D_{j i}^{\sigma \sigma^{\prime}}(\Delta)=\left[D_{j i}^{\sigma \sigma^{\prime}}(-\Delta)\right]^{*}, \\
& X^{\sigma \sigma}, D^{\sigma \sigma} \in \mathbb{R} .
\end{aligned}
$$

As a result, only four independent symmetric frequencydependent matrices are left, which we define as follows:

$$
\begin{aligned}
P_{j i}^{\Lambda}(\Pi) & =P_{j i}^{\sigma \bar{\sigma}}(\Pi), \quad X_{j i}^{\Lambda}(\mathrm{X})=X_{j i}^{\sigma \bar{\sigma}}(\mathrm{X}), \\
D_{j i}^{\sigma \Lambda}(\Delta) & =D_{j i}^{\sigma \sigma}(\Delta),
\end{aligned}
$$


where the superscript $\Lambda$ signifies a dependence on the flow parameter. At zero magnetic field, the number of independent matrices reduces to three since in this case $D^{\uparrow}=D^{\downarrow}$.

The flow equations for these matrices can be derived starting from Eqs. (12). The replacement (20) restricts the internal quantum numbers on the right-hand side of the flow equation $q_{3}, q_{4}, q_{3}^{\prime}$, and $q_{4}^{\prime}$ according to the definitions (18):

$$
\begin{aligned}
\dot{P}_{j i}^{\Lambda}(\Pi)= & \dot{\gamma}_{p}^{\Lambda}\left(j \sigma \Pi-\omega_{n^{\prime}}, j \bar{\sigma} \omega_{n^{\prime}} ; i \sigma \Pi-\omega_{n}, i \bar{\sigma} \omega_{n}\right) \\
= & T \sum_{k l, n^{\prime \prime}}\left[\gamma_{2}^{\Lambda}\left(j \sigma \Pi-\omega_{n^{\prime}}, j \bar{\sigma} \omega_{n^{\prime}} ; k \sigma \omega_{n^{\prime \prime}}, k \bar{\sigma} \Pi-\omega_{n^{\prime \prime}}\right) \mathcal{S}_{k l}^{\sigma \Lambda}\left(\omega_{n^{\prime \prime}}\right) \mathcal{G}_{k l}^{\bar{\sigma} \Lambda}\left(\Pi-\omega_{n^{\prime \prime}}\right) \gamma_{2}^{\Lambda}\left(l \sigma \omega_{n^{\prime \prime}}, l \bar{\sigma} \Pi-\omega_{n^{\prime \prime}} ; i \sigma \Pi-\omega_{n}, i \bar{\sigma} \omega_{n}\right)\right. \\
& \left.+\gamma_{2}^{\Lambda}\left(j \sigma \Pi-\omega_{n^{\prime}}, j \bar{\sigma} \omega_{n^{\prime}} ; k \bar{\sigma} \omega_{n^{\prime \prime}}, k \sigma \Pi-\omega_{n^{\prime \prime}}\right) \mathcal{S}_{k l}^{\bar{\sigma} \Lambda}\left(\omega_{n^{\prime \prime}}\right) \mathcal{G}_{k l}^{\sigma \Lambda}\left(\Pi-\omega_{n^{\prime \prime}}\right) \gamma_{2}^{\Lambda}\left(l \bar{\sigma} \omega_{n^{\prime \prime}}, l \sigma \Pi-\omega_{n^{\prime \prime}} ; i \sigma \Pi-\omega_{n}, i \bar{\sigma} \omega_{n}\right)\right],
\end{aligned}
$$

$$
\begin{aligned}
\dot{X}_{j i}^{\Lambda}(\mathrm{X})= & \dot{\gamma}_{x}^{\Lambda}\left(j \sigma \mathrm{X}+\omega_{n^{\prime}}, i \bar{\sigma} \omega_{n} ; i \sigma \mathrm{X}+\omega_{n}, j \bar{\sigma} \omega_{n^{\prime}}\right) \\
= & T \sum_{k l, n^{\prime \prime}}\left[\gamma_{2}^{\Lambda}\left(j \sigma \mathrm{X}+\omega_{n^{\prime}}, k \bar{\sigma} \omega_{n^{\prime \prime}} ; k \sigma \mathrm{X}+\omega_{n^{\prime \prime}}, j \bar{\sigma} \omega_{n^{\prime}}\right) \mathcal{S}_{k l}^{\bar{\sigma} \Lambda}\left(\omega_{n^{\prime \prime}}\right) \mathcal{G}_{l k}^{\sigma \Lambda}\left(\mathrm{X}+\omega_{n^{\prime \prime}}\right) \gamma_{2}^{\Lambda}\left(l \sigma \mathrm{X}+\omega_{n^{\prime \prime}}, i \bar{\sigma} \omega_{n} ; i \sigma \mathrm{X}+\omega_{n}, l \bar{\sigma} \omega_{n^{\prime \prime}}\right)\right. \\
& \left.+\gamma_{2}^{\Lambda}\left(j \sigma \mathrm{X}+\omega_{n^{\prime}}, k \bar{\sigma} \omega_{n^{\prime \prime}} ; k \sigma \mathrm{X}+\omega_{n^{\prime \prime}}, j \bar{\sigma} \omega_{n^{\prime}}\right) \mathcal{G}_{k l}^{\bar{\sigma} \Lambda}\left(\omega_{n^{\prime \prime}}\right) \mathcal{S}_{l k}^{\sigma \Lambda}\left(\mathrm{X}+\omega_{n^{\prime \prime}}\right) \gamma_{2}^{\Lambda}\left(l \sigma \mathrm{X}+\omega_{n^{\prime \prime}}, i \bar{\sigma} \omega_{n} ; i \sigma \mathrm{X}+\omega_{n}, l \bar{\sigma} \omega_{n^{\prime \prime}}\right)\right]
\end{aligned}
$$

$$
\begin{aligned}
\dot{D}_{j i}^{\sigma \Lambda}(\Delta)= & \dot{\gamma}_{d}^{\Lambda}\left(j \sigma \Delta+\omega_{n^{\prime}}, i \sigma \omega_{n} ; j \sigma \omega_{n^{\prime}}, i \sigma \Delta+\omega_{n}\right) \\
= & -T \sum_{k l, n^{\prime \prime}}\left[\gamma_{2}^{\Lambda}\left(j \sigma \Delta+\omega_{n^{\prime}}, k \sigma \omega_{n^{\prime \prime}} ; j \sigma \omega_{n^{\prime}}, k \sigma \Delta+\omega_{n}\right) \mathcal{S}_{k l}^{\sigma \Lambda}\left(\omega_{n^{\prime \prime}}\right) \mathcal{G}_{k l}^{\sigma \Lambda}\left(\Delta+\omega_{n^{\prime \prime}}\right) \gamma_{2}^{\Lambda}\left(l \sigma \Delta+\omega_{n^{\prime \prime}}, i \sigma \omega_{n} ; l \sigma \omega_{n^{\prime \prime}}, i \sigma \Delta+\omega_{n}\right)\right. \\
& +\gamma_{2}^{\Lambda}\left(j \sigma \Delta+\omega_{n^{\prime}}, k \sigma \omega_{n^{\prime \prime}} ; j \sigma \omega_{n^{\prime}}, k \sigma \Delta+\omega_{n}\right) \mathcal{G}_{k l}^{\sigma \Lambda}\left(\omega_{n^{\prime \prime}}\right) \mathcal{S}_{k l}^{\sigma \Lambda}\left(\Delta+\omega_{n^{\prime \prime}}\right) \gamma_{2}^{\Lambda}\left(l \sigma \Delta+\omega_{n^{\prime \prime}}, i \sigma \omega_{n} ; l \sigma \omega_{n^{\prime \prime}}, i \sigma \Delta+\omega_{n}\right) \\
& +\gamma_{2}^{\Lambda}\left(j \sigma \Delta+\omega_{n^{\prime}}, j \bar{\sigma} \omega_{n^{\prime \prime}} ; j \sigma \omega_{n^{\prime}}, j \bar{\sigma} \Delta+\omega_{n}\right) \mathcal{S}_{j i}^{\bar{\sigma} \Lambda}\left(\omega_{n^{\prime \prime}}\right) \mathcal{G}_{i j}^{\bar{\sigma} \Lambda}\left(\Delta+\omega_{n^{\prime \prime}}\right) \gamma_{2}^{\Lambda}\left(i \bar{\sigma} \Delta+\omega_{n^{\prime \prime}}, i \sigma \omega_{n} ; i \bar{\sigma} \omega_{n^{\prime \prime}}, i \sigma \Delta+\omega_{n}\right) \\
& \left.+\gamma_{2}^{\Lambda}\left(j \sigma \Delta+\omega_{n^{\prime}}, j \bar{\sigma} \omega_{n^{\prime \prime}} ; j \sigma \omega_{n^{\prime}}, j \bar{\sigma} \Delta+\omega_{n}\right) \mathcal{G}_{j i}^{\bar{\sigma} \Lambda}\left(\omega_{n^{\prime \prime}}\right) \mathcal{S}_{i j}^{\bar{\sigma} \Lambda}\left(\Delta+\omega_{n^{\prime \prime}}\right) \gamma_{2}^{\Lambda}\left(i \bar{\sigma} \Delta+\omega_{n^{\prime \prime}}, i \sigma \omega_{n} ; i \bar{\sigma} \omega_{n^{\prime \prime}}, i \sigma \Delta+\omega_{n}\right)\right]
\end{aligned}
$$

As is the case for the diagrams (18), these equations do not depend on $\omega_{n}$ and $\omega_{n^{\prime}}$, if the same holds for $\gamma_{2}$ on the righthand side. The latter is of course not the case without the replacement (20). The initial conditions are

$$
P^{\Lambda_{i}}=X^{\Lambda_{i}}=D^{\sigma \Lambda_{i}}=0
$$

Performing the replacement (20), these equations can be compactly written in matrix form

$$
\begin{aligned}
\frac{d}{d \Lambda} P^{\Lambda}(\Pi)= & \tilde{P}^{\Lambda}(\Pi) W^{p \Lambda}(\Pi) \tilde{P}^{\Lambda}(\Pi), \\
\frac{d}{d \Lambda} X^{\Lambda}(\mathrm{X})= & \tilde{X}^{\Lambda}(\mathrm{X}) W^{x \Lambda}(\mathrm{X}) \tilde{X}^{\Lambda}(\mathrm{X}), \\
\frac{d}{d \Lambda} D^{\sigma \Lambda}(\Delta)= & -\tilde{D}^{\sigma \Lambda}(\Delta) W^{\sigma d \Lambda}(\Delta) \tilde{D}^{\sigma \Lambda}(\Delta) \\
& -I^{\Lambda} W^{\bar{\sigma} d \Lambda}(\Delta) I^{\Lambda},
\end{aligned}
$$

where we have introduced the definitions

$$
\begin{aligned}
\tilde{P}_{j i}^{\Lambda}(\Pi) & =P_{j i}^{\Lambda}(\Pi)+\delta_{j i}\left(X_{j j}^{\Lambda}(0)+U_{j}\right), \\
\tilde{X}_{j i}^{\Lambda}(\mathrm{X}) & =X_{j i}^{\Lambda}(\mathrm{X})+\delta_{j i}\left(P_{j j}^{\Lambda}(0)+U_{j}\right), \\
\tilde{D}_{j i}^{\sigma \Lambda}(\Delta) & =D_{j i}^{\sigma \Lambda}(\Delta)+\delta_{j i} X_{j j}^{\sigma \Lambda}(0) \\
& =D_{j i}^{\sigma \Lambda}(\Delta)-\delta_{j i} D_{j j}^{\sigma \Lambda}(0), \\
I_{j i}^{\Lambda} & =\delta_{j i}\left[P_{j j}^{\Lambda}(0)+X_{j j}^{\Lambda}(0)+U_{j}\right],
\end{aligned}
$$

which account for the interchannel feedback contained in Eq. (20). $W^{p}, W^{x}$, and $W^{\sigma d}$ each represent a specific bubble, i.e., a product of two propagators summed over an internal frequency:

$$
\begin{aligned}
W_{j i}^{p \Lambda}(\Pi)= & T \sum_{\sigma n} \mathcal{S}_{j i}^{\sigma \Lambda}\left(\omega_{n}\right) \mathcal{G}_{j i}^{\bar{\sigma} \Lambda}\left(\Pi-\omega_{n}\right), \\
W_{j i}^{x \Lambda}(\mathrm{X})= & T \sum_{n}\left[\mathcal{S}_{j i}^{\uparrow \Lambda}\left(\omega_{n}\right) \mathcal{G}_{i j}^{\downarrow \Lambda}\left(\mathrm{X}+\omega_{n}\right)\right. \\
& \left.+\mathcal{S}_{i j}^{\downarrow \Lambda}\left(\omega_{n}\right) \mathcal{G}_{j i}^{\uparrow \Lambda}\left(\omega_{n}-\mathrm{X}\right)\right], \\
W_{j i}^{\sigma d \Lambda}(\Delta)= & T \sum_{n}\left[\mathcal{S}_{j i}^{\sigma \Lambda}\left(\omega_{n}\right) \mathcal{G}_{i j}^{\sigma \Lambda}\left(\Delta+\omega_{n}\right)\right. \\
& \left.+\mathcal{S}_{i j}^{\sigma \Lambda}\left(\omega_{n}\right) \mathcal{G}_{j i}^{\sigma \Lambda}\left(\omega_{n}-\Delta\right)\right] .
\end{aligned}
$$

Using the above definitions, the flow equation of the selfenergy (8) can be written explicitly as

$$
\begin{aligned}
\frac{d}{d \Lambda} \Sigma_{j i}^{\sigma \Lambda}\left(\omega_{n}\right)= & -T \sum_{n^{\prime}}\left[\left(\delta_{j i} U_{j}+P_{j i}\left(\omega_{n}+\omega_{n^{\prime}}\right)\right.\right. \\
& \left.+X_{j i}\left(\sigma\left(\omega_{n}-\omega_{n^{\prime}}\right)\right)\right) \mathcal{S}_{j i}^{\bar{\sigma}}\left(\omega_{n^{\prime}}\right) \\
& -D_{j i}^{\sigma}\left(\omega_{n}-\omega_{n^{\prime}}\right) \mathcal{S}_{j i}^{\sigma}\left(\omega_{n^{\prime}}\right) \\
& \left.+\delta_{j i} \sum_{k} D_{j k}^{\sigma}(0) \mathcal{S}_{k k}^{\sigma}\left(\omega_{n^{\prime}}\right)\right]
\end{aligned}
$$


To summarize, dfRG2 is defined by the flow equations (27) and (30), together with the definitions (9), (18), (24), (28), and (29).

\section{E. Magnetic susceptibility}

In this section, we demonstrate how the fRG approach can be used to derive expressions for linear response theory. We start by defining the magnetic susceptibility $\chi_{i}$ at a given site $i$ as the linear response of the local magnetization $m_{i}$ to a magnetic field $B$ :

$$
\chi_{i}=\left.\partial_{B} m_{i}\right|_{B=0}=\left.\frac{1}{2} \partial_{B}\left(n_{i}^{\uparrow}-n_{i}^{\downarrow}\right)\right|_{B=0},
$$

where $n_{i}^{\sigma}$ is the local occupation of site $i$ with spin $\sigma$. Using the Matsubara sum representation of the local density $n_{i}^{\sigma}=$ $T \sum_{n} \mathcal{G}_{i i}^{\sigma}\left(\omega_{n}\right)$, we explicitly calculate the derivative w.r.t. the magnetic field:

$$
\begin{aligned}
\chi_{i}= & \left.\frac{T}{2} \sum_{n \sigma} \sigma \partial_{B} \mathcal{G}_{i i}^{\sigma}\left(\omega_{n}\right)\right|_{B=0} \\
= & \frac{T}{2} \sum_{n \sigma}-\left.\sigma \mathcal{G}^{\sigma}\left(\omega_{n}\right) \partial_{B}\left[\sigma B / 2-\Sigma^{\sigma}\left(\omega_{n}\right)\right] \mathcal{G}^{\sigma}\left(\omega_{n}\right)\right|_{B=0} \\
= & -\frac{T}{2} \sum_{n j} \mathcal{G}_{i j}\left(\omega_{n}\right) \mathcal{G}_{j i}\left(\omega_{n}\right) \\
& +\left.\frac{T}{2} \sum_{n k l \sigma} \sigma \mathcal{G}_{i k}\left(\omega_{n}\right) \partial_{B} \Sigma_{k l}^{\sigma}\left(\omega_{n}\right)\right|_{B=0} \mathcal{G}_{l i}\left(\omega_{n}\right) .
\end{aligned}
$$

Note that the derivative of the self-energy w.r.t. the magnetic field $B$ has the structure of the fRG flow equation of the selfenergy (8). So, we perform the derivative by setting $\Lambda=B$ instead of the $\Lambda$ dependence defined in Eq. (7). The singlescale propagator (9) with $\Lambda=B$ set to zero then is

$$
\mathcal{S}^{\sigma, B=0}=\mathcal{G} \partial_{B}\left[\mathcal{G}_{0}^{\sigma}\right]_{B=0}^{-1} \mathcal{G}=\frac{\sigma}{2} \mathcal{G}^{2} .
$$

Using this in combination with the flow equation of the self-energy (8),

$$
\begin{aligned}
\partial_{B} \Sigma_{k l}^{\sigma}\left(\omega_{n}\right)= & \frac{T}{2} \sum_{n^{\prime} j_{1} j_{2} j_{3} \sigma^{\prime}} \sigma^{\prime} \mathcal{G}_{j_{1} j_{2}}^{\sigma^{\prime}}\left(\omega_{n^{\prime}}\right) \mathcal{G}_{j_{2} j_{3}}^{\sigma^{\prime}}\left(\omega_{n^{\prime}}\right) \\
& \times \gamma_{2}\left(j_{3} \sigma^{\prime} \omega_{n^{\prime}}, k \sigma \omega_{n} ; j_{1} \sigma^{\prime} \omega_{n^{\prime}}, l \sigma \omega_{n}\right),
\end{aligned}
$$

one directly arrives at the well-known Kubo formula for the magnetic susceptibility, which is exact if the self-energy and the vertex are known exactly. For the coupled-ladder approximation, we directly use the explicit flow equation for the self-energy (30), which yields

$$
\begin{aligned}
\chi_{i}= & -\frac{T}{2} \sum_{n, j} \mathcal{G}_{i j}\left(\omega_{n}\right) \mathcal{G}_{j i}\left(\omega_{n}\right) \\
& +\frac{T^{2}}{4} \sum_{n n^{\prime} k l j}\left(\mathcal{G}_{i k}\left(\omega_{n}\right) \mathcal{G}_{l i}\left(\omega_{n}\right) \mathcal{G}_{l j}\left(\omega_{n^{\prime}}\right) \mathcal{G}_{j k}\left(\omega_{n^{\prime}}\right)\right. \\
& \times\left[P_{k l}\left(\omega_{n}+\omega_{n^{\prime}}\right)+X_{k l}\left(\omega_{n}-\omega_{n^{\prime}}\right)+D_{k l}\left(\omega_{n}-\omega_{n^{\prime}}\right)\right] \\
& \left.-\mathcal{G}_{i k}\left(\omega_{n}\right) \mathcal{G}_{k i}\left(\omega_{n}\right) D_{k l}(0) \mathcal{G}_{l j}\left(\omega_{n}\right) \mathcal{G}_{j l}\left(\omega_{n}\right)\right) .
\end{aligned}
$$

\section{F. Zero-temperature limit}

For the numerical data presented in Sec. IV, we focused exclusively on the case of zero temperature. For the fRG scheme defined by Eq. (7), the limit $T \rightarrow 0$ has to be performed carefully [7]: $\omega_{n} \rightarrow i \omega(\omega \in \mathbb{R})$ becomes a continuous variable and $\Theta_{T}$ a sharp step function, with $\Theta(0)=\frac{1}{2}$ and $\partial_{\omega} \Theta(\omega)=\delta(\omega)$. For this combination of $\delta$ and $\Theta$ functions, Morris' lemma [21] can be applied, which yields

$$
\begin{aligned}
& \mathcal{S}^{\Lambda}(i \omega) \stackrel{T=0}{=} \delta(|\omega|-\Lambda) \widetilde{\mathcal{G}}^{\Lambda}(i \omega), \\
& \widetilde{\mathcal{G}}^{\Lambda}(i \omega)= {\left[\left[\mathcal{G}_{0}(i \omega)\right]^{-1}-\Sigma^{\Lambda}(i \omega)\right]^{-1}, } \\
& \mathcal{S}_{i, j}^{\Lambda}\left(i \omega_{1}\right) \mathcal{G}_{k, l}^{\Lambda}\left(i \omega_{2}\right) \stackrel{T=0}{=} \delta\left(\left|\omega_{1}\right|-\Lambda\right) \Theta\left(\left|\omega_{2}\right|-\Lambda\right) \\
& \times \widetilde{\mathcal{G}}_{i, j}^{\Lambda}\left(i \omega_{1}\right) \widetilde{\mathcal{G}}_{k, l}^{\Lambda}\left(i \omega_{2}\right) .
\end{aligned}
$$

\section{G. Static fRG}

A further possible approximation is to completely neglect the frequency dependence of the vertex. This is done by setting all three bosonic frequencies $\Pi, X$, and $\Delta$ to zero throughout. As a result, the self-energy is frequency independent, too. This approach, called static fRG2 (sfRG2), loses the property of being exact to second order. It leads to reliable results only for the zero-frequency Green's function at zero temperature. If knowing the latter suffices (such as when studying the magnetic field dependence at $T=0$ ), sfRG2 is a very flexible and efficient tool, computationally cheaper than our full coupled-ladder scheme.

\section{H. Numerical implementation}

Due to the slow decay of $\mathcal{S}^{\Lambda}$ for $\Lambda \rightarrow \infty$, integrating the flow equation (8) of the one-particle vertex $\gamma_{1}$ from $\Lambda=\infty$ to a large but finite value $\Lambda=\Lambda_{0}$ yields a finite contribution. For numerical implementations, the initial condition thus has to be changed to [5]

$$
\gamma_{1}^{\Lambda_{0}}\left(q_{1}^{\prime}, q_{1}\right)=-\frac{1}{2} \sum_{q} v\left(q, q_{1}^{\prime} ; q, q_{1}\right) .
$$

All numerically costly steps can be expressed as matrix operations, for which the optimized toolboxes BLAS and LAPACK can be used. The calculation time scales as $\mathcal{O}\left(N^{3}\right)$, due to the occurrence of matrix inversions (9) and matrix products (27). In the case of sfRG2 there are six matrix functions, each depending only on $\Lambda$. As a result, the integration is straightforward, and can be done, e.g., by a standard fourth-order Runge-Kutta with adaptive step-size control. We used the more efficient Dormand-Prince method [24], and mapped the infinite domain of $\Lambda \in[0, \infty)$ onto a finite domain using the substitution $\Lambda=\frac{x}{1-x}$ with $x \in[0,1)$. The upper bound for $N$, the maximal number of sites where $U_{j} \neq 0$, is mainly set by accessible memory. In the case of several gigabytes, $N$ should not exceed $10^{4}$ to $10^{5}$. We note in passing that for the one-dimensional Hubbard model [which is a special case of the model studied below, see Eq. (40)], $N$ values of that magnitude would not yet be large enough to reach the Luttiger-liquid regime for the case of small interactions $U$. The reason is that for the Hubbard model the spectral weight and the conductance have a nonmonotonic dependence on energy: 
as the energy is decreased, there is an intermediate regime in which they first increase, before the power-law decrease characteristic of Luttinger-liquid behavior finally sets in at very low energy scales, i.e. very large system sizes [6,25]. For small interactions $U \lesssim 0.5 \tau$, the latter crossover only becomes accessible for system sizes well beyond $10^{5}$ sites (see, e.g., Fig. 6 in Ref. [6]). To be able to see the low-energy decrease of spectral weight for system sizes of order $10^{5}$, interactions would have to be chosen to be as large as $U \simeq 4 \tau$, for which, however, the CLA can no longer be trusted.\}

For dfRG2, all matrices depend additionally on the Matsubara frequency, which is, in the case of zero temperature, a continuous variable. This variable has to be discretized in the numerical implementation. A good and safe choice is a logarithmic discretization since analytic functions have most structure close to their branch cuts, i.e., small Matsubara frequencies. Another possible choice, used in Ref. [7], is a geometric mesh. Since an appropriate discretization consists of at least 100 frequencies, the upper bound for $N$ is reduced to $10^{3}$, for which the run time already becomes quite large.

For frequency values in-between the discrete frequencies on the mesh, the functions have to be interpolated. Intuitively, one might expect that a nonlinear interpolation, e.g., a cubic spline, would lead to better results. However, in our implementations this led to a self-enhanced oscillatory behavior of the selfenergy as a function of frequency, even for a very dense discretization mesh. To avoid such numerical artifacts, the safest choice is a linear interpolation, where the density of the discretization is increased until the desired accuracy is reached.

\section{Relation between fRG2 and RPA}

In this section, we show that in the ladder approximation proposed here, fRG retains the quality of being closely related to parquet-type equations. This can be seen by considering a simplified version thereof, in which the coupling between the three channels is neglected, i.e., using replacement (19) instead of (20), and so is the feedback of the self-energy by replacing $\tilde{\mathcal{G}}^{\Lambda}$ by $\mathcal{G}_{0}$ in Eq. (29). In this case, each of the three differential equations (27) reduces to the generic form

$$
\frac{d}{d \Lambda} \Gamma^{\Lambda}(v)=\Gamma^{\Lambda}(v) W^{\Lambda}(v) \Gamma^{\Lambda}(v)
$$

with initial condition $\Gamma^{\Lambda_{i}}=\mathcal{U}=\delta_{i j} U_{j}$ (with $U_{j} \geqslant 0$, for present purposes). If Eq. (38) converges, its solution is given by

$$
\Gamma(v)=\mathcal{U}[\mathbb{I}+W(v) \mathcal{U}]^{-1},
$$

with

$$
W(v)=\int_{0}^{\infty} d \Lambda W^{\Lambda}(\nu) .
$$

Now note that Eq. (39) is also obtained if each channel is separately treated in the random phase approximation (RPA). Consequently, the full fRG2 scheme (either dynamic or static), described by Eqs. (27), amounts to a simultaneous treatment of all RPA channels with interchannel coupling via (28), and a feedback of Hartree-type diagrams via (9).

\section{IV. fRG RESULTS}

In this section, we will discuss some properties of the results obtained with the fRG equations stated in Sec. III, for the case of a QPC geometry. We will compare the results for the linear response conductance for the three approximation schemes and discuss the spin susceptibility within dfRG2.

\section{A. Model for a QPC}

We note that Eq. (1) applies to systems of arbitrary spatial dimensions. However, in this work we only present and discuss results for QPCs, thus restricting the model to one dimension. The lowest one-dimensional subband of the QPC is modeled by an inhomogeneous tight-binding chain, with onsite interactions:

$$
H=\sum_{j \sigma}\left[E_{j}^{\sigma} n_{j \sigma}-\tau\left(d_{j \sigma}^{\dagger} d_{j+1 \sigma}+\text { H.c. }\right)\right]+\sum_{j} U_{j} n_{j \uparrow} n_{j \downarrow},
$$

with $E_{j}^{\sigma}=V_{j}+2 \tau-\frac{\sigma B}{2}$ where $B$ is a Zeeman field. For low kinetic energies, this tight-binding model is a good approximation for a continuum model with mass $\frac{m}{\hbar^{2}}=\frac{1}{2 \tau a^{2}}$ (where $\hbar$ is Plank's constant) and potential $V_{j}=V(x=$ $j a$ ) [26], provided that the lattice spacing $a$ is much smaller than the length scales on which the potential changes. In order to keep computational time small, the model should always be chosen in such a way that the number of sites $N$ where $V_{j}$ or $U_{j}$ are nonzero is as small as possible. In other words: The inhomogeneity should be incorporated within as few sites as possible, without loss of adiabaticity.

We model the QPC as a smooth one-dimensional potential barrier which is purely parabolic around its maximum at $x=0$ :

$$
V(x)=V_{g}+\mu-\frac{m}{2 \hbar^{2}} \Omega_{x}^{2} x^{2}
$$

or in discrete version

$$
V_{j}=V_{g}+\mu-\frac{\Omega_{x}^{2}}{4 \tau} j^{2} \quad\left(|j|<j_{c}\right)
$$

Here, $j_{c}$ defines the range of pure parabolicity, $\mu$ is the chemical potential, and $\Omega_{x}$ is the relevant energy scale for the QPC [16], which we define such that it has the dimension of an energy (not frequency). The condition that $a$ has to be much smaller then the length scales on which the potential changes implies the condition $\Omega_{x} \ll \tau$. $V_{g}$ is the gate voltage, which controls the height of the potential. For $|j|>j_{c}$, the potential is smoothly connected to homogenous semi-infinite noninteracting leads. The potential can be considered as purely parabolic regarding its low-energy transport properties if $j_{c} \gg$ $\sqrt{\tau / \Omega_{x}}$. In the following, we use $\mu=0.5 \tau, \Omega_{x}=0.04 \tau$, $j_{c}=\sqrt{2 \tau \mu} / \Omega_{x}$, and $N=81$. These values optimize the conditions on $\Omega_{x}, j_{c}$, and the smoothness of the potential on the one hand and the smallness of the number of sites $N$ on the other hand. Typical experimental values for GaAs QPCs are $\Omega_{x}=1 \mathrm{meV}$ and $m=0.067 m_{e}$, where $m_{e}$ is the electron mass. The latter fixes the hopping to $\tau=25 \mathrm{meV}$ and thus the length unit to $a=\sqrt{\hbar^{2} / 2 \tau m} \simeq 5 \mathrm{~nm}$. These values should give a rough estimate for comparison with experiment, however, in the following we will use the system of measurement defined by $\tau$ and $a$, without referring to SI units. 

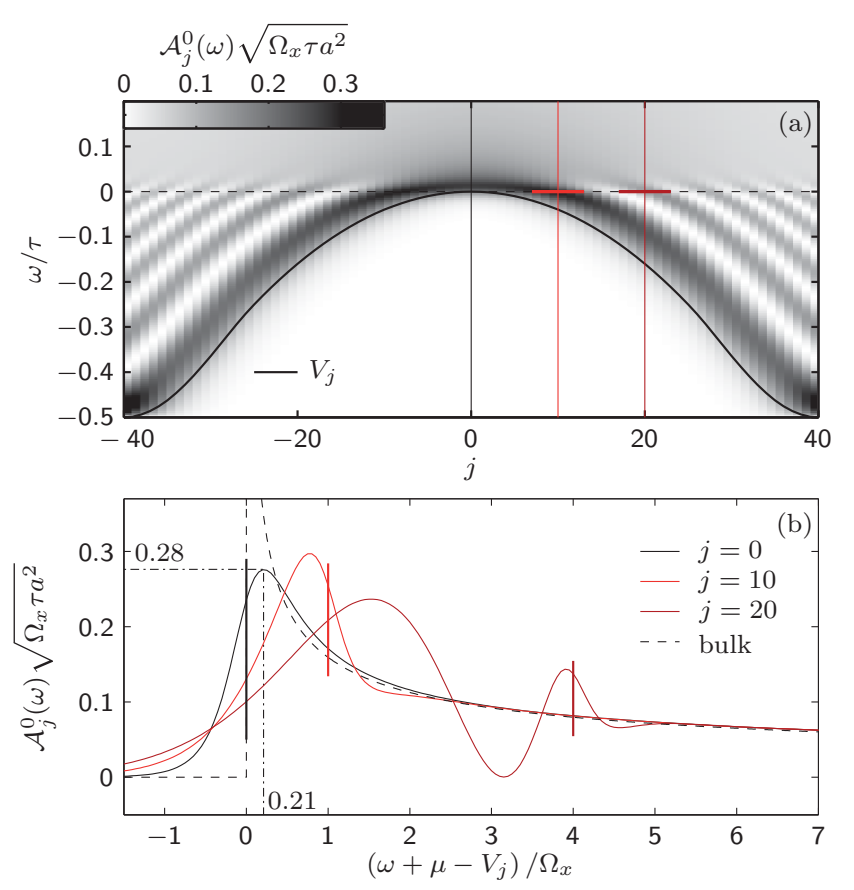

FIG. 1. (Color online) (a) Local density of states $\mathcal{A}_{j}(\omega)$ (color scale) for the noninteracting $U_{j}=0$, Hamiltonian Eq. (40) with potential (42) at $V_{g}=0$ (thick black line). (b) Local density of states $\mathcal{A}_{j}(\omega)$ as a function of $\left(\omega-V_{j}\right) / \Omega_{x}$ for a homogeneous tight-binding chain ( $V_{j}=0$, gray line) and for the potential (42) at fixed site $j=0$ (blue), $j=10$ (green), and $j=20$ (red), indicated in (a) by vertical lines with corresponding colors.

\section{B. Model properties}

Having defined the model we first discuss its noninteracting $(U=0)$ properties. Figure 1 shows the local density of states (LDOS)

$$
\mathcal{A}_{j}(\omega)=-\frac{1}{a \pi} \operatorname{Im} \mathcal{G}_{j j}\left(\omega+i 0^{+}\right)
$$

both in a grayscale plot as a function of site index and frequency [Fig. 1(a)] and at several fixed sites as a function of frequency [Fig. 1(b)]. Note that just above the potential [black line in Fig. 1(a)] the LDOS is enhanced [dark region in Fig. 1(a)]. This property originates from the fact that the density of states (DOS) of a one-dimensional system shows a divergence at zero velocity: indeed the DOS for the homogenous version [ $V_{j}=0$, i.e., $V_{g}=\mu=\Omega_{x}=0$ in Eq. (42)] of our model [black dashed line in Fig. 1(b)] reads as

$$
\mathcal{A}(\omega)=\frac{1}{\pi a \sqrt{\omega(4 \tau-\omega)}} \stackrel{\omega \ll \tau}{\approx} \frac{1}{2 \pi a \sqrt{\tau \omega}} \propto \frac{1}{v^{\text {clas }}},
$$

where $v^{\text {clas }}$ is the classical velocity of the electron. Quantum mechanically, this divergence is smeared out by the inhomogeneity $\left(V_{j} \neq 0\right)$ of a potential. Following Ref. [1], we call this smeared van Hove singularity in the LDOS that follows the potential a "van Hove ridge." In the case of a parabolic barrier with curvature given by $\Omega_{x}$ [Eq. (42)], the maximum of the LDOS is at an energy of $\mathcal{O}\left(\Omega_{x}\right)$ bigger than $V_{j}$ and has a height of $\mathcal{O}\left(\sqrt{\tau \Omega_{x}}\right)$ [see dashed-dotted line in Fig. 1(b)]. For energies below the potential maximum, electrons get reflected.
This leads to standing waves, altering the LDOS by oscillations around its bulk value [white striped area in Fig. 1(a) and oscillations in dark red line Fig. 1(b)].

\section{Conductance of a QPC}

Having discussed the properties of the noninteracting model, we continue with the fRG results at finite interaction. For this we first define the spatial dependence of the interaction $U_{j}$, which, for the one-dimensional model is an effective one-dimensional interaction resulting from integrating out two space dimensions. Its strength depends on the geometry, and is larger if the spatial confinement perpendicular to the one-dimensional system is smaller. We assume that this confinement is independent of the position in the transport direction in the center of the QPC, with $U_{j=0}=U$. This is a fair assumption for a saddle-point approximation of the two-dimensional QPC potential. For $|j| \rightarrow N^{\prime}=\frac{N-1}{2}, U_{j}$ drops smoothly to zero, describing the adiabatic coupling to the two-dimensional electron system, represented by the semi-infinite tight-binding chain.

In Ref. [1], we showed that the 0.7 anomaly is caused by the van Hove ridge in the LDOS discussed above. Its apex crosses the chemical potential when the QPC is tuned into the subopen regime, i.e., the regime where the conductance takes values $0.5 G_{Q}<G<0.9 G_{Q}$. This high LDOS at the chemical potential enhances effect of interactions by two main mechanisms: first, the effective Hartree barrier depends nonlinearly on gate voltage and magnetic field, causing an enhanced elastic backscattering; and second, due to the high LDOS inelastic backscattering is enhanced once a phase space is opened up by a finite temperature or source-drain voltage. Both effects reduce the conductance in the subopen regime, causing the 0.7 anomaly. Since interactions are enhanced by the LDOS, the relevant dimensionless interaction strength is $U_{j} \mathcal{A}_{j}(\mu) a$, which scales like $U / \sqrt{\Omega_{x} \tau}$ in the subopen regime.

In this paper, we will concentrate on examining how the reliability of the method depends on the interaction, without explaining the physical mechanism underlying the 0.7 anomaly in detail (for the latter, we refer to Ref. [1]). For the model (40), no reliable results are available from other methods to which we could have compared our own. Instead, we here compare the results of the different fRG schemes fRG1, sfRG2, and dfRG2. These schemes differ in the prefactor of the perturbative expansion of terms in order $U^{2}$ and higher. If these terms are important, the three approximation schemes will deviate from each other. Hence, the qualitative and quantitative reliability can be deduced from the qualitative and quantitative deviations between these schemes.

The first observable we discuss is the linear response conductance at zero teperature [27]:

$$
G=\frac{e^{2}}{h} \sum_{\sigma}\left|2 \pi \rho^{\sigma}\left(i 0^{+}\right) \mathcal{G}_{-N^{\prime} N^{\prime}}^{\sigma}\left(i 0^{+}\right)\right|^{2},
$$

where $\rho(\omega)$ is the density of states at the boundary of a semiinfinite tight-binding chain, representing the two-dimensional leads (for a derivation of the boundary Green's function, see the Appendix).

Particularly interesting in studying the 0.7 anomaly in QPCs is the shape of the conductance trace as a function 


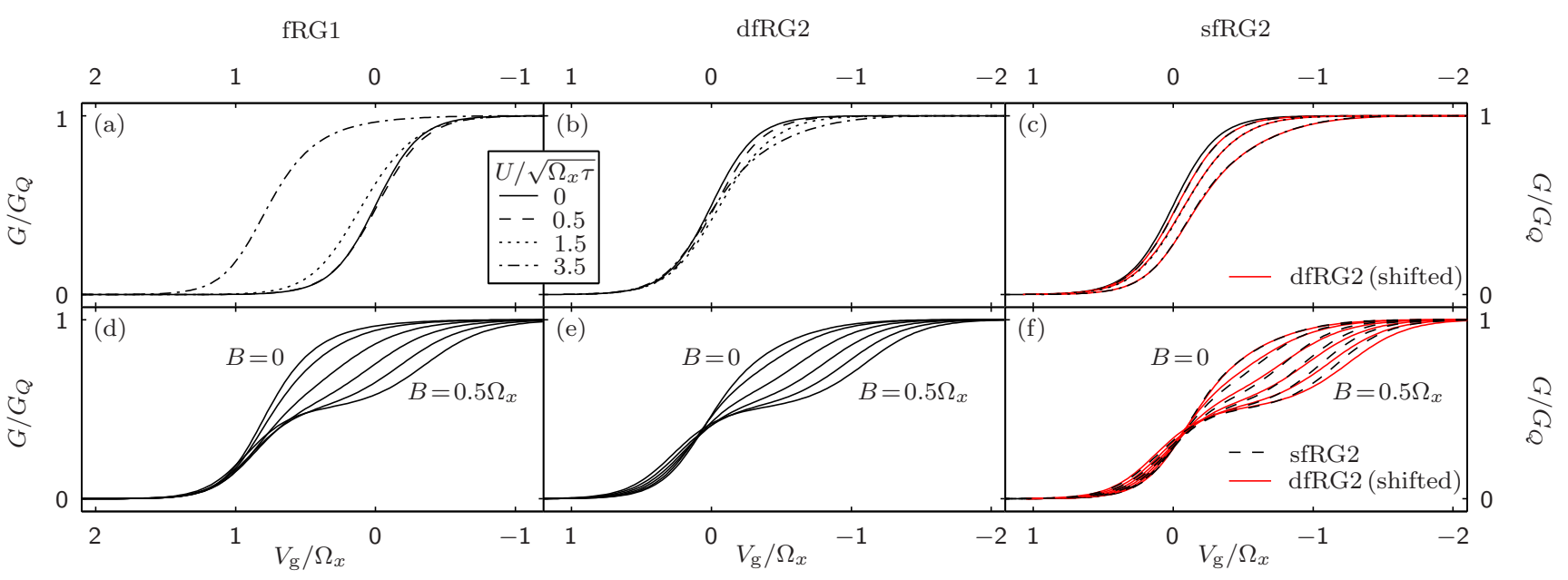

FIG. 2. (Color online) (a)-(c) Conductance $G$, as a function of gate voltage $V_{g}$, at zero magnetic field $B=0$ for different values of interaction $U$. (d)-(f) Conductance $G$ at fixed interaction strength $U=3.5 \sqrt{\Omega_{x}}$, for six equidistant magnetic fields $B$, between 0 and $0.5 \Omega_{x}$. Conductance is calculated using fRG1 [(a), (d)], dfRG2 [(b), (c)], or sfRG2 [black lines in (c) and (f)]. Red lines in (c) and (f) show dfRG2 data repeated from (b) and (e) with a $U$-dependent shift $\Delta V_{g}$ in $V_{g}$ direction $\left(\Delta V_{g}=0,0.001,0.02\right.$ and $0.15 \Omega_{x}$ for $U=0,0.5,1,5$, and 3.5 $\sqrt{\Omega_{x} \tau}$, respectively).

of applied gate voltage in the region where its value (in units of $G_{Q}$ ) changes from zero to one, and how this shape changes with external parameters, such as applied magnetic field. First of all, we emphasize the good qualitative agreement of all three approximation schemes with each other as well as with experimental results [compare Figs. 2(d), 2(e), and 2(f) with Refs. [17,19] (a direct comparison of dfRG2 with experiment is given in Ref. [1])]. This confirms that the method qualitatively captures the physical mechanism with respect to the conductance at zero temperature very well.

For a more quantitative analysis, we first consider the position of the conductance step, say $V_{\mathrm{po}}$; even though the actual position of the step is of minor interest experimentally, it gives information about how accurate Hartree-type correlations are treated. Figures 2(a), 2(b), and 2(c) show the conductance at $B=0$ for increasing values of interaction $U$ for fRG1, dfRG2, and sfRG2, respectively. While for dfRG2 and for sfRG2, $V_{\text {po }}$ decreases with interaction, its behavior for fRG1 is nonmonotonic: $V_{\text {po }}$ decreases slightly at small values of interaction, and increases strongly at larger values of interaction. Hence, the conductance at large interaction is higher than the bare $U=0$ value. This behavior is unphysical: whenever the density is nonzero, an increase in $U$ should cause an increase in the effective barrier height due to Coulomb repulsion, and hence a decrease in the conductance. This artifact is significantly reduced by the vertex flow of both dfRG 2 and sfRG2. For the latter, interactions suppress the conductance more strongly than for the former. Due to these deviations between the three schemes, we can not make a quantitative statement about the exact position of the conductance step $V_{\mathrm{po}}$.

The deviations just discussed make quantitative comparisons between these methods (or with others, such as RPA) difficult if interactions are large. The reason for the difficulty is that the $V_{g}$ position of the conductance step depends sensitively on the precise way in which Hartree-type correlations are treated and hence differ for each of the above schemes. Hence, it would not be meaningful to compare their predictions for physical quantities calculated at a given value of $V_{g}$; instead, it is only meaningful to compare the shape of their evolution with $V_{g}$. Actually, the same is true for physical quantities that are dominated by Fock-type correlations since internal propagators have to be dressed by Hartree diagrams. Doing this is crucial for inhomogeneous systems such as ours since an inhomogeneous density causes these Hartree contributions to have a significant dependence on position and gate voltage. In the fRG approach, the feedback of the self-energy (9) always guarantees that internal lines are dressed in a self-consistent way.

Let us now compare the shapes of the $V_{g}$-dependent conductance curves for dfRG2 and sfRG2. To this end, we replotted the dfRG2 data from Fig. 2(b) in Fig. 2(c) with a $U$-dependent shift $\Delta V_{g}$ in the $V_{g}$ direction (red lines). It can be seen from comparison with sfRG2 data that the shapes of the conductance curves are almost identical.

Next, we analyze the shape of the conductance step at finite interaction, and how it develops with magnetic field. The effect of an increasing magnetic field is qualitatively similar for the three approximation schemes [see Figs. 2(d), 2(e), and 2(f)]: the conductance step develops into a spin-resolved double step, in an asymmetric way: while the curves hardly change for $V_{g}$ values where $G<0.5 G_{Q}$, they are strongly suppressed in the subopen regime, where the LDOS is large. For fRG1, the $V_{g}$ range, where the lowest magnetic field $B=0.1 \Omega_{x}$ significantly reduces the conductance w.r.t. the conductance at $B=0$, is larger than for dfRG2 and sfRG2. This is related to the fact that the magnetoconductance, the change of conductance with magnetic field, within fRG1 is negative even for $V_{g}$ values where conductance is close to zero [this effect is too small to be visible in Fig. 2(d)]. Since this is not the case for dfRG2 and sfRG2 it is not possible to make a reliable statement about the sign of the magnetoconductance in the tunnel regime. Again, we reproduced the dfRG2 data from Fig. 2(e) in Fig. 2(f) with a shift $\Delta V_{g}$ in $V_{g}$ (red line) in order to compare their shape with the sfRG2 data (black dashed line). The effect of the magnetic field on the conductance within 
sfRG is slightly larger for small fields and slightly smaller for large fields than for the dfRG2 results. Based on the facts that, first, the deviations between dfRG2 and sfRG2 are small and, second, sfRG2 is computationally much cheaper than dfRG2, we conclude that for preliminary studies, or when scanning a large parameter space, one should favor sfRG2 whenever it is sufficient to know the static part of vertex functions.

\section{Susceptiblity}

As explained in Ref. [1], the 0.7 anomaly is related to an enhanced spin susceptibility in the subopen regime of the QPC. For this quantity, an estimate of the error is available within the dfRG2 approximation scheme. We note that the spin susceptibility defined in Eq. (31) can be calculated in two ways: by numerical differentiation of the magnetization for a small magnetic field $\chi^{\text {num }}$, or via Eq. (35), $\chi^{\text {Kubo }}$. Like the conductance, the value of $\chi$ is not known exactly. Thus, we argue here as in the previous section. $\chi^{\text {num }}$ and $\chi^{\text {Kubo }}$ are both exact to second order in the interaction, as can easily be checked, but they differ in terms that are of order $U^{3}$ and higher. If the difference of $\chi^{\text {num }}$ and $\chi^{\text {Kubo }}$ is significant, the higher-order terms are non-negligible, and the results can not be trusted.

In Ref. [1] we showed that $\chi_{j}^{\text {num }}$ is enhanced due to the inhomogeneity of the QPC potential and in addition amplified by interactions. It has a strong $V_{g}$ dependence, and is maximal when the QPC is tuned into the subopen regime. In this regime, at $V_{g}=-\Omega_{x} / 4$, we compare $\chi^{\text {num }}$ (Fig. 3 black lines) with $\chi^{\text {Kubo }}$ (Fig. 3 red lines) for different values of interaction. For intermediate values of interaction $U=1.5 \sqrt{\Omega_{x} \tau}$, both results are essentially identical, while for a larger value of interaction $U=3.5 \sqrt{\Omega_{x} \tau}$ deviations are clearly visible, however still not too large. The qualitative features that the susceptibility strongly increases with interaction, and that it is enhanced in the center of the QPC, emphasized in Ref. [1], are confirmed by both results. Furthermore, they coincide in their spatial structure, i.e., two maxima in the center and a decaying

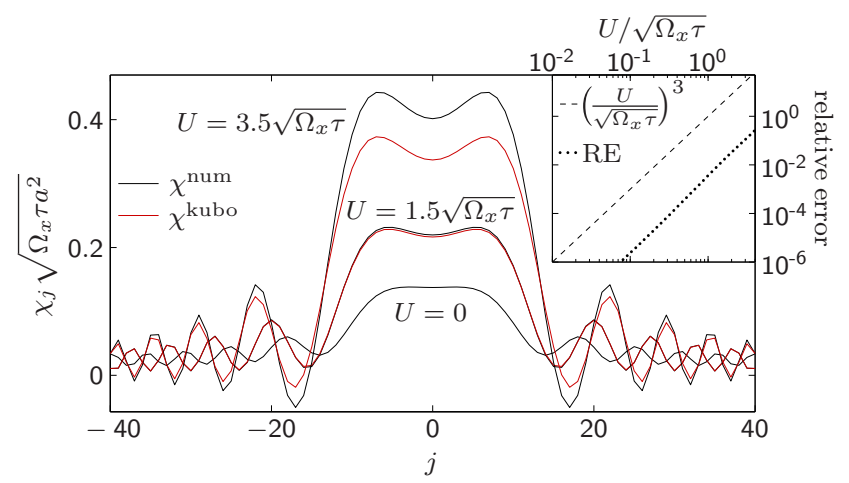

FIG. 3. (Color online) Local spin susceptibility $\chi_{j}$ [Eq. (31)] as a function of site index, for the QPC potential [Eq. (42)] at $V_{g}=$ $-\Omega_{x} / 4$, calculated using dfRG2 via the the numerical derivative of the local magnetization $\chi^{\text {num }}$ (black lines), as well as via the Kubo formula (35), $\chi^{\text {kubo }}$ (red lines), for three different values of interaction. Inset: relative error RE [Eq. (46)] (dots), as a function of interaction $U$, on a log-log scale. The error scales as $U^{3}$ (compare dashed line) since dfRG2 is exact to second order in the interaction $U$. oscillating behavior. This spatial structure is mainly given by the LDOS at the chemical potential (see Sec. IV B) and enhanced by interactions.

For a better quantification, we define the relative error

$$
\mathrm{RE}=2 \frac{\sum_{j}\left|\chi_{j}^{\text {Kubo }}-\chi_{j}^{\text {num }}\right|}{\sum_{j}\left|\chi_{j}^{\text {Kubo }}+\chi_{j}^{\text {num }}\right|} .
$$

This error is shown on a log-log scale in the inset of Fig. 3 (dots). The relative error scales like $U^{3}$ since dfRG2, and thus $\chi^{\text {num }}$ and $\chi^{\text {Kubo }}$, are exact to second order in $U$. For the larger value of interaction $U=3.5 \sqrt{\tau \Omega_{x}}$, the relative error of about $18 \%$ is quite significant and thus the value of $\chi$ is quantitatively not reliable. The reason for this is that the dimensionless interaction strength $U_{j} \mathcal{A}_{j}(\mu) a \approx 3.5 \times 0.3 \approx 1$ is already close to one. Nevertheless, the error is still dominated by the third-order term, implying that it is controlled.

Finally, we note that the spin susceptibility in the RPA approximation

$$
\chi_{i}^{\mathrm{RPA}}=\sum_{j}\left[W^{d}(0)\left[1+\mathcal{U} W^{d}(0)\right]^{-1}\right]_{i j}
$$

diverges at an interaction strength for which fRG is still well behaved. For example, if bare internal propagators are used to calculate $W^{d}, \chi_{i}^{\mathrm{RPA}}\left(V_{g}\right)$ turns out to diverge at $U \simeq 3.3 \sqrt{\Omega_{x} \tau}$. Moreover, the value of $\chi^{\mathrm{RPA}}$ and thus also the $U$ value for which it diverges depends on how one chooses to treat interactions for internal propagators of $W^{d}$. However, RPA itself gives no recipe how to do this. In contrast, the fRG approach gives a systematic framework for computing the two-particle vertex, the self-energy, and their feedback into each other, in a way that moderates competing instabilities in an unbiased way (as mentioned in Sec. III).

\section{CONCLUSION AND OUTLOOK}

We have derived a fRG based approximation scheme, called the coupled-ladder approximation (CLA), for spinful fermionic tight-binding models with a local interaction and an arbitrary potential. The main applications are systems with a significant spatial dependence, in particular, models where the electron density significantly changes with the position in real space.

The CLA is formulated within the context of third-ordertruncated fRG schemes, in which the three-particle vertex is set to zero, while the flow of the two-particle vertex is fully incorporated. The CLA retains two of the main properties of third-order-truncated fRG: it is exact to second order, and sums up diagrams of the RPA in all channels. Since the CLA is based on a perturbative argument, it is reliable only if interactions are not too large.

We analyzed in detail the reliability of this approach for a one-dimensional tight-binding model with a parabolic potential barrier representing a QPC. For this, we compared results for the conductance and the spin susceptibility calculated using different approaches within the fRG for different interactions up to $U=3.5 \sqrt{\Omega_{x} \tau}$. We identified the magnetic field dependence of the conductance and the enhanced susceptibility related to the 0.7 anomaly [1], as robust properties of the model. 
Finally, let us comment briefly on the prospects of using the CLA approach presented here to obtain finite-temperature results. While the formulas for the local density $n$ and the local susceptibility $\chi$ [Eq. (35)] are valid for arbitrary temperature $T$, the conductance formula (45) holds only for the case of zero temperature. The generalization of this formula to finite temperature[28] involves an analytic continuation to the real axis for both self-energy and vertex w.r.t. their frequency arguments. However, performing such an analytic continuation for numerical data is a mathematically ill-defined problem and turns out to be especially difficult for matrix-valued functions.

One possibility to avoid this complication is to formulate our CLA scheme on the Keldysh contour, in which case there are several different possibilities for introducing the fRG flow parameter [29]. (For a fRG treatment of the singleimpurity Anderson model, see Ref. [11].) When using Keldysh fRG to treat equilibrium properties, the number of independent correlators can be reduced by exploiting the Kubo-Martin-Schwinger conditions [30]. Moreover, Keldysh fRG in principle also allows nonequilibrium properties to be calculated. The actual implementation of Keldysh fRG for our model will be nontrivial, though, in particular since numerical integrations along the real axis, where Green's functions can have poles, can be challenging. Another problem at finite temperature is the violation of particle conservation due to the fRG truncation (5) [31]. The extent of this violation might be reduced by implementing the modified vertex flow suggested by Katanin [32]. We believe that it would be worth pursuing work in these directions.

\section{ACKNOWLEDGMENTS}

We thank S. Andergassen, S. Jakobs, V. Meden, and H. Schöller for very helpful discussions. We acknowledge support from the DFG via SFB-631, SFB-TR12, De730/4-3, and the Cluster of Excellence Nanosystems Initiative Munich.

\section{APPENDIX: PROJECTION METHOD}

The propagator in the fRG flow [Eqs. (8) and (12)], in general, lives on an infinite-dimensional chain. However, since the interacting region has finite extent, we only need to evaluate it on an $\mathrm{N}$-dimensional subspace. Furthermore, for the evaluation of Eq. (35) we need to calculate the sum over all site indices $j$, including the infinite number of sites in the leads. To this end, we perform a standard projection procedure $[8,20]$. With this method, the influence of the leads on the propagator and their contribution to the sum can be calculated analytically if the diagonalization of the leads is known analytically. Therefore, we define projection operators $C$ and $L$, with $C^{2}=C, L^{2}=L$, and $L+C=\mathbb{1}$ which divide the Hilbert space into the part that describes the leads $L$ and the finite-dimensional part that describes the central region where interaction is nonzero $C$. Furthermore, we define for a given quadratic Hamiltonian $H$ (for an interacting system $H$ is replaced by $\left.H_{0}+\Sigma\right), H_{c}=C H C, H_{c}=C H C$, $H_{c l}=C H L, H_{l c}=L H C, \omega_{l}=\omega L$, and $\omega_{c}=\omega C$ and write the Hamiltonian in the basis defined by the projections

$$
H=\left(\begin{array}{cc}
H_{c} & H_{c l} \\
H_{l c} & H_{l}
\end{array}\right) .
$$

Consequently, the Green's function in the same basis reads as

$$
\mathcal{G}=\left(\begin{array}{lc}
\omega_{c}-H_{c} & -H_{c l} \\
-H_{l c} & \omega_{l}-H_{l}
\end{array}\right)^{-1}=\left(\begin{array}{ll}
\mathcal{G}_{c} & \mathcal{G}_{c l} \\
\mathcal{G}_{l c} & \mathcal{G}_{l}
\end{array}\right) .
$$

with

$$
\begin{aligned}
\mathcal{G}_{c} & =\frac{1}{\omega_{c}-H_{c}-H_{c l} g_{l} H_{l c}}, \quad g_{l}=\frac{1}{\omega_{l}-H_{l}}, \\
\mathcal{G}_{l} & =\frac{1}{\omega_{l}-H_{l}-H_{l c} g_{c} H_{c l}}, \quad g_{c}=\frac{1}{\omega_{c}-H_{c}}, \\
\mathcal{G}_{c l} & =\mathcal{G}_{c} H_{c l} g_{l}=g_{c} H_{c l} \mathcal{G}_{l}, \\
\mathcal{G}_{l c} & =g_{l} H_{l c} \mathcal{G}_{c}=\mathcal{G}_{l} H_{l c} g_{c} .
\end{aligned}
$$

In the following, we will only use $\mathcal{G}_{l}$ and $g_{c}$ as well as $G_{c l}$ and $G_{l c}$ expressed in terms $\mathcal{G}_{l}$ and $g_{c}$, so we use the shorthand notations $\mathcal{G}=\mathcal{G}_{l}$ and $g=g_{l}$ (whether $\mathcal{G}$ lives on the infinitedimensional Hilbert space, or on the subspace of the central contact, will be clear from its site indices).

For the case of the infinite tight-binding chain defined by Eq. (40), the central region extends from site $-N^{\prime}$ to site $N^{\prime}$, with $N^{\prime}=\frac{N-1}{2}$, and the coupling to the leads can be expressed as

$$
\begin{aligned}
& H_{c l}=-\tau\left(d_{-N^{\prime}}^{\dagger} d_{-N^{\prime}-1}+d_{N^{\prime}}^{\dagger} d_{N^{\prime}+1}\right), \\
& H_{l c}=H_{c l}^{\dagger} .
\end{aligned}
$$

Consequently,

$$
\begin{aligned}
H_{c l} g H_{l c}= & \tau^{2}\left(d_{-N^{\prime}}^{\dagger} d_{-N^{\prime}-1}+d_{N^{\prime}}^{\dagger} d_{N^{\prime}+1}\right) \\
& \times g\left(d_{-N^{\prime}-1}^{\dagger} d_{-N^{\prime}}+d_{N^{\prime}+1}^{\dagger} d_{N^{\prime}}\right) \\
= & \tau^{2} b\left(n_{-N^{\prime}}+n_{N^{\prime}}\right),
\end{aligned}
$$

where $b=g_{N^{\prime}+1, N^{\prime}+1}$ is the boundary Green's function of a half-infinite tight-binding chain. Transforming into $k$ space and using the boundary condition $\left\langle d_{N^{\prime}}^{\dagger} d_{k}\right\rangle=0$ we get $\left\langle d_{N^{\prime}+1}^{\dagger} d_{k}\right\rangle \propto \sin ^{2}(k)$. Together with the dispersion $\varepsilon_{k}=$ $-\mu-2 \tau \cos (k)$ and the proper normalization, this yields for $\operatorname{Im}\left(\omega_{n}\right)>0$

$$
\begin{aligned}
b\left(\omega_{n}\right) & =\frac{1}{\pi} \int_{-\pi}^{\pi} d k \frac{\sin ^{2}(k)}{\omega_{n}+\mu+2 \tau \cos (k)} \\
& =\frac{1}{2 \tau^{2}}\left[\omega_{n}+\mu-i \sqrt{4 \tau^{2}-\left(\omega_{n}+\mu\right)^{2}}\right] .
\end{aligned}
$$

The square root is defined to have a positive real part, and $b\left(-\omega_{n}\right)=b^{*}\left(\omega_{n}\right)$. (For the spin-dependent boundary Green's function at finite magnetic field, $\mu$ has to be replaced by $\mu+\sigma B / 2$.)

Next, we calculate the infinite sum in Eq. (35). We split the sum into three parts and take $k$ and $l$ to be site indices in the central region

$$
\begin{aligned}
\sum_{j=-\infty}^{\infty} \mathcal{G}_{k j} \mathcal{G}_{j l}= & \left(\sum_{j=-\infty}^{-N^{\prime}-1}+\sum_{j=-N^{\prime}}^{N^{\prime}}+\sum_{j=N^{\prime}+1}^{\infty}\right) \mathcal{G}_{j k} \mathcal{G}_{j l} \\
= & \sum_{j=-N^{\prime}}^{N^{\prime}} \mathcal{G}_{j k} \mathcal{G}_{j l}+\tau^{2} \mathcal{G}_{k,-N^{\prime}} h^{L} \mathcal{G}_{-N^{\prime}, l} \\
& +\tau^{2} \mathcal{G}_{k, N^{\prime}} h^{R} \mathcal{G}_{N^{\prime}, l},
\end{aligned}
$$


with

$$
\begin{aligned}
& h^{L}=\sum_{j=-\infty}^{-N^{\prime}-1} g_{-N^{\prime}-1, j} g_{j,-N^{\prime}-1}, \\
& h^{R}=\sum_{j=N^{\prime}+1}^{\infty} g_{N^{\prime}+1, j} g_{j, N^{\prime}+1},
\end{aligned}
$$

where we made use of Eqs. (A3c), (A3d), and (A4).
Finally, we note that the last two terms are identical and given by

$$
\begin{aligned}
h\left(\omega_{n}\right) & =h^{L}\left(\omega_{n}\right)=h^{R}\left(\omega_{n}\right)=\left[g^{2}\left(i \omega_{n}\right)\right]_{N^{\prime}+1, N^{\prime}+1} \\
& =\frac{1}{\pi} \int_{-\pi}^{\pi} d k \frac{\sin (k)^{2}}{\left[\omega_{n}+\mu+2 \tau \cos (k)\right]^{2}} \\
& =\frac{1}{2 \tau^{2}}\left(\frac{\omega_{n}+\mu}{\omega_{n}+\mu-2 \tau} \sqrt{\frac{\omega_{n}+\mu-2 \tau}{\omega_{n}+\mu+2 \tau}}-1\right) .
\end{aligned}
$$

[1] F. Bauer, J. Heyder, E. Schubert, D. Borowsky, D. Taubert, B. Bruognolo, D. Schuh, W. Wegscheider, J. von Delft, and S. Ludwig, Nature (London) 501, 73 (2013).

[2] C. Wetterich, Phys. Lett. B 301, 90 (1993).

[3] W. Metzner, M. Salmhofer, C. Honerkamp, V. Meden, and K. Schönhammer, Rev. Mod. Phys. 84, 299 (2012).

[4] V. Meden, W. Metzner, U. Schollwöck, and K. Schönhammer, Phys. Rev. B 65, 045318 (2002).

[5] S. Andergassen, T. Enss, V. Meden, W. Metzner, U. Schollwöck, and K. Schönhammer, Phys. Rev. B 70, 075102 (2004).

[6] S. Andergassen, T. Enss, V. Meden, W. Metzner, U. Schollwöck, and K. Schönhammer, Phys. Rev. B 73, 045125 (2006).

[7] C. Karrasch, R. Hedden, R. Peters, T. Pruschke, K. Schnhammer, and V. Meden, J. Phys.: Condens. Matter 20, 345205 (2008).

[8] C. Karrasch, Master's thesis, Georg-August Universität Göttingen, 2006, arXiv:cond-mat/0612329.

[9] C. Husemann, K.-U. Giering, and M. Salmhofer, Phys. Rev. B 85, 075121 (2012).

[10] K.-U. Giering and M. Salmhofer, Phys. Rev. B 86, 245122 (2012).

[11] S. G. Jakobs, M. Pletyukhov, and H. Schoeller, Phys. Rev. B 81, 195109 (2010)

[12] C. Honerkamp, Eur. Phys. J. B 21, 81 (2001).

[13] C. Honerkamp and M. Salmhofer, Phys. Rev. B 67, 174504 (2003).

[14] B. J. van Wees, H. van Houten, C. W. J. Beenakker, J. G. Williamson, L. P. Kouwenhoven, D. van der Marel, and C. T. Foxon, Phys. Rev. Lett. 60, 848 (1988).

[15] D. A. Wharam, T. J. Thornton, R. Newbury, M. Pepper, H. Ahmed, J. E. F. Frost, D. G. Hasko, D. C. Peacock, D. A. Ritchie, and G. A. C. Jones, J. Phys. C: Solid State Phys. 21, L209 (1988).

[16] M. Büttiker, Phys. Rev. B 41, 7906 (1990).
[17] K. J. Thomas, J. T. Nicholls, M. Y. Simmons, M. Pepper, D. R. Mace, and D. A. Ritchie, Phys. Rev. Lett. 77, 135 (1996).

[18] N. J. Appleyard, J. T. Nicholls, M. Pepper, W. R. Tribe, M. Y. Simmons, and D. A. Ritchie, Phys. Rev. B 62, R16275 (2000).

[19] S. M. Cronenwett, H. J. Lynch, D. Goldhaber-Gordon, L. P. Kouwenhoven, C. M. Marcus, K. Hirose, N. S. Wingreen, and V. Umansky, Phys. Rev. Lett. 88, 226805 (2002).

[20] J. Taylor, Scattering Theory: The Quantum Theory on Nonrelativistic Collisions (Wiley, New York, 1972).

[21] T. R. Morris, Int. J. Mod. Phys. A 09, 2411 (1994).

[22] S. G. Jakobs, V. Meden, and H. Schoeller, Phys. Rev. Lett. 99, 150603 (2007).

[23] F. Bauer, Master's thesis, LMU-München, 2008.

[24] J. Dormand and P. Prince, J. Comput. Appl. Math. 6, 19 (1980).

[25] D. Schuricht, S. Andergassen, and V. Meden, J. Phys.: Condens. Matter 25, 014003 (2013).

[26] G. Forsythe and W. Wasow, Finite-Difference Methods for Partial Differential Equations, Applied Mathematics Series (Wiley, New York, 1960).

[27] S. Datta, Electronic Transport in Mesoscopic Systems, Cambridge Studies in Semiconductor Physics and Microelectronic Engineering (Cambridge University Press, Cambridge, UK, 1997).

[28] A. Oguri, J. Phys. Soc. Jpn. 70, 2666 (2001).

[29] S. G. Jakobs, Ph.D. thesis, RWTH Aachen, 2009.

[30] S. G. Jakobs, M. Pletyukhov, and H. Schoeller, J. Phys. A: Math. Theor. 43, 103001 (2010).

[31] T. Enss, Ph.D. thesis, Universität Stuttgart, 2005, arXiv:condmat/0504703.

[32] A. A. Katanin, Phys. Rev. B 70, 115109 (2004). 


\section{Chapter 4}

\section{Conductance for Interacting Fermi Systems}

One major theoretical goal in the context of the 0.7 -anomaly is to calculate the conductance of a QPC, i.e. the conductance through an extended interacting system of fermions. Since interactions are crucial (compare section 2.3), one needs to include them as accurately as possible. Furthermore the chosen approach should respect particle conservation, in the sense of respecting the corresponding Ward identities (Ward, 1950, see also section 4.2 Appendix C). The latter are violated in the fRG approach in the order of the truncation (see Enss, 2005; Katanin, 2004). Even if interactions are small enough that the violation of particle conservation is negligible, it turns out that the numerical calculation of the conductance at finite temperature in the Matsubara formalism is unfeasible, as will be explained in section 4.3 .

\section{1 fRG in Keldysh formalism}

The linear conductance is defined as the response of the current to an applied voltage:

$$
G=\left.\frac{d I}{d V_{\mathrm{sd}}}\right|_{V_{\mathrm{sd}}=0} .
$$

To perform this derivative we will follow the same strategy as for the spin susceptibility in section 3.3.6.III.E (see also Bauer et al. (2014)): The current I can be expressed in terms of single particle Green's functions, and thus equation (4.1) leads to derivatives of Green's functions w.r.t. voltage. This derivative can be expressed using the fRG equations, i.e. using derivatives of the one-particle vertex function.

Even though at the end voltage is set to zero, we need to set up the fRG equations in nonequilibrium if the flow parameter is the voltage. This can only be done in the Keldysh formalism. 


\subsubsection{Specifying the Action}

The $\mathrm{fRG}$ approach is defined once the action is specified. We use the Keldysh formalism in the frequency representation and a contour index $a$ (see Keldysh, 1965; Kamenev and Levchenko, 2009; Jakobs, 2009; Jakobs et al., 2010, for details). The composite index $k=(\omega, a, j, \sigma)$ carries information of frequency $\omega$, contour index $a$, site $j$ and spin $\sigma$. The action reads as

$$
\begin{aligned}
\kappa S=\frac{i}{2 \pi} \int d \omega \sum_{i j \sigma a b} \bar{\psi}_{\sigma i}^{a}(\omega)\left[g_{\sigma i j}^{a b}(\omega)\right]^{-1} \psi_{\sigma j}^{b}(\omega) \\
\quad+\frac{i}{(2 \pi)^{3}} \int(d \omega)^{4} \sum_{i j \sigma \sigma^{\prime} a} a_{1} \delta_{a_{1} a_{2}} \delta_{a_{1}^{\prime} a_{2}^{\prime}} \delta_{a_{1} a_{1}^{\prime}} U_{i j} \delta_{\left(\omega_{1}+\omega_{2}-\omega_{1}^{\prime}-\omega_{2}^{\prime}\right)} \bar{\psi}_{\sigma i}^{a}\left(\omega_{1}\right) \bar{\psi}_{\sigma^{\prime} j}^{a}\left(\omega_{2}\right) \psi_{\sigma i}^{a}\left(\omega_{1}^{\prime}\right) \psi_{\sigma^{\prime} j}^{a}\left(\omega_{2}^{\prime}\right)
\end{aligned}
$$

where $g$ is a shorthand for the bare Green's function $\mathcal{G}^{0}$ and $a$ and $b$ are contour indices. In the Keldysh formalism it is not possible give a universal explicit definition for $g^{-1}(\omega)$. It can only be given in some special cases, e.g. in equilibrium. A universal definition is only given in the time representation, where the density-matrix at $t=\infty$ is known.

We take $\kappa=i, v=i$ and $\operatorname{Tr}_{\omega}=\int \frac{d \omega}{2 \pi}$ where $\operatorname{Tr}_{\omega}$ is the summation over frequency degrees of freedom. Because of

$$
\Gamma^{\Lambda_{\text {initial }}}(\{\bar{\phi}\},\{\phi\})=\kappa S_{\text {int }}(\{\bar{\phi}\},\{\phi\})
$$

we get

$$
\begin{aligned}
\gamma_{2}\left(k_{1}^{\prime}, k_{2}^{\prime}, k_{1}, k_{2}\right)= & -a_{1} \delta_{a_{1} a_{2}} \delta_{a_{1}^{\prime} a_{2}^{\prime}} \delta_{a_{1} a_{1}^{\prime}} U_{j_{1} j_{2}} 2 \pi \delta\left(\omega_{1}^{\prime}+\omega_{2}^{\prime}-\omega_{1}-\omega_{2}\right) \\
& \times\left(\delta_{j_{1} j_{1}^{\prime}} \delta_{j_{2} j_{2}^{\prime}} \delta_{\sigma_{1}^{\prime} \sigma_{1}} \delta_{\sigma_{2}^{\prime} \sigma_{2}}-\delta_{j_{1} j_{2}} \delta_{j_{2} j_{1}^{\prime}} \delta_{\sigma_{1}^{\prime} \sigma_{2}} \delta_{\sigma_{2}^{\prime} \sigma_{1}}\right)\left(1-\delta_{j_{1} j_{2}} \delta_{\sigma_{1} \sigma_{2}}\right) .
\end{aligned}
$$

Note that $\gamma_{2}$ has no dimension of energy. The same is true for all other vertex functions. Further the $m$-particle Green's functions have dimension of [energy $]^{-2 m}$. However we defined these objects in equations (3.2), (3.5) and (3.7) such that the fact that they conserve frequency is not made explicit. From time translationally invariance one can easily see that

$$
\begin{aligned}
& \gamma_{m}\left(\omega_{1}^{\prime}, \ldots, \omega_{m}^{\prime}, \omega_{1}, \ldots, \omega_{m}\right)=2 \pi \delta\left(\omega_{1}^{\prime}+\cdots+\omega_{m}^{\prime}-\omega_{1}-\cdots-\omega_{m}\right) \\
& \times \gamma_{m}\left(t_{1}^{\prime}=0, \omega_{2}^{\prime}, \ldots, \omega_{m}^{\prime}, \omega_{1}, \ldots, \omega_{m}\right) \\
& G_{m}\left(\omega_{1}^{\prime}, \ldots, \omega_{m}^{\prime}, \omega_{1}, \ldots, \omega_{m}\right)=2 \pi \delta\left(\omega_{1}^{\prime}+\cdots+\omega_{m}^{\prime}-\omega_{1}-\cdots-\omega_{m}\right) \\
& \times G_{m}\left(t_{1}^{\prime}=0, \omega_{2}^{\prime}, \ldots, \omega_{m}^{\prime}, \omega_{1}, \ldots, \omega_{m}\right)
\end{aligned}
$$

(see Jakobs, 2009, for a derivation). The vertex functions on the r.h.s. do all have dimension of energy, and the Green's function on the r.h.s. have dimension of $[\text { energy }]^{-2 m+1}{ }^{1}$ In the following we derive the flow equations for these vertex functions. So we define

$$
\begin{aligned}
\Sigma(\omega) & =-\gamma_{1}\left(t^{\prime}=0, \omega\right), \\
\mathcal{S}(\omega) & =\mathcal{S}\left(t^{\prime}=0, \omega\right), \\
\mathcal{L}\left(\omega_{1}^{\prime}, \omega_{2}^{\prime} ; \Delta\right) & =\gamma_{2}\left(t_{1}^{\prime}=0, \omega_{2}^{\prime}, \omega_{1}, \omega_{2}\right) \quad\left(\Delta=\omega_{2}-\omega_{2}^{\prime}\right) .
\end{aligned}
$$

\footnotetext{
${ }^{1}$ Note, that this problem did not appear in Matsubara formalism in the previous chapter (see section 3.3), since energy conservation is represented by a Kronecker delta which is dimensionless and either one or zero.
} 
The flow equation for the one-particle vertex function is

$$
\frac{d}{d \Lambda} \gamma_{1}\left(k_{1}^{\prime} \omega_{1}^{\prime}, k_{1} \omega_{1}\right)=i \int \frac{d \omega_{2}^{\prime}}{2 \pi} \frac{d \omega_{2}}{2 \pi} \sum_{k_{2}^{\prime}, k_{2}} \gamma_{2}\left(k_{1}^{\prime} \omega_{1}^{\prime}, k_{2}^{\prime} \omega_{2}^{\prime}, k_{1} \omega_{1}, k_{2} \omega_{2}\right) \mathcal{S}\left(k_{2} \omega_{2}, k_{2}^{\prime} \omega_{2}^{\prime}\right)
$$

Using equation (4.5) and integrating over $\omega_{1}^{\prime}$ to get rid of the delta-function, we get

$$
\frac{d}{d \Lambda} \Sigma_{k_{1}^{\prime} k_{1}}(\omega)=\frac{1}{2 \pi i} \int d \omega^{\prime} \mathcal{S}_{k_{2} k_{2}^{\prime}}\left(\omega^{\prime}\right) \gamma_{2}\left(k_{1}^{\prime} t_{1}^{\prime}=0, k_{2}^{\prime} \omega^{\prime}, k_{1} \omega, k_{2} \omega^{\prime}\right)
$$

In the case of the one-particle Green's and vertex functions, our notation is as follows: whenever they only depend on a single frequency they do not contain the delta-function. For the two-particle we have a certain freedom to parametrise the three independent frequencies. In the previous section we used three bosonic frequencies whereas for the present purpose the parametrisation given in equation (4.6c) is more efficient.

Deriving the flow equation for the other vertex functions is straight forward: since the trace over $\omega$ is defined with a factor $\frac{1}{2 \pi}$ and each delta-function appears with a factor $2 \pi$, we are left with a single frequency integral and the prefactor $\frac{1}{2 \pi}$. Together with $v=i$ we get the prefactor $\frac{i}{2 \pi}$.

\subsection{Conductance Formula - Publication}

The derivation of the conductance formula will be published in a paper giving also additional details of our calculation using second order perturbation theory. The conductance formula derived in this publication is a generalised version of the formula derived by Oguri (2001) who started from the Matsubara formalism. In contrast to the formula of Oguri (2001), our approach is also valid for non-symmetric Hamiltonians, necessary e.g. when spin-orbit interactions are present. This section contains a reprint of the draft (Heyder, 2014). 


\title{
Conductance Formula for Interacting Fermi Systems in Keldysh Formalism
}

\author{
Jan Heyder, Florian Bauer, and Jan von Delft \\ Arnold Sommerfeld Center for Theoretical Physics and Center for NanoScience, \\ Ludwig-Maximilians-Universität München, Theresienstrasse 37, D-80333 München, Germany
}

(Dated: January 27, 2015)

\begin{abstract}
We discuss various options for calculating the conductance through a central, interacting region coupled to non-interacting fermionic leads in the Keldysh formalisms. Our starting point is the wellknown Meir-Wingreen formula for the current, whose derivative w.t.r. to the source-drain voltage yields the conductance. We explore various ways of performing this derivative analytically. They all exploit an exact flow equation from the function renormalization group (fRG), which expresses the flow w.r.t. voltage of the one-particle vertex, i.e. the self-energy, in terms of the two-particle vertex. One of these ways can be used to obtain a Keldysh-based derivation of a formula for the linear conductance that has previously been obtained by Oguri in the Matsubara formalism. We apply this formula to calculate the conductance for a model that has previously been shown to capture the essential physics of a quantum point contact (QPC) in the regime of the 0.7 anomaly. The model involves a tight-binding chain with a one-dimensional potential barrier and onsite interactions, which we treat using second order perturbation theory (SOPT). We show that numerical costs can be reduced significantly by using a non-uniform lattice spacing, chosen such that the occurence of artificial bound states close to the upper band edge is avoided.
\end{abstract}

\section{INTRODUCTION}

Two cornerstones of the theoretical description of transport through a mesoscopic system are the LandauerBüttiker $^{1}$ and Meir-Wingreen ${ }^{2}$ formulas for the conductance. The Landauer-Büttiker formula describes the conductance between two reservoirs connected by a central region in the absence of interactions. The Meir-Wingreen formula applies to the more general case that the central region contains electron-electron interactions: it expresses the current, in beautifully compact fashion, in terms of the Fermi functions of the reservoirs, and the retarded, advanced and Keldysh components of the Green's function for the central region.

To actually apply the Meir-Wingreen formula, these Green's functions have to be calculated explicitly, which in general is a challenging task. Depending on the intended application, a wide range of different theoretical tools have been employed for this purpose. Much attention has been lavished on the case of non-equilibrium transport through a quantum dot described by a Kondo or Anderson model, where the central interacting region consists of just a single localized spin or a single electronic level, see Refs. 3 and 4 for reviews. Here we are interested in the less well-studied case of systems, where the physics of the interacting region cannot be described by just a single site, but rather requires an extended modelling, consisting of many sites.

We have recently used a model of this type in a paper that offers an explanation for the microscopic origin of the 0.7-anomaly in the conductance through a $\mathrm{QPC}^{5}$. The model involves a tight-binding chain with a one-dimensional potential barrier and onsite interactions. In Ref. 5 we used two approaches to treat interactions: second-order perturbation theory (SOPT) and the functional renormalization group (fRG). Our calculations of the linear conductance were based on an exact formula derived by Oguri ${ }^{6}$. He started from the Kubo formula in the Matsubara formalism and performed the required an- alytical continuation of the two-particle vertex function occurring therein using Eliashberg theory ${ }^{7}$.

Since Oguri's formula for the linear conductance is exact, it can also be used by employing methods more powerful and reliable than SOPT, for example fRG, to calculate the self-energy and two-particle vertex. If this is done in the Matsubara formalism, however (as in Ref. 5), one is limited, in practice, to the case of zero temperature, because finite-temperature calculations would require an analytic continuation of numerical data from the imaginary to the real frequency axis, which is a mathematically ill-defined problem. This problem can be avoided by calculating the self-energy and vertex directly on the real axis using the Keldysh formalism ${ }^{8,9}$. However, to then calculate the linear conductance, the ingredients occuring in Oguri's formula would have to be transcribed into Keldysh language, and such a transcription is currently not available in the literature in easily accesible form.

In the present paper, we find a Keldysh version of Oguri's formula by deriving it entirely within the Keldysh formalism. Our starting point is the Meir-Wingreen formula for the current, $J(V)$, with the conductance defined by $\mathrm{g}=\partial_{V} J$. Rather than performing this derivative numerically, we here perform it analytically, based on the following central observation: The voltage derivative of the Green's functions that occur in the Meir-Wingreen formula, $\partial_{V} \mathcal{G}$, all involve the voltage derivative of the self-energy, $\partial_{V} \Sigma$. The latter can be expressed in terms of the two-particle vertex by using an exact flow equation from the function renormalization group (fRG). We show that it is possible to use this observation to derive Oguri's formula for the linear conductance, expressed in Keldysh notation, provided that the Hamiltonian is symmetric and conserves particle number. Our argument evokes a Ward identity ${ }^{10}$, following from $U(1)$-symmetry, which provides a relation between components of the self-energy and components of the vertex.

As an application of our Keldysh version of Oguri's conductance formula, we use Keldysh-SOPT to calcu- 
late the conductance through a QPC using the model of Ref. 5. We show that it is possible to greatly reduce the numerical costs by using a non-monotonic lattice spacing when formulating the discretized model. We present results for the conductance as function of barrier height for different choices of interaction strength $U$, magnetic field $B$ and temperature $T$ and discuss both the successes and limitations of the SOPT scheme.

The paper is organized as follows: After introducing the general interacting model Hamiltonian in Sec. II, we present the Keldysh derivation of Oguri's conductance formula in Sec. III. We set the stage for explicit conductance calculations by expressing the self-energy and the two-particle vertex within Keldysh SOPT in Sec. IV and introduce our the 1D-model of a QPC and show and discuss conductance results in Sec. V. A detailed collection of definitions and properties of both Green's and vertex functions in Keldysh formalism can be found in Appendix A and in Ref. 11 (in fact our paper closely follows the notation used therein). A diagrammatic derivation of the fRG flow-equation for the self-energy is given in Appendix B and the Ward identity resulting from particle conservation is presented in Appendix C. In Appendix $\mathrm{D}$ we perform an explicit calculation to show the fluctuation-dissipation theorem for the vertex-functions within SOPT. Finally, we apply the method of finite differences in Appendix E, to discretize the continuous Hamiltonian using a non-constant discretization scheme.

\section{MICROSCOPIC MODEL}

Within this work we consider a system composed of a finite central interacting region coupled to two non-interacting semi-infinite fermionic leads, a left lead, with chemical potential $\mu^{l}$, temperature $T^{l}$ and Fermidistribution function $f^{l}$, and a right lead, with chemical potential $\mu^{r}$, temperature $T^{r}$ and Fermi-distribution function $f^{r}$. The two leads are not directly connected to each other, but only via the central region. A similar setup was considered in Ref. 2 and Ref. 6.

The general form of the model Hamiltonian reads

$$
\mathcal{H}=\mathcal{H}_{0}+\mathcal{H}_{\text {int }}=\sum_{i j} h_{i j} d_{i}^{\dagger} d_{j}+\sum_{i j} U_{i j} n_{i} n_{j}
$$

where $h_{i j}$ is a hermitian matrix, and $U_{i j}$ is a real, symmetric matrix, non-zero only for states $i, j$ within the central region. $d_{i}^{\dagger} / d_{i}$ creates/destroys an electron in state $i$ and $n_{i}=d_{i}^{\dagger} d_{i}$ counts the number of electrons in state $i$. While in general the index $i$ can represent any set of quantum numbers we will regard it as a composite index, referring, e.g. to the site and spin of an electron for a spinful lattice model. Note, that the Hamiltonian conserves particle number, which is crucial in order to formulate a continuity equation for the charge current in the system.

Defining operators $L / C / R$, which project onto the subspace of the central region/left lead/right lead respectively, we can represent the quadratic part of the Hamil- tonian as

$$
\mathcal{H}_{0}=\left(\begin{array}{ccc}
L \mathcal{H}_{0} L & L \mathcal{H}_{0} C & 0 \\
C \mathcal{H}_{0} L & C \mathcal{H}_{0} C & C \mathcal{H}_{0} R \\
0 & R \mathcal{H}_{0} C & R \mathcal{H}_{0} R
\end{array}\right)=\left(\begin{array}{ccc}
\mathcal{H}_{l} & \mathcal{H}_{l c} & 0 \\
\mathcal{H}_{c l} & \mathcal{H}_{0, c} & \mathcal{H}_{c r} \\
0 & \mathcal{H}_{r c} & \mathcal{H}_{r}
\end{array}\right) .
$$

We note that all matrices on the r.h.s. have the dimension of the full bare Hilbert space, but are non-zero only in the corresponding sub-space, e.g. an entry $\mathcal{H}_{l c, i j}$ of the coupling matrix can differ from zero only if $i$ is a quantum state of the left lead and $j$ is a quantum state of the central region.

\section{TRANSPORT FORMULAS}

We henceforth work in the Keldysh formalism. Our notation for Keldysh indices, which mostly follows that of Ref. 11, is set forth in detail in Appendix A, to allow the main text to focus only on the essential steps of the argument.

\section{A. Current formula}

We begin by retracing the derivation of the MeirWingreen formula. In steady state the number of particles in the central region is constant. Hence, the particle current from the left lead into the central region is equal to the particle current from the central region into the right lead, $J:=J_{l \rightarrow c}=J_{c \rightarrow r}$. We remark that this continuity equation can also be obtained by imposing the invariance of the partition sum under a gauged $U(1)$ transformation, following from particle conservation of the Hamiltonian, see Appendix C]. This allows us to focus on the current through the interface between left lead and central region. Expressing the current in terms of the time-derivative of the total particle number operator of the left lead, $n_{l}=\sum_{i \in L} n_{i}$, we obtain the Heisenberg equation of motion $J=-e\left\langle\dot{n}_{l}\right\rangle=-i e / \hbar\left\langle\left[\mathcal{H}, n_{l}\right]\right\rangle$, where $e$ is the electronic charge and $\hbar$ is Planck's constant. Thus, considering the above definition of the Hamiltonian, Eq.(1), the current reads

$$
\begin{aligned}
J & =-\frac{i e}{\hbar} \sum_{\substack{i \in L \\
j \in C}}\left[h_{i j}\left\langle d_{j}^{-}(t)\left[d_{i}^{+}\right]^{\dagger}(t)\right\rangle-h_{j i}\left\langle d_{i}^{-}(t)\left[d_{j}^{+}\right]^{\dagger}(t)\right\rangle\right] \\
& =\frac{e}{\hbar}\left[\operatorname{Tr}\left\{\left(\mathcal{H}_{l c}-\mathcal{H}_{c l}\right) G^{-\mid+}\right\}\right]
\end{aligned}
$$

with the interacting equal-time lesser Green's function $G_{i \mid j}^{-\mid+}=G_{i \mid j}^{-\mid+}(t \mid t)=-i\left\langle d_{i}^{-}(t)\left[d_{j}^{+}\right]^{\dagger}(t)\right\rangle$ (here we used timetranslational invariance of the steady-state). Fourier transformation of Eq.(3) yields

$$
J=\frac{e}{h} \int d \varepsilon \operatorname{Tr}\left\{\left(\mathcal{H}_{l c}-\mathcal{H}_{c l}\right) \mathcal{G}^{-1+}(\varepsilon)\right\},
$$

with $h=2 \pi \hbar$. We introduced the symbol $\mathcal{G}$ for a Green's function that depends on a single frequency only (as opposed to the Fourier transform of the time-dependent 
Green's function $G$, which, in general, depends on two frequencies, see Appendix A, Eq.(A.7), for details).

Following the strategy of Ref. 2, we use Dyson's equation, Eq.(A.26), to express the current in terms of the central region Green's function $\mathcal{G}_{c}$ and rotate from the contour basis into the Keldysh basis (the explicit Keldysh rotation is given by Eq.(A.10) and Eq.(A.14c)). This yields

$$
J=\frac{i e}{2 h} \int d \varepsilon \operatorname{Tr}\left\{\Gamma^{l}\left[\mathcal{G}_{c}^{2 \mid 2}-\left(1-2 f^{l}\right)\left(\mathcal{G}_{c}^{2 \mid 1}-\mathcal{G}_{c}^{1 \mid 2}\right)\right]\right\},
$$

with retarded, $\mathcal{G}_{c}^{2 \mid 1}(\varepsilon)$, advanced, $\mathcal{G}_{c}^{1 \mid 2}(\varepsilon)$, and Keldysh central region Green's function, $\mathcal{G}_{c}^{2 \mid 2}(\varepsilon)$, and the hybridization function $\Gamma^{l}(\varepsilon)=i H_{c l}\left(g_{l}^{2 \mid 1}(\varepsilon)-g_{l}^{1 \mid 2}(\varepsilon)\right) H_{l c}$, where $g_{l}(\varepsilon)$ is the Green's function of the isolated left lead. Here and below we omit the frequency argument for all quantities that depend on the integration variable only. Eq.(5) is the celebrated Meir-Wingreen formula for the current [c.f. Eq.(6) in Ref. 2 for a symmetrized version].

We now present a version of the Meir-Wingreen formula in terms of the interacting one-particle irreducible self-energy $\Sigma$ (with retarded, $\Sigma^{1 \mid 2}$, advanced, $\Sigma^{2 \mid 1}$ and Keldysh component $\Sigma^{1 \mid 1}$ [Eq.(A.3), Eq.(A.7), Eq.(A.13)]). It can be derived by means of Dyson's equation, Eq.(A.25), which enables a reformulation of the Green's functions in Eq.(5) in terms of the hybridization functions $\Gamma$, the lead distribution functions $f$ and the self-energy $\Sigma$ :

$$
\begin{aligned}
\mathcal{G}_{c}^{2 \mid 1}-\mathcal{G}_{c}^{1 \mid 2} & =\mathcal{G}_{c}^{2 \mid 1}\left(\left[\mathcal{G}_{c}^{1 \mid 2}\right]^{-1}-\left[\mathcal{G}_{c}^{2 \mid 1}\right]^{-1}\right) \mathcal{G}_{c}^{1 \mid 2} \\
& =\mathcal{G}_{c}^{2 \mid 1}\left(-i\left(\Gamma^{l}+\Gamma^{r}\right)+\Sigma^{1 \mid 2}-\Sigma^{2 \mid 1}\right) \mathcal{G}_{c}^{1 \mid 2} \\
\mathcal{G}_{c}^{2 \mid 2}=\mathcal{G}_{c}^{2 \mid 1} & \left(-i \sum_{k=l, r}\left(1-2 f^{k}\right) \Gamma^{k}+\Sigma^{1 \mid 1}\right) \mathcal{G}_{c}^{1 \mid 2}
\end{aligned}
$$

Hence, the current formula can be written as the sum of two terms,

$$
\begin{aligned}
J & =\frac{e}{h} \int d \varepsilon\left(f^{l}-f^{r}\right) \operatorname{Tr}\left\{\Gamma^{l} \mathcal{G}_{c}^{2 \mid 1} \Gamma^{r} \mathcal{G}_{c}^{1 \mid 2}\right\}+ \\
& +\frac{i e}{2 h} \int d \varepsilon \operatorname{Tr}\left\{\Gamma^{l} \mathcal{G}_{c}^{2 \mid 1}\left(\Sigma^{1 \mid 1}-\left(1-2 f^{l}\right)\left(\Sigma^{1 \mid 2}-\Sigma^{2 \mid 1}\right)\right) \mathcal{G}_{c}^{1 \mid 2}\right\} .
\end{aligned}
$$

In equilibrium, i.e. $f:=f^{l}=f^{r}$, the current must fulfill $J=0$. With the first term of Eq.(7) vanishing trivially, this imposes the fluctuation-dissipation theorem (FDT) for the self-energy at zero bias voltage, $\Sigma^{1 \mid 1}=(1-2 f)\left(\Sigma^{1 \mid 2}-\Sigma^{2 \mid 1}\right)$. Note that a similar FDT can be formulated for the Green's function in Eq.(5).

\section{B. Differential conductance formula}

Differentiating Eq.(5) w.r.t. the source-drain voltage $V=\left(\mu_{l}-\mu_{r}\right) / e$, i.e. the voltage drop from the left to the right lead, provides the differential conductance $\mathrm{g}_{V}=$ $\partial_{V} J$. We denote derivatives w.r.t. frequency by a prime, e.g. $f^{l^{\prime}}:=\partial_{\varepsilon} f^{l}$, and derivatives w.r.t. the source-drain voltage by a dot, $\dot{\mathcal{G}}_{c}:=\partial_{V} \mathcal{G}_{c}$. Using Dyson's equation [Eq.(A.25)], we can express the derivative of the Green's function in terms of derivatives of the self-energy:

$$
\begin{aligned}
\dot{\mathcal{G}}_{c}^{\alpha \mid \alpha^{\prime}} & =\sum_{\beta, \beta^{\prime}} \mathcal{G}_{c}^{\alpha \mid \beta^{\prime}} \dot{\Sigma}^{\beta^{\prime} \mid \beta} \mathcal{G}_{c}^{\beta \mid \alpha^{\prime}}+S^{\alpha \mid \alpha^{\prime}}, \\
S^{1 \mid 1} & =S^{1 \mid 2}=S^{2 \mid 1}=0, S^{2 \mid 2}=\mathcal{G}_{c}^{2 \mid 1} \dot{\Sigma}_{\text {lead }}^{1 \mid 1} \mathcal{G}_{c}^{1 \mid 2} .
\end{aligned}
$$

Here we introduced the so called single scale propagator $S$ and the lead self-energy $\Sigma_{\text {lead }}^{1 \mid 1}=-i \sum_{k=l, r}\left(1-2 f^{k}\right) \Gamma^{k}$ [Eq.(A.21)]. Hence, we can write the differential conductance in the form

$$
\begin{aligned}
\mathrm{g}_{V}=\frac{i e}{2 h} \int d \varepsilon \operatorname{Tr} & \left\{\Gamma ^ { l } \left[\sum_{\beta, \beta^{\prime}} \mathcal{G}_{c}^{\alpha \mid \beta^{\prime}} \dot{\Sigma}^{\beta^{\prime} \mid \beta} \mathcal{G}_{c}^{\beta \mid \alpha^{\prime}}+S^{2 \mid 2}\right.\right. \\
& -\left(1-2 f^{l}\right)\left(\mathcal{G}_{c}^{2 \mid 1} \dot{\Sigma}^{1 \mid 2} \mathcal{G}_{c}^{2 \mid 1}-\mathcal{G}_{c}^{1 \mid 2} \dot{\Sigma}^{2 \mid 1} \mathcal{G}_{c}^{1 \mid 2}\right) \\
& \left.\left.+2 \dot{f}^{l}\left(\mathcal{G}_{c}^{2 \mid 1}-\mathcal{G}_{c}^{1 \mid 2}\right)\right]\right\} .
\end{aligned}
$$

We specify the voltage via the chemical potentials in the leads, $\mu^{l}=\mu+\alpha e V$ and $\mu^{r}=\mu+(\alpha-1) e V$, with $\alpha \in[0,1]$. This provides

$$
S^{2 \mid 2}=-2 i e \mathcal{G}_{c}^{2 \mid 1}\left[\alpha f^{l^{\prime}} \Gamma^{l}+(\alpha-1) f^{r \prime} \Gamma^{r}\right] \mathcal{G}_{c}^{1 \mid 2} .
$$

Note that in the special case $\alpha=0$, i.e. if the voltage is applied to the right lead only, the last term in Eq.(9) vanishes and the differential conductance takes a particularly simple form. This is a consequence of our initial choice to express the current via the time derivative of the left lead's occupation.

The above derived formula for the differential conductance of an interacting Fermi system involves derivatives of all self-energy components, $\dot{\Sigma}$. Below, we show how these can be expressed in terms of the irreducible twoparticle vertex $\mathcal{L}$ and the single scale propagator $S$ using the fRG flow equation for the self-energy. While we apply this scheme only to derive a Keldysh Kubo-type linear conductance formula, which for a symmetric Hamiltonian yields a Keldysh version of Oguri's formula, we want to stress that an extension to finite bias is trivial and that Eq.(9) can likewise be written in terms of the two-particle vertex, following the recipe below.

In Ref. 5 we used Eq.(9) (with $\alpha=1 / 2$ ) to calculate the differential conductance of a $1 \mathrm{D}$ parabolic potential barrier in the presence of an onsite electron-electron interaction (see Sec.V for details of the model). There we used second order perturbation theory (SOPT, details are presented in Sec.IV) to evaluate both the self-energy and its derivative with respect to voltage. We showed that the model, which is designed to mimick the lowest transport mode of a quantum point contact (QPC), qualitatively reproduces the main feature of the 0.7 conductance anomaly, including both its typical magnetic field and temperature dependence as well as the zero-bias peak, which usually accompanies the anomaly. 


\section{Conductance for Interacting Fermi Systems}

\section{Linear conductance formula}

In linear response, i.e. $V \rightarrow 0$, the linear conductance $g_{0}$ does not depend on the specific choice of $\alpha$. For the sake of simplicity we use $\alpha=1$, which corresponds to a voltage setup $\mu^{l}=\mu+e V$ and $\mu^{r}=\mu$. Henceforth, a dot implies the derivative at zero bias, e.g. $\dot{f}^{l}=\left.\partial_{V} f^{l}\right|_{V=0}$, and we have $\dot{f}^{l}=-e f^{\prime}$ and $\dot{f}^{r}=0$. Differentiating Eq.(7) w.r.t. the voltage, followed by setting $V=0$, yields the linear conductance formula

$$
\begin{aligned}
\mathrm{g}_{0}= & \left.\partial_{V} J\right|_{V=0} \\
= & -\frac{e^{2}}{h} \int d \varepsilon f^{\prime} \operatorname{Tr}\left\{\Gamma^{l} \mathcal{G}_{c}^{2 \mid 1}\left(\Gamma^{r}+i\left(\Sigma^{1 \mid 2}-\Sigma^{2 \mid 1}\right)\right) \mathcal{G}_{c}^{1 \mid 2}\right\} \\
& +\frac{e^{2}}{h} \int d \varepsilon \operatorname{Tr}\left\{\Gamma^{l} \mathcal{G}_{c}^{2 \mid 1} \Phi^{l} \mathcal{G}_{c}^{1 \mid 2}\right\} .
\end{aligned}
$$

All quantities in the integrand are evaluated in equilibrium. The voltage derivatives of the self-energy are combined in the expression

$$
\Phi^{l}=\frac{i}{2 e}\left[\dot{\Sigma}^{1 \mid 1}-(1-2 f)\left(\dot{\Sigma}^{1 \mid 2}-\dot{\Sigma}^{2 \mid 1}\right)\right] .
$$

Provided that all components of the self-energy and its derivative in Eq.(12) are known at zero bias, Eq.(11) is sufficient to calculate the linear conductance. But, as is shown below, it is possible to express the voltage derivatives of $\Sigma$ directly in terms of the two-particle vertex $\mathcal{L}$, i.e. the rank-four tensor defined as the sum of all one-particle irreducible diagrams with four external amputated legs (see Appendix A). This not only reduces the numbers of objects to be calculated, but more importantly, it completely eliminates the voltage from the linear conductance formula: whereas the derivative $\dot{\Sigma}$ needs information of the self-energy at finite bias, the two-particle vertex does not. Finally, it allows for a very compact representation of the linear conductance formula with a clear interpretation of the individual terms.

To this end we use the fact that an exact expression for the derivative of the self-energy w.r.t. some parameter $\Lambda$ is provided by the so called flow equation of the functional renormalization group (fRG) (for a diagrammatic derivation of this equation see Appendix B and Ref. 12. A rigorous functional derivation of the full set of coupled fRG equations for all $1 \mathrm{PI}$ vertex functions is given in e.g. Ref. 13). Usually, $\Lambda$ is taken to be some high-energy cutoff, but it can equally well be a physical parameter of the system, such as magnetic field, temperature or, as in the case in question, voltage: $\Lambda=V$. The general flow equation reads

$$
\partial_{\Lambda} \Sigma_{i \mid j}^{\alpha^{\prime} \mid \alpha}(\varepsilon)=\frac{1}{2 \pi i} \int d \varepsilon^{\prime} \sum_{\substack{\beta \beta^{\prime} \\ k l \in C}} S_{\Lambda, k \mid l}^{\beta \mid \beta^{\prime}}\left(\varepsilon^{\prime}\right) \mathcal{L}_{\Lambda, i k \mid j l}^{\alpha^{\prime} \beta^{\prime} \mid \alpha \beta}\left(\varepsilon^{\prime}, \varepsilon ; 0\right)
$$

where $\mathcal{L}\left(\varepsilon^{\prime}, \varepsilon ; 0\right)$ is the irreducible two-particle vertex, defined via Eq.(A.4) and Eq.(A.7). The specific form of this equation for a given flow-parameter $\Lambda$ is encoded in the single-scale propagator $S$, which is given by

$$
S_{\Lambda}=-\mathcal{G}_{c} \partial_{\Lambda}\left[\mathcal{G}_{0, c}\right]^{-1} \mathcal{G}_{c}=\mathcal{G}_{c} \mathcal{G}_{0, c}^{-1}\left[\partial_{\Lambda} \mathcal{G}_{0, c}\right] \mathcal{G}_{0, c}^{-1} \mathcal{G}_{c}
$$

with bare central region Green's function $\mathcal{G}_{0, c}(\varepsilon)$. According to Eq.(A.22) only its Keldysh component, $\mathcal{G}_{0, c}^{2 \mid 2}$, depends explicitly on the voltage. Additionally, we use $\left[\mathcal{G}_{0, c}^{-1}\right]^{2 \mid 2}=0$, following from causality, Eq.(A.12), which yields:

$$
\begin{aligned}
S_{V=0}^{2 \mid 2} & =\mathcal{G}_{c}^{2 \mid 1}\left[\mathcal{G}_{0, c}^{-1}\right]^{1 \mid 2} \partial_{V=0} \mathcal{G}_{0, c}^{2 \mid 2}\left[\mathcal{G}_{0, c}^{-1}\right]^{2 \mid 1} \mathcal{G}_{c}^{1 \mid 2} \\
& =-2 i e f^{\prime} \mathcal{G}_{c}^{2 \mid 1} \Gamma^{l} \mathcal{G}_{c}^{1 \mid 2}, \\
S_{V=0}^{1 \mid 1} & =S_{V=0}^{1 \mid 2}=S_{V=0}^{2 \mid 1}=0 .
\end{aligned}
$$

It is instructive to realize that this is indeed the singlescale propagator already introduced in the derivation of the differential conductance via Eq.(10). The trivial Keldysh structure of $S$ now implies, that the $\alpha^{\prime} \mid \alpha$ dependence of the self-energy derivatives only enters via that of the two-particle vertex:

$$
\dot{\Sigma}_{i \mid j}^{\alpha^{\prime} \mid \alpha}(\varepsilon)=\frac{1}{2 \pi i} \int d \varepsilon^{\prime} \sum_{k l \in C} S_{V=0, k \mid l}^{2 \mid 2}\left(\varepsilon^{\prime}\right) \mathcal{L}_{i l \mid j k}^{\alpha^{\prime} 2 \mid \alpha 2}\left(\varepsilon^{\prime}, \varepsilon ; 0\right)
$$

This allows us to write Eq.(12) in the form

$$
\Phi_{i \mid j}^{l}(\varepsilon)=\frac{1}{2 \pi i} \int d \varepsilon^{\prime} f^{\prime}\left(\varepsilon^{\prime}\right) \sum_{k l \in C}\left[\mathcal{G}_{c}^{2 \mid 1}\left(\varepsilon^{\prime}\right) \Gamma^{l}\left(\varepsilon^{\prime}\right) \mathcal{G}_{c}^{1 \mid 2}\left(\varepsilon^{\prime}\right)\right]_{k \mid l} K_{i l \mid j k}\left(\varepsilon^{\prime}, \varepsilon ; 0\right)
$$

with vertex response part

$$
K_{i l \mid j k}\left(\varepsilon^{\prime}, \varepsilon ; 0\right)=\mathcal{L}_{i l \mid j k}^{12 \mid 12}\left(\varepsilon^{\prime}, \varepsilon ; 0\right)-(1-2 f(\varepsilon))\left(\mathcal{L}_{i l \mid j k}^{12 \mid 22}\left(\varepsilon^{\prime}, \varepsilon ; 0\right)-\mathcal{L}_{i l \mid j k}^{22 \mid 12}\left(\varepsilon^{\prime}, \varepsilon ; 0\right)\right)
$$

We use the invariance of the trace under a cyclic permutation, $\operatorname{Tr}\left\{\Gamma^{l} \mathcal{G}_{c}^{2 \mid 1} \Phi^{l} \mathcal{G}_{c}^{1 \mid 2}\right\}=\operatorname{Tr}\left\{\Phi^{l} \mathcal{G}_{c}^{1 \mid 2} \Gamma^{l} \mathcal{G}_{c}^{2 \mid 1}\right\}$, and interchange the frequency labels, $\varepsilon \leftrightarrow \varepsilon^{\prime}$, to obtain the linear conductance formula 


$$
\mathrm{g}_{0}=-\frac{e^{2}}{h} \int d \varepsilon f^{\prime} \operatorname{Tr}\left\{\Gamma^{l} \mathcal{G}_{c}^{2 \mid 1}\left(\Gamma^{r}+i\left(\Sigma^{1 \mid 2}-\Sigma^{2 \mid 1}\right)\right) \mathcal{G}_{c}^{1 \mid 2}\right\}+\frac{e^{2}}{h} \int d \varepsilon f^{\prime} \operatorname{Tr}\left\{\Gamma^{l} \mathcal{G}_{c}^{1 \mid 2} \tilde{\Phi}^{l} \mathcal{G}_{c}^{2 \mid 1}\right\},
$$

with the resorted vertex correction term

$$
\tilde{\Phi}_{l \mid k}^{l}(\varepsilon)=\frac{1}{2 \pi i} \int d \varepsilon^{\prime} \sum_{i j \in C}\left[\mathcal{G}_{c}^{1 \mid 2}\left(\varepsilon^{\prime}\right) \Gamma^{l}\left(\varepsilon^{\prime}\right) \mathcal{G}_{c}^{2 \mid 1}\left(\varepsilon^{\prime}\right)\right]_{j \mid i} K_{i l \mid j k}\left(\varepsilon, \varepsilon^{\prime} ; 0\right) .
$$

In appendix $\mathrm{C}$ we show that particle conservation requires, that the imaginary part of the self-energy is connected to the vertex correction part via a Ward identity

$$
i\left[\Sigma^{1 \mid 2}(\varepsilon)-\Sigma^{2 \mid 1}(\varepsilon)\right]=\tilde{\Phi}^{l}+\tilde{\Phi}^{r} .
$$

This result is obtained by demanding the invariance of the physics under a gauged, local $U(1)$ transformation, which must hold for any Hamiltonian that conserves the particle number in the system. This symmetry implies an infinite hierarchy of relations connecting different Green's functions. The first equation in this hierarchy reproduces the continuity equation used in the beginning of the above derivation. The second equation in the hierarchy is Eq.(21), which connects parts of one-particle and two-particle Green's function. Inserting the ward identity in Eq.(19) yields

$$
\mathrm{g}_{0}=-\frac{e^{2}}{h} \int d \varepsilon f^{\prime}(\varepsilon) \operatorname{Tr}\left\{\Gamma^{l}(\varepsilon) \mathcal{G}_{c}^{2 \mid 1}(\varepsilon)\left[\Gamma^{r}(\varepsilon)+\tilde{\Phi}^{l}(\varepsilon)+\tilde{\Phi}^{r}(\varepsilon)\right] \mathcal{G}_{c}^{1 \mid 2}(\varepsilon)\right\}+\frac{e^{2}}{h} \int d \varepsilon f^{\prime}(\varepsilon) \operatorname{Tr}\left\{\Gamma^{l}(\varepsilon) \mathcal{G}_{c}^{1 \mid 2}(\varepsilon) \tilde{\Phi}^{l}(\varepsilon) \mathcal{G}_{c}^{2 \mid 1}(\varepsilon)\right\} .
$$

This formula is the central result of this chapter. It expresses the linear conductance in terms of the two-particle vertex $\mathcal{L}$, which enters via the vertex part $\Phi$ [Eq.(20)] and the response vertex $K$ [Eq.(18)]. Note that the two terms in Eq.(22) differ in their Keldysh structure via the Keldysh indexing of the full Green's functions, which prevents further compactification of Eq.(22) for a non-symmetric Hamiltonian (e.g. in the presence of finite spin-orbit interactions, see. e.g. Ref. 14). If, in contrast, the Hamiltonian of Eq.(1) is symmetric (i.e. $h_{i j}=h_{j i}$ ), Eq.(22) can be compactified significantly using the following argument: A symmetric Hamiltonian implies that the Green's function $\mathcal{G}$, the self-energy $\Sigma$ and the hybridization $\Gamma$ are symmetric, too. This in turn gives a symmetric $\tilde{\Phi}$ via Eq.(21). Hence, the trace in the first term of Eq.(22) is taken over the product of four symmetric matrices, and transposing yields $\operatorname{Tr}\left\{\Gamma^{l} \mathcal{G}_{c}^{2 \mid 1}\left[\Gamma^{r}+\tilde{\Phi}^{l}+\tilde{\Phi}^{r}\right] \mathcal{G}_{c}^{1 \mid 2}\right\}=\operatorname{Tr}\left\{\Gamma^{l} \mathcal{G}_{c}^{1 \mid 2}\left[\Gamma^{r}+\tilde{\Phi}^{l}+\tilde{\Phi}^{r}\right] \mathcal{G}_{c}^{2 \mid 1}\right\}$. Hence, all contributions involving $\tilde{\Phi}^{l}$ cancel in Eq. (22) and the linear conductance now simply reads

$$
\mathrm{g}_{0}=-\frac{e^{2}}{h} \int_{-\infty}^{\infty} d \varepsilon f^{\prime}(\varepsilon) \operatorname{Tr}\left\{\Gamma^{l}(\varepsilon) \mathcal{G}_{c}^{1 \mid 2}(\varepsilon)\left[\Gamma^{r}(\varepsilon)+\tilde{\Phi}^{r}(\varepsilon)\right] \mathcal{G}_{c}^{2 \mid 1}(\varepsilon)\right\} .
$$

A Matsubara version of this linear conductance formula for a symmetric Hamiltonian has been derived before in Ref. 6 using Eliashberg theory in order to perform the analytic continuation of the vertex from Matsubara space to the real axis. Comparison of the two formulas allows for a connection between the three Keldysh vertex components in Eq.(18) and the ones used in Oguri's derivation.

\section{Thermal conductance formula}

We end this chapter with some considerations regarding thermal conductance, i.e. the conductance induced by a temperature difference between the leads. In the following we assume zero bias voltage, $V=0$. The left lead is in thermal equilibrium with $T^{l}=T+\tilde{T}$ and the right lead in thermal equilibrium with temperature $T^{r}=T$. Thus, the temperature gradient between the leads will provide a charge current through the central region. Similar to above, we are now interested in the linear response thermal conductance formula, $\mathrm{g}_{0, T}=\partial_{\tilde{T}=0} J$, which we could calculate in similar fashion as the linear conductance $\mathrm{g}_{0}$. Much easier is the following though: all terms in Eq.(22) were obtained by once time taking the derivative of the Fermi distribution $f^{l}$ w.r.t. the voltage, partly explicitly in Eq.(7) and partly from evaluating the single-scale propagator in Eq.(15). Now note, that $\partial_{\tilde{T}=0} f^{l}=\frac{\varepsilon-\mu}{T} f^{\prime}=-\frac{(\varepsilon-\mu)}{e T} \partial_{V=0} f^{l}$. For a symmetric Hamiltonian this directly implies, that the linear thermal conductance is given by

$$
\mathrm{g}_{0, T}=\frac{e}{h T} \int_{-\infty}^{\infty} d \varepsilon(\varepsilon-\mu) f^{\prime}(\varepsilon) \operatorname{Tr}\left\{\Gamma^{l}(\varepsilon) \mathcal{G}_{c}^{1 \mid 2}(\varepsilon)\left[\Gamma^{r}(\varepsilon)+\tilde{\Phi}^{r}(\varepsilon)\right] \mathcal{G}_{c}^{2 \mid 1}(\varepsilon)\right\} .
$$




\section{Conductance for Interacting Fermi Systems}

\section{VERTEX FUNCTIONS IN SOPT}

In order to apply the above defined conductance formulas we calculate the self-energy $\Sigma$ and the two-particle vertex $\mathcal{L}$ in second order perturbation theory (SOPT). Both are defined in Eq. (A.7) and occur in the conductance formula (22). The SOPT-strategy is to approximate them by a diagrammatic series truncated beyond second order in the bare interaction vertex $\nu$, defined below.

Within this section the compact composite index notation used above is dropped in favor of a more explicit one. We henceforth use blue roman subscripts $\left(i_{1}, i_{2}, \ldots\right)$ for site indices only and explicitly denote spin dependencies using $\sigma \in\{\uparrow, \downarrow\}=\{+,-\}$. A green number subscript denotes an object's order in the interaction, e.g. $\Sigma_{2}$ is the desired self-energy to second order in the bare vertex $\nu$.

Below, the quadratic part of the model Hamiltonian, Eq.(1), is is represented by a real matrix that is symmetric in position basis and diagonal in spin space

$$
h_{i j}^{\sigma}=h_{j i}^{\sigma} \in \mathbb{R}, \quad h=h^{\uparrow}+h^{\downarrow} .
$$

In consequence, the bare Green's function, too, is diagonal in spin space and symmetric in position space:

$$
\mathcal{G}_{0, i \sigma \mid j \sigma^{\prime}}=\delta_{\sigma \sigma^{\prime}} \mathcal{G}_{0, i \mid j}^{\sigma}, \quad \mathcal{G}_{0, i \mid j}^{\sigma}=\mathcal{G}_{0, j \mid i}^{\sigma} .
$$

We distinguish between composite quantum numbers including contour indices $k_{n}=\left(a_{n}, i_{n}, \sigma_{n}\right)$ and composite quantum numbers including Keldysh indices $\kappa_{n}=$ $\left(\alpha_{n}, i_{n}, \sigma_{n}\right)$. The noninteracting Green's function is represented by a directed line

$$
\mathcal{G}_{0, k_{1} \mid k_{1}^{\prime}}(\varepsilon)=\underline{k_{1} \quad \varepsilon \quad k_{1}^{\prime}} .
$$

We choose an onsite interaction, which reduces the quartic term in Eq.(1) to a single sum

$$
\mathcal{H}_{i n t}=\sum_{i \in C} U_{i} n_{i \uparrow} n_{i \downarrow}
$$

i.e. we evaluate the vertex functions for the case of an onsite electron-electron interaction. Since the two-particle interaction is instantaneous in time, we construct the anti-symmetrized bare interaction vertex as

$$
\begin{aligned}
\nu_{k_{1}^{\prime}, k_{2}^{\prime} \mid k_{1}, k_{2}}\left(t_{1}^{\prime}, t_{2}^{\prime} \mid\right. & \left.t_{1}, t_{2}\right) \\
= & U_{i_{1}} \delta_{i_{1} i_{2}} \delta_{i_{1} i_{1}^{\prime}} \delta_{i_{1} i_{2}^{\prime}}\left(-a_{1}\right) \delta_{a_{1} a_{2}} \delta_{a_{1} a_{1}^{\prime}} \delta_{a_{1} a_{2}^{\prime}} \\
& \times \delta\left(t_{1}-t_{2}\right) \delta\left(t_{1}-t_{1}^{\prime}\right) \delta\left(t_{1}-t_{2}^{\prime}\right) \\
& \times \delta_{\sigma_{1} \bar{\sigma}_{2}} \delta_{\sigma_{1}^{\prime} \bar{\sigma}_{2}^{\prime}}\left(\delta_{\sigma_{1}^{\prime} \sigma_{1}}-\delta_{\sigma_{1}^{\prime} \sigma_{2}}\right)
\end{aligned}
$$

with $\bar{\sigma}=-\sigma$. Note that its spin-dependence is determined by Pauli's exclusion principle and the Slaterdeterminant character of the fermionic state. After Fourier transformation [ Eq.(A.6), Eq.(A.7)] and Keldysh rotation [Eq.(A.10), Eq.(A.11)] we find

$\nu_{\kappa_{1}^{\prime}, \kappa_{2}^{\prime} \mid \kappa_{1} \kappa_{2}}\left(\varepsilon_{1}^{\prime}, \varepsilon_{2}^{\prime} \mid \varepsilon_{1}, \varepsilon_{2}\right)=2 \pi \delta\left(\varepsilon_{1}+\varepsilon_{2}-\varepsilon_{1}^{\prime}-\varepsilon_{2}^{\prime}\right) \bar{u}_{\kappa_{1}^{\prime}, \kappa_{2}^{\prime} \mid \kappa_{1} \kappa_{2}}$,

where we introduced the bare vertex

$$
\begin{aligned}
\bar{u}_{\kappa_{1}^{\prime}, \kappa_{2}^{\prime} \mid \kappa_{1} \kappa_{2}}= & u_{i_{1}} \delta_{i_{1} i_{2}} \delta_{i_{1} i_{1}^{\prime}} \delta_{i_{1} i_{2}^{\prime}} \xi^{\alpha_{1}^{\prime} \alpha_{2}^{\prime} \mid \alpha_{1} \alpha_{2}} \\
& \times \delta_{\sigma_{1} \bar{\sigma}_{2}} \delta_{\sigma_{1}^{\prime} \bar{\sigma}_{2}^{\prime}}\left(\delta_{\sigma_{1}^{\prime} \sigma_{1}}-\delta_{\sigma_{1}^{\prime} \sigma_{2}}\right) \\
= & { }_{\kappa_{1}}^{\kappa_{1}},
\end{aligned}
$$

with $u_{i}=U_{i} / 2$ and the modulo operation

$$
\xi^{\alpha_{1}^{\prime} \alpha_{2}^{\prime} \mid \alpha_{1} \alpha_{2}}= \begin{cases}1, & \text { if } \alpha_{1}^{\prime}+\alpha_{2}^{\prime}+\alpha_{1}+\alpha_{2}=\text { odd } \\ 0, & \text { else. }\end{cases}
$$

\section{A. The two-particle vertex in SOPT}

Our goal is to approximate the vertex part, Eq.(18), to second order in the interaction. The fully interacting two-particle vertex, $\mathcal{L}\left(\varepsilon, \varepsilon^{\prime} ; 0\right)$, has the following diagrammatic representation:

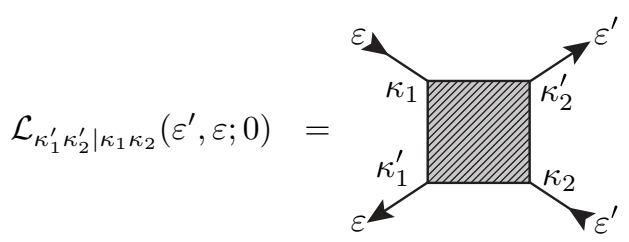

In SOPT, the vertex $\mathcal{L}_{2}$ is given by the sum of all 1PI diagrams with four external amputated legs and not more than two bare vertices. Defining the frequencies

$$
p=\varepsilon+\varepsilon^{\prime}, \quad x=\varepsilon-\varepsilon^{\prime},
$$

the vertex reads

$$
\mathcal{L}_{2}\left(\varepsilon^{\prime}, \varepsilon ; 0\right)=\bar{u}+\mathcal{L}_{2}^{p}(p)+\mathcal{L}_{2}^{x}(x)+\mathcal{L}_{2}^{d}(0),
$$

with particle-particle channel $\mathcal{L}_{2}^{p}$, particle-hole channel $\mathcal{L}_{2}^{x}$ and direct channel $\mathcal{L}_{2}^{d}$ defined as 


$$
\mathcal{L}_{2, \kappa_{1}^{\prime} \kappa_{2}^{\prime} \mid \kappa_{1} \kappa_{2}}^{d}(0)=\mathcal{L}_{2, \kappa_{1}^{\prime} \kappa_{2}^{\prime} \mid \kappa_{1} \kappa_{2}}^{p}(p)=\kappa_{\kappa_{1}^{\prime}}^{\infty} d \varepsilon^{\prime \prime} \sum_{q_{1} q_{2} q_{1}^{\prime} q_{2}^{\prime}} \bar{u}_{\kappa_{1}^{\prime} \kappa_{2}^{\prime} \mid q_{1} q_{2}} \mathcal{G}_{0, q_{1} \mid q_{1}^{\prime}}\left(p-\varepsilon^{\prime \prime}\right) \mathcal{G}_{0, q_{2} \mid q_{2}^{\prime}}\left(\varepsilon^{\prime \prime}\right) \bar{u}_{q_{1}^{\prime} q_{2}^{\prime} \mid \kappa_{1} \kappa_{2}},
$$

These expressions can be derived by a straightforward perturbation theory.

Using Eq.(26) and Eq.(31), we can identify the only non-vanishing components in spin- and real space,

$$
\begin{aligned}
& \Pi_{i j}^{\sigma \bar{\sigma}}(p)=\mathcal{L}_{2, i \sigma i \bar{\sigma} \mid j \sigma j \bar{\sigma}}^{p}(p), \\
& X_{i j}^{\sigma \sigma^{\prime}}(x)=\mathcal{L}_{2, i \sigma j \sigma^{\prime} \mid j \sigma i \sigma^{\prime}}^{x}(x), \\
& \Delta_{i j}^{\sigma \sigma^{\prime}}(0)=\mathcal{L}_{2, i \sigma j \sigma^{\prime} \mid i \sigma^{\prime} j \sigma}^{d}(0) .
\end{aligned}
$$

Eq.(25) and the channel definitions, Eq.(35), imply the symmetries

$$
\begin{aligned}
& \Pi_{i j}=\Pi_{j i}, X_{i j}=X_{j i}, \Delta_{i j}=\Delta_{j i}, \\
& \Pi(p)=\Pi^{\sigma \bar{\sigma}}(p)=\Pi^{\bar{\sigma} \sigma}(p), \\
& X^{\sigma \sigma^{\prime}}(x)=X^{\sigma^{\prime} \sigma}(-x), \\
& \Delta^{\sigma \sigma^{\prime}}(0)=\Delta^{\sigma^{\prime} \sigma}(0) .
\end{aligned}
$$

Moreover, and directly following from the Keldysh structure of the bare vertex in Eq.(31), we are left with only four non-zero components per channel in Keldysh space. This is best seen from realizing, that the internal Keldysh structure of the diagrams in Eq.(35) only depends on whether the sum of external indices belonging to the same bare vertex is even/odd. Furthermore, from the Keldysh structure of the bare vertex, combined with $\mathcal{G}^{1 \mid 1}=0$ and the analytic properties of $\mathcal{G}$, it follows that $\mathcal{L}^{22 \mid 22}=0$. Hence, SOPT preserves the theorem of causality, Eq.(A.12), as it should. (this has also been shown for a wide range of approximation schemes in Ref. 15). Thus, the Keldysh structure of the channels $Y=\Pi, X, \Delta$ is given by the matrix representation

$$
Y=\left(\begin{array}{cc}
Y^{K} & Y^{R} \\
Y^{A} & 0
\end{array}\right)=\left(\begin{array}{ll}
Y^{1 \mid 1} & Y^{1 \mid 2} \\
Y^{2 \mid 1} & Y^{2 \mid 2}
\end{array}\right) .
$$

We define the individual components according to the
Keldysh structure of the full vertex,

$$
\begin{aligned}
\mathcal{L}_{2}^{\alpha_{1}^{\prime} \alpha_{2}^{\prime} \mid \alpha_{1} \alpha_{2}} & =\Pi^{\psi\left(\alpha_{1}^{\prime}, \alpha_{2}^{\prime}\right) \mid \psi\left(\alpha_{1}, \alpha_{2}\right)} \\
& +X^{\psi\left(\alpha_{1}^{\prime}, \alpha_{2}\right) \mid \psi\left(\alpha_{1}, \alpha_{2}^{\prime}\right)} \\
& +\Delta^{\psi\left(\alpha_{1}^{\prime}, \alpha_{1}\right) \mid \psi\left(\alpha_{2}, \alpha_{2}^{\prime}\right)},
\end{aligned}
$$

where we introduced the modified modulo operation

$$
\psi\left(\alpha_{1}, \alpha_{2}, \ldots, \alpha_{n}\right)=\left\{\begin{array}{l}
1, \text { if } \sum_{\mathrm{i}=1, \ldots, \mathrm{n}} \alpha_{\mathrm{i}}=\text { odd } \\
2, \text { else. }
\end{array}\right.
$$

That leaves us with the following explicit formulas

$$
\begin{aligned}
& \Pi_{i j}^{1 \mid 2}(p)=-\frac{u_{i} u_{j}}{2 \pi i} \int d \varepsilon\left[\mathcal{G}_{0, i \mid j}^{\sigma, 2 \mid 1}(p-\varepsilon) \mathcal{G}_{0, i \mid j}^{\bar{\sigma}, 2 \mid 2}(\varepsilon)\right. \\
& \left.+\mathcal{G}_{0, i \mid j}^{\sigma, 2 \mid 2}(p-\varepsilon) \mathcal{G}_{0, i \mid j}^{\bar{\sigma}, 2 \mid 1}(\varepsilon)\right], \\
& \Pi^{2 \mid 1}=\left[\Pi^{1 \mid 2}\right]^{*}, \\
& \Pi_{i j}^{1 \mid 1}(p)=-\frac{u_{i} u_{j}}{2 \pi i} \int d \varepsilon\left[\mathcal{G}_{0, i \mid j}^{\sigma, 2 \mid 2}(p-\varepsilon) \mathcal{G}_{0, i \mid j}^{\bar{\sigma}, 2 \mid 2}(\varepsilon)\right. \\
& +\mathcal{G}_{0, i \mid j}^{\sigma, 2 \mid 1}(p-\varepsilon) \mathcal{G}_{0, i \mid j}^{\bar{\sigma}, 2 \mid 1}(\varepsilon) \\
& \left.+\mathcal{G}_{0, i \mid j}^{\sigma, 1 \mid 2}(p-\varepsilon) \mathcal{G}_{0, i \mid j}^{\bar{\sigma}, 1 \mid 2}(\varepsilon)\right], \\
& \left.\Pi^{1 \mid 1}(p)\right|_{V=0}=[1+2 b(p-\mu)]\left[\Pi^{1 \mid 2}(p)-\Pi^{2 \mid 1}(p)\right]_{V=0},
\end{aligned}
$$




$$
\begin{aligned}
& X_{i j}^{\sigma \sigma^{\prime}, 1 \mid 2}(x)=-\frac{u_{i} u_{j}}{2 \pi i} \int d \varepsilon\left[\mathcal{G}_{0, i \mid j}^{\bar{\sigma}, 1 \mid 2}(\varepsilon) \mathcal{G}_{0, i \mid j}^{\bar{\sigma}^{\prime}, 2 \mid 2}(\varepsilon+x)\right. \\
& \left.+\mathcal{G}_{0, i \mid j}^{\bar{\sigma}, 2 \mid 2}(\varepsilon) \mathcal{G}_{0, i \mid j}^{\bar{\sigma}^{\prime}, 2 \mid 1}(\varepsilon+x)\right], \\
& X^{2 \mid 1}=\left[X^{1 \mid 2}\right]^{*}, \\
& X_{i j}^{\sigma \sigma^{\prime}, 1 \mid 1}(x)=-\frac{u_{i} u_{j}}{2 \pi i} \int d \varepsilon\left[\mathcal{G}_{0, i \mid j}^{\bar{\sigma}, 2 \mid 2}(\varepsilon) \mathcal{G}_{0, i \mid j}^{\bar{\sigma}^{\prime}, 2 \mid 2}(\varepsilon+x)\right. \\
& \left.+\mathcal{G}_{0, i \mid j}^{\bar{\sigma}, 2 \mid 1}(\varepsilon) \mathcal{G}_{0, i \mid j}^{\bar{\sigma}^{\prime}, 1 \mid 2}(\varepsilon+x)\right] \\
& \left.+\mathcal{G}_{0, i \mid j}^{\bar{\sigma}, 1 \mid 2}(\varepsilon) \mathcal{G}_{0, i \mid j}^{\bar{\sigma}^{\prime}, 2 \mid 1}(\varepsilon+x)\right], \\
& \left.X^{1 \mid 1}(x)\right|_{V=0}=[1+2 b(x+\mu)]\left[X^{1 \mid 2}(x)-X^{2 \mid 1}(x)\right]_{V=0},
\end{aligned}
$$

$$
\begin{aligned}
& \begin{array}{l}
\Delta_{i j}^{\sigma \sigma^{\prime}, 1 \mid 2}(0)=\frac{u_{i} u_{j}}{2 \pi i} \int d \varepsilon\left[\mathcal{G}_{0, i \mid j}^{\bar{\sigma}, 1 \mid 2}(\varepsilon) \mathcal{G}_{0, i \mid j}^{\bar{\sigma}^{\prime}, 2 \mid 2}(\varepsilon)\right. \\
\left.+\mathcal{G}_{0, i \mid j}^{\bar{\sigma}, 2 \mid 2}(\varepsilon) \mathcal{G}_{0, i \mid j}^{\bar{\sigma}^{\prime}, 2 \mid 1}(\varepsilon)\right],
\end{array} \\
& \begin{array}{l}
\Delta=\Delta^{2 \mid 1}=\Delta^{1 \mid 2}, \\
\Delta^{1 \mid 1}=0 .
\end{array}
\end{aligned}
$$

Here, we introduced the Bose distribution function, $b(z)=1 /\left(e^{(z-\mu) / T}-1\right)$, with chemical potential $\mu$ and temperature $T$. []* denotes the complex conjugate. Note that the components of every individual channel fulfill a fluctuation dissipation theorem (FDT) in equilibrium [Eqs. (40d,41d,42c)], warranting the choice of notation introduced in Eq.(38). We derive this FDT in detail in Appendix D.

Finally we write down the three components of the SOPT two-particle vertex that occur in the vertex- correction part, Eq.(18):

$$
\begin{aligned}
& \mathcal{L}_{2, i \sigma, l \sigma^{\prime} \mid j \sigma, k \sigma^{\prime}}^{12 \mid 22}\left(\varepsilon^{\prime}, \varepsilon ; 0\right)= \\
& \quad \delta_{\sigma \bar{\sigma}^{\prime}} \delta_{i j} \delta_{i k} \delta_{i l} u_{i}+\delta_{\sigma \bar{\sigma}^{\prime}} \delta_{i l} \delta_{j k} \Pi_{i j}^{1 \mid 2}(p) \\
& \quad+\delta_{i k} \delta_{j l} X_{i j}^{\sigma \sigma^{\prime}, 1 \mid 2}(x)+\delta_{\sigma \sigma^{\prime}} \delta_{i j} \delta_{k l} \Delta_{i k}^{\sigma \sigma^{\prime}}(0), \\
& \mathcal{L}_{2}^{22 \mid 12}=\bar{u}+\Pi^{2 \mid 1}+X^{2 \mid 1}+\Delta, \\
& \mathcal{L}_{2}^{12 \mid 12}=\Pi^{1 \mid 1}+X^{1 \mid 1} .
\end{aligned}
$$

Utilizing the equilibrium's FDT for the $\Pi-$, and $X$ channel [Eq.(40d), Eq.(41d)], we find

$$
\begin{aligned}
& K_{i \sigma, l \sigma^{\prime} \mid j \sigma, k \sigma^{\prime}}\left(\varepsilon^{\prime}, \varepsilon ; 0\right)= \\
& \quad \delta_{\sigma \bar{\sigma}^{\prime}} \delta_{i l} \delta_{j k}[2 f(\varepsilon)+2 b(p-\mu)]\left(\Pi_{i j}^{1 \mid 2}(p)-\Pi_{i j}^{2 \mid 1}(p)\right) \\
& +\delta_{i k} \delta_{j l}[2 f(\varepsilon)+2 b(x+\mu)]\left(X_{i j}^{\sigma \sigma^{\prime}, 1 \mid 2}(x)-X_{i j}^{\sigma \sigma^{\prime}, 2 \mid 1}(x)\right) .
\end{aligned}
$$

We note, that this result (for $\mu=0$ ) has been obtained before by Oguri (see Eq.(4.7) of Ref. 6) using Matsubara formalism and an analysis of the two-particle vertex following Eliashberg? .

\section{B. The self-energy in SOPT}

Our goal is to approximate the self-energy to second order in the interaction. The fully interacting self-energy, $\Sigma(\varepsilon)$, has the following diagrammatic representation:

$$
\Sigma_{\kappa_{1}^{\prime} \mid \kappa_{1}}(\varepsilon)=\underset{\kappa_{1}^{\prime}}{\kappa_{1}}
$$

In SOPT, the self-energy $\Sigma_{2}$ is given by the sum of all 1PI diagrams with two external amputated legs and not more than two bare vertices. This amounts to three topologically different diagrams (the static first and second order Hartree diagrams and the frequency-dependent second order Fock diagram):

$$
\begin{aligned}
\Sigma_{2, \kappa_{1}^{\prime} \mid \kappa_{1}}(\varepsilon) & =\kappa_{1}^{\kappa_{1}} \\
& =\frac{-i}{2 \pi} \int_{-\infty}^{\infty} d \varepsilon^{\prime} \sum_{q_{1} q_{1}^{\prime}}\left[\bar{u}_{k_{1}^{\prime} q_{1}^{\prime} \mid k_{1} q_{1}}+\gamma_{2, k_{1}^{\prime} q_{1}^{\prime} \mid k_{1} q_{1}}^{d}(0)+\gamma_{2, k_{1}^{\prime} q_{1}^{\prime} \mid k_{1} q_{1}}\left(\varepsilon+\varepsilon^{\prime}\right)\right] \mathcal{G}_{0, q_{1} \mid q_{1}^{\prime}}\left(\varepsilon^{\prime}\right) .
\end{aligned}
$$


[Eq.(41a), Eq.(48a)] of the particle-hole vertex channel $\gamma_{2}^{x}$ instead of the particle-particle channel $\gamma_{2}^{p}$.

As a consequence of the spin-dependence of both the noninteracting Green's function and the bare vertex, Eq.(26) and Eq.(31), as well as the real space symmetry of the Hamiltonian, Eq.(25), the self-energy, too, is spin-diagonal and symmetric in real space:

$$
\Sigma_{i \sigma \mid j \sigma^{\prime}}=\delta_{\sigma \sigma^{\prime}} \Sigma_{i \mid j}^{\sigma}, \quad \Sigma_{i \mid j}^{\sigma}=\Sigma_{j \mid i}^{\sigma} .
$$

The Keldysh structure of the self-energy is given by matrix structure [Eq.(A.13)] with $\Sigma^{R}=\Sigma^{1 \mid 2}$. The theorem of causality demands $\Sigma^{2} 2=0$ [Eq.(A.12)]. Finally, explicit evaluation of the diagrams in Eq.(46) yields

$$
\begin{aligned}
& \Sigma_{2, i \mid j}^{\sigma, 1 \mid 2}(\varepsilon)=\frac{-i}{2 \pi} \int d \varepsilon^{\prime}\left[\delta_{i j} u_{i} \mathcal{G}_{0, i \mid i}^{\sigma, 2 \mid 2}\left(\varepsilon^{\prime}\right)+\delta_{i j} \sum_{k} \mathcal{G}_{0, k \mid k}^{\sigma, 2 \mid 2}\left(\varepsilon^{\prime}\right) \Delta_{i k}^{\sigma \sigma}(0)+\mathcal{G}_{0, i \mid j}^{\sigma, 2 \mid 2}\left(\varepsilon^{\prime}\right) X_{i j}^{\sigma, 1 \mid 2}\left(\varepsilon-\varepsilon^{\prime}\right)+\mathcal{G}_{0, i \mid j}^{\sigma, 2 \mid 1}\left(\varepsilon^{\prime}\right) X_{i j}^{\sigma \sigma, 1 \mid 1}\left(\varepsilon-\varepsilon^{\prime}\right)\right], \\
& \Sigma_{2}^{21}=\left[\Sigma^{12}\right]^{*}, \\
& \Sigma_{2, i \mid j}^{\sigma, 1 \mid 1}(\varepsilon)=\frac{-i}{2 \pi} \int d \varepsilon^{\prime}\left[\mathcal{G}_{0, i \mid j}^{\sigma, 2 \mid 2}\left(\varepsilon^{\prime}\right) X_{i j}^{\sigma \sigma, 1 \mid 1}\left(\varepsilon-\varepsilon^{\prime}\right)+\mathcal{G}_{0, i \mid j}^{\sigma, 2 \mid 1}\left(\varepsilon^{\prime}\right) X_{i j}^{\sigma \sigma, 1 \mid 2}\left(\varepsilon-\varepsilon^{\prime}\right)+\mathcal{G}_{0, i \mid j}^{\sigma, 1 \mid 2}\left(\varepsilon^{\prime}\right) X_{i j}^{\sigma \sigma, 21}\left(\varepsilon-\varepsilon^{\prime}\right)\right] \\
& \left.\Sigma_{2, i \mid j}^{\sigma, 1 \mid 1}(\varepsilon)\right|_{V=0}=(1-2 f(\varepsilon))\left[\Sigma_{2, i \mid j}^{\sigma, 1 \mid 2}(\varepsilon)-\Sigma_{2, i \mid j}^{\sigma, 2 \mid 1}(\varepsilon)\right]_{V=0} .
\end{aligned}
$$

We derive the FDT, Eq.(48d), in Appendix D.

\section{Voltage derivative of the self-energy in SOPT}

In order to calculate the differential conductance via Eq.(9) we now provide explicit formulas for the voltage derivative of the self-energy components. In principle we could use the natural approach and differentiate the r.h.s. of the self-energy expressions, Eq.(48), with the corresponding vertex components given by Eqs.(40)-(42). To illustrate the power of the fRG flow equation we choose an alternative, more direct route, by expanding Eq.(16) up to second order in the bare interaction and allow for arbitrary values of the voltage $V$.

To first order in the interaction the single-scale propagator, Eq.(14), reads

$$
S_{1, V}^{2 \mid 2}=\dot{\mathcal{G}}_{0}^{2 \mid 2}+\mathcal{G}_{0}^{2 \mid 1} \Sigma_{1}^{1 \mid 2} \dot{\mathcal{G}}_{0}^{2 \mid 2}+\dot{\mathcal{G}}_{0}^{2 \mid 2} \Sigma_{1}^{2 \mid 1} \mathcal{G}_{0}^{1 \mid 2} .
$$

Inserting both Eq.(49) and the SOPT vertex, Eq.(43c), in Eq.(16) directly yields

$$
\begin{aligned}
\dot{\Sigma}_{2, i \mid j}^{\sigma, 1 \mid 2}(\varepsilon)=\frac{-i}{2 \pi} \int d \varepsilon^{\prime}[ & \delta_{i j} u_{i} \dot{\mathcal{G}}_{0, i \mid i}^{\bar{\sigma}, 2 \mid 2}+\delta_{i j} \sum_{k}\left[u_{i}\left(\mathcal{G}_{0, i \mid k}^{\bar{\sigma} 2 \mid 1} \Sigma_{1, k \mid k}^{\bar{\sigma} 1 \mid 2} \dot{\mathcal{G}}_{0, k \mid i}^{\bar{\sigma} 2 \mid 2}+\dot{\mathcal{G}}_{0, i \mid k}^{\bar{\sigma}, 2 \mid 2} \Sigma_{1, k \mid k}^{\bar{\sigma}, 2 \mid 1} \mathcal{G}_{0, k \mid i}^{\bar{\sigma}, 1 \mid 2}\right)+\dot{\mathcal{G}}_{0, k \mid k}^{\sigma, 2 \mid 2} \Delta_{i k}^{\sigma \sigma}(0)\right] \\
& \left.+\dot{\mathcal{G}}_{0, i \mid j}^{\sigma, 2 \mid 2} X_{i j}^{\sigma \sigma, 1 \mid 2}(x)+\dot{\mathcal{G}}_{0, i \mid j}^{\bar{\sigma}, 2 \mid 2}\left(X_{i j}^{\sigma \bar{\sigma}, 1 \mid 2}(x)+\Pi_{i j}^{1 \mid 2}(p)\right)\right], \\
\dot{\Sigma}_{i \mid j}^{\sigma, 2 \mid 1}(\varepsilon)= & {\left[\dot{\Sigma}_{i \mid j}^{\sigma, 1 \mid 2}(\varepsilon)\right]^{*} } \\
\dot{\Sigma}_{i \mid j}^{\sigma, 1 \mid 1}(\varepsilon)= & \frac{-i}{2 \pi} \int d \varepsilon^{\prime}\left[\dot{\mathcal{G}}_{0, i \mid j}^{\sigma, 2 \mid 2} X_{i j}^{\sigma \sigma, 1 \mid 1}(x)+\dot{\mathcal{G}}_{0, i \mid j}^{\bar{\sigma}, 2 \mid 2}\left(X_{i j}^{\sigma \bar{\sigma}, 1 \mid 1}(x)+\Pi_{i j}^{1 \mid 1}(p)\right)\right],
\end{aligned}
$$

where the derivative of the Keldysh bare Green's function is given by [e.g. Eq.(A.22)]

$$
\dot{\mathcal{G}}_{0}^{2 \mid 2}=\mathcal{G}_{0}^{2 \mid 1} \dot{\Sigma}_{\text {lead }}^{1 \mid 1} \mathcal{G}_{0}^{1 \mid 2}=2 i \mathcal{G}_{0}^{2 \mid 1}\left(\sum_{k \in l, r} \dot{f}^{k} \Gamma^{k}\right) \mathcal{G}_{0}^{1 \mid 2} .
$$

For compactness, we dropped all arguments that match the integration frequency in Eq.(50).

It is important to note that the energy integral $\int d \varepsilon^{\prime}$ in Eq. (50) can be performed trivially for the special case of zero temperature, $T=0$ : Then the derivative of the
Fermi functions in $\dot{\mathcal{G}}_{0}^{2 \mid 2}$ are Dirac delta functions [for the definition of the voltage see Sec.(IIIB)]:

$$
\begin{aligned}
& \dot{f}^{l}\left(\varepsilon^{\prime}\right) \stackrel{T=0}{=} e \alpha \cdot \delta\left(\varepsilon^{\prime}-\mu-e \alpha V\right) \\
& \dot{f}^{r}\left(\varepsilon^{\prime}\right) \stackrel{T \equiv}{=} e(\alpha-1) \cdot \delta\left(\varepsilon^{\prime}-\mu-e(\alpha-1) V\right) .
\end{aligned}
$$

This reduces the integration in Eq.(50) to evaluating the integrand at the chemical potentials of the left and right lead, respectively. Naturally, this simplification proves extremely beneficial: We can express the self-energy at 
arbitrary voltage as

$$
\Sigma(V)=\Sigma(0)+\int_{0}^{V} d V^{\prime} \dot{\Sigma}\left(V^{\prime}\right) .
$$

Numerically calculating this voltage integration provides both the self-energy $\Sigma\left(V^{\prime}\right)$ and its derivative $\dot{\Sigma}\left(V^{\prime}\right)$ within the whole intervall $0 \leq V^{\prime} \leq V$. Hence, this procedure can save orders of magnitude of calculation time compared to the direct evaluation of the self-energy and its voltage derivative via Eq.(48) and Eq.(50), respectively.

\section{1D MODEL OF A QPC}

As an application of the above formalism, we now study the influence of electron-electron interactions on the linear conductance of a one-dimensional symmetric potential barrier of height $V_{c}$ (measured w.r.t. the chemical potential $\mu$ ) and parabolic near the top,

$$
V(x)=V_{c}+\mu-\frac{m \Omega_{x}^{2}}{2 \hbar^{2}} x^{2},
$$

where $m$ is the electron's mass. The geometry of the barrier is determined by the energy scale $\Omega_{x}$ and the length scale $l_{x}=\hbar / \sqrt{2 m \Omega_{x}}$. While the system extends to infinity, the potential is non-zero only within the central region $C$, defined by $-\ell / 2<x<\ell / 2$, and drops smoothly to zero as $|x|$ approaches $|\ell| / 2$. We call the outer homogeneous regions the left lead $L(x<-\ell / 2)$ and the right lead $R(x>\ell / 2)$.

Numerics cannot deal with the infinite Hilbert space of this continuous system. Hence, we discretize real space using the method of finite differences (see Appendix E for details), which maps the system onto a discrete set of space points $\left\{x_{j}\right\}$. This results in the tight-binding representation

$H=\sum_{j \sigma}\left[E_{j}^{\sigma} n_{j \sigma}-\tau_{j}\left(d_{j \sigma}^{\dagger} d_{j+1 \sigma}+\right.\right.$ h.c. $\left.)\right]+\sum_{j \in C} U_{j} n_{j \uparrow} n_{j \downarrow}$,

with spin-dependent onsite energy $E_{j}^{\sigma}=E_{j}-\sigma B / 2=$ $V_{j}+\tau_{j-1}+\tau_{j}-\sigma B / 2$, site-dependent hopping amplitude $\tau_{j}=\hbar^{2} /\left(2 m a_{j}^{2}\right)$, spacing $a_{j}=x_{j+1}-x_{j}$ and potential energy $V_{j}=V\left(x_{j}\right)$. Note that we included a homogeneous Zeeman-field $B$ to investigate magnetic field dependencies, as well as an onsite-interaction, whose strength is tuned by the site-dependent parameter $U_{j}$.

In Ref.5 we have used this model to investigate the physics of a quantum point contact (QPC), a short onedimensional constriction We showed that the model suffices to reproduce the main features of the 0.7 anomaly, including the strong reduction of conductance as function of magnetic field, temperature and source-drain voltage in a sub-open QPC (see below). We argued, that the appearance of the 0.7 anomaly is due to an interplay of a maximum in the local density of states (LDOS) just above the potential barrier (the "van-Hove ridge") and electron-electron interactions.
Here, we show that a proper choice of real space discretization scheme can minimize numerical costs. We discuss both the noninteracting physics of the model as well as the magnetic field and temperature dependence of the linear conductance in the presence of interactions using SOPT.

\section{A. The choice of discretization}

For a proper description of the continuous case it is essential to choose the spacing much smaller than the length scale on which the potential changes (condition of adiabatic discretization). We model the central region by $N=2 N^{\prime}+1$ sites, located at the space points $\left\{x_{-N^{\prime}}, x_{-N^{\prime}+1}, \ldots, x_{N^{\prime}-1}, x_{N^{\prime}}\right\}$, where $N \gtrsim 100$ proves sufficient for a potential of the form Eq. (54). Due to the parity symmetry of the barrier we always choose $x_{0}=0$ and $x_{j}=-x_{-j}$.

The discretization of real space introduces an upper bound, $E^{\max }=\max \left(V_{j}+2 \tau_{j-1}+2 \tau_{j}\right)$, for the eigenenergies of the bare Hamiltonian. In addition, it causes the formation of a site-dependent energy band, defined as the energy intervall where the local density of states (LDOS) is non-negligible, i.e. where eigenstates have non-negligible weight. In case of an adiabatic discretization this energy band follows the shape of the potential. At a site $j$ it is defined within the upper and lower band edge

$$
\varepsilon_{j}^{\min }=V_{j}, \quad \varepsilon_{j}^{\max }=V_{j}+w_{j},
$$

where the band width depends on the local spacing, i.e. on the choice of discretization (see Appendix E for additional information):

$$
w_{j}=2 \tau_{j-1}+2 \tau_{j}=\frac{\hbar^{2}}{m}\left(\frac{1}{a_{j-1}^{2}}+\frac{1}{a_{j}^{2}}\right) .
$$

Note that a larger distance between successive sites leads to a narrowing of the energy band and vice versa; while the lower band edge is, for any adiabatic discretization, directly given by the potential, the upper band edge depends sensitively on the applied discretization scheme.

In the following we discuss and compare two different discretization procedures: The standard approach of equidistant discretization (constant hopping $\tau$ ) causes a local maximum $\varepsilon_{0}^{\max }=V_{0}+2 \tau$ of the upper band edge in the vicinity of the barrier center. This approach leads to artificial bound states far above the potential barrier, which complicate numerical implementation and calculation. Hence, we recommend and apply an alternative adaptive scheme where the spacing increases (the band width decreases) with increasing potential, i.e. towards $j=0$. Note that this still implies a constant hopping $\tau_{|j|>N^{\prime}}=\tau$ in the leads.

\section{Constant discretization}



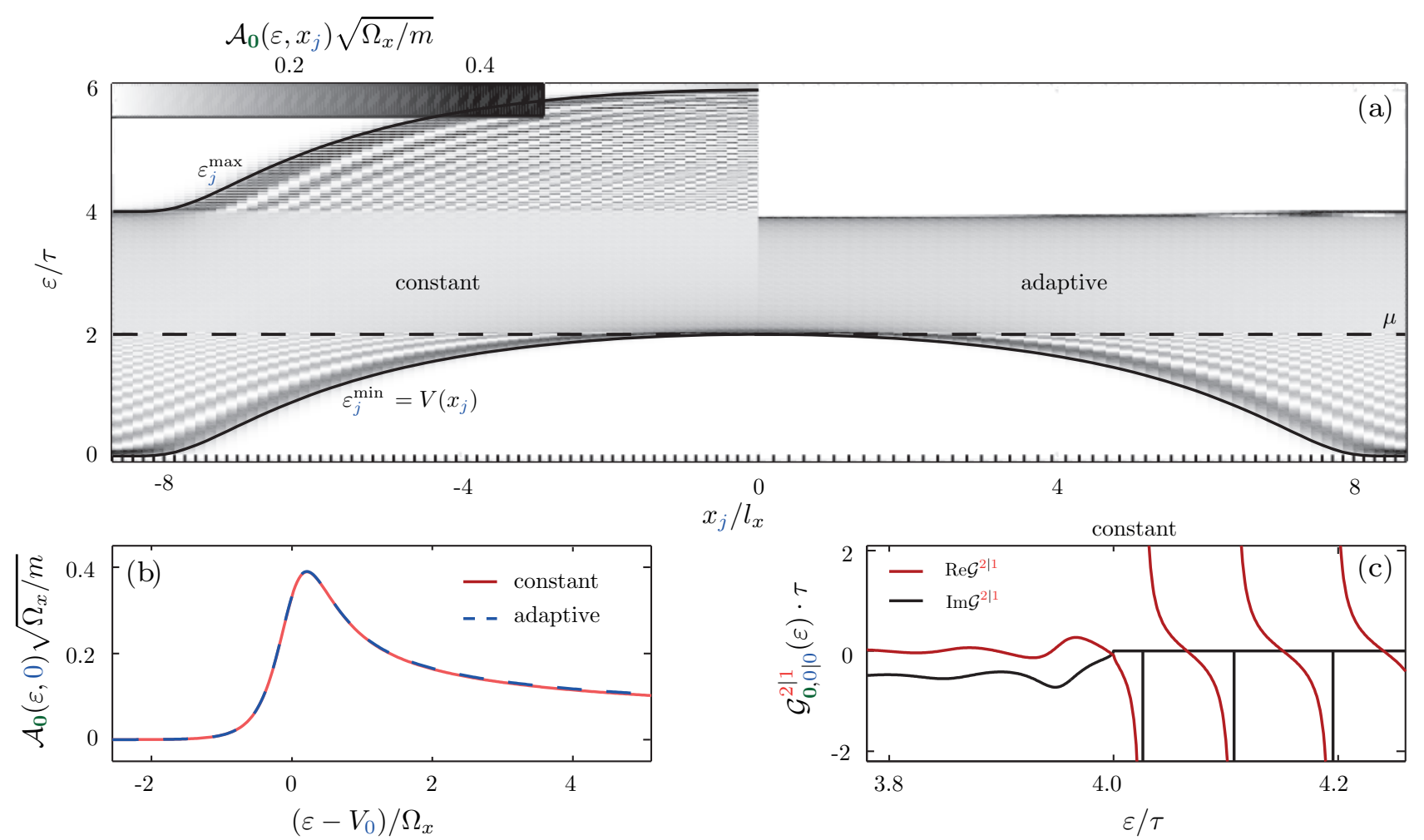

Figure 1. (a), left half: The non-interacting LDOS of the central region, $\mathcal{A}_{0}\left(\varepsilon, x_{j}\right)$, resulting from a constant real-space discretization. The position of the discrete points $x_{j}$ is indicated by the x-axis ticks. Both the lower and upper band edge follow the shape of the potential: $\varepsilon_{j}^{\min }=V\left(x_{j}\right)$ and $\varepsilon_{j}^{\max }=V\left(x_{j}\right)+4 \tau$. The local maximum of $\varepsilon_{j}^{\max }$ at $j=0$ causes the formation of bound states for energies $\varepsilon>4 \tau$. (c), their discrete spectrum shows up as poles in the non-interacting Green's function $\mathcal{G}_{0,0 \mid 0}(\varepsilon)$. (a), right half: The non-interacting LDOS of the central region resulting from an adaptive real-space discretization with $c=0.55$ [Eq.(60)], i.e. the spacing $a_{j}$ increases towards the barrier center (see x-axis ticks). Hence, the band width decreases with increasing barrier height, resulting in a local minimum of $\varepsilon_{j}^{\max }$ at $j=0$. (b), the LDOS at the central site, $\mathcal{A}_{0}(\varepsilon, 0)$, for both schemes.

We discuss the case of constant spacing $a=a_{j}$, implying grid points $x_{j}=a j$ and a constant hopping $\tau=\hbar^{2} /\left(2 m a^{2}\right)$. In a homogeneous system, $V\left(x_{j}\right)=0$, the energy eigenstates are Bloch waves $\psi_{k}\left(x_{j}\right)=e^{i k a j}$, which form an energy band $\varepsilon_{k}=2 \tau[1-\cos (k a)]$ of width $w=4 \tau$. Adding the parabolic potential,

$$
V\left(x_{j}\right)=V_{c}+\mu-\frac{\Omega_{x}^{2}}{4 \tau} j^{2},
$$

these states are now subject to scattering at the barrier which causes the formation of standing wave patterns for energies $\varepsilon<V_{0}=V(0)=V_{c}+\mu$ below the barrier top. The left half $\left(x_{j}<0\right)$ of Fig. $1(\mathrm{a})$ shows the noninteracting central region's local density of states (LDOS), $\mathcal{A}_{0}^{\sigma}\left(x_{j}, \varepsilon\right)=-1 /(\pi a) \cdot \operatorname{Im} \mathcal{G}_{0, j \mid j}^{\sigma, 2 \mid 1}(\varepsilon)$ at $B=0$, as a function of position $x_{j}$ and energy $\varepsilon$. Due to the condition of adiabaticity the energy band smoothly follows the shape of the potential, implying a site-dependent upper band edge, $\varepsilon^{\max }\left(x_{j}\right)=V_{j}+4 \tau$.

The local maximum of $\varepsilon^{\max }\left(x_{j}\right)$ in the central region's center generates artificial bound states, owed to the discretization scheme, in the energy interval $\varepsilon \in\left[4 \tau, 4 \tau+V_{0}\right]$. This is illustrated in Figure 1(c), where the real and imag- inary parts of the bare Green's function of the central site, $\mathcal{G}_{0,0 \mid 0}^{2 \mid 1}(\varepsilon)$, are plotted. These bound states result from the shape of the upper band edge: Since the band in the homogeneous leads is restricted to energies below $4 \tau$ (unlike in the continuous case), all states with higher energy are spacially confined to within the central region, have an infinite lifetime and form a discrete spectrum, determined by the shape of the applied potential $V\left(x_{j}\right)$.

The calculation of self-energy and two particle vertex, Eq.(48) and Eq.(42), is performed by ad-infinitum frequency integrations over products of Green's functions. Thus, the energy region of the upper band edge and the local bound states must be included in their calculation with adequate care. This involves determining the exact position and weigth of the bound states, which requires high numerical effort, as well as dealing with the numerical evaluation of principal value integrals and convolutions, where one function has poles and the other one is continuous. While all this is doable with sufficient dedication, we can avoid such complications entirely by adapting the discretization scheme, discussed next. 


\section{Conductance for Interacting Fermi Systems}

\section{Adaptive discretization}

According to Eq. (56) and Eq. (57) we can modify the band width locally by choosing non-equidistant discretization points. In the following we discuss a nonconstant discretization scheme that reduces the band width within the central region enough so that the upper band edge exhibits a local minimum at $x_{0}$ rather than a local maximum (as in the case of constant spacing). In consequence the Green's functions are continuous within the whole energy band, which facilitates a numerical treatment of interactions.

For a non-constant real space discretization it proves useful to first define the onsite energy $E_{j}$ and the hopping $\tau_{j}$ of the discrete tight-binding Hamiltonian Eq. (55) and then use these expressions to calculate the geometry of the corresponding physical barrier, i.e. its height $V_{c}$ and curvature $\Omega_{x}$.

We specify the onsite energy to be quadratic near the top with

$$
E_{j}=\tilde{E}_{j}+2 \tau \simeq \tilde{E}_{0}\left[1-\frac{j^{2}}{N^{\prime 2}}\right]+2 \tau,
$$

where $\tilde{E}_{0}$ is positive. We use the shape of $\tilde{E}_{j}$ within $C$ (which, apart from its height and the quadratic shape around the top does not influence transport properties, as long as $\tilde{E}_{j}$ goes adiabatically to zero upon approaching $j=\left|N^{\prime}\right|$ ) to define a site-dependent hopping (amounting to a site-dependent spacing)

$$
\tau_{j}=\tau\left[1-\frac{c}{2 \tau}\left(\tilde{E}_{j}+\tilde{E}_{j+1}\right)\right],
$$

where we have introduced a dimensionless positive parameter $c<\tau / \tilde{E}_{0}$ that determines how strongly the band width is to be reduced. Note that Eq. (60) describes a hopping, that is constant $(=\tau)$ in the leads, where $\tilde{E}_{j}=V_{j}=0$, and decreases with increasing $\tilde{E}_{j}$ in the central region. This corresponds to a site-dependent lattice spacing $a_{j}=a \sqrt{\tau / \tau_{j}}$, which increases towards the center of the central region. The real space position $x_{j}$ that corresponds to a site $j$ is given by

$$
x_{j}=\operatorname{sgn}(j) \sum_{j^{\prime}=1}^{|j|} a_{j^{\prime}}=a \sqrt{\tau} \operatorname{sgn}(j) \sum_{j^{\prime}=1}^{|j|} \frac{1}{\sqrt{\tau_{j}}},
$$

where $\operatorname{sgn}(x)$ is the sign function. Following Eq. (56), the construction introduced in Eq.(59) and Eq.(60) leads to an upper band edge given by

$$
\varepsilon_{j}^{\max } \simeq E_{j}+\tau_{j-1}+\tau_{j} \simeq 4 \tau+(1-2 c) \tilde{E}_{j},
$$

which for the choice $c>0.5$ indeed exhibits a smooth local minimum at $j=0$, thus avoiding the bound states discussed above for the constant discretization, $c=0$.

Despite the drastic manipulation of $\varepsilon_{j}^{\max }$, the lower band edge still serves as a proper potential barrier,

$$
\varepsilon_{j}^{\min }=V_{j} \simeq(1+2 c) \tilde{E}_{j}
$$

with a quadratic potential barrier top whose height now depends on the compensation factor $c$ :

$$
V_{j} \simeq(1+2 c) \tilde{E}_{j}\left[1-\frac{j^{2}}{N^{\prime 2}}\right]
$$

Finally, we write the potential barrier in the form given in Eq.(58), i.e. express the curvature $\Omega_{x}$ in units of the constant lead-hopping $\tau$. By comparison we find

$$
V_{c}=V_{0}-\mu, \quad \Omega_{x}=\frac{2}{N^{\prime}} \sqrt{V_{0} \tau_{0}} .
$$

The right half $\left(x_{j}>0\right)$ of Fig. 1(a) shows the LDOS of the central region for an adaptive discretization with $c=0.55$. All additional parameters are chosen such that the resulting potential barrier matches the case of constant discretization (plotted for $x_{j}<0$ ). Most importantly, the minimum of $\varepsilon_{j}^{\max }$ at $j=0$ prevents the occurance of bound states above the barrier, which allows for a faster numerical evaluation of the vertex functions. Importantly, both discretization schemes approximate the same physical system; their differences are non-neglegible only for energies far above the barrier, i.e. far away from the energies relevant for transport. This can be seen from the matching grey scale at the interface $j=0$ for energies $\varepsilon<V_{0}+\mathcal{O}\left(\Omega_{x}\right)$, as well as from comparison of the central site's LDOS in Fig. 1(c).

\section{B. The choice of system parameters}

To ensure that the discrete model reflects the transport properties of the continuous barrier, Eq. (54), the chemical potential of the system (or of both leads in nonequilibrium) must be chosen far enough below the global minimum of $\varepsilon^{\max }\left(x_{j}\right)$. Only in this case the unphysical upper band edge does not contribute to the results. The onsite-energy is chosen as

$$
\tilde{E}_{j}=\theta\left(N^{\prime}-|j|\right) \tilde{E}_{0} \exp \left(-\frac{\left(\frac{j}{N^{\prime}}\right)^{2}}{1-\left(\frac{j}{N^{\prime}}\right)^{2}}\right),
$$

where $\theta(x)$ is the Heavyside step function. Note, that this definition is consistent with Eq.(59). In order to calculate the site-dependent coupling we use $c=0.55$ in Eq. (60). Hence, for a barrier height $V_{0}=\mu$ (corresponding to a noninteracting transmission $\mathcal{T}_{0}=0.5$, see Eq.(68) below), we get a potential curvature $\Omega_{x}=0.039 \tau$. Finally, the shape of the onsite interaction is chosen as

$$
U_{j}=\theta\left(N^{\prime}-|j|\right) U_{0} \exp \left(-\frac{\left(\frac{j}{N^{\prime}}\right)^{6}}{1-\left(\frac{j}{N^{\prime}}\right)^{2}}\right) .
$$

\section{Non-interacting properties of the model}

In Ref. 5 we argued that the model of Eq.(55), combined with a potential with parabolic barrier top, 


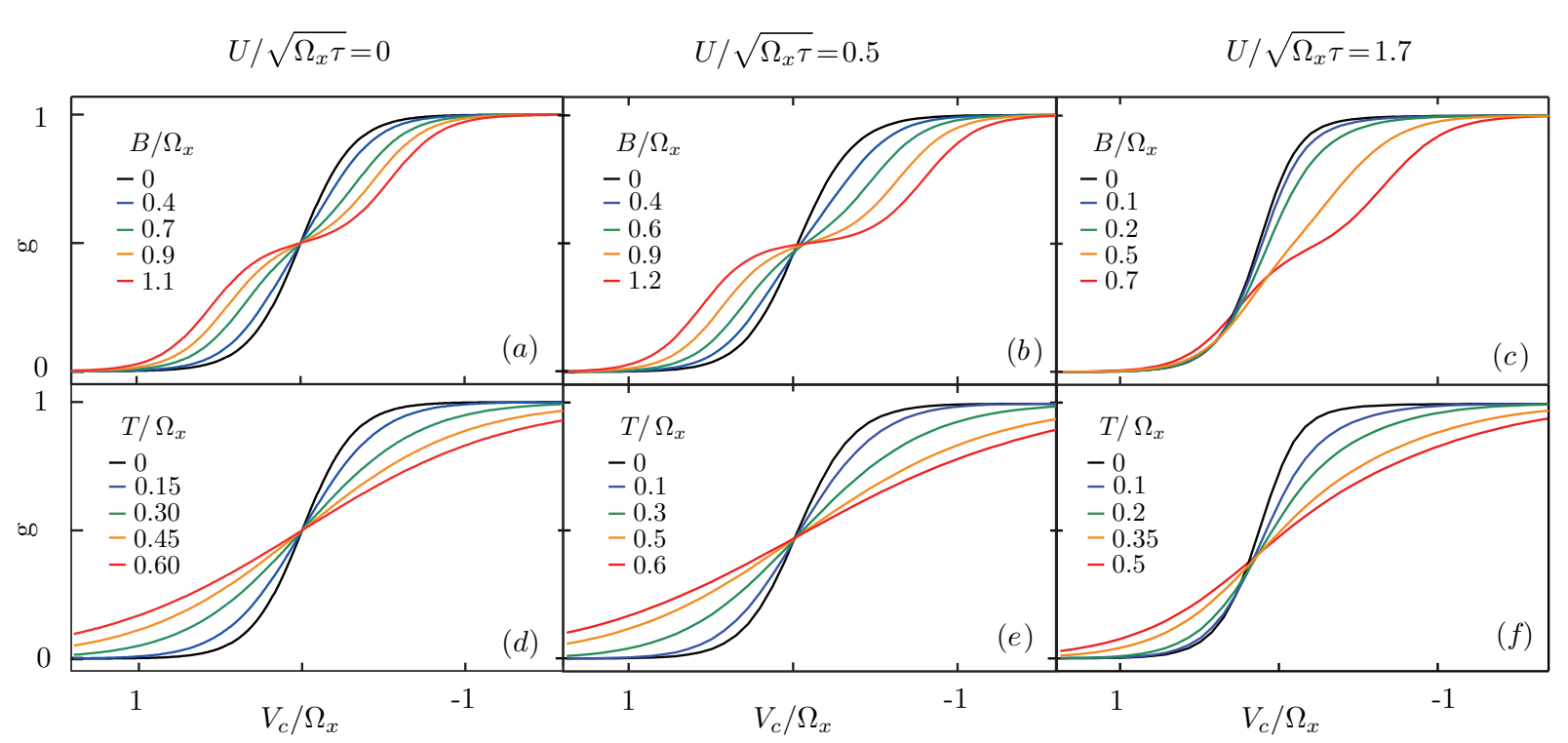

Figure 2. : (a)-(c), Linear conductance as a function of barrier height $V_{c}$ for some values of magnetic field $B$ with interaction strength $U$ increasing from left to right. (d)-(f), Linear conductance as a function of barrier height $V_{c}$ for some values of temperature $T$ with interaction strength $U$ increasing from left to right. Interactions cause an asymmetric evolution of conductance with magnetic field and temperature due to the interaction-enhanced reduction of conductance in the sub-open regime - the 0.7 anomaly.

Eq.(54), is sufficient to describe the physics of the lowest subband of a QPC: Making a saddle-point ansatz for the electrostatic potential caused by voltages applied to a typical QPC gate structure provides an effective 1Dpotential of the form Eq.(54). Information about the transverse geometry of the QPCs potential can be incorporated into the site-dependent effective interaction strength $U_{j}$, see Eq.(67).

The non-interacting, spin-dependent transmission through a quadratic barrier of height $V_{0}=V_{c}+\mu$ and curvature $\Omega_{x}$, Eq. (54), in the presence of a magnetic field $B$ can be derived analytically ${ }^{16}$ and is given by

$$
\mathcal{T}_{0}^{\sigma}(\varepsilon)=\frac{1}{e^{-2 \pi\left(\varepsilon-V_{0}+\sigma B / 2\right) / \Omega_{x}}+1} .
$$

Hence, according to the Landauer-Büttiker formula, the non-interacting (bare) linear conductance,

$$
\mathrm{g}_{0}=-\frac{e^{2}}{h} \sum_{\sigma} \int_{-\infty}^{\infty} f^{\prime}(\varepsilon) \mathcal{T}_{0}^{\sigma}(\varepsilon),
$$

is a step function of width $\Omega_{x}$ at $B=T=0$, changing from 0 to 1 , when the barrier top is shifted through $\mu$ from above. This step gets broadened with temperature [see Figure 2(d)] and develops a double-step structure with magnetic field [see Figure 2(a)]. For all $B$ and $T$ the bare conductance obeys the symmetry $\mathrm{g}_{0}\left(V_{c}\right)=1-\mathrm{g}_{0}\left(-V_{c}\right)$.

Furthermore, an analytic expression for the noninteracting LDOS at the chemical potential in the barrier center as function of barrier height $V_{c}$ can be calculated [see e.g. Ref. 17],

$$
\mathcal{A}_{0}(\varepsilon=\mu, 0)=\frac{\left|\Gamma\left(1 / 4+i V_{c} /\left(2 \Omega_{x}\right)\right)\right|^{2}}{4 \sqrt{2} \pi^{2} e^{\pi V_{c} /\left(2 \Omega_{x}\right)}},
$$

where $\Gamma(z)$ is the complex gamma-function. This is a smeared and shifted version of the $1 \mathrm{D}$ van Hove singularity [see Ref. 5 for further details], peaked at $V_{c}=-\mathcal{O}\left(\Omega_{x}\right)$, i.e. if the barrier top lies sightly below the chemical potential. Here, the value of the noninteracting conductance is given by $\mathrm{g}_{0} \approx 0.8$. Hence, we call this parameter regime sub-open.

\section{Interacting results}

As was discussed in Ref. 5, the shape of the LDOS in the barrier center lies at the heart of the mechanism causing the 0.7 conductance anomaly: Semiclassically, the LDOS can be interpreted as being inversely proportional to the velocity $v$ of the charge carriers, $\mathcal{A}_{0}\left(\varepsilon, x_{j}\right) \propto 1 / v_{j}(\varepsilon)$. Hence, the average time that a non-interacting electron with energy $\varepsilon=\mu$ spends in the vicinity of the barrier center is maximal in the sub-open regime (where $\mathcal{A}_{0}(\mu, 0)$ is maximal), resulting in an enhanced scattering probability and thus a strong reduction of conductance at finite interaction strength in this parameter regime.

Figure 2 compares the bare conductance, calculated via the Landauer-Büttiker formula [Eq.(69)], with the conductance obtained by taking into account interactions using SOPT, calculated via the Keldysh version of Oguri's formula [Eq.(23)], as a function of barrier height 
$V_{c}$ for several values of magnetic field (panels (a)-(c)) and temperature (panels (d)-(f)), for three interaction strengths increasing from left to right. For small but finite interactions, $U \sqrt{\Omega_{x} \tau}=0.5$, the shape of the LDOS causes a slight asymmetry in the conductance curves at (b) finite magnetic field or (e) finite temperature: A finite magnetic field induces an imbalance of spin-species in the vicinity of the barrier center. This imbalance is enhanced by exchange interactions via Stoner-type physics, where the disfavoured spin species (say spin down) is pushed out of the center region by the coulomb blockade of the the favoured spin-species (say spin up). Hence, transport is dominated by the spin-up channel, resulting in a strong reduction of total conductance in the sub-open regime even for a small magnetic field. A finite temperature, on the other hand, opens phase-space for inelastic scattering, which, again, is strongest for large LDOS, again resulting in the reduction of conductance in the subopen regime. This interaction-induced trend continues with increasing interactions, and gives rise to a weak 0.7 anomaly at $B \neq 0$, Figure 2(c), or $T \neq 0$, Figure 2(f), for intermediate interaction strength, $U \sqrt{\Omega_{x} \tau}=1.7$. Upon a further increase of interactions, SOPT breaks down (see below), and more elaborate methods are needed to obtain qualitatively correct results. This was done in Ref. 5 and Ref. 18, where we used fRG to reach interaction strength of up to $U \sqrt{\Omega_{x} \tau}=3.5$; they yielded a pronounced 0.7 anomaly even at $B=T=0$ and its typical magnetic field development into the spin-resolved conductance steps at high field.

The main limitations of SOPT when treating the inhomogeneous system, introduced in Eq.(54), can be explained as follows: Upon increasing interactions, the LDOS is shifted towards higher energy, as Hartree contributions cause an effective higher potential barrier compared to the non-interaction case. As a consequence, a proper description of interactions requires information about this shift to be incorporated into the calculation of the vertex functions via feed-back of the self-energy into all propagators. However, SOPT calculates the selfenergy and the two-particle vertex [Section IV] using only bare propagators, which only carry information of the bare LDOS. Together with the drastic truncation of the perturbation series beyond second order, this limits the quantitative validity of SOPT to weak interaction strength and the qualitative validity of SOPT to intermediate interaction strength. Nevertheless, SOPT suffices to illustrate the essential physics involved in the appearance of the 0.7 conductance anomaly.

\section{CONCLUSION AND OUTLOOK}

In this paper we discuss electronic transport through an interacting region of arbitrary shape using the Keldysh formalism. Starting from the well-established Meir-Wingreen formula for the system's current we derive formulas for both the differential and linear conductance. In the latter case we use the fRG flow-equation for the self-energy as well as a Ward identity, following from the
Hamiltonian's particle conservation, to obtain a Keldysh version of Oguri's linear conductance formula. As an application we calculate the conductance of the lowest subband of a QPC, which we model by a one-dimensional parabolic potential barrier and onsite interactions - a setup we have recently used to explore the microscopic origin of the 0.7 conductance anomaly ${ }^{5}$. We present detailed discussion of the model's properties and argue that an adaptive, non-constant real space discretization scheme greatly facilitates numerical effort. We treat the influence of interactions using SOPT, presenting all details that are necessary to employ the derived conductance formulas. Our SOPT-results for the linear conductance as function of magnetic field and temperature illustrate that the anomalous reduction of conductance in the sub-open regime of a QPC is due to an interplay of the van-Hove ridge and electron-electron interactions.

In order to improve on the interacting results we are currently setting up Keldysh fRG. Since approximation schemes within the fRG violate particle conservation at finite excitation energies, future studies must prioritize to quantify how severe this violation influences the validity of transport results. A possible alternative are self-consistent approximation schemes, where the $U(1)$ symmetry of the Hamiltonian is preserved by construction.

\section{Appendix A: Properties of Green's and vertex functions in Keldysh formalism}

To investigate transport properties of the system in and out of equilibrium, we apply the well-established Keldysh formalish ${ }^{8,9}$. Here we collect some of its standard ingredients. In large parts, we follow the definitions and conventions given in Ref.[11].

All operators carry Keldysh time-contour indices, $a_{1}, a_{1}^{\prime}, a_{2}, \ldots=\{+,-\}$, marking the position of the time argument $t$ of an operator as lying on the forward $(-)$ or backward $(+)$ branch of the Keldysh contour. We use Keldysh indices with or without a prime, $a$ or $a^{\prime}$, to label the time arguments of annihilation or creation operators, respectively. Since the model Hamiltonian, Eq.(1), is time-independent, the only non-zero matrix elements of the Hamiltonian in contour space have equal contour indices:

$$
\begin{aligned}
\mathcal{H}_{0}^{a_{1} a_{1}^{\prime}} & =-a_{1} \cdot \delta_{a_{1} a_{1}^{\prime}} H_{0}, \\
\mathcal{H}_{\text {int }}^{a_{1} a_{2} \mid a_{1}^{\prime} a_{2}^{\prime}} & =-a_{1} \cdot \delta_{a_{1} a_{2}} \delta_{a_{1} a_{1}^{\prime}} \delta_{a_{1} a_{2}^{\prime}} H_{\text {int }},
\end{aligned}
$$

with $\{a\}$ labeling the time arguments of annihilation operators and $\left\{a^{\prime}\right\}$ labeling the time arguments of creation operators. Note that a calligraphic $\mathcal{H}$ carries contour indices, while a capital $H$ does not.

We define time-dependent, $n$-particle Keldysh Green's functions as the expectation values

$$
\begin{aligned}
& G_{\boldsymbol{i} \mid \boldsymbol{i}^{\prime}}^{n, a \mid a^{\prime}}\left(\boldsymbol{t} \mid \boldsymbol{t}^{\prime}\right)=G_{i_{1}, \ldots, i_{n} \mid i_{1}^{\prime}, \ldots i_{n}^{\prime}}^{a_{1}, \ldots, a_{n} \mid a_{1}^{\prime}, \ldots a_{n}^{\prime}}\left(t_{1}, \ldots, t_{n} \mid t_{n}^{\prime}, \ldots, t_{1}^{\prime}\right)= \\
& (-i)^{n}\left\langle\mathcal{T}_{c} d_{i_{1}}^{a_{1}}\left(t_{1}\right) \ldots d_{i_{n}}^{a_{n}}\left(t_{n}\right)\left[d_{i_{n}^{\prime}}^{a_{n}^{\prime}}\right]^{\dagger}\left(t_{n}^{\prime}\right) \ldots\left[d_{i_{1}^{\prime}}^{a_{1}^{\prime}}\right]^{\dagger}\left(t_{1}^{\prime}\right)\right\rangle, \quad
\end{aligned}
$$


where we use boldface notation for multi-indices $\boldsymbol{x}=$ $\left(x_{1}, \ldots, x_{n}\right)$. The operator $d_{i}^{a}(t) /\left[d_{i}^{a}\right]^{\dagger}(t)$ destroys/creates an electron at time $t$ on contour branch $a$ in quantum state $i$, and the time-ordering operator $\mathcal{T}_{c}$ moves later contour times to the left. In case of equal time arguments, annihilation operators are always arranged to the right of creation operators. The bare, non-interacting Green's function, whose time-dependence is governed by the quadratic part of the Hamiltonian, $\mathcal{H}_{0}$, carries an additional subscript, $G_{0}$.

We define anti-symmetrized, irreducible, $n$-particle vertex functions, $\gamma_{\boldsymbol{i}^{\prime} \mid \boldsymbol{i}}^{n, \boldsymbol{a}^{\prime} \mid a}\left(\boldsymbol{t}^{\prime} \mid \boldsymbol{t}\right)$, as the sum of all 1-particle irreducible (1PI) diagrams with $n$ amputated ingoing and $n$ amputated outgoing legs. For an explicit series representation of the one- and two-particle vertex, see Eq.(B.1). A formula for the prefactor of every single diagram is given by Eq.(20) of Ref.[11].

The Dyson equation provides a direct relation between the one-particle Green's and vertex function:

$G\left(t_{1} \mid t_{1}^{\prime}\right)=G_{0}\left(t_{1} \mid t_{1}^{\prime}\right)-\int d \tau_{1} d \tau_{1}^{\prime} G_{0}\left(t_{1} \mid \tau_{1}^{\prime}\right) \gamma\left(\tau_{1}^{\prime} \mid \tau_{1}\right) G\left(\tau_{1} \mid t_{1}^{\prime}\right)$.

Here and below, whenever quantum state indices $i$ and contour indices $a /$ Keldysh indices $\alpha$ are implicit, they are understood to be summed over in products.

Decomposing the two-particle Green's yields a connection to the two-particle vertex function via

$$
\begin{aligned}
& G\left(t_{1}, t_{2} \mid t_{1}^{\prime}, t_{2}^{\prime}\right)=G\left(t_{1} \mid t_{1}^{\prime}\right) G\left(t_{2} \mid t_{2}^{\prime}\right)-G\left(t_{1} \mid t_{2}^{\prime}\right) G\left(t_{2} \mid t_{1}^{\prime}\right) \\
& -i \int d \tau G\left(t_{1} \mid \tau_{1}^{\prime}\right) G\left(t_{2} \mid \tau_{2}^{\prime}\right) \gamma\left(\tau_{1}^{\prime}, \tau_{2}^{\prime} \mid \tau_{1}, \tau_{2}\right) G\left(\tau_{1} \mid t_{1}^{\prime}\right) G\left(\tau_{2} \mid t_{2}^{\prime}\right) .
\end{aligned}
$$

Since the Hamiltonian, Eq.(1), is time-independent, the Green's/vertex functions are translationally invariant in time, implying that $n$-particle functions depend on $2 n-1$ time arguments only:

$$
\begin{aligned}
G\left(t_{1}, \ldots, t_{n} \mid t_{1}^{\prime}, \ldots t_{n}^{\prime}\right) & =G\left(0, \ldots, t_{n}-t_{1} \mid t_{1}^{\prime}-t_{1}, \ldots, t_{n}^{\prime}-t_{1}\right), \\
\gamma\left(t_{1}^{\prime}, \ldots, t_{n}^{\prime} \mid t_{1}, \ldots t_{n}\right) & =\gamma\left(0, \ldots, t_{n}^{\prime}-t_{1}^{\prime} \mid t_{1}-t_{1}^{\prime}, \ldots, t_{n}-t_{1}^{\prime}\right) .
\end{aligned}
$$

As a consequence, the Fourier-transform,

$$
\begin{aligned}
& G\left(\varepsilon \mid \varepsilon^{\prime}\right)=\int d \mathbf{t} d \mathbf{t}^{\prime} e^{i \varepsilon t} e^{-i \varepsilon^{\prime} t^{\prime}} G\left(\mathbf{t} \mid \mathbf{t}^{\prime}\right), \\
& \gamma\left(\varepsilon^{\prime} \mid \varepsilon\right)=\int d \mathbf{t} d \mathbf{t}^{\prime} e^{i \varepsilon^{\prime} \boldsymbol{t}^{\prime}} e^{-i \varepsilon t} \gamma\left(\mathbf{t}^{\prime} \mid \mathbf{t}\right),
\end{aligned}
$$

fulfills energy conservation. In particular, this allows for the following representation for the one- and two-particle functions, where calligraphic letters $\mathcal{G}$ and $\mathcal{L}$ are used when a $\delta$-function has been split off:

$$
\begin{aligned}
& G\left(\varepsilon_{1} \mid \varepsilon_{1}^{\prime}\right)=2 \pi \delta\left(\varepsilon_{1}-\varepsilon_{1}^{\prime}\right) \mathcal{G}\left(\varepsilon_{1}\right), \\
& G\left(\varepsilon_{1}, \varepsilon_{2} \mid \varepsilon_{1}^{\prime}, \varepsilon_{2}^{\prime}\right)=2 \pi \delta\left(\varepsilon_{1}+\varepsilon_{2}-\varepsilon_{1}^{\prime}-\varepsilon_{2}^{\prime}\right) \mathcal{G}\left(\varepsilon_{2}, \varepsilon_{1}^{\prime} ; \varepsilon_{1}-\varepsilon_{1}^{\prime}\right), \\
& \gamma\left(\varepsilon_{1}^{\prime} \mid \varepsilon_{1}\right)=-2 \pi \delta\left(\varepsilon_{1}^{\prime}-\varepsilon_{1}\right) \Sigma\left(\varepsilon_{1}^{\prime}\right), \\
& \gamma\left(\varepsilon_{1}^{\prime}, \varepsilon_{2}^{\prime} \mid \varepsilon_{1}, \varepsilon_{2}\right)=2 \pi \delta\left(\varepsilon_{1}^{\prime}+\varepsilon_{2}^{\prime}-\varepsilon_{1}-\varepsilon_{2}\right) \mathcal{L}\left(\varepsilon_{2}^{\prime}, \varepsilon_{1} ; \varepsilon_{1}^{\prime}-\varepsilon_{1}\right) .
\end{aligned}
$$

The one-particle vertex-function $\Sigma$, introduced above, is called the interacting irreducible self-energy. We Fouriertransform Dyson's equation, Eq.(A.3), which provides

$$
\mathcal{G}(\varepsilon)=\mathcal{G}_{0}(\varepsilon)+\mathcal{G}_{0}(\varepsilon) \Sigma(\varepsilon) \mathcal{G}(\varepsilon)=\left[\left[\mathcal{G}_{0}(\varepsilon)\right]^{-1}-\Sigma(\varepsilon)\right]^{-1} .
$$

Note that this is a matrix equation in both Keldysh and position space.

The four single-particle Green's functions and selfenergies in contour space are called chronological $\left(\mathcal{G}^{-1-}\right.$, $\left.\Sigma^{-\mid-}\right)$, lesser $\left(\mathcal{G}^{-\mid+}, \Sigma^{-\mid+}\right)$, greater $\left(\mathcal{G}^{+\mid-}, \Sigma^{+\mid-}\right)$and anti-chronological $\left(\mathcal{G}^{+\mid+}, \Sigma^{+\mid+}\right)$. As a consequence of the definition, Eq.(A.2), the single-particle Green's functions fulfill the contour-relation

$$
\mathcal{G}^{+\mid+}+\mathcal{G}^{-1-}=\mathcal{G}^{-1+}+\mathcal{G}^{+\mid-}
$$

We define the transformation from contour space $(a=$ $\{-,+\})$ into Keldysh space $(\alpha=\{1,2\})$ by the rotation

$$
R=\left(\begin{array}{ll}
R^{-\mid 1} & R^{-\mid 2} \\
R^{+\mid 1} & R^{+\mid 2}
\end{array}\right)=\frac{1}{\sqrt{2}}\left(\begin{array}{cc}
1 & 1 \\
-1 & 1
\end{array}\right) .
$$

Hence, any $n$-th rank tensor $A^{n, \alpha^{\prime} \mid \alpha}$ in Keldysh space is represented in contour space by

$$
A^{n, \alpha \mid \alpha^{\prime}}=\sum_{a, a^{\prime}}\left[R^{-1}\right]^{\alpha \mid a} A^{n, a \mid a^{\prime}} R^{a^{\prime} \mid \alpha^{\prime}} .
$$

As can be shown explicitly (see Chapter 3.3 of Ref.11) the Green's and vertex functions fulfill a theorem of causality:

$$
\begin{aligned}
& \mathcal{G}^{1 \ldots 1 \mid 1 \ldots 1}=0, \\
& \mathcal{L}^{2 \ldots 2 \mid 2 \ldots 2}=0 .
\end{aligned}
$$

The remaining three non-zero Keldysh components of the single-particle functions are called retarded $\left(\mathcal{G}^{2 \mid 1}, \Sigma^{1 \mid 2}\right)$, advanced $\left(\mathcal{G}^{1 \mid 2}, \Sigma^{2 \mid 1}\right)$ and Keldysh $\left(\mathcal{G}^{2 \mid 2}, \Sigma^{1 \mid 1}\right)$ :

$$
\begin{aligned}
& \mathcal{G}=\left(\begin{array}{cc}
0 & \mathcal{G}^{A} \\
\mathcal{G}^{R} & \mathcal{G}^{K}
\end{array}\right)=\left(\begin{array}{cc}
0 & \mathcal{G}^{1 \mid 2} \\
\mathcal{G}^{2 \mid 1} & \mathcal{G}^{2 \mid 2}
\end{array}\right), \\
& \Sigma=\left(\begin{array}{cc}
\Sigma^{K} & \Sigma^{R} \\
\Sigma^{A} & 0
\end{array}\right)=\left(\begin{array}{cc}
\Sigma^{1 \mid 1} & \Sigma^{1 \mid 2} \\
\Sigma^{2 \mid 1} & 0
\end{array}\right) .
\end{aligned}
$$

The transformation, Eq.(A.11), provides the identities

$$
\begin{aligned}
\mathcal{G}^{-\mid+} & =\frac{1}{2}\left[\mathcal{G}^{2 \mid 2}-\left(\mathcal{G}^{2 \mid 1}-\mathcal{G}^{1 \mid 2}\right)\right] \\
\mathcal{G}^{+\mid-}-\mathcal{G}^{-\mid+} & =\mathcal{G}^{2 \mid 1}-\mathcal{G}^{1 \mid 2} \\
\mathcal{H}_{0}^{1 \mid 2} & =\mathcal{H}_{0}^{2 \mid 1}=H_{0}, \mathcal{H}_{0}^{1 \mid 1}=\mathcal{H}_{0}^{2 \mid 2}=0
\end{aligned}
$$

all of which are used in the derivation of the conductance formula in Sec.I. Note that a calligraphic $\mathcal{H}$ carries Keldysh indices, while a capital $H$ does not. The retarded/advanced components are analytic in the upper/lower half plane of the complex frequency plane. Hence, the following notation is always implied,

$$
\begin{aligned}
& \mathcal{G}^{2 \mid 1}(\varepsilon)=\mathcal{G}^{2 \mid 1}(\varepsilon+i \delta), \Sigma^{1 \mid 2}(\varepsilon)=\Sigma^{1 \mid 2}(\varepsilon+i \delta), \\
& \mathcal{G}^{1 \mid 2}(\varepsilon)=\mathcal{G}^{1 \mid 2}(\varepsilon-i \delta), \Sigma^{2 \mid 1}(\varepsilon)=\Sigma^{2 \mid 1}(\varepsilon-i \delta),
\end{aligned}
$$




\section{Conductance for Interacting Fermi Systems}

with real, infinitesimal, positive $\delta$. In contrast, the Keldysh component is a response function, restricted to the real frequency axis. In equilibrium, the single-particle functions fulfill a fluctuation dissipation theorem (FDT):

$$
\begin{aligned}
& \Sigma^{1 \mid 1}(\varepsilon)=(1-2 f(\varepsilon))\left[\Sigma^{1 \mid 2}(\varepsilon)-\Sigma^{2 \mid 1}(\varepsilon)\right], \\
& \mathcal{G}^{2 \mid 2}(\varepsilon)=(1-2 f(\varepsilon))\left[\mathcal{G}^{2 \mid 1}(\varepsilon)-\mathcal{G}^{1 \mid 2}(\varepsilon)\right],
\end{aligned}
$$

where $f(\varepsilon)=1 /(1+\exp [(\varepsilon-\mu) / T])$ is the Fermi distribution function.

Within this work we consider a system composed of a finite central interacting region coupled to two noninteracting fermionic leads: The left lead $L$, with chemical potential $\mu_{L}$ and temperature $T_{L}$, and the right lead $R$, with chemical potential $\mu_{R}$ and temperature $T_{R}$. Defining operators $C / L / R$, which project onto the subspace of the central region/left lead/right lead respectively, we can represent the quadratic part of the Hamiltonian as

$\mathcal{H}_{0}=\left(\begin{array}{ccc}L \mathcal{H}_{0} L & L \mathcal{H}_{0} C & 0 \\ C \mathcal{H}_{0} L & C \mathcal{H}_{0} C & C \mathcal{H}_{0} R \\ 0 & R \mathcal{H}_{0} C & R \mathcal{H}_{0} R\end{array}\right)=\left(\begin{array}{ccc}\mathcal{H}_{l} & \mathcal{H}_{l c} & 0 \\ \mathcal{H}_{c l} & \mathcal{H}_{0, c} & \mathcal{H}_{c r} \\ 0 & \mathcal{H}_{r c} & \mathcal{H}_{r}\end{array}\right)$.

where the matrices $\mathcal{H}_{l}$ and $\mathcal{H}_{r}$ fully define the properties of the isolated leads, and the matrix $\mathcal{H}_{0, c}$ describes the non-interacting physics of the isolated central region. Finally, $\mathcal{H}_{c l}$ and $\mathcal{H}_{c r}$ specify the coupling of the central region to the corresponding lead. Similarly, we write the system's Green's function, $\mathcal{G}(\varepsilon)$ [Eq.(A.8)], in the same basis (for the bare, non-interacting Green's function $\mathcal{G}_{0}$ we set $\Sigma=0)$ :

$$
\mathcal{G}=\left(\begin{array}{ccc}
L \mathcal{G} L & L \mathcal{G} C & 0 \\
C \mathcal{G} L & C \mathcal{G} C & C \mathcal{G} R \\
0 & R \mathcal{G} C & R \mathcal{G} R
\end{array}\right)=\left(\begin{array}{ccc}
\mathcal{G}_{l} & \mathcal{G}_{l c} & 0 \\
\mathcal{G}_{c l} & \mathcal{G}_{c} & \mathcal{G}_{c r} \\
0 & \mathcal{G}_{r c} & \mathcal{G}_{r}
\end{array}\right)
$$

We use the small letter $g$ to denote the Green's function of an isolated subsystem, e.g. $g_{l}(\varepsilon)$ is the Green's function of the isolated left lead $L$. The non-interacting Green's function of the central region is given by Dyson's equation

$$
\mathcal{G}_{0, c}=g_{0, c}+g_{0, c} \Sigma_{\mathrm{lead}} \mathcal{G}_{0, c}=\left[\left[g_{0, c}\right]^{-1}-\Sigma_{\mathrm{lead}}\right]^{-1} .
$$

Again note that this is a matrix equation in Keldysh and position space. We incorporated environment contributions into the lead self-energy

$$
\Sigma_{\text {lead }}=\sum_{k=l, r} \mathcal{H}_{c k} g_{k} \mathcal{H}_{k c}
$$

The individual Keldysh components of the non- interacting Green's function are given by

$$
\begin{aligned}
\mathcal{G}_{0, c}^{1 \mid 2}(\varepsilon) & =\left(\varepsilon-H_{0, c}-\Sigma_{\text {lead }}^{2 \mid 1}(\varepsilon)\right)^{-1} \\
\mathcal{G}_{0, c}^{2 \mid 1}(\varepsilon) & =\left(\varepsilon-H_{0, c}-\Sigma_{\text {lead }}^{1 \mid 2}(\varepsilon)\right)^{-1} \\
\mathcal{G}_{0, c}^{2 \mid 2}(\varepsilon) & =\mathcal{G}_{0, c}^{2 \mid 1}(\varepsilon) \Sigma_{\text {lead }}^{1 \mid 1}(\varepsilon) \mathcal{G}_{0, c}^{1 \mid 2}(\varepsilon) \\
& =-i \sum_{k=l, r}\left[1-2 f^{k}(\varepsilon)\right] \mathcal{G}_{0, c}^{2 \mid 1}(\varepsilon) \Gamma^{k}(\varepsilon) \mathcal{G}_{0, c}^{2 \mid 1}(\varepsilon),
\end{aligned}
$$

where we introduced the hybridization function, $\Gamma^{k}(\varepsilon)=$ $i H_{c k}\left(g_{k}^{2 \mid 1}(\varepsilon)-g_{k}^{1 \mid 2}(\varepsilon)\right) H_{k c}$.

With the interaction being restricted to the central region we use the notation $\Sigma=\Sigma_{c}=C \Sigma C$ for the interacting self-energy. Dyson's equation, Eq.(A.8), and the real space structure, Eq.(A.19), yields

$$
\mathcal{G}_{c}(\varepsilon)=\left[\left[\mathcal{G}_{0, c}(\varepsilon)\right]^{-1}-\Sigma(\varepsilon)\right]^{-1}
$$

The matrix representation of its Keldysh structure is given by

$$
\left(\begin{array}{cc}
0 & \mathcal{G}_{c}^{1 \mid 2} \\
\mathcal{G}_{c}^{2 \mid 1} & \mathcal{G}_{c}^{2 \mid 2}
\end{array}\right)=\left[\left(\begin{array}{cc}
0 & \mathcal{G}_{0, c}^{1 \mid 2} \\
\mathcal{G}_{0, c}^{2 \mid 1} & \mathcal{G}_{0, c}^{2 \mid 2}
\end{array}\right)^{-1}-\left(\begin{array}{cc}
\Sigma^{1 \mid 1} & \Sigma^{1 \mid 2} \\
\Sigma^{2 \mid 1} & 0
\end{array}\right)\right]^{-1}
$$

Block matrix inversion then provides the components

$$
\begin{aligned}
& \mathcal{G}_{c}^{1 \mid 2}(\varepsilon)=\left(\varepsilon-H_{0, c}-\Sigma_{\text {lead }}^{2 \mid 1}(\varepsilon)-\Sigma^{2 \mid 1}(\varepsilon)\right)^{-1}, \\
& \mathcal{G}_{c}^{2 \mid 1}(\varepsilon)=\left(\varepsilon-H_{0, c}-\Sigma_{\text {lead }}^{1 \mid 2}(\varepsilon)-\Sigma^{1 \mid 2}(\varepsilon)\right)^{-1}, \\
& \mathcal{G}_{c}^{2 \mid 2}(\varepsilon)=\mathcal{G}_{c}^{2 \mid 1}(\varepsilon)\left[\Sigma_{\text {lead }}^{1 \mid 1}+\Sigma^{1 \mid 1}\right] \mathcal{G}_{c}^{1 \mid 2}(\varepsilon) .
\end{aligned}
$$

From Eq.(A.8), we can show, that the off-diagonal components of the full Green's function, are given by

$$
\mathcal{G}_{k c}=g_{k} \mathcal{H}_{k c} \mathcal{G}_{c}, \mathcal{G}_{c k}=\mathcal{G}_{c} \mathcal{H}_{c k} g_{k}
$$

For a symmetric, real Hamiltonian, the following symmetries hold

$$
\begin{aligned}
& \mathcal{G}_{0, i \mid j}^{1 \mid 2}=\left[\mathcal{G}_{0, i \mid j}^{2 \mid 1}\right]^{*}, \mathcal{G}_{i \mid j}^{1 \mid 2}=\left[\mathcal{G}_{i \mid j}^{2 \mid 1}\right]^{*}, \Sigma_{i \mid j}^{1 \mid 2}=\left[\Sigma_{i \mid j}^{2 \mid 1}\right]^{*}, \\
& \mathcal{G}_{0, i \mid j}^{1 \mid 2}=\mathcal{G}_{0, j \mid i}^{1 \mid 2}, \mathcal{G}_{i \mid j}^{1 \mid 2}=\mathcal{G}_{j \mid i}^{1 \mid 2}, \Sigma_{i \mid j}^{1 \mid 2}=\Sigma_{j \mid i}^{1 \mid 2}, \\
& \mathcal{G}_{0, i \mid j}^{2 \mid 1}=\mathcal{G}_{0, j \mid i}^{2 \mid 1}, \mathcal{G}_{i \mid j}^{2 \mid 1}=\mathcal{G}_{j \mid i}^{2 \mid 1}, \Sigma_{i \mid j}^{2 \mid 1}=\Sigma_{j \mid i}^{2 \mid 1}, \\
& \mathcal{G}_{0, i \mid j}^{2 \mid 2}=-\left[\mathcal{G}_{0, j \mid i}^{2 \mid 2}\right]^{*}, \mathcal{G}_{i \mid j}^{2 \mid 2}=-\left[\mathcal{G}_{j \mid i}^{2 \mid 2}\right]^{*}, \Sigma_{i \mid j}^{1 \mid 1}=-\left[\Sigma_{j \mid i}^{1 \mid 1}\right]^{*} .
\end{aligned}
$$


Appendix B: Diagrammatic derivation of the fRG flow-equation of the self-energy

Here, we provide a diagrammatic derivation of the $f R G$ flow-equation for the self-energy, Eq.(13). We use the observation, that every diagram in the diagrammatic series of the self-energy contains a sub-diagram which appears in the diagrammatic series of the two-particle vertex. As a consequence, taking the derivative of the self-energy, $\partial_{\Lambda} \Sigma$, w.r.t. some parameter $\Lambda$ allows for a resummation of diagrams, such that the full two-particle vertex series can be factorized. Hence, we get an equation which can formally be written as $\partial_{\Lambda} \Sigma_{\Lambda}=\int S_{\Lambda} \mathcal{L}_{\Lambda}$, with the so called single-scale propagator $S$ and the two-particle vertex $\mathcal{L}$, both depending on the parameter $\Lambda$.

The self-energy $\Sigma$ and two-particle vertex $\mathcal{L}$ are diagrammatically defined as the sum of all one-particle irreducible diagrams with two and four amputated external legs, respectively. Using the graphical representation of the bare Green's function, Eq.(27), and the bare vertex, Eq.(31), the first terms of their perturbation series are (we omit the arrows for the sake of simplicity)

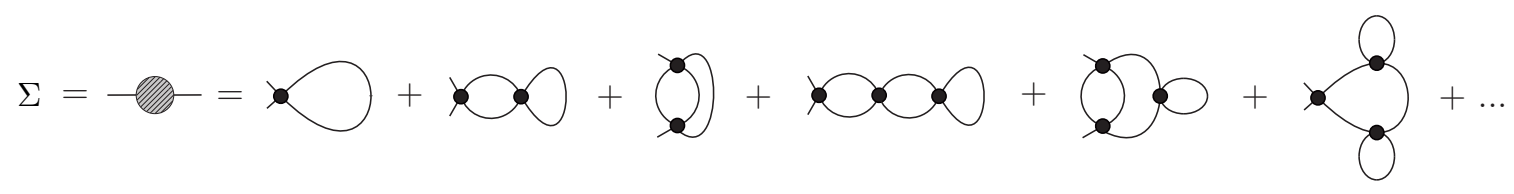

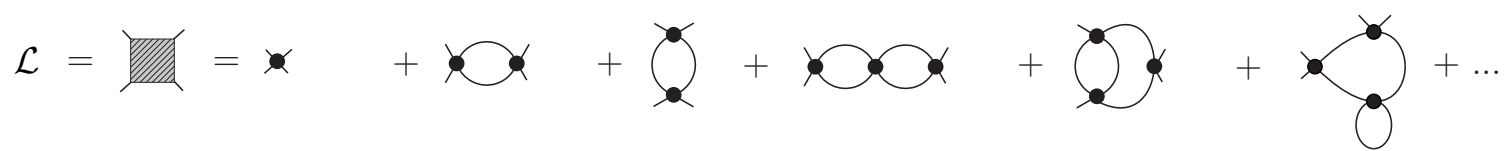

We introduce a parameter $\Lambda$ into the bare propagator, $\mathcal{G}_{0} \rightarrow \mathcal{G}_{0}^{\Lambda}$, and represent its derivative w.r.t. $\Lambda$ by a crossed-out line, $\partial_{\Lambda} \mathcal{G}_{0}^{\Lambda}=\longleftarrow$. Hence, the derivative of the self-energy is given by

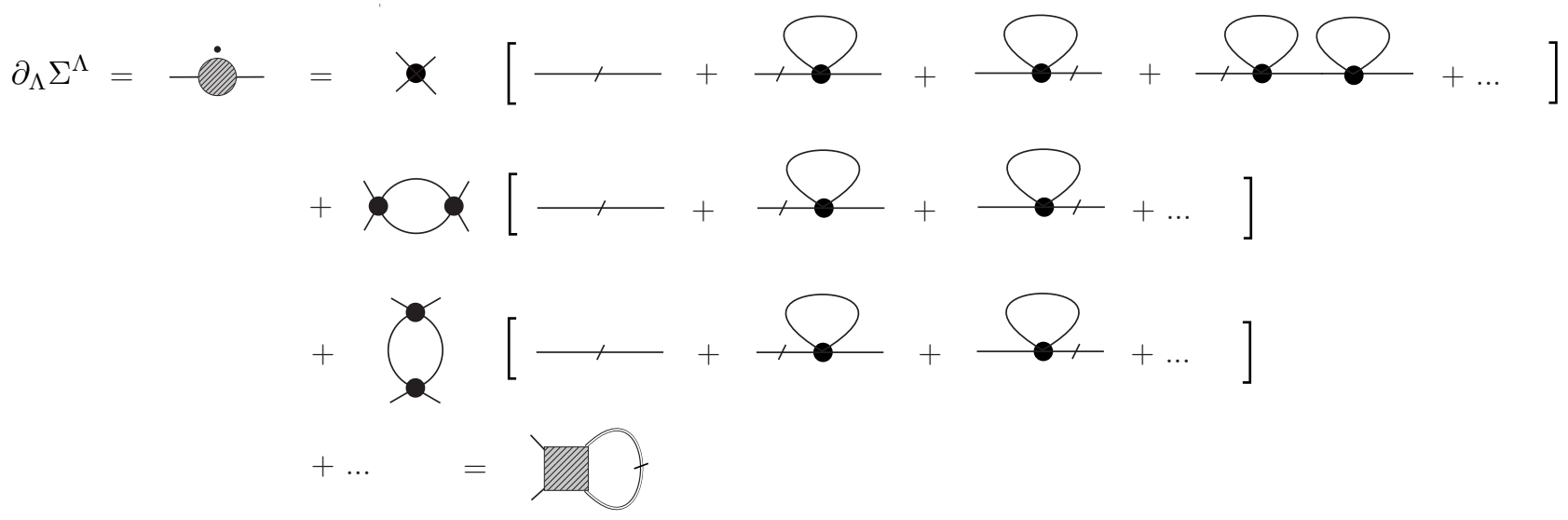

where we introduced the so called single scale propagator

$$
\begin{aligned}
& S_{\Lambda}=\frac{1}{=}+1+1+\ldots \\
& =\left[1+\mathcal{G}_{0} \Sigma+\mathcal{G}_{0} \Sigma \mathcal{G}_{0} \Sigma+\ldots\right] \partial_{\Lambda} \mathcal{G}_{0}^{\Lambda}\left[1+\Sigma \mathcal{G}_{0}+\Sigma \mathcal{G}_{0} \Sigma \mathcal{G}_{0}+\ldots\right] \\
& =\mathcal{G}\left[\mathcal{G}_{0}\right]^{-1} \partial_{\Lambda} \mathcal{G}_{0}^{\Lambda}\left[\mathcal{G}_{0}\right]^{-1} \mathcal{G}
\end{aligned}
$$

Finally, we fix the prefactor of the diagram on the r.h.s. in Eq.(B.2) by the following argument: The first order selfenergy, $\Sigma_{1}$, is of Hartree-type and hence purely determined by the local density $n_{j}$ and the local interaction strength $U_{j}$. We use the bare local density of states, $\mathcal{A}_{0, j}(\varepsilon)=-1 / \pi \cdot \operatorname{Im} \mathcal{G}_{0, j \mid j}^{2 \mid 1}(\varepsilon)$, and calculate the first order self-energy in equilibrium

$$
\left.\Sigma_{1, j}\right|_{V=0}=n_{j} U_{j}=2 \bar{u}_{j} \int d \varepsilon f(\varepsilon) \mathcal{A}_{0, j}(\varepsilon)=\pi i \bar{u}_{j} \int d \varepsilon f(\varepsilon)\left(\mathcal{G}_{0, j \mid j}^{2 \mid 1}-\mathcal{G}_{0, j \mid j}^{1 \mid 2}\right)=\frac{1}{2 \pi i} \bar{u}_{j} \int d \varepsilon \mathcal{G}_{0, j \mid j}^{2 \mid 2}(\varepsilon) .
$$


Hence, we end up with Eq.(13) for the derivative of the self-energy:

$$
\partial_{\Lambda} \Sigma_{i \mid j}^{\alpha^{\prime} \mid \alpha}(\varepsilon)=\frac{1}{2 \pi i} \int d \varepsilon^{\prime} \sum_{\substack{\beta \beta^{\prime} \\ k l \in C}} S_{\Lambda, k \mid l}^{\beta \mid \beta^{\prime}}\left(\varepsilon^{\prime}\right) \mathcal{L}_{i k \mid j l}^{\alpha^{\prime} \beta^{\prime} \mid \alpha \beta}\left(\varepsilon^{\prime}, \varepsilon ; 0\right)
$$

\section{Appendix C: Charge conservation - Ward identity}

Here we derive the Ward identity, Eq.(21), from variational principles; since the action corresponding to the Hamiltonian, Eq.(1), is invariant under a global U(1) symmetry, it satisfies a conservation law. Starting from the path integral representation of expectation values using Grassmann variables, the requirement of vanishing variation under the gauged $U(1)$ transformation yields both a continuity equation for particle current and the desired connection between the interacting self-energy $\Sigma$, introduced in Eq.(A.7), and the vertex part $\Phi$, defined in Eq.(20).

Within this Appendix we combine the left and right lead to $\mathbb{L}=L \otimes R$, thus representing the Hamiltonian and the Green's function by

$$
\mathcal{H}_{0}=\left(\begin{array}{cc}
C \mathcal{H}_{0} C & C \mathcal{H}_{0} \mathbb{} \\
\mathbb{\mathcal { H } _ { 0 }} C & \mathbb{\mathcal { H } _ { 0 }} \mathbb{L}
\end{array}\right)=\left(\begin{array}{cc}
\mathcal{H}_{0, c} & \mathcal{H}_{c \ell} \\
\mathcal{H}_{\ell c} & \mathcal{H}_{\ell}
\end{array}\right), \mathcal{G}=\left(\begin{array}{cc}
C \mathcal{G} C & C \mathcal{G} \mathbb{} \\
\mathbb{L} C & \mathbb{G} \mathbb{G}
\end{array}\right)=\left(\begin{array}{cc}
\mathcal{G}_{c} & \mathcal{G}_{c \ell} \\
\mathcal{G}_{\ell c} & \mathcal{G}_{\ell}
\end{array}\right) .
$$

Let $\{\psi\},\{\bar{\psi}\}$ be sets of Grassmann variables, i.e. fermionic fields. We write $n$-particle expectation values in terms of the functional path integral,

$$
G_{\boldsymbol{i} \mid \boldsymbol{i}^{\prime}}^{n, a \mid a^{\prime}}\left(\boldsymbol{t} \mid \boldsymbol{t}^{\prime}\right)=(-i)^{n}\left\langle\psi_{i_{1}}^{a_{1}}\left(t_{1}\right) \ldots \psi_{i_{n}}^{a_{n}}\left(t_{n}\right) \bar{\psi}_{i_{n}^{\prime}}^{a_{n}^{\prime}}\left(t_{n}^{\prime}\right) \ldots \bar{\psi}_{i_{1}^{\prime}}^{a_{1}^{\prime}}\left(t_{1}^{\prime}\right)\right\rangle=(-i)^{n} \int \mathcal{D}(\overline{\boldsymbol{\psi}} \boldsymbol{\psi}) \psi_{i_{1}}^{a_{1}}\left(t_{1}\right) \ldots \psi_{i_{n}}^{a_{n}}\left(t_{n}\right) \bar{\psi}_{i_{n}^{\prime}}^{a_{n}^{\prime}}\left(t_{n}^{\prime}\right) \ldots \bar{\psi}_{i_{1}^{\prime}}^{a_{1}^{\prime}}\left(t_{1}^{\prime}\right) e^{i S[\overline{\boldsymbol{\psi}}, \boldsymbol{\psi}]}
$$

where the Keldysh action is given by the Keldysh contour time integral

$$
\begin{aligned}
S[\overline{\boldsymbol{\psi}}, \boldsymbol{\psi}] & =\int_{\mathcal{C}} d t \sum_{i i^{\prime}} \bar{\psi}_{i^{\prime}}(t) \overbrace{\left.i \delta_{i^{\prime} i} \partial_{t}-\mathcal{H}_{0, i^{\prime} i}\right)}^{\left[G_{0}(t)^{-1}\right]_{i^{\prime} i}} \psi_{i}(t)+S_{\mathrm{int}}[\overline{\boldsymbol{\psi}}, \boldsymbol{\psi}]=\int_{-\infty}^{\infty} d t \sum_{a, i i^{\prime}}(-a) \bar{\psi}_{i^{\prime}}^{a}(t)\left(i \delta_{i^{\prime} i} \partial_{t}-H_{0, i^{\prime} i}\right) \psi_{i}^{a}(t)+S_{\mathrm{int}}[\overline{\boldsymbol{\psi}}, \boldsymbol{\psi}] \\
& =\int_{-\infty}^{\infty} d t \sum_{a}(-a) \overline{\boldsymbol{\psi}}^{a}(t)\left(i \boldsymbol{\partial}_{t}-H_{0}\right) \boldsymbol{\psi}^{a}(t)+S_{\mathrm{int}}[\overline{\boldsymbol{\psi}}, \boldsymbol{\psi}] .
\end{aligned}
$$

In the last line we introduced the vector notation $\boldsymbol{\psi}=\left(\begin{array}{c}\psi_{1} \\ \psi_{2} \\ \vdots\end{array}\right)$ and $\overline{\boldsymbol{\psi}}=\left(\psi_{1}, \psi_{2}, \ldots\right)$. Note that $\boldsymbol{\partial}_{t}$ is a diagonal matrix.

\section{Gauge transformation}

The action, Eq.(C.3), is invariant under the global $U(1)$ transformation $\boldsymbol{\psi} \rightarrow \boldsymbol{\psi} e^{i \alpha}$ and $\overline{\boldsymbol{\psi}} \rightarrow \overline{\boldsymbol{\psi}} e^{-i \alpha}$, where $\alpha$ is a real constant. Gauging this transformation, i.e. making $\alpha$ space-, and time-dependent, yields to linear order in $\alpha$

$$
\delta \psi_{i}^{a}(t)=i \alpha_{i}^{a}(t) \psi_{i}^{a}(t), \delta \bar{\psi}_{i^{\prime}}^{a}\left(t^{\prime}\right)=-i \alpha_{i^{\prime}}^{a}\left(t^{\prime}\right) \bar{\psi}_{i^{\prime}}^{a}\left(t^{\prime}\right)
$$

Since we are interested in the current through the system, from one lead to another, it is convenient to pick $\alpha$ non-vanishing only in the central region:

$$
\alpha_{i}^{a}(t)= \begin{cases}\alpha^{a}(t), & \text { if } \quad i \in C \\ 0, & \text { if } \quad i \in \mathbb{L} .\end{cases}
$$

This is equivalent to first deriving the Ward identity using an arbitrary $\alpha$ and then summing over the central region. The requirement of vanishing variation under the gauged $U(1)$ transformation now reads:

$$
\delta G_{i \mid i^{\prime}}^{n, a \mid a^{\prime}}\left(\boldsymbol{t} \mid \boldsymbol{t}^{\prime}\right)=0
$$




\section{The continuity equation (zeroth order Ward identity)}

For $n=0$, Eq.(C.6) sets a condition on the variation of the partition sum. Since the measure of the path integral is invariant under the transformation in Eq.(C.4) (implying that the $U(1)$-symmetry is not anomalous), this in turn sets a condition on the variation of the action:

$$
0=\delta\left[\int \mathcal{D}(\overline{\boldsymbol{\psi}} \boldsymbol{\psi}) e^{i S[\overline{\boldsymbol{\psi}}, \boldsymbol{\psi}]}\right]=i \int \mathcal{D}(\overline{\boldsymbol{\psi}} \boldsymbol{\psi}) \delta S[\overline{\boldsymbol{\psi}}, \boldsymbol{\psi}] e^{i S[\overline{\boldsymbol{\psi}}, \boldsymbol{\psi}]}
$$

The quartic term, $S_{\text {int }}$, describes a density-density interaction. Hence, its variation vanishes trivially and the variation of the total action reduces to the variation of the quadratic term:

$$
\begin{aligned}
\delta S[\overline{\boldsymbol{\psi}}, \boldsymbol{\psi}] & =\int_{-\infty}^{\infty} d t \sum_{a, i}(-a)\left[\alpha_{i}^{a}(t) \bar{\psi}_{i}^{a}(t) \partial_{t} \psi_{i}^{a}(t)-\bar{\psi}_{i}^{a}(t) \partial_{t}\left(\alpha_{i}^{a}(t) \psi_{i}^{a}(t)\right)+\sum_{i^{\prime}}\left[i \alpha_{i^{\prime}}^{a}(t)-i \alpha_{i}^{a}(t)\right] \bar{\psi}_{i^{\prime}}^{a}(t) H_{0, i^{\prime} i} \psi_{i}^{a}(t)\right] \\
& =\int_{-\infty}^{\infty} d t \sum_{a}(-a) \alpha^{a}(t)\left[\partial_{t}\left(\overline{\boldsymbol{\psi}}_{c}^{a}(t) \boldsymbol{\psi}_{c}^{a}(t)\right)-i \overline{\boldsymbol{\psi}}_{c}^{a}(t) H_{c l} \boldsymbol{\psi}_{l}^{a}(t)+i \overline{\boldsymbol{\psi}}_{l}^{a}(t) H_{l c} \boldsymbol{\psi}_{c}^{a}(t)\right] \\
& =\int_{-\infty}^{\infty} d t \sum_{a}(-a) \alpha^{a}(t)\left[-\partial_{t}\left(\boldsymbol{\psi}_{c}^{a}(t) \overline{\boldsymbol{\psi}}_{c}^{a}(t)\right)+i \operatorname{Tr}\left\{H_{c l} \boldsymbol{\psi}_{l}^{a}(t) \overline{\boldsymbol{\psi}}_{c}^{a}(t)\right\}-i \operatorname{Tr}\left\{H_{l c} \boldsymbol{\psi}_{c}^{a}(t) \overline{\boldsymbol{\psi}}_{l}^{a}(t)\right\}\right]
\end{aligned}
$$

where we used integration by parts in the first term Since Eq.(C.7) must hold for arbitrary $\alpha(t)$ this provides the continuity equation

$$
-\partial_{t}\left\langle\boldsymbol{\psi}_{c}^{a}(t) \overline{\boldsymbol{\psi}}_{c}^{a}(t)\right\rangle=i \operatorname{Tr}\left\{H_{l c}\left\langle\boldsymbol{\psi}_{c}^{a}(t) \overline{\boldsymbol{\psi}}_{l}^{a}(t)\right\rangle\right\}-i \operatorname{Tr}\left\{H_{c l}\left\langle\boldsymbol{\psi}_{l}^{a}(t) \overline{\boldsymbol{\psi}}_{c}^{a}(t)\right\rangle\right\}
$$

In steady-state, the time derivative of the density term on the 1.h.s. vanishes and Eq.(C.9) reduces to current conservation, i.e. the current into the central region equals the current out of the central region:

$$
\operatorname{Tr}\left\{H_{l c} G_{c l}^{-1+}(0)\right\}=\operatorname{Tr}\left\{H_{c l} G_{l c}^{-1+}(0)\right\} .
$$

Here we made use of the time-translational invariance of the Green's function, Eq.(A.5), and the equivalence of the contour Green's function components for equal-time arguments $G^{-1+}(t, t)=G^{-\mid-}(t, t)=G^{+\mid+}(t, t)$.

\section{Relation between self-energy and two-particle vertex (first order Ward identity)}

For $n=1$, Eq.(C.6) reads

$$
0=\delta\left\langle\psi_{i}^{a}(t) \bar{\psi}_{i^{\prime}}^{a^{\prime}}\left(t^{\prime}\right)\right\rangle=\int \mathcal{D}(\overline{\boldsymbol{\psi}} \boldsymbol{\psi})\left[\left(\delta \psi_{i}^{a}(t)\right) \bar{\psi}_{i^{\prime}}^{a^{\prime}}\left(t^{\prime}\right)+\psi_{i}^{a}(t)\left(\delta \bar{\psi}_{i^{\prime}}^{a^{\prime}}\left(t^{\prime}\right)\right)+i \psi_{i}^{a}(t) \bar{\psi}_{i^{\prime}}^{a^{\prime}}\left(t^{\prime}\right)(\delta S[\overline{\boldsymbol{\psi}}, \boldsymbol{\psi}])\right] e^{i S[\overline{\boldsymbol{\psi}}, \boldsymbol{\psi}]}
$$

Since the r.h.s. contains both terms quadratic and quartic in $\psi$, this equation will eventually lead to a relation between the self-energy and the two-particle vertex. For states $i, i^{\prime} \in C$ Eq.(C.11) can be written as

$$
\begin{aligned}
0=\int_{\infty}^{\infty} d t^{\prime \prime} \sum_{a^{\prime \prime}}\left(-a^{\prime \prime}\right) i \alpha^{a^{\prime \prime}}\left(t^{\prime \prime}\right)\{ & \int \mathcal{D}(\overline{\boldsymbol{\psi}} \psi) \psi_{i}^{a}(t) \bar{\psi}_{i^{\prime}}^{a^{\prime}}\left(t^{\prime}\right)\left[(-a) \delta\left(t^{\prime \prime}-t\right) \delta_{a a^{\prime \prime}}+a^{\prime} \delta\left(t^{\prime \prime}-t^{\prime}\right) \delta_{a^{\prime} a^{\prime \prime}}\right. \\
& +\sum_{j \in C} \partial_{t^{\prime \prime}}\left(\bar{\psi}_{j}^{a^{\prime \prime}}\left(t^{\prime \prime}\right) \psi_{j}^{a^{\prime \prime}}\left(t^{\prime \prime}\right)\right) \\
& \left.+i \sum_{j_{1}, j_{2}}\left(\bar{\psi}_{j_{1}}^{a^{\prime \prime}}\left(t^{\prime \prime}\right) H_{\ell c, j_{1} \mid j_{2}} \psi_{j_{2}}^{a^{\prime \prime}}\left(t^{\prime \prime}\right)-\bar{\psi}_{j_{2}}^{a^{\prime \prime}}\left(t^{\prime \prime}\right) H_{c \ell, j_{2} \mid j_{1}} \psi_{j_{1}}^{a^{\prime \prime}}\left(t^{\prime \prime}\right)\right)\right] e^{i S[\overline{\boldsymbol{\psi}}, \boldsymbol{\psi}]\} .}
\end{aligned}
$$

Again, this must be true for arbitrary $\alpha(t)$, providing

$$
\begin{aligned}
& {\left[(-a) \delta\left(t^{\prime \prime}-t\right) \delta_{a a^{\prime \prime}}+a^{\prime} \delta\left(t^{\prime \prime}-t^{\prime}\right) \delta_{a^{\prime} a^{\prime \prime}}\right] G_{i \mid i^{\prime}}^{a \mid a^{\prime}}\left(t \mid t^{\prime}\right)} \\
& =\sum_{j_{1}, j_{2}}\left[H_{\ell c, j_{1} \mid j_{2}} G_{j_{2} i \mid j_{1} i^{\prime}}^{a^{\prime \prime} a \mid a^{\prime \prime} a^{\prime}}\left(t^{\prime \prime} t \mid t^{\prime \prime} t^{\prime}\right)-H_{c \ell, j_{2} \mid j_{1}} G_{j_{1} i \mid j_{2} i^{\prime}}^{a^{\prime \prime} a \mid a^{\prime \prime} a^{\prime}}\left(t^{\prime \prime} t \mid t^{\prime \prime} t^{\prime}\right)\right]-i \partial_{t^{\prime \prime}} \sum_{j \in C} G_{j i \mid j i^{\prime}}^{a^{\prime \prime} a \mid a^{\prime \prime} a^{\prime}}\left(t^{\prime \prime} t \mid t^{\prime \prime} t^{\prime}\right) .
\end{aligned}
$$


We proceed by decomposing the 2-particle Green's function in the first term of the r.h.s. according to Eq.(A.4). Since the first disconnected term, $G\left(t^{\prime \prime} \mid t^{\prime \prime}\right) G\left(t \mid t^{\prime}\right)$, vanishes due to the current conservation, Eq.(C.9), we get

$$
\begin{aligned}
& {\left[(-a) \delta\left(t^{\prime \prime}-t\right) \delta_{a a^{\prime \prime}}+a^{\prime} \delta\left(t^{\prime \prime}-t^{\prime}\right) \delta_{a^{\prime} a^{\prime \prime}}\right] G_{i \mid i^{\prime}}^{a \mid a^{\prime}}\left(t \mid t^{\prime}\right)} \\
& =-\sum_{j_{1}, j_{2}}\left[G_{i \mid j_{1}}^{a \mid a^{\prime \prime}}\left(t \mid t^{\prime \prime}\right) H_{\ell c, j_{1} \mid j_{2}} G_{j_{2} \mid i^{\prime}}^{a^{\prime \prime} \mid a^{\prime}}\left(t^{\prime \prime} \mid t^{\prime}\right)-G_{i \mid j_{2}}^{a \mid a^{\prime \prime}}\left(t \mid t^{\prime \prime}\right) H_{c \ell, j_{2} \mid j_{1}} G_{j_{1} \mid i^{\prime}}^{a^{\prime \prime} \mid a^{\prime}}\left(t^{\prime \prime} \mid t^{\prime}\right)\right]-i \partial_{t^{\prime \prime}} \sum_{j \in C} G_{j i \mid j i^{\prime}}^{a^{\prime \prime} a \mid a^{\prime \prime} a^{\prime}}\left(t^{\prime \prime} t \mid t^{\prime \prime} t^{\prime}\right) \\
& -i \sum_{j_{1}, j_{2}} \sum_{\boldsymbol{k}, b} \int d \tau G_{i \mid k_{2}^{\prime}}^{a \mid b_{2}^{\prime}}\left(t \mid \tau_{2}^{\prime}\right)\left[G_{k_{1} \mid j_{1}}^{b_{1} \mid a^{\prime \prime}}\left(\tau_{1} \mid t^{\prime \prime}\right) H_{\ell c, j_{1} \mid j_{2}} G_{j_{2} \mid k_{1}^{\prime}}^{a^{\prime \prime} \mid b^{\prime}}\left(t^{\prime \prime} \mid \tau_{1}^{\prime}\right)-\left(j_{1} \leftrightarrow j_{2}, H_{\ell c} \leftrightarrow H_{c \ell}\right)\right] \gamma_{k_{1}^{\prime} k_{2}^{\prime} \mid k_{1} k_{2}}^{b_{\prime}^{\prime} b_{2}^{\prime} \mid b_{1} b_{2}}\left(\tau_{1}^{\prime}, \tau_{2}^{\prime} \mid \tau_{1}, \tau_{2}\right) G_{k_{2} \mid i^{\prime}}^{b_{2} \mid a^{\prime}}\left(\tau_{2} \mid t^{\prime}\right) .
\end{aligned}
$$

We find the corresponding relation in frequency domain after Fourier transformation w.r.t. all time arguments $t, t^{\prime}, t^{\prime \prime}$,

$$
\begin{aligned}
& (-a) \delta_{a a^{\prime \prime}} \mathcal{G}_{i \mid i^{\prime}}^{a \mid a^{\prime}}(\varepsilon+\omega)+a^{\prime} \delta_{a^{\prime} a^{\prime \prime}} \mathcal{G}_{i \mid i^{\prime}}^{a \mid a^{\prime}}(\varepsilon) \\
& =-\sum_{j_{1}, j_{2}}\left[\mathcal{G}_{i \mid j_{1}}^{a \mid a^{\prime \prime}}(\varepsilon+\omega) H_{\ell c, j_{1} \mid j_{2}} \mathcal{G}_{j_{2} \mid i^{\prime}}^{a^{\prime \prime} \mid a^{\prime}}(\varepsilon)-\left(j_{1} \leftrightarrow j_{2}, H_{\ell c} \leftrightarrow H_{c \ell}\right)\right]-\frac{\omega}{2 \pi} \int d \varepsilon^{\prime} \sum_{j \in C} \mathcal{G}_{j i \mid j i^{\prime}}^{a^{\prime \prime} a \mid a^{\prime \prime} a^{\prime}}\left(\varepsilon, \varepsilon^{\prime} ; \omega\right) . \\
& -\frac{i}{2 \pi} \sum_{\substack{\boldsymbol{k}, \boldsymbol{b} \\
j_{1}, j_{2}}} \mathcal{G}_{i \mid k_{2}^{\prime}}^{a \mid b_{2}^{\prime}}(\varepsilon)\left\{\int d \varepsilon^{\prime}\left[\mathcal{G}_{k_{1} \mid j_{1}}^{b_{1} \mid a^{\prime \prime}}\left(\varepsilon^{\prime}\right) H_{\ell c, j_{1} \mid j_{2}} \mathcal{G}_{j_{2} \mid k_{1}^{\prime}}^{a^{\prime \prime} \mid b_{1}^{\prime}}\left(\varepsilon^{\prime}+\omega\right)-\left(j_{1} \leftrightarrow j_{2}, H_{\ell c} \leftrightarrow H_{c \ell}\right)\right] \mathcal{L}_{k_{1}^{\prime} k_{2}^{\prime} \mid k_{1} k_{2}}^{b_{1}^{\prime} b_{2}^{\prime} \mid b_{1} b_{2}}\left(\varepsilon, \varepsilon^{\prime} ; \omega\right)\right\} \mathcal{G}_{k_{2} \mid i^{\prime}}^{b_{2} \mid a^{\prime}}(\varepsilon+\omega) .
\end{aligned}
$$

We set $\omega=0$ and sum over $a^{\prime \prime}$ on both sides to get the matrix equation

$$
\sum_{a^{\prime \prime}}\left[(-a) \delta_{a a^{\prime \prime}}+a^{\prime} \delta_{a^{\prime} a^{\prime \prime}}\right] \mathcal{G}_{c}^{a \mid a^{\prime}}(\varepsilon)=Y^{a \mid a^{\prime}}(\varepsilon)
$$

where we defined the response object

$$
\begin{aligned}
Y_{i \mid i^{\prime}}^{a \mid a^{\prime}}(\varepsilon)= & -\sum_{a^{\prime \prime}} \sum_{j_{1}, j_{2}}\left[\mathcal{G}_{i \mid j_{1}}^{a \mid a^{\prime \prime}}(\varepsilon) H_{\ell c, j_{1} \mid j_{2}} \mathcal{G}_{j_{2} \mid i^{\prime}}^{a^{\prime \prime} \mid a^{\prime}}(\varepsilon)-\left(j_{1} \leftrightarrow j_{2}, H_{\ell c} \leftrightarrow H_{c \ell}\right)\right] \\
& -\frac{i}{2 \pi} \sum_{a^{\prime \prime}} \sum_{\substack{k, b \\
j_{1}, j_{2}}} \mathcal{G}_{i \mid k_{2}^{\prime}}^{a \mid b_{2}^{\prime}}(\varepsilon)\left\{\int d \varepsilon^{\prime}\left[\mathcal{G}_{k_{1} \mid j_{1}}^{b_{1} \mid a^{\prime \prime}}\left(\varepsilon^{\prime}\right) H_{\ell c, j_{1} \mid j_{2}} \mathcal{G}_{j_{2} \mid k_{1}^{\prime \prime}}^{a^{\prime \prime} \mid b^{\prime}}\left(\varepsilon^{\prime}\right)-\left(j_{1} \leftrightarrow j_{2}, H_{\ell c} \leftrightarrow H_{c \ell}\right)\right] \mathcal{L}_{k_{1}^{\prime} k_{2}^{\prime} \mid k_{1} k_{2}}^{b_{1}^{\prime} b_{2}^{\prime} \mid b_{1} b_{2}}\left(\varepsilon, \varepsilon^{\prime} ; 0\right)\right\} \mathcal{G}_{k_{2} \mid i^{\prime}}^{b_{2} \mid a^{\prime}}(\varepsilon) .
\end{aligned}
$$

With two independent contour arguments, $a$ and $a^{\prime}$, Eq.(C.16) results in four independent contour space relations

$$
0=Y^{+\mid+}=Y^{-1-},-2 \mathcal{G}_{c}^{+1-}=Y^{+\mid-}, 2 \mathcal{G}_{c}^{-1+}=Y^{-1+} .
$$

Adding up all equations and transforming into Keldysh space [Eq.(A.10)] yields

$$
\begin{aligned}
2\left(\mathcal{G}_{c}^{+\mid-}-\mathcal{G}_{c}^{-\mid+}\right) & =Y^{+\mid+}+Y^{-\mid-}-Y^{+\mid-}-Y^{-\mid+} \\
\text {Eq.(A.10) } \quad \mathcal{G}_{c}^{2 \mid 1}-\mathcal{G}_{c}^{1 \mid 2} & =Y^{1 \mid 1} .
\end{aligned}
$$

As a consequence of the theorem of causality [Eq.(A.12)] we have $\mathcal{G}^{1 \mid 1}=0$. Hence, only the summand with $a^{\prime \prime}=2$ in $Y^{1 \mid 1}$ is non-zero:

$$
Y^{1 \mid 1}(\varepsilon)=b^{1 \mid 1}(\varepsilon)-i \mathcal{G}_{c}^{1 \mid 2}(\varepsilon) \tilde{\Phi}(\varepsilon) \mathcal{G}_{c}^{2 \mid 1}(\varepsilon)
$$

where we defined the coupling term

$$
\begin{aligned}
b^{\alpha \mid \alpha^{\prime}} & =\mathcal{G}_{c}^{\alpha \mid 2} H_{c \ell} \mathcal{G}_{\ell c}^{2 \mid \alpha^{\prime}}-\mathcal{G}_{c \ell}^{\alpha \mid 2} H_{\ell c} \mathcal{G}_{c}^{2 \mid \alpha^{\prime}} \\
& \stackrel{\text { Eq.(A. }{ }^{26)}}{=} \mathcal{G}_{c}^{\alpha \mid 2} H_{c \ell} \sum_{\beta, \gamma} g_{\ell}^{2 \mid \beta} \mathcal{H}_{\ell c}^{\beta \mid \gamma} \mathcal{G}_{c}^{\gamma \mid \alpha^{\prime}}-\sum_{\beta, \gamma} \mathcal{G}_{c}^{\alpha \mid \beta} \mathcal{H}_{c \ell}^{\beta \mid \gamma} g_{\ell}^{\gamma \mid 2} H_{\ell c} \mathcal{G}_{c}^{2 \mid \alpha^{\prime}},
\end{aligned}
$$

and the response function

$$
\tilde{\Phi}_{k_{2}^{\prime} \mid k_{2}}(\varepsilon)=\frac{1}{2 \pi} \int d \varepsilon^{\prime} \sum_{\substack{b_{1}, b_{1}^{\prime} \\ k_{1}, k_{1}^{\prime}}} b_{k_{1} \mid k_{1}^{\prime}}^{b_{1} \mid b_{1}^{\prime}}\left(\varepsilon^{\prime}\right) \mathcal{L}_{k_{1}^{\prime} k_{2}^{\prime} \mid k_{1} k_{2}}^{b_{1}^{\prime} 2 \mid b_{1} 2}\left(\varepsilon, \varepsilon^{\prime} ; 0\right)
$$


Using the hybridization, $\Gamma=i H_{c \ell}\left(g_{\ell}^{2 \mid 1}-g_{\ell}^{1 \mid 2}\right) H_{\ell c}$, we find

$$
b^{1 \mid 1}=-i \mathcal{G}_{c}^{1 \mid 2} \Gamma \mathcal{G}_{c}^{2 \mid 1}, b^{1 \mid 2}=-b^{2 \mid 1}=(1-2 f) b^{1 \mid 1} .
$$

Hence, the response function reads (since $\gamma^{22 \mid 22}=0$ )

$$
\begin{aligned}
\tilde{\Phi}_{k_{2}^{\prime} \mid k_{2}}(\varepsilon)=\frac{1}{2 \pi i} \int d \varepsilon^{\prime} & \sum_{\substack{j_{1}, j_{1}^{\prime} \\
k_{1}, k_{1}^{\prime}}} \mathcal{G}_{k_{1} \mid j_{1}^{\prime}}^{1 \mid 2}\left(\varepsilon^{\prime}\right) \Gamma_{j_{1}^{\prime} \mid j_{1}}\left(\varepsilon^{\prime}\right) \mathcal{G}_{j_{1} \mid k_{1}^{\prime}}^{2 \mid 1}\left(\varepsilon^{\prime}\right) \\
& \times\left[\mathcal{L}_{k_{1}^{\prime} k_{2}^{\prime} \mid k_{1} k_{2}}^{12 \mid 12}\left(\varepsilon, \varepsilon^{\prime} ; 0\right)-\left(1-2 f\left(\varepsilon^{\prime}\right)\right)\left(\mathcal{L}_{k_{1}^{\prime} k_{2}^{\prime} \mid k_{1} k_{2}}^{12 \mid 22}\left(\varepsilon, \varepsilon^{\prime} ; 0\right)-\mathcal{L}_{k_{1}^{\prime} k_{2}^{\prime} \mid k_{1} k_{2}}^{22 \mid 12}\left(\varepsilon, \varepsilon^{\prime} ; 0\right)\right)\right],
\end{aligned}
$$

in accord with Eq.(20). Finally, we multiply $\left[\mathcal{G}^{1 \mid 2}\right]^{-1}$ from the left and $\left[\mathcal{G}^{2 \mid 1}\right]^{-1}$ from the right in Eq.(C.19), which provides

$$
\left[\mathcal{G}^{1 \mid 2}(\varepsilon)\right]^{-1}-\left[\mathcal{G}^{2 \mid 1}(\varepsilon)\right]^{-1}=-i[\Gamma(\varepsilon)+\tilde{\Phi}(\varepsilon)]
$$

Inserting Eq.(A.25) and using $\Sigma_{\text {lead }}^{1 \mid 2}(\varepsilon)-\Sigma_{\text {lead }}^{2 \mid 1}(\varepsilon)=-i \Gamma(\varepsilon)$ [see Eq.(A.21)] the hybridization terms cancel and we recover Eq.(21) (note that we combined the left and right lead, which implies $\tilde{\Phi}=\tilde{\Phi}^{l}+\tilde{\Phi}^{r}$ ):

$$
i\left[\Sigma^{1 \mid 2}(\varepsilon)-\Sigma^{2 \mid 1}(\varepsilon)\right]=\tilde{\Phi}(\varepsilon) .
$$

This equation is a necessary condition that any method for describing the influence of interactions has to satisfy in order to produce quantitative reliable results for transport properties of the system. If Eq.(C.26), and therefore particle conservation, is violated by a chosen approach (such as e.g. truncated fRG schemes) one should exercise great caution in interpreting the results.

\section{Appendix D: Derivation of the fluctuation-dissipation theorem for the vertex channels and the self-energy}

Below, we derive the fluctuation-dissipation theorem in SOPT for both the frequency-dependent vertex channels, Eq.(40d) and Eq.(41d), and the self-energy, Eq.(48d).

\section{FDT for the П-channel}

We use the FDT for the bare Green's function, Eq.(A.17), to write the Keldysh Green's function in terms of the difference between the retarded and advanced Green's function. With that we can write the Keldysh component of the ПI-channel as

$$
\begin{aligned}
\Pi_{i j}^{1 \mid 1}(p) & =-\frac{u_{i} u_{j}}{2 \pi i} \int d \varepsilon\left[\mathcal{G}_{0, i \mid j}^{\sigma, 2 \mid 2}(p-\varepsilon) \mathcal{G}_{0, i \mid j}^{\bar{\sigma}, 2 \mid 2}(\varepsilon)+\mathcal{G}_{0, i \mid j}^{\sigma, 2 \mid 1}(p-\varepsilon) \mathcal{G}_{0, i \mid j}^{\bar{\sigma}, 2 \mid 1}(\varepsilon)+\mathcal{G}_{0, i \mid j}^{\sigma, 1 \mid 2}(p-\varepsilon) \mathcal{G}_{0, i \mid j}^{\bar{\sigma}, 1 \mid 2}(\varepsilon)\right] \\
& =-\frac{u_{i} u_{j}}{\pi i} \int d \varepsilon[1-f(\varepsilon)-f(p-\varepsilon)+2 f(\varepsilon) f(p-\varepsilon)]\left(\mathcal{G}_{0, i \mid j}^{\sigma, 2 \mid 1}(\varepsilon)-\mathcal{G}_{0, i \mid j}^{\sigma, 1 \mid 2}(\varepsilon)\right)\left(\mathcal{G}_{0, i \mid j}^{\bar{\sigma}, 2 \mid 1}(p-\varepsilon)-\mathcal{G}_{0, i \mid j}^{\bar{\sigma}, 1 \mid 2}(p-\varepsilon)\right),
\end{aligned}
$$

where we added zeros $\int d \varepsilon \mathcal{G}_{0}^{2 \mid 1}(\varepsilon) \mathcal{G}_{0}^{1 \mid 2}(p-\varepsilon)=\int d \varepsilon \mathcal{G}_{0}^{1 \mid 2}(\varepsilon) \mathcal{G}_{0}^{2 \mid 1}(p-\varepsilon)=0$. We then use the relation

$$
2 f(\varepsilon) f(p-\varepsilon)=2 b(p-\mu)[1-f(p-\varepsilon)-f(\varepsilon)],
$$

which yields

$$
\begin{aligned}
\Pi_{i j}^{1 \mid 1}(p) & =-\frac{u_{i} u_{j}}{\pi i}[1+2 b(p-\mu)] \int d \varepsilon[1-f(\varepsilon)-f(p-\varepsilon)]\left(\mathcal{G}_{0, i \mid j}^{\sigma, 2 \mid 1}(\varepsilon)-\mathcal{G}_{0, i \mid j}^{\sigma, 1 \mid 2}(\varepsilon)\right)\left(\mathcal{G}_{0, i \mid j}^{\bar{\sigma}, 2 \mid 1}(p-\varepsilon)-\mathcal{G}_{0, i \mid j}^{\bar{\sigma}, 1 \mid 2}(p-\varepsilon)\right) \\
& =[1+2 b(p-\mu)]\left[\Pi_{i j}^{1 \mid 2}(p)-\Pi_{i j}^{2 \mid 1}(p)\right] .
\end{aligned}
$$

This proves Eq.(40d). 


\section{FDT for the $X$-channel}

A similar calculation as above shows the FDT for the $x$-channel:

$$
\begin{aligned}
X_{i j}^{\sigma \sigma^{\prime}, 1 \mid 1}(x) & =-\frac{u_{i} u_{j}}{2 \pi i} \int d \varepsilon\left[\mathcal{G}_{0, i \mid j}^{\bar{\sigma}, 2 \mid 2}(\varepsilon) \mathcal{G}_{0, i \mid j}^{\bar{\sigma}^{\prime}, 2 \mid 2}(\varepsilon+x)+\mathcal{G}_{0, i \mid j}^{\bar{\sigma}, 2 \mid 1}(\varepsilon) \mathcal{G}_{0, i \mid j}^{\bar{\sigma}^{\prime}, 1 \mid 2}(\varepsilon+x)+\mathcal{G}_{0, i \mid j}^{\bar{\sigma}, 1 \mid 2}(\varepsilon) \mathcal{G}_{0, i \mid j}^{\bar{\sigma}^{\prime}, 2 \mid 1}(\varepsilon+x)\right] \\
& =-\frac{u_{i} u_{j}}{\pi i} \int d \varepsilon[f(\varepsilon)-f(\varepsilon+x)-2 f(\varepsilon) f(-\varepsilon-x+2 \mu)]\left(\mathcal{G}_{0, i \mid j}^{\bar{\sigma}, 2 \mid 1}(\varepsilon)-\mathcal{G}_{0, i \mid j}^{\bar{\sigma}, 1 \mid 2}(\varepsilon)\right)\left(\mathcal{G}_{0, i \mid j}^{\bar{\sigma}^{\prime}, 2 \mid 1}(\varepsilon+x)-\mathcal{G}_{0, i \mid j}^{\bar{\sigma}^{\prime}, 1 \mid 2}(\varepsilon+x)\right) \\
& =-\frac{u_{i} u_{j}}{\pi i}[1+2 b(x+\mu)] \int d \varepsilon[f(\varepsilon+x)-f(\varepsilon)]\left(\mathcal{G}_{0, i \mid j}^{\bar{\sigma}, 2 \mid 1}(\varepsilon)-\mathcal{G}_{0, i \mid j}^{\bar{\sigma}, 1 \mid 2}(\varepsilon)\right)\left(\mathcal{G}_{0, i \mid j}^{\bar{\sigma}^{\prime}, 2 \mid 1}(\varepsilon+x)-\mathcal{G}_{0, i \mid j}^{\bar{\sigma}^{\prime}, 1 \mid 2}(\varepsilon+x)\right) \\
& =[1+2 b(x+\mu)]\left[X_{i j}^{\sigma \sigma^{\prime}, 1 \mid 2}(x)-X_{i j}^{\sigma \sigma^{\prime}, 2 \mid 1}(x)\right] .
\end{aligned}
$$

\section{FDT for the self-energy}

Finally we show the FDT for the self-energy: Using the FDT for both the $X$-channel of the vertex as well as of the bare Green's function, we can rewrite the Keldysh component of the self-energy:

$$
\begin{aligned}
\Sigma_{2, i \mid j}^{\sigma, 1 \mid 1}(\varepsilon) & =-\frac{1}{2 \pi i} \int d \varepsilon^{\prime}\left[\mathcal{G}_{0, i \mid j}^{\sigma, 2 \mid 2}\left(\varepsilon^{\prime}\right) X_{i j}^{\sigma \sigma, 1 \mid 1}\left(\varepsilon-\varepsilon^{\prime}\right)+\mathcal{G}_{0, i \mid j}^{\sigma, 2 \mid 1}\left(\varepsilon^{\prime}\right) X_{i j}^{\sigma \sigma, 1 \mid 2}\left(\varepsilon-\varepsilon^{\prime}\right)+\mathcal{G}_{0, i \mid j}^{\sigma, 1 \mid 2}\left(\varepsilon^{\prime}\right) X_{i j}^{\sigma \sigma, 2 \mid 1}\left(\varepsilon-\varepsilon^{\prime}\right)\right] \\
& =-\frac{1}{2 \pi i} \int d \varepsilon^{\prime}\left(\left[1-2 f\left(\varepsilon^{\prime}\right)\right]\left[1+2 b\left(\varepsilon-\varepsilon^{\prime}+\mu\right)\right]+1\right)\left(\mathcal{G}_{0, i \mid j}^{\sigma, 21}\left(\varepsilon^{\prime}\right)-\mathcal{G}_{0, i \mid j}^{\sigma, 12}\left(\varepsilon^{\prime}\right)\right)\left(X_{i j}^{\sigma \sigma, 1 \mid 2}\left(\varepsilon-\varepsilon^{\prime}\right)-X_{i j}^{\sigma \sigma, 2 \mid 1}\left(\varepsilon-\varepsilon^{\prime}\right)\right) \\
& =-\frac{1}{2 \pi i}[1-2 f(\varepsilon)] \int d \varepsilon^{\prime}\left[2-2 f\left(\varepsilon^{\prime}\right)+2 b\left(\varepsilon-\varepsilon^{\prime}+\mu\right)\right]\left(\mathcal{G}_{0, i \mid j}^{\sigma, 21}\left(\varepsilon^{\prime}\right)-\mathcal{G}_{0, i \mid j}^{\sigma, 12}\left(\varepsilon^{\prime}\right)\right)\left(X_{i j}^{\sigma, 1 \mid 2}\left(\varepsilon-\varepsilon^{\prime}\right)-X_{i j}^{\sigma \sigma, 2 \mid 1}\left(\varepsilon-\varepsilon^{\prime}\right)\right) \\
& .=[1-2 f(\varepsilon)]\left[\Sigma_{2, i \mid j}^{\sigma, 1 \mid 2}(\varepsilon)-\Sigma_{2, i \mid j}^{\sigma, 2 \mid 1}(\varepsilon)\right]
\end{aligned}
$$

Here we added zeros, $\int d \varepsilon^{\prime} \mathcal{G}_{0}^{2 \mid 1}\left(\varepsilon^{\prime}\right) X^{2 \mid 1}\left(\varepsilon-\varepsilon^{\prime}\right)=\int d \varepsilon^{\prime} \mathcal{G}_{0}^{1 \mid 2}\left(\varepsilon^{\prime}\right) X^{1 \mid 2}\left(\varepsilon-\varepsilon^{\prime}\right)=0$, to get to the second line. Furthermore we used the relation

$$
b\left(\varepsilon-\varepsilon^{\prime}+\mu\right)\left[f(\varepsilon)-f\left(\varepsilon^{\prime}\right)\right]=-f(\varepsilon) f\left(-\varepsilon^{\prime}+2 \mu\right)=-f(\varepsilon)+f(\varepsilon) f\left(\varepsilon^{\prime}\right)
$$

\section{Appendix E: Method of finite differences for non-uniform grid}

Our goal in this appendix is to derive a discrete description of a continuous system having the Hamiltonian $\mathcal{H}(x)=\hbar^{2} /(2 m) \partial_{x}^{2}+V(x)$. While the standard precedure usually involves discretization via a grid with constant spacing, we focus on the more general case, where the spacing is non-constant. This bypasses, for a proper choice of non-monotonic discretization, the occurence of artificial bound states close to the upper band edge, which are a consequence of the inhomogeneity $V(x)$.

We discretize real space using a set of grid points $\left\{x_{j}\right\}$ (see Fig.(3)). The distance between two successive points is given by $a_{j}=x_{j+1}-x_{j}$. Now, a function $\psi(x)$ and its first and second derivatives $\psi^{\prime}(x)$ and $\psi^{\prime \prime}(x)$ are discretized as

$$
\begin{gathered}
\psi_{j}=\psi\left(x_{j}\right), \\
\psi_{j+1 / 2}^{\prime}=\frac{\psi\left(x_{j+1}\right)-\psi\left(x_{j}\right)}{a_{j}},
\end{gathered}
$$

$$
\begin{aligned}
\psi_{j}^{\prime \prime} & =\frac{\psi_{j+1 / 2}^{\prime}-\psi_{j-1 / 2}^{\prime}}{\frac{a_{j}+a_{j-1}}{2}}=2 \frac{\frac{\psi_{j+1}-\psi_{j}}{a_{j}}-\frac{\psi_{j}-\psi_{j-1}}{a_{j-1}}}{a_{j}+a_{j-1}} \\
& \simeq \frac{1}{a_{j-1}^{2}} \psi_{j-1}-\left(\frac{1}{a_{j-1}^{2}}+\frac{1}{a_{j}^{2}}\right) \psi_{j}+\frac{1}{a_{j}^{2}} \psi_{j+1},
\end{aligned}
$$

where we demanded that the spacing changes smoothly

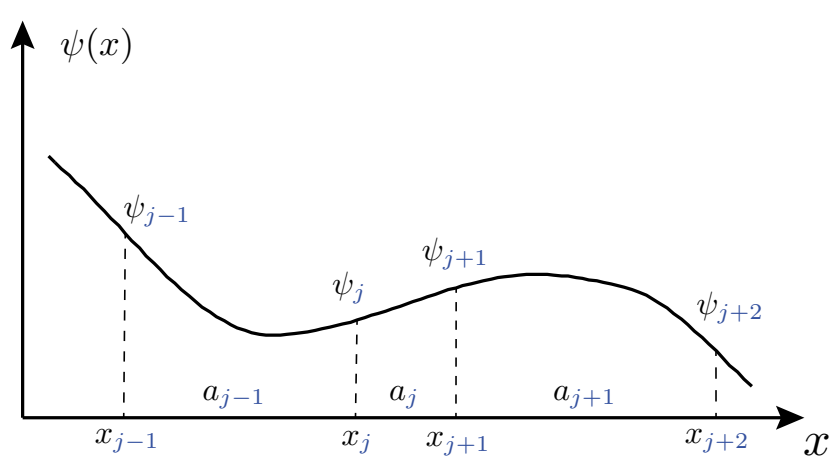

Figure 3. Illustration of the choice of notation used to discretize real space. 
as a function of $j$, implying $\left(a_{j}+a_{j-1}\right) a_{j} \simeq 2 a_{j}^{2}$ and $\left(a_{j}+a_{j-1}\right) a_{j-1} \simeq 2 a_{j-1}^{2}$. Note that the first derivative is defined 'in between' grid points.

Hence, the discretized version of the Hamiltonian $H(x)=-\frac{\hbar^{2}}{2 m} \partial_{x}^{2}+V(x)$ at a point $x_{j}$ is given by

$$
H \psi_{j}=-\tau_{j-1} \psi_{j-1}-\tau_{j} \psi_{j+1}+E_{j} \psi_{j}
$$

1 R. Landauer. Spatial Variation of Currents and Fields Due to Localized Scatterers in Metallic Conduction. IBM Journal of Research and Development 1, 223-231

${ }^{2}$ Y. Meir \& N. S. Wingreen. Landauer formula for the current through an interacting electron region. Phys. Rev. Lett. 68, 2512-2515 (1992).

3 J. Eckel, F. Heidrich-Meisner, S. G. Jakobs, M. Thorwart, M. Pletyukhov, and R. Egger, New J. Phys. 12, 043042 (2010).

${ }^{4}$ S. Andergassen, V. Meden, H. Schoeller, J. Splettstoesser, and M. R. Wegewijs, Nanotechnology 21, 272001 (2010).

${ }^{5}$ F. Bauer, J. Heyder, E. Schubert, D. Borowsky, D. Taubert, B. Bruognolo, D. Schuh, W. Wegscheider, J. von Delft, and S. Ludwig, Nature 501, 73 (2013).

${ }^{6}$ A. Oguri, Transmission Probability for Interacting Electrons Connected to Reservoirs. J. Phys. Soc. Jap. 70, 2666 (2001).

7 G.M.Eliashberg, Transport equation for a degenerate system of fermi particles, JETP41,1241-1251 (1962)

${ }^{8}$ L.V.Keldysh. Zh. Eksp. Teor. Fiz. 47, 1515 (1964).

9 A.Kamenev, A. Levchenko. arXiv: 0901.3586

10 J.C. Ward, Phys. Rev. 78,182 (1950)

11 S. G. Jakobs et al. Properties of multi-particle Green's and vertex functions within Keldysh formalism J. Phys. with site-dependent hopping $\tau_{j}=1 /\left(2 m a_{j}^{2}\right)$ (here and below we set $\hbar=1$ ) and the onsite-energy $E_{j}=\tau_{j-1}+$ $\tau_{j}+V_{j}$.
A: Math. Theor. 43,103001 (2010)

12 S. G. Jakobs, M. Pletyukhov, H.Schoeller, Nonequilibrium functional renormalization group with frequencydependent vertex function: A study of the single-impurity Anderson model Phys. Rev. B 81,195109 (2010)

13 Walter Metzner, Manfred Salmhofer, Carsten Honerkamp, Volker Meden, and Kurt Schnhammer. Functional renormalization group approach to correlated fermion systems. Rev. Mod. Phys., 84,299-352

14 O.Goulko, F.Bauer, J.Heyder, J. von Delft, Effect of SpinOrbit Interactions on the 0.7 Anomaly in Quantum Point Contacts, Phys. Rev. Lett., 113, 266402 (2014)

15 S.Jakobs, Functional renormalization group studies of quantum transport through mesoscopic systems, Dissertation

${ }_{16}$ M. Buettiker, Phys. Rev. B41, 7906 (1990).

17 C. Sloggett, A. I. Milstein, \& O. P. Sushkov, Correlated electron current and temperature dependence of the conductance of a quantum point contact. Eur. Phys. J. B 61, 427432 (2008).

18 F.Bauer, J.Heyder, J.von Delft, Functional Renormalization Group Approach for Inhomogeneous Interacting Fermi-Systems arXiv:1311.3210 


\subsection{Problems of the Matsubara Formalism}

In the last section we derived a conductance formula which requires retarded and advanced Green's and vertex function on the real frequency axis. The vertex functions calculated using the approach described in section 3.3 are given numerically on a finite subset of Matsubara frequencies, i.e. purely imaginary frequencies. Performing an analytic continuation of the vertex functions from the imaginary axis to the real axis numerically is an ill-posed problem (see e.g. Jarrell and Gubernatis, 1996): If the number of frequencies for which the vertex functions are given is finite, as is the case in numerics, the number of analytic functions intersecting the given points is infinite.

\subsubsection{Zero Temperature}

Fortunately the analytic continuation is only necessary at finite temperature. At zero temperature the derivative of the Fermi functions is a $\delta$-function:

$$
\lim _{T \rightarrow 0} f^{\prime}(\omega)=-\delta(\omega) .
$$

As a consequence the Green's and vertex functions only need to be evaluated at $\omega= \pm i 0^{+}$ which at zero temperature are fermionic Matsubara frequencies. Further the vertex correction $P$ and the self energy terms vanishes at zero frequency:

$$
\Sigma^{R}(0)-\Sigma^{A}(0)=P^{s}(0)=0 \quad(T=0) .
$$

So the conductance can be written as

$$
G=\operatorname{Tr} \Gamma^{L} \mathcal{G}\left(i 0^{+}\right) \Gamma^{R} \mathcal{G}\left(-i 0^{+}\right)
$$

\subsubsection{Finite Temperature}

At finite temperature one needs to perform the analytic continuation to the real axis. Although the problem is ill-posed a wide range of different methods exist which approach it. For scalar functions the most commonly used technique is the maximum entropy method (MEM) (Silver et al., 1990; Gubernatis et al., 1991). In the context of the fRG, where the numerical data has no statistical error, one usually represents the function using continuous fractions, also known as Padé approximation (Vidberg and Serene, 1977; Karrasch et al., 2008). Karrasch et al. (2010) made additional use of the proportional coupling $\Gamma^{L} \propto \Gamma^{R}$ given for the single impurity Anderson model (SIAM). In this case the conductance can be expressed in terms of single particle Green's functions (Meir and Wingreen, 1992; Oguri, 2001).

In principle it is possible to extend all these approaches to matrix valued functions given by the fRG equations described in section 3.3. However, it turns out that the result using continued fraction is very unstable, especially for the two particle vertex. Since this then results in an uncontrolled error in the conductance we stopped pursuing this approach. Despite these problems Aryanpour and Han (2009) chose this approach for Monte Carlo data and they claim that it works. 
We decided to follow the direct approach, i.e. to calculate the vertex functions directly on the real axis. The first step in this direction was to calculate them using SOPT. This approach is explained in detail in section 4.2. A brief summary can also be fond in section S-7 in the supplementary information of our NATURE article (chapter 5, Bauer et al., 2013). Higher order correlations can be included by using fRG in Keldysh formalism. This in principle is possible following Jakobs et al. (2010), and is the topic of ongoing research in our group. 


\section{Chapter 5}

\section{Microscopic origin of the 0.7 anomaly - Publication}

In section 2.4 we gave an intuitive picture for the origin of the 0.7 anomaly in QPCs. In this chapter we now set this intuitive picture on solid ground by corroborating everything with detailed theoretical calculations using fRG and SOPT introduced in chapter 3 and 4 and comparing the results with experimental data made in the group of Stefan Ludwig. The detailed explanations of the microscopic mechanism has been published in NATURE (Bauer et al., 2013). The publication consists of two parts: The main article containing a summarized version of the most important aspects, and extensive supplementary information containing detaild explanations and additional data. The supplementary information also contains a summary of the fRG approach and SOPT, which can be skipped by those who have read chapter 3 and 4 . Below we include a reprint of the publication including the supplementary information. 


\title{
Microscopic origin of the ' 0.7 -anomaly' in quantum point contacts
}

\author{
Florian Bauer $^{1,2 *}$, Jan Heyder ${ }^{1,2 *}$, Enrico Schubert ${ }^{1}$, David Borowsky ${ }^{1}$, Daniela Taubert ${ }^{1}$, Benedikt Bruognolo ${ }^{1,2}$, Dieter Schuh $^{3}$,
} Werner Wegscheider ${ }^{4}$, Jan von Delft ${ }^{1,2} \&$ Stefan Ludwig ${ }^{1}$

Quantum point contacts are narrow, one-dimensional constrictions usually patterned in a two-dimensional electron system, for example by applying voltages to local gates. The linear conductance of a point contact, when measured as function of its channel width, is quantized ${ }^{1-3}$ in units of $G_{Q}=2 e^{2} / h$, where $e$ is the electron charge and $h$ is Planck's constant. However, the conductance also has an unexpected shoulder at $\sim 0.7 G_{\mathrm{Q}}$, known as the ' 0.7 -anomaly ${ }^{\prime-12}$, whose origin is still subject to debate $\mathrm{e}^{11-21}$. Proposed theoretical explanations have invoked spontaneous spin polarization ${ }^{4,17}$, ferromagnetic spin coupling ${ }^{19}$, the formation of a quasi-bound state leading to the Kondo effect ${ }^{13,14}$, Wigner crystallization ${ }^{16,20}$ and various treatments of inelastic scattering ${ }^{18,21}$. However, explicit calculations that fully reproduce the various experimental observations in the regime of the 0.7 -anomaly, including the zero-bias peak that typically accompanies it ${ }^{6,9-11}$, are still lacking. Here we offer a detailed microscopic explanation for both the 0.7 -anomaly and the zero-bias peak: their common origin is a smeared van Hove singularity in the local density of states at the bottom of the lowest one-dimensional subband of the point contact, which causes an anomalous enhancement in the Hartree potential barrier, the magnetic spin susceptibility and the inelastic scattering rate. We find good qualitative agreement between theoretical calculations and experimental results on the dependence of the conductance on gate voltage, magnetic field, temperature, source-drain voltage (including the zero-bias peak) and interaction strength. We also clarify how the low-energy scale governing the 0.7 -anomaly depends on gate voltage and interactions. For low energies, we predict and observe Fermi-liquid behaviour similar to that associated with the Kondo effect in quantum dots ${ }^{22}$. At high energies, however, the similarities between the 0.7 -anomaly and the Kondo effect end.

In our measurements, we use the multigate layout on the surface of a $\mathrm{GaAs} / \mathrm{AlGaAs}$ heterostructure shown in Fig. 1a. By suitably tuning the central- and side-gate voltages, $V_{\mathrm{c}}$ and $V_{\mathrm{s}}$, at a fixed top-gate voltage, $V_{\mathrm{t}}$, we can use the device to define a short, one-dimensional (1D) channel, containing a smooth, symmetric barrier, in the two-dimensional electron system (2DES) buried in the heterostructure. To describe such a quantum point contact (QPC), we adopt a $1 \mathrm{D}$ model with local interactions and a smooth potential barrier. We treat interactions perturbatively, using either second-order perturbation theory ${ }^{23}$ (SOPT) or the functional renormalization group ${ }^{24-26}$ (FRG) approach (Supplementary Information, sections 7 and 6, respectively). The lowest 1D subband of the device is modelled by

$$
\hat{H}=\sum_{j \sigma}\left[E_{j \sigma} \hat{n}_{j \sigma}-\tau_{j}\left(d_{j+1}^{\dagger} d_{j \sigma}+\text { h.c. }\right)\right]+\sum_{j} U_{j} \hat{n}_{j \uparrow} \hat{n}_{j \downarrow}
$$

Here $\hat{n}_{j \sigma}=d_{j \sigma}^{\dagger} d_{j \sigma}$ counts the number of electrons with spin $\sigma$ (spin up, $\sigma=\uparrow$ or + ; spin down, $\sigma=\downarrow$ or - ) at site $j$ of an infinite, tight-binding chain with hopping amplitude $\tau_{j}$, on-site interaction $U_{j}$ and potential energy $E_{j \sigma}=E_{j}-\sigma \tilde{B} / 2$ (Supplementary Fig. 8), and 'h.c.' denotes Hermitian conjugate. The Zeeman energy, $\tilde{B}=\left|g_{\mathrm{el}}\right| \mu_{\mathrm{B}} B$, describes the effect of a uniform external parallel magnetic field $B$, where $\mu_{\mathrm{B}}$ is the Bohr magneton and $g_{\text {el }}$ is the effective $g$ factor $(<0$ in GaAs). (When similar symbols are used for model parameters and experimental parameters, we add tildes to the former to distinguish them from the latter.) We neglect spin-orbit interactions and other orbital effects. The parameters $E_{j}, U_{j}$ and $\tau_{j}$ vary smoothly with $j$ and differ from their bulk values, $E_{\text {bulk }}=U_{\text {bulk }}=0$ and $\tau_{\text {bulk }}=\tau$ (taken as the unit of energy), only within a central constriction region (CCR) of $N$ sites around $j=0$, representing the QPC. Sites $j<-N / 2$ and $j>N / 2$ represent two non-interacting leads, each with bandwidth $4 \tau$, chemical potential $\mu$ and bulk Fermi energy $\varepsilon_{\mathrm{F}}=2 \tau+\mu$; we choose $\mu=0$, implying halffilled leads (Fig. 1b). We set $U_{j}$ to a fixed value, $U$, for all but the outermost sites of the CCR, where it drops smoothly to zero.

Within the CCR, we define the QPC barrier by specifying the shape of the 'band bottom' as $\omega_{j}^{\min }=E_{j}-\left(\tau_{j-1}+\tau_{j}\right)-\mu$ (Fig. 1b, solid black line). We choose $\omega_{j}^{\text {min }}$ to define a smooth, symmetric barrier within the CCR, parabolic near the top ${ }^{3}$, where we parameterize it as $\omega_{j}^{\min } \approx \tilde{V}_{\mathrm{c}}-\Omega_{x}^{2} j^{2} / 4 \tau_{0}$ (Supplementary Information, section 4D). Here $\tilde{V}_{\mathrm{c}}$ sets the barrier height with respect to $\mu$ (Fig. 1b, dashed black line), and $\Omega_{x} \ll \tau$ characterizes its curvature. We first consider the theoretical case of zero temperature, $\tilde{T}=k_{\mathrm{B}} T\left(k_{\mathrm{B}}\right.$, Boltzmann's constant), sourcedrain voltage, $\tilde{V}_{\text {sd }}=|e| V_{\text {sd }}$, and field, $\tilde{B}$ : $\tilde{T}=\tilde{V}_{\text {sd }}=\tilde{B}=0$. As $\tilde{V}_{\text {c }}$ is decreased below 0 , the conductance, $g=G / G_{\mathrm{Q}}$, increases from 0 to 1 , showing a step of width $\sim \Omega_{x}$ (about $1.5 \mathrm{meV}$ in our experiment), whose shape depends on $U$ (Fig. 1k). In the upper part of the step, say $0.5 \lesssim g \lesssim 0.9$, we say that the QPC is 'sub-open'; the sub-open regime is of special interest because for measured $g\left(V_{\mathrm{c}}\right)$ curves it contains the 0.7 -anomaly.

The bare local density of states (LDOS), $A_{j}^{0}(\omega)$, for equation (1) has a strong maximum just above the band bottom $^{18}$, seen as a yellow-red ridge-like structure in Fig. 1b. In a semiclassical picture, $A_{j}^{0}(\omega) \propto 1 / v_{j}(\omega)$, where $v_{j}(\omega)$ is the velocity at site $j$ of an electron with energy $\omega$ with respect to $\mu$. The ridge-like maximum of $A_{j}^{0}(\omega)$ above the barrier reflects the fact that electrons move slowest there. In the CCR's outer flanks, this ridge develops smoothly into the van Hove singularity, $A_{\text {bulk }}^{0} \propto\left[\left(\omega-\omega_{\text {bulk }}^{\text {min }}\right) \tau\right]^{-1 / 2}$, in the bulk LDOS at the bulk band bottom in the leads, $\omega_{\text {bulk }}^{\min }=-\varepsilon_{\mathrm{F}}$. We therefore call this LDOS structure a 'van Hove ridge'. Near the barrier's centre, its curvature causes the singularity to be smeared out on a scale set by $\Omega_{x}$. This limits the amplitude of the van Hove ridge to $\max \left[A_{j}^{0}(\omega)\right] \propto \mathcal{O}\left(\Omega_{x} \tau_{0}\right)^{-1 / 2}$ and shifts it upwards in frequency relative to the band by $\mathcal{O}\left(\Omega_{x}\right)$ (Fig. 1f-h).

The van Hove ridge has a strong, $\tilde{V}_{\mathrm{c}}$-dependent effect on numerous QPC properties. Near those spatial locations where the ridge intersects the chemical potential $(\omega=0)$, the LDOS is enhanced, thus amplifying the effects of interactions by $\mathcal{O}\left(\Omega_{x} \tau_{0}\right)^{-1 / 2}$ (which grows with QPC 


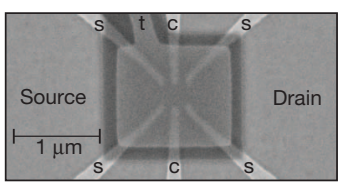

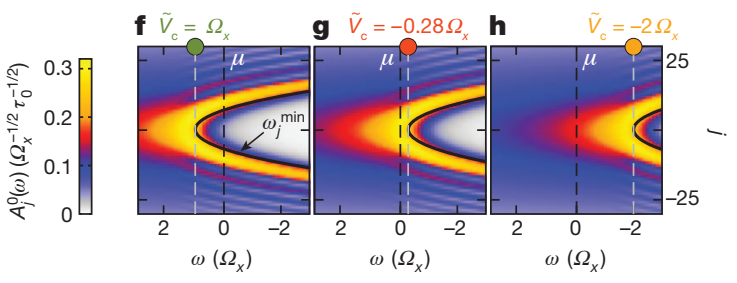

$\chi_{j}\left(\Omega_{x}^{-1 / 2} \tau_{0}^{-1 / 2}\right)$

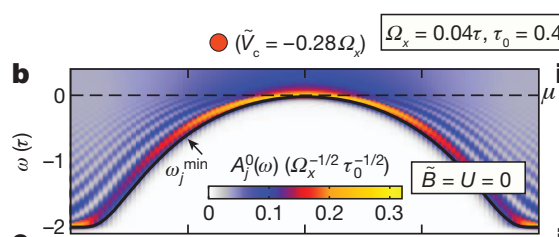

c

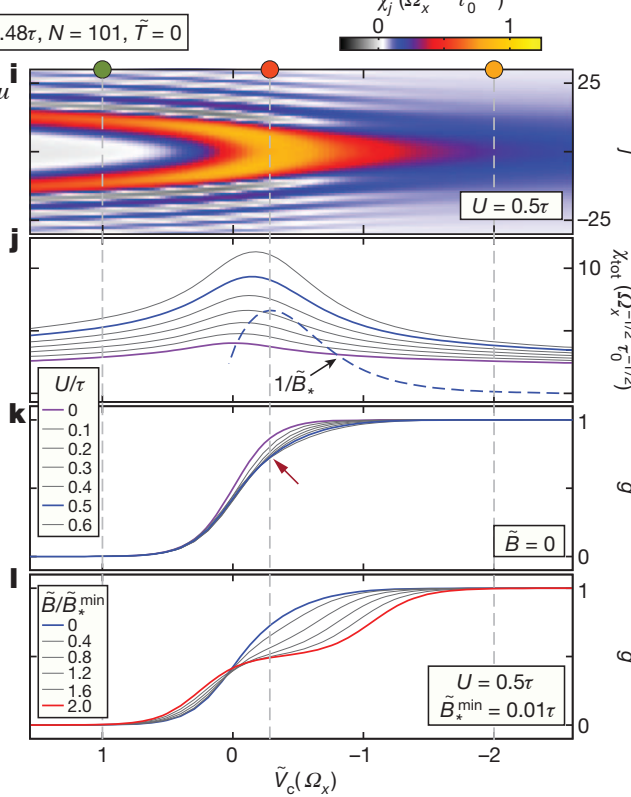

Figure $1 \mid$ Experimental set-up and model. a, Scanning electron microscope picture of the gate layout, featuring a top gate $(\mathrm{t})$ at voltage $V_{\mathrm{t}}$, two central gates (c) at voltage $V_{\mathrm{c}}$ and four side gates (s) at voltage $V_{\mathrm{s}}$. Negative voltages $V_{\mathrm{c}}$ and $V_{\mathrm{s}}$ locally deplete the 2DES, which is $85 \mathrm{~nm}$ beneath the sample surface. Together with $V_{\mathrm{t}}$, they induce a tunable electrostatic potential landscape in the 2DES. b, Barrier shape and LDOS. The bare $\left(U_{j}=0, \tilde{B}=0\right) 1 \mathrm{D}$ LDOS per spin species, $A_{j}^{0}(\omega)$ (colour scale), as a function of energy, $\omega$, and site index, $j$, for $\tilde{V}_{\mathrm{c}}=-0.28 \Omega_{x}$. The barrier shape is defined by the solid black line, showing the band bottom, $\omega_{j}^{\min }$. The LDOS vanishes exponentially rapidly below $\omega_{j}^{\min }$ (Supplementary Fig. 11), and has a van Hove ridge (yellow-red) just above it, followed by Friedel oscillations (white fringes) at higher energies (up to $\omega \lesssim \tilde{V}_{\mathrm{c}}$ ). c-e, Local properties of a sub-open QPC: FRG results for the subopen barrier shown in b. c, d, The local density, $n_{j}(\mathbf{c})$, and the magnetization, $m_{j}$ (d), for several values of magnetic field, $\tilde{B}$. Inset of $\mathbf{d}, m_{\Sigma}=\sum_{|j| \leq 10} m_{j}$ as a function of $\tilde{B}$. e, The local spin susceptibility, $\chi_{j}$, for several values of interaction

strength, $U$. The shapes of $m_{j}$ and $\chi_{j}$ are modulated by Friedel oscillations inherited from the bare LDOS (b), with locally varying wavelength, $\lambda \approx 1 / n_{j}$. $\mathbf{f}-\mathbf{l}$, Changing barrier height. $\mathbf{f}-\mathbf{h}$, The bare LDOS, $A_{j}^{0}(\omega)$, for three successively lower barrier heights, $\tilde{V}_{\mathrm{c}} / \Omega_{x}=1(\mathbf{f}),-0.28(\mathbf{g})$ and $-2(\mathbf{h})$. The LDOS pattern is fixed with respect to $\tilde{V}_{c}$ (grey dashed lines) but shifts with respect to $\mu$ (black dashed lines). i-l, FRG results for the $\tilde{V}_{c}$ dependence of the local spin susceptibility, $\chi_{j}$ (colour scale), at fixed $U=0.5 \tau(\mathbf{i})$; the total spin susceptibility, $\chi_{\text {tot }}=\sum_{j}^{C C R} \chi_{j}$, for several $U$ values (solid lines), and the inverse low-energy scale, $1 / \tilde{B}_{*}$, for $U=0.5 \tau$ (dashed line) (j); the zero-temperature linear-response $\left(V_{\text {sd }}=0\right)$ conductance, $g=G / G_{\mathrm{Q}}$, for several $U$ values (at fixed $\left.\tilde{B}=0\right)(\mathbf{k})$ and for several $\tilde{B}$ values (at fixed $U=0.5 \tau)(\mathbf{l})$. For a large enough interaction,

$U=0.5 \tau$, even for $\tilde{B}=\tilde{T}=\tilde{V}_{\text {sd }}=0$ (blue lines in $\mathbf{k}$ and $\mathbf{l}$ ), $g\left(\tilde{V}_{\mathrm{c}}\right)$ has a shoulder (red arrow) at $g \approx 0.7$, the 0.7 -anomaly. Three vertical dashed lines in $\mathbf{i}-\mathbf{l}$ mark the three $\tilde{V}_{\mathrm{c}}$ values used in $\mathbf{f}-\mathbf{h}$, as indicated by dots of matching colours.

length). In semiclassical terms, slow electrons feel interactions particularly strongly. When lowering the barrier top, $\tilde{V}_{c}$, to open the QPC, the van Hove ridge sweeps downwards (Fig. 1f-h); its interactionamplifying effects are strongest in the $\tilde{V}_{\mathrm{c}}$ regime where its apex, which has most weight, crosses $\mu$. This happens for $0 \gtrsim \tilde{V}_{\mathrm{c}} \gtrsim-\mathcal{O}\left(\Omega_{x}\right)$ (Fig. 1g), which, very importantly, encompasses the sub-open regime containing the 0.7 -anomaly. Below, we show that the 0.7 -anomaly and the zero-bias peak (ZBP) stem precisely from the amplification of interaction effects where the van Hove ridge intersects $\mu$. The relevant implications are enhancements in the effective Hartree barrier governing elastic transmission, the spin susceptibility and the inelastic scattering rate, all of which lead to an anomalous reduction of $g$ in the sub-open regime, especially for $T, B, V_{\text {sd }}>0$.

Figure 1c-e illustrates several local properties, calculated at $\tilde{T}=0$ using FRG, for the sub-open QPC barrier shown in Fig. 1b. We note four salient features, all intuitively expected. First, the local density, $n_{j}=\left\langle\hat{n}_{j \uparrow}+\hat{n}_{j \downarrow}\right\rangle$, is minimal at the barrier centre (Fig. 1c). Second, the local magnetization, $m_{j}=\left\langle\hat{n}_{j \uparrow}-\hat{n}_{j \downarrow}\right\rangle / 2$, vanishes at $\tilde{B}=0$ (Fig. 1d, blue line); this reflects a physical assumption entailed in our calculations (Supplementary Information, section 6), namely that no spontaneous

magnetization occurs, in contrast to the spontaneous spin splitting scenario advocated in refs $4,8,17$. Third, $m_{j}$ increases without saturation when $\tilde{B}$ becomes large (Fig. 1d, inset), indicating a smooth redistribution of spin, as expected for an open structure. Fourth, the local spin susceptibility, $\chi_{j}=\left(\partial m_{j} / \partial \tilde{B}\right)_{\tilde{B}=0}$, is strongly enhanced with increasing $U$ (Fig. 1e), because interactions amplify any field-induced spin imbalance.

The $j$ dependence of $\chi_{j}$ is governed by that of $A_{j}^{0}(0)$ (in fact, $\left.\chi_{j}^{U=0}=A_{j}^{0}(0) / 2\right)$, which is maximal near those sites where the van Hove ridge intersects $\mu$. When $\tilde{V}_{\mathrm{c}}$ is decreased through 0 (Figs 1f-h), these intersection points sweep out a parabolic arch in the $\tilde{V}_{c}-j$ plane, along which $\chi_{j}\left(\tilde{V}_{\mathrm{c}}\right)$ (Fig. 1i, colour scale) is peaked, with most weight near the arch's apex. This leads to a corresponding peak in the total spin susceptibility, $\chi_{\text {tot }}=\sum_{j}^{\mathrm{CCR}} \chi_{j}$, as a function of $\tilde{V}_{\mathrm{c}}$ (Fig. $1 \mathrm{j}$ ). This peak is strongly enhanced by increasing $U$ (in accordance with the fourth feature above) and is located near the $\tilde{V}_{\mathrm{c}}$ value where $\mathrm{g} \approx 0.7$ (Fig. $1 \mathrm{k}$ ). We will see further below that this peak strongly affects the $\tilde{B}$ dependence of the conductance (Fig. 11).

Note that the spatial structure for $\chi_{j}\left(\tilde{V}_{c}\right)$ in Fig. 1i, namely two peaks merging into one as $\tilde{V}_{\mathrm{c}}$ is lowered, is consistent with that, shown 
in fig. $2 \mathrm{~b}$ of ref. 14 , for the density of spin-up electrons calculated using spin-density-functional theory, initialized in a small applied field to break spin symmetry. In ref. 14, the local maximum in the spin-up density was interpreted as evidence for a 'quasi-bound state' that was argued to host a spin-1/2 local moment; in contrast, features one and, especially, three above imply that our model yields no local moment.

Next we discuss the effect of the van Hove ridge on the conductance, $g\left(\tilde{V}_{\mathrm{c}}\right)$, starting with its $U$ dependence at $\tilde{B}=\tilde{T}=0$ (Fig. $1 \mathrm{k}$ ). Increasing $U$ skews the shape of the step in $g\left(\tilde{V}_{\mathrm{c}}\right)$, which eventually develops a shoulder near $g \approx 0.7$ (red arrow). This shoulder develops because the increase in local density with decreasing $\tilde{V}_{\mathrm{c}}$ is slightly nonlinear when the apex of the van Hove ridge drops past $\mu$, causing a corresponding nonlinear upward shift in the effective Hartree barrier. For a parabolic barrier top, this occurs for $g \approx 0.7$. If the shape of the barrier top is changed to be non-parabolic, both the shape of the bare conductance step and the energy distance between the van Hove ridge apex and $\mu$ will change, which can cause the interaction-induced shoulder in $g$ to shift away from 0.7. This explains the experimentally observed spread $^{6,12}$ of shoulders (that is, plateau values of the 0.7 -anomaly) for $0.5 \lesssim g \lesssim 1$.

On increasing $\tilde{B}$ for fixed $U$ and $\tilde{T}=0$ (Figs 11 and $2 \mathrm{a}$ ), the shoulder in $g\left(\tilde{V}_{\mathrm{c}}\right)$ becomes more pronounced, eventually developing into a spin-split plateau. Comparison of Fig. 2a with Fig. 2e shows that this development qualitatively agrees with experiment; the agreement was optimized by using $U$ as fit parameter. Inspecting how the corresponding spin-resolved conductances, $g_{\uparrow}$ and $g_{\downarrow}$, change with $\tilde{B}$ (Fig. 2b), we note a strong asymmetry: although the bare barrier heights for spins $\uparrow$ and $\downarrow$ are shifted symmetrically by $-\tilde{B} / 2$ and $\tilde{B} / 2$, respectively, $g_{\downarrow}$ is decreased much more strongly than $g_{\uparrow}$ is increased. This is due to exchange interactions: increasing the spin-up density near the CCR centre (Fig. 1d) strongly raises the Hartree barrier, and more so for spin-down electrons than spin-up, owing to Pauli's exclusion principle. The consequences are most pronounced in the sub-open regime, owing to the van-Hove-ridgeinduced peak in $\chi_{\text {tot }}$ there (Fig. 1j). We note, however, that $g_{\uparrow}=g_{\downarrow}$ at $\tilde{B}=0$, reflecting our above-mentioned assumption that no spontaneous spin splitting occurs.

Our FRG approach is limited to the case of zero temperature and zero source-drain voltage, for which no inelastic scattering occurs. To access qualitatively the effects of the latter at fixed $U$, we have instead used SOPT (Supplementary Information, section 7). Figure 2c-h shows a comparison of our SOPT results for the linear conductance, $g\left(\tilde{V}_{\mathrm{c}}\right)$, calculated for several values of magnetic field, $\tilde{B}$, and temperature, $\tilde{T}=k_{\mathrm{B}} T$, and our experimental data for $g\left(V_{\mathrm{c}}\right)$. The measured conductance step shows a shoulder (Fig. 2e, f, red arrows) that becomes increasingly more pronounced with both increasing field, $B$ (Fig. 2e), and increasing temperature, $T$ (Fig. 2f), which is the hallmark of the 0.7 -anomaly. Our perturbative calculations qualitatively reproduce both trends remarkably well. The only caveat is that the experimental curves in Fig. 2e, f show more pronounced shoulders than do the respective SOPT curves in Fig. 2c, d. This failure of SOPT to
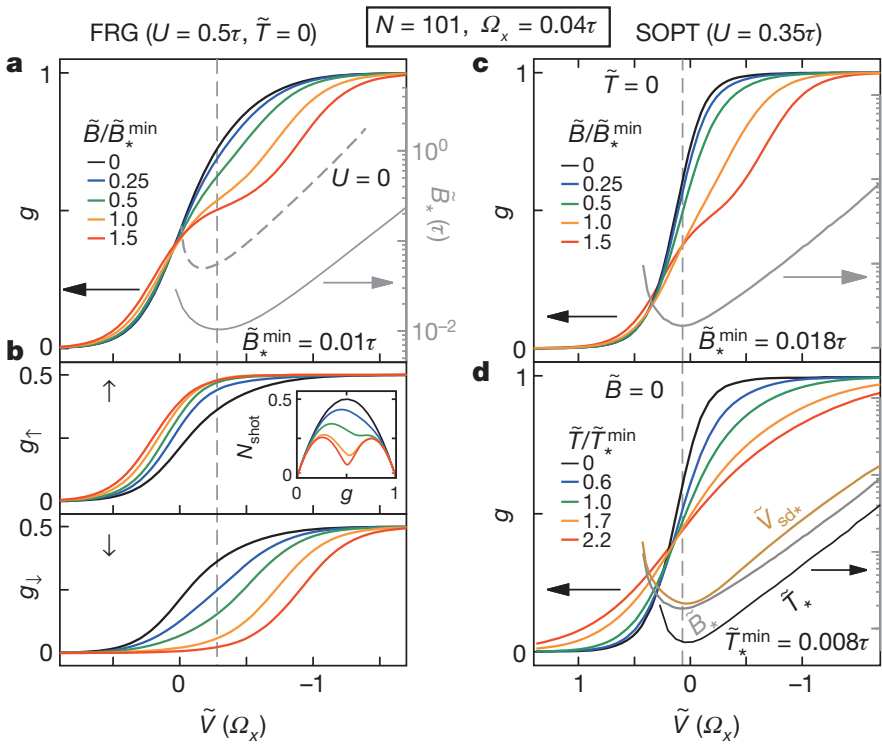

Figure $2 \mid$ Conductance: theory versus experiment. a, b, FRG results: the linear response conductance, $g\left(\tilde{V}_{\mathrm{c}}, \tilde{B}\right)$, of a QPC $(\mathbf{a})$, and its spin-resolved components, $g_{\uparrow}$ and $g_{\downarrow}(\mathbf{b})$, plotted as functions of $\tilde{V}_{\mathrm{c}} / \Omega_{x}$ for several values of $\tilde{B}$ at $T=0$ (but finite interaction $U$ ). The grey dashed and solid lines in a show the low-energy scale $\tilde{B}_{*}\left(\tilde{V}_{c}\right)$ for $U=0$ and $U=0.5$, respectively, plotted on the loglinear scale indicated on the right-hand axis (as also done in $\mathbf{c - f}$ ). The smallfield magnetoresponse in a is strongest when $\tilde{B}_{*}$ takes its smallest value, $\tilde{B}_{*}^{\min }$ (vertical dashed lines). Inset of $\mathbf{b}$, the shot noise factor,

$N_{\text {shot }}=\sum_{\sigma} g_{\sigma}\left(1-g_{\sigma}\right) / 2$, plotted as function of $g$. Its asymmetric development with $\tilde{B}$, which reflects that of $g_{\uparrow}$ and $g_{\downarrow}$, agrees qualitatively with experiment (see fig. $4 \mathrm{~d}$ of ref. 7). c, d, SOPT results: $g\left(\tilde{V}_{\mathrm{c}}, \tilde{B}\right)$ at $\tilde{T}=0$ for several values of $\tilde{B}$ (c) and $g\left(\tilde{V}_{\mathrm{c}}, \tilde{T}\right)$ at $\tilde{B}=0$ for several values of $\tilde{T}(\mathbf{d})$, both plotted as functions of $\tilde{V}_{\mathrm{c}} / \Omega_{x}$. The low-energy scale $\tilde{B}_{*}\left(\tilde{V}_{\mathrm{c}}\right)$ is shown as a thin grey line in $\mathbf{c}$ and repeated in d; $\tilde{T}_{*}\left(\tilde{V}_{\mathrm{c}}\right)$ and $\tilde{V}_{\mathrm{sd} *}\left(\tilde{V}_{\mathrm{c}}\right)$ are respectively shown as thin black and brown lines in $\mathbf{d}$. The vertical dashed line indicates where $\tilde{B}_{*}$ takes its minimal value, $\tilde{B}_{*}^{\min }$. For $\tilde{V}_{\mathrm{c}}$ values below this dashed line, the lines for $\tilde{B}_{*}, \tilde{T}_{*}$ and $\tilde{V}_{\mathrm{sd}}$ in $\mathbf{d}$ are nearly straight on the log-linear scale, implying the behaviour summarized by equation (3), and are nearly parallel to each other, implying that the ratios $\tilde{B}_{*} / \tilde{T}_{*}$ and $\tilde{V}_{\mathrm{sd} *} / \tilde{T}_{*}$ are essentially independent of $\tilde{V}_{\mathrm{c}}$ there. e, f, Experiments-pinch-off curves. e, $g\left(V_{c}\right)$ measured at a low 2DES

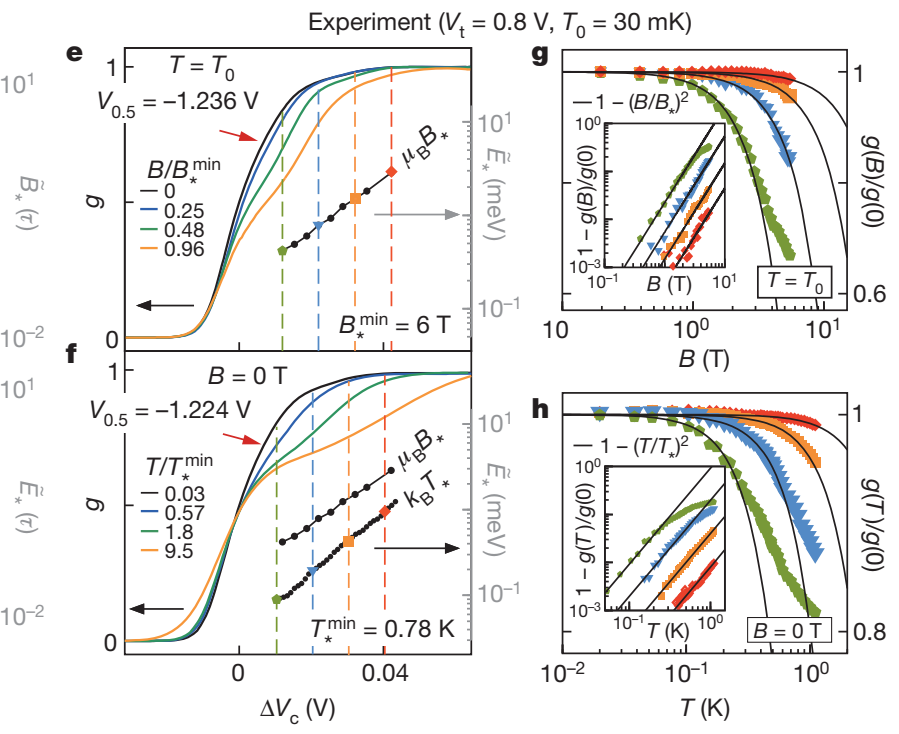

temperature, $T_{0}$, for various magnetic fields parallel to the 2DES, plotted as a function of $\Delta V_{c}=V_{c}-V_{0.5}$, where $V_{0.5}$ is the gate voltage for which the conductance at $B=0$ and $T=T_{0}$ is $g\left(V_{0.5}\right)=0.5$. f, Analogous to e, but for $B=0$ and various temperatures $T$. Colours in $\mathbf{e}$ and $\mathbf{f}$ are chosen to provide comparability with theory curves in $\mathbf{a}, \mathbf{c}$ and $\mathbf{d}$ (with the correspondence $\left.|e| \Delta V_{\mathrm{c}} \propto-\tilde{V}_{\mathrm{c}}\right)$. g, h, Experiments-Fermi-liquid behaviour: $g(B) / g(0)$ as function of $B$ at temperature $T_{0}(\mathbf{g})$, and $g(T) / g\left(T_{0}\right)$ as function of $T$ at $B=0$ (h), shown on log-linear scales (insets show their differences from unity on log$\log$ scales) to emphasize small values of $B$ and $T$. Coloured symbols distinguish data taken at different fixed $V_{\mathrm{c}}$ values, indicated by dashed lines of corresponding colour in $\mathbf{e}$ and $\mathbf{f}$. The quadratic $B$ and $T$ dependences observed in $\mathbf{g}$ and $\mathbf{h}$ for each fixed $V_{\mathrm{c}}$ value confirm equation (2) and were used to determine the corresponding scales $B_{*}\left(V_{\mathrm{c}}\right)$ and $T_{*}\left(V_{\mathrm{c}}\right)$. (Black lines in $\mathbf{g}$ and h show $1-\left(B / B_{*}\right)^{2}$ and $1-\left(T / T_{*}\right)^{2}$, respectively.) The resulting energies, $E_{*}=\mu_{\mathrm{B}} B_{*}\left(\mathrm{~V}_{\mathrm{c}}\right)$ and $E_{*}=k_{\mathrm{B}} T_{*}\left(V_{\mathrm{c}}\right)$, are shown as functions of $V_{\mathrm{c}}$ in e (for $B_{*}$ ) and $\mathbf{f}$ (for both $B_{*}$ and $T_{*}$ ) on a log-linear scale. The shape of these measured functions agrees qualitatively with the SOPT predictions in $\mathbf{c}$ and $\mathbf{d}$, confirming the nearly exponential $\tilde{V}_{\mathrm{c}}$ dependences and the nearly $V_{\mathrm{c}}$-independent $B_{*} / T_{*}$ ratio, discussed above. (For additional data, similar to that in $\mathbf{g}$ and $\mathbf{h}$, see Supplementary Information, section 2B.) 
produce real shoulders is present both in the low-field dependence at low temperature (compare Fig. 2e with Fig. 2c; the former, but not the latter, shows a weak shoulder even at zero field) and in the temperature dependence at zero field (compare Fig. $2 \mathrm{f}$ and Fig. $2 \mathrm{~d}$ ). In contrast, the more powerful FRG approach does reproduce the weak shoulder even for $\tilde{B}=\tilde{T}=0$, as discussed above; compare the black $g\left(\tilde{V}_{\mathrm{c}}\right)$ curves in Fig. 2a (FRG) and Fig. 2c (SOPT). (That the latter curve, in contrast to the former, lies above its non-interacting version, $g^{0}\left(\tilde{V}_{\mathrm{c}}\right)$, is an artefact of SOPT; see Supplementary Information, section $7 \mathrm{D}$.)

We next focus on the limit of small energies $\tilde{B}, \tilde{T}$ and $\tilde{V}_{\text {sd }}$. Here our SOPT calculations yield three predictions, enumerated below, that are all consistent with our measurements. First, for fixed $\tilde{V}_{c}$, the leading dependence of the nonlinear conductance, $g_{\mathrm{nl}}=\left(\mathrm{d} I / \mathrm{d} \tilde{V}_{\mathrm{sd}}\right) / G_{\mathrm{Q}}$, on $\tilde{B}$, $\tilde{T}$ and $\tilde{V}_{\text {sd }}$ is predicted to be quadratic, as confirmed by the measured data in Figs 2g, $\mathrm{h}$ and 3a. This implies an expansion of the form

$$
\frac{g_{\mathrm{nl}}\left(\tilde{B}, \tilde{T}, \tilde{V}_{\mathrm{sd}}\right)}{g_{\mathrm{nl}}(0,0,0)} \approx 1-\frac{\tilde{B}^{2}}{\tilde{B}_{*}^{2}}-\frac{\tilde{T}^{2}}{\tilde{T}_{*}^{2}}-\frac{\tilde{V}_{\mathrm{sd}}^{2}}{\tilde{V}_{\mathrm{sd} *}^{2}}
$$

for $\tilde{B} / \tilde{B}_{*}, \tilde{T} / \tilde{T}_{*}, \tilde{V}_{\text {sd }} / \tilde{V}_{\text {sd } *} \ll 1$, where $\tilde{B}_{*}, \tilde{T}_{*}$ and $\tilde{V}_{\text {sd } *}$ are $\tilde{V}_{\text {c }}$-dependent crossover scales that govern the 'strength' of the 0.7 -anomaly for $U \neq 0$ : the smaller these scales, the stronger the dependence on $\tilde{B}, \tilde{T}$ and $\tilde{V}_{\text {sd }}$ for a given $\tilde{V}_{\mathrm{c}}$. Our SOPT results for these crossover scales are shown as thin lines on log-linear scales in Fig. $2 c$ and Fig. 2 d, respectively. Second, in that part of the sub-open regime where $g_{\text {nl }}(0,0,0) \approx 1$, they all depend exponentially on $\tilde{V}_{c}$ :

$$
\tilde{B}_{*}, \tilde{T}_{*}, \tilde{V}_{\mathrm{sd} *} \propto \exp \left(-\pi \tilde{V}_{\mathrm{c}} / \Omega_{x}\right)
$$

Third, and again for $g_{\mathrm{nl}}(0,0,0) \approx 1$, the ratios $\tilde{B}_{*} / \tilde{T}_{*}$ and $\tilde{V}_{\mathrm{sd} *} / \tilde{T}_{*}$ are essentially independent of $\tilde{V}_{c}$ (Supplementary Fig. 4). Remarkably, both the second and third predictions are confirmed by our experimental results (Fig. 2e for $B *$, Fig. 2f for $T *$ and Supplementary Fig. 3 for

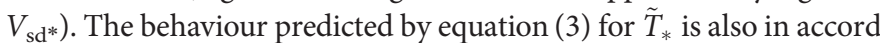
with previous experiments ${ }^{6}$ and with a perturbative treatment of interactions using Wentzel-Kramers-Brillouin wavefunctions ${ }^{21}$. Remarkably, the exponential $\tilde{V}_{\mathrm{c}}$ dependence of the crossover scales stated in equation (3) can be understood from a non-interacting $(U=0)$ theory, by using the bare transmission probability ${ }^{3}$

$$
T_{\sigma}^{0}(\omega)=\left[\mathrm{e}^{-2 \pi\left(\omega-\tilde{V}_{\mathrm{c}}+\sigma \tilde{B} / 2\right) / \Omega_{x}}+1\right]^{-1}
$$

in the Landauer-Büttiker formula. A detailed analysis (Supplementary Information, section 5) shows that the crossover scales experience a further exponential reduction with increasing effective interaction strength, $U / \sqrt{\Omega_{x} \tau_{0}}$.

When plotted as a function of $\tilde{V}_{c}, 1 / \tilde{B}_{*}$ has a peak in the sub-open regime just before the onset of the exponential dependence of equation (3) (Fig. 1j). This peak is roughly similar in shape and position to that in $\chi_{\text {tot }}\left(\tilde{V}_{c}\right)$ (compare dashed and solid blue lines in Fig. 1j), except that the latter has a finite offset, reflecting the non-zero spin susceptibility of an open QPC. Thus, we predict, fourth, that $1 / \tilde{B}_{*}$, which characterizes the strength of the low-field magnetoconductance, is roughly proportional to the spin susceptibility, $\chi_{\text {tot }}$, of the CCR.

Next we address the remarkable experimental fact ${ }^{6}$ that many lowenergy properties of the 0.7 -anomaly (including our first and third predictions) are similar to those seen in transport through a Kondo quantum dot (KQD). This led to the proposal ${ }^{13,14}$ that a QPC harbours
Experiment

a

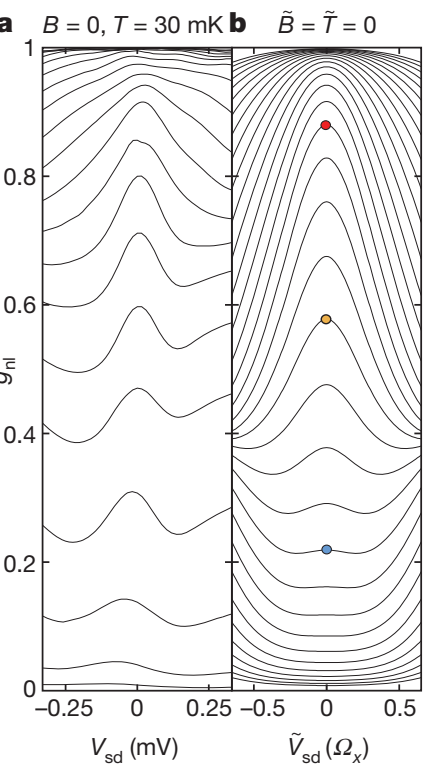

c
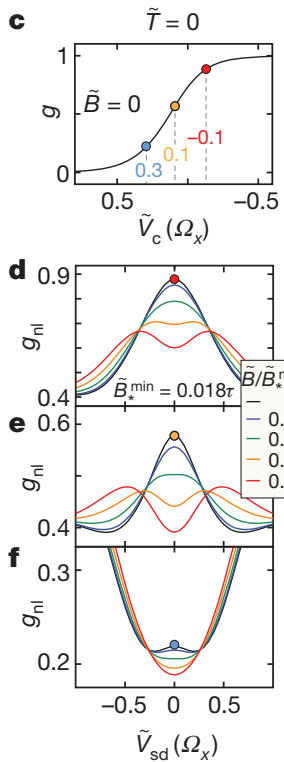

$\operatorname{SOPT}\left(U=0.35 \tau, N=101, \Omega_{x}=0.04 \tau, B=0, \tau_{0}=0.48 \tau\right)$
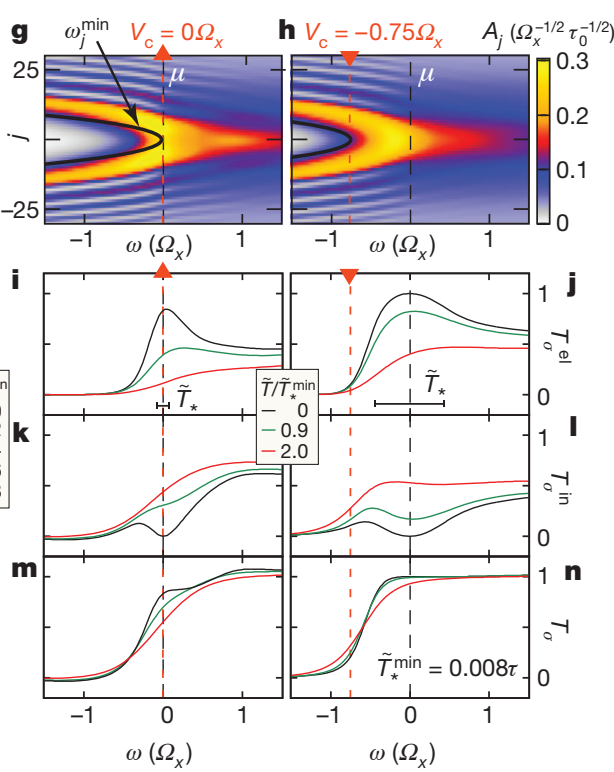

Figure 3 Finite excitation energies. a-f, Zero-bias peak. a, Experimental data for the nonlinear conductance, $g_{\mathrm{nl}}$, as a function of source-drain voltage, measured for several $V_{c}$ values at a fixed low temperature and zero field.

b, Keldysh SOPT results for $g_{\mathrm{nl}}\left(\tilde{V}_{\mathrm{sd}}\right)$ for several $\tilde{V}_{\mathrm{c}}$ values at $\tilde{T}=\tilde{B}=0$, showing qualitative agreement with a. c, The linear-response conductance,

$g=g_{\mathrm{nl}}\left(\tilde{V}_{\mathrm{sd}}=0\right)$, as a function of $\tilde{V}_{\mathrm{c}} \cdot \mathbf{d}-\mathbf{f}, g_{\mathrm{nl}}\left(\tilde{V}_{\mathrm{sd}}\right)$ as in $\mathbf{b}$, but for three different $\tilde{V}_{\mathrm{c}}$ values (compare colour-matched dots in $\mathbf{c}$ and $\mathbf{b}$ ) and five different magnetic field values in each panel. Increasing $B$ causes the $Z B P$ to split into two subpeaks once $\tilde{B} \gtrsim \tilde{B}_{*}$; the splitting is therefore most pronounced in e, for which $\widetilde{B}_{*}$ is smallest. A detailed discussion of the ZBP, including its $T$ dependence, will be published elsewhere. Here we would like to point out the qualitative agreement of $\mathbf{d}-\mathbf{f}$ with published data; see, for example, fig. $2 \mathrm{~d}$ of ref. $6 . \mathbf{g}, \mathbf{h}$, Interacting LDOS: $A_{j}(\omega)$, calculated using SOPT, shown for two fixed gate voltage values, $\tilde{V}_{\mathrm{c}} / \Omega_{x}=0(\mathbf{g})$ and -0.75 (h) (red dashed lines). i-n, Equilibrium transmission probabilities: the corresponding elastic, inelastic and total transmission probabilities, $T_{\sigma}^{\mathrm{el}}(\mathbf{i}, \mathbf{j}), T_{\sigma}^{\mathrm{in}}(\mathbf{k}, \mathbf{l})$ and $T_{\sigma}(\mathbf{m}, \mathbf{n})$, calculated using SOPT and shown as functions of energy, $\omega$, for three different temperatures. At $\tilde{T}=0$ (black curves) $T_{\sigma}^{\text {in }}(\omega)$ vanishes at $\omega=0$, where there is no phase space for inelastic scattering. However, it increases as $\omega$ changes from zero, causing a corresponding reduction in the elastic transmission for $\omega \neq 0$, such that $T_{\sigma}^{\mathrm{el}}(\omega)$ has a narrow 'low-energy peak' around $\omega=0$. On increasing the temperature, the probability of inelastic scattering increases, causing the minimum in $T_{\sigma}^{\text {in }}(\omega)$ and the peak in $T_{\sigma}^{\mathrm{el}}(\omega)$ to be smeared out. This leads to a net $\tilde{T}$-induced reduction in the total transmission, $T_{\sigma}(\omega)$ near $\omega=0$, causing a corresponding reduction in the conductance (Fig. 2d, f). This reduction is stronger for $\tilde{V}_{\mathrm{c}} / \Omega_{x}=0(\mathbf{m})$ than for $\tilde{V}_{\mathrm{c}} / \Omega_{x}=-0.75(\mathbf{n})$, because the probability of electron-hole pair creation during inelastic scattering is largest when apex of the van Hove ridge lies closest to $\mu$ (compare $\mathbf{g}$ and $\mathbf{h}$ ). 
a quasi-bound state, whose local moment gives rise to the Kondo effect. In contrast, our van-Hove-ridge scenario fully explains the 0.7 -anomaly without invoking the Kondo effect. In particular, we find no indications that a smooth parabolic barrier hosts a discrete, localized spin (compare with the third feature above), and no Kondo effect/0.7-anomaly similarities (experimentally or theoretically) at high energies $\left(\gtrsim \tilde{B}_{*}\right)$, where the Kondo effect is governed by an unscreened local moment. Nevertheless, the two phenomena do have similar low-energy behaviour. This is because both involve a spin-singlet ground state featuring spatially confined spin fluctuations. For a KQD they result from screening of the localized spin, whereas for a QPC they result from the extended structure of the van Hove ridge (Fig. 1i); but this distinction, which is important on short length scales (high energies), does not matter on long ones (low energies). These spin fluctuations are characterized by exponentially small energy scales, the Kondo temperature for a KQD, and $\tilde{T}_{*}$ for a QPC, both scaling inversely with the local spin susceptibility (for a QPC, this follows from prediction four). For a KQD, the local spin fluctuations can be described by Nozières-Fermi-liquid theory ${ }^{27,28}$ in terms of scattering phase shifts, which determine its lowenergy properties. Because a QPC, like a KQD, harbours spatially confined spin fluctuations, a similar Nozières-Fermi-liquid framework applies, explaining why its low-energy transport properties are similar to those of a KQD.

We next study finite excitation energies $\left(\tilde{T}, \tilde{V}_{\text {sd }}>0\right)$, where inelastic scattering becomes important (Fig. 3). We begin by considering the nonlinear differential conductance, $g_{\mathrm{n}}$, as a function of source-drain voltage, $V_{\mathrm{sd}}$. Experimentally, $g_{\mathrm{nl}}$ shows a narrow peak at $V_{\mathrm{sd}}=0$ (Fig. 3a; see also refs $6,9,10)$. This ZBP appears strongest in the sub-open regime, but remains visible even very close to pinch off ${ }^{10}(g \rightarrow 0)$. It splits with increasing field once $B$ exceeds a $V_{\mathrm{c}}$-dependent crossover value that is smallest when $g \approx 0.7$ (see fig. $2 \mathrm{~d}$ of ref. 6 ). Remarkably, our model, treated using Keldysh SOPT (Supplementary Information, section 7B), yields a ZBP (Fig. 3b, d-f) that qualitatively reproduces this behaviour. In the sub-open regime $(0.5 \lesssim g \lesssim 0.9)$, a ZBP arises even without interaction (this follows from equation (4)), but interactions modify it in two ways (Supplementary Information, section 7C): a finite $V_{\text {sd }}$ causes a net charge enhancement at the barrier, resulting in a reduction of transmission due to Coulomb repulsion; and opens up a finite phase space for inelastic backscattering. Both effects strongly depend on the LDOS near $\mu$ (Fig. 3g, h), and are thus strongest when the apex of the van Hove ridge lies near $\mu$ (as in Figs $3 g$ and $1 \mathrm{~g}$ ). However, the van Hove ridge intersects $\mu$ also for $g<0.5$ (as in Fig. 1f), which explains why a ZBP is experimentally observed even close to pinch off ${ }^{10}$.

The two modification mechanisms just discussed also apply to the case of increasing temperature. To highlight the role of inelastic scattering, we now discuss (for $\tilde{B}=\tilde{V}_{\text {sd }}=0$ ) the transmission probability $T_{\sigma}(\omega)=T_{\sigma}^{\mathrm{el}}(\omega)+T_{\sigma}^{\mathrm{in}}(\omega)$, written as the sum of elastic and inelastic contributions corresponding respectively to transmission without or with the creation of electron-hole pairs (see Supplementary Information, section 7A, for their precise definition). Figure 3i-n shows examples of these quantities. With increasing temperature, the probability for inelastic scattering increases, causing $T_{\sigma}^{\text {in }}(\omega)$ to increase (Fig. 3k, l) and $T_{\sigma}^{\mathrm{el}}(\omega)$ to decrease (Fig. $3 \mathrm{i}, \mathrm{j}$ ). This leads to a net temperatureinduced reduction in the total transmission, $T_{\sigma}(\omega)$ (Fig. $3 \mathrm{~m}, \mathrm{n}$ ), near $\omega=0$, causing a corresponding reduction in the conductance (Fig. $2 \mathrm{~d}, \mathrm{f}$ ). Importantly, this reduction is $\tilde{V}_{\mathrm{c}}$ dependent: it is strongest when the apex of the van Hove ridge lies near $\mu$ (as in Fig. 3m) and decreases away from this point (as in Fig. 3n), because the probability for electron-hole pair creation during inelastic scattering increases with the LDOS near $\mu$. The fact that $T_{\sigma}(\omega)$ acquires a non-trivial, interaction-induced dependence on $\tilde{T}$ in the sub-open regime is consistent with the fact that near $\mathrm{g} \approx 0.7$ the measured thermopower violates the Mott relation ${ }^{5}$, which is based on the assumption of non-interacting electrons.

Finally, we note that we have studied the magnetic field dependence of the transconductance, $\mathrm{d} G / \mathrm{d} V_{\mathrm{c}}$, both experimentally and by using FRG. We obtain excellent qualitative agreement between experiment and theory, showing that such measurements can be understood without invoking spontaneous spin polarization, as is often advocated to explain them ${ }^{4,8,17}$. A detailed analysis (Supplementary Information, section 2C, and Supplementary Fig. 5) establishes that the $g$ factor is enhanced significantly by interactions, and that interaction strength can be tuned experimentally using a top gate.

We have presented detailed microscopic calculations that qualitatively reproduce the full phenomenology of the 0.7 -anomaly. We argued that a van Hove ridge in the LDOS, combined with interactions, provides a natural explanation for the anomalous behaviour of the conductance of a sub-open $(g \gtrsim 0.5)$ QPC. The experimentally observed $^{6}$ similarities between the 0.7 -anomaly and the Kondo effect at low energies arise because both phenomena involve spatially localized spin fluctuations; at high energies, the similarities cease. We verified our Fermi-liquid predictions for the QPC conductance by systematic measurement of the conductance as a function of $V_{c}, B$ and T. Strikingly, we demonstrated that the zero-bias peak in a QPC arises from the interplay of interactions and geometry. By implication, anomalous zero-bias behaviour might also arise in other systems involving interacting electrons traversing $1 \mathrm{D}$ low-density regions with slowly varying spatial inhomogeneities, such as the gated nanowires being studied in the search for Majorana fermions ${ }^{29}$.

\section{METHODS SUMMARY}

The nanostructure is laterally defined in a 2DES located $85 \mathrm{~nm}$ beneath the surface of a GaAs/AlGaAs heterostructure. The low-temperature carrier density and mobility are $1.9 \times 10^{11} \mathrm{~cm}^{-2}$ and $1.2 \times 10^{6} \mathrm{~cm}^{2} \mathrm{~V}^{-1} \mathrm{~s}^{-1}$, respectively. Electronbeam lithography was used to create the Ti/Au gates. The top gate is electrically insulated from the others by cross-linked poly(methyl methacrylate). Perfect alignment of magnetic fields parallel to the 2DES and the 1D channel defining the QPC was ensured by using a two-axis magnet and was controlled by magnetotransport measurements. We used a dilution refrigerator and reached electron temperatures as low as $T_{2 \mathrm{DES}} \approx 30 \mathrm{mK}$

Our most accurate theoretical results were obtained by using FRG $^{24-26}$ to calculate $T=0$ properties. FRG amounts to doing renormalization-group-enhanced perturbation theory in the interaction $U$. In setting up our FRG flow equations, we made two approximations, both exact to second order in $U$ : we truncated the infinite hierarchy of flow equations by neglecting the flow of the three-particle vertex; and we set to zero all components of the two-particle vertex that are not already generated to second order in the interaction (coupled-ladder approximation).

To access the effects of inelastic scattering for $\tilde{T}>0$ or $\tilde{V}_{\text {sd }}>0$ at fixed $U$, we used SOPT: we dressed bare Green's functions by evaluating the self-energy perturbatively to second order in the interaction. For $\tilde{V}_{\text {sd }}=0$, we calculated the linear conductance following the strategy in ref. 23 , generalized to $\tilde{B} \neq 0$ and broken electron-hole symmetry. For $\tilde{V}_{\mathrm{sd}}>0$, we calculated the nonlinear conductance, $g_{\mathrm{nl}}=\left(\mathrm{d} I / \mathrm{d} \tilde{V}_{\mathrm{sd}}\right) / G_{\mathrm{Q}}$, using the Meir-Wingreen formula for the current (equation (6) of ref. 30)

\section{Received 16 November 2012; accepted 26 June 2013.}

\section{Published online 28 August 2013.}

1. van Wees, B. J. etal. Quantized conductance of point contacts in a two-dimensional electron gas. Phys. Rev. Lett. 60, 848-850 (1988).

2. Wharam, D. A. etal. One-dimensional transport and the quantisation of the ballistic resistance. J. Phys. C 21, L209-L214 (1988).

3. Büttiker, M. Quantized transmission of a saddle-point constriction. Phys. Rev. B 41, 7906(R) (1990)

4. Thomas, K. J. et al. Possible spin polarization in a one-dimensional electron gas. Phys. Rev. Lett. 77, 135-138 (1996)

5. Appleyard, N. J. et al. Direction-resolved transport and possible many-body effects in one-dimensional thermopower. Phys. Rev. B 62, R16275-R16278 (2000).

6. Cronenwett, S. M. et al. Low-temperature fate of the 0.7 structure in a point contact: a Kondo-like correlated state in an open system. Phys. Rev. Lett. 88, 226805 (2002).

7. DiCarlo, L. et al. Shot-noise signatures of 0.7 structure and spin in a quantum point contact. Phys. Rev. Lett. 97, 036810 (2006)

8. Koop, E. J. et al. The influence of device geometry on many-body effects in quantum point contacts: signatures of the 0.7-anomaly, exchange and Kondo. J. Supercond. Nov. Magn. 20, 433-441 (2007).

9. Sarkozy, S. et al. Zero-bias anomaly in quantum wires. Phys. Rev. B 79, 161307R (2009).

10. Ren, Y.etal. Zero-bias anomaly of quantum point contacts in the low-conductance limit. Phys. Rev. B 82, 045313 (2010). 
11. Micolich, A. P. What lurks below the last plateau: experimental studies of the $0.7 \times 2 \mathrm{e}^{2} / \mathrm{h}$ conductance anomaly in one-dimensional systems. J. Phys. Condens. Matter 23, 443201 (2011).

12. Burke, A. etal. Extreme sensitivity of the spin-splitting and 0.7 anomaly to confining potential in one-dimensional nanoelectronic devices. Nano Lett. 12, 4495-4502 (2012).

13. Meir, Y., Hirose, K. \& Wingreen, N. S. Kondo model for the 0.7 anomaly in transport through a quantum point contact. Phys. Rev. Lett. 89, 196802 (2002)

14. Rejec, T.\& Meir, Y. Magnetic impurity formation in quantum point contacts. Nature 442, 900-903 (2006)

15. Ihnatsenka, S. \& Zozoulenko, I. V. Conductance of a quantum point contact based on spin density-functional theory. Phys. Rev. B 76, 045338 (2007).

16. Matveev, K. A. Conductance of a quantum wire at low electron density. Phys. Rev. B 70, 245319 (2004)

17. Reilly, D. J. Phenomenological model for the 0.7 conductance feature in quantum wires. Phys. Rev. B 72, 033309 (2005).

18. Sloggett, C. Milstein, A. I. \& Sushkov, O. P. Correlated electron current and temperature dependence of the conductance of a quantum point contact. Eur. Phys. J. B 61, 427-432 (2008).

19. Aryanpour, K. \& Han, J. E. Ferromagnetic spin coupling as the origin of 0.7 anomaly in quantum point contacts. Phys. Rev. Lett. 102, 056805 (2009).

20. Güçlü, A. D. etal. Localization in an inhomogeneous quantum wire. Phys. Rev. B 80 , 201302(R) (2009).

21. Lunde, A. M. et al. Electron-electron interaction effects in quantum point contacts. New J. Phys. 11, 023031 (2009).

22. Goldhaber-Gordon, D. et al. Kondo effect in a single-electron transistor. Nature 391, 156-159 (1998).

23. Oguri, A. Transmission probability for interacting electrons connected to reservoirs. J. Phys. Soc. Jpn 70, 2666-2681 (2001).

24. Andergassen, S. et al. Renormalization-group analysis of the one-dimensional extended Hubbard model with a single impurity. Phys. Rev. B 73, 045125 (2006).

25. Karrasch, C., Enss, T. \& Meden, V. Functional renormalization group approach to transport through correlated quantum dots. Phys. Rev. B 73, 235337 (2006).

26. Metzner, W. et al. Functional renormalization group approach to correlated fermion systems. Rev. Mod. Phys. 84, 299-352 (2012).
27. Nozières, P. A "fermi-liquid" description of the Kondo problem at low temperatures. J. Low Temp. Phys. 17, 31-42 (1974).

28. Glazman, L\&. Pustilnik, M. in Nanophysics: Coherence and Transport (eds Bouchiat, H. et al.) 427-478 (Elsevier, 2005).

29. Mourik, V. et al. Signatures of Majorana fermions in hybrid superconductor-semiconductor nanowire devices. Science 336, 1003-1007 (2012)

30. Meir, Y. \& Wingreen, N. S. Landauer formula for the current through an interacting electron region. Phys. Rev. Lett. 68, 2512-2515 (1992).

Supplementary Information is available in the online version of the paper.

Acknowledgements We thank B. Altshuler, P. Brouwer, R. Egger, J. Folk, L. Glazman, V. Golovach, A. Hamilton, A. Högele, Y. Imry, M. Kiselev, J. Kotthaus, D. Logan, D. Loss, C. Marcus, Y. Meir, H. Monien, M. Pepper, M. Pustilnik, A. Rosch, K. Schönhammer, B. Spivak and A. Yacoby for discussions, and, in particular, S. Andergassen, C. Honerkamp, S. Jakobs, C. Karrasch, V. Meden, M. Pletyukhov and H. Schoeller for FRG-related help and advice. We acknowledge support from the DFG through SFB-631, SFB-TR12, De730/3-2, De730/4-1, De730/4-2, De730/4-3, HO 4687/1-3, LU819/ 4-1 and the Cluster of Excellence Nanosystems Initiative Munich; from the Center for NanoScience; and from the US National Science Foundation under grant no. NSF PHY05-51164. S.L. acknowledges support through a Heisenberg fellowship of the DFG.

Author Contributions J.v.D. and S.L. coordinated the project: J.v.D. initiated and supervised the theoretical work, and S.L. planned and supervised the experiments and their analysis. F.B. and J.H. carried out the calculations using FRG, and J.H., F.B. and B.B. carried out the calculations using perturbation theory. D.S. and W.W. provided the wafer material, and D.B. fabricated the nanostructure. E.S., D.B., D.T. and S.L. carried out the measurements, and E.S., D.B., F.B. and J.H. carried out the experimental data analysis. J.H. and F.B. prepared the figures, and J.v.D., S.L., F.B., J.H. and E.S. wrote the paper.

Author Information Reprints and permissions information is available at www.nature.com/reprints. The authors declare no competing financial interests. Readers are welcome to comment on the online version of the paper. Correspondence and requests for materials should be addressed to J.v.D. (vondelft@lmu.de) or S.L. (ludwig@Imu.de). 


\section{Contents}

\section{S-1. Overview}

\section{PART I: EXPERIMENT VS. THEORY}

S-2. Our experimental data vs. theory

A. Experimental setup

B. Fermi liquid properties

C. Top-gate tuning of effective $g_{\mathrm{ss}}$-factor

\section{S-3. Other experimental data vs. fRG}
A. Spin-resolved conductance, shot noise
B. Compressibility and charge susceptibility

\section{PART II: THEORETICAL DETAILS}

S-4. Models used for barrier shape
A. Hamiltonian, chemical potential, $U_{j}$
B. Model I
C. Bare local density of states (LDOS)
D. Model II
E. Comparison: bare LDOS of QPC and QD

S-5. The low-energy scale $\widetilde{B}_{*}$
A. Exponential $\widetilde{V}_{\mathrm{c}}$-dependence of $\widetilde{B}_{*}$
B. Effects of interactions on $\widetilde{B}_{*}$ and $\widetilde{T}_{*}$
C. Geometry-dependence of interaction $U$

S-6. Functional renormalization group
A. Observables
B. fRG strategy and approximations
C. fRG Flow equations
D. fRG for non-uniform systems
E. Zero-temperature limit
F. Static fRG

\section{S-7. Second-order perturbation theory}
A. Equilibrium SOPT
B. Nonequilibrium SOPT
C. $\widetilde{B}$-, $\widetilde{T}$ - , and $\widetilde{V}_{\text {sd }}$-dependence of $g\left(\widetilde{V}_{\mathrm{c}}\right)$
D. SOPT artefact arising for increasing $U$

\section{References}

\section{S-1. OVERVIEW}

The following supplementary material provides additional information related to various aspects of the main article. Its sections can be read independently and in arbitrary order. They are grouped into two parts: Part I (Secs. S-2 and S-3) is devoted to experiments and their comparison with theory; Part II (Secs. S-4 to S-7 C) provides further theoretical details.

Section S-2 gives supplementary information about our measurements discussed in the main article. Section S-2 A describes the experimental setup. In Sec. S$2 \mathrm{~B}$ we present the raw data on which the experimental tests of Fermi liquid predictions in the main article are based, together with corresponding results obtained by the functional renormalization group (fRG) (Fig. S2). We also present additional data (Fig. S3) illustrating the gate-voltage dependence of the crossover scales in magnetic field, temperature and source-drain-voltage, $B_{*}, T_{*}$ and $V_{\mathrm{sd} *}$, together with corresponding calculations using second-order perturbation theory (SOPT). Sec. S-2 C explains in detail how the effective $g$-factor $g_{\mathrm{ss}}$ is extracted from the transconductance for large fields (Fig. S5), and offers some comments on the much-discussed scenario that the 0.7-anomaly is due to spontaneous spin polarization in the QPC region.

Sec. S-3 presents further $T=0$ fRG results (Figs. S6 and S7) that demonstrate qualitative agreement with shot noise and compressibility measurements by other groups. These fRG results, and those in Sec. S-5, were calculated using "static" fRG, which differs from the "dynamic" fRG approach used in the main text by neglecting the frequency dependence of the self-energy and all vertices (see Sec. S-6 F). Static fRG yields results that are very similar to those of dynamic fRG (see Fig. S15), while being numerically cheaper by a factor $\sim 10^{3}$.

Section S-4 describes our theoretical model in detail. We have used two slightly different parametrizations of the QPC barrier shape, called "model I" and "model II", which both describe parabolic barrier tops and hence give essentially equivalent results for QPC properties. They are defined in Secs. S-4B and S-4D, respectively (the main article uses only model II). Sections S-4 C and S$4 \mathrm{E}$ explain how the effects of geometry are encoded in the bare local density of states (LDOS), focussing in particular on the van Hove ridge of a QPC, which is key to understanding the 0.7-anomaly.

Section S-5 focuses on the low-energy scale $\widetilde{B}_{*}\left(\widetilde{V}_{\mathrm{c}}\right)$ for a QPC: it shows that its exponential $\widetilde{V}_{\text {c-dependence has }}$ a purely geometric origin, and that the strength of its $U$ dependence likewise depends on the shape of the barrier.

Sections S-6 and S-7 discuss details of the two theoretical methods used here to incorporate the effect of interaction: the functional Renormalization Group (fRG) and second order perturbation theory (SOPT), respectively. Section S-7 C is devoted to a detailed description of our 
SOPT results for finite temperature or finite source-drain voltage, offering a summary of the features of the 0.7 anomaly which SOPT does or does not capture qualitatively. Finally, Sec. S-7 D discusses an SOPT artefact that arises with increasing $U$.

Equation and figure and section numbers from the main article or the supplementary material are pref- aced below by A (for "article") vs. S (for "supplementary"), respectively, e.g. Eq. (A1), Fig. A1f, Sec. A2 vs. Eq. (S1), Fig. S2b, Sec. S-4. As in the main article, we use tildes to distinguish theory parameters from those used in experiment, writing, e.g. $\widetilde{T}=k_{\mathrm{B}} T$, $\widetilde{B}=\left|g_{\mathrm{el}}\right| \mu_{\mathrm{B}} B$, and $\widetilde{V}_{\mathrm{c}, \mathrm{s}} \propto-|e| V_{\mathrm{c}, \mathrm{s}}$.

\section{PART I: EXPERIMENT VS. THEORY}

\section{S-2. OUR EXPERIMENTAL DATA VS. THEORY}

\section{A. Experimental setup}

The gate layout of our sample, shown in Fig. A1a for a dummy sample whose layout is identical to that of the actual sample, provides a particularly high tunability of the central constriction region (CCR). The gates can be used to laterally define either a quantum point contact (QPC) or a quantum dot (QD) in the two-dimensional electron system (2DES) $85 \mathrm{~nm}$ beneath the surface of a GaAs/AlGaAs heterostructure. In this work, we focus exclusively on the QPC geometry; a study of the crossover from QD to QPC will be published elsewhere. ${ }^{1}$ More information about the experimental conditions is provided in the methods summary section of the main article.

In our two-terminal transport measurements the current $I_{\text {sd }}$ flows through the nanostructure between ohmic contacts marked by "source" and "drain" in Fig. A1a, and we measure the differential conductance $g=$ $\left(\mathrm{d} I_{\mathrm{sd}} / \mathrm{d} V_{\mathrm{sd}}\right) / G_{\mathrm{Q}}$ (henceforth simply called conductance) using lockin methods. In all measurements discussed in this paper we apply a negative voltage $V_{\mathrm{c}}$ to both center gates and a negative voltage $V_{\mathrm{s}}$ to all four side gates. This depletes the 2DES in the vicinity of the gates, so that propagation between source and drain through the CCR is confined to a narrow channel, leading to the quantization of transverse modes. (Further variations of individual gate voltages allow additional control of the lateral symmetry properties of the CCR, but such studies are not included in this work.) Moreover, our sample also contains a global top gate (see Fig. A1a).

In this work, we focus on gate voltages such that transport is carried solely by the first subband, corresponding to the lowest transverse mode. Its behavior can be described by a one-dimensional effective model for motion in the longitudinal (say $x$-) direction. The shape of the effective potential $V_{\text {eff }}(x)$ in the CCR can be changed by tuning $V_{\mathrm{c}}, V_{\mathrm{s}}$ and $V_{\mathrm{t}}$. Increasing the top gate voltage $V_{\mathrm{t}}$ increases the carrier density of the 2DES in the contacts of the CCR and hence the chemical potential, thereby deepening (w.r.t. $\mu$ ) the trenches between the regions of high potential energy caused by the central and side gates $^{4}$. This changes not only the shape of $V_{\text {eff }}(x)$, but also causes the transverse wave functions to be more lo- calized and hence increases the effective one-dimensional on-site interaction strength $U$ within the CCR. For future reference, we summarize this trend as follows:

The effective interaction strength $U$ can

be increased experimentally by increasing $V_{\mathrm{t}}$.

For a QPC geometry, increasing $V_{\mathrm{t}}$ has an additional effect: due to the deepened trenches in the potential landscape, the energy spacing of the transverse eigenmodes increases, resulting in an increased subband spacing ${ }^{5}$. This trend is demonstrated in Fig. S1 based on measured pinch-off curves of our QPC for varying top-gate voltages. It can be used, in principle, to quantify the $V_{\mathrm{t}^{-}}$ induced increase in $U$ in terms of the $V_{\mathrm{t}}$-induced increase in subband spacing ${ }^{6}$, as will be elaborated in Sec. S-5 C below.

\section{B. Fermi liquid properties}

Figs. S2b and S2c show the raw experimental data for the measured linear response conductance of our QPC (a constant lead resistance has already been subtracted for all data). They show how the pinch-off curves depend on magnetic field and temperature, respectively. For comparison, Fig. S2a shows corresponding fRG data calculated for zero temperature as a function of the magnetic field $\widetilde{B}$. Both calculated and measured data exhibit the expected transition from a weak kink at $g \simeq 0.7$ at small $T$ and $B$ to a pronounced 0.7-anomaly if either magnetic field (measured and calculated data) or temperature (measured data) is substantially increased.

The raw data from Figs. S2b and S2c underly the experimental results presented in Figs. A2e-h of the main article. Figs. S3a-d shows additional data sets, plotted in the same way as in Figs. A2g and A2h, but displaying data not shown there for lack of space. Together, these data confirm the Fermi-liquid behavior expected theoretically for sufficiently low fields and temperatures: Figures $\mathrm{A} 2 \mathrm{~g}$ and $\mathrm{S} 3 \mathbf{a}, \mathbf{b}$ show that at sufficiently low temperatures, $T_{0} \ll T_{*}$ (in our measurements $T_{0}=T_{2 \mathrm{DES}} \simeq 30 \mathrm{mK}$ ), the leading magnetic fielddependence of the linear conductance is quadratic,

$$
g(B) / g(0)=1-\left(B / B_{*}\right)^{2}, \quad B \ll B_{*},
$$



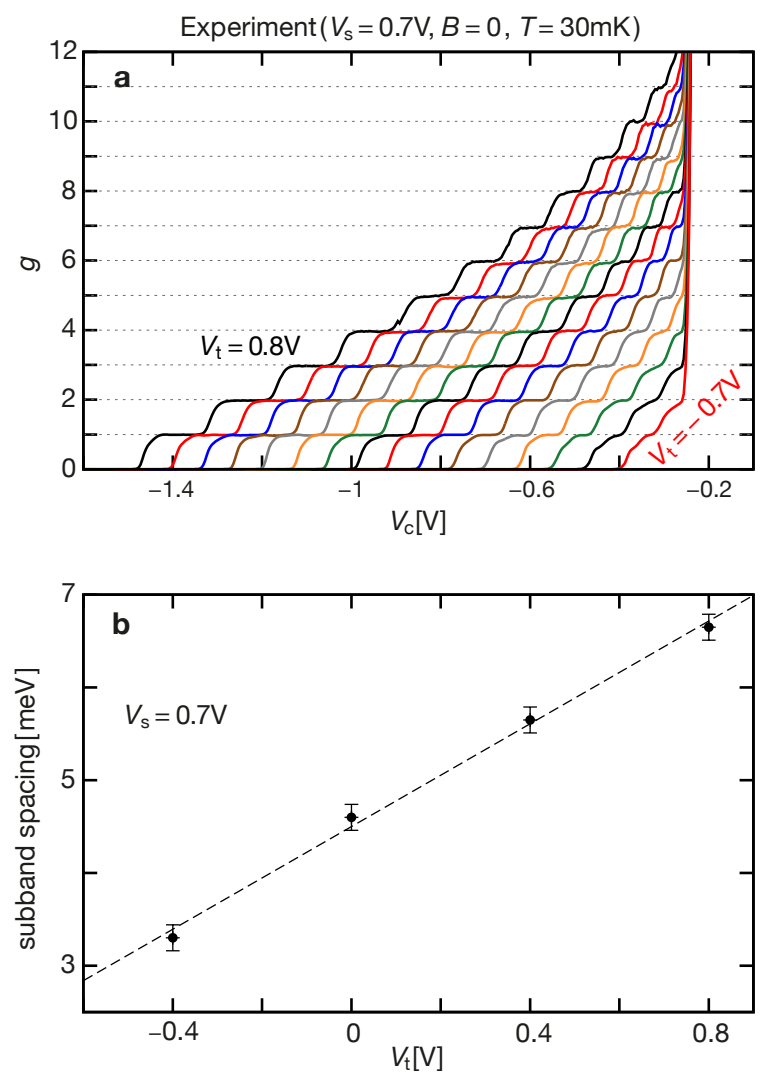

Figure S1: $V_{\mathrm{t}}$-dependence of subband spacing. a, Measured pinch-off curves $g\left(V_{\mathrm{c}}\right)$ of our QPC for a series of top-gate voltages in the range $-0.7 \mathrm{~V} \leq V_{\mathrm{t}} \leq 0.8 \mathrm{~V}$. As $V_{\mathrm{t}}$ is decreased the carrier density also becomes smaller which, in turn, results in a larger pinch-off voltage $V_{\mathrm{c}}$ and, clearly, in more narrow plateaus at integer $g$. The steep increase of $g\left(V_{\mathrm{c}}\right)$ independent of $V_{\mathrm{t}}$ at $V_{\mathrm{c}} \simeq-0.25 \mathrm{~V}$ indicates the transition from $1 \mathrm{D}$ to $2 \mathrm{D}$ transport as the 2DES directly below the center gates is no longer depleted. b, Energy spacing between the lowest two $1 \mathrm{D}$ subbands as a function of $V_{\mathrm{t}}$. The data points were evaluated from finite- $V_{\text {sd }}$ measurements (raw data ${ }^{2}$ not shown), using a procedure described in Refs. 2,3, whose uncertainty is indicated by the error bars. The resulting subband spacing is approximately proportional to the width of the first conductance plateau in a. As expected from a simple capacitive model, it is also proportional to $V_{\mathrm{t}}$ (the dashed straight line is a guide for the eye).

as expected from Eq. (A2). Similarly, Figs. A2h and S3c,d show that at zero field $(B=0)$, the leading temperature dependence is likewise quadratic,

$$
g(T) / g\left(T_{0}\right)=1-\left(T / T_{*}\right)^{2}, \quad T \ll T_{*} .
$$

Fitting Eqs. (S2a) and (S2b) to the data in Figs. S2b and S2c, respectively, yields the low-energy scales $B_{*}\left(V_{\mathrm{c}}\right)$ and $T_{*}\left(V_{\mathrm{c}}\right)$ used in Figs. A2g and A2h and depicted by colored symbols in Figs. A2e and A2f, respectively (and similarly for Figs. S3a-d). The scaled conductance curves displayed in Figs. S3a-d are plotted only in the restricted ranges $g(B) / g(0) \gtrsim 0.8$ and
$g(T) / g\left(T_{0}\right) \gtrsim 0.8$, respectively. For smaller conductances, where the conditions $B \ll B_{*}$ or $T \ll T_{*}$ no longer hold, the measured $B$ - and $T$-dependences of the conductance deviate from quadratic behavior by bending upward, tending toward saturation (as shown in Figs. A2g,h ).

The fit parameters $B_{*}$ and $T_{*}$ are compared in the half-logarithmic presentation in Fig. A2f as functions of the center gate voltage $\Delta V_{\mathrm{c}}$. For convenience, this data is shown again in Fig. S3f, together with the low-energy source-drain voltage scale $V_{\mathrm{sd} *}$. The latter was obtained by determining the curvature of the nonlinear conductance curves $g_{\text {nl }}\left(V_{\text {sd }}\right)$ (shown in Fig. A3i) at $V_{\text {sd }}=0$ :

$$
g_{\mathrm{nl}}\left(V_{\mathrm{sd}}\right) / g_{\mathrm{nl}}(0)=1-\left(V_{\mathrm{sd}} / V_{\mathrm{sd} *}\right)^{2}, \quad V_{\mathrm{sd}} \ll V_{\mathrm{sd} *} .
$$

Compared to our determinations of $B_{*}$ and $T_{*}$ from linear-response data, those for $V_{\mathrm{sd} *}$ have rather larger error margins, since for technical reasons the non-linear conductance data was measured with a smaller signal-tonoise ratio.

As mentioned in the main article, SOPT makes two predictions for the $\widetilde{V}_{\mathrm{c}}$-dependence of the crossover scales $\widetilde{B}_{*}, \widetilde{T}_{*}$ and $\widetilde{V}_{\mathrm{sd} *}$ in the $V_{\mathrm{c}}$-range where $g \rightarrow 1$ : first, all three scales depend exponentially on $V_{\mathrm{c}}$ (Fig. S3e); and second, the ratios $\widetilde{B}_{*} / \widetilde{T}_{*}$ and $\widetilde{V}_{\mathrm{sd} *} / \widetilde{T}_{*}$ are, to within a few \%, independent of $\widetilde{V}_{\mathrm{c}}$ (as illustrated in Fig. S4 for a range of $U$-values). The experimental results for $B_{*}$, $T_{*}$ and $V_{\mathrm{sd} *}$ shown in Fig. S3f confirm both predictions. This demonstrates that at low energies a QPC shows Fermi-liquid behavior, as argued in detail in the main article.

\section{Top-gate tuning of effective $g_{\mathrm{ss}}$-factor}

In a QPC geometry, interactions are known to enhance the effective electronic $g$-factor ${ }^{7-9}$. For large fields $(B \gg$ $\left.B_{*}\right)$, an effective $g$-factor, say $g_{\mathrm{ss}}$, can be extracted from the transconductance $d g / d V_{c}$, by exploiting the fact that the measured field-induced subband splitting of the first conductance step, say $\Delta E$, increases linearly with field, $\Delta E=g_{\mathrm{ss}} B$. In previous experiments with in-plane fields ( $B$ in the 2DES plane), $\left|g_{\text {ss }}\right|$-values have been observed exceeding the bulk value $\left(g_{\mathrm{GaAs}} \simeq-0.45\right)$ by up to a factor of $6^{9,10}$, an increase that was attributed by Koop et al. to interaction effects ${ }^{9}$.

In Fig. S5 we present the results of fRG calculations and measurements of the transconductance and the topgate dependence of $g_{\mathrm{ss}}$ that confirm this interpretation. We numerically deduce the transconductance $\mathrm{d} G / \mathrm{d} V_{c}$ $\left(\mathrm{d} G / \mathrm{d} \widetilde{V}_{\mathrm{c}}\right)$ from both the measured and calculated conductance data. Typical experimental results are plotted in Fig. S5a for the range $0<g<1$ as a function of $V_{\mathrm{c}}$. They show two peaks whose splitting $\Delta E$ increases linearly for large fields, as $\Delta E \simeq g_{\mathrm{ss}} B+\Delta_{\mathrm{hfo}}$ (Fig. S5b), where both the slope $g_{\mathrm{ss}}$ and the "high-field offset" $\Delta_{\text {hfo }}$ are found to increase with top-gate voltage $V_{\mathrm{t}}$ (Fig. S5c). 
$\operatorname{fRG}\left(U=0.5 \tau, N=101, \Omega_{x}=0.04 \tau\right)$

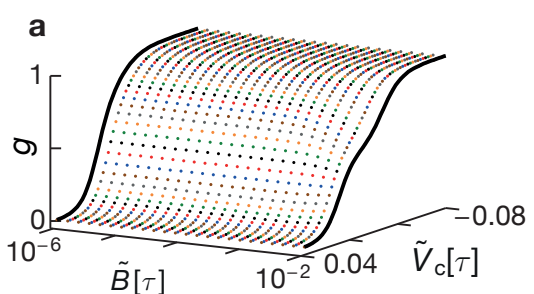

Experiment $\left(V_{\mathrm{s}}=-0.4 \mathrm{~V}, T_{0}=30 \mathrm{mK}\right)$

b

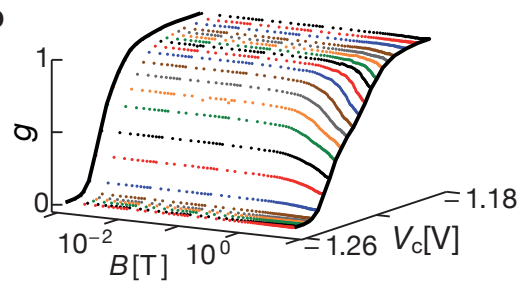

Experiment $\left(V_{\mathrm{s}}=-0.4 \mathrm{~V}, B=0 \mathrm{~T}\right)$

C

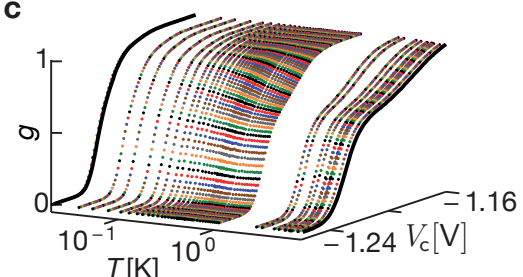

Figure S2: QPC theory versus experiment, raw data: a, fRG data (model II) for the normalized conductance $g=G / G_{\mathrm{Q}}$, calculated at $T=0$ and fixed side gate voltage $\widetilde{V}_{\mathrm{s}}=1.75 \tau$ as function of center gate voltage $\widetilde{V}_{\mathrm{c}}$ and magnetic field $\widetilde{B}_{\text {. }} \mathbf{b}, \mathbf{c}$ Experimental data for the normalized linear response conductance $g=\left(\mathrm{d} I / \mathrm{d} V_{\mathrm{sd}}\right) / G_{\mathrm{Q}}$ (lead resistance subtracted), measured at fixed side and top gate voltages, $V_{\mathrm{s}}=-0.4 \mathrm{~V}$ and $V_{\mathrm{t}}=0.8 \mathrm{~V}$. b $g$ at $T_{2 \mathrm{DES}}=T_{0}=30 \mathrm{mK}$, measured as function of center gate voltage $V_{\mathrm{c}}$ and inplane magnetic field $B$ aligned along the narrow constriction. $\mathbf{c}, g$ at $B=0$, measured as function of $V_{\mathrm{c}}$ and temperature $T$. The data presented here are the raw data used for Figs. 2e-h in the main article and in Figs. S3a-d below. For better visibility, the pinch-off curves at minimal and maximal magnetic field / temperature have been highlighted by thick black lines, serving as guides for the eyes. The best signal-to-noise ratio was achieved by slowly sweeping $B$ at constant $V_{\mathrm{c}}$ in b, and by sweeping $V_{\mathrm{c}}$ at constant $T$ in $\mathbf{c}$.
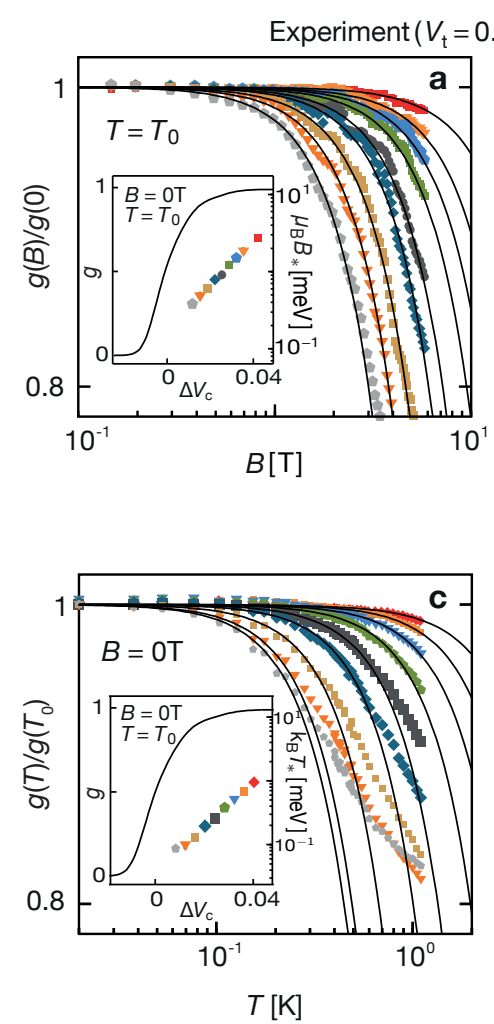

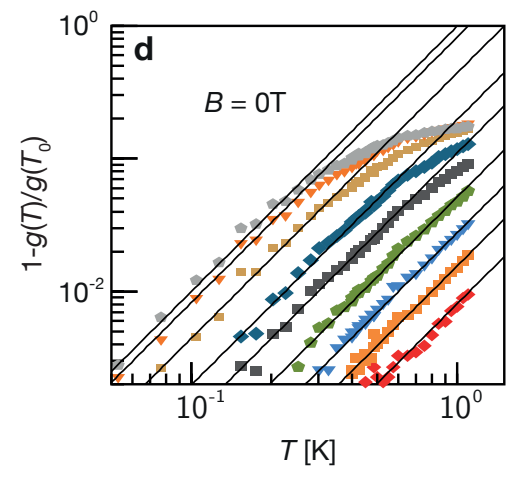

$\operatorname{SOPT}\left(U=0.35 \tau, N=101, \Omega_{\mathrm{x}}=0.04 \tau\right)$

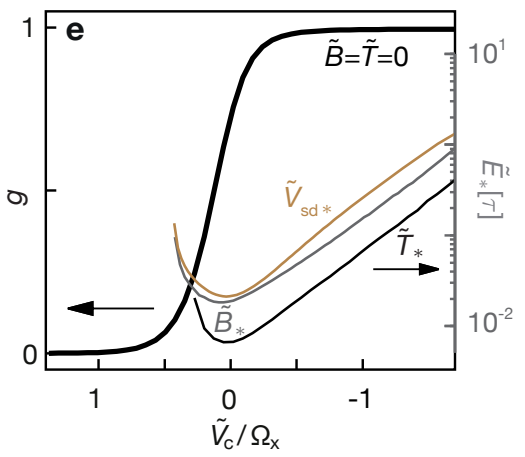

Experiment $\left(V_{\mathrm{t}}=0.8 \mathrm{~V}, V_{\mathrm{s}}=-0.4 \mathrm{~V}\right)$

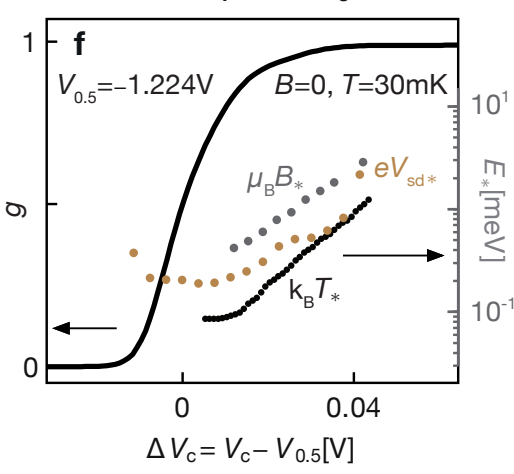

Figure S3: a-d, Experiments; Fermi-liquid behavior. a, $g(B) / g(0)$ on a lin-log-scale and b, $1-g(B) / g(0)$ on a log$\log$-scale. $\mathbf{c}$ and $\mathbf{d}$, as in $\mathbf{a}$ and $\mathbf{b}$ but for temperature instead of magnetic field dependence. These data supplement similar data shown in Figs. A2g and A2h, not repeated here. Black lines are fits of the form $g(X) / g(0)=1-X^{2} / X_{\star}^{2}$ and express the leading quadratic decrease in both temperature and magnetic field. Insets demonstrate the exponential dependence of the scaling energies $\mu_{\mathrm{B}} B_{*}$ and $k_{\mathrm{B}} T_{*}$ (extracted from the fits) on $V_{\mathrm{c}}$, respectively. Colored symbols in the main plots and corresponding insets have the same $V_{\mathrm{c}}$-values. e, f, Comparison of low-energy scales from theory and experiment: e SOPT results for model II, for the conductance $g$ (thick black line) and the low-energy scales $\widetilde{B}_{*}, \widetilde{T}_{*}$ and $\widetilde{V}_{\text {sd } *}($ thin grey, black and brown lines, respectively), as functions of $\widetilde{V}_{\mathrm{c}}$. f Corresponding experimental results for $g, \mu_{\mathrm{B}} B_{*}$ and $k_{\mathrm{B}} T_{*}$ (data repeated from insets of $\mathbf{a}$ and $\mathbf{c}$ ) and $e V_{\mathrm{sd} *}$ as functions of $\Delta V_{\mathrm{c}}=V_{\mathrm{c}}-V_{0.5}$, where $V_{0.5}$ is the gate voltage for which the conductance at $B=0$ and $T=T_{0}$ is $g\left(V_{0.5}\right)=0.5$. $V_{\mathrm{sd} *}$ has been extracted from the leading quadratic dependence of the ZBP (as in a-d for $\left.X=V_{\mathrm{sd}}\right)$. Note the strikingly good qualitative agreement between the SOPT results in $\mathbf{e}$ and the experimental results in $\mathbf{f}$, for the gate-voltage dependence of all three low-energy scales. 


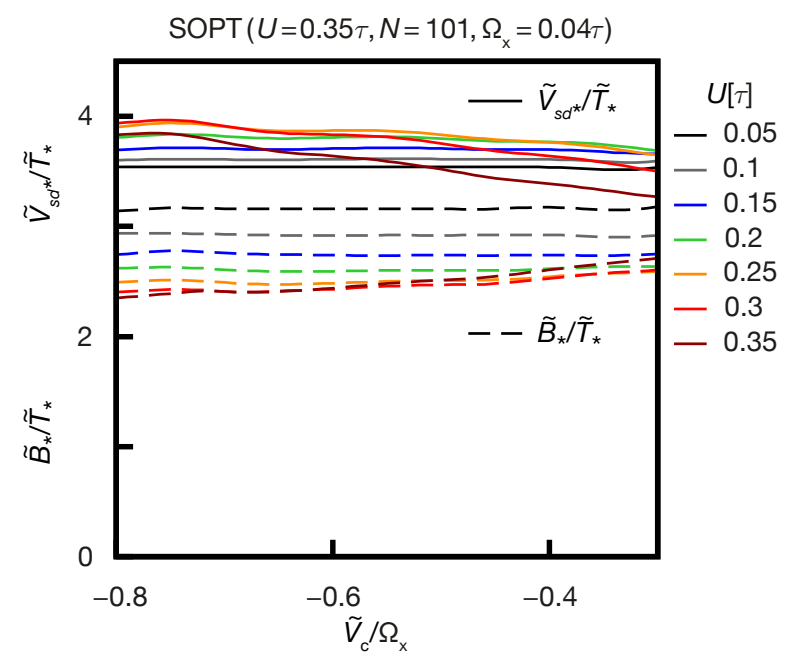

Figure S4: The ratios $\widetilde{V}_{\mathrm{sd} *} / \widetilde{T}_{*}$ (solid lines) and $\widetilde{B}_{*} / \widetilde{T}_{*}$ (dashed lines) as functions of $\widetilde{V}_{\mathrm{c}} / \Omega_{x}$ for several different $U$ values, calculated by SOPT for model II, using the same QPC barrier shape as used for Figs. A2c, d.

The effect of increasing $V_{\mathrm{t}}$ can be mimicked in our model by increasing $U$ (for reasons explained in Supplementary Sec. S-2 A). Indeed, the results of our fRG calculations, shown in Fig. S5d-f, qualitatively match the trends shown by the experimental data in Fig. S5a-c. This establishes several important points. First, interactions are the reason why the $g$-factor extracted from $\Delta E(B)$ is anomalously large. Second, the effective interaction strength can be tuned experimentally via a top gate voltage. Third, the experimental observation of $\Delta_{\text {hfo }} \neq 0$ can be understood without adopting the spontaneous spin polarization scenario that is often advocated ${ }^{7,9,31}$ to explain it. Let us now elaborate these points in more detail.

We theoretically studied the $U$-dependence of $g_{\text {ss }}$ by using fRG to calculate pinch-off curves for parabolic QPC barrier shapes such as that of Fig. A1b, for a range of fields $\widetilde{B}$ and interaction strengths $U$. Fig. S5d plots the transconductance, i. e. the derivative $-d g\left(\widetilde{V}_{\mathrm{c}}\right) / d \widetilde{V}_{\mathrm{c}}$ as function of $\widetilde{V}_{\mathrm{c}}$ (varied over a range corresponding to $0 \leq g \leq 1$ ), for a large number of different $\widetilde{B}$-fields, at $U=0.5 \tau$. In such a plot the field-induced spin splitting of the conductance step manifests itself as a pair of local maxima ${ }^{7-9,11}$. The $\widetilde{V}_{\mathrm{c}}$-separation of their peaks, say $\Delta E$, is proportional to the effective $\widetilde{B}$-induced subband splitting. Evidently $\Delta E$ increases with $\widetilde{B}$. Fig. S5e shows $\Delta E(\widetilde{B})$ vs. $\widetilde{B}$ for six values of $U$, including the data extracted from Fig. S5d. For large fields $\left(\widetilde{B} \gg \widetilde{B}_{*}\right)$ we find a linear relation,

$$
\Delta E(\widetilde{B}) \simeq\left(g_{\mathrm{ss}} / g_{\mathrm{el}}\right) \widetilde{B}+\Delta_{\mathrm{hfo}},
$$

where $\Delta_{\text {hfo }}$ represents the "high-field offset" as defined by Koop et al. ${ }^{9}$, i. e. the linear extrapolation of the highfield behavior to $\widetilde{B}=0$. Fig. S5f and its inset show that both the slope and the offset increase with $U$, implying that both $g_{\mathrm{ss}}$ and $\Delta_{\text {hfo }}$ serve as measures of the effective interaction strength.

Koop et al. have reported a strong enhancement of the $g$-factor as the spacing $\omega_{12}$ between the electronic subbands of the QPC is increased ${ }^{9}$. Our theory nicely explains this finding: an increase in $\omega_{12}$ corresponds to a smaller transverse channel width, implying an enhanced interaction strength (as argued at the end of section S$2 \mathrm{~A}$ ) and hence an increase in $g_{\mathrm{ss}}$ (see Fig. S5f).

This interpretation is confirmed by the experimental data shown in Fig. S5a-c. This data was measured using a second sample ("sample 2"), of similar design than that used to study the Fermi-liquid properties of Figs. A2e-h discussed in the main text ("sample 1"). For sample 2, we measured $\Delta E(B)=a \cdot \Delta V_{c}$ (for the values of the conversion factor $a$ see table in Fig. S5b) as function of top gate voltage $V_{\mathrm{t}}$, which corresponds to tuning the effective interaction strength. According to our theoretical considerations, increasing $V_{\mathrm{t}}$ causes increasing $U$ [see Eq. (S1)] and hence increasing $g_{\text {ss }}$ (by Fig. S5f). Fig. S5a-c present experimental results corresponding to the predictions in Fig. S5d-f (using $V_{\mathrm{t}}$ instead of $U$ ). They qualitatively confirm our numerical results, especially that both $g_{\mathrm{ss}}$ and $\Delta_{\text {hfo }}$ increase with $V_{\mathrm{t}}$ and, therefore, the interaction strength. (In contrast to us, Koop et al. did not observe a systematic correlation between $g_{\mathrm{ss}}$ and $\Delta_{\text {hfo }}$. A possible reason is that their study varied the shape of the QPC potential by varying the width and length of the QPC, whereas we varied $V_{\mathrm{t}}$. Our studies thus differ from theirs in the detailed shape of the $2 \mathrm{D}$ potential landscape. The effective interaction strength is very sensitive towards the latter, as discussed in more detail in Sec. S-5 C.)

We conclude our discussion on $\Delta E(B)$ with an important comment on the high-field offset $\Delta_{\text {hfo }}$. In several experimental studies of the 0.7 -anomaly ${ }^{7-9}$, the observation of a nonzero value for $\Delta_{\text {hfo }}$ was interpreted as evidence "that there is a possible spin polarization of the $1 \mathrm{D}$ electron gas in zero magnetic field" (the quote is from Thomas et al. ${ }^{7}$ ). Our fRG results show that this interpretation is not compelling, since we obtain $\Delta_{\text {hfo }} \neq 0$ without any spontaneous spin polarization. $\quad \Delta_{\mathrm{hfo}} \neq 0$ simply implies that the $\widetilde{B}=0$ conductance step $g\left(\widetilde{V}_{\mathrm{c}}\right)$ is somewhat skewed (see Figs. A1k, A1l, A2a), so that the peak in the transconductance is not symmetric (as seen in Fig. S5d); as shown here, this can be achieved with a magnetization that is strictly zero. Indeed, our fRG approach assumes from the outset that the magnetization per site, $m_{j}=\frac{1}{2}\left(n_{j \uparrow}-n_{j \downarrow}\right)$, is strictly zero at $\widetilde{B}=0$ (see blue line in Fig. A1d, and introduction of Sec. S-6). This a priori assumption is justified a posteriori by the good qualitative agreement between $\mathrm{ARG}$ and experiment found throughout this work, and in Fig. S5 in particular. Moreover, this assumption is a prerequisite for understanding the low-energy Fermi-liquid properties of the 0.7 -anomaly discussed in the main text, and the resulting analogies between the 0.7-anomaly and the Kondo effect: for the latter, there is zero spin polarization at 


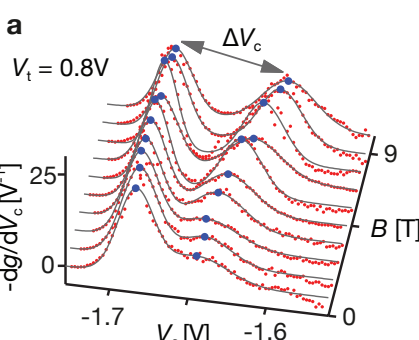

b

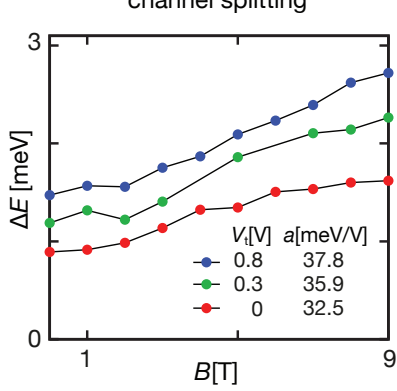

C

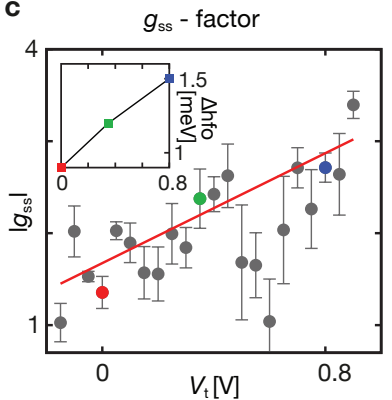

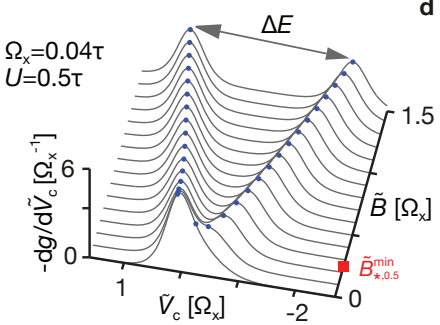

channel splitting

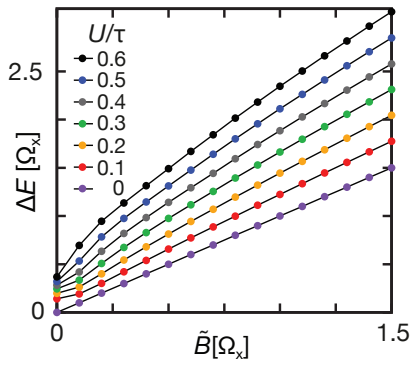

$g_{\text {ss }}$ - factor

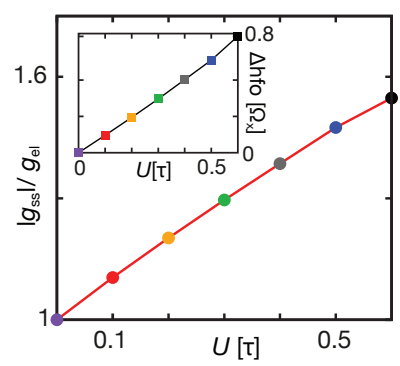

d $\widetilde{B}=T=0$, because lead electrons screen the local spin into a spin singlet.

It is noteworthy, though, that the linear increase in $\Delta E(\widetilde{B})$ in Fig. S5e sets in already at rather small fields, of order $\mathcal{O}\left(\widetilde{B}_{*}\right)$ and similarly for $\Delta E(B)$ in Fig. S5b. The reason is that at small fields the spin polarization rapidly grows with field, since the spin susceptibility is large. It is large because it is strongly enhanced by interactions (Fig. A1j), as recognized and emphasized by Thomas et $a l . .^{7}$, and because the effects of interactions are further enhanced by the van Hove ridge in the QPC, as discussed in the main article. According to our analysis, the large spin susceptibility goes hand in hand with a strong interaction-induced enhancement in the inverse scale $1 / B_{*}\left(\propto \chi_{\text {tot }}\right)[$ Fig. A1 $\mathbf{j}$, as discussed in the main article, and also in Sec. S-5 B below.

The scale $\widetilde{B}_{*}$ governs the "strength" of the 0.7 anomaly, in that the conductance is significantly reduced once $\widetilde{B}$ or $\widetilde{T}$ increase past $\widetilde{B}_{*}$. In an alternative model proposed by Reilly et $a l .{ }^{12}$, one of the advocates of spontaneous spin polarization, the strength of the 0.7anomaly is governed by the size of the spin gap. This model was used successfully, for example, to model the shot noise measurements of Ref. 13. The Reilly model assumes that the spin gap increases strongly with decreasing $V_{\mathrm{c}}$, i. e. with increasing density in the QPC-region. Note that this $\widetilde{V}_{\mathrm{c}}$-dependence of the proposed spin gap shows the same tendency as that shown by the Hartreeenhancement of the barrier size in our work, which likewise increases linearly with increasing density as $\widetilde{V}_{\mathrm{c}}$ is made more negative. (The density near the CCR center also increases as temperature or source-drain voltage is increased, and becomes strongly spin-asymmetry as $\widetilde{B}$ increases.) In this sense, our work sheds light on why the Reilly model is phenomenologically successful at large energies: it makes qualitatively correct assumptions about the $V_{\mathrm{c}}$-dependence of the effective barrier height that governs the strength of the conductance's $B$ - or $T$ dependence. That having been said, we emphasize once more that our Hartree-shift in barrier height is not a spin gap, and that our scenario differs decidedly from that of the Reilly model for energies below $B_{*}$ : there we assert the appearance of Fermi-liquid behavior that is not compatible with spontaneous spin polarization. In our theory, a spin splitting sets in only once spin symmetry is broken by finite $\widetilde{B}$ (though a spin-symmetric Hartreeshift in barrier height is present even at $\widetilde{B}=0$ ). The spin gap predicted by our theory for $\widetilde{B} \neq 0$ does increase with the density in the QPC, as in the Reilly model, since it arises from Hartree contributions to the self-energy (see Eq. (S42) in our fRG scheme, or the first two diagrams in Eq. (S53) when doing perturbation theory). 


\section{S-3. OTHER EXPERIMENTAL DATA VS. FRG}

This section presents additional fRG results on the zero-temperature behavior of the conductance, the shot noise, and the charge susceptibility. Their $\widetilde{V}_{\mathrm{c}^{-}}$and $\widetilde{B}$-dependence is found to be in qualitative agreement with that observed experimentally by other groups (DiCarlo et al. for the shot noise ${ }^{13}$, Smith et al. for the compressibility $^{14}$.

The fRG results presented below were obtained using "static" fRG, a simplified version of the "dynamic" fRG scheme used in the main text. Static fRG neglects the frequency dependence of the self-energy and all vertices (see Sec. S-6 F). This simplification reduces computational costs by a factor of $10^{3}$. Nevertheless, for the model studied here the results of static fRG turn out to be qualitatively very similar to those of dynamic fRG (see Fig. S15 below). Hence we have opted to use static fRG for the results presented in Secs. S-3 and S-5.

\section{A. Spin-resolved conductance, shot noise}

This subsection presents a detailed discussion of the spin-resolved conductance. It is based on calculations using model I (defined in Sec. S-4 B), but the results are fully analogous to those shown in Figs. A2a, $\mathbf{b}$ for model II (defined in Sec. S-4D).

The role of interactions for the magnetoconductance of a QPC at zero temperature can be very clearly revealed by studying the spin-resolved conductance $g_{\sigma}=\mathcal{T}_{\sigma}$ and the shot noise factor ${ }^{15}$

$$
\mathcal{N}=\frac{1}{2} \sum_{\sigma} g_{\sigma}\left(1-g_{\sigma}\right) .
$$

Fig. S6 shows these quantities together with the full conductance $g=g_{\uparrow}+g_{\downarrow}$, all calculated at $T=0$ as functions of $\widetilde{V}_{\mathrm{c}}$, for various fields. To highlight the effect of interactions, we also show corresponding results for the bare $(U=0)$ model, which we discuss first.

We begin with some elementary observations: First, the bare transmission probability $\mathcal{T}_{\sigma}^{0}\left(\widetilde{V}_{\mathrm{c}}, 0\right)$ at zero field, studied as function of $\widetilde{V}_{\mathrm{c}}$, is antisymmetric w.r.t. the point $\mathcal{T}_{\sigma}^{0}(0,0)=0.5$ [cf. Eq. (S30) below]:

$$
\mathcal{T}_{\sigma}^{0}\left(\widetilde{V}_{\mathrm{c}}, 0\right)=1-\mathcal{T}_{\sigma}^{0}\left(-\widetilde{V}_{\mathrm{c}}, 0\right) .
$$

A finite field $\widetilde{B}$ shifts the bare potential in opposite directions for opposite spins, $\delta \widetilde{V}_{j}=-\frac{\sigma}{2} \widetilde{B}$ (with $\sigma= \pm 1$ for $\uparrow$, $\downarrow)$. Thus the bare spin-resolved transmission probability $\mathcal{T}_{\sigma}^{0}$ at finite $\widetilde{B}$ is equal to that at $\widetilde{B}=0$ but for a shifted value of $\widetilde{V}_{\mathrm{c}}$ :

$$
\mathcal{T}_{\sigma}^{0}\left(\widetilde{V}_{\mathrm{c}}, \widetilde{B}\right)=\mathcal{T}_{\sigma}^{0}\left(\widetilde{V}_{\mathrm{c}}-\frac{\sigma}{2} \widetilde{B}, 0\right) .
$$

This implies that $\widetilde{B}$ induces a shift (but not a change in shape) for the spin-resolved conductance step in $g_{\sigma}$ by $\frac{\sigma}{2} \widetilde{B}$ (see Figs. S6b-c). Nevertheless, since Eqs. (S6) and (S5) together imply

$$
\mathcal{T}_{\sigma}^{0}\left(\widetilde{V}_{\mathrm{c}}, \widetilde{B}\right)=1-\mathcal{T}_{\bar{\sigma}}^{0}\left(-\widetilde{V}_{\mathrm{c}}, \widetilde{B}\right)
$$

the full conductance remains antisymmetric w.r.t. the point $\mathcal{T}_{\sigma}^{0}(0, \widetilde{B})=0.5$ even for finite $\widetilde{B}$ (see Fig. S6a):

$$
g^{0}\left(\widetilde{V}_{\mathrm{c}}, \widetilde{B}\right)=1-g^{0}\left(-\widetilde{V}_{\mathrm{c}}, \widetilde{B}\right) .
$$

Eq. (S7) also implies that the bare shot noise, $\mathcal{N}^{0}$, is symmetric w.r.t. $\widetilde{V}_{\mathrm{c}}=0$, or $g=0.5$ (see Fig. S6j).

The above antisymmetry of $g\left(\widetilde{V}_{\mathrm{c}}\right)$ w.r.t. $\widetilde{V}_{\mathrm{c}}=0$ is broken in the presence of interactions, in a manner that becomes increasingly more pronounced with increasing field, see Figs. S6d and S6g, for $U / \tau=0.2$ and 0.45 , respectively. In the latter case the broken antisymmetry is visible already at zero field, in that the fRG conductance curve shows a slight 0.7-shoulder, in agreement with experiment (cf. Fig. A2e). This shoulder at $\widetilde{B}=\widetilde{T}=0$ occurs because the interaction-induced increase of the effective potential barrier is enhanced by the van Hove ridge in the local density of states (LDOS) and hence is nonuniform in $\widetilde{V}_{\mathrm{c}}$ (see the main article for a detailed explana-

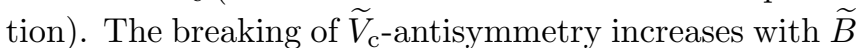
because (exchange) interactions amplify the field-induced asymmetry in the population of spin-up and -down electrons in the CCR, in particular near the top of the barrier: a small $\widetilde{B}$-induced surplus of spin-up electrons leads to a significantly increased Hartree barrier, and more so for spin-down electrons than for spin-up electrons (due to the Pauli principle), causing a strong decrease of $g_{\downarrow}$ relative to $g_{\uparrow}$. This effect, whose strength increases with $U$ (compare 2nd and 3rd columns of Fig. S6) results in the field-induced strengthening of the 0.7-shoulder that is characteristic of the 0.7-anomaly, and its evolution into a double step for large fields.

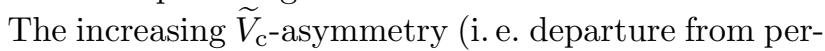
fect antisymmetry) in $g_{\sigma}\left(\widetilde{V}_{\mathrm{c}}\right)$ as $\widetilde{B}$ increases is also reflected in the shot noise factor $\mathcal{N}(g)$ [Eq. (S4)], see Figs. S6k and S6l, for $U / \tau=0.2$ and 0.45 , respectively. For zero applied field, $\mathcal{N}(g)$ is symmetric w.r.t. $g=0.5$; this follows directly from the form of Eq. (S4) (which holds whenever a Fermi-liquid description applies), and our assumption that there is no spontaneous breaking of spin symmetry at $\widetilde{B}=0$, implying $g_{\uparrow}=g_{\downarrow}$. With increasing field, $\mathcal{N}(g)$ develops an $g$-asymmetry w.r.t. $g=0.5$, being somewhat suppressed in the range $g>0.5$ relative to its values in the range $g<0.5$. This field-induced $g$-asymmetry is in good qualitative agreement with the experimental measurements of the noise factor by DiCarlo et al., cf. Fig. 4(d) of Ref. 13. Note, though, that the measured noise factor shows an $g$-asymmetry even at zero field, in contrast to our fRG predictions; we believe that this remnant $g$-asymmetry is a finite-temperature effect that will gradually disappear if the experimental temperature is lowered further. Reproducing this behavior explicitly by a finite-temperature calculation of the 

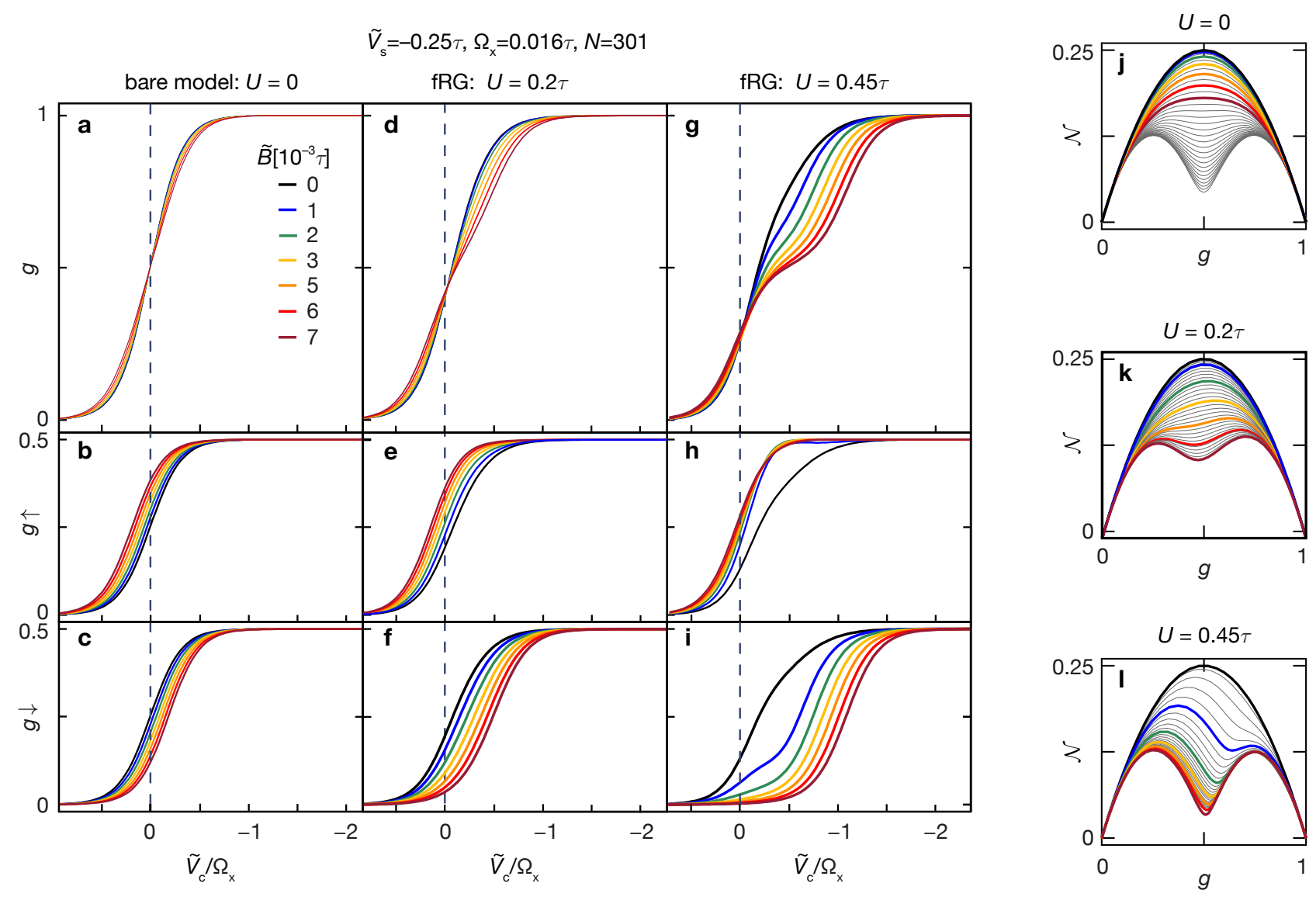

Figure S6: Comparison of results for model I, for its bare $U=0$ version (first column), or treated using static fRG for $U / \tau=0.2$ and 0.45 (second and third columns, respectively). The top, middle and bottom rows show, respectively, the full QPC conductance $g=g_{\uparrow}+g_{\downarrow}$ and its spin-resolved contributions $g_{\uparrow}$ and $g_{\downarrow}$, all plotted as functions of $\widetilde{V}_{\mathrm{c}} / \Omega_{x}$ for several values of magnetic field $\widetilde{B}$. The fourth column shows a similar comparison for the shot noise factor $\mathcal{N}$ [Eq. (S4)], plotted as function of $g$.

noise factor for our model is left as a task for future study.

\section{B. Compressibility and charge susceptibility}

Recently, Smith et al. ${ }^{14}$ have experimentally studied the compressibility of the electron gas of a QPC. In particular, they measured the $V_{\mathrm{c}}$-dependence of the compressibility in the vicinity of the 0.7 -anomaly and studied its evolution with increasing temperature and magnetic field. The compressibility is a measure of the density of states at the chemical potential. In a QPC geometry, its $V_{\mathrm{c}}$-dependence is thus governed by that of the LDOS maxima at the bottom of the $1 \mathrm{D}$ band, i. e. by the van Hove ridge discussed in detail in the main article and in Sec. S-4 C below (see the yellow ridges in Fig. A1b and Fig. S10d); and its $B$-dependence is governed by the spin splitting of this van Hove ridge.

Within our model, the compressibility can be associ- ated with the charge susceptibility of the CCR,

$$
\chi_{\mu}=\frac{d n_{\mathrm{tot}}}{d \mu}, \quad n_{\mathrm{tot}}=\sum_{j \sigma}^{\mathrm{CCR}} n_{j \sigma},
$$

where $n_{\text {tot }}$ is the total charge in the CCR and $\mu$ the chemical potential. Figs. S7a and S7b show zero-temperature fRG results for the conductance $g\left(\widetilde{V}_{\mathrm{c}}\right)$ and the charge susceptibility $\chi_{\mu}\left(\widetilde{V}_{\mathrm{c}}\right)$, respectively. The results exhibit a number of features, enumerated below, that are qualitatively consistent with features observed by Smith et $a l .{ }^{14}$.

Consider first the noninteracting case, $U=0$ (black dashed lines for $g^{0}$ and $\left.\chi_{\mu}^{0}\right)$ : When $\widetilde{V}_{\mathrm{c}}$ is lowered past 0 , the bare charge susceptibility $\chi_{\mu}^{0}\left(\widetilde{V}_{\mathrm{c}}\right)$ in Fig. S7b traverses a single broad peak, aligned with the center of the corresponding conductance step in Fig. S7a. This peak arises because the bare charge susceptibility equals the bare total density of states at the chemical potential [cf. 


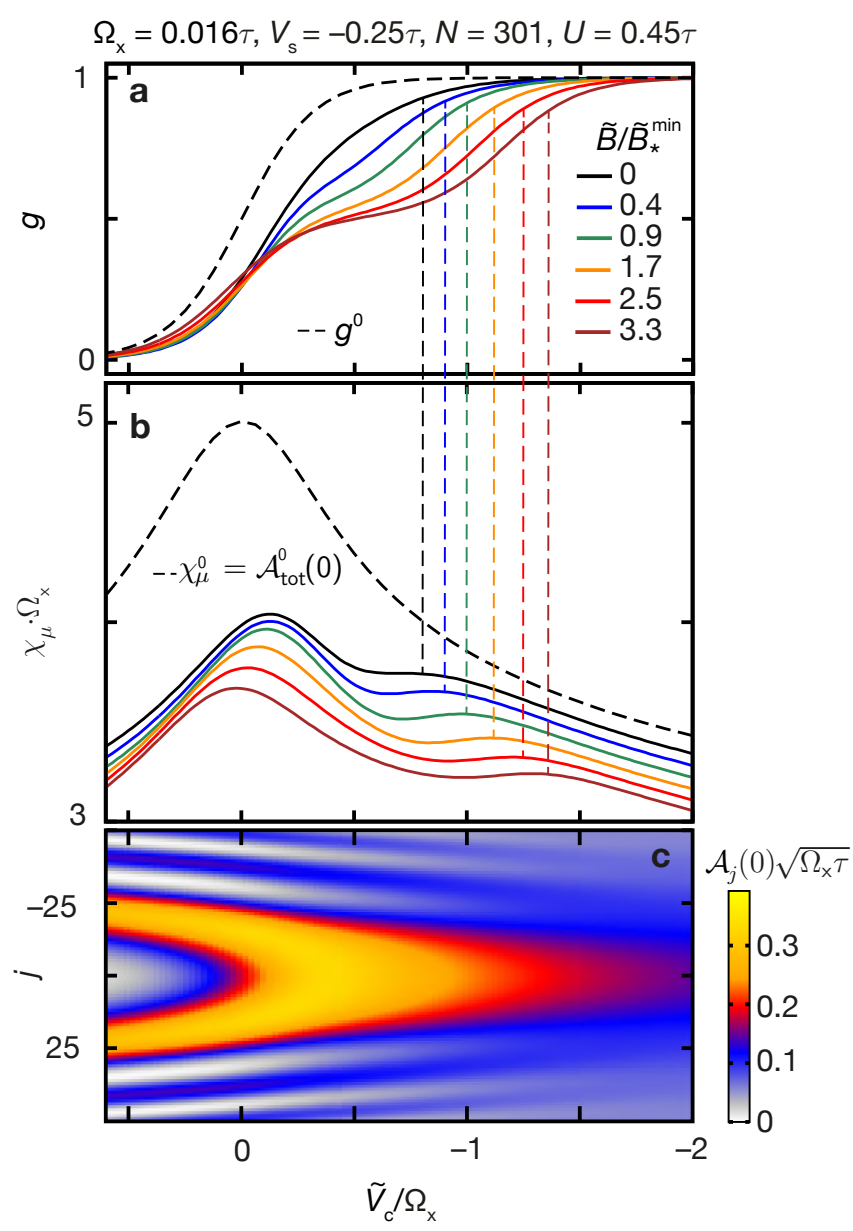

Figure S7: Charge Susceptibility. Static fRG results (model I) for (a) the conductance $g\left(\widetilde{V}_{\mathrm{c}}\right)$ and (b) the charge susceptibility $\chi_{\mu}\left(\widetilde{V}_{\mathrm{c}}\right)\left[\right.$ [Eq. S9] as function of $\widetilde{V}_{\mathrm{c}}$, calculated for six values of $\widetilde{B}$ at a fixed $\widetilde{V}_{\mathrm{s}}$ and $\widetilde{T}=0$. Black dashed lines in $\mathbf{a}$ and $\mathbf{b}$ show the bare $(U=0, \widetilde{B}=0)$ curves, $g^{0}$ and $\chi_{\mu}^{0}=\mathcal{A}_{\mathrm{tot}}^{0}(0)$, respectively. Vertical dashed lines are a guide for the eyes and mark the weak shoulder or second maximum of $\chi_{\mu}\left(\widetilde{V}_{\mathrm{c}}\right)$. c, The full $(U \neq 0)$ LDOS at the chemical potential, $\mathcal{A}_{j}(0)$, as function of gate voltage $\widetilde{V}_{\mathrm{c}}$ and site index $j$.

Eq. (S39)],

$$
\chi_{\mu}^{0}=\sum_{j \sigma}^{\mathrm{CCR}} \mathcal{A}_{j \sigma}^{0}(0)=\mathcal{A}_{\mathrm{tot}}^{0}(0),
$$

which traverses a peak when the spin-degenerate van Hove ridge is lowered past $\mu$. For nonzero $U$ but still $\widetilde{B}=0$ (black solid lines), $\chi_{\mu}\left(\widetilde{V}_{\mathrm{c}}\right)$ is reduced, since interactions tend to counteract the (infinitesimal) increase in charge induced by an (infinitesimal) increase in $\mu$ [Eq. (S9)]. This reduction occurs in such a way that (i) $\chi_{\mu}\left(\widetilde{V}_{\mathrm{c}}\right)$ retains a dominant peak, with (ii) a weak shoulder developing on its right (even though $\widetilde{B}=0$ ), roughly aligned with the roll-over of $g\left(\widetilde{V}_{\mathrm{c}}\right)$ towards the first conductance plateau. This shoulder arises because when $\widetilde{V}_{\text {c }}$ decreases into the open-channel regime, the van Hove ridge apex drops so far below $\mu$ that $\mathcal{A}_{j}(0)$, the LDOS at $\mu$, decreases rapidly (Fig. S7c). As a result, its interaction-enhancing effects, and hence also the Coulomb-blockade reduction in $\chi_{\mu}$, weaken rapidly, resulting in a shoulder in $\chi_{\mu}$.

The colored lines in Fig. S7 show the evolution of the conductance $g(\widetilde{B})$ and charge susceptibility $\chi_{\mu}\left(\widetilde{V}_{\mathrm{c}}\right)$ with magnetic field for $U=0.45 \tau$. While the conductance step evolves into the familiar spin-split double step with increasing field (Fig. S7a), (iii) the dominant peak in $\chi_{\mu}\left(\widetilde{V}_{\mathrm{c}}\right)$ (Fig. S7b) remains aligned with the center of the first conductance step, while (iv) the shoulder in $\chi_{\mu}\left(\widetilde{V}_{\mathrm{c}}\right)$ develops into a weak peak that shifts towards the right, remaining roughly aligned with the roll-over to the second conductance plateau (as indicated by dashed colored lines between Figs. S7a and S7b). This reflects the field-induced spin-splitting of the van Hove ridge into two spin-resolved sub-ridges, which get lowered past $\mu$ at different $\widetilde{V}_{\mathrm{c}}$-values. As a result, $(\mathrm{v}) \chi_{\mu}\left(\widetilde{V}_{\mathrm{c}}\right)$ develops a weak minimum between the two peaks.

Features (i)-(v) can also be found, on a qualitative level, in Figs. 2 and 3(a) of Smith et al.. Their measured signal, called $d V_{\mathrm{sg}} / d V_{\text {mid }}$ there, has minima when the compressibility has maxima, and vice versa. In their Fig. 2(a), the red curve shows a strong dip at $V_{\text {mid }}=0.14 \mathrm{~V}$ and a very weak minimum at $0.22 \mathrm{~V}$. We associate these, respectively, with the dominant peak (i) and the weak shoulder (ii) in $\chi_{\mu}\left(\widetilde{V}_{\mathrm{c}}\right)$ discussed above. In their Fig. 2(b), the two dips in the red curve at $V_{\text {mid }}=0.12 \mathrm{~V}$ and $0.19 \mathrm{~V}$, correspond, respectively, to the two maxima mentioned in (iii) and (iv) above. And in their Fig. 3(a), the peak marked by an arrow corresponds to the dip mentioned in (v). We thus conclude that the measured compressibility maxima accompanying the conductance steps are indeed due to maxima in the density of states at the band bottom, as suggested by Smith et al. themselves (and in Ref. 16). This supports our contention that van Hove ridges play a central role in the physics of the 0.7-anomaly. By implication it also confirms the presence of the "quasi-bound states" advocated by Meir and collaborators ${ }^{17-19}$, provided that we identify their "quasi-bound states" with our van Hove ridges - as argued in Sec. S-4 E below, both names refer to the same peaked structures in the LDOS.

This identification was not clear at the time of writing of Ref. 14, however. Instead, Smith et al. argued that they see "no evidence of the formation of the quasibound state predicted by the Kondo model". This statement was based on a comparison of their $B=0$ data for $d V_{\mathrm{sg}} / d V_{\text {mid }}$ to simulations ${ }^{16}$ using density-functional theory (DFT) combined with the local spin density approximation (LSDA). These data and the simulation results are shown, respectively, as black and red curves in Fig. 4(b) of Ref. 14. The simulations yielded an additional strong dip [indicated by an arrow in Fig. 4(b)], 
aligned with the onset of the conductance plateau, that had no counterpart in the measured data. We suspect that this additional strong dip might be an artefact of the tendency of DFT+LSDA calculations, when initialized using a small nonzero magnetic field ${ }^{19,20}$, to yield a nonzero spin polarization in regions where the spin susceptibility is large (as is the case in the QPC). We assert, however, that at $B=0$ the spin polarization is strictly zero (in contrast to views expressed in Refs. 7,8,14), since this is a prerequisite for understanding the Fermi-liquid properties discussed in the main article. Our fRG calculations for $\widetilde{B}=0$ thus assume zero spin polarization from the outset. Remarkably they yield, instead of the strong additional peak found by DFT+LSDA, only the weak shoulder (ii) mentioned above, which is consistent with the compressibility data of Smith et al. Further arguments in support of the absence of spontaneous spin polarization at zero field are offered at the end of Sec. S$2 \mathrm{C}$.

\section{PART II: THEORETICAL DETAILS}

\section{S-4. MODELS USED FOR BARRIER SHAPE}

In the course of our studies of the 0.7-anomaly, we have explored many different parametrizations of smooth, symmetric QPC barrier shapes. We found that as long as the barrier top is parabolic, characterized by a barrier height $\widetilde{V}_{\text {c }}$ (w.r.t. to the chemical potential) and a curvature parameter $\Omega_{x}$, the details of the parametrization of the barrier do not matter.

In this section we present the details of two different parametrizations for parabolic barriers, to be called "model I" and "model II", whose results for QPC properties are fully equivalent when expressed as functions of $\widetilde{V}_{\mathrm{c}}$ and $\Omega_{x}$. Both models use the same Hamiltonian, choice of chemical potential and local interaction strength $U_{j}$, specified in Sec. S-4 A, but differ in their choices for the hopping amplitude $\tau_{j}$ (which is $j$-independent for Model I but not for Model II) and the on-site potential $E_{j}$.

Model I is presented in Sec. S-4 B: its hopping amplitude is $j$-independent, $\tau_{j}=\tau$, and the barrier shape is specified by parametrizing $E_{j}$ in terms of a central gate voltage $\widetilde{V}_{\mathrm{c}}$ and a side gate voltage $\widetilde{V}_{\mathrm{s}}$. It is designed to allow a theoretic study of the crossover between a Kondo quantum dot (KQD) and a QPC by continuously deforming the $1 \mathrm{D}$ potential from a double-barrier to a single-barrier shape (see Figs. S9c and S9d below, respectively). (The results of a corresponding study will be published elsewhere ${ }^{1}$.) Here we use model I to calculate numerous QPC properties presented in various parts of the supplementary material (Figs. S6, S7, S10, S11, S13 and S14). Moreover, model I allows instructive insights into the similarities and differences between the bare density of states of a QD and a QPC, which are key to understanding the similarities and differences between the Kondo effect and the 0.7-anomaly, as briefly discussed in Sec. S-4 C.

For model II, presented in Sec. S-4 D, $\tau_{j}$ depends nontrivially on $j$, and the barrier shape is specified solely in terms of a central gate voltage $\widetilde{V}_{\text {c }}$ and the barrier curvature $\Omega_{x}$ (adjusted via the length $N$ of the CCR, but without reference to a side gate voltage). Compared to model I, model II has technical advantages when treated using SOPT (as explained below). For clarity, model II was used for all numerical results (both from fRG and SOPT) presented in the main article. It was also used for Figs. S2, S4, S12, S16 in the supplementary material. We emphasize that the results obtained using models I and II are qualitatively consistent.

To conclude our introductory comments on the models used here, we remark that the idea of studying the 0.7anomaly using an effective 1D model with a smoothly varying QPC potential and local interactions has of course been pursued previously by numerous authors. For example, a model with local exchange interactions was studied in Refs. 21 and 22, a model with an unscreened Coulomb interaction in Ref. 6, and a model with a point like interaction restricted to the center of the QPC potential in Ref. 23. Our work is similar in spirit to these, but our use of fRG allows us to treat the effects of interactions more systematically than Refs. 21 and 6, and for longer chains than Ref. 22, which also did not have access to the limit $T \rightarrow 0$. Works based on $2 \mathrm{D}$ or $3 \mathrm{D}$ density-functional theory calculations ${ }^{16-20}$ treat the potential landscape more realistically than we do, but at the expense of not treating correlation effects as accurately as fRG does. In particular, our fRG treatment allows accurate predictions for the conductance at zero temperature, which is beyond the scope of all previous treatments. Moreover, our SOPT calculations at finite source-drain voltages are first to give a detailed description of the origin of the ZBP.

\section{A. Hamiltonian, chemical potential, $U_{j}$}

The model Hamiltonian defined in the main article,

$$
\begin{aligned}
\hat{H}= & \sum_{j \sigma}\left[E_{j \sigma} \hat{n}_{j \sigma}-\tau_{j}\left(d_{j+1 \sigma}^{\dagger} d_{j \sigma}+\text { h.c. }\right)\right]+ \\
& \sum_{j} U_{j} \hat{n}_{j \uparrow} \hat{n}_{j \downarrow}
\end{aligned}
$$

with $E_{j \sigma}=E_{j}-\frac{\sigma}{2} \widetilde{B}$, is depicted schematically in Fig. S8. It shows a tight-binding chain divided into two semiinfinite, non-interacting, uniform leads on the left and right, connected to the central constriction region (CCR), 


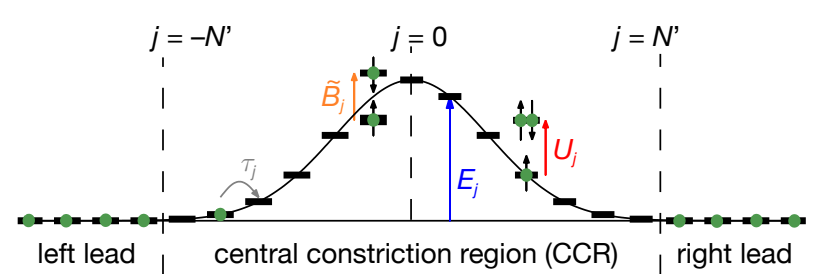

Figure S8: Schematic depiction of the one-dimensional model of Eq. (S11) (for a QPC barrier shape). It represents an infinite tight-binding chain with hopping matrix element $\tau_{j}$ (gray); the prescribed local potential $E_{j}$ (blue) and on-site interaction $U_{j}$ (red) are nonzero only within a central constriction region (CCR) of $N=2 N^{\prime}+1$ sites. The CCR is connected to two semi-infinite non-interacting leads on the left and right. A homogeneous Zeeman magnetic field $\widetilde{B}$ (orange) can be switched on along the whole chain.

consisting of an odd number $N=2 N^{\prime}+1$ of sites centered on $j=0$. The lattice does not represent actual atomic sites, but instead is merely used to obtain a discrete, coarse-grained description of transport in the lowest subband. The position-dependent parameters $U_{j}$ and $E_{j}$, nonzero only within the CCR, are taken to vary slowly on the scale of the lattice spacing $a$. (We set $a=1$ in our calculations.)

Choice of $\mu$ : Since the chemical potential is a property of the bulk, we begin by considering our model for $E_{j}=U_{j}=0$ and $\tau_{j}=\tau$, representing a bulk tightbinding chain (infinite, homogeneous). The eigenergies $\epsilon_{k}$ corresponding to wave number $k$ have dispersion

$$
\epsilon_{k}=-2 \tau \cos (k a) \in[-2 \tau, 2 \tau],
$$

plotted in Fig. S9a. To describe the phenomena of present interest, the chemical potential $\mu$ should lie somewhere within this band, not too close to its edges; the precise value does not matter. All our numerical calculations (fRG and SOPT) used $\mu=0$, implying half-filled leads; but for the sake of generality, we keep $\mu$ arbitrary below, particularly in Figs. S9a,b and S10a,b.) The energy difference between the chemical potential and the bulk band bottom defines the bulk Fermi energy,

$$
\varepsilon_{\mathrm{F}}=2 \tau+\mu \quad(>0) .
$$

Choice of $U_{j}$ : In choosing a purely on-site interaction in Eq. (S11), we implicitly assume that screening is strong enough to render the interaction short-ranged. (A more realistic treatment of screening is beyond the scope of this work.) We set the on-site interaction $U_{j}$ equal to $U$ throughout the CCR, except near its edges, where it drops smoothly to zero to avoid spurious backscattering effects (Fig. S9e):

$$
U_{j}=\left\{\begin{array}{lll}
0, & \forall & N^{\prime} \leq|j|, \\
U \exp \left[-\frac{\left(\frac{j}{N^{\prime}}\right)^{6}}{1-\left(\frac{j}{N^{\prime}}\right)^{2}}\right] & \forall & |j| \leq N^{\prime} .
\end{array}\right.
$$

$U$ is to be regarded as an effective parameter, whose value is influenced by the transverse modes not treated explicitly in our model. In particular, the effect of increasing
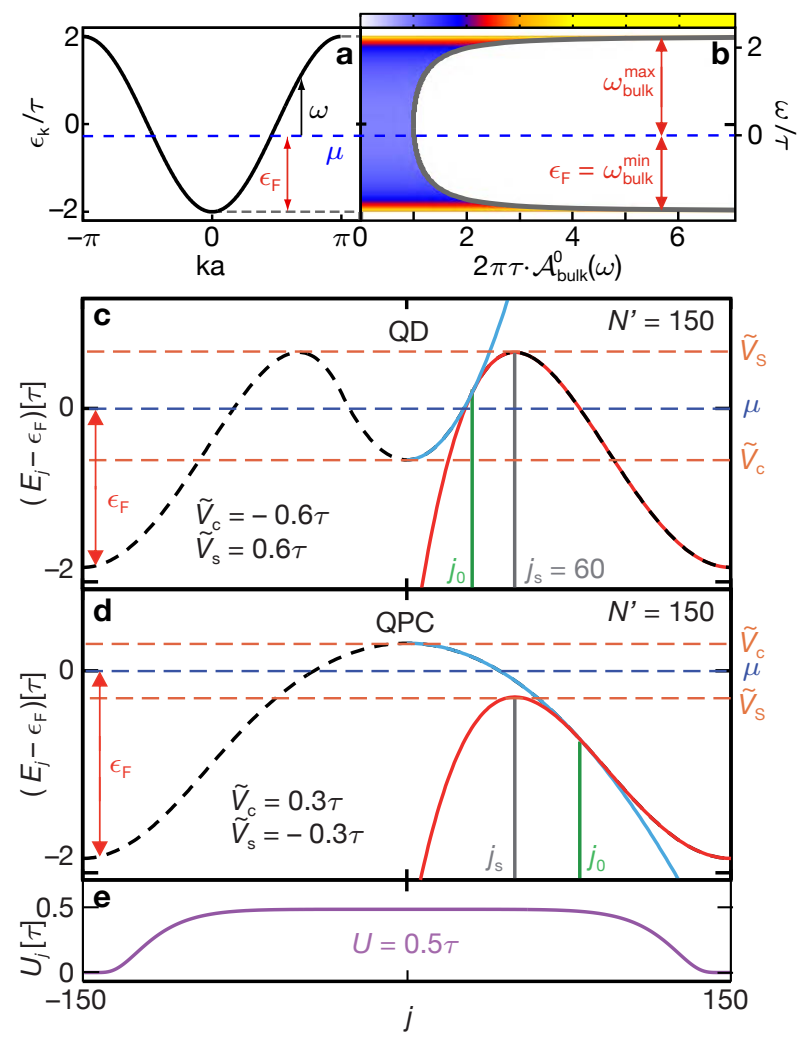

Figure S9: a, Dispersion relation $\epsilon_{k}$ vs. $k$ [Eq. (S12)] for a bulk non-interacting tight-binding chain without magnetic field (infinite, homogeneous, $E_{j \sigma}=U_{j}=0$ ). The filling factor in the leads is controlled by the global chemical potential $\mu$ (blue dashed line); it is here drawn at $\mu \neq 0$ for generality, although our fRG calculations use $\mu=0$. b. The corresponding $j$-independent bulk LDOS [Eq. (S19)], shown both as $\mathcal{A}_{\text {bulk }}^{0}(\omega)$ (on $x$-axis) versus $\omega=\epsilon_{k}-\mu$ (on $y$-axis), and using a color scale. The distance from the chemical potential to the bulk band bottom $\omega_{\text {bulk }}^{\min }$ is $\varepsilon_{\mathrm{F}}=2 \tau+\mu=-\omega_{\text {bulk }}^{\min }(>0)$. c and $\mathbf{d}$, Model I: The one-dimensional potential $E_{j}$ of Eq. (S15) (thick dashed black line) for a QD potential $\left(\widetilde{V}_{\mathrm{s}}>\widetilde{V}_{\mathrm{c}}\right)$ and a QPC potential $\left(\widetilde{V}_{\mathrm{c}}>\widetilde{V}_{\mathrm{s}}\right)$, respectively. In the outer region of the CCR $\left(j_{0} \leq|j| \leq N^{\prime}\right), E_{j}$ is described by quartic polynomial, in the inner region $\left(|j|<j_{0}\right)$ by a quadratic one (thin red and blue lines, respectively, shown only for $j>0$.) For given $N^{\prime}, j_{\mathrm{s}}, \widetilde{V}_{\mathrm{s}}$ and $\widetilde{V}_{\mathrm{c}}$, the parameters $j_{0}$ and $\bar{\Omega}_{x}$ are adjusted such that the resulting potential $E_{j}$ depends smoothly on $j$ throughout the CCR. e, The on-site interaction $U_{j}$ of Eq. (S14).

the top gate voltage $V_{\mathrm{t}}$ can be mimicked by increasing $U$ [Eq. (S1)], as will be discussed in more detail in Sec. S5 C. We typically take $U$ to be somewhat smaller than the maximum value of the inverse bare LDOS, since if $U \cdot \max \left[\mathcal{A}_{j}^{0}(\omega)\right]$, is too large, the fRG calculations do not converge. We remark that we have also explored the option of taking $U_{j}$ to be proportional to $E_{j}$, or of taking the range of sites where $U_{j}=U$ to be several times larger than that where $E_{j} \neq 0$. Such modifications change details of the results, such as the precise shape of the con- 
ductance $g\left(\widetilde{V}_{\mathrm{c}}, \widetilde{V}_{\mathrm{s}}\right)$ as function of $\widetilde{V}_{\mathrm{c}}$ or $\widetilde{V}_{\mathrm{s}}$, but not the qualitative trends discussed in the main article, as long as $U_{j}$ drops smoothly to zero near the edges of the CCR.

\section{B. Model I}

For model I, we choose the the hopping amplitude to be $j$-independent, $\tau_{j}=\tau$, while the on-site potential $E_{j}$ describes a reflection-symmetric barrier within the CCR. Its shape is tunable between a double barrier describing a QD (Fig. S9c) and a single barrier describing a QPC (Fig. S9d) (thick dashed black lines). We have parametrized it as follows:

$$
E_{j}=\left\{\begin{array}{lr}
0, & \forall|j| \geq N^{\prime}, \\
\left(\widetilde{V}_{\mathrm{s}}+\varepsilon_{\mathrm{F}}\right)\left[2\left(\frac{|j|-N^{\prime}}{j_{\mathrm{s}}-N^{\prime}}\right)^{2}-\left(\frac{|j|-N^{\prime}}{j_{\mathrm{s}}-N^{\prime}}\right)^{4}\right], & \\
& \forall j_{0} \leq|j| \leq N^{\prime}, \\
\widetilde{V}_{\mathrm{c}}+\varepsilon_{\mathrm{F}}+\frac{\bar{\Omega}_{x}^{2} j^{2}}{4 \tau} \operatorname{sgn}\left(\widetilde{V}_{\mathrm{s}}-\widetilde{V}_{\mathrm{c}}\right), & \forall 0 \leq|j|<j_{0} .
\end{array}\right.
$$

The sites $\pm j_{0}$ divide the CCR into two "outer regions", where the potential is a quartic polynomial in $j$, and an "inner region", where it is quadratic in $j$. In the latter, the magnitude of the curvature is governed by the parameter $\bar{\Omega}_{x}(\geq 0)$, which has units of energy. (The quadratic term for the inner region was chosen to have the form $\frac{1}{2} m \omega_{x}^{2} x^{2}$ used in Ref. 5 , with $\omega_{x}=\bar{\Omega}_{x} / \hbar, x=a j$ and $m=\hbar^{2} /\left(2 \tau a^{2}\right)$ corresponding to the effective mass at the bottom of a tight-binding chain.) The shape of $E_{j}$ is controlled by four independent parameters: (i) $N^{\prime}$, which sets the halfwidth of the CCR; (ii) $j_{s}$, which governs the width of the outer flanks of the potential; (iii) $\widetilde{V}_{\mathrm{s}}$ and (iv) $\widetilde{V}_{\mathrm{c}}$, which give the potential's height w.r.t. $\varepsilon_{\mathrm{F}}$ at the sites $j= \pm j_{\mathrm{s}}$ and 0 , respectively:

$$
E_{ \pm j_{\mathrm{s}}}=\widetilde{V}_{\mathrm{s}}+\varepsilon_{\mathrm{F}} ; \quad E_{j=0}=\widetilde{V}_{\mathrm{c}}+\varepsilon_{\mathrm{F}} .
$$

Once the four parameters $N^{\prime}, j_{\mathrm{s}}, \widetilde{V}_{\mathrm{s}}$ and $\widetilde{V}_{\mathrm{c}}$ have been specified, the dependent parameters $j_{0}$ and $\bar{\Omega}_{x}$ are chosen such that $E_{j}$ is a smooth function of $j$ at the boundaries $\pm j_{0}$ between the inner and outer regions.

An electron incident at the chemical potential has energy $\varepsilon_{\mathrm{F}}$ w.r.t. to the bulk band bottom and hence sees a relative potential of height $E_{j}-\varepsilon_{\mathrm{F}}$ at site $j$. For $\widetilde{V}_{\mathrm{s}}>\widetilde{V}_{\mathrm{c}}$, the relative potential describes a QD potential with two maxima of height $\widetilde{V}_{\mathrm{s}}$ at $j= \pm j_{\mathrm{s}}$ and a local parabolic minimum of height $\widetilde{V}_{\mathrm{c}}$ at $j=0$. For $\widetilde{V}_{\mathrm{c}}>\widetilde{V}_{\mathrm{s}}$ (the case of present interest), it describes a QPC potential with a single parabolic maximum at $j=0$, of height $\widetilde{V}_{\mathrm{c}}$. The crossover point between QD and QPC lies at $\widetilde{V}_{\mathrm{s}}=\widetilde{V}_{\mathrm{c}}$ (for which $\bar{\Omega}_{x}=0$ ). Evidently $\widetilde{V}_{\mathrm{c}}$ and $\widetilde{V}_{\mathrm{s}}$ respectively mimick the role of the voltages applied to the central gates $\left(V_{\mathrm{c}}\right)$ and side gates $\left(V_{\mathrm{s}}\right)$ in the experiment (with $\left.\widetilde{V}_{\mathrm{c}, \mathrm{s}} \propto-|e| V_{\mathrm{c}, \mathrm{s}}\right)$.

\begin{tabular}{|c||c|c|c|c|c|}
\hline Figure & $N^{\prime}$ & $\widetilde{V}_{\mathrm{c}}[\tau]$ & $\widetilde{V}_{\mathrm{s}}[\tau]$ & $\Omega_{x}$ & $j_{\mathrm{s}}$ \\
\hline \hline Fig. S6 & 150 & -0.035 to 0.015 & -0.25 & 0.016 & 60 \\
Fig. S7 & 150 & -0.032 to 0.01 & -0.25 & 0.016 & 60 \\
Fig. S9a-b & 150 & -2 & -2 & 0 & - \\
Fig. S9c & 150 & -0.6 & 0.6 & 0.0416 & 60 \\
Fig. S9d & 150 & 0.3 & -0.3 & 0.023 & 60 \\
Fig. S10a & 150 & -0.5 & 0.5 & 0.037 & 60 \\
Fig. S10b & 150 & 0.5 & -0.5 & 0.027 & 60 \\
Fig. S10c & 150 & -0.025 & 0 & 0.005 & 60 \\
Fig. S10d & 150 & 0.008 & -0.25 & 0.016 & 60 \\
Fig. S11 & 150 & 0 & -0.25 & 0.016 & 60 \\
Fig. S13 & 150 & -0.016 to 0.006 & -0.25 & 0.016 & 60 \\
Fig. S14 & 150 & -0.02 to 0.02 & -0.25 & $0.016-0.048$ & 60
\end{tabular}

Table I: Parameters used for model I [defined in Eq. (S15)] for the fRG results shown in various figures of the supplementary information.

We emphasize that the QPC barriers studied in this work are all parabolic near the top. For quantitative studies of the 0.7-anomaly using model I, we fix $N^{\prime}, j_{\mathrm{s}}$ and $V_{\mathrm{s}}$, and tune the QPC from closed to open by lowering $\widetilde{V}_{\text {c }}$ past 0 , at which the bare $(U=0)$ conductance $g^{0}$ equals 0.5 . The width of the conductance step [see Fig. A1k, and Eq. (S30)] is governed by the curvature parameter at this point, $\Omega_{x}=\left.\bar{\Omega}_{x}\right|_{\widetilde{V}_{c}=0}$, which we will simply call "curvature" henceforth. $\left(\bar{\Omega}_{x}\right.$ itself changes slightly during this crossover, but for the barrier shapes used in this work this change is typically less than $10 \%$ between $\widetilde{V}_{\mathrm{c}}= \pm \Omega_{x}$.) The curvature $\Omega_{x}$ also governs the exponential $\widetilde{V}_{\mathrm{c}}$-dependence of $\widetilde{B}_{*}$ [Eq. (S35a)]. Note that formulas such as Eqs. (S30) and (S35a) would change for non-parabolic QPC barriers, e.g. barriers with a flat top. Studying the 0.7 -anomaly for such situations would be an interesting extension of the present work, which we leave for the future ${ }^{24}$.

\section{Bare local density of states (LDOS)}

In the main article we have argued that geometry strongly influences the 0.7 -anomaly, via its effect on the local density of states (LDOS) and the van Hove ridge of the latter. Here we elaborate this in detail, by discussing the geometry-dependence of the noninteracting LDOS (for model I). We do so not only for the QPC barrier shape of present interest, but also for a QD barrier shape. This lays the ground for a subsequent comparison, presented in Sec. S- $4 \mathrm{E}$ below, of the LDOS structures of a QPC and a QD, which sheds light on the similarities and differences between the 0.7-anomaly and the Kondo effect.

The LDOS per spin species $\sigma$ at energy $\omega$ (measured 

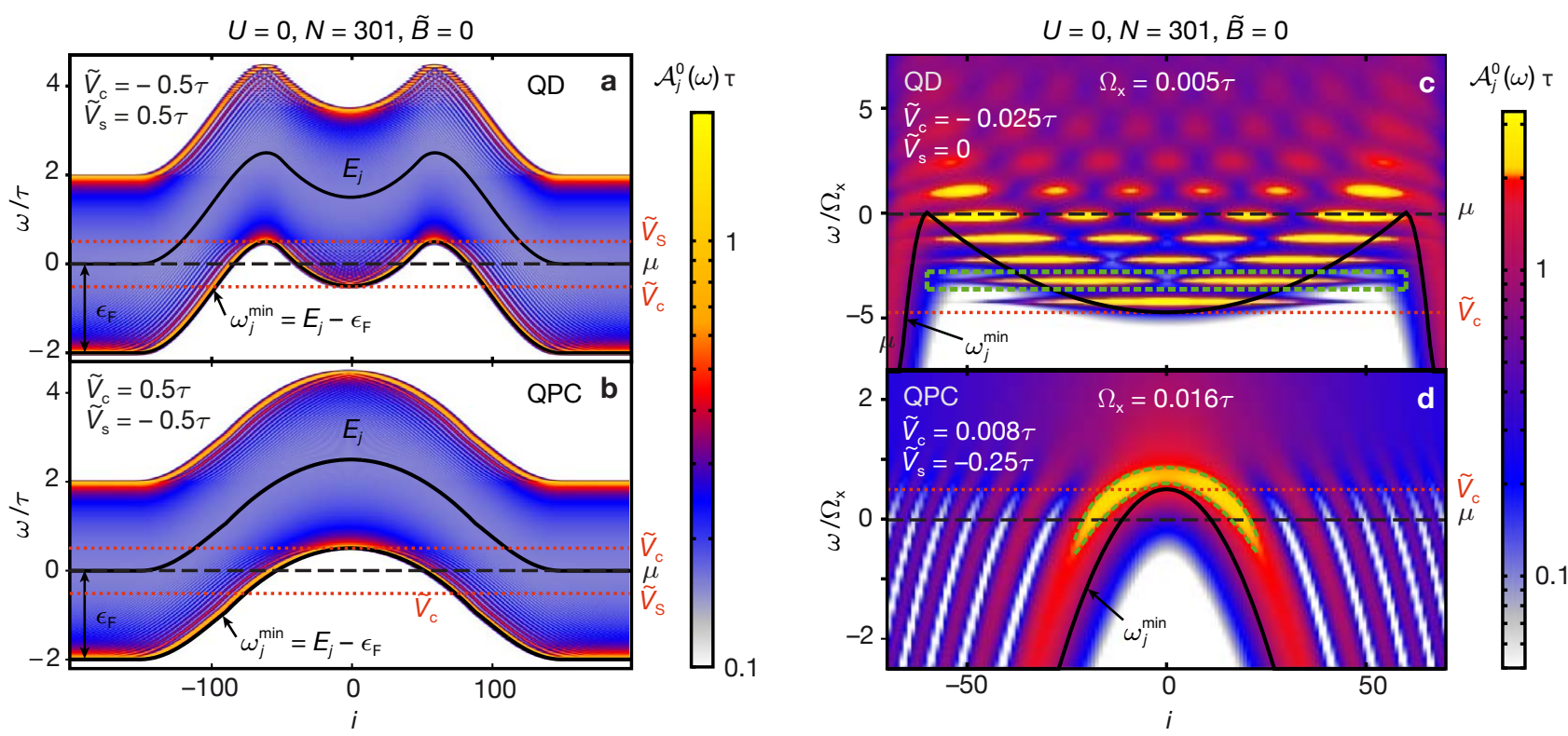

Figure S10: Model I: Noninteracting zero-field LDOS per spin species of a,c, a QD, and b,d, a QPC, for potential shapes shown by thin black lines (marked by black arrows) for $\omega_{j}^{\min }=E_{j}-\varepsilon_{\mathrm{F}}$. (The logarithmic color scale shows $\mathcal{A}_{j}^{0}(\omega)$ smeared by a Lorentzian of width $\delta=0.001 \tau$, in order to render very sharp structures visible.) Panels c,d focus on the central region of the CCR and energies close to $\mu$ (black dashed lines); the $\widetilde{V}_{\mathrm{c}^{-}}$and $\widetilde{V}_{\mathrm{s}}$-choices differ from those used in a,b. Thin green dashed lines in c,d indicate the shape of the "LDOS ridges" discussed in the text. For the KQD, they enclose an area in the $j$ - $\omega$ plane on which the corresponding LDOS ridge has weight 1 ; for the QPC, they trace a contour along which $\mathcal{A}_{j}^{0, \mathrm{QPC}}(\omega)=0.7$.

relative to the chemical potential $\mu$ ) is defined as

$$
\mathcal{A}_{j}^{\sigma}(\omega)=-\frac{1}{\pi} \operatorname{Im} \mathcal{G}_{j j}^{\sigma R}(\omega),
$$

where $\mathcal{G}_{i j}^{\sigma R}(\omega)$ is the Fourier transform of the retarded $T=0$ propagator $^{25}$,

$$
\mathcal{G}_{i j}^{\sigma R}(t)=-i \theta(t)\left\langle G\left|\left\{d_{i \sigma}(t), d_{j \sigma}^{\dagger}(0)\right\}\right| G\right\rangle,
$$

where $|G\rangle$ is the model's ground state. In this subsection we will discuss only the spin-degenerate case of zero field $(\widetilde{B}=0)$ and zero interaction $\left(U_{j}=0\right)$. We thus drop the spin index $\sigma$ (as in the main article) and instead put a superscript 0 on $\mathcal{A}_{j}^{0}(\omega)$ to denote the bare LDOS.

For $E_{j}=0$, representing an infinite, homogenous, bulk tight-binding chain, the LDOS of Eq. (S17) is $j$ independent and equal to the 1D bulk LDOS,

$$
\mathcal{A}_{\mathrm{bulk}}^{0}(\omega)=\frac{a}{\pi}\left[\frac{\partial k}{\partial \epsilon_{k}}\right]_{\epsilon_{k}=\omega+\mu} .
$$

This is nonzero only for $\omega_{\text {bulk }}^{\min }<\omega<\omega_{\text {bulk }}^{\max }$, where

$$
\omega_{\mathrm{bulk}}^{\min }=-\varepsilon_{\mathrm{F}}, \quad \omega_{\mathrm{bulk}}^{\max }=-\varepsilon_{\mathrm{F}}+4 \tau,
$$

denote the bottom and top of the band, measured w. r. t. $\mu$, respectively. Within these limits, it has the form

$$
\mathcal{A}_{\mathrm{bulk}}^{0}(\omega)=\frac{1}{\pi \sqrt{\left(\omega_{\mathrm{bulk}}^{\max }-\omega\right)\left(\omega-\omega_{\mathrm{bulk}}^{\min }\right)}},
$$

shown in Fig. S9b, featuring square-root van Hove singularities near the band edges (yellow fringes in Fig. S9b). While the upper van Hove singularity (of unoccupied states) may be viewed as an artefact of describing the lowest subband using a tight-binding chain, the lower one is realistic for effective one-dimensional geometries; it would also arise, e. g., when using a free-electron model.

Now consider a nonzero potential $E_{j}$ that is smooth on the scale of the lattice spacing, modelling a $\mathrm{QD}$ or QPC in the CCR, as shown by the thick black lines in Figs. S10a-d. The color scale in these figures indicates the corresponding $j$-dependent $\operatorname{LDOS}, \mathcal{A}_{j}^{0}(\omega)$. The latter has an $\omega$-dependence that, for fixed $j$, is reminiscent of the bulk case, but with several differences, caused by the spatial structure in $E_{j}$. First, the band edges now are $j$-dependent and follow the shape of the potential, with

$$
\omega_{j}^{\min }=E_{j}-\varepsilon_{\mathrm{F}}, \quad \omega_{j}^{\max }=E_{j}-\varepsilon_{\mathrm{F}}+4 \tau .
$$

In particular, the band bottom at the CCR center, $j=0$, is given by $\omega_{0}^{\min }=\widetilde{V}_{\mathrm{c}}$. Second, $\mathcal{A}_{j}^{0}(\omega)$ exhibits narrow fringes (visible clearly in Figs. S10a-d), due to the fact that the electronic wave functions form standing wave patterns. In the central part of the QD potential (Fig. S10a), and in the central part of the QPC potential for energies $\omega>\omega_{\text {bulk }}^{\max }$ (Fig. S10b), these standing waves correspond to bound state wave functions. (For the case of the QPC these bound states are artefacts and they are avoided in model II.) In the outer regions of both QD and QPC potentials they correspond to Friedel oscilla- 


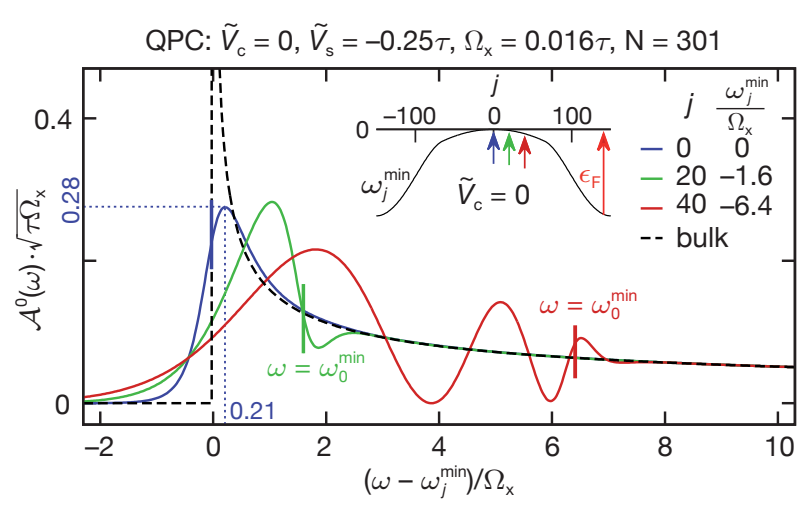

Figure S11: Inset: The band bottom $\omega_{j}^{\text {min }}$ (black line) as function of $j$, for a 301-site CCR with a parabolic QPC barrier (model I) with curvature $\Omega_{x}$ and height $\widetilde{V}_{\mathrm{c}}=\omega_{0}^{\min }=0$. Main plot: Energy dependence of the LDOS near the band bottom, showing $\mathcal{A}_{\text {bulk }}^{0}(\omega)$ (dashed), and $\mathcal{A}_{j}^{0, \mathrm{QPC}}(\omega)$ (solid) for three $j$-values near the center, all plotted as functions of $\left(\omega-\omega_{j}^{\min }\right) / \Omega_{x}$ (blue line corresponds to Fig. 1 in Ref. 23). Arrows of matching colors in the inset indicate the corresponding values of $j$ (namely 0,20 and 40). The short, heavy, colored vertical lines in the main panel indicate where the energy coincides with the barrier top, $\omega=\omega_{0}^{\mathrm{min}}$; the corresponding values of the $x$-coordinate $\left(\omega_{0}^{\min }-\omega_{j}^{\min }\right) / \Omega_{x}$ (namely $0,1.6$ and 6.4 ) give the remaining barrier height as seen from site $j$. In the bulk, $\omega_{j}^{\text {min }}=-\varepsilon_{\mathrm{F}}$. The peak of $\mathcal{A}_{j}^{0, \mathrm{QPC}}(\omega)$ lies at an energy $\omega_{j}^{\mathrm{H}}=\omega_{j}^{\mathrm{min}}+\mathcal{O}\left(\Omega_{x}\right)$. For $j=0$ it lies at $\omega_{0}^{\mathrm{H}}=0.21 \Omega_{x}$ and has height $0.28 / \sqrt{\tau \Omega_{x}}$ (dotted blue lines). Note that $\mathcal{A}_{j}^{0, \mathrm{QPC}}(\omega)$ matches $\mathcal{A}_{\text {bulk }}^{0}(\omega)$ once the energy $\omega$ lies above $\omega_{0}^{\min }$ by more than $\mathcal{O}\left(\Omega_{x}\right)$, corresponding to free propagation above the barrier.

tions. Third, the van Hove singularities are somewhat smeared out on the outer flanks of the QD, and throughout the entire QPC, in the latter case on a scale set by $\Omega_{x}$ (see Fig. S11).

For the rest of this subsection, we focus on the QPC barrier of Figs. S10b,d. (The QD barrier of Figs. S10a,c is revisited in Sec. S-4 E below, where we compare its LDOS to that of a QPC.) For a QPC, $\mathcal{A}_{j}^{0, \mathrm{QPC}}(\omega)$ depends smoothly on $\omega$ and $j$ near the center of the CCR, its weight being concentrated along a curved, broad "van Hove ridge" (framed by the green dashed lines in Fig. S10d). This ridge originates from a van Hove singularity just above the band bottom that has been pushed upward by the QPC potential barrier. The van Hove ridge has limited spatial extent when traversed at constant $\omega$, reflecting the limited spatial size of the QPC. At the outside flanks of the CCR barrier, the tails of the ridge split up into discrete fringes, representing Friedel oscillations associated with standing waves that build up near the barrier (as also seen in Figs. S10a,b). For given $j$, the $\omega$-dependence of the van Hove ridge, shown in Fig. S11, is asymmetric w.r.t. to its maximum, with a steep, exponentially decaying flank below the maximum, and above it a long tail, whose envelope decays as

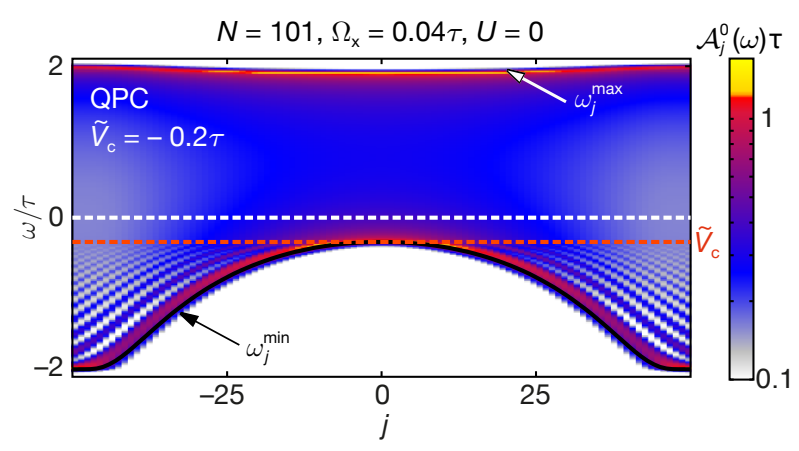

Figure S12: Noninteracting zero-field LDOS per spin species, $\mathcal{A}_{j}^{0}(\omega)$, shown on a logarithmic color scale, for the QPC model II defined by Eqs. (S22) and (S23). The thin black line (marked by black arrow) indicates the lower band edge, $\omega_{j}^{\text {min }}$ [Eq. (S24)]. The curvature of the lower and upper band edges is, respectively, negative and positive throughout the CCR, ensuring that no bound states occur.

$\left[\tau\left(\omega-\omega_{j}^{\min }\right)\right]^{-1 / 2}$, reflecting the $\omega$-asymmetry of the bulk van Hove singularity of Eq. (S19). The divergence of the latter is cut off here, due to the absence of translational invariance, on a scale set by the barrier curvature. Indeed, the maximum value taken by the van Hove peak in $\mathcal{A}_{j}^{0, \mathrm{QPC}}(\omega)$ occurs at an energy, say $\omega_{j}^{\mathrm{H}}$, that lies above the lower band edge by an amount of order $\Omega_{x}$,

$$
\omega_{j}^{\mathrm{H}}=\omega_{j}^{\min }+\mathcal{O}\left(\Omega_{x}\right) .
$$

For example, for a purely parabolic barrier top, the van Hove peak in $\mathcal{A}_{j=0}^{0, \mathrm{QPC}}(\omega)$, the LDOS at the center of the $\mathrm{QPC}$, lies at $\omega_{0}^{\mathrm{H}}=\omega_{0}^{\mathrm{min}}+0.21 \Omega_{x}$. In that case, the van Hove peak lies precisely at the chemical potential, $\omega_{0}^{\mathrm{H}}=0$, when $\widetilde{V}_{\mathrm{c}}=-0.21 \Omega_{x}$.

Eq. (S21) implies not only that the van Hove peak energy depends on $\Omega_{x}$, but also that its height (i.e. the maximum value of the LDOS) scales as $1 / \sqrt{\tau \Omega_{x}}$. As a consequence, all local quantities that depend on $\mathcal{A}_{j}^{0, \mathrm{QPC}}(\omega)$, such as the local magnetic susceptibility $\chi_{j}$, depend on $\Omega_{x}$, too. In this way they acquire an explicit dependence on the shape of the QPC barrier.

\section{Model II}

In this section we describe model II, used for all numerical results (fRG and SOPT) presented in the main article. For model II, designed to model exclusively a QPC, we have modified the choice of $E_{j}$ and $\tau_{j}$ in two minor ways relative to model I of Sec. S- 4 B, which turn out to facilitate SOPT calculations. The two changes, described below, are designed (i) to allow using a shorter CCR while maintaining a small curvature $\Omega_{x}$ at the barrier top, and (ii) to avoid the occurrence of artificial bound states in the bare density of states of the QPC (such as those seen in Fig. S10b near the upper band edge, for energies $\omega>\omega_{\text {bulk }}^{\max }$ ). 
(i) Modified potential shape: We define the onsite potential $E_{j}$ by

$$
E_{j}=\left\{\begin{array}{lll}
0, & \forall & N^{\prime} \leq|j|, \\
\frac{\left(\widetilde{V}_{\mathrm{c}}+\varepsilon_{\mathrm{F}}\right)}{1+2 b} \exp \left[-\frac{\left(\frac{j}{N^{\prime}}\right)^{2}}{1-\left(\frac{j}{N^{\prime}}\right)^{2}}\right] & \forall & |j| \leq N^{\prime},
\end{array}\right.
$$

where the parameter $b$ is defined in (ii) below. This yields a smooth parabolic barrier near the CCR center and rapidly decaying flanks, allowing the CCR to be chosen shorter than for the potential of Eq. (S15). Using a short CCR is advantageous in particular for SOPT calculations: due to the matrix structure of the Greens function and the summation/integration over internal frequencies, the computation of the self-energy $\Sigma$ and the vertex correction $P$ needed for SOPT [see Eq. (S53) and Eq. (S52)] is rather time-consuming.

(ii) Modified hopping: For model I, the QPC potential barrier of Eq. (S15) (at $\widetilde{B}=0$ ) yields a bare band whose upper edge has a maximum in energy at $j=0$, causing a large number of bound states in the energy range $\omega \in\left[\omega_{\mathrm{bulk}}^{\max }, \omega_{\mathrm{bulk}}^{\max }+\widetilde{V}_{\mathrm{c}}\right]$ (visible as narrow fringes in Fig. S10). Though these artificial bound states are completely irrelevant for the physics of the 0.7 -anomaly, the corresponding poles in the bare Green's functions nevertheless would have to be treated with due accuracy in the energy integrals involved in SOPT. To avoid the occurrence of such poles, model II takes the hopping matrix element $\tau_{j}$ in Eq. (S11) to be site-dependent within the $\mathrm{CCR}, \tau_{j}=\tau-\delta \tau_{j}$, involving a smooth (adiabatic) reduction proportional to the local barrier height:

$$
\delta \tau_{j}=\frac{1}{2}\left(E_{j}+E_{j+1}\right) b \quad(>0), \quad-N^{\prime} \leq j<N^{\prime},
$$

and $\delta \tau_{j}=0$ otherwise. Then the lower and upper band edges are approximately given by

$$
\left.\begin{array}{l}
\omega_{j}^{\min } \\
\omega_{j}^{\max }
\end{array}\right\}=-\mu \mp 2 \bar{\tau}_{j}+E_{j}, \quad \bar{\tau}_{j}=\frac{1}{2}\left(\tau_{j}+\tau_{j-1}\right) .
$$

Here $\bar{\tau}_{j}$, the average hopping matrix element involving site $j$, is approximately equal to $\bar{\tau}_{j} \simeq \tau-b E_{j}$, since the potential varies smoothly with $j$. Eq. (S24) implies a $j$-dependent bandwidth, $4 \bar{\tau}_{j}$. For the upper band edge $\omega_{j}^{\max }$, the upward shift contributed by $E_{j}$ inside the CCR is counteracted by a downward shift, contributed by $2 \bar{\tau}_{j}$, of $-2 \delta \bar{\tau}_{j} \simeq-2 b E_{j}$. The latter can be ensured to overcompensate the former by choosing the numerical factor $b$ to be larger than $1 / 2$ (we choose $b=0.55$ ). Then the upper band edge $\omega_{j}^{\max }$ throughout the CCR lies below the bulk band edge $\omega_{\text {bulk }}^{\max }$, ensuring that no bound states occur near the upper band edge. This is illustrated in Fig. S12, which is to be contrasted to the bound states seen in Fig. S10b for model I, with $j$-independent hopping.

The prefactor $1 /(1+2 b)$ in Eq. (S22) ensures that $\widetilde{V}_{\mathrm{c}}$ corresponds to the effective barrier height w.r.t. the chemical potential, $\widetilde{V}_{\mathrm{c}}=\omega_{j=0}^{\min }$ [as is the case for Eq. (S15)]. Finally, the parameter $\Omega_{x}$ is defined as the curvature of the band bottom at $\widetilde{V}_{\mathrm{c}}=0$, obtained by expanding Eq. (S24) to second order in $j$ [in analogy to Eq. (S15)]: $\left.\omega_{j}^{\min }\right|_{\widetilde{V}_{c}=0} \simeq-\Omega_{x}^{2} j^{2} /\left(4 \bar{\tau}_{0}\right)$. For the choice $\mu=0$ used here, $\bar{\tau}_{0} \simeq \tau /(1+2 b)$. We have checked that with this definition of $\Omega_{x}$, the bare transmission probability for model II, calculated numerically, agrees well with the analytic prediction of Eq. (S30) below (and that of model I). For all calculations performed in this with with model II, we chose $N^{\prime}=50$ and $b=0.55$, in which case $\Omega_{x}=0.04 \tau$.

We emphasize that transport and local properties are not modified in any essential way by the changes (i) and (ii) of model II w.r.t. model I. Their effect is solely to reduce the computation time.

\section{E. Comparison: bare LDOS of QPC and QD}

In this subsection, we offer a detailed comparison of the bare LDOS structures for a QPC and a QD. They are shown in Figs. S10d and S10c, respectively, which focus on the CCR-center and energies near $\omega=0$. They evidently exhibit numerous differences, but also some similarities. These are key to understanding the differences and similarities between the 0.7 -anomaly and the Kondo effect.

For a $\mathrm{QPC}, \mathcal{A}_{j}^{0, \mathrm{QPC}}(\omega)$ exhibits a prominent, smooth van Hove ridge (Fig. S10d), as discussed in detail in Sec. S-4 C. In contrast, for a $\mathrm{QD}, \mathcal{A}_{j}^{0, \mathrm{QD}}(\omega)$ has appreciable weight only along a set of "ridges" at discrete energies, one of which is marked by the green box in Fig. S10c. Each ridge is associated with a discrete eigenstate of the bare QD potential: it is characterized by a discrete eigenenergy, say $\omega_{\alpha}$, and its spatially confined, oscillatory $j$-dependence reflects that of $\left|\psi_{\alpha}(j)\right|^{2}$, where the wavefunction $\psi_{\alpha}(j)$ represents a confined standing wave. Its spatial extent is approximately set by the classical turning points (where $\omega_{\alpha}=\omega_{j}^{\min }$ ), though it tunnels a bit beyond these. Each ridge has a small but nonzero width in $\omega$, due to tunneling into the leads outside the $\mathrm{QD}$, and a quantized total weight of 1 when $j$-summed over the range of $\psi_{\alpha}(j)$ and $\omega$-integrated over its width, as indicated by the green box in Fig. S10c.

(Parenthetic remark: When interactions are turned on, the detailed shape of the LDOS will change, since barrier heights and energy levels will be renormalized. Nevertheless, the full $\mathcal{A}_{j}(\omega)$ will retain the generic properties illustrated in Figs. S10c,d, namely discrete ridges for the QD and a single broad ridge for the QPC. Many-body correlations may lead to additional fine structures in the full LDOS, such as a narrow Kondo resonance at the Fermi energy for $\mathcal{A}_{j}^{\mathrm{KQD}}(\omega)$. However, such many-body effects do not concern us at the present qualitative level of argumentation, which merely seeks to identify the geometric 
prerequisites for their occurrence.)

The most important difference between the bare LDOS of a QD and a QPC lies in the following fact, evident from Figs. S10a-d: near the center of the CCR, $\mathcal{A}_{j}^{0, \mathrm{QD}}(\omega)$ consists of a series of discrete ridges of quantized weight, whereas $\mathcal{A}_{j}^{0, \mathrm{QPC}}(\omega)$ does not, being dominated by just a single ridge of nonquantized weight. The physical reason for this difference clearly is that a QD constitutes a closed structure that hosts discrete, localized states, whereas a QPC, being truly open, does not. This difference is responsible for the different behavior between a KQD and a QPC for large fields: for $\widetilde{B} \gg \widetilde{B}_{*}$, the magnetization of a KQD saturates, whereas that of a QPC does not (as seen in Fig. A1d and its inset). This behavior reflects the fact that for the KQD the spin of only the single odd electron in the topmost nonempty level is being polarized in a large field, whereas for the QPC, whose LDOS has no discrete structure, there is no intrinsic limit for the magnitude of the magnetization.

While the differences between the LDOS ridge structures of KQD and QPC matter at high energies, the lowenergy behavior $\left(\widetilde{B}, \widetilde{T} \ll B_{*}\right)$ is governed by a generic common feature shared by the LDOS of both geometries: the very existence of a $\widetilde{V}_{\mathrm{c}}$-tunable ridge with a strongly peaked dependence on both $\omega$ and $j$. (Details such as the number of such ridges or their internal spatial structure are irrelevant for the ensuing argument.) The existence of such a ridge guarantees a strong magnetic response in both the conductance and the magnetization when $\widetilde{V}_{\mathrm{c}}$ is tuned such that the (interaction-shifted version of the) ridge is located energetically somewhat below $\mu$. For a $\mathrm{KQD}$, this is the local moment regime; for the QPC, it is the regime where $g \simeq 0.7$. This situation is particularly inductive to a strong local magnetic response, for two reasons: First, when spin symmetry is broken by turning on a magnetic field (say $\widetilde{B}>0$ ), the $\widetilde{B}$-induced surplus of spin-up over spin-down electrons is enhanced by the presence of an LDOS ridge below the chemical potential, because this ridge constitutes a large density of states in a confined region of space. Second, interactions will generally act to further increase this surplus by repelling spin-down electrons, and will be aided in this by the fact that the ridge, and hence the region in which the surplus is large, has a limited spatial extent.

This microscopic mechanism generates a strong, local magnetic response irrespective of whether the LDOS ridge has quantized weight or not. Thus, this mechanism applies equally to a KQD and a QPC, and in this respect the low-energy behavior of the Kondo effect and the 0.7-anomaly are indeed similar. This similarity was first pointed out in Ref. 26 and emphasized, in particular, by Meir and collaborators ${ }^{17-19}$ : the "narrow transmission resonances above the barrier" or "quasi-bound state" evoked in their arguments correspond to the van Hove ridge in $\mathcal{A}_{j}^{0, \mathrm{QPC}}(\omega)$ described above. Indeed, the asymmetric bare LDOS peak at the QPC barrier center found by us [Fig. S11, blue line for $\mathcal{A}_{j=0}^{0, \mathrm{QPC}}(\omega)$ is qualitatively similar to that found in Ref. 17 [see Fig. 3a there, right inset, solid line for $\left.\nu_{\uparrow}(\epsilon)\right]$ by spin-density-functional theory (SDFT) in a small applied field. Moreover, the van Hove ridge in our Figs. S10b,d corresponds to the bright spot seen in the center of Fig. 3a of Ref. 19 by Rejec and Meir, which shows the full spin-up $\operatorname{LDOS} \mathcal{A}_{j}(\omega)$ as function of position and energy, again calculated by SDFT in a small applied field. Though SDFT includes interactions and our bare LDOS does not, interactions affect the minority species much more strongly than the majority species. We therefore expect that the geometry-dependence of the majority LDOS obtained from SDFT should be similar to that of a noninteracting theory. Thus, we believe that Meir and Rejec's "quasi-bound states" are synonymous to our "van Hove ridges". (We somewhat prefer the latter nomenclature, since it indicates the origin of these LDOS structures.) It would be highly desirable to have a plot similar to Fig. S10d for the full $\mathcal{A}_{j}^{\mathrm{QPC}}(\omega)$ calculated using $\mathrm{fRG}$, but its energy dependence is not accessible by static fRG. To obtain a first impression, we have calculated it using perturbation theory, see Fig. A3g,h; calculating it with Keldysh fRG would be an interesting goal for future studies.

We wish to emphasize that the details of the magnetic response of a KQD and a QPC will be similar only as long as the conditions $\widetilde{B}, \widetilde{T} \ll \widetilde{B}_{*}$ hold; once they are violated, the differences in the LDOS ridges, discrete for QD vs. continuous for QPC, begin to matter. This caveat, not discussed in Refs. 17-19, prevents the similarity between Kondo effect and 0.7-anomaly from extending to the regime of large energies.

A detailed comparative study of the similarities and differences in the behavior of a KQD and QPC, all originating from the similarities and differences between their LDOS ridges, will be published elsewhere ${ }^{1}$.

\section{S-5. THE LOW-ENERGY SCALE $\widetilde{B}_{*}$}

This section covers the influence of geometry and interactions on the low-energy scale $\widetilde{B}_{*}$ for a QPC. In Sec. S$5 \mathrm{~A}$ we show that the exponential dependence of the lowenergy scale $\widetilde{B}_{*}\left(\widetilde{V}_{\mathrm{c}}\right)$ has a purely geometric origin, and contrast this to the more complicated case of the Kondo temperature for a KQD. Sec. S-5 B discusses the effects of interactions on $\widetilde{B}_{*}$ and $\widetilde{T}_{*}$ for a QPC. Finally, Sec. S-5C discusses the extent to which the interaction parameter $U$ itself depends on the 2D potential landscape.

\section{A. Exponential $\widetilde{V}_{\mathrm{c}}$-dependence of $\widetilde{B}_{*}$}

In the main article we reported that for a QPC the low-energy scale $B_{*}\left(V_{\mathrm{c}}\right)$ depends exponentially on $V_{\mathrm{c}}$ (see Eq. (A3), Figs. A2a, c,e). The same is true for $T_{*}\left(V_{\mathrm{c}}\right)$ (see Eq. (A3), Figs. A2d, f), as was first found in Ref. 26. In this subsection, we explain the origin of this exponential $V_{\mathrm{c}}$-dependence. It is present already for the nonin- 
teracting $(U=0)$ version of our model, hence we begin by discussing the latter.

According to the Landauer-Büttiker formula, the noninteracting differential conductance $g_{\mathrm{nl}}^{0}\left(\widetilde{T}, \widetilde{B}, \widetilde{V}_{\mathrm{sd}}\right)$ as a function of temperature $\widetilde{T}=k_{\mathrm{B}} T$, magnetic field $\widetilde{B}=$ $\left|g_{\mathrm{el}}\right| \mu_{\mathrm{B}} B$ and source-drain voltage $\widetilde{V}_{\mathrm{sd}}=-|e| V_{\mathrm{sd}}$ is given by

$$
\begin{aligned}
g_{\mathrm{nl}}^{0}\left(\widetilde{T}, \widetilde{B}, \widetilde{V}_{\mathrm{sd}}\right)= & \frac{d}{d \widetilde{V}_{\mathrm{sd}}} \frac{1}{2} \sum_{\sigma= \pm} \int_{-\infty}^{\infty} d \omega \mathcal{T}\left(\omega+\frac{1}{2} \sigma \widetilde{B}\right) \\
& \times\left[f\left(\omega-\frac{1}{2} \widetilde{V}_{\mathrm{sd}}\right)-f\left(\omega+\frac{1}{2} \widetilde{V}_{\mathrm{sd}}\right)\right]
\end{aligned}
$$

where $f(\omega)=\left[e^{\omega / \widetilde{T}}+1\right]^{-1}$ is the Fermi function, and $\mathcal{T}(\omega)$ is the noninteracting transmission probability across the QPC barrier of a lead electron incident with energy $\omega$ w.r.t. $\mu$, at $\widetilde{B}=0$. Let us expand it in powers of energy:

$$
\mathcal{T}(\omega)=\mathcal{T}^{(0)}+\mathcal{T}^{(1)} \omega+\frac{1}{2} \mathcal{T}^{(2)} \omega^{2}+\ldots,
$$

where $\mathcal{T}^{(0)}=\mathcal{T}(0)$ is the transmission probability at the chemical potential. Inserting Eq. (S26) into (S25) leads to the following expression for the leading dependence of the bare conductance on $\widetilde{T}^{2}, \widetilde{B}^{2}$ and $\widetilde{V}_{\text {sd }}^{2}$ (at fixed $\widetilde{V}_{\text {c }}$ and $\left.\widetilde{V}_{\mathrm{s}}\right)$

$$
\frac{g_{\mathrm{nl}}^{0}\left(\widetilde{T}, \widetilde{B}, \widetilde{V}_{\mathrm{sd}}\right)}{\mathcal{T}^{(0)}}=\left[1-\left(\frac{\widetilde{T}}{\widetilde{T}_{*}^{0}}\right)^{2}-\left(\frac{\widetilde{B}}{\widetilde{B}_{*}^{0}}\right)^{2}-\left(\frac{\widetilde{V}_{\mathrm{sd}}}{\widetilde{V}_{\mathrm{sd} *}^{0}}\right)^{2}\right],
$$

with low-energy scales $\widetilde{T}_{*}^{0}, \widetilde{B}_{*}^{0}$ and $\widetilde{V}_{\mathrm{sd} *}^{0}$ given by

$$
-\frac{8 \mathcal{T}^{(0)}}{\mathcal{T}^{(2)}}=\left(\widetilde{B}_{*}^{0}\right)^{2}=\left(\widetilde{V}_{\mathrm{sd} *}^{0}\right)^{2}=\frac{4 \pi^{2}}{3}\left(\widetilde{T}_{*}^{0}\right)^{2} .
$$

Their mutual ratios hence are independent of $\widetilde{V}_{\mathrm{c}}$ :

$$
\frac{\widetilde{B}_{*}^{0}}{\widetilde{T}_{*}^{0}}=\frac{2 \pi}{\sqrt{3}}, \quad \frac{\widetilde{V}_{\mathrm{sd} *}^{0}}{\widetilde{B}_{*}^{0}}=1 .
$$

(Remark: Depending on the height and shape of the potential barrier, $\mathcal{T}^{(2)}$ can be either negative or positive; in the latter case, the scales $\widetilde{B}_{*}^{0}, \widetilde{T}_{*}^{0}$ and $\widetilde{V}_{\text {sd* }}^{0}$ as defined here would be imaginary. In the following, we are interested only in the former case.)

Now consider a purely parabolic QPC potential barrier with height $\widetilde{V}_{\text {c }}$ and longitudinal curvature $\frac{1}{4 \tau} \Omega_{x}^{2}$ [as in Eq. (S15)]. Then the bare transmission $\mathcal{T}(\omega)$ at $\widetilde{B}=0$ is given by ${ }^{5}$

$$
\mathcal{T}(\omega) \simeq \frac{1}{e^{-2 \pi\left(\omega-\widetilde{V}_{\mathrm{c}}\right) / \Omega_{x}}+1} .
$$

Recall that $\widetilde{V}_{\mathrm{c}}=\omega_{j=0}^{\min }$ is the height of the band bottom's maximum at the central site w.r.t. the chemical potential. When $\widetilde{V}_{\text {c }}$ is decreased to open up the QPC, the bare transmission of an electron incident at the chemical potential $(\omega=0)$ increases past $\mathcal{T}(0)=0.5$ when $\widetilde{V}_{\text {c }}$ decreases past 0. [We obtained Eq. (S30) from Eq. (4) of Ref. 5, which in turn was derived by a semiclassical treatment of transmission through a parabolic barrier ${ }^{27,28}$, assuming a quadratic dispersion of the form $p^{2} / 2 m$. The latter assumption is applicable for our situation in the limit that our tight-binding band is much wider than the energy range over which the transmission changes rapidly, $\tau \gg \Omega_{x}$. This allows a quadratic approximation for the dispersion [Eq. (S12)] near the band bottom, $\epsilon_{k} \simeq-2 \tau+\tau k^{2} a^{2}$, implying an effective mass of $m=\hbar^{2} /\left(2 \tau a^{2}\right)$.]

The bare dimensionless conductance at $\widetilde{B}=\widetilde{T}=\widetilde{V}_{\mathrm{sd}}=$ 0 , viewed as function of $\widetilde{V}_{\mathrm{c}}$, is then given by

$$
g_{\mathrm{nl}}^{0}=g_{\mathrm{nl}}^{0}(0,0,0)=\mathcal{T}^{(0)}=\frac{1}{e^{-2 \pi \widetilde{V}_{\mathrm{c}} / \Omega_{x}}+1} .
$$

Let us now focus on the regime of negative $\widetilde{V}_{\mathrm{c}}=-\left|\widetilde{V}_{\mathrm{c}}\right|$, where for the quadratic potential top considered here the bare magnetoconductance is strictly negative. Evaluating Eq. (S28) for $\widetilde{B}_{*}^{0}$ using Eq. (S30), one finds:

$$
\begin{aligned}
\widetilde{B}_{*}^{0} & =\frac{\Omega_{x}}{\pi} \sqrt{2 \operatorname{coth}\left(\pi\left|\widetilde{V}_{\mathrm{c}}\right| / \Omega_{x}\right)} e^{\pi\left|\widetilde{V}_{\mathrm{c}}\right| / \Omega_{x}} \\
& =\frac{\Omega_{x}}{\pi} \frac{1}{\sqrt{g^{0}-1 / 2}} \sqrt{\frac{g^{0}}{1-g^{0}}} .
\end{aligned}
$$

(The second line follows from the first by inverting Eq. (S31).) Expression (S32a) for the low-energy scale in the absence of interactions, $\widetilde{B}_{*}^{0}$, agrees to within a few percent with our numerical calculations for $U=0$, shown by the black dashed line in Fig. S13a. It states that $\widetilde{B}_{*}^{0}$ diverges both when $\left|\widetilde{V}_{\mathrm{c}}\right| \rightarrow 0^{+}$(i. e. $g^{0} \rightarrow 1 / 2$ from above) and when $\left|\widetilde{V}_{\mathrm{c}}\right| / \Omega_{x} \gg 1$ (i.e. $g^{0} \rightarrow 1$ from below). Between these two limiting cases $\widetilde{B}_{*}^{0}$ has a minimum, which turns out to occur at a bare conductance of $g_{*}^{0}=1 / \sqrt{2} \simeq 0.707$.

The message of the above analysis is that the experimentally observed exponential $V_{\mathrm{c}}$-dependence of the lowenergy scales $B_{*}$ and $T_{*}$ reported in the main article (and for $T_{*}$ also in Ref. 26 ) has a purely geometric origin, which can already be understood within a noninteracting model. It arises simply because for a quadratic barrier the transmission amplitude above the barrier depends exponentially on its height (as can be made explicit in a semiclassical WKB treatment of the transmission problem ${ }^{27,28}$ ). Moreover, the scale for the $\widetilde{V}_{\mathrm{c}}$-dependence is set solely by $\Omega_{x}$, the curvature at the top of the barrier [Eq. (S15)].

The fact that for a QPC the exponential $\widetilde{V}_{\mathrm{c}}$-dependence of $\widetilde{B}_{*}$ can be found without considering interactions at all stands in striking contrast to the case of a KQD: there $\widetilde{B}_{*}$ is proportional to the Kondo temperature, which likewise depends exponentially on $\widetilde{V}_{\mathrm{c}}$, but the exponent is quadratic in $\widetilde{V}_{\mathrm{c}}$, and the scale of its $\widetilde{V}_{\mathrm{c}^{-}}$ dependence is set by the interaction strength $U$ and effective level width $\Gamma$. To be explicit, for the single-impurity 
Anderson model, with level position $\varepsilon_{d}=\widetilde{V}_{\mathrm{c}}$, the Kondo temperature corresponding to $\widetilde{T}_{*}$ is given by ${ }^{29}$

$$
\widetilde{T}_{\mathrm{K}}=\sqrt{U \Gamma / 2} \exp \left[\frac{\pi \widetilde{V}_{\mathrm{c}}\left(\widetilde{V}_{\mathrm{c}}+U\right)}{2 \Gamma U}\right] .
$$

It arises as the low-energy scale $\widetilde{T}_{\mathrm{K}} \propto e^{-1 / j_{\text {eff }}}$ characterizing the onset of a logarithmic infrared divergence that occurs when doing perturbation theory in the effective exchange interaction between the spins of a local moment and a conduction band. The form of the corresponding effective dimensionless exchange coupling $j_{\text {eff }}$, given by

$$
j_{\mathrm{eff}}=\frac{2 \Gamma}{\pi}\left[\frac{1}{\widetilde{V}_{\mathrm{c}}+U}-\frac{1}{\widetilde{V}_{\mathrm{c}}}\right],
$$

is found by a Schrieffer-Wolff transformation. Evidently, such KQD results can not be obtained without considering the role of interactions from the outset. In contrast, for a QPC the origin of the exponential $\widetilde{V}_{\mathrm{c}}$-dependence of $\widetilde{B}_{*}$ is decidedly different and can be understood already for a noninteracting theory, as described above. In particular, at high energies a QPC does not display local-moment behavior, so that the corresponding logarithmic infrared divergence characteristic of the Kondo effect does not occur.

Having made this point, we hasten to add that $\widetilde{B}_{*}$ is of course affected by interactions for a QPC too, albeit less severely than for a KQD. The role of interactions is discussed in the next subsection.

\section{B. Effects of interactions on $\widetilde{B}_{*}$ and $\widetilde{T}_{*}$}

While the fact that the low-energy scales $\widetilde{B}_{*}, \widetilde{T}_{*}$ and $\widetilde{V}_{\mathrm{sd} *}$ for a QPC depend exponentially on gate voltage, as $e^{\pi\left|\widetilde{V}_{\mathrm{c}}\right| / \Omega_{x}}$, has an elementary geometric origin, the behavior of the pre-exponential factor is more subtle: quite generally this pre-exponential factor will depend on the interaction strength $U$ and gate voltage $\widetilde{V}_{\text {c }}$. A detailed theoretical analysis of this issue is beyond the scope of the present paper. Here we just want to make two points, the first regarding the ratio $\widetilde{B}_{*} / \widetilde{T}_{*}$, the second regarding the $U$-dependence of $\widetilde{B}_{*}$.

The ratio $\widetilde{B}_{*} / \widetilde{T}_{*}$ : When interactions are turned on, the effects of finite $\widetilde{B}$ or finite $\widetilde{T}$ are, in general, not equivalent: Finite $\widetilde{B}$ shifts the effective barrier height seen by spin-up and spin-down electrons in opposite directions, in a way that is enhanced by interactions (which amplifies the imbalance between spin up and spin down), however without opening up the possibility of inelastic scattering. Finite $\widetilde{T}$ causes an effective increase in barrier height, too, due to an increase in density near the barrier center (because the LDOS is $\omega$-asymmetric there), but it does not involve any imbalance between spin-up and -down. Moreover, finite $\widetilde{T}$ also leads to inelastic scattering. In lowestorder perturbation theory for the self-energy (Sec. S-7), shifts in barrier height (both $\widetilde{B}$-induced, spin-asymmetric and $\widetilde{T}$-induced, spin-symmetric shifts) are described by the Hartree contribution, and $\widetilde{T}$-induced inelastic scattering by the Fock contribution [see Eq. (S53)]. In general, the relative strength of these two effects will depend not only on $U$ but also on gate voltage. Since the strength of the (negative) conductance response to increasing $\widetilde{B}$ or $\widetilde{T}$ is characterized by $1 / \widetilde{B}_{*}$ or $1 / \widetilde{T}_{*}$, respectively, the ratio $\widetilde{B}_{*} / \widetilde{T}_{*}$, too, will in general likewise depend not only on $U$, but also directly on $\widetilde{V}_{\mathrm{c}}$.

In the light of the above comments, it is all the more remarkable that the experimentally observed ratio $B_{*} / \widetilde{T}_{*}$ does, in fact, become essentially independent of $V_{\mathrm{c}}$ in the $V_{\mathrm{c}}$-regime well below $V_{\mathrm{c} 0}$, where $g \rightarrow 1$ (compare thin grey and black lines in Fig. A2f). In the main article we have already pointed out that this $V_{\mathrm{c}}$-independence of $B_{*} / T_{*}$ for $g \simeq 1$ is characteristic of the Fermi-liquid behavior expected from Nozières' treatment of the Kondo problem in the limit $B, T \ll T_{\mathrm{K}}$.

Once the condition $g \simeq 1$ is relaxed, the experimentally determined $B_{*} / T_{*}$ does acquire a dependence on $V_{\mathrm{c}}$, in accord with the expectations stated above. Indeed, in Fig. A2f the measured ratio $B_{*} / T_{*}$ increases with increasing $\widetilde{V}_{\text {c }}$ as $B_{*}$ and $T_{*}$ approach their minimal values. Remarkably, our model qualitatively reproduces this behavior when we treat interactions using SOPT (compare the lines for $\widetilde{B}_{*}$ and $\widetilde{T}_{*}$ in Fig. A2d). An increase in $\widetilde{B}_{*} / \widetilde{T}_{*}$ means that the conductance reduction induced by increasing $\widetilde{T}$ grows relative to that induced by increasing $\widetilde{B}$, implying that inelastic scattering [Fock term, diagram c in Eq. (S53)] gains importance relative to the $\widetilde{B}$-induced enhancement of the barrier height [Hartree terms, diagrams a and b in Eq. (S53)]. Moreover, Fig. S4 above shows that $\widetilde{B}_{*} / \widetilde{T}_{*}$ decreases with increasing $U$, implying that in general interactions have a stronger effect on the low- $\widetilde{B}$ dependence of the conductance than on its low- $\widetilde{T}$ dependence.

$U$-dependence of $\widetilde{B}_{*}$ : We have used fRG to explore in some more detail how interactions affect the $\widetilde{V}_{\mathrm{c}^{-}}$ dependence of $\widetilde{B}_{*}$ for a QPC. (Similar studies of $\widetilde{T}_{*}$ are not possible using static fRG, but would be worth pursuing by Keldysh fRG). As in Sec. S-5 A above, we focus on the regime $g \simeq 1$ (say $\left.\left|\widetilde{V}_{\mathrm{c}}\right| \gtrsim 0.75 \Omega_{x}\right)$. Our results for this regime can be summarized by stating that for $U$ small enough to be treatable by fRG, $\widetilde{B}_{*}$ shows the following behavior:

$$
\begin{aligned}
\widetilde{B}_{*}\left(\widetilde{V}_{\mathrm{c}}, U\right) & \simeq \frac{\sqrt{2} \Omega_{x}}{\pi} e^{-F(U)} e^{\pi\left|\widetilde{V}_{\mathrm{c}}\right| / \Omega_{x}} \\
F(U) & \simeq(0.8 \pm 0.05) U / \sqrt{\tau \Omega_{x}}
\end{aligned}
$$

The behavior of Eq. (S35a) is illustrated in Fig. S13a, which shows $\ln \left(\widetilde{B}_{*}\right)$ as function of $\left|\widetilde{V}_{\mathrm{c}}\right| / \Omega_{x}$ for several values of $U$ : for $\left|\widetilde{V}_{\mathrm{c}}\right| / \Omega_{x} \gtrsim 0.75$ the resulting lines all have roughly the same slope, but are shifted downward 

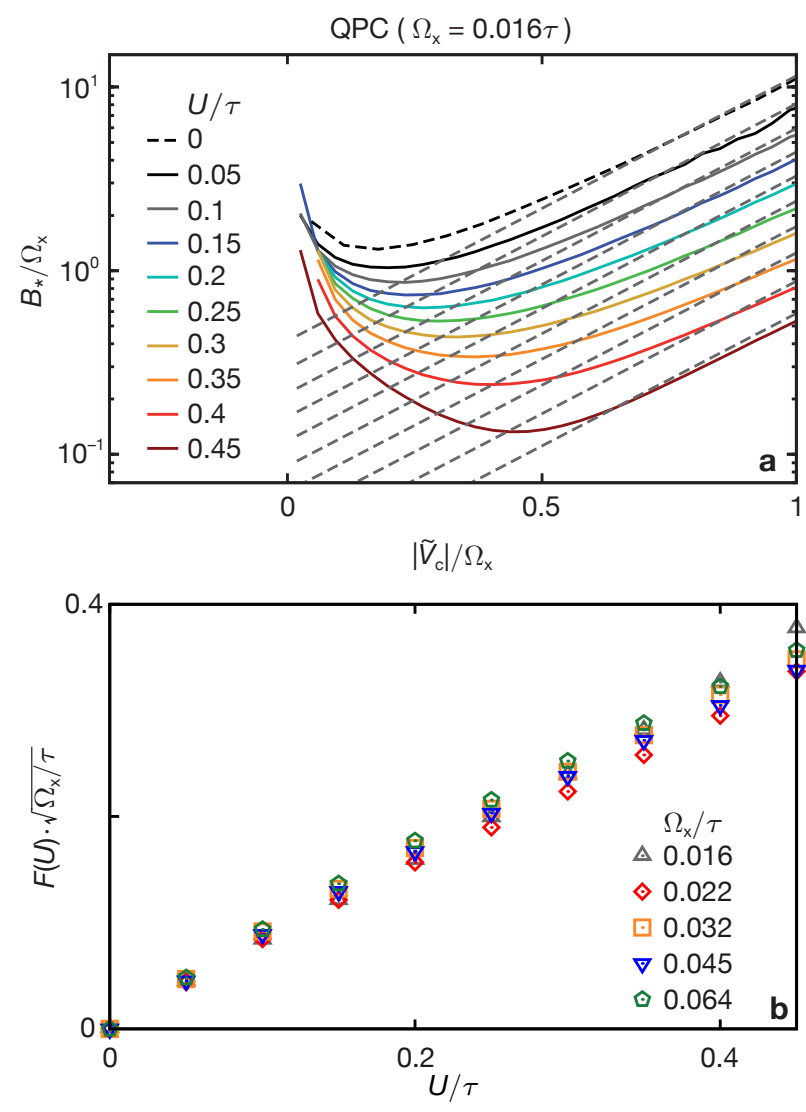

Figure S13: Effect of interaction strength $U$ on the lowenergy scale $\widetilde{B}_{*}$, in the regime of negative $\widetilde{V}_{\mathrm{c}}=-\left|\widetilde{V}_{\mathrm{c}}\right|$ (calculated for model I using static fRG). a, $\widetilde{B}_{*} / \Omega_{x}$ as function of $\left|\widetilde{V}_{\mathrm{c}}\right| / \Omega_{x}$. Grey dashed lines indicate linear fits of $\ln \left(\widetilde{B}_{*}\right)$ vs. $\left|\widetilde{V}_{\mathrm{c}}\right| / \Omega_{x}$ in the range $0.8 \leq\left|\widetilde{V}_{\mathrm{c}}\right| / \Omega_{x}<1$, the offsets of which yield $F(U)$. b, The function $F(U)$ vs. $U$ for several choices of $\Omega_{x}$, plotted in scaled fashion to illustrate the behavior of Eq. (S35b).

in uniformly-spaced steps when $U$ is increased in uniform steps. This implies that $\widetilde{B}_{*}$ is exponentially suppressed with increasing $U$ (which also shifts the minimum in $\widetilde{B}_{*}$ towards more positive $\left|\widetilde{V}_{\mathrm{c}}\right|$-values $)$. The function $F(U)$ that characterizes the exponential suppression can be obtained from the offsets of linear fits to $\ln \left(\widetilde{B}_{*}\right)$ vs. $\pi\left|\widetilde{V}_{\mathrm{c}}\right| / \Omega_{x}$, repeated for various $U$-value. The resulting function $F(U)$, shown in Fig. S13b for several values of the curvature $\Omega_{x}$, exhibits the behavior described by Eq. (S35b) quite well: it increases linear with $U$, on a scale set by $\sqrt{\tau \Omega_{x}}$. This reflects the fact that in static $\mathrm{fRG}$, the dimensionless quantity that governs the effective interaction strength is $U \mathcal{A}_{j}(0)$ (cf. Sec. S-6), and at the barrier top we have [cf. Eq. (S19c) and Fig. S11]

$$
U \mathcal{A}_{j=0}(0) \propto U / \sqrt{\tau \Omega_{x}} .
$$

Paraphrasing Eq. (S35a), we can formulate the following conclusions for how the $\widetilde{V}_{\mathrm{c}}$-dependence of $\widetilde{B}_{*}$ in the regime $g \simeq 1$ is affected by turning on $U$ : (i) The factor $e^{\pi\left|\widetilde{V}_{\mathrm{c}}\right| / \Omega_{x}}$ from Eq. (S32) persists, essentially without a change in the numerical prefactor $\pi / \Omega_{x}$ of $\left|\widetilde{V}_{\mathrm{c}}\right|$ in the exponent. (ii) The pre-exponential factor decreases exponentially with $U$, in a fashion that corresponds to shifting $\widetilde{V}_{\mathrm{c}} \rightarrow \widetilde{V}_{\mathrm{c}}+\Omega_{x} F(U) / \pi$. The physical interpretation is that local interactions increase the Hartree potential and hence the effective barrier height [causing (ii)], but do not significantly change its effective curvature [resulting in (i)]. Of course, the latter statements are true only approximately, in that Fig. S13 does exhibit slight deviations between the actual data and the behavior stated by Eqs. (S35).

Together, points (i) and (ii) suggest that for a QPC, the qualitative effect of interactions on $\widetilde{B}_{*}$ can already be found by perturbatively calculating the Hartree potential. We have done so, obtaining results (not shown here) in qualitative agreement with those just discussed. A simpler treatment of the same effects might be possible using semiclassical WKB wave functions, as done in Ref. 6 in a calculation of the Fock contribution to $\widetilde{T}_{*}$, but this is left as a topic for future study. (We remark that when the calculation of $\widetilde{T}_{*}$ in Ref. 6 , extractable from their Eq. (33), is specialized to a point-like interaction with range zero, the result yields precisely the same $e^{\pi\left|\widetilde{V}_{\mathrm{c}}\right| / \Omega_{x}}$ dependence for $\widetilde{T}_{*}$ as found by us in Eq. (S32) above.)

Note from Eq. (S35) that decreasing the curvature $\Omega_{x}$ at the top of the QPC barrier or increasing the interaction strength $U$ (e.g. using a top gate) have qualitatively similar effects, in that both tend to decrease $\widetilde{B}_{*}$ and hence to strengthen the low- $\widetilde{B}$ response of the conductance. Likewise, decreasing $\Omega_{x}$ or increasing $U$ also cause similar changes in the conductance step at $\widetilde{B}=\widetilde{T}=0$, in that both tend to make the 0.7 -shoulder more prominent. This is illustrated in Fig. S14, whose panels $\mathbf{b}$ and $\mathbf{c}$ offer a succinct summary of how the 0.7-anomaly depends on geometry and interactions, respectively.

Since $\Omega_{x}$ sets both the width of the conductance step [Eq. (S31)] and the slope of $\ln \left(\widetilde{B}_{*}\right)$ vs. $\left|\widetilde{V}_{\mathrm{c}}\right|$ [Eq. (S35a)], an experimental consistency check is possible: We have determined the said step width and slope from Fig. A2e and extracted $\Omega_{x}$-values from each, finding $\Omega_{x}^{\text {step }} \simeq a \times$ $0.026 \mathrm{~V}$ from the step width and $\Omega_{x}^{\text {slope }} \simeq a \times 0.048 \mathrm{~V}$ from the slope $(a \simeq 37 \mathrm{meV} / \mathrm{V}$ is a geometric conversion factor between applied gate voltage (in V) and the resulting electrostatic potential energy (in $\mathrm{meV}$ ), such that $\left.\widetilde{V}_{\mathrm{c}}=-a V_{\mathrm{c}}\right)$. The fact that $\Omega_{x}^{\text {step }}$ and $\Omega_{x}^{\text {slope }}$ agree within a factor of two is quite satisfactory, given the fact that we made no attempt to realistically model the shape of the QPC potential. Possible reasons for why the agreement is not perfect are that the experimental QPC potential was not perfectly parabolic, and that our use of a purely on-site (instead of longer-range) interaction is an oversimplification. (See also Sec. S-5 C below.)

To conclude this subsection, let us emphasize once more its most important qualitative conclusion: inter- 
$\operatorname{QPC}(B=0)$

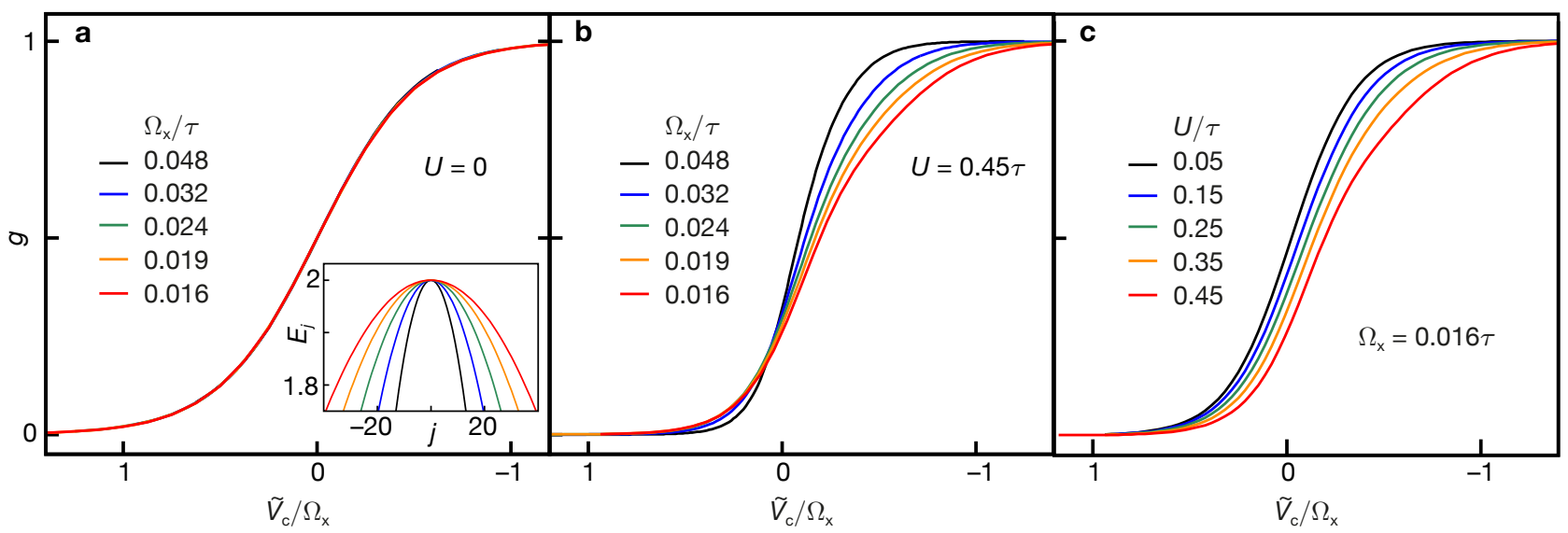

Figure S14: Effect of barrier curvature $\Omega_{x}$ (panels a,b) and interaction strength $U$ (panel c) on the conductance through a QPC (model I), calculated by static fRG. The inset to a shows the top of a parabolic QPC barrier for several values of the curvature $\Omega_{x}$. a, In the absence of interactions $(U=0)$ the conductance curves $g\left(\widetilde{V}_{\mathrm{c}}\right)$, calculated for different $\Omega_{x}$, all collapse onto a single curve when plotted as function of $\widetilde{V}_{\mathrm{c}} / \Omega_{x}$, as expected from Eq. (S30). b, Similar plot as a, but for $U \neq 0$, which prevents a scaling collapse. c, Conductance curves $g\left(\widetilde{V}_{\mathrm{c}}\right)$ calculated for fixed $\Omega_{x}$ but several different values of $U$, and scaled as in $\mathbf{a}$ and $\mathbf{b}$. As explained in the main article, the combination of enhanced spectral weight at the Fermi energy $\mathcal{A}_{j=0}(0)$ and interaction $U$ lowers the conductance in the vicinity of $\widetilde{V}_{\mathrm{c}} \simeq 0$, resulting in an asymmetric and broadened conductance step. The strength of this effect is governed by the product $U \mathcal{A}_{j=0}(0) \propto U / \sqrt{\tau \Omega_{x}}$ [cf. Eq. (S36)]. This increases with decreasing $\Omega_{x}$ at fixed $U$ (panel b) or likewise with increasing $U$ at fixed $\Omega_{x}$ (panel c), causing an enhancement of the 0.7-shoulder in both cases.

actions cause an exponential reduction in $\widetilde{B}_{*}$, which can thus be significantly smaller than the QPC's natural energy scale $\Omega_{x}$. (In Fig. A2f, the smallest values reported for $\mu_{\mathrm{B}} B_{*}$ and $k_{\mathrm{B}} T_{*}$ are $0.3 \mathrm{meV}$ and $0.08 \mathrm{meV}$, respectively, significantly smaller than the above estimates of $\Omega_{x}^{\text {step }} \simeq 1 \mathrm{meV}$.) While the detailed form of the function $F(U)$ describing this suppression may be modeldependent, we believe the strong suppression of $\widetilde{B}_{*}$ with increasing $U$ to be generic. This is a crucial ingredient for understanding the 0.7 -anomaly, since it becomes more pronounced the smaller this crossover scale.

\section{Geometry-dependence of interaction $U$}

It would be interesting to experimentally study the interaction- and geometry dependence of $B_{*}$ more systematically, by using the side- and top-gate voltages $V_{\mathrm{s}}$ and $V_{\mathrm{t}}$ to vary the effective barrier shape and interaction strength. Of course numerous studies of the 0.7anomaly in varying geometries do exist ${ }^{4,8-10,12,30,31}$, but to systematically check the predictions of Eq. (S35) for $\widetilde{B}_{*}\left(\widetilde{V}_{\mathrm{c}}, \Omega_{x}, U\right)$, it would be necessary to simultaneously monitor the $V_{\mathrm{t}^{-}}$and $V_{\mathrm{s}}$-dependence of $B_{*}\left(V_{\mathrm{c}}\right), \Omega_{x}$ and $U$. Indeed, whereas our model treats $U$ as a fixed, given constant, in reality the effective interaction strength is geometry-dependent. We have already pointed out in Sec. S-2 A that it depends on the lateral confinement in the QPC region; more specifically, the effective interaction constant for a 1D model will depend on the spatial extent, say $l_{y}$, of the transverse wave-function, which, in turn, can depend quite delicately on the amount of screening, etc.

If no realistic modelling of the latter is available (we have not attempted any), the evolution of interaction strength with geometry is best gauged by tracking the evolution of experimentally accessible quantities such as $g_{\mathrm{ss}}$ and $\Delta_{\text {hfo. }}$. To be specific, the conductance $g\left(V_{\mathrm{c}}, B, T\right)$ could be measured for various settings of $V_{\mathrm{t}}$ and $V_{\mathrm{s}}$. A measure for the resulting changes in the effective interaction strength $U$ could be obtained from the transconductance $d G / d V_{\mathrm{c}}$ at low $T$ by monitoring the corresponding changes in $g_{\mathrm{ss}}$ or $\Delta_{\mathrm{hfo}}$ (as in Fig. S5b, c). Simultaneously, estimates for $\Omega_{x}$ and $l_{y}$ could be extracted, respectively, from the widths of the first step and first plateau of the conductance curve at low $T$; and $B_{*}$ and $T_{*}$ from the low-energy $B$ - and $T$-dependence of the conductance (as in Figs. A2e,f). This would yield enough information to check Eq. (S35) in detail. We leave such a study for future work.

\section{S-6. FUNCTIONAL RENORMALIZATION GROUP}

In this section and the next, we describe the details of the two theoretical approaches used here: The present section is devoted to the functional renormalization group (fRG) which we used to study the $\widetilde{B}$ dependent quantities at $\widetilde{T}=\widetilde{V}_{\text {sd }}=0$. Section S-7 outlines the second-order perturbation theory (SOPT) ap- 
proach which we used to explore the properties of our model for fixed $U$ at $\widetilde{T} \neq 0$ or $\widetilde{V}_{\text {sd }} \neq 0$.

Both fRG and SOPT are set up as perturbation expansions with respect to a noninteracting ground state that has zero magnetization in the absence of a magnetic field, hence both yield perturbed ground states that also have this property. The possibility of spontaneous breaking of spin symmetry is thus ruled out a priori within both approaches. In choosing to set them up in this way, we therefore make the physical assumption that spontaneous symmetry breaking need not be invoked to explain the 0.7 -anomaly. This assumption is justified a posteriori by the agreement between our fRG results and our experiments for the magnetic-field dependence of the 0.7anomaly (as discussed in detail in Sec. S-2 C).

The present section summarizes the central ingredients of the fRG approach in the one-particle irreducible version $^{32}$ used here. The details of our approach, using the Matsubara formalism, are very similar to those of Refs. 33,34,40 and 41; technical aspects going beyond the latter works will be presented in detail elsewhere ${ }^{36}$. The main purpose here is to explicitly formulate the approximations that we have employed for the translationally nonuniform system with on-site interactions defined by Eq. (A1) of the main text [or Eq. (S11)]. (For completeness, we remark that the fRG approach described below is also capable of dealing with the Kondo effect in a 1Dmodel of a quantum dot, described by a double-barrier potential. Corresponding results will be presented elsewhere, in a comparative study of the Kondo effect and the 0.7 -anomaly. ${ }^{1}$ )

\section{A. Observables}

Our goal is to calculate the conductance $g$ through the CCR and the average number $n_{j \sigma}$ of spin- $\sigma$ electrons at site $j$, at zero temperature. Following Ref. 33,34, we proceed in three steps. (i) We integrate out the two semi-infinite, noninteracting leads to the left and right of the CCR, using a standard projection technique; this results in a bare Matsubara Green's function for the CCR, $\left(\mathcal{G}_{0}\right)_{j i}^{\sigma}(i \omega)$, with a matrix structure in real space, $j, i \in\left[-N^{\prime}, N^{\prime}\right]$ being site indices. (ii) We incorporate interactions in the CCR by using fRG to calculate the full Matsubara Green's function of the CCR, $\mathcal{G}_{j i}^{\sigma}(i \omega)$; this step will be described in more detail in the next subsection. (iii) We calculate $g$ and $n_{j \sigma}$ at $T=0$ using

$$
\begin{aligned}
g & =\frac{1}{2} \sum_{\sigma} \mathcal{T}_{\sigma}(0), \\
\mathcal{T}_{\sigma}(\omega) & =\left|2 \pi \tau^{2} \rho_{0}^{\sigma}(\omega) \mathcal{G}_{-N^{\prime}, N^{\prime}}^{\sigma}\left(\omega+i 0^{+}\right)\right|^{2}, \\
n_{j \sigma} & =\left\langle\hat{n}_{j \sigma}\right\rangle_{T}=\int_{-\infty}^{\infty} d \omega f(\omega) \mathcal{A}_{j \sigma}(\omega) \\
& =T \sum_{n} \mathcal{G}\left(i \omega_{n}\right)+\frac{1}{2},
\end{aligned}
$$

$$
\mathcal{A}_{j \sigma}(\omega)=-\frac{1}{\pi} \operatorname{Im} \mathcal{G}_{j j}^{\sigma}\left(\omega+i 0^{+}\right) .
$$

Here $\mathcal{T}_{\sigma}(\omega)$ is the spin-dependent transmission probability for a spin- $\sigma$ electron incident with energy $\omega$ relative to the chemical potential $\mu$, and $\rho_{0}^{\sigma}(\omega)$ is the local density of states at the first site of a semi-infinite noninteracting tight-binding chain, representing a lead. For our fRG calculations we have chosen $\mu=0$, implying half-filled leads.

\section{B. fRG strategy and approximations}

fRG may be viewed as RG-enhanced perturbation theory in the interaction. It is based on solving a hierarchy of coupled ordinary differential equations, the flow equations, for the system's $n$-particle vertex functions, $\gamma_{n}^{\Lambda}$. The flow parameter $\Lambda$ controls the RG flow from an initial cutoff $\Lambda_{i}$, at which all vertex functions are known and simple, to a final cutoff $\Lambda_{f}$, at which the full theory is recovered. Solving the full hierarchy of flow equations, however, is impossible in practice and simplifying approximations are needed to render them tractable. When setting up our flow equations, we make two technical approximations, which are both exact to second order in the interaction $U$. We briefly summarize them here, and provide more details in the subsequent technical discussions.

(i) We truncate the fRG hierarchy by setting $\gamma_{n>3}^{\Lambda}=0$. This standard approximation ${ }^{32}$ offers a systematic way of summing up parquet-type diagrams (i.e. diagrams that result from coupled RPA-equations) ${ }^{32}$ for the twoparticle vertex. However, due to the neglect of higher order terms, it fails if the interaction becomes too large (on a scale set by the local density of states at the chemical potential).

(ii) We apply the coupled-ladder approximation ${ }^{40,41}$ to treat the frequency dependence of the vertex, and extend this scheme to also treat the real space structure of the vertex. The coupled-ladder approximation sets to zero all components of the vertex except those that are generated already to second order in the bare (onsite) interaction, but retains the latter components throughout the flow.

\section{C. fRG Flow equations}

We introduce $\Lambda$ as an infrared cut-off in the bare Matsubara propagator,

$$
\mathcal{G}_{0}^{\Lambda}(i \omega)=\Theta_{T}(|\omega|-\Lambda) \mathcal{G}_{0}(i \omega), \Lambda_{i}=\infty, \Lambda_{f}=0,
$$

where $\Theta_{T}$ is a step function that is broadened on the scale of the temperature $T$ (we discuss the limit $T=$ 0 in Sec. S-6 E below). The fRG approach in the oneparticle irreducible version then leads to the following set of equations. (For a derivation, see e.g. Refs. 32,37; very detailed discussions are given e. g. in Refs. 33,38, for 
a diagrammatic derivation see Ref. 39.) The flow of the self-energy $\Sigma^{\Lambda}=-\gamma_{1}^{\Lambda}$ is given by

$$
\frac{d}{d \Lambda} \gamma_{1}^{\Lambda}\left(q_{1}^{\prime}, q_{1}\right)=T \sum_{q_{2}^{\prime}, q_{2}} \mathcal{S}_{q_{2}, q_{2}^{\prime}}^{\Lambda} \gamma_{2}^{\Lambda}\left(q_{2}^{\prime}, q_{1}^{\prime} ; q_{2}, q_{1}\right)
$$

Here $\mathcal{S}^{\Lambda}$ is defined in terms of the scale-dependent full propagator $\mathcal{G}^{\Lambda}$,

$$
\begin{aligned}
\mathcal{S}^{\Lambda} & =\mathcal{G}^{\Lambda} \partial_{\Lambda}\left[\mathcal{G}_{0}^{\Lambda}\right]^{-1} \mathcal{G}^{\Lambda}=\longleftarrow \\
\mathcal{G}^{\Lambda} & =\left[\left[\mathcal{G}_{0}^{\Lambda}\right]^{-1}-\Sigma^{\Lambda}\right]^{-1}=\longleftarrow
\end{aligned}
$$

and $\gamma_{2}^{\Lambda}$ is the two-particle irreducible vertex.

The flow of the latter can be arranged into three contributions (or parquet channels),

$$
\frac{d}{d \Lambda} \gamma_{2}^{\Lambda}=\frac{d}{d \Lambda}\left(\gamma_{p}^{\Lambda}+\gamma_{x}^{\Lambda}+\gamma_{d}^{\Lambda}\right)
$$
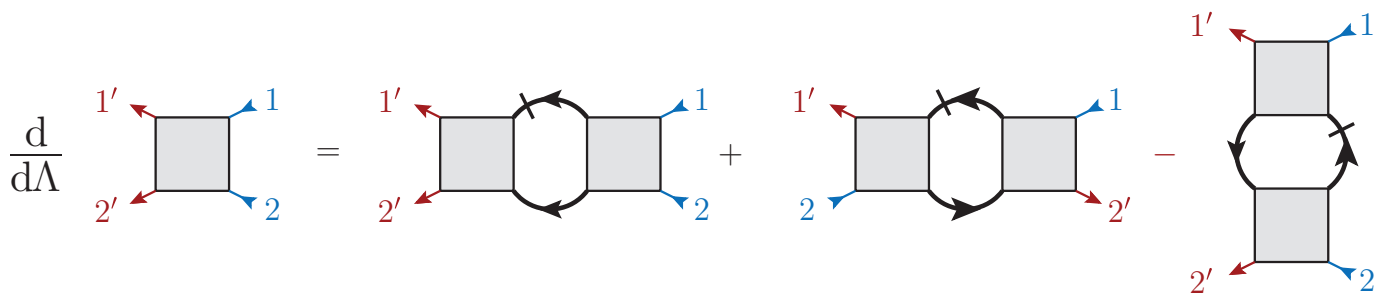

called the particle-particle channel $(\mathrm{P})$, and the exchange $(\mathrm{X})$ and direct $(\mathrm{D})$ contributions to the particle-hole channel, respectively, with the following explicit forms:

$$
\begin{aligned}
\frac{d}{d \Lambda} \gamma_{p}^{\Lambda}\left(q_{1}^{\prime}, q_{2}^{\prime} ; q_{1}, q_{2}\right) & =T \sum_{q_{3}^{\prime}, q_{3}, q_{4}^{\prime}, q_{4}} \gamma_{2}^{\Lambda}\left(q_{1}^{\prime}, q_{2}^{\prime} ; q_{3}, q_{4}\right) \mathcal{S}_{q_{3}, q_{3}^{\prime}}^{\Lambda} \mathcal{G}_{q_{4}, q_{4}^{\prime}}^{\Lambda} \gamma_{2}^{\Lambda}\left(q_{3}^{\prime}, q_{4}^{\prime} ; q_{1}, q_{2}\right), \\
\frac{d}{d \Lambda} \gamma_{x}^{\Lambda}\left(q_{1}^{\prime}, q_{2}^{\prime} ; q_{1}, q_{2}\right) & =T \sum_{q_{3}^{\prime}, q_{3}, q_{4}^{\prime}, q_{4}} \gamma_{2}^{\Lambda}\left(q_{1}^{\prime}, q_{4}^{\prime} ; q_{3}, q_{2}\right)\left[\mathcal{S}_{q_{3}, q_{3}^{\prime}}^{\Lambda} \mathcal{G}_{q_{4}, q_{4}^{\prime}}^{\Lambda}+\mathcal{G}_{q_{3}, q_{3}^{\prime}}^{\Lambda} \mathcal{S}_{q_{4}, q_{4}^{\prime}}^{\Lambda}\right] \gamma_{2}^{\Lambda}\left(q_{3}^{\prime}, q_{2}^{\prime} ; q_{1}, q_{4}\right), \\
\frac{d}{d \Lambda} \gamma_{d}^{\Lambda}\left(q_{1}^{\prime}, q_{2}^{\prime} ; q_{1}, q_{2}\right) & =-T \sum_{q_{3}^{\prime}, q_{3}, q_{4}^{\prime}, q_{4}} \gamma_{2}^{\Lambda}\left(q_{1}^{\prime}, q_{3}^{\prime} ; q_{1}, q_{4}\right)\left[\mathcal{S}_{q_{4}, q_{4}^{\prime}}^{\Lambda} \mathcal{G}_{q_{3}, q_{3}^{\prime}}^{\Lambda}+\mathcal{G}_{q_{4}, q_{4}^{\prime}}^{\Lambda} \mathcal{S}_{q_{3}, q_{3}^{\prime}}^{\Lambda}\right] \gamma_{2}^{\Lambda}\left(q_{4}^{\prime}, q_{2}^{\prime} ; q_{3}, q_{2}\right) .
\end{aligned}
$$

All higher order vertices $\gamma_{n>3}$ have been set to zero. For the purpose of treating the inhomogeneous chain model of Eq. (S11), the quantum numbers $q_{i}$ denote a composite index of site, spin and Matsubara-frequency, $q_{1}=\left(j_{1}, \sigma_{1}, i \omega_{n}^{1}\right)$, etc.

\section{D. fRG for non-uniform systems}

A standard strategy for getting an initial impression of the system's behavior is to neglect the flow of the twoparticle vertex completely. For the present model, the results so obtained ${ }^{36}$ turn out to be similar to those obtained from SOPT - they capture the effects of interactions quite well qualitatively, but not quantitatively. To allow quantitative comparisons to experiment, we have therefore included the flow of the two-particle vertex for all fRG results shown in this work. We now describe how this was done.

Since the bare propagators are not site-diagonal, the number of independent variables needed to describe the vertex $\gamma_{2}^{\Lambda}\left(q_{1}^{\prime}, q_{2}^{\prime} ; q_{1}, q_{2}\right)$ generated by Eq. (S46) is very large, $\mathcal{O}\left(N^{4} N_{\mathrm{f}}^{3}\right)\left(N_{\mathrm{f}}\right.$ is the number of Matsubarafrequencies used in the numerics). To deal with this complication we use the coupled-ladder approximation ${ }^{40,41}$ for the frequency dependence of $\gamma_{2}^{\Lambda}$ and treat its sitedependence in a similar manner. Given the structure of the flow equation (S46) for $\gamma_{2}^{\Lambda}$, it is natural to divide the flowing vertex into four parts ${ }^{41}$ :

$$
\gamma_{2}^{\Lambda}=v+\gamma_{p}^{\Lambda}+\gamma_{x}^{\Lambda}+\gamma_{d}^{\Lambda}, \quad \gamma_{p}^{\Lambda_{i}}=\gamma_{x}^{\Lambda_{i}}=\gamma_{d}^{\Lambda_{i}}=0 .
$$

Here $v$ is the bare vertex, and $\gamma_{2}^{p}, \gamma_{2}^{x}$ and $\gamma_{2}^{d}$, whose flows by definition are given by Eqs. (S46a), (S46b) and (S46c), sum up the $\mathrm{P}-\mathrm{X}$ - and D-channels, respectively (see Sec. 
S-6 C).

Now, since the bare vertex is site-diagonal, only $\mathcal{O}\left(N^{2} N_{\mathrm{f}}\right)$ of the $\mathcal{O}\left(N^{4} N_{\mathrm{f}}^{3}\right)$ different components in each channel are generated already to order $v^{2}$ [i.e. if, at the beginning of the flow, $\gamma_{2}^{\Lambda}$ is replaced by $v$ on the r.h.s. of Eq. (S46)]. We exploit this fact by making the following simplifying approximation in the spatial structure of $\gamma_{2}$ : in each channel we set to zero all components except those that are generated already to order $v^{2}$, but retain the latter components throughout the flow. The dropped components are all of order $v^{3}$ or higher, which justifies their neglect as long as $U_{j}$ is not too large. Furthermore we only keep the intrinsic frequency dependence of each channel (i.e. the frequency-dependence generated to 2nd order). Each channel thus depends only on a single bosonic frequency, denoted by $\Pi, \mathrm{X}$ and $\Delta$ for the $\mathrm{P}-$, $\mathrm{X}$ - and D-channels, respectively. The feed-back into the other channels is performed using only the static part of each channel, i. e. its value evaluated at zero frequency ${ }^{41}$. By exploiting various symmetry relations, the retained components of $\gamma_{2}^{\Lambda}$ can be parametrized in terms of four frequency-dependent matrices, $P_{i j}^{\Lambda}(\Pi), X_{i j}^{\Lambda}(\mathrm{X}), D_{i j}^{\uparrow \Lambda}(\Delta)$ and $D_{i j}^{\downarrow \Lambda}(\Delta)$, defined as follows (and shown together with the diagrams that generate them to lowest order):

$$
\begin{aligned}
& P_{j i}^{\Lambda}(\Pi):=\gamma_{p}^{\Lambda}\left(j \sigma \Pi-\omega_{n}^{\prime}, j \bar{\sigma} \omega_{n}^{\prime} ; i \sigma \Pi-\omega_{n}, i \bar{\sigma} \omega_{n}\right)=-\gamma_{p}^{\Lambda}\left(j \sigma \Pi-\omega_{n}^{\prime}, j \bar{\sigma} \omega_{n}^{\prime} ; i \bar{\sigma} \Pi-\omega_{n}, i \sigma \omega_{n}\right),
\end{aligned}
$$

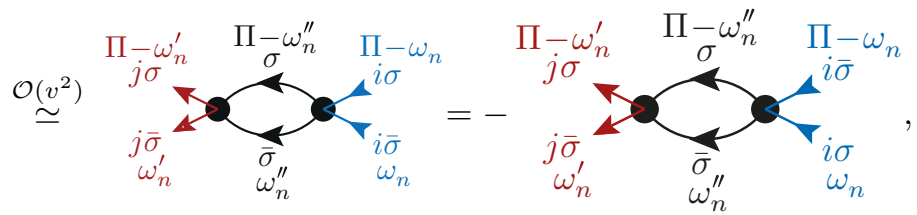

$$
\begin{aligned}
& X_{j i}^{\Lambda}(\mathrm{X}):=\gamma_{x}^{\Lambda}\left(j \sigma \mathrm{X}+\omega_{n}^{\prime}, i \bar{\sigma} \omega_{n} ; i \sigma \mathrm{X}+\omega_{n}, j \bar{\sigma} \omega_{n}^{\prime}\right)=-\gamma_{d}^{\Lambda}\left(j \sigma \mathrm{X}+\omega_{n}^{\prime}, i \bar{\sigma} \omega_{n} ; j \bar{\sigma} \omega_{n}^{\prime}, i \sigma \mathrm{X}+\omega_{n}\right),
\end{aligned}
$$

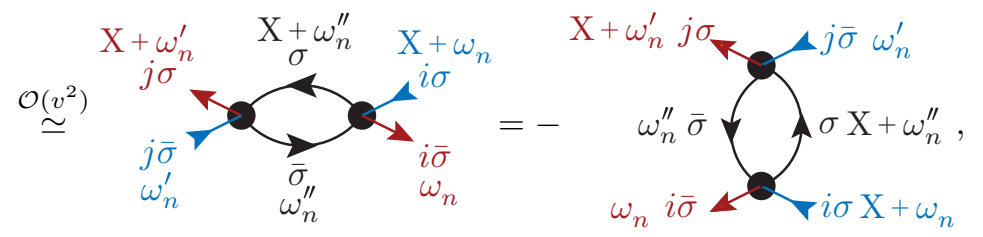

$$
\begin{aligned}
& D_{j i}^{\sigma \Lambda}(\Delta):=\gamma_{d}^{\Lambda}\left(j \sigma \Delta+\omega_{n}^{\prime}, i \sigma \omega_{n} ; j \sigma \omega_{n}^{\prime}, i \sigma \Delta+\omega_{n}\right)=-\gamma_{x}^{\Lambda}\left(j \sigma \Delta+\omega_{n}^{\prime}, i \sigma \omega_{n} ; i \sigma \Delta+\omega_{n}, j \sigma \omega_{n}^{\prime}\right), \\
& \stackrel{\mathcal{O}\left(v^{2}\right)}{\sim} \omega_{\omega_{n}}^{\prime \prime \sigma} \bar{\sigma} \overbrace{i \sigma \Delta+\omega_{n}}^{j \sigma}
\end{aligned}
$$

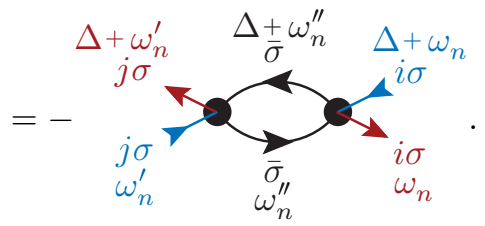

Note that these diagrams do not depend on $\omega_{n}$ and $\omega_{n}^{\prime}$; this is the reason why the coupled-ladder approximation allows each channel to be parametrized by just a single frequency. A detailed analysis of the flow of $P_{i j}^{\Lambda}, X_{i j}^{\Lambda}$ and $D_{i j}^{\sigma \Lambda}$, to be published elsewhere ${ }^{36}$, shows that the exchange channel $X_{i j}^{\Lambda}$, which grows significantly during the flow, is the dominant one. This lends $a$ posteriori support to an assertion made in numerous works ${ }^{4,7-9,12,14,20-22,30,31,42}$, namely that exchange interactions in the low-density inner region of the QPC play a dominant role for the 0.7 -anomaly.

The parameter controlling the convergence of the fRG equations is $U \cdot \max _{\widetilde{V}_{\mathrm{c}}, j}\left[\mathcal{A}_{j}(0)\right]$; if it is too large, these equations do not converge. For a QPC, the maximum value of the bare $\operatorname{LDOS} \mathcal{A}_{j}^{0}(0)$ scales as $1 / \sqrt{\tau \Omega_{x}}$ (see Sec. S-4 C).

\section{E. Zero-temperature limit}

The fRG flow equations discussed above apply to an arbitrary temperature $T$. However, the conductance at $T \neq 0$ depends on the retarded correlator $\mathcal{G}^{R}(\omega)=$ $\mathcal{G}\left(i \omega_{n} \rightarrow \omega+i 0^{+}\right)$as well as the retarded parts of the vertex channels (e.g. $P\left(i \Pi_{n} \rightarrow \Pi+i 0^{+}\right)$), which have to be obtained by analytic continuation from the imag- 
inary to the real frequency axis. In numerical practice, this analytical continuation turned out to be unfeasible for the present problem. Therefore, we have here studied only the $T=0$ limit, in which the Matsubara frequencies form a continuum and the conductance [Eq. (S37)] is expressed in terms of the zero-energy transmission $\mathcal{T}_{\sigma}(0)$. For numerical computations, we represented the continuum of Matsubara frequencies by a discrete set, and used its smallest positive member to evaluate the Matsubara Green's function $\mathcal{G}_{-N^{\prime}, N^{\prime}}^{\sigma}\left(i 0^{+}\right)$needed for $\mathcal{T}_{\sigma}(0)$ [Eq. (S38)].

In the limit $T \rightarrow 0$, the cut-off function $\Theta_{T}$ in Eq. (S41) becomes a sharp step function, with $\Theta_{0}(0)=\frac{1}{2}$ and $\partial_{\omega} \Theta_{0}(\omega)=\delta(\omega)$. Since a combination of $\delta$ - and $\Theta$ functions occurs in the fRG flow equations, the limit $T \rightarrow 0$ has to be taken with care, with the result ${ }^{40}$ :

$$
\begin{aligned}
\mathcal{S}^{\Lambda}(i \omega) \stackrel{T \equiv}{=} & \delta(|\omega|-\Lambda) \widetilde{\mathcal{G}}^{\Lambda}(i \omega), \\
\widetilde{\mathcal{G}}^{\Lambda}(i \omega)= & {\left[\left[\mathcal{G}_{0}(i \omega)\right]^{-1}-\Sigma^{\Lambda}(i \omega)\right]^{-1}, } \\
\mathcal{S}_{i, j}^{\Lambda}(i \omega) \mathcal{G}_{k, l}^{\Lambda}\left(i \omega^{\prime}\right) \stackrel{T=0}{=} & \delta(|\omega|-\Lambda) \widetilde{\mathcal{G}}_{i, j}^{\Lambda}(i \omega) \\
& \times \Theta\left(\left|\omega^{\prime}\right|-\Lambda\right) \widetilde{\mathcal{G}}_{k, l}^{\Lambda}\left(i \omega^{\prime}\right) .
\end{aligned}
$$

\section{F. Static fRG}

Most of our exploratory work on the zero-temperature properties of the 0.7-anomaly was done using "static" fRG (here denoted by $\mathrm{fRG}^{0}$ ). It entails a further approximation relative to the "dynamic" fRG approach described above (here denoted by $\mathrm{fRG}^{\omega}$ ), in that $\mathrm{fRG}^{0}$ neglects the frequency dependence of the self-energy and all vertices. This is done by setting all three bosonic frequencies $\Pi, \mathrm{X}$ and $\Delta$ to zero. As a result the self-energy is frequency-independent, too. $\mathrm{fRG}^{0}$ leads to reliable results only for the zero-frequency Green's function at zero temperature. If knowing the latter suffices (such as when studying the magnetic field-dependence at $\widetilde{T}=0$ ), $\mathrm{fRG}^{0}$ is a very flexible and efficient tool, computationally cheaper than our full coupled-ladder scheme $\mathrm{fRG}^{\omega}$ by a factor of $10^{3}$. Moreover, for the model studied here its results turn out to be qualitatively very similar to those of $\mathrm{fRG}^{\omega}$. This is illustrated in Fig. S15, from which we note the following salient features.

The main difference in the conductance curves calculated by the two methods is an overall interactioninduced, $U$-dependent shift of the position of the $\mathrm{fRG}^{0}$ conductance step w.r.t. to that of $\mathrm{fRG}^{\omega}$, towards somewhat smaller values of $\widetilde{V}_{\mathrm{c}}$ (compare Figs. S15a,b); however the shapes of the corresponding curves (modulo the shift) are essentially identical (Fig. S15c). The shift itself merely amounts to a small change in overall chemical potential and can be regarded as an insignificant detail, in particular in the context of the 0.7-anomaly, where both in theoretical and experimental studies, the focus is on the shape of the step, not its position.
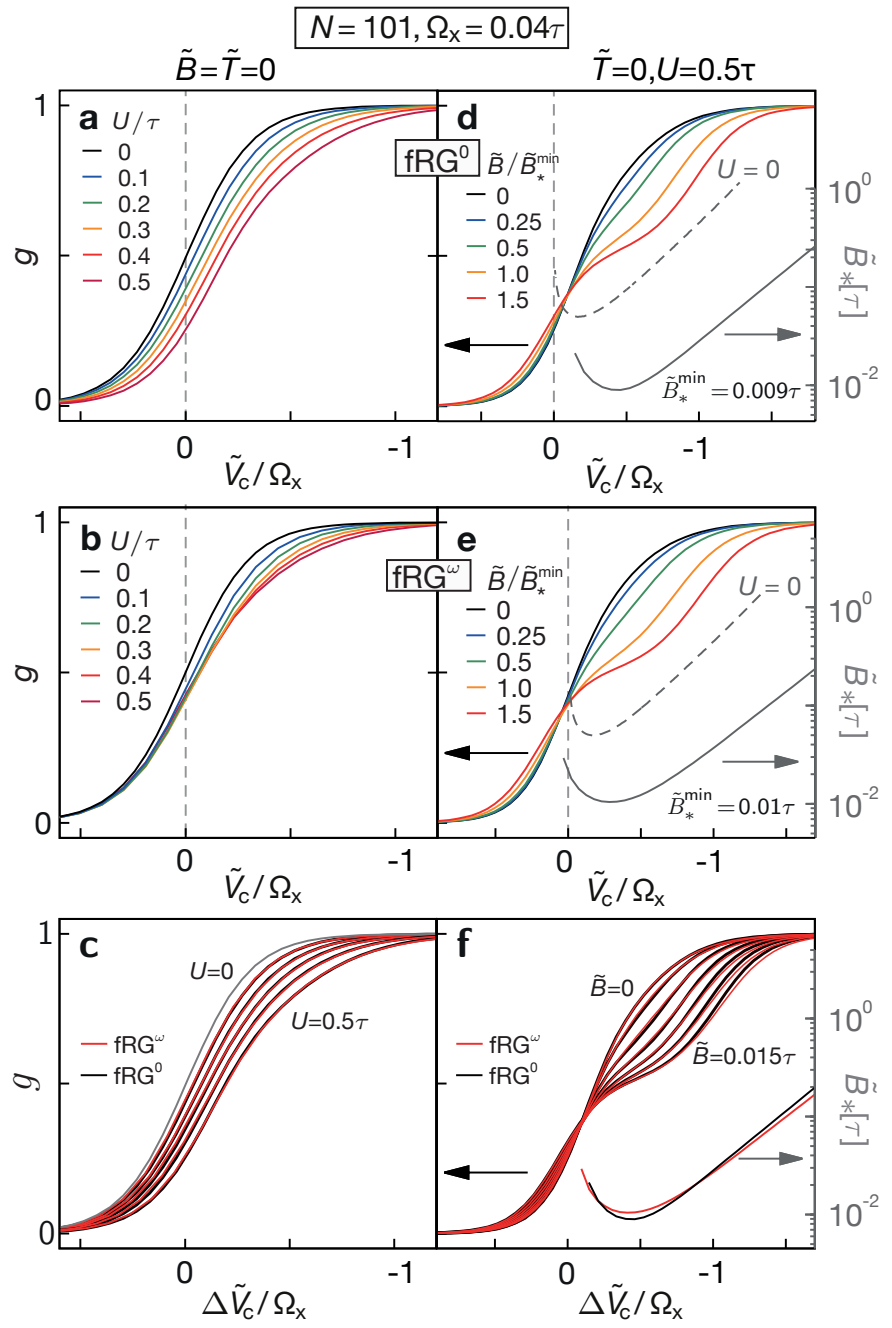

Figure S15: Comparison of results from static fRG $\left(\mathrm{fRG}^{0}\right.$, top panels) and dynamic fRG (fRG ${ }^{\omega}$, middle panels, reproduced from Figs. A1k and A2a, respectively). The bottom panels show that after compensating for a $U$-dependent shift (here applied to the $\mathrm{fRG}^{\omega}$ curves to get best overlap with the $\mathrm{fRG}^{0}$ curves) both sets of curves have almost identical shapes. Left panels: The interaction dependence of the conductance $g\left(\widetilde{V}_{\mathrm{c}}\right)$ at $\widetilde{B}=\widetilde{T}=0$. Right panels: The magnetic-field dependence of the $\widetilde{T}=0$ conductance $g\left(\widetilde{V}_{\mathrm{c}}\right)$ at fixed $U=0.5 \tau$.

Closer inspection reveals that the magnetic field dependence (at fixed $U$ ) of the $\mathrm{fRG}^{0}$ conductance curves is slightly stronger for small fields and slightly weaker for large fields, compared to that of $\mathrm{fRG}^{\omega}$ (see Figs. S15df). This implies small quantitative differences in the lowenergy scale $\widetilde{B}_{*}$ and the effective g-factor $g_{\mathrm{ss}}$.

All in all, for the purposes of exploring the fielddependence of the 0.7 -anomaly at fixed $U$, the differences in results between $\mathrm{fRG}^{\omega}$ and $\mathrm{fRG}^{0}$ are evidently very small. Hence we have opted to use the computationally much cheaper $\mathrm{fRG}^{0}$ for the results presented in Secs. S-3 and S-5. 


\section{S-7. SECOND-ORDER PERTURBATION THEORY}

The main limitation of our calculations using static fRG is their restriction to $\omega=0$ and hence to zero temperature and zero source-drain voltage. As a first step toward exploring the properties of our model at $\widetilde{T} \neq 0$ and $\widetilde{V}_{\text {sd }} \neq 0$, we have calculated the conductance of a QPC using second-order perturbation theory (SOPT), applied to model II (see Supplementary Sec. S-4D). Sec. S-7 A presents the diagrams used for this purpose, and Sec. S$7 \mathrm{~B}$ discusses how we treat non-equilibrium transport using Keldysh-SOPT. Sec. S-7 C elaborates the main article's discussion of the SOPT results for the $\widetilde{B}$-, $\widetilde{T}$ - and $\widetilde{V}_{\text {sd }}$-dependence of the conductance, which are in fairly good qualitative agreement with experiment. Sec. S-7 D concludes with some comments regarding an SOPT artefact that arises with increasing $U$.

\section{A. Equilibrium SOPT}

We follow the strategy of Oguri ${ }^{43}$, who has carried out a similar calculation for a particle-hole symmetric version of our model (with $N \leq 4$ ) at zero field. It is straightforward to generalize his equations to the case of present interest, with broken particle-hole symmetry and nonzero field. The conductance is calculated from

$$
g=\frac{1}{2} \sum_{\sigma} \int_{-\infty}^{\infty} d \omega\left[-\frac{\partial f(\omega)}{\partial \omega}\right] \mathcal{T}_{\sigma}(\omega) .
$$

where $\mathcal{T}_{\sigma}(\omega)$ is calculated using Oguri's ${ }^{43}$ equations (2.36-38) and (4.10). They can be graphically depicted as

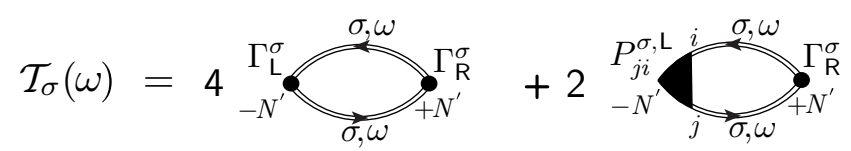

$(\mathrm{S} 51)$

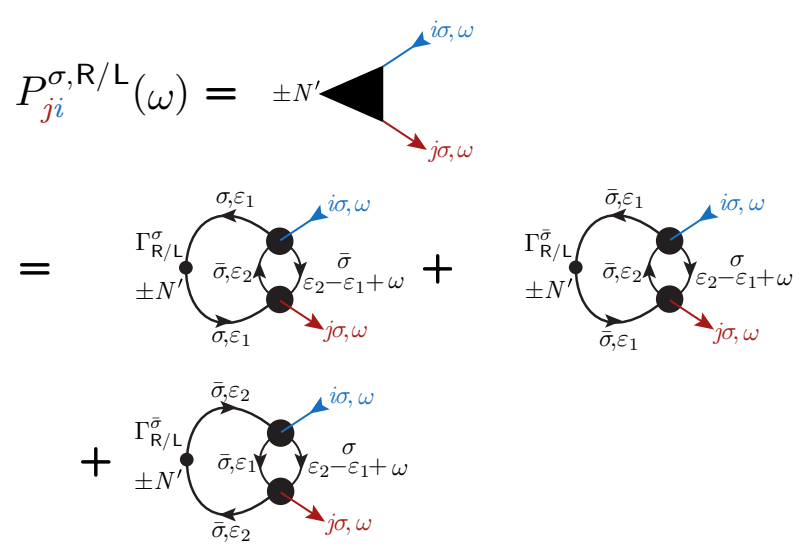

(S52)

where large black dots depict the bare interaction vertex, small black dots the coupling $\Gamma_{R / L}^{\sigma}(\epsilon)$ to the reservoirs, and the double lines represent the retarded interacting Green's function $\mathcal{G}^{R}=\left[\left(\mathcal{G}^{0} R\right)^{-1}-\Sigma^{R}\right]^{-1}$. Its self-energy $\Sigma^{R}$ is calculated to second order using the following diagrams:

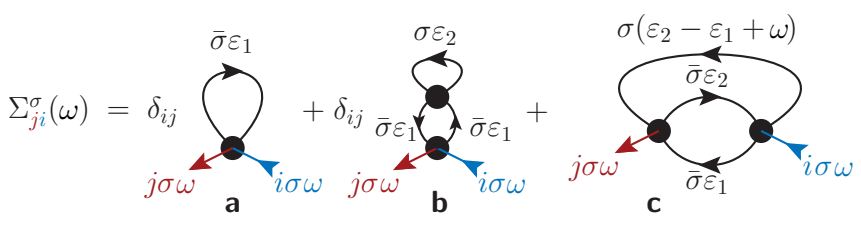

Diagram c corresponds to Oguri's ${ }^{43}$ Fig. 6, which represent the Fock contribution. Our treatment differs from Oguri's only regarding the Hartree diagrams a and b. Whereas he incorporates their effects in an implicit manner by exploiting particle-hole symmetry, this symmetry is not present in our problem, hence we include the Hartree diagrams explicitly in the self-energy.

The diagrams in Eqs. (S52) and (S53) involve Matsubara frequencies; they have to be analytically continued to real frequencies before being used in Eq. (S51) for $\mathcal{T}_{\sigma}(\omega)$, as discussed in detail by Oguri. The resulting formulas, obtained by generalizing Oguri's ${ }^{43}$ equations [his (4.2), (4.3) for the Fock diagram, and (4.10) for the current vertex] to the spin-dependent case of non-zero field, will be presented elsewhere ${ }^{36}$.

In the main article, the transmission probability is written as

$$
\mathcal{T}_{\sigma}(\omega)=\mathcal{T}_{\sigma}^{\mathrm{el}}(\omega)+\mathcal{T}_{\sigma}^{\mathrm{in}}(\omega),
$$

where $\mathcal{T}_{\sigma}^{\mathrm{el}}(\omega)$ and $\mathcal{T}_{\sigma}^{\text {in }}(\omega)$, given by the first and second terms of Eq. (S51), describe the elastic and inelastic contributions to the transmission probability, respectively. They are related by a generalized Ward identity that is respected within the approximation scheme described above (Eq. (3.120) in Ref. 43):

$$
-\operatorname{Im} \Sigma_{j i}^{\sigma, R}(\omega)=\sum_{\alpha=L / R} P_{j i}^{\sigma, \alpha}(\omega) .
$$

This relation links the current vertex to the inelastic decay rate, governed by the imaginary part of the selfenergy. An increase in the contribution of the current vertex, therefore, goes hand in hand with an increase in inelastic scattering.

SOPT calculations turn out to be computationally significantly more costly than fRG calculations. Therefore, all our SOPT calculations were done using model II, which has some computational advantages over model I, as explained in Supplementary Sec. S-4 D.

\section{B. Nonequilibrium SOPT}

In order to calculate the differential conductance

$$
g_{\mathrm{nl}}=\frac{d I}{d \widetilde{V}_{\mathrm{sd}}}
$$


at finite bias voltage $\left(\widetilde{V}_{\text {sd }} \neq 0\right)$ we use the Meir-Wingren formula for the current through a region of interacting electrons $^{44}$ :

$$
\begin{aligned}
& I=\frac{i e}{2 h} \int d \varepsilon\left(\operatorname{Tr}\left\{\left[f_{L} \Gamma^{L}-f_{R} \Gamma^{R}\right]\left(\mathcal{G}^{R}-\mathcal{G}^{A}\right)\right\}\right. \\
&\left.+\operatorname{Tr}\left\{\left[\Gamma^{L}-\Gamma^{R}\right] \mathcal{G}^{<}\right\}\right),
\end{aligned}
$$

with $\mathcal{G}^{<}=\frac{1}{2}\left(\mathcal{G}^{K}-\mathcal{G}^{R}+\mathcal{G}^{A}\right)$. The finite bias $\widetilde{V}_{\text {sd }}$ enters via the occupation functions of the left and right lead:

$$
f_{R / L}(\omega)=f\left(\omega \mp \widetilde{V}_{\mathrm{sd}} / 2\right) .
$$

The retarded, advanced and Keldysh Green's functions $\mathcal{G}^{R / A / K}$ are given by Dyson equations:

$$
\begin{aligned}
\mathcal{G}^{R / A} & =\frac{1}{\left(G^{R / A}\right)^{-1}-\Sigma^{R / A}} . \\
\mathcal{G}^{K} & =\mathcal{G}^{R}\left[\left(G^{R}\right)^{-1} G^{K}\left(G^{A}\right)^{-1}+\Sigma^{K}\right] \mathcal{G}^{A},
\end{aligned}
$$

The non-equilibrium retarded, advanced and Keldysh self-energies occurring herein, $\Sigma^{R / A / K}$, are calculated to second order in the interaction, using standard Keldysh techniques. The corresponding diagrams are again given by Eq. (S53), but now feature an additional Keldysh index.

\section{C. $\widetilde{B}-, \widetilde{T}-$, and $\widetilde{V}_{\mathrm{sd}}$-dependence of $g\left(\widetilde{V}_{\mathrm{c}}\right)$}

In this section, we give a detailed discussion of the SOPT results presented in the main text for the $\widetilde{B}$-, $\widetilde{T}$ and $\widetilde{V}_{\text {sd }}$-dependence of the conductance $g\left(\widetilde{V}_{\mathrm{c}}\right)$. In particular, we analyse their similarities and differences w.r.t. our fRG results and experimental measurements.

Dependence on magnetic field at zero temperature: To gauge the reliability of SOPT, we begin by comparing its results for the magnetic field dependence of the conductance (Fig. A2c) to those obtained from fRG (Fig. A2a) and from experimental measurements (Fig. A2e). Though some details differ, the qualitative agreement is very good. It includes, in particular, the following two important features: (i) The conductance $g\left(\widetilde{V}_{\mathrm{c}}\right)$ is strongly suppressed with increasing $\widetilde{B}$ for $\widetilde{V}_{\mathrm{c}}<0$, leading to the evolution of a kink around 0.5 (thick red line in Fig. A2c). (ii) $\ln \left(\widetilde{B}_{*}\right)$ increases nearly linearly with decreasing $\widetilde{V}_{\mathrm{c}}$ in the regime where $g \rightarrow 1$ (thin grey line in Fig. A2c); in fact, even the slope of the linear increase is nearly the same as that found by fRG (grey line in Fig. A2a). This remarkable agreement between SOPT and fRG for the $\widetilde{V}_{\mathrm{c}}$-dependence of $\widetilde{B}_{*}$ implies that the latter is determined mainly by geometry (corroborating a similar conclusion from Sec. S-5), i. e. interactions, which are underestimated in SOPT, influence the

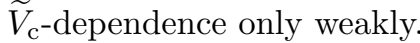

As an aside, we note that both of the above-mentioned features (i) and (ii) survive ${ }^{45}$ (data not shown) even if

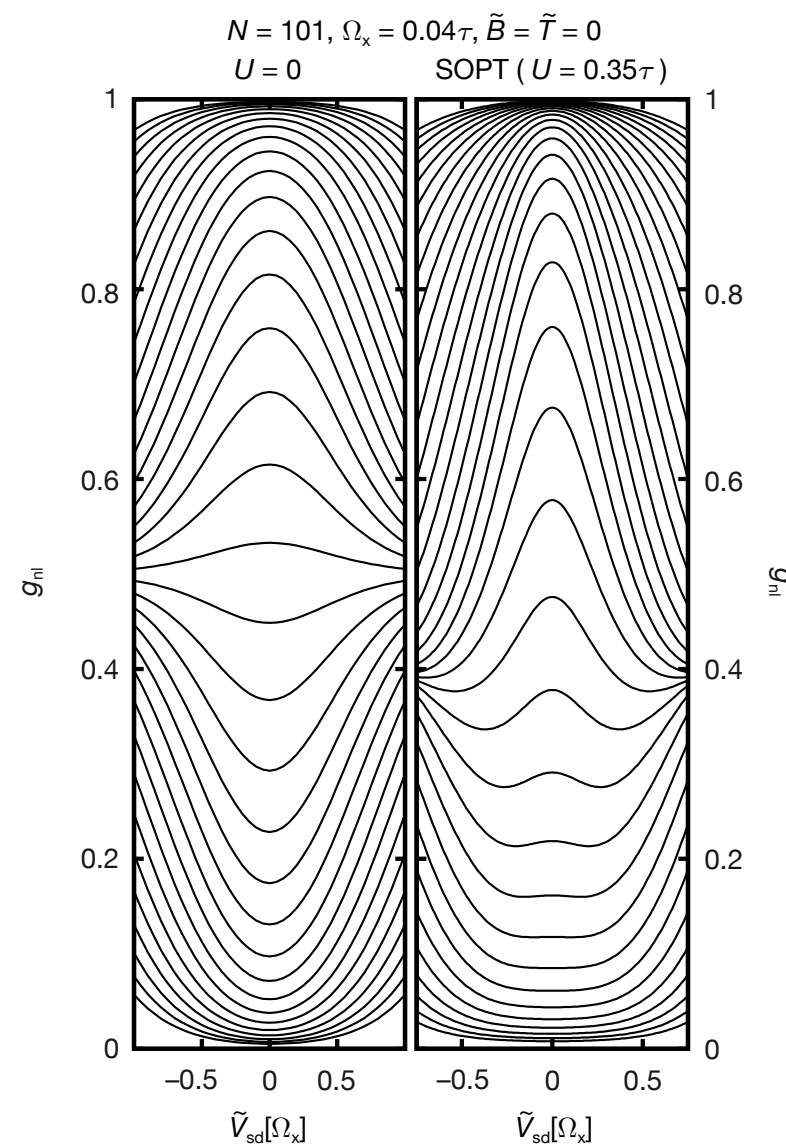

Figure S16: Differential conductance for model II at $\widetilde{B}=$ $\widetilde{T}=0$, plotted as a function of bias voltage for several $\widetilde{V}_{\mathrm{c}^{-}}$ values, calculated $\mathbf{a}$ without and $\mathbf{b}$ with interactions, the latter treated using second order perturbation theory (SOPT) (see Sec. S-7 B).

SOPT is simplified by neglecting the Fock contribution to the self-energy [diagram c of Eq. (S53)], retaining only the first- and second-order Hartree terms [diagrams a and b of Eq. (S53)]. Thus, the $\widetilde{B}$-dependence is dominated by Hartree terms (describing shifts in the barrier heights for spin-up vs. spin-down electrons), rather than Fock terms (describing inelastic scattering, which is relevant only at finite temperatures and finite bias voltage). This conclusion is consistent with the fact that the approach of Lunde et al., Ref. 6, which properly incorporated the (model) system's geometry-dependence by using WKB wave functions, is nevertheless unable to reproduce the energy scale $\widetilde{B}_{*}$ from the magnetic field dependence as long as only Fock-like diagrams are considered ${ }^{46}$.

Next, we mention an important instance in which SOPT fails to agree with experimental and fRG results for the conductance (compare Fig. A2c to Fig. A2e and Fig. A2a): SOPT does not yield the 0.7-shoulder in the conductance at $\widetilde{T}=\widetilde{B}=0$. (A shoulder does develop for larger $U(\gtrsim 0.5 \tau)$, for which, however, SOPT can no longer be trusted.) 
To summarize: SOPT correctly captures several important features of the field dependence of the conductance at zero temperature, but not all details. The same turns out to be true for the temperature dependence at zero field, as we discuss next:

Dependence on temperature at zero magnetic field: Fig. A2d presents SOPT results for the temperaturedependence of the conductance at zero field. Comparing these to the corresponding experimental results of Fig. A2f, we note that SOPT correctly reproduces the main effect of increasing temperature, namely to reduce the conductance. However, SOPT does not fully succeed in reproducing the detailed shape of the experimental pinch-off curves: the SOPT curves lack the tendency of the experimental curves to show a well-defined shoulder that is amplified by increasing temperature.

Dependence on bias voltage: Fig. S16a shows the bare $(U=0)$ differential conductance $g_{\mathrm{nl}}^{0}$ as a function of

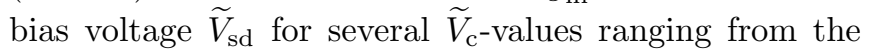
open channel $(g \simeq 1)$ to the pinched-off regime $(g \simeq 0)$. The bare conductance can easily be calculated from the Landauer-Büttiker formula (S25), using Büttiker's formula (S30) for the transmission. The resulting $g_{\mathrm{nl}}^{0}\left(\widetilde{V}_{\mathrm{sd}}\right)$ exhibits a zero bias peak (ZBP) for linear conductance $g>0.5\left(\widetilde{V}_{\mathrm{c}}<0\right)$, and a zero bias minimum in the tunneling regime, where $\widetilde{V}_{\mathrm{c}}>0$ and $g<0.5$.

Turning on interaction (see Fig. A3i, as well as Fig. S16b) causes the following effects on $g_{\mathrm{nl}}$ : First, a ZBP forms even when the linear conductance is $g<0.5$, and second, the width of the ZBP is reduced across the whole $\widetilde{V}_{\mathrm{c}}$-range. These two interaction-induced characteristics can be understood in terms of two main mechanisms: (i) Applying finite bias generates a net charge flow into the barrier region (since there the LDOS is $\omega$ asymmetric around $\omega=0$ ), thereby enhancing the effective barrier height for electrons entering the CCR. For sufficiently large interaction this leads to a reduction of conductance (Hartree effect). (ii) Turning on $\widetilde{V}_{\text {sd }}$ opens phase space for inelastic scattering. Consequently the combination of a large LDOS in the vicinity of the classical turning points (where $\omega_{j}^{\text {min }} \simeq 0$ ), interactions, and $\widetilde{V}_{\text {sd }}>0$, leads to a high probability for backscattering, hence a reduction of conductance. We note that both mechanisms (i) and (ii) also apply when the temperature is increased; in this sense, the temperature- and biasdependencies of the 0.7-anomaly are manifestations of the similar physical processes.

We take the SOPT results shown in Fig. A2d and Figs. A3b,d-f as encouraging indications that our model has the potential to properly describe properties of the 0.7 -anomaly at finite temperature and bias. To summarize: the anomalous conductance decrease with increasing $\widetilde{T}$ or $\widetilde{V}_{\text {sd }}$ in the sub-open regime originates from the enhancement, by the van Hove ridge apex near $\mu$, of the $\widetilde{T}$ - or $\widetilde{V}_{\text {sd }}$-induced increase of (i) the net charge and (ii) the amount of inelastic scattering in the CCR.

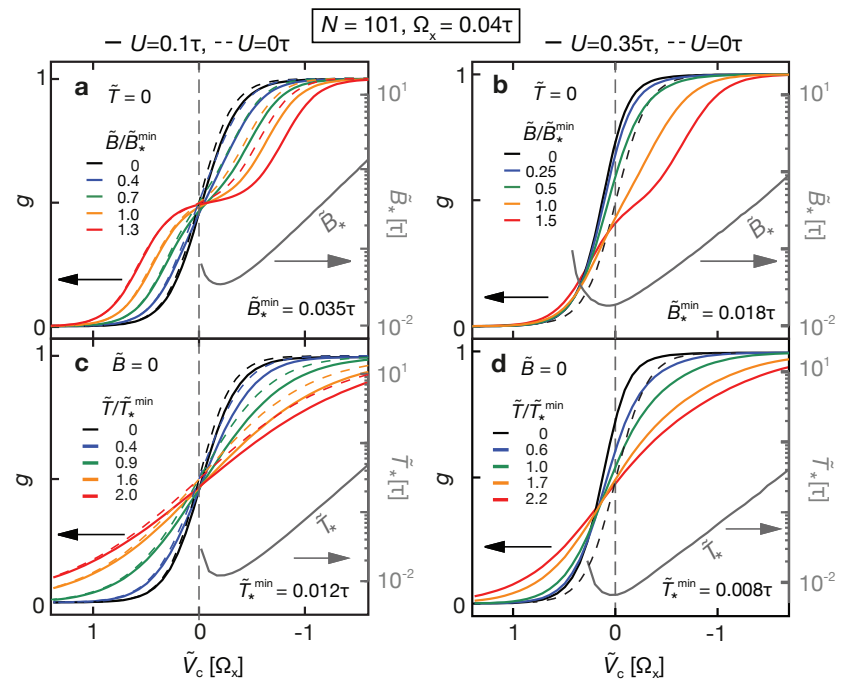

Figure S17: SOPT results (solid lines) for the conductance $g\left(\widetilde{V}_{\mathrm{c}}\right)$ as function of $\widetilde{V}_{\mathrm{c}}$, illustrating the qualitative changes incurred when interactions are increased from being weak (left panels) to rather strong (right panels, reproduced from Figs. A2c, d). Panels $(\mathrm{a}, \mathrm{b})$ show the $\widetilde{B}$-dependence of the conductance at $\widetilde{T}=0$, panels $(\mathrm{c}, \mathrm{d})$ show its $\widetilde{T}$-dependence at $\widetilde{B}=0$. Dashed lines show corresponding curves for the bare $U=0$ conductance, $g^{0}\left(\widetilde{V}_{\mathrm{c}}\right)$.

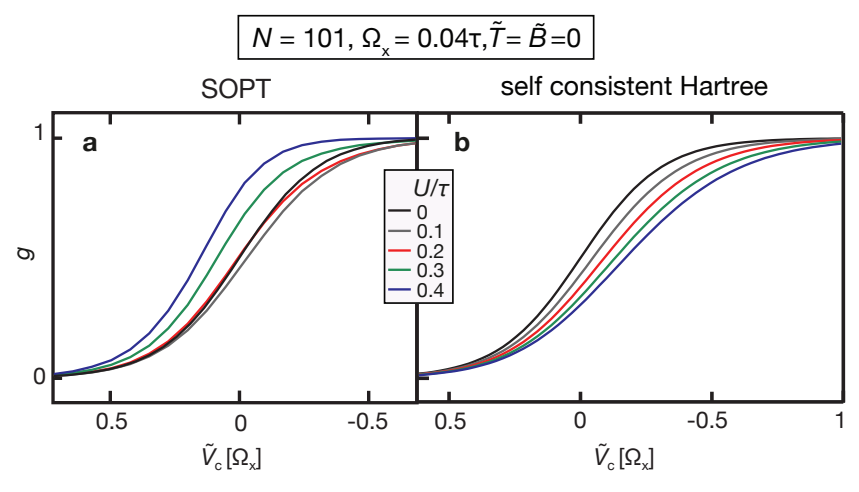

Figure S18: The zero-temperature linear conductance $g\left(\widetilde{V}_{\mathrm{c}}\right)$ as function of $\widetilde{V}_{\mathrm{c}}$, for several values of $U$, calculated a, using pure SOPT, and $\mathbf{b}$, using a self-consistent Hartree approach (without Fock contributions). The non-monotonic behavior of $g\left(\widetilde{V}_{\mathrm{c}}\right)$ as function of increasing $U$ seen in a is an SOPT artefact, caused by the neglect of terms beyond 2nd order; this artefact is avoided by the self-consistent Hartree approach, as seen in $\mathbf{b}$.

\section{SOPT artefact arising for increasing $U$}

We conclude with some comments on the choice of interaction strength used for our SOPT calculations. Fig. S17 compares the SOPT results for the $\widetilde{B}$ - and $\widetilde{T}$ dependence of $g\left(\widetilde{V}_{\mathrm{c}}\right)$ calculated at $U=0$ (left panels, dashed lines), $U=0.1 \tau$ (left panels, solid lines) and $U=0.35 \tau$ (right panels, solid lines). The left pan- 
els show that upon turning on a weak interaction (solid lines), the conductance $g$ at fixed values of $\widetilde{V}_{\mathrm{c}}, \widetilde{B}$ and $\widetilde{T}$ is shifted slightly downward due to the increase of the Hartree barrier, leading to a slight skewing of the shape of the conductance step relative to the corresponding noninteracting value $g^{0}\left(\widetilde{V}_{\mathrm{c}}\right)$ (dashed lines). However, significantly larger values of $U$ are needed (right panels) to yield the strong type of skewing characteristic for measured conductance curves that exhibit the 0.7-anomaly.

Note that due to this large choice of $U$ in the right panels, the SOPT conductance curve $g\left(\widetilde{V}_{\mathrm{c}}\right)$ at $\widetilde{B}=\widetilde{T}=0$ (solid black) has been shifted upwards to actually lie above its non-interacting value (dashed black). This non-monotonic behavior of $g\left(\widetilde{V}_{\mathrm{c}}\right)$ for increasing $U$ (the shift being initially downwards, then upwards, illustrated clearly in Fig. S18a) is an artefact of SOPT's neglect of terms beyond 2nd order: the signs $(+$ or -$)$ of Hartree contributions are known to alternate with the order of expansion, hence truncating the latter beyond 2nd order generates non-monotonic behavior for the shift with increasing $U$ once the 2 nd-order term becomes larger than the 1st-order term. (fRG avoids this problem by summing up, in effect, a series of diagrams to all orders, resulting in a monotonic dependence of $g\left(\widetilde{V}_{\mathrm{c}}\right)$ on $U$, see Fig. A1k.)

We emphasize that this SOPT artefact is problematic only if one is interested in following the evolution of physical properties with increasing $U$ (examples of such evo- lution, calculated by fRG, are shown in Figs. A1e,j,k). However, for the purpose of studying physical properties at fixed $U$, SOPT does quite well: it succeeds in qualitatively illustrating the generic, experimentally observed trends of how interactions affect the $\widetilde{B}_{-}^{-}, \widetilde{T}_{-}^{-}$, and $\widetilde{V}_{\mathrm{sd}^{-}}$ dependence of the conductance even if the (fixed) value of $U$ is rather large, because the physical origin of these trends is robust. A detailed discussion of this point will be published elsewhere.

Finally, we note that the above-mentioned artefact can be avoided by adopting an approach similar in spirit to SOPT, but using a self-consistently-determined Hartree potential (thus treating Hartree and Fock terms on unequal footing): For $\widetilde{T}=\widetilde{V}_{\text {sd }}=0$, calculate the self-energy from just the first-order Hartree diagram Eq. (S53)a to obtain a Hartree-shifted local potential $E_{j \sigma}+U_{j} n_{j \sigma}$, determine the local charge $n_{j \sigma}$ self-consistently, and calculate the QPC transmission using Hartree-dressed Green's functions (see Fig. S18b).

For nonzero $\widetilde{T}$ or $\widetilde{V}_{\text {sd }}$, use Hartree-dressed (instead of bare) Green's functions for all thin lines in the SOPT Eqs. (S51) to Eq. (S53), but include only the Fock diagram in the latter, to avoid double-counting Hartree contributions. The Ward identity [Eq. (S55)] relating the current vertex to the self-energy would remain intact in this approach. Pursuing it in detail is left as a topic for future study.
${ }^{1}$ Heyder, J., Bauer, F., E. Schubert, D. Borowsky, D. Taubert, D. Schuh, W. Wegscheider, J. von Delft \& S. Ludwig. Comparative study of the Kondo effect and the 0.7-anomaly. To be published.

${ }^{2}$ D. Borowski. Transportmessungen zur Untersuchung der 0.7-Struktur in Quantenpunktkontakten. Diploma Thesis, Ludwig-Maximilians-Universität München (2011).

${ }^{3}$ Patel, N. K., Nicholls, J. T., Martín-Moreno, L., Pepper, M., Frost, J. E. F., Ritchie, D. A. \& Jones, G. A. C. Evolution of half plateaus as a function of electric field in a ballistic quasi-one-dimensional constriction. Phys. Rev. B, 44, 13549-13555 (1991).

${ }^{4}$ Reilly, D. J., Facer, G. R., Dzurak, A. S., Kane, B. E., \& Clark, R. G. Many-body spin-related phenomena in ultra low-disorder quantum wires. Phys. Rev. B 63, 121311(R) (2001).

5 Büttiker, M. Quantized transmission of a saddle-point constriction. Phys. Rev. B 41, 7906(R) (1990).

${ }^{6}$ Lunde, A. M., De Martino, A., Schulz, A., Egger, R., and Flensberg, K., Electron-electron interaction effects in quantum point contacts. New J. Phys. 11, 023031 (2009).

7 Thomas, K. J., Nicholls, J. T., Simmons, M. Y., Pepper, M., Mace, D. R. Possible Spin Polarization in a OneDimensional Electron Gas. Phys. Rev. Lett. 77, 135-138 (1996).

8 Thomas, K. J., Nicholls, J. T., Appleyard, N. J., Simmons, M. Y., Pepper, M., Mace, D. R., Tribe, W. R., \& Ritchie, D. A. Interaction effects in a one-dimensional constriction.
Phys. Rev. B 58, 4846-4852 (1998)

${ }^{9}$ Koop, E., Lerescu, A., Liu, J., van Wees, B., Reuter, D., Wieck A.D. \& van der Wal, C. The Influence of Device Geometry on Many-Body Effects in Quantum Point Contacts: Signatures of the 0.7 Anomaly, Exchange and Kondo J. Supercond. Nov. Magn. 20, 433-441 (2007).

10 Burke A. M., Klochan O., Farrer I., Ritchie D. A., Hamilton A. R. \& Micolich A. P. Extreme Sensitivity of the SpinSplitting and 0.7 Anomaly to Confining Potential in OneDimensional Nanoelectronic Devices. Nano Letters, Article ASAP, DOI: 10.1021/nl301566d (2012).

11 Patel, N. K., Nicholls, J. T., Martn-Moreno, L., Pepper, M., Frost, J. E. F., Ritchie, D. A. \& Jones, G. A. C. Properties of a ballistic quasi-one-dimensional constriction in a parallel high magnetic field. Phys. Rev. B 44, 10973-10975 (1991).

12 Reilly, D. J., Buehler, T. M., O'Brien, J. L., Hamilton, A. R., Dzurak, A. S., Clark, R. G., Kane, B. E., Pfeiffer, L. N. \& West, K. W. Density-Dependent Spin Polarization in Ultra-Low-Disorder Quantum Wires. Phys. Rev. Lett. 89, 246801 (2002)

13 DiCarlo, L., Zhang, Y., McClure, D. T., Reilly, D. J., Marcus, C. M., Pfeiffer, L. N. \& West, K. W. Shot-Noise Signatures of 0.7 Structure and Spin in a Quantum Point Contact. Phys. Rev. Lett., 97, 036810 (2006).

14 Smith, L. W., Hamilton, A. R., Thomas, K. J., Pepper, M., Farrer, I., Griffths, J. P., Jones, G. A. C. \&Ritchie, D. A. Compressibility measurements of quasi-one-dimensional 
quantum wires Phys. Rev. Lett. 107, 126801 (2011).

15 Blanter, Ya. M. \& Büttiker. Shot noise in mesoscopic conductors. Physics Reports, 336 1-166, (2000).

${ }^{16}$ Lüscher, S., Moore, L. S., Rejec, T., Meir, Y., Shtrikman, H. \& Goldhaber-Gordon, D. Charge Rearrangement and Screening in a Quantum Point Contact. Phys. Rev. Lett. 98, 196805 (2007).

17 Meir, Y., Hirose, K. \& Wingreen, N. S. Kondo Model for the 0.7 Anomaly in Transport through a Quantum Point Contact. Phys. Rev. Lett. 89, 196802 (2002).

18 Golub, A., Aono, T. \& Meir, Y. Suppression of Shot Noise in Quantum Point Contacts in the "0.7 Regime". Phys. Rev. Lett. 97, 186801 (2006).

19 Rejec, T. \& Meir, Y. Magnetic impurity formation in quantum point contacts. Nature 442, 900-903 (2006).

${ }^{20}$ Wang, C.-K. \& Berggren, K.-F. Local spin polarization in ballistic quantum point contacts. Phys. Rev. B 57, 45524556 (1998)

${ }^{21}$ Tokura, Y. \& Khaetski, A. Towards a microscopic theory of the 0.7 anomaly. Physica E: Low-dimensional Systems and Nanostructures, 12, 711 (2002).

${ }^{22}$ Aryanpour, K. \& Han, J. E. Ferromagnetic Spin Coupling as the Origin of 0.7 Anomaly in Quantum Point Contacts. Phys. Rev. Lett., 102, 056805 (2009).

${ }^{23}$ C. Sloggett, C., Milstein, A. I., \& Sushkov, O. P. Correlated electron current and temperature dependence of the conductance of a quantum point contact. Eur. Phys. J. B 61, 427432 (2008).

24 Theoretical work on nonparabolic barriers is being pursued in the group of $\mathrm{H}$. Baranger, private communication (2012).

25 Mahan, D. Many-Particle Physics. Kluwer Academic, New York (2000).

${ }^{26}$ Cronenwett, S. M., Lynch, H. J., Goldhaber-Gordon, D., Kouwenhoven, L. P., Marcus, C. M. Low-Temperature Fate of the 0.7 Structure in a Point Contact: A Kondolike Correlated State in an Open System. Phys. Rev. Lett. 88, 226805 (2002)

27 Connor, J. N. L. On the semiclassical approximation for double well potentials. Mol. Phys., 15, 37 (1968).

28 Miller, W. H. Semiclassical Treatment of Multiple TurningPoint Problems - Phase Shifts and Eigenvalues. J. Chem. Phys., 48, 1651 (1968).

29 Haldane, F. D. M. Scaling Theory of the Asymmetric Anderson Model. Phys. Rev. Lett. 40, 416-419 (1978).

30 Thomas, K. J., Nicholls, J. T., Pepper, M., Tribe, W. R., Simmons, M. Y. \& Ritchie, D. A. Spin properties of lowdensity one-dimensional wires. Phys. Rev. B 61, R1336513368 (2000).

${ }^{31}$ Reilly, D. J. Phenomenological model for the 0.7 conductance feature in quantum wires. Phys. Rev. B, 72, 033309 (2005).
32 Metzner, W., Salmhofer, M., Honerkamp, C., Meden, V. \& Schönhammer, K. Functional renormalization group approach to correlated fermion systems. Rev. Mod. Phys. 84, 299 (2012).

33 Karrasch, C. Transport through Correlated Quantum Dots - a Functional Renormalization Group Approach. Diploma thesis, Universität Götingen (2006).

34 Karrasch, C., Enss, T. \& Meden, V. Functional renormalization group approach to transport through correlated quantum dots. Phys. Rev. B, 73, 235337 (2006).

35 Karrasch, C., Hecht, T., Weichselbaum, A., Oreg, Y. \& von Delft, J. Mesoscopic to Universal Crossover of the Transmission Phase of Multilevel Quantum Dots. Phys. Rev. Lett. 98, 186802 (2007).

${ }^{36}$ Bauer, F., Heyder, J. \& von Delft, J. Functional Renormalization Group Treatment of a Quantum Chain Model for the Kondo Effect and the 0.7-Anomaly. to be published.

37 Andergassen, S., Enss, T., Meden, V., Metzner, W. \& Schollwöck, U. Functional renormalization group for Luttinger liquids with impurities. Phys. Rev. B 70, 075102 (2004).

38 Bauer, F. 0.7 Anomaly of Quantum Point Contacts: Treatment of Interactions with Functional Renormalization Group. Diploma thesis, LMU Munich (2008).

39 Jakobs, S. G., Meden, V. \& Schoeller, H. Nonequilibrium Functional Renormalization Group for Interacting Quantum Systems. Phys. Rev. Lett. 99, 150603 (2007).

${ }^{40}$ Karrasch, C., Hedden, R., Peters, R., Pruschke, T., Schönhammer, K. \& Meden, V. A finite-frequency functional RG approach to the single impurity Anderson model. J. Phys.: Condensed Matter, 20, 345205 (2008).

41 Jakobs, S. G., Pletyukhov, M. \& Schoeller, H. Nonequilibrium functional $R G$ with frequency dependent vertex function - a study of the single impurity Anderson model. Phys. Rev. B, 81, 195109 (2010).

42 Wang, C.-K. \& Berggren, K.-F. Spin splitting of subbands in quasi-one-dimensional electron quantum channels. Phys. Rev. B 54, R14257-14260 (1996).

43 Oguri, A. Transmission Probability for Interacting Electrons Connected to Reservoirs. J. Phys. Soc. Jap. 70, 2666 (2001).

${ }^{44}$ Y. Meir \& N. S. Wingreen. Landauer formula for the current through an interacting electron region. Phys. Rev. Lett. 68, 2512-2515 (1992).

45 Bruognolo, B. Störungstheoretische Analyse der Magnetfeldabhängigkeit der 0.7 Anomalie in Quantenpunktkontakten. Bachelor Thesis, LMU Munich (2011).

46 Gangkofner, D. WKB-Calculations on the 0.7Conductance Anomaly of Quantum Point Contacts. Bachelor Thesis, LMU Munich (2010). 


\section{Chapter 6}

\section{The 0.7 Anomaly in the Presence of Spin Orbit Interactions - Publication}

Until now we mainly explained and reproduced data that has already been measured. A crucial test for every theory is the prediction of new phenomena, which then can be tested experimentally. In this section we present such a prediction, namely the influence of spin orbit interaction (SOI) on the shape of the conductance curve at finite magnetic field depending on the relative direction of SOI and magnetic file. This section contains a draft of a paper to be submitted to Physical Review Letters (Goulko et al., 2014). 


\title{
Effect of Spin-Orbit Interactions on the 0.7 Anomaly in Quantum Point Contacts
}

\author{
Olga Goulko, ${ }^{1,2}$ Florian Bauer, ${ }^{1}$ Jan Heyder, ${ }^{1}$ and Jan von Delft ${ }^{1}$ \\ ${ }^{1}$ Physics Department, Arnold Sommerfeld Center for Theoretical Physics, and Center for NanoScience, \\ Ludwig-Maximilians-Universität, Theresienstraße 37, 80333 Munich, Germany \\ ${ }^{2}$ Department of Physics, University of Massachusetts, Amherst, Massachusetts 01003, USA
}

(Received 6 August 2014; published 24 December 2014)

\begin{abstract}
We study how the conductance of a quantum point contact is affected by spin-orbit interactions, for systems at zero temperature both with and without electron-electron interactions. In the presence of spin-orbit coupling, tuning the strength and direction of an external magnetic field can change the dispersion relation and hence the local density of states in the point contact region. This modifies the effect of electron-electron interactions, implying striking changes in the shape of the 0.7 -anomaly and introducing additional distinctive features in the first conductance step.
\end{abstract}

DOI: $10.1103 /$ PhysRevLett.113.266402

PACS numbers: 71.70.Ej, 73.40.-c

Spin-orbit interactions (SOI) play an important role in a variety of fields within mesoscopic physics, such as spintronics and topological quantum systems. In this Letter we study the effects of SOI on the conductance of a quantum point contact (QPC), a one-dimensional constriction between two reservoirs $[1,2]$. The linear conductance $G$ of a QPC is quantized in multiples of $G_{Q}=2 e^{2} / h$, showing the famous staircase as a function of gate voltage. In addition, at the onset of the first plateau, measured curves show a shoulderlike structure near $0.7 G_{Q}$ [3]. In this regime QPCs exhibit anomalous behavior in the electrical and thermal conductance, noise, and thermopower [3-11]. The microscopic origin of this 0.7 -anomaly has been the subject of a long debate [12-18]. It has recently been attributed to a strong enhancement of the effects of electron-electron interactions (EEI) by a smeared van Hove singularity in the local density of states (LDOS) at the bottom of the lowest QPC subband $[15,18]$. While this explains the 0.7-anomaly without evoking SOI, the presence of SOI can change the dispersion relation and hence the LDOS, thus strongly affecting the shape of the 0.7-anomaly. Previous studies of SOI in QPCs exist [19-23], but not with the present emphasis on their interplay with the QPC barrier shape and EEI, which are crucial for understanding the effect of SOI on the 0.7-anomaly.

Setup.-We consider a heterostructure forming a twodimensional electron system (2DES) in the $x y$ plane. Gate voltages are used to define a smooth, symmetric potential which splits the 2DES into two leads, connected by a short, one-dimensional channel along the $x$ axis: the QPC $[1,2]$. The transition between the leads and the QPC is adiabatic. We also assume the confining potential in the transverse direction to be so steep that the subband spacing is much larger than all other energy scales relevant for transport, in particular those related to the magnetic field and SOI, and consider only transport in the first subband, corresponding to the lowest transverse mode. This can be described by a one-dimensional model with a smooth potential barrier and local EEI [18]. The magnetic field $\boldsymbol{B}$ is assumed to be in the $x y$ plane, acting as a pure Zeeman field, without orbital effects.

A moving electron in an electric field can experience an effective magnetic field $\boldsymbol{B}_{\text {SOI }}$ proportional to its momentum $\hbar k$. Depending on the origin of the electric field one distinguishes between Rashba and Dresselhaus terms, the former resulting from the gradient of the external potential, and the latter from the asymmetry of the ionic lattice [24]. To be able to rotate $\boldsymbol{B}$ through any angle $\varphi$ w.r.t. $\boldsymbol{B}_{\mathrm{SOI}}$ we require that $\boldsymbol{B}_{\text {SOI }}$ also lies in the $x y$ plane. Without loss of generality (see the Supplemental Material [25]), we choose the $y$ axis to be parallel to $\boldsymbol{B}_{\mathrm{SOI}}$, such that the SOI contribution to the Hamiltonian is $-\alpha \sigma_{y} k$, where $\alpha$ characterizes the strength of the (Rashba) SOI and $\sigma_{y}$ is a Pauli matrix [26]. We only consider the leading SOI contribution proportional to $k$ and choose the spin quantization direction along $\boldsymbol{B}$.

Without SOI, the dispersion relation $\hbar^{2} k^{2} / 2 m$ of a homogeneous one-dimensional model with effective mass $m$ splits in the presence of a Zeeman field into two identical branches offset in energy by $\pm B / 2$. On the other hand, without a Zeeman field, the momentumdependent SOI splits the dispersion in $k$ direction and also yields a negative spin-independent energy offset of magnitude $\Delta E_{\mathrm{SOI}}=\alpha^{2} m / 2 \hbar^{2}$. In the following, we shift the energy origin by $-\Delta E_{\mathrm{SOI}}$ and quote all energies w.r.t. the new origin. If both $\boldsymbol{B}$ and $\boldsymbol{B}_{\text {SOI }}$ are nonzero, their interplay depends on $\varphi$, as illustrated in Fig. 1(a1)-(a3). In (a1), where the fields are parallel $(\varphi=0)$, the energy offsets simply add, while for nonparallel fields a spin mixing occurs, resulting in an avoided crossing [27]. For orthogonal fields $(\varphi=\pi / 2)$, the lower dispersion branch exhibits either one broader minimum at $k=0$ if $B \geq 4 E_{\mathrm{SOI}}$, or two minima at finite $k$ and a maximum at $k=0$ otherwise. The latter case is shown in Fig. 1(a2)-(a3).

Model.-For the lowest subband we model the QPC by a symmetric potential barrier which is quadratic around its maximum, 


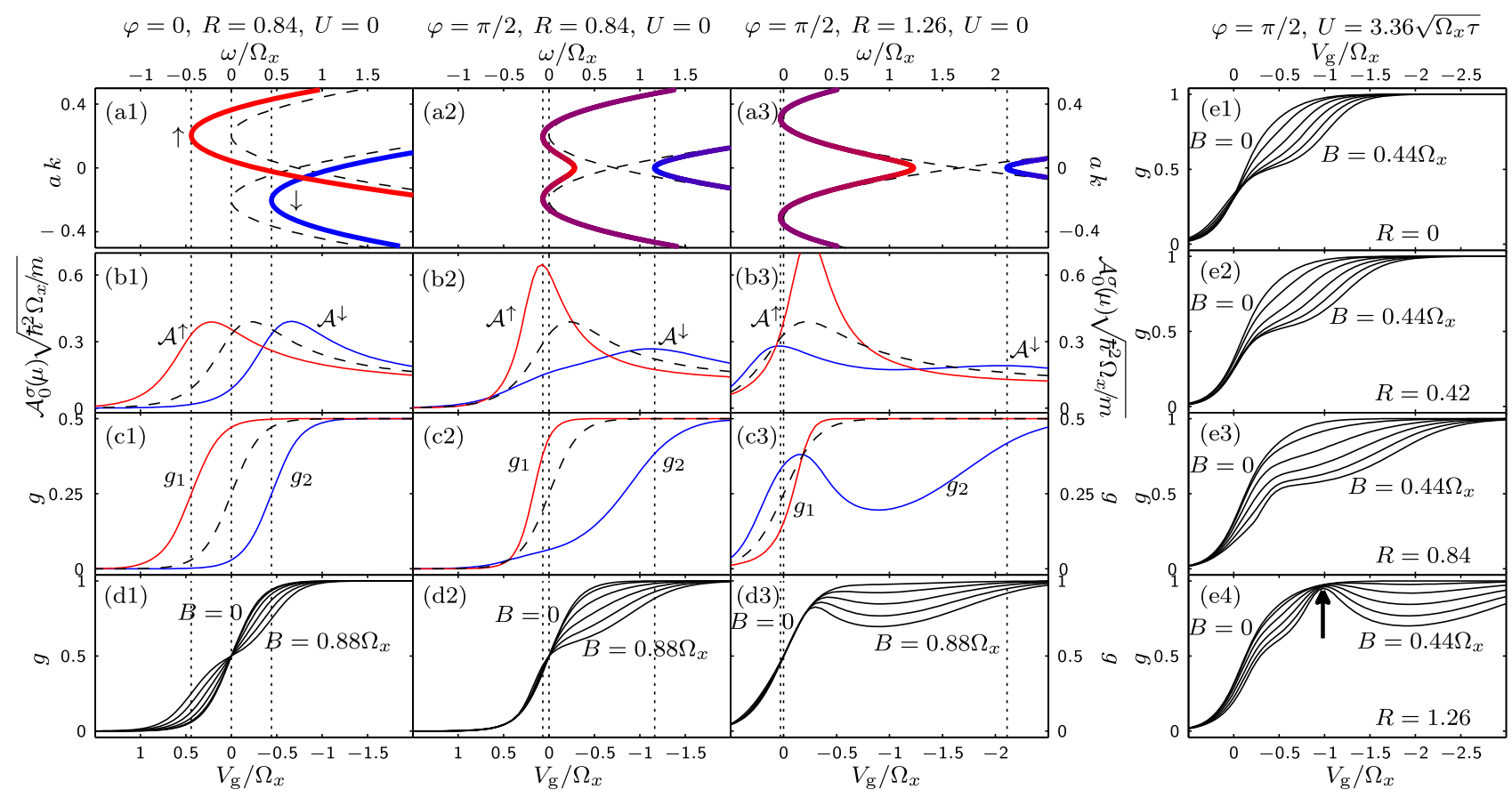

FIG. 1 (color online). Effect of SOI on the model without EEI, left columns (a1)-(d3), and with EEI, right column (e1)-(e4). The left columns (a1)-(d1), (a2)-(d2), and (a3)-(d3) represent different combinations of SOI strength $R$ and angle $\varphi$ between $\boldsymbol{B}$ and $\boldsymbol{B}_{\text {SOI. }}$. They highlight the correspondence between the dispersion relation $\omega(k)$ in a homogeneous system (a1)-(a3), the LDOS for fixed $\omega=\mu$ as function of $V_{g}$ on the central site of a QPC with potential barrier (b1)-(b3), the conductances of the two QPC transmission channels (c1)-(c3), and the total conductance of the QPC for several equally spaced magnetic field values between $B=0$ and $B=0.88 \Omega_{x}$ (d1)-(d3). In (a1)-(c3), the magnetic field is fixed at $B=0.88 \Omega_{x}$, with dashed lines showing the case $B=0$ for comparison. The line colors in (a1)-(a3) quantify the contribution of each spin state $($ red $=\uparrow$, blue $=\downarrow$ ) in the dispersion branches, to illustrate the spin mixing at $\varphi \neq 0$. The right column (e1)-(e4) shows the total conductance for $U>0$, with $\varphi=\pi / 2$ and several combinations of $R$ and $B$ [the latter were chosen smaller than in (d1)-(d3), since EEI enhance the $g$-factor [18]].

$$
V(x) \simeq V_{g}+\mu-C_{b} x^{2} / 2,
$$

and vanishes smoothly at the boundary of the QPC. The barrier height $V_{g}$, measured w.r.t. the chemical potential $\mu$, mimics the role of the gate voltage. If $V_{g}$ is swept downwards through zero, the conductance $g=G / G_{Q}$ increases from 0 to 1 . For $B=0$ this occurs in a single step whose width is given by the energy scale $\Omega_{x}=\sqrt{C_{b} C_{d}}$, which is set by the fixed curvature of the barrier $C_{b}$ and the curvature of the bulk dispersion at its minimum $C_{d}$ [28]. For $\varphi=0, C_{d}=\hbar^{2} / m$.

For numerical purposes, we discretize real space and obtain an infinite tight-binding chain with spacing $a$, taking $\boldsymbol{B}$ and $\alpha$ constant throughout the chain. The noninteracting Hamiltonian is

$$
\begin{aligned}
H_{0}= & \sum_{j, \sigma, \sigma^{\prime}} d_{j \sigma}^{\dagger}\left[\left(V_{j}+2 \tau\right) \delta_{\sigma \sigma^{\prime}}-\frac{1}{2}(\boldsymbol{\sigma} \cdot \boldsymbol{B})_{\sigma \sigma^{\prime}}\right] d_{j \sigma^{\prime}} \\
& +\sum_{j, \sigma, \sigma^{\prime}}\left[d_{j+1 \sigma}^{\dagger}\left(-\tau_{0} \delta_{\sigma \sigma^{\prime}}+\frac{i \alpha}{2}\left(\sigma_{y}\right)_{\sigma \sigma^{\prime}}\right) d_{j \sigma^{\prime}}+\text { H.c. }\right],
\end{aligned}
$$

where $d_{j \sigma}$ annihilates an electron with spin $\sigma \in\{\uparrow, \downarrow\} \equiv$ $\{+,-\}$ at site $j$. The effective mass of the charge carrier is $m=\hbar^{2} / 2 \tau a^{2}$ with $\tau=\sqrt{\tau_{0}^{2}+\alpha^{2}}$ [29]. We keep $\tau$ fixed when varying $\alpha$. The QPC barrier potential $V_{j}=V(j a)$ (and later EEI) are nonzero only in a region of length $L=2 N a$ centered around $j=0$, representing the QPC. All results shown are for $N=50$. We use the smooth function $V(x)=\left(V_{g}+\mu\right) \exp \left\{-(2 x / L)^{2} /\left(1-[2 x / L]^{2}\right)\right\}$ for the potential, with $\mu=2 \tau$. Sites $j<-N$ and $j>N$ represent two leads with bandwidth $4 \tau$. The strength of SOI in a QPC is determined by the dimensionless parameter

$$
R=\sqrt{\frac{\Delta E_{\mathrm{SOI}}}{\Omega_{x}}}=\frac{\alpha}{\hbar} \sqrt{\frac{m}{2 \Omega_{x}}} .
$$

SOI strengths of up to $\alpha \simeq 10^{-11} \mathrm{eVm}$ have been reported in the literature [19,27,30,31]. Typical values of $\Omega_{x} \simeq 1 \mathrm{meV}$ and $m \simeq 0.05 m_{e}$ for InGaAs yield $R \simeq 0.2$. A stronger spinorbit effect due to an enhancement of the anisotropic Lande $g$-factor is reported in Ref. [32]. Hole quantum wires have been used to observe the spin-orbit gap [27] and the anisotropic Zeeman splitting [33]. For hole QPCs, the larger effective hole mass and the resulting smaller $\Omega_{x}$ imply larger values of $R$. Here we consider both small and large $R$, where $R \lesssim 0.4$ is a realistic scale for electron systems and $R \gtrsim 1$ is accessible using hole systems [34], for QPCs with small barrier curvature $C_{b}$ and hence small $\Omega_{x}$.

System without EEI.-Many insights on the interplay between SOI and geometry can already be gained from the model withoutEEI, as shown in the left part (a1)-(d3) of Fig. 1 . 
We discuss exact results for two physical quantities, which we also relate to the bulk dispersion relation: the linear conductance $g$ and the $\operatorname{LDOS} \mathcal{A}_{j}^{\sigma}(\omega)=-\operatorname{Im} \mathcal{G}_{j j}^{\sigma \sigma}(\omega) / \pi a$, where $\mathcal{G}_{j j^{\prime}}^{\sigma \sigma^{\prime}}$ is the retarded propagator from site $j^{\prime}$ with spin $\sigma^{\prime}$ to site $j$ with $\operatorname{spin} \sigma$. Due to SOI, spin is not conserved for $\varphi \neq 0$ and hence $\mathcal{G}_{j j}^{\sigma \sigma^{\prime}}$ is not spin-diagonal. However at $j=0$ its off-diagonal elements turn out to be negligible compared to the diagonal ones. Thus it is meaningful to analyze the LDOS at $j=0$ for given $\sigma$. The linear conductance at zero temperature can be calculated via $g=g_{1}+g_{2} \propto \operatorname{Tr}\left(t^{\dagger} t\right)$ [35], where $t^{\sigma \sigma^{\prime}}=$ $\mathcal{G}_{-N, N}^{\sigma \sigma^{\prime}}(\mu)$ is the transmission matrix of the QPC and $\operatorname{Tr}\left(t^{\dagger} t\right)$ equals the sum of the eigenvalues of $t^{\dagger} t$. The spin structure of $t$ depends on $N$, but the eigenvalues of $t^{\dagger} t$, which yield the conductances $g_{1}$ and $g_{2}$ of the two transmission channels, do not.

For $\varphi=0$ (Fig. 1, left column) spin is conserved and SOI have no influence on the LDOS and the conductance. This case is analogous to the one discussed in Ref. [18]. The bulk [i.e., $V(x)=0$ ] LDOS,

$$
\left.\mathcal{A}_{\text {bulk }}^{\sigma}(\omega) \propto \frac{\partial k}{\partial \omega}\right|_{\sigma}=\sqrt{\frac{m}{2 \hbar^{2}(\omega+\sigma B / 2)}},
$$

has a van Hove singularity, diverging at the minimum $\omega=-\sigma B / 2$ of the corresponding dispersion branch, where the electron velocity vanishes. In the QPC, the $x$-dependent LDOS is shifted in energy by the barrier potential $V(x)$. Since the barrier breaks translational invariance, the van Hove singularity is smeared out on a scale set by $\Omega_{x}$ [15], forming a ridgelike structure, called van Hove ridge in [18]. The LDOS height becomes finite, of order $\mathcal{O}\left(\sqrt{m /\left(\hbar^{2} \Omega_{x}\right)}\right)$, determined by $\Omega_{x}$ and the curvature $\hbar^{2} / m$ of the bulk dispersion. At a given position $x$, the LDOS maximum occurs at an energy which is $\mathcal{O}\left(\Omega_{x}\right)$ larger than the corresponding potential energy $V(x)-\sigma B / 2$. Here and henceforth we quote the LDOS as a function of $V_{g}$ at fixed $\omega=\mu$. Figure 1(b1) shows it at the central site $j=0$; the spatially resolved LDOS is shown in Fig. 1 of the Supplemental Material [25]. The LDOS has the same shape for both spins. Its structure is clearly inherited from that of the dispersion in (a1), with peak energies aligned with the dispersion minima up to the shift of $\mathcal{O}\left(\Omega_{x}\right)$. Similarly, the conductances $g_{1}\left(V_{g}\right)$ and $g_{2}\left(V_{g}\right)$ of the two channels in (c1) show steps of the same shape with widths $\propto \Omega_{x}$ [28], split by $B$ and aligned with the dispersion minima. This causes the total conductance $g\left(V_{g}\right)$ in (d1) to split symmetrically into a double step with increasing field, just as for a QPC without SOI.

Next consider the case $\varphi=\pi / 2$ shown in Fig. 1(a2)-(d3). Spin mixing leads to an avoided crossing with spin gap $\propto B$, which splits the dispersion into an upper branch with a narrow minimum and a lower branch with two minima and one maximum (for $B<4 E_{\mathrm{SOI}}$ ). Note that bulk LDOS structures separated in energy by less than $\Omega_{x}$ are not resolved within the QPC. In the following, we give an intuitive explanation of how the dispersion minima relate to the properties of the LDOS peaks and the conductance steps.
The curvatures of the lower and upper dispersion branches are, respectively, smaller or larger than in (a1), $C_{d 1}<C_{d}<$ $C_{d 2}$ (loosely speaking, $C_{d 1}$ is the effective curvature obtained by smearing the double dispersion minimum by $\Omega_{x}$, yielding a single minimum). Because the barrier curvature $C_{b}$ is fixed, this results in two modified energy scales $\Omega_{x i}=\sqrt{C_{b} C_{d i}}$, with $\Omega_{x 1}<\Omega_{x}<\Omega_{x 2}$, which determine the LDOS peak heights and widths, as well as the conductance step widths. Consequently, in (b2) the LDOS peak for $\mathcal{A}_{0}^{\downarrow}$ is lower and wider than for $\mathcal{A}_{0}^{\uparrow}$. Likewise, in (c2) the conductance step for $g_{2}\left(V_{g}\right)$ is wider than for $g_{1}\left(V_{g}\right)$, causing $g\left(V_{g}\right)$ in $(\mathrm{d} 2)$ to show a striking asymmetry for its $B$-induced evolution from a single to a double step. This asymmetry is reminiscent of but unrelated to that known for the 0.7-anomaly - the latter is driven by EEI, as discussed below-but should be observable in higher conductance steps, where EEI are weaker.

For $R \gtrsim 1$ more structures emerge, see Fig. 1(a3)-(b3). Spin-mixing produces an additional "emergent" peak in $\mathcal{A}_{0}^{\downarrow}$ (b3) and an additional step in $g_{2}\left(V_{g}\right)$ (c3) near $V_{g} \simeq 0$. Between the two steps, the transmission $g_{2}\left(V_{g}\right)$ has a minimum, corresponding to the spin gap, and the total conductance $g\left(V_{g}\right)$ in (d3) likewise develops a spin gap minimum with increasing $B$. These features can be understood by looking at the spin composition of the two bulk dispersion branches, depicted quantitatively through the colors in Fig. 1(a1)-(a3). At $k=0$ the SOI field is zero and we have pure spin-states w.r.t. the chosen quantization. At larger $|k|$ the SOI field increases, leading to spin-mixing. In fact in the limit $k \rightarrow \infty$ we find a fully mixed state with equal up and down contributions. Since the upper branch minimum at $k=0$ is in a pure spin-down state, it corresponds to a peak only in $\mathcal{A}^{\downarrow}$. But the minima of the lower branch are shifted away from $k=0$ and have a spindown share besides the dominant spin-up contribution. This causes the emergent peak in $\mathcal{A}^{\downarrow}$ at low frequencies, whose height increases with $R$, due to the stronger spin-mixing.

Interacting system.-We now include EEI via $H_{\text {int }}=$ $\sum_{j} U_{j} d_{j \uparrow}^{\dagger} d_{j \uparrow} d_{j \downarrow}^{\dagger} d_{j \downarrow}$. The on-site interaction $U_{j}=U(j a)$ is switched on smoothly over the QPC according to $U(x)=U \exp \left\{-(2 x / L)^{6} /\left[1-(2 x / L)^{2}\right]\right\}$. We set $U_{j}=0$ for $|j|>N$, because outside the QPC region transverse confinement is weak or absent, and screening strong $[18,36]$. We calculate the conductance at zero temperature with the functional renormalization group technique in the one-particle irreducible version [29,37-40] using the coupled ladder approximation, which was presented in Ref. [36] for a model without SOI. Generalizations necessary in the presence of SOI are described in the Supplemental Material [25].

The $B$ dependence of the conductance for $\varphi=\pi / 2$ and different $R$ in the presence of EEI is shown in the right column (e1)-(e4) of Fig. 1 and the corresponding transconductance $d g / d V_{g}$ in Fig. 2(b)-(f). The case $R=0$ [see Figs. 1(e1) and $2(\mathrm{a})-(\mathrm{c})]$, which is equivalent to $\varphi=0$, has been discussed in Refs. [18,36]: once a finite magnetic field breaks 
the spin degeneracy a surplus of spin-up electrons develops in the QPC, so that spin-down electrons experience both a Zeeman and a Coulomb energy cost. This Stoner-type effect depends on the LDOS at $\mu$ and hence is strongest when the apex of the van Hove ridge touches the chemical potential, i.e., when $V_{g}$ is within $\simeq 0.5 \Omega_{x}$ below 0 [18]. This causes an asymmetry w.r.t. $V_{g}=0$ in the $B$-induced evolution of $g\left(V_{g}\right)$ from a single to a double step in Fig. 1(e1), in contrast to the case without EEI in Fig. 1(d1). This asymmetry is characteristic of the 0.7-anomaly. The corresponding transconductance in Fig. 2(b)-(c) shows a double peak whose spacing increases roughly linearly with $B$ (with an EEI-enhanced $g$-factor), as seen in numerous experiments $[3,10,18]$.

The Stoner-type Coulomb enhancement of a fieldinduced population imbalance is amplified when $R \neq 0$, as shown in Figs. 1(e2)-(e4) and 2(d)-(f), because of the height imbalance for the spin-up and spin-down LDOS peaks caused by SOI. Correspondingly, with increasing $R$ the double-step structure in the conductance becomes more pronounced, the second substep becoming much broader than the first [see Figs. 1(e2)-(e3)] and the transconductance in Fig. 2(d)-(e) shows a weakening of the lower- $V_{g}$
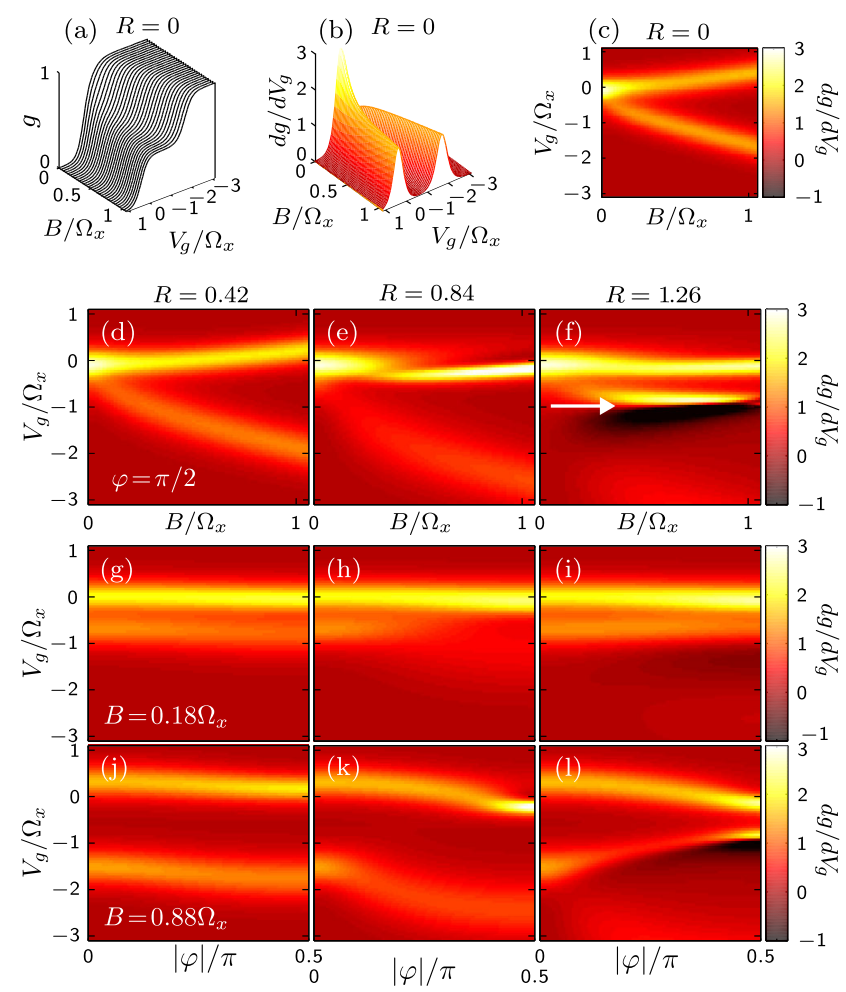

FIG. 2 (color online). Functional renormalization group results for the conductance $g$ and transconductance $d g / d V_{g}$, for $U=$ $3.36 \sqrt{\Omega_{x} \tau}$ at zero temperature. Top row: $3 \mathrm{~d}$ or color-scale plots of the conductance (a) and the transconductance (b) and (c) as functions of $V_{g}$ and $B$, for $R=0$. Three bottom rows (d)-(l): Color-scale plots of the transconductance for three choices of $R$ (three columns), plotted as a function of $V_{g}$ and either $B$ for fixed $\varphi=\pi / 2$ (second row) or of $\varphi$ for fixed $B=0.18 \Omega_{x}$ (third row) and $B=0.88 \Omega_{x}$ (fourth row). peak with increasing $R$. This reflects the increasing curvature $C_{d 2}$ of the upper dispersion branch (and hence larger step width $\Omega_{x 2}$ ). For $R \gtrsim 1$, additional features, inherited from the noninteracting case, emerge for $g\left(V_{g}\right)$ in Fig. 1(e4): a local maximum (marked by an arrow), followed by a spin gap minimum at lower $V_{g}$. For the transconductance, Fig. 2(f), these features show up as a strong secondary peak around $V_{g} / \Omega_{x} \simeq-1$ (marked by an arrow), followed by a region of negative transconductance (black). EEI also induce a secondary 0.7-type double-step feature in $g\left(V_{g}\right)$ for $V_{g} / \Omega_{x}$ between 0 and -1 , Fig. 1(e4), which is similar to, but narrower than that for $R=0$. It originates from the main LDOS peak in $\mathcal{A}_{0}^{\uparrow}$ and the emergent peak in $\mathcal{A}_{0}^{\downarrow}$. Unlike the regular $\mathcal{A}_{0}^{\downarrow}$ peak aligned with the upper dispersion branch, whose $V_{g}$ position is governed by the magnetic field, the emergent $\mathcal{A}_{0}^{\downarrow}$ peak occurs, due to strong spin-mixing, at nearly $B$-independent energy close to the $\mathcal{A}_{0}^{\uparrow}$ peak. As a result, the two transconductance maxima in Fig. 2(f) remain parallel with increasing $B$, in strong contrast to the situation for $R<1$ in Fig. 2(c)-(e).

Figures 2(g)-(1) show, for two fixed values of $B$, how the transconductance evolves as $|\varphi|$ is increased from 0 to $\pi / 2$, thus switching on the effects of SOI. The decrease in peak spacing with increasing $|\varphi|$ in Fig. 2(1) strikingly reflects the increasing importance of spin mixing. The strong angle dependence predicted here is a promising candidate for an experimental test of our theory [41].

At small nonzero temperature, inelastic scattering causes a Fermi-liquid-type reduction of the conductance, $g\left(T, V_{g}\right) / g\left(0, V_{g}\right)=1-\left(T / T_{*}\right)^{2}$ for $T \ll T_{*}$, with a $V_{g}$-dependent low-energy scale $T_{*}\left(V_{g}\right)$. We expect its magnitude to be similar to the case without SOI, typically $18] \simeq 1 \mathrm{~K}]$. Thus, for $T \lesssim 0.1 \mathrm{~K}$, the $T$-dependence should be very weak and the $T=0$ predictions applicable.

In summary, we have shown that in the presence of SOI, the changes in the dispersion induced by the interplay of $\boldsymbol{B}$ and $\boldsymbol{B}_{\text {SOI }}$ can strongly affect the shape of the 0.7-anomaly. In the absence of EEI, SOI cause an anisotropic response of the spin splitting to the applied in-plane magnetic field. With EEI, the 0.7-anomaly also develops an anisotropic response to magnetic field, and if SOI are strong, the conductance develops additional features due to the interplay of EEI and SOI: for $\varphi=\pi / 2$ these include a fieldinduced double step in the conductance that does not split linearly with $B$, followed by a spin gap minimum. The dependence of the conductance on the angle between $\boldsymbol{B}$ and $\boldsymbol{B}_{\mathrm{SOI}}$ is already apparent for $R \simeq 0.4$, which is accessible in experiments with electron QPCs. Hole QPCs with $R \gtrsim 1$ would allow access to regimes with strong SOI.

An experimental verification of our predictions would highlight the influence of LDOS features on the conductance and thus lend further support to the van Hove scenario of Ref. [18] as microscopic explanation for the 0.7anomaly. More generally, our work lays out a conceptual framework for analyzing the interplay of SOI, EEI, and 
barrier shape in quasi-1D geometries: examine how SOI and barrier shape modify the (bare) LDOS near $\mu-$ whenever the LDOS is large, EEI effects are strong. We expect this to be relevant for the more complicated hybrid superconductor-semiconductor junctions currently studied by seekers of Majorana fermions [42-44]. A proper analysis of such systems would require a generalization of our approach to include superconducting effects.

We thank S. Fischer, A. Hamilton, K. Hudson, S. Ludwig, C. Marcus, A. Micolich and A. Srinivasan for interesting and useful discussions and acknowledge support from the DFG via SFB-631, SFB-TR12, De730/4-3, and the Cluster of Excellence Nanosystems Initiative Munich. O. G. acknowledges support from the NSF under the Grant No. PHY-1314735.

[1] D. A. Wharam, T. J. Thornton, R. Newbury, M. Pepper, H. Ahmed, J. E. F. Frost, D. G. Hasko, D. C. Peacock, D. A. Ritchie, and G. A. C. Jones, J. Phys. C 21, L209 (1988).

[2] B. J. van Wees, H. van Houten, C. W. J. Beenakker, J. G. Williamson, L. P. Kouwenhoven, D. van der Marel, and C. T. Foxon, Phys. Rev. Lett. 60, 848 (1988).

[3] K. J. Thomas, J. T. Nicholls, M. Y. Simmons, M. Pepper, D. R. Mace, and D. A. Ritchie, Phys. Rev. Lett. 77, 135 (1996).

[4] N. J. Appleyard, J. T. Nicholls, M. Pepper, W. R. Tribe, M. Y. Simmons, and D. A. Ritchie, Phys. Rev. B 62, R16275 (2000).

[5] A. Kristensen, H. Bruus, A. E. Hansen, J. B. Jensen, P. E. Lindelof, C. J. Marckmann, J. Nygård, C. B. Sørensen, F. Beuscher, A. Forchel, and M. Michel, Phys. Rev. B 62, 10950 (2000).

[6] S. M. Cronenwett, H. J. Lynch, D. Goldhaber-Gordon, L. P. Kouwenhoven, C. M. Marcus, K. Hirose, N. S. Wingreen, and V. Umansky, Phys. Rev. Lett. 88, 226805 (2002).

[7] L. DiCarlo, Y. Zhang, D. T. McClure, D. J. Reilly, C. M. Marcus, L. N. Pfeiffer, and K. W. West, Phys. Rev. Lett. 97, 036810 (2006).

[8] O. Chiatti, J. T. Nicholls, Y. Y. Proskuryakov, N. Lumpkin, I. Farrer, and D. A. Ritchie, Phys. Rev. Lett. 97, 056601 (2006).

[9] L. W. Smith, A. R. Hamilton, K. J. Thomas, M. Pepper, I. Farrer, J. P. Griffiths, G. A. C. Jones, and D. A. Ritchie, Phys. Rev. Lett. 107, 126801 (2011).

[10] A. P. Micolich, J. Phys. Condens. Matter 23, 443201 (2011).

[11] R. Danneau, O. Klochan, W. R. Clarke, L. H. Ho, A. P. Micolich, M. Y. Simmons, A. R. Hamilton, M. Pepper, and D. A. Ritchie, Phys. Rev. Lett. 100, 016403 (2008).

[12] D. J. Reilly, T. M. Buehler, J. L. O'Brien, A. R. Hamilton, A. S. Dzurak, R. G. Clark, B. E. Kane, L. N. Pfeiffer, and K. W. West, Phys. Rev. Lett. 89, 246801 (2002).

[13] K. F. Berggren and I. I. Yakimenko, Phys. Rev. B 66, 085323 (2002).

[14] Y. Meir, K. Hirose, and N. S. Wingreen, Phys. Rev. Lett. 89, 196802 (2002).

[15] C. Sloggett, A. I. Milstein, and O. P. Sushkov, Eur. Phys. J. B 61, 427 (2008).

[16] A. M. Lunde, A. D. Martino, A. Schulz, R. Egger, and K. Flensberg, New J. Phys. 11, 023031 (2009).

[17] K. Aryanpour and J. E. Han, Phys. Rev. Lett. 102, 056805 (2009).
[18] F. Bauer, J. Heyder, E. Schubert, D. Borowsky, D. Taubert, B. Bruognolo, D. Schuh, W. Wegscheider, J. von Delft, and S. Ludwig, Nature (London) 501, 73 (2013).

[19] M. Kohda, S. Nakamura, Y. Nishihara, K. Kobayashi, T. Ono, J.-i. Ohe, Y. Tokura, T. Mineno, and J. Nitta, Nat. Commun. 3, 1082 (2012).

[20] J.-H. Hsiao and T.-M. Hong, Phys. Rev. B 82, 115309 (2010).

[21] M. P. Nowak, K. Kolasiński, and B. Szafran, Phys. Rev. B 90, 035301 (2014).

[22] R. Danneau, W. R. Clarke, O. Klochan, A. P. Micolich, A. R. Hamilton, M. Y. Simmons, M. Pepper, and D. A. Ritchie, Appl. Phys. Lett. 88, 012107 (2006).

[23] F. Nichele, S. Chesi, S. Hennel, A. Wittmann, C. Gerl, W. Wegscheider, D. Loss, T. Ihn, and K. Ensslin, Phys. Rev. Lett. 113, 046801 (2014).

[24] R. Winkler, Spin-Orbit Coupling Effects in Two-Dimensional Electron and Hole Systems (Springer, Berlin, 2003).

[25] See Supplemental Material at http://link.aps.org/ supplemental/10.1103/PhysRevLett.113.266402 for geometric details of the model, plots of the spatially resolved LDOS, and details on the functional renormalization group formalism with SOI.

[26] L. Meier, G. Salis, I. Shorubalko, E. Gini, S. Schön, and K. Ensslin, Nat. Phys. 3, 650 (2007).

[27] C. H. L. Quay, T. L. Hughes, J. A. Sulpizio, L. N. Pfeiffer, K. W. Baldwin, K. W. West, D. Goldhaber-Gordon, and R. de Picciotto, Nat. Phys. 6, 336 (2010).

[28] M. Büttiker, Phys. Rev. B 41, 7906(R) (1990).

[29] J. E. Birkholz, Ph.D. thesis, Georg-August-Universität zu Göttingen, 2008.

[30] J. Nitta, T. Akazaki, H. Takayanagi, and T. Enoki, Phys. Rev. Lett. 78, 1335 (1997).

[31] T. Kita, T. Kakegawa, M. Akabori, and S. Yamada, Solid State Commun. 136, 479 (2005).

[32] T. P. Martin, A. Szorkovszky, A. P. Micolich, A. R. Hamilton, C. A. Marlow, R. P. Taylor, H. Linke, and H. Q. Xu, Phys. Rev. B 81, 041303 (2010).

[33] J. C. H. Chen, O. Klochan, A. P. Micolich, A. R. Hamilton, T. P. Martin, L. H. Ho, U. Zülicke, D. Reuter, and A. D. Wieck, New J. Phys. 12, 033043 (2010).

[34] A. R. Hamilton, T. Li, O. P. Sushkov, and R. Winkler, private communication. For weakly 1D-confined holes, it can be argued that the dominant SOI term has the same $k \sigma_{y}$ form as used here.

[35] S. Datta, Electronic Transport in Mesoscopic Systems (Cambridge University Press, Cambridge, England, 1995).

[36] F. Bauer, J. Heyder, and J. von Delft, Phys. Rev. B 89, 045128 (2014).

[37] C. Wetterich, Phys. Lett. B 301, 90 (1993).

[38] J. E. Birkholz and V. Meden, Phys. Rev. B 79, 085420 (2009).

[39] F. Bauer, Master's thesis, LMU-München, 2008.

[40] W. Metzner, M. Salmhofer, C. Honerkamp, V. Meden, and K. Schönhammer, Rev. Mod. Phys. 84, 299 (2012).

[41] A. R. Hamilton, K. Hudson, A. P. Micolich, and A. Srinivasan, private communication.

[42] R. M. Lutchyn, J. D. Sau, and S. Das Sarma, Phys. Rev. Lett. 105, 077001 (2010).

[43] Y. Oreg, G. Refael, and F. von Oppen, Phys. Rev. Lett. 105, 177002 (2010).

[44] V. Mourik, K. Zuo, S. M. Frolov, S. R. Plissard, E. P. A. M. Bakkers, and L. P. Kouwenhoven, Science 336, 1003 (2012). 


\section{Supplemental Material to "The effect of spin-orbit interactions on the 0.7-anomaly in quantum point contacts"}

\section{GEOMETRIC DETAILS OF THE MODEL}

In our model the 2DES is in the $x y$-plane and the QPC is directed along the $x$-axis (this is the direction of motion of the charge carrier). For the directions of the $\boldsymbol{B}$ and $\boldsymbol{B}_{\mathrm{SOI}}$ fields we impose the following restrictions. To avoid orbital effects we require the magnetic field $\boldsymbol{B}$ to be in the $x y$-plane of the 2DES. We also want to be able to rotate $\boldsymbol{B}$ through any angle $\varphi$ w.r.t. $\boldsymbol{B}_{\mathrm{SOI}}$, which implies that $\boldsymbol{B}_{\mathrm{SOI}}$ also must lie in the $x y$-plane. With the latter condition, the $\boldsymbol{B}_{\mathrm{SOI}}$ field can be either parallel to the direction of motion of the electrons (pure Dresselhaus contribution), or orthogonal to it (pure Rashba contribution), or a combination of the two. But for our mathematical model, the end results depend only on the relative angle $\varphi$ between $\boldsymbol{B}$ and $\boldsymbol{B}_{\mathrm{SOI}}$. This means that we can choose the direction of $\boldsymbol{B}_{\mathrm{SOI}}$ without loss of generality. We choose $\boldsymbol{B}_{\mathrm{SO}}$ to be parallel to the $y$-axis, c.f. Eq. (2) of the main text.

\section{THE SPATIALLY RESOLVED LDOS}

In Fig. 1(b1-b3) of the main text we show the LDOS at fixed $\mu$ as a function of $V_{\mathrm{g}}$ on the central site of the QPC. The behavior at the center captures all relevant features. For completeness we include here in Fig. 1 the spatially resolved plots of the $\operatorname{LDOS} \mathcal{A}_{j}^{\sigma}$ for both spin states and the same parameter values as in Fig. 1 of the main text.

\section{SECOND ORDER FRG}

The functional Renormalization Group (fRG) method is an improved perturbation technique [1-5]. Rather than expanding the Green's function in orders of the coupling and truncating the expansion, fRG introduces a flow parameter $\Lambda$ into the free Green's function $\mathcal{G}_{0}$. At zero temperature we define

$$
\mathcal{G}_{0}(i \omega) \rightarrow \theta(|\omega|-\Lambda) \mathcal{G}_{0}(i \omega) \equiv \mathcal{G}_{0}^{\Lambda}(i \omega)
$$

where $\theta(\omega)$ is the Heaviside step function. At the beginning of the flow, setting $\Lambda=\infty$ yields $\mathcal{G}_{0}^{\Lambda}=0$, which means that the only contribution to the full Green's function comes from the bare vertex. At the end of the flow, $\Lambda=0$ recovers the full model. The technical details of the one-particle irreducible version of the fRG employed in this work are presented in depth in [6]. We use the static approximation at zero temperature, which will be described below in Sec. III B. Since [6] does not deal with spin-orbit interactions, no spin-mixing is possible, which introduces additional symmetries into the system. In our

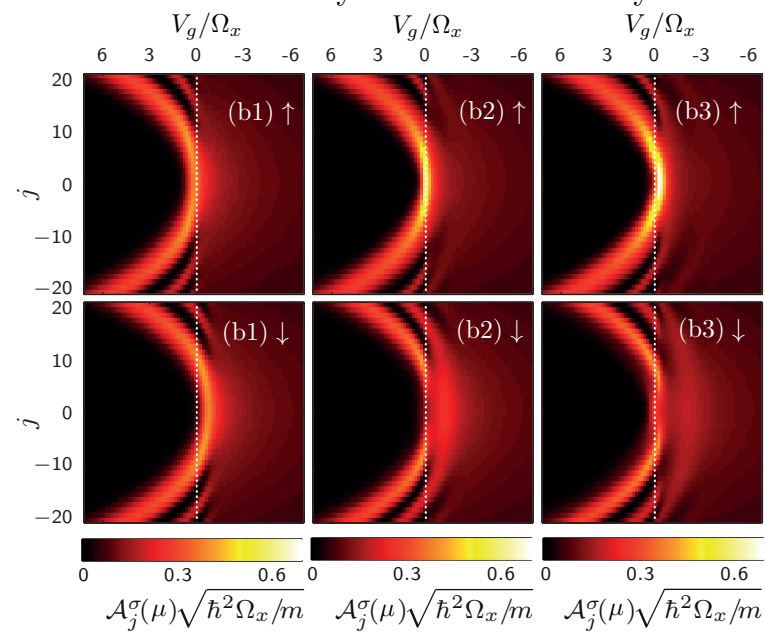

FIG. 1. Spatially resolved plots of the noninteracting LDOS $\mathcal{A}_{j}^{\sigma}$ at fixed $\omega=\mu$, plotted as a function of gate voltage $V_{\mathrm{g}}$ and site index $j$, for $B=0.88 \Omega_{x}$ and for spin $\sigma=\uparrow$ (top row) and $\sigma=\downarrow$ (bottom row). Left column: $R=0.84, \varphi=0$. Middle column: $R=0.84, \varphi=\pi / 2$. Right column: $R=1.26$, $\varphi=\pi / 2$. All results shown are for $N=50$.

case these symmetries are no longer present. In this Supplement we focus on the generalizations necessary to account for such spin-mixing terms.

The second-order fRG flow equations in the oneparticle irreducible version and in the static approximation are given by 


$$
\begin{aligned}
\frac{\mathrm{d}}{\mathrm{d} \Lambda} \gamma_{1}^{\Lambda}\left(q_{1}^{\prime}, q_{1}\right) & =\frac{1}{2 \pi} \sum_{\omega= \pm \Lambda} \sum_{q_{2}^{\prime}, q_{2}} \widetilde{\mathcal{G}}_{q_{2}, q_{2}^{\prime}}^{\Lambda}(i \omega) \gamma_{2}^{\Lambda}\left(q_{2}^{\prime}, q_{1}^{\prime} ; q_{2}, q_{1}\right), \\
\frac{\mathrm{d}}{\mathrm{d} \Lambda} \gamma_{2}^{\Lambda} & =\frac{\mathrm{d}}{\mathrm{d} \Lambda}\left(\gamma_{p}^{\Lambda}+\gamma_{x}^{\Lambda}+\gamma_{d}^{\Lambda}\right), \text { where } \\
\frac{\mathrm{d}}{\mathrm{d} \Lambda} \gamma_{p}^{\Lambda}\left(q_{1}^{\prime}, q_{2}^{\prime} ; q_{1}, q_{2}\right) & =\frac{1}{2 \pi} \sum_{\omega= \pm \Lambda} \sum_{q_{3}^{\prime}, q_{3}, q_{4}^{\prime}, q_{4}} \frac{1}{2} \gamma_{2}^{\Lambda}\left(q_{1}^{\prime}, q_{2}^{\prime} ; q_{3}, q_{4}\right) \widetilde{\mathcal{G}}_{q_{3}, q_{3}^{\prime}}^{\Lambda}(i \omega) \widetilde{\mathcal{G}}_{q_{4}, q_{4}^{\prime}}^{\Lambda}(-i \omega) \gamma_{2}^{\Lambda}\left(q_{3}^{\prime}, q_{4}^{\prime} ; q_{1}, q_{2}\right), \\
\frac{\mathrm{d}}{\mathrm{d} \Lambda} \gamma_{x}^{\Lambda}\left(q_{1}^{\prime}, q_{2}^{\prime} ; q_{1}, q_{2}\right) & =\frac{1}{2 \pi} \sum_{\omega= \pm \Lambda} \sum_{q_{3}^{\prime}, q_{3}, q_{4}^{\prime}, q_{4}} \gamma_{2}^{\Lambda}\left(q_{1}^{\prime}, q_{4}^{\prime} ; q_{3}, q_{2}\right) \widetilde{\mathcal{G}}_{q_{3}, q_{3}^{\prime}}^{\Lambda}(i \omega) \widetilde{\mathcal{G}}_{q_{4}, q_{4}^{\prime}}^{\Lambda}(i \omega) \gamma_{2}^{\Lambda}\left(q_{3}^{\prime}, q_{2}^{\prime} ; q_{1}, q_{4}\right), \\
\frac{\mathrm{d}}{\mathrm{d} \Lambda} \gamma_{d}^{\Lambda}\left(q_{1}^{\prime}, q_{2}^{\prime} ; q_{1}, q_{2}\right) & =-\frac{1}{2 \pi} \sum_{\omega= \pm \Lambda} \sum_{q_{3}^{\prime}, q_{3}, q_{4}^{\prime}, q_{4}} \gamma_{2}^{\Lambda}\left(q_{1}^{\prime}, q_{3}^{\prime} ; q_{1}, q_{4}\right) \widetilde{\mathcal{G}}_{q_{4}, q_{4}^{\prime}}^{\Lambda}(i \omega) \widetilde{\mathcal{G}}_{q_{3}, q_{3}^{\prime}}^{\Lambda}(i \omega) \gamma_{2}^{\Lambda}\left(q_{4}^{\prime}, q_{2}^{\prime} ; q_{3}, q_{2}\right),
\end{aligned}
$$

where $-\gamma_{1}^{\Lambda}$ is the self-energy and $\gamma_{2}^{\Lambda}$ is the two-particle irreducible vertex. All higher order vertices $\gamma_{n \geq 3}$ have been set to zero. Here $\widetilde{\mathcal{G}}^{\Lambda}$ is defined as

$$
\widetilde{\mathcal{G}}^{\Lambda}=\left[\mathcal{G}_{0}^{-1}+\gamma_{1}^{\Lambda}\right]^{-1}=\frac{1}{i \omega-H_{0}+\gamma_{1}^{\Lambda}},
$$

where $H_{0}$ is the (known) Hamiltonian of the noninteracting system. The quantum numbers $q_{i}$ encode the spin and spatial degrees of freedom $q \equiv(\sigma, j)$. The flow of $\gamma_{2}^{\Lambda}$ was split into three contributions called the particleparticle channel $(P)$, and the exchange $(X)$ and direct $(D)$ contributions to the particle-hole channel, respectively. This will allow us to simplify the flow equations later.

For a derivation of Eqs. (2-6) see for instance [4, 6, 7].

\section{A. Initial condition}

For the numerical treatment we cannot set the initial value of the flow parameter $\Lambda_{\text {init }}$ to infinity, but it is sufficient that it is much larger than all relevant energy scales. We have the following initial condition at $\Lambda_{\text {init }}$ $[4,6,7]$,

$$
\begin{aligned}
\gamma_{2}^{\Lambda_{\text {init }}}\left(q_{1}, q_{2}, q_{3}, q_{4}\right) & =v_{q_{1}, q_{2}, q_{3}, q_{4}} \\
\gamma_{1}^{\Lambda_{\text {init }}}\left(q_{1}, q_{2}\right) & =-\frac{1}{2} \sum_{q} v_{q_{1}, q, q_{2}, q}
\end{aligned}
$$

where the vertex $v_{q_{1}, q_{2}, q_{3}, q_{4}}$ is site diagonal and at site $j \equiv j_{1}=j_{2}=j_{3}=j_{4}$ is given by

$$
v_{q_{1}, q_{2}, q_{3}, q_{4}}=U_{j} \delta_{\sigma_{1} \bar{\sigma}_{2}}\left(\delta_{\sigma_{1} \sigma_{3}} \delta_{\sigma_{2} \sigma_{4}}-\delta_{\sigma_{1} \sigma_{4}} \delta_{\sigma_{2} \sigma_{3}}\right) .
$$

This means that the spins $q_{1}$ and $q_{2}$, as well as the spins $q_{3}$ and $q_{4}$ must be opposite. This leaves two possibilities: $\sigma_{1}=\sigma_{3}=\bar{\sigma}_{2}=\bar{\sigma}_{4}$ that has positive sign, and $\sigma_{1}=\sigma_{4}=$ $\bar{\sigma}_{2}=\bar{\sigma}_{3}$ that has negative sign. Inserting this into the initial condition for $\gamma_{1}^{\Lambda}$ yields

$$
\gamma_{1}^{\Lambda_{\text {init }}}\left(q^{\prime}, q\right)=-\left(U_{j} / 2\right) \delta_{\sigma^{\prime} \sigma}
$$

\section{B. Approximations}

We use the following approximations, see [7] and references thereof. Firstly, we neglect the frequency dependence of $\gamma_{2}^{\Lambda}$. This is called the static approximation and is known to give good results at $T=0$ [6]. Given the structure of the flow equation for $\gamma_{2}^{\Lambda}$ above, it is natural to divide the flowing vertex into four parts as follows:

$$
\gamma_{2}^{\Lambda}=v+\gamma_{p}^{\Lambda}+\gamma_{x}^{\Lambda}+\gamma_{d}^{\Lambda} .
$$

Here $v$ is shorthand for the bare vertex, and the flows of $\gamma_{p}^{\Lambda}, \gamma_{x}^{\Lambda}$ and $\gamma_{d}^{\Lambda}$ were given above. The initial value for $\gamma_{2}^{\Lambda}$ is simply the bare vertex. If we insert the bare vertex into the flow equations for the components of $\gamma_{2}^{\Lambda}$ we observe that some of its symmetries remain preserved in the derivative on the left hand side. For instance, for $\gamma_{p}^{\Lambda}$ we see that the first two and the second two site indices must be identical and also that the first and the second pair of spin indices must be opposite, respectively. Similarly, for $\gamma_{x}^{\Lambda}$ we see that the first and fourth site index, as well as the the second and third site index must be equal. For $\gamma_{d}^{\Lambda}$ the first and the third, as well as the second and the fourth site indices are equal. There is no restriction on the any of the spin indices for either $\gamma_{x}^{\Lambda}$ or $\gamma_{d}^{\Lambda}$

The next iteration would break the remaining symmetries, since all three channels contribute to the derivative of $\gamma_{2}^{\Lambda}$ and then back-feed into the differential equations for each channel. If instead we choose to only backfeed each channel into its own differential equation, we can preserve the symmetries described above. This immensely simplifies the treatment of the equations. By doing so, we only neglect contributions of order $v^{3}$ and higher, which justifies their neglect as long as $U_{j}$ is not too large. Altogether we obtain the following contributions:

$$
\begin{aligned}
P_{j i}^{\sigma \bar{\sigma}} & :=\gamma_{p}^{\Lambda}(j \sigma, j \bar{\sigma} ; i \sigma, i \bar{\sigma}) \\
\bar{P}_{j i}^{\sigma \bar{\sigma}} & :=\gamma_{p}^{\Lambda}(j \sigma, j \bar{\sigma} ; i \bar{\sigma}, i \sigma) \\
X_{j i}^{\sigma_{1} \sigma_{2} \sigma_{3} \sigma_{4}}: & :=\gamma_{x}^{\Lambda}\left(j \sigma_{1}, i \sigma_{2} ; i \sigma_{3}, j \sigma_{4}\right) \\
D_{j i}^{\sigma_{1} \sigma_{2} \sigma_{3} \sigma_{4}} & :=\gamma_{d}^{\Lambda}\left(j \sigma_{1}, i \sigma_{2} ; j \sigma_{3}, i \sigma_{4}\right)
\end{aligned}
$$


Note that some elements of a channel can also satisfy the symmetries of another channel. So for instance the diagonal element $P_{j j}^{\sigma \bar{\sigma}}$ has the same symmetries as the diagonal elements $X_{j j}^{\sigma \bar{\sigma} \sigma \bar{\sigma}}$ and $D_{j j}^{\sigma \bar{\sigma} \sigma \bar{\sigma}}$. If we back-feed such elements too we preserve the symmetries in each channel, but obtain a more accurate approximation. Therefore, in each of the three flow equations for the channels of $\gamma_{2}^{\Lambda}$ we replace $\gamma_{2}^{\Lambda}$ on the right hand side by the appropriate channels plus the site diagonal contributions of the other channels that obey the same symmetries. The initial conditions for the three channels follow immediately from (12): $\gamma_{p}^{\Lambda_{\text {init }}}=\gamma_{x}^{\Lambda_{\text {init }}}=\gamma_{d}^{\Lambda_{\text {init }}}=0$. Of course, for the differential equation for $\gamma_{1}^{\Lambda}$ we need the full $\gamma_{2}^{\Lambda}$ which is the sum of all three channels and the bare vertex.

\section{Symmetries}

Due to the hermiticity of the Hamiltonian the following relation holds for the Green's function

$$
\mathcal{G}(i \omega)=\mathcal{G}^{\dagger}(-i \omega) \Leftrightarrow \mathcal{G}_{i j}(i \omega)=\mathcal{G}_{j i}^{*}(-i \omega)
$$

We assume that this relation also holds for $\tilde{\mathcal{G}}^{\Lambda}$. If $\gamma_{1}^{\Lambda}$ is hermitian then the assumption is obviously justified. Numerical results indeed confirm that $\gamma_{1}^{\Lambda}$ is hermitian. We also have the following symmetries of $\gamma_{2}$ :

$$
\begin{aligned}
\gamma_{2}\left(q_{1}, q_{2}, q_{3}, q_{4}\right) & =-\gamma_{2}\left(q_{2}, q_{1}, q_{3}, q_{4}\right) \\
& =-\gamma_{2}\left(q_{1}, q_{2}, q_{4}, q_{3}\right) \\
& =\gamma_{2}\left(q_{2}, q_{1}, q_{4}, q_{3}\right)
\end{aligned}
$$

This follows directly from the equation defining the twoparticle vertex, see e.g. [4]. Again we assume that these relations hold also for $\gamma_{2}^{\Lambda}$ and moreover for each of the separate channels. Their consistency with the numerical results will be demonstrated below. Altogether this yields the following symmetry relations for the different channels:

$$
\begin{aligned}
& P_{j i}^{\sigma \bar{\sigma}}=P_{j i}^{\bar{\sigma} \sigma}=-\bar{P}_{j i}^{\sigma \bar{\sigma}} \\
& D_{j i}^{\sigma_{1} \sigma_{2} \sigma_{3} \sigma_{4}}=D_{i j}^{\sigma_{2} \sigma_{1} \sigma_{4} \sigma_{3}}=-X_{j i}^{\sigma_{1} \sigma_{2} \sigma_{4} \sigma_{3}} \\
& X_{j i}^{\sigma_{1} \sigma_{2} \sigma_{3} \sigma_{4}}=X_{i j}^{\sigma_{2} \sigma_{1} \sigma_{4} \sigma_{3}}=-D_{j i}^{\sigma_{1} \sigma_{2} \sigma_{4} \sigma_{3}}
\end{aligned}
$$

We observe that $P^{\uparrow \downarrow}=P^{\downarrow \uparrow}$ and hence the spin indices for $P$ will be dropped from now on, leaving only the site index. The alternative configuration $\bar{P}$ follows completely from $P$ and does not need to be kept track of separately. Same applies to $X$ and $D$ which completely define each other. We choose to work with $D$. There are various symmetries of $D$ but there is no restriction on the spin index. This means that there are $2^{4}=16$ different submatrices corresponding to 16 different spin configurations of $D$. We choose to arrange them as follows

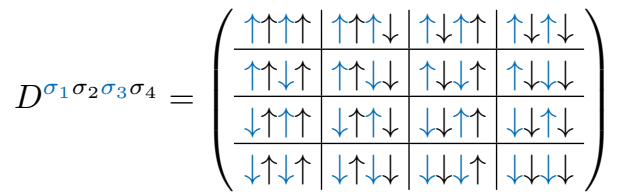

Note that the first and third spin index are fixed along a row and correspondingly the second and fourth index are fixed along one column. This form of the matrix will prove convenient later. From the symmetries of $D$ it follows that this matrix is symmetric. Numerically we also confirm the following relations between the different blocks, schematically

\begin{tabular}{|c|c|c|c|}
\hline$A_{s}$ & $B$ & $B^{*}$ & $C$ \\
\hline$B^{T}$ & $D_{s}$ & $E_{h}$ & $F$ \\
\hline$\left(B^{*}\right)^{T}$ & $E_{h}^{T}$ & $D_{s}^{*}$ & $F^{*}$ \\
\hline$C^{T}$ & $F^{T}$ & $\left(F^{*}\right)^{T}$ & $G_{s}$ \\
\hline
\end{tabular}

where identical symbols denote equal blocks and symmetric (hermitian) submatrices are labeled by the subscript $s(h)$. There are only seven different blocks in total. Numerically we also show that the corner submatrices $A_{s}$, $G_{s}, C$ and $C^{T}$ are real. The other submatrices are complex in general. For a hermitian $\gamma_{1}^{\Lambda}$, the first flow equation implies that $\gamma_{2}^{\Lambda}\left(q_{2}^{\prime}, q_{1}^{\prime} ; q_{2}, q_{1}\right)=\gamma_{2}^{\Lambda *}\left(q_{2}, q_{1} ; q_{2}^{\prime}, q_{1}^{\prime}\right)$. Translated to the separate channels this confirms that $P$ must indeed be hermitian, since $P_{i j}=P_{j i}^{*}$, as well as all the remaining relations between the different submatrices of $D$. 


\section{Flow equation for the P-channel}

Restricting $\gamma_{p}^{\Lambda}$ according to the symmetries of the $P$-channel we obtain the following simplified equation for the derivative of $P$ :

$$
\begin{array}{r}
\frac{\mathrm{d}}{\mathrm{d} \Lambda} P_{j i}=\frac{\mathrm{d}}{\mathrm{d} \Lambda} \gamma_{p}^{\Lambda}(j \sigma, j \bar{\sigma} ; i \sigma, i \bar{\sigma}) \\
=\frac{1}{2 \pi} \sum_{\omega= \pm \Lambda} \sum_{k, l} \frac{1}{2} \cdot\left[\gamma_{2}^{\Lambda}(j \sigma, j \bar{\sigma} ; k \sigma, k \bar{\sigma}) \widetilde{\mathcal{G}}_{k l}^{\Lambda \sigma \sigma}(i \omega) \widetilde{\mathcal{G}}_{k l}^{\Lambda \bar{\sigma} \bar{\sigma}}(-i \omega) \gamma_{2}^{\Lambda}(l \sigma, l \bar{\sigma} ; i \sigma, i \bar{\sigma})\right. \\
\quad+\gamma_{2}^{\Lambda}(j \sigma, j \bar{\sigma} ; k \bar{\sigma}, k \sigma) \widetilde{\mathcal{G}}_{k l}^{\Lambda \bar{\sigma} \bar{\sigma}}(i \omega) \widetilde{\mathcal{G}}_{k l}^{\Lambda \sigma \sigma}(-i \omega) \gamma_{2}^{\Lambda}(l \bar{\sigma}, l \sigma ; i \sigma, i \bar{\sigma}) \\
+\gamma_{2}^{\Lambda}(j \sigma, j \bar{\sigma} ; k \sigma, k \bar{\sigma}) \widetilde{\mathcal{G}}_{k l}^{\Lambda \sigma \bar{\sigma}}(i \omega) \widetilde{\mathcal{G}}_{k l}^{\Lambda \bar{\sigma} \sigma}(-i \omega) \gamma_{2}^{\Lambda}(l \bar{\sigma}, l \sigma ; i \sigma, i \bar{\sigma}) \\
\left.+\gamma_{2}^{\Lambda}(j \sigma, j \bar{\sigma} ; k \bar{\sigma}, k \sigma) \widetilde{\mathcal{G}}_{k l}^{\Lambda \bar{\sigma} \sigma}(i \omega) \widetilde{\mathcal{G}}_{k l}^{\Lambda \sigma \bar{\sigma}}(-i \omega) \gamma_{2}^{\Lambda}(l \sigma, l \bar{\sigma} ; i \sigma, i \bar{\sigma})\right]
\end{array}
$$

Note that the first two terms and the last two terms in the sum are equivalent after summation over $\omega$, due to the symmetry relations (19) and (20). We can thus keep one of the terms respectively and cancel the factor of $1 / 2$. With the definitions

$$
\begin{aligned}
& \Pi_{k l}^{p \Lambda(1)}=\frac{1}{2 \pi} \sum_{\omega= \pm \Lambda} \widetilde{\mathcal{G}}_{k l}^{\Lambda \bar{\sigma} \bar{\sigma}}(i \omega) \widetilde{\mathcal{G}}_{k l}^{\Lambda \sigma \sigma}(-i \omega) \\
& \Pi_{k l}^{p \Lambda(2)}=\frac{1}{2 \pi} \sum_{\omega= \pm \Lambda} \widetilde{\mathcal{G}}_{k l}^{\Lambda \bar{\sigma} \sigma}(i \omega) \widetilde{\mathcal{G}}_{k l}^{\Lambda \sigma \bar{\sigma}}(-i \omega)
\end{aligned}
$$

the flow equation can be written more succinctly as

$$
\begin{aligned}
\frac{\mathrm{d}}{\mathrm{d} \Lambda} P_{j i} & =\sum_{k l}\left[\gamma_{2}^{\Lambda}(j \sigma, j \bar{\sigma} ; k \sigma, k \bar{\sigma}) \Pi_{k l}^{p \Lambda(1)} \gamma_{2}^{\Lambda}(l \sigma, l \bar{\sigma} ; i \sigma, i \bar{\sigma})+\gamma_{2}^{\Lambda}(j \sigma, j \bar{\sigma} ; k \sigma, k \bar{\sigma}) \Pi_{k l}^{p \Lambda(2)} \gamma_{2}^{\Lambda}(l \bar{\sigma}, l \sigma ; i \sigma, i \bar{\sigma})\right] \\
& =\sum_{k l} \gamma_{2}^{\Lambda}(j \sigma, j \bar{\sigma} ; k \sigma, k \bar{\sigma})\left[\Pi_{k l}^{p \Lambda(1)}-\Pi_{k l}^{p \Lambda(2)}\right] \gamma_{2}^{\Lambda}(l \sigma, l \bar{\sigma} ; i \sigma, i \bar{\sigma}),
\end{aligned}
$$

where in the last step we used symmetry relation (19). If we now define

$$
\Pi_{k l}^{p \Lambda} \equiv \Pi_{k l}^{p \Lambda(1)}-\Pi_{k l}^{p \Lambda(2)}=\frac{1}{2 \pi} \sum_{\omega= \pm \Lambda}\left[\widetilde{\mathcal{G}}_{k l}^{\Lambda \bar{\sigma} \bar{\sigma}}(i \omega) \widetilde{\mathcal{G}}_{k l}^{\Lambda \sigma \sigma}(-i \omega)-\widetilde{\mathcal{G}}_{k l}^{\Lambda \bar{\sigma} \sigma}(i \omega) \widetilde{\mathcal{G}}_{k l}^{\Lambda \sigma \bar{\sigma}}(-i \omega)\right]
$$

we arrive at

$$
\frac{\mathrm{d}}{\mathrm{d} \Lambda} P_{j i}=\tilde{P}_{j k} \Pi_{k l}^{p \Lambda} \tilde{P}_{l i}
$$

where $\tilde{P}$ equals $P$ plus the diagonal contributions from the other channels which have the same symmetries as $P$. Explicitly we get

$$
\tilde{P}_{j k}=P_{j k}+\delta_{j k}\left(X_{j j}^{\sigma \bar{\sigma} \sigma \bar{\sigma}}+D_{j j}^{\sigma \bar{\sigma} \sigma \bar{\sigma}}+U_{j}\right)=P_{j k}+\delta_{j k}\left(-D_{j j}^{\sigma \bar{\sigma} \bar{\sigma} \sigma}+D_{j j}^{\sigma \bar{\sigma} \sigma \bar{\sigma}}+U_{j}\right)
$$

Note also that the matrix $\Pi_{k l}^{p \Lambda}$ is hermitian, due to the symmetry (17) of the Green's function.

\section{E. Flow equation for the D-channel}

Restricting $\gamma_{d}^{\Lambda}$ according to the symmetries of the $D$-channel we obtain the following simplified equation for the derivative of $D$ :

$$
\begin{aligned}
\frac{\mathrm{d}}{\mathrm{d} \Lambda} D_{j i}^{\sigma_{1} \sigma_{2} \sigma_{3} \sigma_{4}} & =\frac{\mathrm{d}}{\mathrm{d} \Lambda} \gamma_{d}^{\Lambda}\left(j \sigma_{1}, i \sigma_{2} ; j \sigma_{3}, i \sigma_{4}\right) \\
& =-\frac{1}{2 \pi} \sum_{\omega= \pm \Lambda} \sum_{k l} \sum_{\sigma, \sigma^{\prime}, \sigma^{\prime \prime}, \sigma^{\prime \prime \prime}} \gamma_{2}^{\Lambda}\left(j \sigma_{1}, k \sigma ; j \sigma_{3}, k \sigma^{\prime}\right) \widetilde{\mathcal{G}}_{k l}^{\Lambda \sigma^{\prime} \sigma^{\prime \prime}}(i \omega) \widetilde{\mathcal{G}}_{l k}^{\Lambda \sigma^{\prime \prime \prime} \sigma}(i \omega) \gamma_{2}^{\Lambda}\left(l \sigma^{\prime \prime}, i \sigma_{2} ; l \sigma^{\prime \prime \prime}, i \sigma_{4}\right)
\end{aligned}
$$


Observe that the summation goes over the second and fourth index of the first $\gamma_{2}^{\Lambda}$ matrix and over the first and third index of the second $\gamma_{2}^{\Lambda}$ matrix, while the other indices remain fixed. If we want to recast this expression as a matrix multiplication this indeed implies that the first and third spin index should be fixed along a row and the second and fourth index along one column. This justifies the matrix scheme (24). If we arrange the spin configurations according to this scheme we obtain the matrix equation

$$
\frac{\mathrm{d}}{\mathrm{d} \Lambda} D_{j i}^{\sigma_{1} \sigma_{2} \sigma_{3} \sigma_{4}}=\sum_{k l} \sum_{\sigma, \sigma^{\prime}, \sigma^{\prime \prime}, \sigma^{\prime \prime \prime}} \gamma_{2}^{\Lambda}\left(j \sigma_{1}, k \sigma ; j \sigma_{3}, k \sigma^{\prime}\right) \Pi_{k l}^{d \Lambda \sigma \sigma^{\prime \prime} \sigma^{\prime} \sigma^{\prime \prime \prime}} \gamma_{2}^{\Lambda}\left(l \sigma^{\prime \prime}, i \sigma_{2} ; l \sigma^{\prime \prime \prime}, i \sigma_{4}\right)
$$

where

$$
\Pi_{k l}^{d \Lambda \sigma \sigma^{\prime \prime} \sigma^{\prime} \sigma^{\prime \prime \prime}} \equiv-\frac{1}{2 \pi} \sum_{\omega= \pm \Lambda} \widetilde{\mathcal{G}}_{k l}^{\Lambda \sigma^{\prime} \sigma^{\prime \prime}}(i \omega) \widetilde{\mathcal{G}}_{l k}^{\Lambda \sigma^{\prime \prime \prime} \sigma}(i \omega)
$$

Note that the order of the spin indices on $\Pi$ is not the same as on the Green's functions. The symmetries from (24) remain valid. With our approximation we get

$$
\frac{\mathrm{d}}{\mathrm{d} \Lambda} D_{j i}^{\sigma_{1} \sigma_{2} \sigma_{3} \sigma_{4}}=\tilde{D}_{j k}^{\sigma_{1} \sigma \sigma_{3} \sigma^{\prime}} \Pi_{k l}^{d \sigma \sigma^{\prime \prime} \sigma^{\prime} \sigma^{\prime \prime \prime}} \tilde{D}_{l i}^{\sigma^{\prime \prime} \sigma_{2} \sigma^{\prime \prime \prime} \sigma_{4}}
$$

where $\tilde{D}$ equals $D$ plus the diagonal contributions from the other channels which have the same symmetries as $D$. Explicitly we get

$$
\begin{aligned}
\tilde{D}_{j k}^{\sigma_{1} \sigma_{2} \sigma_{3} \sigma_{4}} & =D_{j k}^{\sigma_{1} \sigma_{2} \sigma_{3} \sigma_{4}}+\delta_{j k}\left(X_{j j}^{\sigma_{1} \sigma_{2} \sigma_{3} \sigma_{4}}+\left(P_{j j}+U_{j}\right) \delta_{\sigma_{1} \bar{\sigma}_{2}}\left(\delta_{\sigma_{1} \sigma_{3}} \delta_{\sigma_{2} \sigma_{4}}-\delta_{\sigma_{1} \sigma_{4}} \delta_{\sigma_{2} \sigma_{3}}\right)\right) \\
& =D_{j k}^{\sigma_{1} \sigma_{2} \sigma_{3} \sigma_{4}}+\delta_{j k}\left(-D_{j j}^{\sigma_{1} \sigma_{2} \sigma_{4} \sigma_{3}}+\left(P_{j j}+U_{j}\right) \delta_{\sigma_{1} \bar{\sigma}_{2}}\left(\delta_{\sigma_{1} \sigma_{3}} \delta_{\sigma_{2} \sigma_{4}}-\delta_{\sigma_{1} \sigma_{4}} \delta_{\sigma_{2} \sigma_{3}}\right)\right)
\end{aligned}
$$

Just like $D$ itself, the matrix $\Pi_{k l}^{d \Lambda \sigma \sigma^{\prime \prime} \sigma^{\prime} \sigma^{\prime \prime \prime}}$ is symmetric, however in general not real. The structure of $\Pi_{k l}^{d \Lambda \sigma \sigma^{\prime \prime} \sigma^{\prime} \sigma^{\prime \prime \prime}}$ in terms of its submatrices is the same as for $D$.

\section{F. Flow equation for $\gamma_{1}$}

For the self-energy equation

$$
\frac{\mathrm{d}}{\mathrm{d} \Lambda} \gamma_{1}^{\Lambda}\left(k^{\prime} \sigma^{\prime}, k \sigma\right)=\frac{1}{2 \pi} \sum_{\omega= \pm \Lambda} \sum_{k_{1}, k_{2}, \sigma_{1}, \sigma_{2}} \widetilde{\mathcal{G}}_{k_{2} k_{1}}^{\Lambda \sigma_{2} \sigma_{1}}(i \omega) \gamma_{2}^{\Lambda}\left(k_{1} \sigma_{1}, k^{\prime} \sigma^{\prime} ; k_{2} \sigma_{2}, k \sigma\right)
$$

we need the full $\gamma_{2}^{\Lambda}=v+\gamma_{p}^{\Lambda}+\gamma_{x}^{\Lambda}+\gamma_{d}^{\Lambda}$. We abbreviate

$$
\mathcal{S}_{k_{2} k_{1}}^{\sigma_{2} \sigma_{1}}=\frac{1}{2 \pi} \sum_{\omega= \pm \Lambda} \widetilde{\mathcal{G}}_{k_{2} k_{1}}^{\Lambda \sigma_{2} \sigma_{1}}(i \omega)
$$

Taking into account the symmetry of each channel we obtain

$$
\begin{aligned}
\frac{\mathrm{d}}{\mathrm{d} \Lambda} \gamma_{1}^{\Lambda}\left(k^{\prime} \sigma^{\prime}, k \sigma\right)= & \delta_{\sigma \sigma^{\prime}} \mathcal{S}_{k k^{\prime}}^{\bar{\sigma} \bar{\sigma}}\left(P_{k^{\prime} k}+\delta_{k k^{\prime}} U_{k}\right)-\delta_{\sigma \bar{\sigma}^{\prime}} \mathcal{S}_{k k^{\prime}}^{\bar{\sigma} \sigma}\left(P_{k^{\prime} k}+\delta_{k k^{\prime}} U_{k}\right) \\
& -\sum_{\sigma_{1} \sigma_{2}} \mathcal{S}_{k^{\prime} k}^{\sigma_{2} \sigma_{1}} D_{k k^{\prime}}^{\sigma_{1} \sigma^{\prime} \sigma \sigma_{2}}+\delta_{k k^{\prime}} \sum_{l, \sigma_{1}, \sigma_{2}} \mathcal{S}_{l l}^{\sigma_{2} \sigma_{1}} D_{l k}^{\sigma_{1} \sigma^{\prime} \sigma_{2} \sigma}
\end{aligned}
$$

The first line accounts for the bare vertex and the $P / \bar{P}$-channel, while the second line contains the contribution from the $X$-channel and then the $D$-channel. Note that the $D$-channel only influences the diagonal elements of $\gamma_{1}^{\Lambda}$, due to its symmetry.

[1] C. Wetterich, Physics Letters B 301, 90 (1993).
[2] J. E. Birkholz and V. Meden, Phys. Rev. B 79, 085420 (2009). 
[3] J. E. Birkholz, Spin-orbit interaction in quantum dots and quantum wires of correlated electrons - A way to spintronics?, Ph.D. thesis, Georg-August-Universität zu Göttingen (2008).

[4] F. Bauer, 0.7 Anomaly of Quantum Point Contacts: Treatment of Interactions with Functional Renormalization Group., Master's thesis, LMU-München (2008).
[5] W. Metzner, M. Salmhofer, C. Honerkamp, V. Meden, and K. Schönhammer, Rev. Mod. Phys. 84, 299 (2012).

[6] F. Bauer, J. Heyder, and J. von Delft, Phys. Rev. B 89, 045128 (2014).

[7] F. Bauer, J. Heyder, E. Schubert, D. Borowsky, D. Taubert, B. Bruognolo, D. Schuh, W. Wegscheider, J. von Delft, and S. Ludwig, Nature 501, 73 (2013). 


\section{Chapter 7}

\section{Conclusion \& Outlook}

In this thesis a detailed microscopic explanation for the origin of the 0.7 anomaly in QPCs was given. We showed that the 0.7-anomaly can be explained by a one-dimensional model with interaction and a potential barrier representing the QPC. This model was derived from first principles and we explained and justified every assumption in detail. We argued that interactions are crucial in regions of large local density of states (LDOS). The LDOS is inversely proportional to the velocity of the electrons, and electrons move particularly slowly in a QPC in the parameter-regime where its conductance is around $0.7 G_{Q}$. This mechanism explains the anomalous behaviour.

We offered detailed theoretical calculations showing excellent agreement with experiments. This gives strong corroboration that the derived model adequately describes QPCs in the regime of the 0.7 anomaly. These calculations where made using two perturbative approaches, the functional renormalization group ( $\mathrm{fRG}$ ) and second order perturbation theory (SOPT). The fRG was formulated in the language of functional path integrals using Grassmann variables. We showed that the fRG can be used to derive formulas for linear response quantities, such as the spin-susceptibility and the linear conductance.

We made detailed predictions for the 0.7 anomaly in the presence of spin orbit interactions (SOI). Thereby the relevant dimensionless strength of SOI was specified, and a prediction was given for how conductance curves change when the relative direction between the external magnetic field and the effective spin-orbit field is changed.

In this work we investigated QPCs represented by a parabolic potential barrier. In order to understand the geometric differences between the various QPCs, other potential forms have to be studied in detail. This can be done with the same theoretical and numerical tools, used for this work. The main challenge here is to define the different potential form, and systematically analyse their differences.

At the current state of our research, the main drawback is the quality of the results using SOPT, which is not good enough to reliably predict shape of a conductance curve. Although SOPT can give a qualitative insight into the microscopic mechanism, it would be desirable to have a method whose results at finite temperature are of a similar quality as the results of fRG at zero temperature. One candidate to achieve this goal would be the fRG approach in Keldysh formalism following Jakobs et al. (2010). Another possibility is to do a diagrammatic 
calculation in the spirit of Janiš (1999) and Janiš and Augustinský (2008). Both approaches have been applied to a single-impurity Anderson model. Extending them to the chain model of a QPC is challenging due to numerical issues: One has to perform a double integral over matrix-valued functions, which can have poles, or at least sharp structures.

Another interesting aspect is to extend the model to a long ranged interaction. A generalization of the coupled ladder approximation in fRG is possible. However, depending on the system size hardware limitations set upper bounds on the range of interaction. In the static approximation this issue is less severe, since no frequency representation of the vertex functions is needed. 


\section{Bibliography}

Aryanpour, K. and J. E. Han, 2009: Ferromagnetic spin coupling as the origin of 0.7 anomaly in quantum point contacts. Phys. Rev. Lett., 102, 056805.

Bauer, F., 2008: 0.7 anomaly of quantum point contacts: Treatment of interactions with functional renormalization group. Master's thesis, Ludwig Maximilians Universiät München.

Bauer, F., J. Heyder, E. Schubert, D. Borowsky, D. Taubert, B. Bruognolo, D. Schuh, W. Wegscheider, J. von Delft, and S. Ludwig, 2013: Microscopic origin of the '0.7-anomaly' in quantum point contacts. Nature, 501(7465), 73-78.

Bauer, F., J. Heyder, and J. von Delft, 2014: Functional renormalization group approach for inhomogeneous interacting fermi systems. Phys. Rev. B, 89, 045128.

Berggren, K. F. and I. I. Yakimenko, 2002: Effects of exchange and electron correlation on conductance and nanomagnetism in ballistic semiconductor quantum point contacts. Phys. Rev. B, 66, 085323.

Blanter, Y. M. and M. Büttiker, 2000: Shot noise in mesoscopic conductors. Physics Reports, 336(1-2), 1-166.

Borowsky, D., 2011: Transportmessungen zur untersuchung der 0.7-struktur in quantenpunktkontakten. Master's thesis, Ludwig Maximilians Universiät München.

Büttiker, M., 1990: Quantized transmission of a saddle-point constriction. Phys. Rev. B, 41, 7906-7909.

Cronenwett, S. M., H. J. Lynch, D. Goldhaber-Gordon, L. P. Kouwenhoven, C. M. Marcus, K. Hirose, N. S. Wingreen, and V. Umansky, 2002: Low-temperature fate of the 0.7 structure in a point contact: A kondo-like correlated state in an open system. Phys. Rev. Lett., 88(22), 226805.

DiCarlo, L., Y. Zhang, D. T. McClure, D. J. Reilly, C. M. Marcus, L. N. Pfeiffer, and K. W. West, 2006: Shot-noise signatures of 0.7 structure and spin in a quantum point contact. Physical Review Letters, 97(3), 036810.

Enss, T., 2005: Renormalization, Conservation Laws and Transport in Correlated Electron Systems. PhD thesis, University of Stuttgart. 
Field, M., C. G. Smith, M. Pepper, D. A. Ritchie, J. E. F. Frost, G. A. C. Jones, and D. G. Hasko, 1993: Measurements of coulomb blockade with a noninvasive voltage probe. Phys. Rev. Lett., 70, 1311-1314.

Goldhaber-Gordon, D., H. Shtrikman, D. Mahalu, D. Abusch-Magder, U. Meirav, and M. A. Kastner, 1998: Kondo effect in a single-electron transistor. Nature, 391, 156-159.

Goulko, O., F. Bauer, J. Heyder, and J. von Delft, 2014: Effect of spin-orbit interactions on the 0.7 anomaly in quantum point contacts. Phys. Rev. Lett., 113, 266402.

Gubernatis, J. E., M. Jarrell, R. N. Silver, and D. S. Sivia, 1991: Quantum monte carlo simulations and maximum entropy: Dynamics from imaginary-time data. Phys. Rev. B, 44, 6011-6029.

Güçlü, A. D., C. J. Umrigar, H. Jiang, and H. U. Baranger, 2009: Localization in an inhomogeneous quantum wire. Phys. Rev. B, 80, 201302.

Heinzel, T., 2003: Mesoscopic Electronics in Solid State Nanostructures. Wiley-VCH.

Heyder, J., 2014: Conductance formula for interacting fermi systems in keldysh formalism.

Heyder, J., F. Bauer, E. Schubert, D. Borowsky, D. Taubert, D. Schuh, W. Wegscheider, J. von Delft, and S. Ludwig, 2014: On the relation between the 0.7-anomaly and the kondo effect: Geometric crossover between a quantum point contact and a kondo quantum dot. arXiv:1409.3415.

Honerkamp, C., D. Rohe, S. Andergassen, and T. Enss, 2004: Interaction flow method for many-fermion systems. Phys. Rev. B, 70, 235115.

Jakobs, S. G., 2009: Functional renormalization group studies of quantum transport through mesoscopic systems. PhD thesis, RWTH Aachen.

Jakobs, S. G., M. Pletyukhov, and H. Schoeller, 2010: Properties of multi-particle green's and vertex functions within keldysh formalism. Journal of Physics A: Mathematical and Theoretical, 43(10), 103001.

Janiš, V., 1999: Stability of self-consistent solutions for the hubbard model at intermediate and strong coupling. Phys. Rev. B, 60(16), 11345-11360.

Janiš, V. and P. Augustinský, 2008: Kondo behavior in the asymmetric anderson model: Analytic approach. Phys. Rev. B, 77(8), 085106.

Jarrell, M. and J. Gubernatis, 1996: Bayesian inference and the analytic continuation of imaginary-time quantum monte carlo data. Physics Reports, 269(3), 133 - 195.

Ji, Y., Y. Chung, D. Sprinzak, M. Heiblum, D. Mahalu, and H. Shtrikman, 2003: An electronic mach-zehnder interferometer. Nature, 422(6930), 415-418.

Kamenev, A. and A. Levchenko, 2009: Keldysh technique and non-linear $\sigma$-model: basic principles and applications. Advances in Physics, 58(3), 197-319. 
Karrasch, C., 2006: Transport through correlated quantum dots - a functional renormalization group approach. Master's thesis, Georg-August Universität Göttingen.

Karrasch, C., 2010: The Functional Renormalization Group for Zero-Dimensional Quantum Systems in and out of Equilibrium. PhD thesis, RWTH Aachen.

Karrasch, C., R. Hedden, R. Peters, T. Pruschke, K. Schönhammer, and V. Meden, 2008: A finite-frequency functional renormalization group approach to the single impurity anderson model. Journal of Physics: Condensed Matter, 20(34), 345205.

Karrasch, C., V. Meden, and K. Schönhammer, 2010: Finite-temperature linear conductance from the matsubara greenÕs function without analytic continuation to the real axis. Phys. Rev. B, 82, 125114 .

Katanin, A. A., 2004: Fulfillment of ward identities in the functional renormalization group approach. Phys. Rev. B, 70, 115109.

Keldysh, L. V., 1965: Diagram thechnique for nonequilibrium processes. Sov. Phys.-JETP, 20(4), 1018.

Koop, E. J., A. I. Lerescu, J. Liu, B. J. van Wees, D. Reuter, A. D. Wieck, and C. H. van der Wal, 2007: The influence of device geometry on many-body effects in quantum point contacts: Signatures of the 0.7 anomaly, exchange and kondo. Journal of Superconductivity Incorporating Novel Magnetism, 20, 433.

Kouwenhoven, L. and L. Glazman, 2001: Revival of the kondo effect. IOP Publishing.

Landauer, R., 1957: Spatial variation of currents and fields due to localized scatterers in metallic conduction. IBM Journal of Research and Development, 1(3), 223-231.

Lunde, A. M., A. D. Martino, A. Schulz, R. Egger, and K. Flensberg, 2009: Electron-electron interaction effects in quantum point contacts. New Journal of Physics, 11(2), 023031.

Meden, V., 2002: Funktionale renormierungsgruppe. Technical report, Georg-August Universität Göttingen.

Meir, Y., K. Hirose, and N. S. Wingreen, 2002: Kondo model for the "0.7 anomaly" in transport through a quantum point contact. Phys. Rev. Lett., 89(19), 196802.

Meir, Y. and N. S. Wingreen, 1992: Landauer formula for the current through an interacting electron region. Phys. Rev. Lett., 68(16), 2512-2515.

Metzner, W., M. Salmhofer, C. Honerkamp, V. Meden, and K. Schönhammer, 2012: Functional renormalization group approach to correlated fermion systems. Rev. Mod. Phys., 84, 299-352.

Micolich, A. P., 2011: What lurks below the last plateau: experimental studies of the $0.7 \times 2 e^{2} / h$ conductance anomaly in one-dimensional systems. Journal of Physics: Condensed Matter, 23(44), 443201. 
Morris, T. R., 1994: The exact renormalizatio group and approximate solutions. International Journal of Modern Physics A, 09(14), 2411-2449.

Negele, J. W. and H. Orland, 1988: Quantum Many-Particle Systems. Eddison-Wesley.

Oguri, A., 2001: Transmission probability for interacting electrons connected to reservoirs. Journal of the Physical Society of Japan, 70(9), 2666-2681.

Sakurai, J. J., 1993: Modern Quantum Mechanics (Revised Edition). Addison Wesley, 1 edition.

Silver, R. N., D. S. Sivia, and J. E. Gubernatis, 1990: Maximum-entropy method for analytic continuation of quantum monte carlo data. Phys. Rev. B, 41, 2380-2389.

Sloggett, C., A. I. Milstein, and O. P. Sushkov, 2008: Correlated electron current and temperature dependence of the conductance of a quantum point contact. The European Physical Journal $B, 61(4), 427-432$.

Thomas, K. J., J. T. Nicholls, N. J. Appleyard, M. Y. Simmons, M. Pepper, D. R. Mace, W. R. Tribe, and D. A. Ritchie, 1998: Interaction effects in a one-dimensional constriction. Phys. Rev. B, 58, 4846-4852.

Thomas, K. J., J. T. Nicholls, M. Y. Simmons, M. Pepper, D. R. Mace, and D. A. Ritchie, 1996: Possible spin polarization in a one-dimensional electron gas. Phys. Rev. Lett., 77(1), 135-138.

van der Wiel, W. G., S. D. Franceschi, T. Fujisawa, J. M. Elzerman, S. Tarucha, and L. P. Kouwenhoven, 2000: The kondo effect in the unitary limit. Science, 289(5487), 2105-2108.

van Wees, B. J., H. van Houten, C. W. J. Beenakker, J. G. Williamson, L. P. Kouwenhoven, D. van der Marel, and C. T. Foxon, 1988: Quantized conductance of point contacts in a two-dimensional electron gas. Phys. Rev. Lett., 60(9), 848-850.

Vidberg, H. and J. Serene, 1977: Solving the eliashberg equations by means ofn-point pad Ãl' approximants. Journal of Low Temperature Physics, 29(3-4), 179-192.

Ward, J. C., 1950: An identity in quantum electrodynamics. Phys. Rev., 78, 182-182.

Wharam, D. A., T. J. Thornton, R. Newbury, M. Pepper, H. Ahmed, J. E. F. Frost, D. G. Hasko, D. C. Peacock, D. A. Ritchie, and G. A. C. Jones, 1988: One-dimensional transport and the quantisation of the ballistic resistance. Journal of Physics C: Solid State Physics, 21(8), L209.

Winkler, R., 2003: Spin-orbit Coupling Effects in Two-Dimensional Electron and Hole Systems. Springer. 


\section{List of Publications}

Microscopic Origin of the 0.7-anomaly in Quantum Point Contacts

Florian Bauer, Jan Heyder, Enrico Schubert, David Borowsky, Daniela Taubert, Benedikt Bruognolo, Dieter Schuh, Werner Wegscheider, Jan von Delft, Stefan Ludwig

Nature, 2013, 501, 73-78

Functional Renormalization Group Approach for Inhomogeneous Interacting Fermi-Systems Florian Bauer, Jan Heyder, Jan von Delft

Phys. Rev. B, 2014, 89, 045128

Toward combined transport and optical studies of the 0.7 -anomaly in a quantum point contact

Enrico Schubert, Jan Heyder, Florian Bauer, Bernd Waschneck, Wolfgang Stumpf, Werner Wegscheider, Jan von Delft, Stefan Ludwig, and Alexander Högele

Phys. Status Solidi B, 1-7, 2014

Effect of Spin-Orbit Interactions on the 0.7 Anomaly in Quantum Point Contacts Olga Goulko, Florian Bauer, Jan Heyder, Jan von Delft

Phys. Rev. Lett., 2014, 113, 266402

Conductance Formula for Interacting Fermi Systems in Keldysh Formalism Jan Heyder, Florian Bauer, Jan von Delft

to be published

On the relation between the 0.7-anomaly and the Kondo effect: Geometric Crossover between a Quantum Point Contact and a Kondo Quantum Dot

Jan Heyder, Florian Bauer, Enrico Schubert, David Borowsky, Daniela Taubert, Dieter Schuh, Werner Wegscheider, Jan von Delft, and Stefan Ludwig

to be published 


\section{Curriculum Vitae}

Florian Bauer

born on June $3^{\text {rd }} 1982$ in Ulm

\section{Education}

Diploma, with distinction, Ludwig-Maximilians-Universität München, 2008

Minor: Mathematics, Focus: Solid State Physics

Thesis: 0.7 Anomaly of Quantum Point Contacts - Treatment of Interaction with functional Renormalization Group

Study Abroad: Erasmus, Sevilla, Spain, 2006

Abitur (A-Level), Descartes-Gymnasium Neuburg, 2002,

Main subjects: Mathematics, Physics,

Mark 2.3 


\section{Danksagung}

Als erstes möchte ich Jan von Delft danken, der mir ermöglicht hat eine Doktorarbeit mit solch einem interessantem Thema zu machen. Ich konnte stets mit seiner Hilfe rechnen, und seine Beharrlichkeit hat letztendlich dazu geführt, dass wir unsere Ergebnisse bei NATURE veröffentlichen konnten.

Ganz besonders möchte ich auch Jan Heyder danken, ohne ihn hätte ich den enormen Arbeitsaufwand nie bewältigt. Ihm ist es ausserdem zu verdanken, dass mich die teils sehr wiiieederliche Arbeit nicht in den Wahnsinn getrieben hat!

Eine außerordentliche Bereicherung war die überaus gute Zusammenarbeit mit der experimentellen Gruppe von Stefan Ludwig. Dafür danke ich David Borowsky, Enrico Schubert, Daniela Taubert und insbesondere Stefan Ludwig, der einen unermüdlichen Einsatz beim Verfassen unserer gemeinsamen Veröffentlichung zeigte.

Wichtig für den Erfolg der Weiterentwicklung der fRG waren auch die präzisen und sehr hilfreichen Ratschläge von Sabine Andergassen, Volker Meden, Severin Jakobs und Herbert Schöller. Vielen Dank dafür.

Ganz besonder Dank geht auch an die Verwaltung, Stéphane Schoonover, Frau Kaiser, Frau Schmitt und Frau Bieber.

Eine schöne Abwechslung war das betreuen anderer Projekte. Für eine gute Zusammenarbeit möchte ich mich bei Katharina, Olga, Benedikt, Lukas, Dennis, Kevin und Nikolai bedanken. 
